Sicherheit und Gesellschaft.

Freiburger Studien des Centre for Security and Society

Benjamin Scharte

\title{
Resilience Engineering
}

Oder von der Kunst, in der zivilen Sicherheitsforschung mit Komplexität umzugehen 
Sicherheit und Gesellschaft.

Freiburger Studien des Centre for Security and Society

herausgegeben von

Prof. Dr. Hans-Helmuth Gander

Prof. Dr. Walter Perron

Prof. Dr. Ralf Poscher

Prof. Dr. Gisela Riescher

Prof. Dr. Thomas Würtenberger

Band 14 
Benjamin Scharte

\section{Resilience Engineering}

Oder von der Kunst, in der zivilen Sicherheitsforschung mit Komplexität umzugehen

\section{Nomos}


The book processing charge was funded by the Baden-Württemberg Ministry of Science, Research and Arts in the funding programme Open Access Publishing and the University of Freiburg.

Die Deutsche Nationalbibliothek verzeichnet diese Publikation in der Deutschen Nationalbibliografie; detaillierte bibliografische Daten sind im Internet über http://dnb.d-nb.de abrufbar.

Zugl.: Freiburg, Univ., Diss., 2020

1. Auflage 2021

(๖) Benjamin Scharte

Publiziert von

Nomos Verlagsgesellschaft mbH \& Co. KG Waldseestraße 3-5 | 76530 Baden-Baden www.nomos.de

Gesamtherstellung:

Nomos Verlagsgesellschaft $\mathrm{mbH} \& \mathrm{Co}$. KG

Waldseestraße 3-5 | 76530 Baden-Baden

ISBN (Print): 978-3-8487-8030-3

ISBN (ePDF): 978-3-7489-2422-7

DOI: https://doi.org/10.5771/9783748924227

Onlineversion Nomos eLibrary

\section{(c) $\underset{\mathrm{BY}}{\mathrm{P}} \mathrm{O}$}

Dieses Werk ist lizenziert unter einer Creative Commons Namensnennung Weitergabe unter gleichen Bedingungen 4.0 International Lizenz. 
Für Stephan Scharte und Klaus Thoma 


\section{Vorwort}

Die Komplexität und Unsicherheit der Welt machen Resilienz notwendig. Deshalb müssen wir wissen, was unter Resilienz zu verstehen ist. Als Ausgangsbasis für eine sozialwissenschaftliche Doktorarbeit ist das motivierend. Als zutreffende Beschreibung der gegenwärtigen Lage der Welt inmitten der COVID-19-Pandemie - ist es hingegen erschreckend. Als ich Anfang 2017 mit der Arbeit begann, hätte ich mir nicht vorstellen können, wie wichtig und für meinen eigenen Alltag unmittelbar relevant diese werden würde. Auch nach Fertigstellung der Analyse Mitte 2019 schienen meine Ergebnisse teils recht akademisch.

Das Jahr 2020 hat uns jedoch eindrücklich gezeigt, dass wir Krisen wie die COVID-19-Pandemie nur dann bewältigen können, wenn wir Komplexität und Unsicherheit explizit in Rechnung stellen. Wenn wir Überraschungen einplanen und unsere Anstrengungen im Krisenmanagement darauf richten, Flexibilität und Anpassungsfähigkeit aufzubauen. Wenn wir als Experten ehrlich über die Grenzen unseres Wissens kommunizieren und nicht Sicherheit vortäuschen, wo Komplexität zu unintendierten und kontraintuitiven Effekten führt. Wenn wir gleichzeitig in fairer und transparenter Weise gesellschaftlich aushandeln, welche Freiheiten wir zugunsten der Krisenbewältigung temporär einzuschränken bereit sind. Mit anderen Worten: wenn wir Resilienz zeigen.

Eine gewisse persönliche Resilienz benötigt man auch, um eine Doktorarbeit zu schreiben. Deshalb möchte ich mich an dieser Stelle ganz herzlich bei all denjenigen bedanken, die das möglich gemacht haben. Ohne sie wäre ich weder fachlich-wissenschaftlich noch persönlich dazu in der Lage gewesen, neben der Arbeit im Team des Strategischen Managements am Fraunhofer EMI ein Promotionsvorhaben voranzutreiben und letztlich erfolgreich abzuschließen. Und das in einer Zeit, die für mich im privaten wie beruflichen Umfeld von Unsicherheit und großen Veränderungen geprägt war.

Zuvorderst gilt mein Dank meiner Doktormutter und Erstgutachterin, Frau Prof. Dr. Gisela Riescher, für ihre stetige und motivierende Begleitung des Prozesses von den ersten Ideen bis zur abgeschlossenen Promotion. Ihre Betreuung wies die ideale Mischung aus fachlicher Anleitung und notwendigem Freiraum auf. In gleicher Weise danken möchte ich meinem Zweitgutachter Prof. Dr.-Ing. habil. Stefan Hiermaier, der mich 
in meiner Zeit am Fraunhofer EMI jederzeit darin bestärkt hat, den Weg in Richtung Promotion zu gehen. Von unserer gemeinsamen Arbeit an Resilienz habe ich enorm profitiert. Selbiges gilt für meine ehemaligen Kolleginnen und Kollegen am Fraunhofer EMI. Ohne sie wäre ich nie zum Resilienzforscher geworden.

Selbstverständlich möchte ich auch meiner Familie und meinen Freunden danken. Es sind unsere sozialen Netzwerke, die uns resilient machen. Die es uns ermöglichen, auch schwere Zeiten gemeinsam zu überstehen und daran zu wachsen. Danke, dass ich mich immer auf euch verlassen kann. Und Silja, einfach nur danke.

Zürich, November 2020 


\section{Inhaltsverzeichnis}

$\begin{array}{ll}\text { Abbildungsverzeichnis } & 11\end{array}$

Tabellenverzeichnis 13

1. Einleitung 15

1.1. Resilienz als interdisziplinäres boundary object 15

1.2. Einordnung in die zivile Sicherheitsforschung 18

1.3. Kritische Infrastrukturen 21

1.4. Notwendigkeit für Resilienz und Resilience Engineering 27

1.5. Aufbau der Arbeit 28

2. Stand der Forschung - Identifikation relevanter konzeptioneller Zugänge 31

2.1. Resilienz als wissenschaftliches Konzept 31

2.2. Psychologie 48

2.3. Ökologie und sozial-ökologische Forschung 58

2.4. Sozialwissenschaften 72

2.5. Organisationswissenschaften - „Resilience Engineering“ 98

2.6. Ingenieurwissenschaften - „engineering resilience” 111

3. Forschungslücken und wissenschaftliche Fragestellung 135

4. Resilienz - Normative sowie system- und komplexitätstheoretische Analyse 139

4.1. Diskussion von Resilienz anhand konzeptioneller Zugänge 139

4.2. Normative Analyse von Resilienz 143

4.2.1. Resilienz und Neoliberalismus 143

4.2.2. Resilienz und Freiheit 161

4.2.3. Resilienz und Terrorismus 181

4.2.4. Resilienz und Vertrauen 190

4.3. System- und komplexitätstheoretische Analyse von Resilienz 202

4.3.1. Resilienz und Systeme 202 
4.3.2. Resilienz und Komplexität

4.3.3. Resilienz und Unsicherheit 262

4.3.4. Resilienz und Vulnerabilität

4.3.5. Resilienz und Anpassungsfähigkeit

5. Eigenständiges Resilienz-Konzept der zivilen Sicherheitsforschung

5.1. Zusammenführung der konzeptionellen Zugänge

5.2. Definition relevanter Begrifflichkeiten für das ResilienzKonzept

5.3. Bestandteile des Resilienz-Konzepts der zivilen Sicherheitsforschung

5.4. Diskussion der Grenzen des Konzepts

6. Resilience Engineering - Hypothesen für die zivile Sicherheitsforschung

6.1. Diskussion von Resilience Engineering anhand von Leitfragen

6.2. Art des Konzepts

6.3. Notwendigkeit für Resilience Engineering

6.4. Notwendige Bedingungen für Resilience Engineering

6.5. Resilience Engineering aus system- und komplexitätstheoretischer Sicht

6.6. Normative Aspekte für Resilience Engineering

6.7. Zusammenfassung der Hypothesen für Resilience Engineering

6.8. Grenzen für Resilience Engineering aus sozialwissenschaftlicher Sicht

7. Fazit und Ausblick 448

7.1. Zusammenfassung der Ergebnisse 448

7.2. Offene Fragestellungen und Kritik 461

7.3. Interdisziplinär-forschungsleitender Ausblick 464 


\section{Abbildungsverzeichnis}

Abbildung 1: Das resilience triangle

Abbildung 2: Resilienz in systemtheoretischer Sichtweise

Abbildung 3: Der adaptive cycle 308

Abbildung 4: Der Resilienz-Zyklus 381

Abbildung 5: Abrupt vs. graceful degradation 400

Abbildung 6: Hypothesen für Resilience Engineering und konzeptionelle Zugänge zu Resilienz 


\section{Tabellenverzeichnis}

Tabelle 1: Relevante Begrifflichkeiten für das Resilienz-Konzept der zivilen SiFo

Tabelle 2: Hypothesen für Resilience Engineering

Tabelle 3: Art des Konzepts

Tabelle 4: Notwendigkeit für Resilienz und Resilience

Engineering

Tabelle 5: Notwendige Bedingungen für Resilienz und Resilience Engineering

Tabelle 6: Resilienz und Resilience Engineering aus system- und komplexitätstheoretischer Sicht

Tabelle 7: Normative Aspekte von Resilienz und Resilience

Engineering 


\section{Einleitung}

\subsection{Resilienz als interdisziplinäres boundary object}

„Manchmal kommt es besser, als man denkt“

(Zander/Roemer 2016: 47).

In einer Welt, in der täglich von Krisen, Problemen und Gefahren die Rede ist, ist ein solcher Satz ermutigend. Und als Ermutigung soll er auch durchaus gelesen und verstanden werden. Deshalb wird er der nun folgenden Arbeit vorangestellt. Krisen können gemeistert, Probleme gelöst und Gefahren gebannt werden. Für eine wissenschaftliche Arbeit stellen sich dann unmittelbar drei Arten von Fragen. Erstens, um welche Art von Krisen, Problemen und Gefahren handelt es sich? Zweitens, wie können diese gemeistert, gelöst und gebannt werden? Und drittens, in welchem Kontext erfolgt das alles, welche Nebenbedingungen müssen beachtet werden und welche sonstigen Auswirkungen entstehen? All diese Fragen werden in den letzten Jahren in den unterschiedlichsten wissenschaftlichen Disziplinen zunehmend unter Zuhilfenahme eines immer prominenter werdenden Begriffs diskutiert: „Resilienz“. Was ist Resilienz? Resilienz lässt sich ganz grundsätzlich zweifach fassen. Zunächst auf der einen Seite als „ordinary magic“, als Fähigkeit dafür zu sorgen, dass es besser kommt, als man denkt, als eine Art Metapher (Metzl 2009: 113). Auf der anderen Seite ist Resilienz ein fundamental theoretisches Konzept (Dinh et al. 2012: 234, Prior 2014: 4). Und als solches bildet es den wesentlichen Untersuchungsgegenstand der vorliegenden Arbeit.

Deren Grundlage ist eine jahrelange Beschäftigung mit Resilienz als wissenschaftlichem Konzept, aus der sich eine Reihe von Fragestellungen ergeben haben, die im Folgenden beantwortet werden sollen. Den Beginn markierte das Forschungsprojekt „Resilien-Tech - ,Resilience-by-Design': Strategie für die technologischen Zukunftsthemen“, das gemeinsam von der Deutschen Akademie der Technikwissenschaften acatech und dem Fraunhofer-Institut für Kurzzeitdynamik, Ernst-Mach-Institut, EMI, durchgeführt wurde. Ziel des Projekts, das von 2012 bis 2014 lief, war es, ein besseres Verständnis des Konzepts Resilienz für den Bereich der sogenannten „zivilen Sicherheitsforschung“ ( $\mathrm{SiFo}$ ) zu erarbeiten, und daraus konkrete Handlungsempfehlungen für Entscheidungsträger in Politik, Wirtschaft und Gesellschaft zu generieren. Die Grundannahme dahinter war, dass 
Resilienz zwar in der zivilen SiFo immer häufiger als Konzept verwendet wird, aber kein nationaler Konsens über eine Definition besteht. Durch Literaturrecherchen, drei Workshops mit nationalen und internationalen Experten sowie einer Zusammenarbeit mit weiteren Partnern wie dem Forschungsforum Öffentliche Sicherheit und der Wirtschaftsprüfungsgesellschaft KPMG konnte eine Studie erstellt werden, deren Ergebnisse in Form von zehn Handlungsempfehlungen an entsprechende Entscheidungsträger herangetragen wurden (siehe dazu Thoma 2014). Im Anschluss an Resilien-Tech konnte der Autor der vorliegenden Arbeit gemeinsam mit Kollegen am Fraunhofer EMI, insbesondere dem damaligen Institutsleiter und Gründungsvater der deutschen wie europäischen zivilen SiFo, Prof. Dr. Klaus Thoma, die theoretisch-konzeptionelle Beschäftigung mit Resilienz fortführen und in einigen weiteren Publikationen vertiefen (siehe Häring et al. 2016, 2016b, Hiermaier et al. 2019, Hiermaier/Scharte 2018, Linkov et al. 2014, Scharte/Thoma 2016, Thoma et al. 2016, Tho$\mathrm{ma} /$ Scharte 2015). Mit einem Verständnis von Wissenschaft als Suche nach dem "Geheimnis der Transformation von Chaos in Ordnung“, wurden dabei zwei Aspekte in Bezug auf Resilienz zunehmend klarer (Antonovsky 1997: 149). Zum einen lässt sich nicht sinnvoll an Resilienz forschen, ohne die Grundherausforderung der Komplexität explizit in Rechnung zu stellen. Der britische Resilienzforscher Charlie Edwards formuliert das treffend am Beispiel einer Tasse Kaffee: „My simple cup of coffee rests on an awe-inspiringly complex system that is global in scale and made up of networks of suppliers, transportation routes, production facilities, distribution centres, warehouses, inventory management processes and cash flows" (Edwards 2009: 27). Die zentrale Frage bei der Beschäftigung mit Resilienz lautet deshalb auch „how to respond to the complexity challenge“ (Helbing 2015)? Noch prägnanter fasst es das englische Begriffspaar „navigating complexity“ zusammen (IRGC 2018: 18). Wie zentral Komplexität im Zusammenhang mit Resilienz ist, zeigt auch bereits der Titel der vorliegenden Arbeit.

Zum anderen wurde aber auch immer klarer, dass Resilienz ein Konzept ist, das sich an den Schnittstellen verschiedener wissenschaftlicher Disziplinen bewegt. Über disziplinäre Grenzen hinweg wird mit dem Konzept Resilienz gearbeitet. Zumal Resilienz auch eher eine „family of related ideas" darstellt, als einen konkret definierten Begriff (Prior/Roth 2013: 61, Westrum 2006: 65). Das Konzept enthält eine Vielzahl an deskriptiven Ideen darüber, was damit konkret gemeint sein könnte, wann etwas oder jemand resilient ist oder nicht, wie Resilienz erhöht oder vermindert werden könnte und auch, wie sie sich von anderen Konzepten abgrenzen lässt. 
Gleichzeitig ist das Konzept so breit, dass es auch Dimensionen normativer Art umfassen kann, also Fragen danach, ob es gut oder schlecht ist, wünschenswert oder nicht wünschenswert ist, wenn jemand oder etwas resilient ist - oder zumindest resilienter als jemand oder etwas anderes (Brand/Jax 2007: 8). Dank seiner Breite gilt Resilienz als ein Konzept oder Ansatz, mit dessen Hilfe Wissenschaft integrativ und interdisziplinär umgesetzt werden kann, insbesondere mit Blick auf fundamental wichtige gesellschaftliche Bereiche, in denen rein disziplinäre Ansätze notwendig aufgrund der Komplexität der zu lösenden Fragestellungen versagen (Folke 2006: 260). Insofern kann Resilienz als interdisziplinäres „boundary object" charakterisiert werden. Als boundary object, in der vorliegenden Arbeit auch „Brückenkonzept“, werden Begriffe bezeichnet, die durch die Schaffung eines begrenzten gemeinsamen Vokabulars dazu in der Lage sind, die Kommunikation zwischen verschiedenen wissenschaftlichen Disziplinen zu ermöglichen, obwohl in der spezifischen Ausgestaltung die Bedeutung des Begriffs weiter stark unterschiedlich sein kann (Bartl et al. 2014: 37, Brand/Jax 2007: 7).

Die für die vorliegende Arbeit wichtigste Schnittstelle ist die zwischen den Sozialwissenschaften einerseits und den Ingenieurwissenschaften andererseits. Das resultiert aus der gerade nachgezeichneten Genese der Beschäftigung mit dem Resilienz-Konzept. Die Projektpartner in ResilienTech, acatech und Fraunhofer EMI, sind primär im ingenieurwissenschaftlichen Bereich zu verorten. Sie haben ein Interesse daran herauszufinden, wie man ingenieurwissenschaftlich dazu beitragen kann, dass es besser kommt, als man denkt. Dieser ingenieurwissenschaftliche Beitrag zu Resilienz wurde, beginnend mit den Ausführungen in der Studie zu ResilienTech und darauf aufbauend in allen weiteren Publikationen, an denen der Autor der vorliegenden Arbeit mitgewirkt hat, als „Resilience Engineering“" bezeichnet (siehe vor allem Scharte et al. 2014c: 125, Scharte/Thoma 2016: 136, Thoma et al. 2016: 14). Gleichzeitig waren die Publikationen selbst in ihrer Ausrichtung sehr konzeptionell-theoretisch geprägt und zwar aus einem stärker sozialwissenschaftlichen Blickwinkel heraus. Interessanterweise stammt zudem der Begriff des Resilience Engineering selbst aus einer sozialwissenschaftlichen Forschungsrichtung, wie an späterer Stelle noch zu zeigen sein wird (siehe 2.5). Die vorliegende Arbeit forscht auf den genannten, vorherigen Publikationen aufbauend, mithilfe sozialwissenschaftlicher Methoden konzeptionell-theoretisch an den beiden Konzepten Resilienz und Resilience Engineering und zwar an der Schnittstelle zwischen den Sozialwissenschaften und den Ingenieurwissenschaften. Dabei ist sie sich der Herausforderung bewusst, die damit verbunden 


\section{Einleitung}

ist. Interdisziplinäre Forschung wird zwar allgemein als wünschenswert angesehen, ist in ihrer konkreten Umsetzung aber wie erwähnt durchaus schwierig. „Zunächst beeindrucken [...] die negativen Konsequenzen der Beteiligung einer Vielzahl von Disziplinen und Fächern. [...] Deshalb muss man schon für die einzelnen Fächer, erst recht aber in der interdisziplinären Zusammenarbeit, Zweifel haben, ob überhaupt bekannt ist, worüber gesprochen wird“ (Luhmann 2003: 14). Integrative Forschung zwischen den Sozialwissenschaften und den Ingenieurwissenschaften gilt sogar als besonders anspruchsvoll (Alderson 2019: 69, Chang 2009: 3). Gerade deshalb wird im Folgenden der Versuch unternommen, die beiden Disziplinen aus einer sozialwissenschaftlichen Perspektive heraus mithilfe des boundary objects Resilienz näher zueinander zu bringen und auf diese Weise einen gegenseitigen Lernprozess zu ermöglichen. Denn es gilt: „Wir können keine Experten in dem Gebiet der jeweils anderen werden. Aber wir können Vorschläge machen, die die anderen verwerten mögen. In diesem Sinne erlaube ich mir, die folgenden Bemerkungen zu machen“ (Antonovsky 1997: 142).

\subsection{Einordnung in die zivile Sicherheitsforschung}

Der interdisziplinäre Charakter von Resilienz als Brückenkonzept ist unter anderem deshalb sinnvoll und notwendig, weil sich die vorliegende Arbeit in einem Forschungsfeld verortet, das durch seine interdisziplinäre Ausrichtung gekennzeichnet ist, nämlich innerhalb der sogenannten zivilen Sicherheitsforschung. Der Begriff Sicherheit wird in der Wissenschaft selbst sehr intensiv diskutiert. Seine Bedeutung ist keinesfalls eindeutig (siehe dazu z.B. Riescher 2010, Winzer et al. 2012). Im Folgenden steht allerdings nicht der Begriff der Sicherheit im Vordergrund. Daher wird er hier im Rahmen der zivilen SiFo als Abwesenheit von Schäden bzw. schnelle Behebung entstandenen Schadens verstanden. Das dient als Ausgangspunkt der gesamten weiteren Diskussion. Das Forschungsgebiet der zivilen SiFo gibt es in Deutschland und auf europäischer Ebene explizit erst seit wenig mehr als einem Jahrzehnt, beginnend mit dem im siebten Forschungsrahmenprogramm der Europäischen Union vorhandenen Teilbereich "Secure Societies" und dem ersten Rahmenprogramm der Deutschen Bundesregierung „Forschung für die zivile Sicherheit“, jeweils aus dem Jahr 2007 (Thoma/Scharte 2015: 32). Es lässt sich kaum als etablierte, eigenständige Disziplin wie etwa Politikwissenschaft, Soziologie oder Psychologie auf der sozialwissenschaftlichen Seite oder Materialwis- 
senschaft, Bauingenieurwesen oder Maschinenbau auf der ingenieurwissenschaftlichen Seite beschreiben. Vielmehr vereint die zivile SiFo als sehr anwendungsnahes Gebiet in sich Lösungsansätze aus den verschiedensten Disziplinen. Gemeinsam ist allen Ansätzen eine Grundannahme, die von der Bundesministerin für Bildung und Forschung im Kabinett Merkel IV, Anja Karliczek, im Vorwort des dritten Rahmenprogramms „Forschung für die zivile Sicherheit“ der Deutschen Bundesregierung - im Folgenden Sicherheitsforschungsprogramm - formuliert wird: "Sicherheit ist nicht alles, aber ohne Sicherheit ist alles nichts“ (BMBF 2018: 2). Gleichzeitig herrscht in der Forschung Einigkeit darüber, dass hundertprozentige Sicherheit, verstanden als Abwesenheit sämtlicher Störungen, Disruptionen und widriger Ereignisse, niemals zu erreichen ist. „Things break; disaster happens; failure is real“ (Robbins et al. 2012). „Irgendetwas kann immer passieren" (Luhmann 2003: 28). So oder so ähnlich wird diese Art Eingeständnis der Unmöglichkeit totaler oder umfassender Sicherheit von vielen Forschern formuliert (siehe z.B. Al-Khudhairy et al. 2012: 581, Brunner/ Giroux 2009: 6, CSS 2009: 1, Flynn/Burke 2011: 11, Pursiainen 2009: 727, Sterbenz et al. 2010: 1252, Tobin 1999: 15, Wachsmuth 2014: 6).

Wenn umfassende Sicherheit niemals garantiert werden kann, leitet sich daraus mindestens implizit das Phänomen der Unsicherheit ab. Schlimme, unerwünschte, mit negativen Auswirkungen verbundene Dinge bzw. Ereignisse passieren. In der zivilen SiFo werden vor allen Dingen drei Arten von disruptiven oder widrigen Ereignissen betrachtet. ${ }^{1}$ Das sind zunächst bewusst von Menschen herbeigeführte Ereignisse, die der Gesellschaft direkt und in intendierter Weise oder aber als nicht explizit intendierte Nebenfolge Schäden zufügen. Das prominenteste Beispiel für die erste Kategorie ist der internationale Terrorismus. Insbesondere die Terroranschläge vom 11. September 2001 gelten nach wie vor als eine Art Initialzündung für eine Vielzahl darauffolgender Entwicklungen in den Bereichen Sicherheitspolitik und Sicherheitsforschung. Die Anschläge auf die beiden Türme des World Trade Centers in New York City, das Pentagon in Washington, D.C., sowie die in Pennsylvania abgestürzte Passagiermaschine forderten beinahe 3000 Menschenleben. Vor allem die Bilder der einstürzenden Türme des World Trade Centers haben sich tief in das kollektive Gedächtnis sowohl der amerikanischen als auch der europäischen Gesellschaft eingegraben. Die für die USA neue Erfahrung der Verwundbarkeit auf eigenem Boden löste ein hohes $\mathrm{Maß}$ an Aktivitäten aus, unter anderem

1 Die beiden Begriffe "disruptiv“ und „widrig“ werden im weiteren Verlauf der Arbeit synonym verwendet. 


\section{Einleitung}

die Gründung des Ministeriums für Heimatschutz (Homeland Security) und die Verabschiedung des sogenannten USA PATRIOT Act, mitsamt der Beschränkung von Bürgerrechten (Kaufmann/Blum 2012: 235f, Thoma et al. 2012b: 321). Der vom damaligen Präsidenten der Vereinigten Staaten, George W. Bush, ausgerufene Krieg gegen den Terrorismus (war on terror) soll hier nicht bewertet werden. Normative Implikationen derartiger Maßnahmen werden an späterer Stelle in Bezug auf das Konzept der Resilienz intensiver diskutiert (siehe 4.2). Die Genese der zivilen SiFo lässt sich aber auch für Deutschland und Europa nicht nachzeichnen, ohne auf dieses Ereignis zu verweisen. Und die Bedrohung durch den internationalen Terrorismus stellt nach wie vor einen der Hauptbeweggründe für die Bundesregierung dar, ein Forschungsprogramm für zivile Sicherheit zu finanzieren. Im aktuellen, dritten Sicherheitsforschungsprogramm wird der internationale Terrorismus erneut als erstes genannt, wenn es um die verschiedenen Arten von Bedrohungen geht, denen gegenüber die Gesellschaft verwundbar ist (BMBF 2018: 3). Ebenfalls als bewusst von Menschen verursacht wird das Phänomen der organisierten Kriminalität gesehen. Auch wenn sich diese weniger durch abrupte, disruptive Ereignisse auszeichnet als vielmehr durch ihr konstantes Auftreten, soll die zivile SiFo dazu beitragen, die durch organisierte Kriminalität verursachten Schäden für die Gesellschaft zu minimieren. Die zweite Art disruptiver Ereignisse stellen Naturkatastrophen dar. Inwiefern der Katastrophenbegriff dazu geeignet ist, diese heterogene Gruppe von Ereignissen zusammenzufassen, wird noch zu diskutieren sein. Er hat sich aber im allgemeinen Sprachgebrauch etabliert und wird auch im Sicherheitsforschungsprogramm verwendet (BMBF 2018: 3). Unter Naturkatastrophen werden unterschiedlichste, natürlich verursachte Extremereignisse verstanden, von Erdbeben und Vulkanausbrüchen, über Hochwasser und Überflutungen, Stürme und Hurrikans bis hin zu Hitze- oder Kältewellen und Dürren. Die Schäden, die durch solche Ereignisse verursacht werden, nehmen weltweit, aber auch in Europa und Deutschland ständig zu. Eine wichtige Ursache dafür ist der Klimawandel, der mindestens mittelbar zu einer Zunahme von Extremwetterereignissen führt. Neben einer erhöhten Häufigkeit führt er zudem $\mathrm{zu}$ intensiveren Extremwetterereignissen (Renn et al. o.J.: 1f). Daneben gibt es noch eine dritte Art von Ereignissen, nämlich schwere Unfälle, etwa im Bereich der Industrie oder des Transportwesens, die für die zivile SiFo eine Rolle spielen.

Beim Blick auf die unterschiedlichen Arten von Ereignissen, von Terroranschlägen über Naturkatastrophen bis hin zu schweren Industrieunfällen, fällt eine Gemeinsamkeit auf, die die zivile SiFo von anderen Forschungs- 
bereichen trennt, in denen es um Sicherheit geht. Die Ereignisse müssen eine gewisse gesellschaftliche Relevanz aufweisen, um im Rahmen der zivilen SiFo aufzutauchen. So sind beispielsweise Autounfälle für die unmittelbar Betroffenen ohne jeden Zweifel ein disruptives Ereignis. Für die Gesellschaft sind sie jedoch als Einzelfall weniger von Bedeutung. In der zivilen SiFo geht es dann darum, die aus disruptiven Ereignissen resultierenden Verwundbarkeiten der Gesellschaft und ihrer relevanten Systeme zu erkennen und analysieren. Daraus sollen Vorschläge bzw. Technologien zur Minderung oder Vermeidung der Schäden entwickelt werden, die durch solche Ereignisse verursacht werden. Wichtig ist die gleichzeitige Achtung der Rechte und Freiheiten der Bürger (Scharte et al. 2014: 10, Thoma et al. 2012: 13f). Im aktuellen Sicherheitsforschungsprogramm wird das wie folgt formuliert: „Mit dem Rahmenprogramm ,Forschung für die zivile Sicherheit 2018 - 2023`stellt sich die Bundesregierung der aktuellen und zukünftigen Verantwortung, Sicherheit und Ordnung in einer vernetzten Welt zu fördern. Im neuen Rahmenprogramm werden dazu innovative Lösungen erforscht und praxisnah umgesetzt, die die Sicherheit und Lebensqualität der Bürgerinnen und Bürger erhöhen und den Schutz lebenswichtiger Infrastrukturen gewährleisten“" (BMBF 2018: 3). Gegeben diese Zielstellung ist das Kriterium der gesellschaftlichen Relevanz geeigneter, zivile SiFo abzugrenzen, als der häufig zitierte Gegensatz zwischen „safety“ und „security“. Beide Begriffe lassen sich als „Sicherheit“ ins Deutsche übersetzen. Bei safety geht es eher um Fehler, die innerhalb eines Systems oder Prozesses auftreten, bei security eher um Bedrohungen, die ein System von außen treffen (Scharte/Thoma 2016: 134). Gerade wenn es in der zivilen SiFo um den Schutz wichtiger gesellschaftlicher Systeme, wie beispielsweise bestimmter Infrastrukturen, geht, verschwimmen die Grenzen zwischen safety und security. Nichtsdestotrotz wird der Begriff der Sicherheit in der vorliegenden Arbeit im Wesentlichen im Sinne von security verwendet, da dieses Verständnis - ohne safety-Aspekte auszuschließen - im Fokus der zivilen SiFo steht. Entscheidend bleibt für die zivile SiFo aber das Kriterium der gesellschaftlichen Relevanz der durch disruptive Ereignisse möglicherweise verursachten, negativen Auswirkungen.

\subsection{Kritische Infrastrukturen}

In der zivilen SiFo geht es auch und gerade um den Schutz gesellschaftlich relevanter Systeme. Den Ingenieurwissenschaften wird dabei immer wieder die Aufgabe zugeschrieben, zur Sicherheit und zum Schutz soge- 


\section{Einleitung}

nannter „kritischer Infrastrukturen“ (critical infrastructures) beizutragen. Deshalb ist es für die vorliegende Arbeit wichtig, diese Begrifflichkeit zu definieren. Das Konzept der kritischen Infrastrukturen ist relativ neu und taucht unter dieser Bezeichnung erstmals Mitte der 1990er Jahre in den USA auf (Pursiainen 2009: 722). Nachdem bereits im Lauf der 1980er Jahre festgestellt wurde, dass ein großer Teil der öffentlichen Infrastrukturen Alterungsprozessen mit ungewissen Auswirkungen ausgesetzt war, führte ein schwerer Terroranschlag zu einer Neubewertung der Bedeutung von Infrastrukturen. Der Bombenanschlag auf das Murrah Federal Building in Oklahoma City am 19. April 1995, durchgeführt von einem einheimischen Terroristen, kostete 168 Menschenleben und war der schwerwiegendste Anschlag, den die USA bis zu diesem Zeitpunkt erleiden mussten. Im Anschluss daran richtete die Administration unter dem damaligen Präsidenten Bill Clinton eine „Kommission zum Schutz kritischer Infrastrukturen“ (Commission on Critical Infrastructure Protection) ein. Deren Ergebnisse wurden in Form eines Reports veröffentlicht. Darauf aufbauend hat sich das Konzept der kritischen Infrastrukturen sehr schnell international verbreitet und wird mittlerweile in den meisten industrialisierten Ländern, von Japan über Australien bis hin zur gesamten EU, genutzt. Das Konzept dient als politisches Programm, zur Rechtfertigung von Gesetzgebungsverfahren und als Grundlage für vielfältige Notfall- und Rettungspläne. Insgesamt ist es zu einem dominanten Narrativ innerhalb der Sicherheitspolitik und Sicherheitsforschung weltweit geworden. Der Schutz kritischer Infrastrukturen ist als „Critical Infrastructure Protection (CIP)“ mittlerweile sogar eine Art feststehender Begriff (Alderson 2019: 66, Al-Khudhairy et al. 2012: 576, Fekete et al. 2016: 221f, Fritzon et al. 2007: 31, Galland 2012: 301ff, Kaufmann/Blum 2012: 244, O'Rourke 2007: 22). Auch die wissenschaftliche Beschäftigung mit kritischen Infrastrukturen ist in den letzten Jahren sprunghaft gewachsen. Während es Mitte der 1990er Jahre fast noch keine Publikationen gab, die sich mit dem Thema beschäftigten, waren es im Jahr 2010 alleine über 100 (Galland 2012: 301). Wissenschaft wie auch Politik haben dabei ihren Blick geweitet und betrachten nun neben der Bedrohung des Terrorismus auch weitere disruptive Ereignisse wie Naturkatastrophen und große Unfälle als relevante Phänomene im Bereich des Schutzes kritischer Infrastrukturen (Fritzon et al. 2007: 32, Galland 2012: 308).

Was steckt hinter dem Konzept der kritischen Infrastrukturen? Wann sind Infrastrukturen „kritisch“ und welche Infrastrukturen werden zu den kritischen gezählt? Entscheidend ist ihre Bedeutung für die Funktionsfähigkeit oder sogar Überlebensfähigkeit der Gesellschaft. Mit anderen 
Worten können moderne Gesellschaften nicht ohne kritische Infrastrukturen existieren. Solche Infrastrukturen hat es schon immer gegeben. Gesellschaften waren schon immer darauf angewiesen, genügend Nahrungsmittel und Wasser zur Verfügung zu haben. Seit der industriellen Revolution und spätestens im digitalen Zeitalter ist auch die Versorgung mit Energie buchstäblich überlebensnotwendig für Gesellschaften. ${ }^{2}$ Aber auch der Transport von Menschen und Gütern, das Gesundheitssystem oder das Notfall- und Rettungswesen sind Infrastrukturen, ohne die menschliches Zusammenleben nicht oder zumindest nicht auf einem bestimmten Mindestniveau an Ordnung und Wohlstand funktioniert (Kröger 2008: 1781, Pursiainen 2009: 723). Kritische Infrastrukturen sind so wichtig, dass sie das „Rückgrat der Gesellschaft“ (backbone of society) bilden. Fallen sie aus oder werden beschädigt, führt das sehr schnell zu sehr großen und unter Umständen langanhaltenden Krisen, verbunden mit massiven negativen Auswirkungen auf die Bevölkerung (Attoh-Okine 2016: 32). Obwohl in Nuancen unterschiedlich, umfassen die kritischen Infrastrukturen auch heute noch in den meisten Definitionen die Systeme, die auch bereits bei der Etablierung der Kommission zum Schutz kritischer Infrastrukturen durch die Clinton-Administration genannt wurden. Diese waren Telekommunikation, das Energiesystem mitsamt Gas- und Ölreserven, das Transportsystem, Banken und Finanzsystem, Wasserversorgung, Notfall- und Rettungswesen mitsamt Polizei und Feuerwehr sowie das Fortbestehen der Regierung (continuity of government) (Galland 2012: 302). So oder so ähnlich definieren beispielsweise auch die EU, Kanada, die NATO, die Schweiz, die Niederlande, das Vereinigte Königreich und Norwegen kritische Infrastrukturen (Galland 2012: 306f). In Deutschland hat das Bundesministerium des Innern, seit März 2018 offiziell Bundesministerium des Innern, für Bau und Heimat, im Jahr 2009 die „Nationale Strategie zum Schutz Kritischer Infrastrukturen (KRITIS-Strategie)“ veröffentlicht. Darin definiert das BMI konsistent zur internationalen Diskussion Infrastrukturen dann als kritisch, „wenn sie für die Funktionsfähigkeit moderner Gesellschaften von wichtiger Bedeutung sind und ihr Ausfall oder ihre

2 In diesem Kontext lohnt sich der Verweis auf das Buch „Blackout. Morgen ist es zu spät“ von Marc Elsberg zum Thema eines durch einen koordinierten, terroristischen Hackerangriff verursachten, langfristigen europaweiten Stromausfalls. Obschon fiktional - und dahingehend auch mit einer spannenden Geschichte aufwartend - zeigt es sehr eindrücklich die Auswirkungen eines derartigen Ereignisses und damit die inhärente Verwundbarkeit unserer Gesellschaft aufgrund der Abhängigkeit von der durchgängigen Funktionsfähigkeit kritischer Infrastrukturen (siehe Elsberg 2012). 
Beeinträchtigung nachhaltige Störungen im Gesamtsystem zur Folge hat" (BMI 2009: 5). Das BMI unterscheidet für Deutschland zwischen den technischen Basisinfrastrukturen auf der einen und den sozioökonomischen Dienstleistungsinfrastrukturen auf der anderen Seite. Die technischen Basisinfrastrukturen sind die Energieversorgung, Informations- und Kommunikationstechnologien, Transport und Verkehr sowie die (Trink-)Wasserversorgung und Abwasserentsorgung. Die sozioökonomischen Dienstleistungsinfrastrukturen sind das Gesundheitswesen, die Ernährung, das Notfall- und Rettungswesen, der Katastrophenschutz, Parlament, Regierung, öffentliche Verwaltung und Justizeinrichtungen, das Finanz- und Versicherungswesen sowie Medien und Kulturgüter (BMI 2009: 5).

Die technischen Basisinfrastrukturen werden im internationalen Kontext als „lifeline systems“ bezeichnet. Es handelt sich typischerweise um sehr große, miteinander gekoppelte, geographisch verteilte Netzwerke, die durch dynamische Strukturen und andauernde Veränderungen technologischer und organisatorischer Art gekennzeichnet sind. Sie werden in aller Regel als komplexe Systeme verstanden. Auch die sozioökonomischen Dienstleistungsinfrastrukturen können so verstanden werden (AlKhudhairy et al. 2012: 576, Kröger 2011: 68, O'Rourke 2007: 23). Obwohl die Leistungen, die von diesen kritischen Infrastrukturen erbracht werden, häufig als öffentliches Gut (public good) gesehen werden, befinden sich in vielen westlichen Industrienationen, wie etwa Deutschland, den USA und dem Vereinigten Königreich über 80\% der kritischen Infrastrukturen in privatem Besitz und werden von ihren Betreibern grundsätzlich zum Zwecke der Erzielung wirtschaftlicher Gewinne genutzt. ${ }^{3}$ Auch das gilt sowohl für technische Basisinfrastrukturen, als auch für sozioökonomische Dienstleistungsinfrastrukturen. Das führt unter anderem dazu, dass die verschiedenen Infrastrukturen weder von einer übergeordneten Einheit kontrolliert oder gesteuert werden können, noch einer gemeinsamen Logik folgen. Die Orientierung an der Erzielung wirtschaftlicher Gewinne kann durchaus in Konflikt mit dem Auftrag der Betreiber kritischer Infrastrukturen geraten, der Bevölkerung zuverlässig die Produkte oder Dienstleistungen zur Verfügung zu stellen, auf die diese für ihre Funktionsfähigkeit oder sogar ihr Überleben angewiesen ist (Alderson 2019: 69,

3 Siehe dazu auch Patricia Wiater „Sicherheitspolitik zwischen Staat und Markt. Der Schutz kritischer Infrastrukturen." Die Autorin untersucht darin, wie Anschläge auf kritische Infrastrukturen die Sicherheit der gesamten Gesellschaft gefährden können. Dem Staat mangele es an Kontrollmöglichkeiten, da er auf eine freiwillige Mitwirkung privater Infrastrukturbetreiber angewiesen sei (siehe Wiater 2013). 
Edwards 2009: 9, Kaufmann/Blum 2012: 249, Kröger 2011: 68). Gleichzeitig weist die Dominanz privater Betreiber auf einen weiteren wichtigen Aspekt im Zusammenhang mit kritischen Infrastrukturen hin. Wie erwähnt besteht deren Auftrag darin, die Bevölkerung mit Produkten oder Dienstleistungen zu versorgen. Welcher Mittel und Wege, welcher konkreten physischen und technischen Ausgestaltung ihrer Systeme sie sich dazu bedienen, ist prinzipiell irrelevant. Die physischen, technischen und sozioökonomischen Systeme existieren nicht aus einem Selbstzweck heraus (Park et al. 2013: 357). Entscheidend sind die Funktionen, welche die Infrastrukturen für die Gesellschaft erfüllen: „However, while these technical features are fundamental to the infrastructure, and ensure it works, the purpose of critical infrastructure is to provide a service to society. These services are what society most values about critical infrastructure" (Prior 2014: 12). Diese sind es auch, die kritisch sind und bei deren Ausfall die Gesellschaft schwere Schäden erleidet (Pursiainen 2009: 726).

Großflächige Ausfälle kritischer Infrastrukturen, wie sie in dramatisch zugespitzter Weise von Elsberg beschrieben werden, sind - bisher - glücklicherweise sehr selten (siehe Fußnote 2). Nichtsdestotrotz treten sie auf und zwar schon länger. Bereits 1977 kam es in New York City zu einem stadtweiten Blackout, der unter anderem in gewalttätigen Plünderungen resultierte und der zeigte "how a complex sequence of unforeseen and interactive technical and human failures is not only possible but likely" (Lovins/Lovins 2001: 57). Ein weiteres Beispiel aus dem Energiesystem das aufgrund seiner essentiellen Bedeutung für beinahe alle anderen kritischen Infrastrukturen als das wahrscheinlich kritischste System überhaupt bezeichnet werden kann $^{4}$ - ist der großflächige Stromausfall im Nordosten Nordamerikas vom August 2003, von dem über 50 Millionen Menschen betroffen waren. Erst nach zwei Tagen konnte die Stromversorgung für einen großen Teil dieser Menschen wiederhergestellt werden (Flynn/Burke 2012: 2). Auch Ereignisse wie die Terroranschläge vom 11. September 2001 wirken sich direkt und indirekt auf die Funktionsfähigkeit kritischer Infrastrukturen aus. Direkt etwa auf das Notfall- und Rettungswesen, auf Polizei, Feuerwehr und Rettungskräfte, die nach den Anschlägen in einer

4 Inwiefern in einer durchgängig digitalisierten Welt das Informations- und Kommunikationssystem eine vergleichbare Kritikalität erreicht hat, kann an dieser Stelle nicht ausführlich diskutiert werden. Ähnlich wie das Energiesystem kann auch diese kritische Infrastruktur mittlerweile für sich beanspruchen, dass beinahe alle anderen Infrastrukturen von ihrer Funktionsfähigkeit abhängen. Die Abhängigkeit vom ebenso kritischen Energiesystem ist dabei wechselseitig. 
organisatorischen wie emotionalen Ausnahmesituation weiter funktionieren mussten. Den Einsatz in den zusammenstürzenden Türmen bezahlten hunderte Feuerwehrleute mit ihrem Leben. Aber auch indirekt hatten die Anschläge gravierende Auswirkungen und führten etwa kurzfristig zur Schließung des gesamten amerikanischen Luftraums und mittelfristig zu neuen Bestimmungen im Luftverkehr (siehe Kean et al. 2004). Noch dramatischere Auswirkungen auf quasi alle kritischen Infrastrukturen innerhalb eines bestimmten Gebiets hatte Hurrikan Katrina, der im August 2005 die Stadt New Orleans traf und dort schlimmste Verheerungen anrichtete. Die Versorgung der Bevölkerung mit allen lebenswichtigen Gütern und Dienstleistungen brach für Wochen zusammen (siehe Colten et al. 2008). Neben derart dramatischen widrigen Ereignissen spielen für kritische Infrastrukturen aber auch andere gesellschaftliche Entwicklungen, wie etwa die Energiewende, eine wichtige Rolle was ihre Zuverlässigkeit und Sicherheit angeht (Wachsmuth 2014: 3, siehe Renn 2017).

Für die vorliegende Arbeit und ihre Beschäftigung mit Resilienz und Resilience Engineering sind kritische Infrastrukturen von besonderer Bedeutung. Denn kritische Infrastrukturen können einerseits als Forschungsgebiet aus dem Bereich der Ingenieurwissenschaften verstanden werden. Energienetze, Brücken, Tunnel, das Wasserversorgungssystem und viele andere kritische Infrastrukturen benötigen „engineering knowledge“ um grundsätzlich installiert bzw. entwickelt zu werden und auch um langfristig gewartet und repariert bzw. optimiert zu werden. Gleichzeitig sind kritische Infrastrukturen aber auch als Forschungsgebiet für die Sozialwissenschaften interessant. Und zwar in mehrfacher Hinsicht. Auch die technischen Basisinfrastrukturen erbringen ihre Leistungen immer für die Gesellschaft. Zudem werden sie von Menschen entwickelt, betrieben, gewartet, etc. Die sozioökonomischen Dienstleistungsinfrastrukturen sind ohnehin nicht rein technisch zu verstehen (Hosseini et al. 2016: 49). Kritische Infrastrukturen als Teilgebiet der zivilen SiFo sind also ebenso interdisziplinär angelegt, wie die zivile SiFo an sich. Der Schutz kritischer Infrastrukturen kann mit einem rein disziplinären Ansatz genauso wenig gelingen, wie die erfolgreiche Erhöhung oder Bewahrung der Sicherheit der Gesellschaft gegenüber disruptiven Ereignissen. Dazu wird zwingend ein interdisziplinärer Ansatz benötigt, wie ihn die vorliegende Arbeit verfolgt. Um diesem Ansatz im weiteren Verlauf zu entsprechen, werden daher kritische Infrastrukturen und generell die Systeme, die im Rahmen der zivilen SiFo wichtig sind - also gesellschaftlich relevant - als sogenannte sozio-technische Systeme verstanden (LRF 2015: 27). In sozio-technischen Systemen interagieren technologische und soziale Bestandteile dergestalt 
miteinander, dass sie weder rein technologisch noch rein sozial erklärbar sind. Deren Resilienz und die Frage, was Resilience Engineering für die innerhalb der zivilen SiFo relevanten sozio-technischen Systeme bedeutet, stehen im Mittelpunkt der folgenden Analysen.

\subsection{Notwendigkeit für Resilienz und Resilience Engineering}

Was hat es nun mit all dem auf sich? Warum die Beschäftigung mit Resilienz und Resilience Engineering im Kontext der zivilen SiFo und des Schutzes kritischer Infrastrukturen? Es ist grundsätzlich sehr wünschenswert, dass es besser kommt, als man denkt. Das gilt natürlich in besonderer Weise für die zivile SiFo und für den Schutz kritischer Infrastrukturen. In diesen Bereichen stehen buchstäblich Leben auf dem Spiel. Als Resultat disruptiver Ereignisse sterben jedes Jahr eine Vielzahl an Menschen, noch wesentlich mehr werden verletzt. Und dazu kommen noch Schäden monetärer Art sowie emotionale und psychische Auswirkungen auf Betroffene und teilweise - wie nach den Anschlägen vom 11. September 2001 auf ganze Gesellschaften. Wenn derartige Schäden mithilfe von Resilienz und Resilience Engineering vermieden oder zumindest verringert werden können, ist es nicht nur sinnvoll, sondern nachgerade notwendig, sich im Kontext der zivilen SiFo eingehender mit den beiden Konzepten zu beschäftigen. Zumal beispielsweise kritische Infrastrukturen immer noch häufig ohne Beachtung von Resilienz entwickelt und betrieben werden. Kete et al. formulieren dazu treffend: „[T]he safety and well-being of billions of people depends on infrastructure systems, at present we do not design, deliver, and operate for resilience“ (Kete et al. 2018: 275). Das sollte künftig geändert werden: „To avoid or lessen the shocks that will arise in the future from infrastructure failure, new infrastructure systems must be designed with resilience in mind, and ways must be found to increase the resilience of existing systems" (Rogers et al. 2012: 75).

Dazu muss zunächst eine solide, theoretische Grundlage für das Resilienz-Konzept geschaffen werden, um darauf aufbauend Ideen für Resilience Engineering für sozio-technische Systeme entwickeln zu können (LRF 2015: 33). Die Notwendigkeit für diesen Prozess in zwei Schritten wird aktuell von verschiedenen Forschern gesehen. So schreibt beispielsweise die Lloyd's Register Foundation in dem gerade bereits zitierten Überblick über aktuelle Entwicklungen und Bedarfe in der ingenieurwissenschaftlichen Resilienzforschung von der Notwendigkeit, Resilience Engineering zu definieren und im größeren Kontext des Konzepts Resilienz zu verorten 
(LRF 2015: 33). Dinh et al. formulieren sehr ähnlich. Ihnen zufolge bedarf es der Identifikation grundlegender theoretischer Prinzipien, um Resilienz besser zu verstehen und das Konzept ingenieurwissenschaftlich umsetzen zu können (Dinh et al. 2012: 234). Laut Al-Khudhairy et al. befindet sich die Forschung immer noch sehr am Anfang, wenn es darum geht, Resilienz zu verstehen und konkret umzusetzen (Al-Khudhairy et al. 2012: 587). Auch Farid sieht gerade Resilience Engineering als Konzept noch sehr stark in der Entwicklung. Er spricht von einem Bedarf nach einer stärkeren Formalisierung (Farid 2015: 2006). Smith et al. wiederum sehen noch viel grundsätzlicher ein Fehlen eines systematischeren Blicks auf das Thema Resilienz und gerade deshalb auch einen Mangel an Ideen zur ingenieurwissenschaftlichen Umsetzung von Resilienz (Smith et al. 2011: 88). Letzteres gilt auch für Boin und McConnell, für die weiterhin unklar ist, wie Ingenieurwissenschaften zu Resilienz beitragen können (Boin/McConnell 2007: 51). Brunner und Giroux sowie Altherr et al. wiederum fordern, dass eine Konzeptualisierung von Resilienz und Resilience Engineering zusammengehen sollen und logisch aufeinanderfolgen müssen (Altherr et al. 2018: 189, Brunner/Giroux 2009: 6). All diese Aussagen beruhen auf einer Annahme, die von Kete et al. treffend zusammengefasst wird: „Engineering is placed at the heart of creating and managing resilience" (Kete et al. 2018: 277). Denn die Qualität ingenieurwissenschaftlicher Lösungen entscheidet mit darüber, ob und wie resilient sozio-technische Systeme sind (Rahimi/Madni 2014: 811f, Schaffer/Schneider 2019: 18). Ausgehend von den grundlegenden Annahmen, dass erstens Resilienz und seine ingenieurwissenschaftliche Umsetzung in Form von Resilience Engineering innerhalb der zivilen SiFo eine sinnvolle und notwendige Ergänzung bisheriger Ansätze darstellen könnten und dass zweitens noch kein solides, theoretisch fundiertes Konzept für Resilienz und darauf aufbauend Resilience Engineering für die zivile SiFo existieren, versucht die vorliegende Arbeit, genau dazu einen Beitrag zu leisten. Ähnlich wie von Rogers et al. formuliert, geht es ihr darum, Resilienz und Resilience Engineering aus einer interdisziplinären Perspektive holistisch zu verstehen und so Erkenntnisse aus verschiedenen Bereichen und Forschungszweigen für die zivile SiFo nutzbar zu machen (Rogers et al. 2012: 75).

\subsection{Aufbau der Arbeit}

Resilienz ist kein neues Forschungsthema. In Disziplinen wie der Psychologie oder der Ökologie wird seit vielen Jahrzehnten an dem Konzept 
geforscht. Auch in verschiedenen Bereichen der Sozialwissenschaften und in den Ingenieurwissenschaften existieren mittlerweile eine Vielzahl an Ansätzen und Ideen. Deshalb lässt sich mit Blick auf die gerade postulierte Grundidee der vorliegenden Arbeit die Frage stellen, ob die ihr zugrundeliegenden Annahmen überhaupt zutreffend sind. Ist das Konzept der Resilienz wirklich so unklar? Gibt es einen "lack of systematic view of the resilience problem" (Smith et al. 2011: 88)? Und lässt sich wirklich sagen, dass auch weiterhin unklar ist, wie die Ingenieurwissenschaften dazu beitragen können, dieses zu lösen (Boin/McConnell 2007: 51)? Um diese Fragen zu beantworten, wird im Folgenden in Kapitel 2 zunächst ein ausführlicher und systematischer Überblick über den Stand der Forschung zu Resilienz in den wichtigsten Disziplinen gegeben. Dieser klassische, disziplinäre Zugang erlaubt es, die Genese des Konzepts in unterschiedlichen Bereichen nachzuzeichnen und aktuelle Erkenntnisse darzustellen. Gleichzeitig beschreibt Kapitel 2 nicht einfach nur den Stand der Forschung. Vielmehr stellt es bereits den ersten Teil der tiefergehenden Analyse von Resilienz und Resilience Engineering dar. Es geht um eine systematische Prüfung der existierenden wissenschaftlichen Ergebnisse im Hinblick darauf, wie sie für die zivile SiFo nutzbar gemacht werden können Das geschieht durch die Identifikation disziplinübergreifender Konzepte, die für Resilienz von besonderer Bedeutung sind. Resilienzforschung erfolgt, wie noch zu zeigen sein wird und trotz der Möglichkeit, Resilienz als boundary object zu nutzen, bisher häufig disziplinär und ohne große Beachtung der Erkenntnisse anderer Disziplinen. Nichtsdestotrotz lassen sich eine Reihe an Konzepten ausmachen, die für Resilienz in jeweils mehreren Disziplinen wichtig sind und die daher auch für das Verständnis von Resilienz und Resilience Engineering innerhalb der zivilen SiFo untersucht werden müssen. Zudem wird in diesem Kapitel aufgearbeitet, ob sich die oben skizzierten Annahmen in tatsächlichen Forschungslücken wiederfinden.

Die Forschungslücken werden dann in Kapitel 3 explizit aufgeführt und aus ihnen die konkrete wissenschaftliche Fragestellung der Arbeit abgeleitet. Die drei folgenden, zentralen Kapitel 4, 5 und 6 dienen der Beantwortung der wissenschaftlichen Fragestellung und der Adressierung der Forschungslücken. In Kapitel 4 wird ein umfassendes eigenständiges Resilienz-Konzept für die zivile SiFo entwickelt. Dazu werden die insgesamt zehn konzeptionellen Zugänge genutzt, die in Kapitel 2 identifiziert wurden. Dieses Vorgehen entspricht der interdisziplinären Grundidee der Arbeit. Es ermöglicht eine übergreifende Analyse und Einordnung der im Kontext von Resilienz relevantesten Konzepte. Die Diskussion anhand 


\section{Einleitung}

konzeptioneller Zugänge führt zu jeweils spezifischen Elementen oder Bestandteilen, die für das Resilienz-Konzept der zivilen SiFo wichtig sind. Sie werden am Ende der jeweiligen Absätze, in denen sie erarbeitet wurden, markiert. Als Abschluss der zehn Unterkapitel zu den konzeptionellen $\mathrm{Zu}-$ gängen werden diese Merksätze jeweils aufzählungsartig zusammengefasst. In Kapitel 5 wiederum werden aus den Merksätzen zum einen relevante Begrifflichkeiten für Resilienz definiert und zum anderen die konkreten Bestandteile des Resilienz-Konzepts anhand von sieben Leitfragen systematisch geordnet und zu einem konsistenten Ganzen geführt, mitsamt der Diskussion von Grenzen des Konzepts. Die mithilfe der Leitfragen geordneten Bestandteile des Resilienz-Konzepts der zivilen SiFo bilden den Ausgangspunkt für die Entwicklung von Hypothesen für Resilience Engineering in Kapitel 6. Um die Konsistenz zum vorherigen Kapitel zu wahren, werden auch diese Hypothesen durch die Leitfragen geordnet. $\mathrm{Zu}$ allen Bestandteilen des Resilienz-Konzepts der zivilen SiFo wird eine Analyse durchgeführt, ob sich daraus Hypothesen für Resilience Engineering ableiten lassen und wenn ja, wie diese konkret lauten müssen. Dabei werden auch weitere aktuelle Erkenntnisse aus der ingenieurwissenschaftlichen Resilienzforschung miteinbezogen. Die Hypothesen werden dann ebenfalls zusammengefasst und ihre Verknüpfung zu den konzeptionellen Zugängen grafisch aufgezeigt, bevor über Grenzen für Resilience Engineering aus sozialwissenschaftlicher Sicht gesprochen wird. Abschließend wird das Ergebnis der Arbeit in Kapitel 7 in einem Fazit zusammengefasst, auf offene Fragestellungen und mögliche Kritik verwiesen, sowie ein interdisziplinär-forschungsleitender Ausblick gegeben. 


\section{Stand der Forschung - Identifikation relevanter konzeptioneller Zugänge}

\subsection{Resilienz als wissenschaftliches Konzept}

Die vorliegende Arbeit ordnet sich selbst eindeutig dem Themenfeld der zivilen Sicherheitsforschung zu. Letztere ist in Deutschland - wie auch Europa - als relativ junge Disziplin anzusehen, deren Geschichte kaum fünfzehn Jahre zurückreicht, wie der kurze Abriss der Geschichte der zivilen SiFo zu Beginn der Arbeit gezeigt hat. Die für die SiFo relevanten Themen und Problemstellungen sind allerdings deutlich älter. Extreme Naturereignisse gibt es bereits sehr viel länger als die Menschheit, das Phänomen des Terrorismus, bzw. des Versuchs, politische Ziele mit Hilfe gegen die Zivilbevölkerung gerichteter nichtstaatlicher Gewaltakte durchzusetzen, lässt sich mindestens einige Jahrhunderte zurückverfolgen und gravierende Unfälle in einer hochtechnisierten Welt gibt es seit den Anfängen der Industrialisierung (Hannig 2016: 84, Härter et al. 2014: 374). Damit einher ging auch immer eine mehr oder minder wissenschaftliche Beschäftigung mit diesen von den Menschen häufig als Bedrohung empfundenen Ereignissen. In einer leicht polemischen Zuspitzung ließe sich folgendes festhalten: Die Gründe für Naturkatastrophen wurden lange Zeit primär in einer Strafe durch die jeweiligen Götter für spezifisches Missverhalten der Bevölkerung gesehen (Kneer/Nassehi 2000: 173). Selbiges galt etwa noch im Mittelalter und bis in die Neuzeit hinein für Pandemien wie die wiederholten und ganze Länder verwüstenden Ausbrüche der Pest, der allein zwischen 1346 und 1353 ein Drittel der gesamten europäischen Bevölkerung zum Opfer fielen und die häufig als „Schwarzer Tod" bezeichnet wird (siehe Bergdolt 2011). Wenn die Gründe für derartig widrige Ereignisse und Entwicklungen in der Launenhaftigkeit göttlicher Wesen gesucht werden, ergeben sich die notwendigen Maßnahmen, die zu treffen sind, um von diesen Katastrophen verschont zu bleiben, in zwingender Logik von selbst. Es bedarf dann „nur“ vermehrten Betens, der Opferung immer zahlreicherer und größerer Gaben auf entsprechenden Altären oder etwa - wie häufig im Zuge von Pestepidemien geschehen - des Ausstoßens von vermeintlich Nicht-Zugehörigen aus der Gemeinschaft der Gläubigen. Jenen wurde gleichzeitig in regelmäßiger Wiederkehr zudem die Schuld am Auftreten der widrigen Ereignisse gegeben, was beispielsweise in Euro- 
pa und mit Bezug zur Pest wiederholt Verfolgungen und Pogrome gegenüber der jüdischen Bevölkerung zur Folge hatte (Alcabes 2009: 31ff, Goehl et al. 2000: 127ff).

Gleichzeitig wurden aber auch bereits im Altertum eine Vielzahl an Krankheiten korrekt beschrieben und Naturphänomene, wie das jährlich wiederkehrende Nil-Hochwasser, soweit verstanden, dass sie für die Bevölkerung nutzbar gemacht werden konnten (siehe Allan/Howell 1994, Leven 2005). Die wissenschaftliche Beschäftigung mit stärker gesellschaftlich zu erklärenden Phänomenen wie dem Terrorismus kann prinzipiell ebenfalls bis ins Altertum und mindestens zu den griechischen Philosophen zurückverfolgt werden, da es in diesem Fall darum geht, menschliche Handlungsund Verhaltensweisen - auch und gerade im politischen - zu verstehen und erklären (siehe z.B. Horn 2013). Ursachen- und Verstehens-zentrierteForschung zu extremen Natur- und geologischen Ereignissen - wie Erdbeben, Überflutungen, Stürmen oder Dürren - in modernem Sinne gibt es auch schon sehr lange. Trotz dieser langen Forschungsgeschichte konnte aber zum Beispiel für Erdbeben erst zu Beginn des 20. Jahrhunderts nachgewiesen werden, wie sie entstehen und ablaufen. Bis heute können Erdbeben nicht zuverlässig vorausgesagt werden (Joffe et al. 2018: 82ff).

Nichtsdestotrotz wird deutlich, dass die Wissenschaft sich bereits sehr viel länger mit Extremereignissen der geschilderten Art auseinandersetzt, als es den Begriff der Sicherheitsforschung gibt. Deren Spezifikum ist allerdings wie beschrieben ein anderes. Es geht weniger um die natur- und/ oder sozialwissenschaftlichen Ursachen widriger Ereignisse, als vielmehr darum, welche negativen Auswirkungen diese auf die Gesellschaft und die für deren Funktionieren relevanten Systeme haben. Und darum, wie diese Auswirkungen durch geeignete Instrumente, seien sie psychologischer, gesellschaftlicher, technischer oder sonst einer Art, kontrolliert und in ihrem Ausmaß begrenzt werden können (Scharte et al. 2014: 10). Diese - bewusst verkürzte und zugespitzte - Darstellung soll die Ähnlichkeit mit zwei weiteren verwandten und wiederum deutlich älteren Forschungsbereichen aufzeigen, derer sich die SiFo und in ihr tätige Sicherheitsforscher klar sein sollten. In der Risikoforschung sowie in der Katastrophenforschung (disaster research) beschäftigen sich Wissenschaftler bereits seit vielen Jahrzehnten - unter anderem - mit der Frage, wie Gesellschaften mit Extremereignissen umgehen, welche Handlungsstrategien es gibt und welche Lösungen zur Minimierung negativer Auswirkungen sinnvollerweise gewählt werden können. Die SiFo knüpfte in vielen Aspekten relativ nahtlos an Erkenntnisse aus diesen beiden Forschungsrichtungen an. Daher sind sie für die vorliegende Arbeit ebenfalls von großer Bedeutung. Auf die Ge- 
nese sowie relevante Inhalte der Katastrophenforschung wird im weiteren Verlauf noch detaillierter eingegangen (siehe 2.4). An dieser Stelle soll eine kurze Bemerkung ausreichen, um einer ersten Einordnung Genüge zu tun. Die Katastrophenforschung ist generell ein anwendungsnahes Gebiet, in dem Ansätze aus verschiedenen wissenschaftlichen Disziplinen zusammengebracht werden. Sie ist vor allen Dingen im Hinblick auf ihre Beschäftigung mit den für diese Arbeit zentralen Begrifflichkeiten von Interesse. Ein Grundverständnis der Risikoforschung an sich wiederum ist essentiell für den Bereich der ingenieurwissenschaftlichen Sicherheitsforschung. Darauf wird deshalb im Folgenden jeweils kurz Bezug genommen, wo es zu den jeweiligen disziplinären Ansätzen passt (siehe 2.4 und 2.6).

Dieser kurze Überblick über einige wissenschaftshistorisch relevante Entwicklungen für die moderne zivile SiFo lässt ein Konzept vermissen: Resilienz. Das liegt nicht etwa daran, dass mit „Resilienz“ ein gänzlich neuer Begriff sozusagen aus dem Nichts in die Debatte eingeführt worden wäre. Resilienz kann als Wort wie als wissenschaftliches Konzept auf eine lange Geschichte zurückblicken (Alexander 2013: 2708, Fekete et al. 2014: 4, Hellige 2019: 30ff). Es lässt sich allerdings mit einiger Sicherheit behaupten, dass dieses Konzept bis vor einigen Jahren bzw. maximal wenigen Jahrzehnten nicht im Zentrum der Forscher stand, die sich aus naturoder sozialwissenschaftlicher Sicht mit der Ursache oder, aus Risiko- und Katastrophenforschung heraus mit den Auswirkungen widriger Ereignisse beschäftigen. Das Konzept der Resilienz hat sich sozusagen parallel und eigenständig in einer Fülle verschiedener wissenschaftlicher Disziplinen entwickelt. Um ein eigenständiges Resilienzverständnis für die zivile SiFo gewinnen zu können, bedarf es einer intensiven Beschäftigung mit den unterschiedlichen disziplinären Zugängen, aus denen heraus Resilienz bereits umfassend analysiert und beschrieben wurde. Einen ersten Schritt auf dem Weg dahin bietet eine kurze Begriffsgeschichte. Bevor diese Genese des Begriffs Resilienz und seiner Verwendung als wissenschaftlichem Konzept gleich nachgezeichnet wird, sollen zunächst einige generelle Bemerkungen vorangeschoben werden, die sich auch auf die weiteren Inhalte dieses Kapitels und ihre Einordnung in den Gesamtkontext der Arbeit beziehen.

Übergreifend und zusammenfassend lässt sich tatsächlich, wie in der Einleitung behauptet, ein Fehlen des Vorhandenseins einer allgemeinen und allseits anerkannten Theorie zu/über/von Resilienz konstatieren. Es herrscht einiges an Konfusion darüber, ob Resilienz eigentlich einen Prozess darstellt, einen Zustand oder doch eine Qualität bestimmter Systeme. Für unterschiedliche Disziplinen können teilweise sogar sich widersprechende Charakteristika, Eigenschaften und Handlungsweisen auf Resilienz 
deuten oder diese ausmachen. Der Begriff wird zum Teil ganz einfach als simple Beschreibung im Alltag verwendet (weniger im deutschen als im angelsächsischen Sprachraum) und zum Teil zur Zusammenfassung eines ganzen Theoriegebäudes genutzt. Häufig handelt sich bei Resilienz um einen „konzeptionellen Rahmen“ innerhalb dessen die jeweiligen Wissenschaftler sich mit den unterschiedlichsten Dingen beschäftigen - je nachdem, aus welcher Disziplin und mit welchem Forschungsinteresse sie sich dem Konzept nähern (Alexander 2013: 2711ff, Wink 2011: 112). Neben der Disziplin spielen auch der historische Kontext sowie mögliche ideologische und/oder normative Randbedingungen eine wichtige Rolle für das individuelle Resilienzverständnis (Wink 2016: 5). Es herrscht also einiges an Verwirrung im Resilienzdiskurs. Zudem trägt die zunehmende populärwissenschaftliche und politische Verbreitung und möglicherweise auch Instrumentalisierung des Begriffs einiges zu dieser Verwirrung bei. Kaufmann und Blum zufolge hat sich Resilienz in den letzten Jahren zu einer Art politischem Programm entwickelt, was sie knapp und zugespitzt als „comprehensive mode of governing (in)security" zusammenfassen (Kaufmann/Blum 2012: 236). Der daraus folgenden, politisch gewünschten Entwicklung konkreter und umsetzbarer Handlungsempfehlungen, um von Extremereignissen gefährdete Gesellschaften nun endlich resilienter gestalten zu können, stehen wiederum die gerade schon skizzierten wissenschaftlichen Hürden entgegen, etwa die „Komplexität der Einflussfaktoren“, „historische Kontextabhängigkeiten“ oder die „Notwendigkeit normativer Ausgangsentscheidungen“ (Wink 2016: 7). Im wissenschaftlichen Resilienzdiskurs wird sogar die genannte Verwirrung selbst - oder präziser formuliert die Unschärfe der Begrifflichkeit - als mögliche Stärke des Resilienz-Konzepts als eigenständiges Phänomen mitbedacht (Prior/Roth 2013: 61, MacAskill/Guthrie 2014: 670, Vogt 2015: 2).

Einige Forscher meinen einen gemeinsamen Kern in den unterschiedlichsten disziplinären Resilienz-Konzepten ausmachen zu können. Sowohl Vogt als auch Wink sehen diesen beispielsweise im Auftreten einer Störung - primär von außerhalb des betrachteten Bezugssystems - und der Fähigkeit, diese zu verarbeiten und dabei die eigene Identität möglichst beizubehalten. Es geht demnach um bestimmte Eigenschaften und/oder Prozesse, die helfen können, mit Störungen - relativ - erfolgreich umzugehen (Vogt 2015: 3, Wink 2011: 112). Folgt man Wink sind eben genau die beiden Aspekte der Störung von außen und des Auftretens nur geringer oder sogar gar keiner negativer Effekte über die Zeit von disziplinübergreifender Bedeutung für Resilienz (Wink 2011: 112f). Ohne dieser Beobachtung zur Gänze widersprechen zu wollen, muss festgehalten werden: die beiden 
von Wink identifizierten Aspekte spielen zwar eine herausragende Rolle in der Resilienzforschung, allerdings gibt es durchaus Bereiche, in denen sie nicht so zentral für den Diskurs sind, wie eine derart apodiktische Aussage erwarten lässt. Eine größere Allgemeingültigkeit kann eine andere Aussage von Wink für sich in Anspruch nehmen: „Wesentlich für das Verständnis und die Verwendung des Resilienz-Begriffs ist das Verhältnis zu Veränderungen“" (Wink 2011: 112). Gleichzeitig ist diese Aussage auch ein trefflicher Ausgangspunkt für kontroverse Diskussionen, insbesondere im Kontext der eher ingenieurwissenschaftlich geprägten Resilienzforschung. Resilienz als Qualifizierung der Art, wie Systeme mit Veränderungen umgehen - damit definiert Wink einen Ausgangspunkt, den die vorliegende Arbeit aufgreift und umfassend zu analysieren versucht.

An dieser Stelle befindet man sich bereits mitten in einem Kernbereich der Diskussion, nämlich der Frage danach, ob das eigene Resilienzverständnis eher einer „mechanischen“, einer „systemischen“ oder einer Mischform von Resilienz entspricht, wie Voss und Dittmer formulieren (Voss/Dittmer 2016: 191). Die Formulierung geht dabei auf eine Unterscheidung zurück, die vom über alle Disziplinen hinweg - mit Ausnahme der Psychologie - wohl wichtigsten Resilienzforscher überhaupt, dem kanadischen Ökologen Crawford Stanley (Buzz) Holling, zum ersten Mal getroffen wurde. Er unterscheidet zwischen „engineering resilience“ einem eher mechanischen Resilienz-Verständnis - und „ecological resilience“ - der eher systemischen Sichtweise (Holling 1996). Beide dezidierten Formen und ihre - notwendige - Vermischung werden in den folgenden Kapiteln diskutiert, auf ihre theoretischen Grundlagen hin untersucht und bewertet im Hinblick darauf, wie sie für die zivile Sicherheitsforschung und ingenieurwissenschaftliche Ansätze zu selbiger nutzbar gemacht werden können.

Die Geschichte des Worts „Resilienz“ ist vielfach beschrieben worden. Ein Großteil der konzeptionell-theoretisch argumentierenden Artikel über Resilienz, aber auch viele empirisch arbeitende Publikationen stellen ihrem Werk einen kurzen Abriss über den Ursprung des Begriffs voraus. Dieser beginnt zumeist mit dem Hinweis auf die lateinischen Wurzeln von Resilienz im Wort „resilire“, welches übersetzt etwa „zurückspringen“ oder „abprallen“ bedeuten soll (Alexander 2013: 2708, Fooken 2016: 24). Das Nachzeichnen der Begriffsgeschichte ist auch für die vorliegende Arbeit von einiger Bedeutung. Es erfolgt zunächst unabhängig von konkreten Disziplinen und geht vor allen Dingen auf den Resilienz-Begriff und seine Bedeutung zu verschiedenen Zeiten, in verschiedenen Sprachen und in unterschiedlichen inhaltlichen Kontexten ein. Die Darstellung orientiert 
sich unter anderem an einem Überblicksartikel von Alexander (siehe Alexander 2013). Im Anschluss daran zeigen die folgenden Unterkapitel die Entwicklung des Resilienz-Konzepts in unterschiedlichen Disziplinen, wie der Psychologie, der Ökologie und ganz generell den Sozialwissenschaften auf. Der Blick auf die Organisationswissenschaften, in denen der Begriff „Resilience Engineering“ zuerst aufkam, sowie klassische, ingenieurwissenschaftliche Ideen von Resilienz unter dem gerade genannten Schlagwort „engineering resilience“ zeigt anschließend die Forschungslücken in Bezug auf die zivile Sicherheitsforschung auf.

Um die folgenden Analysen richtig einordnen zu können, ist diese Vorbemerkung von nicht zu unterschätzender Bedeutung: Die vorliegende Arbeit bewegt sich im Kontext der zivilen Sicherheitsforschung in Deutschland. Ihr Anspruch ist es jedoch nicht, ein rein auf deutsche Verhältnisse anwendbares Konzept für Resilienz und Resilience Engineering zu entwickeln. Vielmehr steht auch und gerade eine breite internationale Anwendbarkeit im Fokus. Denn Resilienz ist ohnehin ein wissenschaftlicher Gegenstand, der primär international diskutiert wird. Hier kann eine Dominanz des angelsächsischen Raums festgestellt werden. Zu dieser Dominanz muss festgehalten werden, dass Resilienz im angelsächsischen Raum im Gegensatz zur Situation in Deutschland - Teil der Alltagssprache ist (Fekete et al. 2016: 48, Zander/Roemer 2016: 48). Dort wird der Begriff damit auch ohne jeden wissenschaftlichen Anspruch und ohne zugrundeliegendes theoretisches Konzept als Adjektiv in allgemeinen medialen Veröffentlichungen genutzt (Scharte et al. 2014b: 53). Er ist in einem normalen Alltagsgespräch nicht notwendigerweise erklärungsbedürftig. Ganz im Gegensatz dazu ist der Begriff im Deutschen nicht geläufig. Versuche einer Übersetzung enden sehr häufig mit dem unzureichenden Wort „Widerstandsfähigkeit“, in dem sich auch die lateinische Ursprungsbedeutung wiederfindet (Fooken 2016: 24). Resilienz erweist sich im Deutschen als sperriges und nicht ohne Weiteres intuitiv zu verstehendes Wort, das sich aber gleichzeitig auch nicht gut übersetzen lässt (Fekete et al. 2016: 218). Deshalb greifen gerade populärwissenschaftliche Arbeiten über Resilienz immer wieder auf das bekannte Bild des „Stehaufmännchens" zurück, um das Prinzip zu verdeutlichen: trotz einer Belastung von außen - das Stehaufmännchen wird umgeschubst - kehrt das System wieder in seinen Ausgangszustand zurück - das Stehaufmännchen richtet sich eben wieder auf (Fooken 2016: 24). Auf den ersten Blick scheint dies eine durchaus geeignete Möglichkeit, das Prinzip hinter dem Begriff Resilienz zu veranschaulichen. Allerdings lässt sich - um im Bild zu bleiben - die Geschichte auch anders erzählen. Denn das Stehaufmännchen wirkt nur nach außen 
unverändert nach Ende der Belastung. Ob es etwa ein Schleudertrauma davongetragen hat, welche langfristigen Auswirkungen die Belastung zeitigt oder was es mit dem Stehaufmännchen macht, dass es gar keine andere Wahl hat, als nach Belastungen immer und immer wieder in seinen Ursprungszustand zurückzukehren, sind Fragen, die weit über das scheinbar simple Funktionsprinzip hinausgehen. Aus diesem Grund scheint es auch für den deutschen Resilienzdiskurs wichtig und notwendig, behelfsmäßige Begriffs-Übersetzungen oder Veranschaulichungen als hilfreiche Werkzeuge zu verstehen, nicht aber als Möglichkeit, das eigentliche Konzept Resilienz zu erfassen.

Dazu muss weiter ausgeholt werden. Denn Resilienz als wissenschaftliches Konzept geht nicht etwa auf Buzz Holling zurück. Dieses häufig wiederholte Missverständnis zeigt eindrucksvoll Hollings Bedeutung für den Resilienzdiskurs. Sein 1973 erschienener Artikel Resilience and stability of ecological systems wird von sehr vielen Forschern als Startpunkt der wissenschaftlichen Beschäftigung mit Resilienz genannt (z.B. Berkes 2007: 286, Birkmann 2008: 10, Brand/Jax 2007: 8, Carpenter et al. 2001: 765, Folke 2006: 254, Folke et al. 2010, Gunderson 2000: 425, Longstaff 2012: 264, Thoma et al. 2012b: 331). Dies ist aber begriffsgeschichtlich und auch konzeptionell-theoretisch so nicht richtig. Alexander zufolge lässt sich der Begriff Resilienz bis zu den Schriften römischer Schriftsteller und Philosophen zurückverfolgen. Unter ihnen waren mit dem Philosophen Marcus Tullius Cicero, dem Rhetoriker und Schriftsteller Lucius Annaeus Seneca (Seneca der Ältere), dem Dichter Publius Ovidius Naso (Ovid), dem Gelehrten Gaius Plinius Secundus Maior (Plinius der Ältere) oder auch dem Geschichtsschreiber Titus Livius einige der wichtigsten und berühmtesten geistigen Persönlichkeiten der römischen Antike. In ihren Schriften war mit dem Begriff „resilire“ zumeist eine Form des (Zurück-)Springens gemeint, Alexander nennt die englischen Begriffe „leaping“, „jumping“ und „rebounding“ (Alexander 2013: 2708, Fekete et al. 2014: 4). Damit kommt die reine Wortbedeutung in ihren Ursprüngen bereits dem nahe, was noch 2000 Jahre später Bestandteil vieler Resilienz-Definitionen ist: dem „bouncing back“, dem Zurückspringen in einen Ursprungszustand nach einer Belastung. Insofern ist sofort einsichtig, warum das eben erwähnte Stehaufmännchen sich als Metapher für Resilienz so sehr aufdrängt. Gleichzeitig bleibt die Metapher aber auch bei dieser ursprünglichen Bedeutung stehen - ganz im Gegensatz zum Wort Resilienz, das sich im Anschluss an seine lateinischen Ursprünge über die Zeit gewandelt und beispielsweise auch seine negativen Konnotationen, die es nach Alexander zunächst viel- 
fach beinhaltete, überwiegend in positive Bedeutungen umgewandelt hat (Alexander 2013: 2708). ${ }^{5}$

Ausgehend von seinem lateinischen Ursprung wurde Resilienz als Verb auch ins Französische (résiler) übertragen, wo es sich von seiner Bedeutung her mehr in Richtung „zurückziehen“ (to retract) oder „abbrechen“ (to cancel) entwickelte. Im englischen Sprachgebrauch nutzte König Heinrich VIII. in einem Papier aus dem Jahr 1529 das Verb „to resile“, ebenfalls im Sinne von zurückziehen bzw. „zurückkehren auf eine ursprüngliche Position" (return to a former position) (Alexander 2013: 2708). Im 17. Jahrhundert folgten darauf zwei neue Verwendungen von Resilienz. Der Anwalt und Antiquar Thomas Blunt zeichnete für die erste lexikalische Definition verantwortlich. In seinem 11.000 Begriffe umfassenden Fach-Wörterbuch, der „Glossographia“, zählte er Resilienz damit zu den Wörtern, die einer spezifischen Definition bedürfen, weil sie nicht genügend im alltäglichen Sprachgebrauch verankert sind, um von jedem ohne nähere Definition verstanden werden zu können. Zum Vergleich nennt Alexander an dieser Stelle übrigens zwei deutsche Begriffe, deren Ursprünge er ebenfalls für interessant hält und bei denen er einen Zusammenhang mit Resilienz sieht. Diese Begriffe sind „Elastizität“ und „Unverwüstlichkeit." Blunt wiederum schrieb Resilienz zwei Bedeutungen zu, von denen nur die erste in das bereits wohl bekannte Schema passt, nämlich „zurückprallen" (to rebound). Seine zweite Definition umschreibt einen völlig neuen Aspekt, der sich so auch in keiner weiteren Verwendung mehr findet und begriffsgeschichtlich daher als weniger bedeutsam eingestuft wird, nämlich „ein Versprechen brechen“ (to go back on one's word) (Alexander 2013: 2709). Neben dem Eintrag in ein Wörterbuch findet Resilienz im 17. Jahrhundert auch erstmals den Weg in die Wissenschaft. Sir Francis Bacon, seines Zeichens unter anderem ein Wegbereiter des Empirismus und damit experimenteller Wissenschaft per se, beschrieb in seinem Werk Sylva Sylvarum Resilienz im Zusammenhang mit einer Betrachtung über die Stärke von Echos - und damit in einem Zusammenhang, der zwar durchaus mit zurückprallen, zurückspringen oder eben bounce back in Verbindung gebracht werden kann, dem heutigen Verständnis im Hinblick auf Widerstandsfähigkeit oder ähnliche Phänomene hingegen eher fremd ist (Alexander 2013: 2709, Voss/Dittmer 2016: 184).

5 Das Adjektiv „positiv“ wird in der vorliegenden Arbeit im Sinne von nicht-negativ, also gut und wünschenswert gebraucht. Es geht nicht um positive = deskriptive Wissenschaft. 
Für die vorliegende Arbeit von besonders großem Interesse war dann der nächste Bedeutungswandel, dem Resilienz im Lauf der Jahrhunderte unterlegen war. Im 19. Jahrhundert, genauer gesagt ab dem Jahr 1839, wurde Resilienz als Ausdruck für die Fähigkeit, sich von den Auswirkungen eines widrigen Ereignisses zu erholen, gebraucht (ability to recover from adversity). Nach zwei Erdbebenvorfällen im japanischen Shimoda verwendeten amerikanische Beobachter des Geschehens im Dezember 1854 dann das englische Wort "resiliencey“ um die Tatsache zu beschreiben, dass die japanische Bevölkerung den Auswirkungen der Erdbeben relativ gut zu widerstehen in der Lage schien. Auch heute noch werden sowohl "resilience“ als auch „resiliency“ im Englischen als Begriffe verwendet. Beide meinen „Resilienz“ und werden sehr häufig synonym bzw. austauschbar gebraucht (Alexander 2013: 2709f, Fekete et al. 2014: 4). Inwiefern mit „resiliency“ eher ein Persönlichkeitsmerkmal definiert wird als mit „resilience“, wird an späterer Stelle der vorliegenden Arbeit noch kurz zu diskutieren sein (Luthar/Cicchetti 2000: 862).

Ebenfalls im 19. Jahrhundert wurde Resilienz erstmals im Bereich der Ingenieurwissenschaften, genauer gesagt der Materialwissenschaft, gebraucht. Der schottische Ingenieur William J.M. Rankine nutzte Resilienz demnach im Jahr 1858, um die Stärke und Duktilität von Stahlträgern zu beschreiben (Alexander 2013: 2710). Unter Duktilität versteht man in der Materialwissenschaft die Eigenschaft eines Materials, sich vor dem Versagen plastisch zu verformen, also seine Form dauerhaft und irreversibel zu verändern. Diese Art der Veränderung steht im Gegensatz zu einer elastischen Verformung, wie etwa dem Verhalten einer Feder, die zusammengepresst wird, aber nach Ende der Belastung wieder in ihren ursprünglichen Zustand zurückkehrt - eine Aussage, die nicht nur zufällig an das bereits beschriebene Stehaufmännchen erinnert. Im 19. Jahrhundert wurde Resilienz jedenfalls zunächst für Duktilität und nicht für Elastizität verwendet, was zum Beispiel Plodinecs Aussage widerspricht, wonach im Bereich der Physik die Beschreibung des Verhaltens einer Feder Inhalt der ersten Resilienz-Definition gewesen sei (Plodinec 2009: 1). Begriffsgeschichtlich lässt sich trotzdem festhalten, dass Resilienz in der Materialkunde im Lauf der Zeit als positive Eigenschaft von Werkstoffen verstanden wurde, sich bei mechanischer Belastung so zu verformen, dass nach Ende der Belastung eine Rückkehr in den Ausgangszustand möglich ist und das Material somit durch die Belastung keinen bleibenden, langfristigen Schaden erleidet (Fooken 2016: 24f). Folgt man Rankine, lässt sich Resilienz als eindeutig zu definierende und numerisch beschreibbare Eigenschaft von Werkstoffen auffassen. Diese erste Verwendung des Begriffs im Kontext der Inge- 
nieurwissenschaften kann noch nicht als umfassendes wissenschaftliches Konzept gesehen werden. Sie weist aber bereits den Weg in Richtung eher technisch orientierter Begriffsverständnisse, denen im Rahmen der übergreifenden Resilienzdiskussion sehr häufig, wenigstens implizit, der Vorwurf gemacht wird, sie seien zu sehr damit beschäftigt, Resilienz als mess- und quantifizierbare Systemeigenschaft zu charakterisieren, um den eigentlichen Kern des Konzepts erkennen und nutzen zu können (siehe z.B. MacAskill/Guthrie 2014: 667 oder auch Blum et al. 2016: 165, Edwards 2009: 17, Leach 2008: 7, Rahimi/Madni 2014: 811, Voss/Dittmer 2016: 186, Zinke/Ummenhofer 2014: 75). Im weiteren Verlauf der Arbeit wird dabei klar, dass diese Zuschreibung durchaus zutreffend ist (siehe 2.6 und 3). Mit Blick auf die Bedeutung des Wortes Resilienz und dessen lateinische Ursprünge liegt ein eher technisches Verständnis allerdings nahe. Passend dazu fasst Alexander in seinem Überblick über die Geschichte von Resilienz die gesamte Entwicklung des Begriffs von der Antike bis zum Ende des 19. Jahrhunderts prägnant zusammen: „In synthesis, before the 20th century, the core meaning was ,to bounce back“" (Alexander 2013: 2710).

Als wissenschaftliches Konzept - über die Bedeutung eines einfachen Maßes hinausgehend - wurde Resilienz erstmals in der Entwicklungspsychologie verwendet. In der breiten Öffentlichkeit wird der Begriff im deutschen Sprachraum auch heute primär als aus der Psychologie stammendes Konzept identifiziert. Nach wie vor wird zum Beispiel im Duden für den Gebrauchszusammenhang des Wortes „besonders Psychologie“ angegeben. ${ }^{6}$ Dort bedeutet es auf ein deutsches Pendant zusammengefasst Widerstandskraft bzw. Widerstandsfähigkeit. Ohne zunächst mit dem Wort Resilienz zu arbeiten, wurde in der Traumaforschung bereits in den 1940er und 1950er Jahren der Frage nachgegangen, wie Menschen es schaffen, trotz schwieriger und schwierigster Lebensumstände ihr eigenes Leben erfolgreich zu meistern. Im Mittelpunkt stand die Erforschung einer emotionalen Stärke, einer Fähigkeit von Individuen, Schicksalsschläge zu überwinden und mit Stress und Widrigkeiten umzugehen (Kaufmann/Blum 2012: 237, Ungericht/Wiesner 2011: 188). ${ }^{7}$ Der konkrete Stress bzw. die widrige Bedingung waren in ersten Studien hauptsächlich die Betroffenheit durch Schizophrenie. Die Wissenschaftler waren interessiert daran, wie sich relativ milde Verläufe der Krankheit erklären ließen. Als Ergebnis konnten sie feststellen, dass Menschen, die ihr Leben vor Ausbruch der Krankheit relativ gut meistern konnten, die also einen Job hatten,

6 https://www.duden.de/rechtschreibung/Resilienz [Stand: 27.6.2019].

7 Ebd. 
verheiratet waren, soziale Kompetenzen aufwiesen und in der Lage waren, Verantwortung zu übernehmen, auch mit der Krankheit Schizophrenie relativ besser umgehen konnten. Bei Studien mit Kindern von schizophrenen Müttern fanden Wissenschaftler im nächsten Schritt eine für sie überraschend hohe Anzahl von Kindern, die später zu erfolgreichen Erwachsenen wurden. Den widrigen Umständen, unter denen sie aufwuchsen, konnten sie aufgrund bestimmter individueller Eigenschaften trotzen (Luthar et al. 2000: 543f). Für die weitere Entwicklung des Resilienz-Konzepts in der Psychologie zeichnete vor allen Dingen die amerikanische Entwicklungspsychologin Emmy Werner mit ihren einflussreichen Studien verantwortlich. Deren wichtigste Inhalte sowie ein genereller Überblick über aktuelle Definitionen und Fragestellungen werden im nächsten Unterkapitel näher vorgestellt.

Im Anschluss an die Verwendung in der Psychologie, hat sich Resilienz im Lauf der letzten Jahrzehnte als wissenschaftliches Konzept in einer Vielzahl wissenschaftlicher Disziplinen etabliert. Ein wichtiger Grund dafür kann in der positiven oder optimistischen Konnotation gesehen werden, die dem Begriff laut Luthar und Cicchetti innewohnt und die ohne Zweifel zu seiner steigenden Popularität beigetragen hat (Luthar/ Cicchetti 2000: 863). Wie bereits erwähnt, war Resilienz damit einem Bedeutungswandel unterlegen, da es zunächst eher negativ besetzt war bzw. verstanden wurde (Alexander 2013: 2708). Resilienz als wünschenswerte Eigenschaft von Menschen oder generell Systemen jedweder Art zu begreifen und danach zu fragen, wie Resilienz geschaffen, genutzt und erhöht werden kann, hat Resilienz von einem Nischen- zu einem Mainstreambegriff werden lassen. Im Verlauf der letzten zehn bis zwanzig Jahre wurde Resilienz immer populärer. Zwischen 1995 und 2013 verdoppelte sich beispielsweise die Zahl der Studien, die sich mit dem Konzept in den verschiedensten Disziplinen beschäftigt haben. Es lässt sich ein Trend zur Verwendung von Resilienz ausmachen (Prior/Roth 2013: 61, Righi et al. 2015: 146, Voss/Dittmer 2016: 179 Woods 2015: 5). Im Hinblick auf eher politisch geprägte Debatten halten Lintz et al. sogar fest, dass die Begriffe „Resilienz“ und „Vulnerabilität“ sich anschicken, das Konzept der „nachhaltigen Entwicklung“ insbesondere auf der internationalen Ebene ein Stück weit in den Hintergrund zu drängen (Lintz et al. 2016: 334).

Schwerpunkte der Resilienzforschung liegen zum Beispiel in der Ökologie, den Umwelt- und Nachhaltigkeitswissenschaften, aber auch der Soziologie, den Wirtschaftswissenschaften oder auch der Risiko- und Katastrophenforschung. Besonders einflussreich ist der Resilienz-Begriff im Kontext der so genannten sozial-ökologischen Forschung (Jabaraeen 2013: 
221, Prior/Roth 2013: 61, Righi et al. 2015: 143). Generell lässt sich festhalten, dass Resilienz heute von einer unüberschaubar gewordenen Bandbreite unterschiedlicher Disziplinen sozusagen „ubiquitär“ genutzt wird (Lukesch 2016: 297). Jede Disziplin entwirft dabei ihr jeweils eigenes theoretisches Resilienzverständnis und definiert den Begriff anhand der disziplinären Besonderheiten und spezifischen Untersuchungsgegenstände häufig in Abgrenzung zu älteren, bekannten disziplinären Konzepten neu und anders. Auch und insbesondere innerhalb der einzelnen Disziplinen wird Resilienz zum Teil völlig unterschiedlich verstanden (Luthar et al. 2000: 544, Plodinec 2009). Das macht Resilienz nach Vogt sowohl „bemerkenswert“ als auch „verwirrend“ (Vogt 2015: 2). Und es stellt sich sowohl aus wissenschaftlicher als auch aus politischer Sicht als Herausforderung dar, einen möglichen, eigentlichen Kern von Resilienz auszumachen. Denn es ist schwierig bis unmöglich, aus der unübersichtlichen Menge an Definitionen und Verständnissen eine „beste“ oder gar „richtige“ Definition herauszufiltern. Jeder disziplinäre Zugang trägt durch die Beachtung der jeweils relevanten Fragestellungen zum übergreifenden Resilienzdiskurs bei (Flynn 2011: i, Plodinec 2009: 7). Resilienz tendiert auf diese Weise da$\mathrm{zu}$, zu einer Art „Container-Begriff“ zu werden, dessen Bedeutung immer mehr verwischt und der von wissenschaftlichen wie politischen Akteuren auch im Hinblick auf die Um- und Durchsetzung ihrer jeweiligen Agenden verwendet werden kann. Diese Gefahr eines politischen Missbrauchs des Begriffs hat Resilienz mit Nachhaltigkeit gemein, da beide sowohl als spezifisch definierte wissenschaftliche Konzepte als auch als stärker metaphorisch $\mathrm{zu}$ verstehende Leitprinzipien verwendet werden können (Carpenter et al. 2001: 765, Deppisch 2016: 199). Die Vermischung von wissenschaftlichem Konzept und politischem Programm stellt sich im Rahmen der Resilienz-Diskussion genauso als Schwierigkeit dar, wie die fehlende Vereinheitlichung des Verständnisses innerhalb der Wissenschaft, die daher auch immer wieder Anlass zur Kritik an der Verwendung von Resilienz bietet (Fekete et al. 2016: 219). Gleichzeitig ermöglicht die Vielseitigkeit des Begriffs und die unterschiedliche Herangehensweise der verschiedenen Disziplinen einen disziplinübergreifenden Austausch. Diese Diversität von Resilienz macht seine Rolle als Brückenkonzept und damit die vorliegende Arbeit - erst möglich (Fekete et al. 2016: 220, MacAskill/Guthrie 2014: 670).

Unterschiedliche Disziplinen zu betrachten und jeweils aus deren Resilienzverständnis zu lernen, um für die zivile Sicherheitsforschung ein passendes Konzept ableiten zu können, bildet die Grundherangehensweise dieser Arbeit. Die Vielseitigkeit der existierenden Resilienzverständnis- 
se und -definitionen bietet beides: Chancen und Risiken. Die Chancen liegen darin, für die zivile Sicherheitsforschung spezifische Aspekte von Resilienz aus dem breiten Diskurs herausfinden zu können, die der jungen Forschungsrichtung einen signifikanten Mehrwert bieten. Die Risiken sind ebenfalls klar erkennbar und bestehen vor allen Dingen darin, unterschiedliche Zugänge auf unzulässige Weise zu vermischen. Grundsätzlich unterscheiden lassen sich zum Beispiel die Träger von Resilienz: soll es um Individuen, soziale Gemeinschaften, technische Infrastrukturen oder ganze Gesellschaften gehen? Diese Diskussion wurde bereits im Einleitungskapitel kurz geführt und soll hier lediglich mit dem Verweis auf Wink ergänzt werden, der die Schwierigkeit der Zuschreibung des Trägers von Resilienz damit umgeht, abstrakt den Begriff „System“ zu nutzen und damit ein einigendes Band zwischen den einzelnen Kapiteln des von ihm herausgegebenen Sammelbands zu schaffen (Wink 2016: 2f). Die vorliegende Arbeit folgt Wink in dieser Hinsicht. Es geht zunächst sehr abstrakt um die Resilienz von Systemen. Erst später werden die interessierenden Systeme ein Stück weit spezifiziert und im Kontext der ingenieurwissenschaftlichen zivilen Sicherheitsforschung verortet. Der Begriff ist dabei nicht selbsterklärend, es muss dargelegt werden, was unter System zu verstehen ist, wie Systeme funktionieren und wie sich das Konstrukt „System“ gegenüber Resilienz verhält. Daher bildet „Resilienz und Systeme“ das erste der Begriffspaare, die im Folgenden detailliert im Hinblick auf ihre Beziehung zueinander analysiert werden. Und zwar unter Zuhilfenahme der Systemtheorie.

Neben der Frage danach, wessen Resilienz gemeint ist, kommt auch der Frage, gegenüber was das System resilient sein soll/kann, eine signifikante Bedeutung zu. Einigkeit herrscht hier im Prinzip nur insofern, dass es um eine aus Sicht des jeweiligen Trägers der Resilienz als negativ empfundene Veränderung des gegenwärtigen Systemzustands geht. Wodurch diese hervorgerufen wird, ob sie sich innerhalb oder außerhalb des beobachteten Systems, schnell oder langsam, erwartet oder unerwartet entwickelt und ob sie besonders gravierende, die Überlebensfähigkeit des Systems herausfordernde, oder eher alltägliche, vernachlässigbare Systemzustände hervorruft, ist Gegenstand vielfältiger Debatten. Zum Teil sind diese Debatten innerhalb bestimmter Disziplinen zu finden, zum Teil reichen sie über disziplinäre Grenzen hinaus und zum Teil machen sie gerade den Unterschied im Begriffsverständnis verschiedener Disziplinen aus. In den folgenden Kapiteln wird daher präzise darauf geachtet, die Frage nach den interessierenden Störungen/Belastungen/Veränderungen im jeweiligen disziplinären Kontext zu beantworten. 
An dieser Stelle soll zunächst eine für die zivile Sicherheitsforschung wichtige Unterscheidung näher beleuchtet werden. Zivile Sicherheitsforschung beschäftigt sich primär mit verschiedenen Bedrohungsarten, denen gegenüber eine Gesellschaft verwundbar sein kann. Im vorangegangenen Kapitel wurden hier bereits beispielsweise Dinge wie Naturkatastrophen, Terroranschläge, organisierte Kriminalität und schwere Industrieunfälle genannt. Und es wurde ebenso festgehalten, dass das Kriterium der gesellschaftlichen Relevanz - obgleich dies im Zweifel eher breit zu verstehen ist - einer Störung gegeben sein sollte, damit es sich um einen die SiFo interessierenden Forschungsgegenstand handelt (Scharte et al. 2014: 10). Diese Qualifizierung sagt allerdings noch nichts darüber aus, ob es im Rahmen der SiFo nur um plötzlich auftretende Extremereignisse oder auch um langfristige gesellschaftliche Veränderungen gehen soll. Während erstere, prominent vertreten durch Terroranschläge, als Untersuchungsgegenstand der SiFo sofort einleuchten, haben gerade letztere, etwa in Form des Klimawandels, das Potential, Gesellschaften fundamental zu schädigen.

Der interdisziplinäre Resilienzdiskurs ist hier ebenfalls a priori nicht eindeutig und schlägt, je nach disziplinärer Sichtweise, ein unterschiedliches Vorgehen in Bezug auf die Art der interessierenden Veränderungen vor. Mit Abstand am häufigsten wird Resilienz als Antwortverhalten eines Systems gegenüber einem kurzfristig, vornehmlich überraschend auftretenden Schockereignis definiert (Leach 2008: 3, Woods 2015: 6, Zander/Roemer 2016: 52). Zander und Roemer skizzieren treffend: „Resilienz als ungewöhnliche Reaktionsweise bedarf außergewöhnlicher Risiken, um sich überhaupt zeigen zu können" (Zander/Roemer 2016: 51). Es geht um unerwartet, abrupt eintretende Störungen, die über das hinausgehen, mit dem das System im Normalfall umzugehen in der Lage ist. Die Störungen müssen „erheblich“ sein, um überhaupt Resilienz zu erfordern. Resilienz wird erst sichtbar im Nachgang eines derartig unerwartet auftretenden, die Systemgrenzen übertretenden, massiven Störereignisses. Der amerikanische Organisationspsychologe David Woods spricht in diesem Zusammenhang von einem brutalen und abrupten „Audit“ des Systems (Fooken 2016: 25, Woods 2015: 6, Zander/Roemer 2016: 52). Auf der anderen Seite des Spektrums steht ein Resilienzverständnis, bei dem es nicht mehr primär um diese Art von Störungen geht, sondern auch die Frage nach einer Resilienz im Alltag und gegenüber den täglichen Herausforderungen, mit denen Systeme konfrontiert werden, diskutiert wird. Ein derartiges Resilienz-Verständnis findet sich vor allen Dingen in der psychologischen Forschung wieder (Wadenpohl 2016: 76ff). Verbreitet ist darüber hinaus eine Mischform dieser Ansätze. Resilienz wird als Konzept 
verstanden, mit dessen Hilfe Systemverhalten sowohl im Hinblick auf radikale Schocks als auch langfristig anhaltenden Stress optimiert werden kann (Alexander 2013: 2713, Strambach/Klement 2016: 265f). Allerdings verweist zum Beispiel Lukesch auch auf eine damit in Zusammenhang stehende, paradox anmutende Herausforderung. Bei Systemen, die gleichzeitig gegenüber kurzfristigen, abrupt auftretenden wie auch gegenüber langfristig wirksamen Störungen resilient gestaltet werden sollen, könnten gerade Maßnahmen, die als Abwehr gegen eine Form der Störung hilfreich sind, sich als „Bumerang“ im Hinblick auf andere Störungs-Formen erweisen und so die Gesamt-Resilienz des Systems unter Umständen sogar verringern (Lukesch 2016: 317). Diesem Aspekt folgend, soll an dieser Stelle zunächst darauf verzichtet werden, für das zu entwickelnde Resilienz-Konzept der zivilen SiFo festzulegen, gegenüber welcher Art von Störungen die relevanten Systeme resilient sein sollen.

Die beiden unterschiedlichen Formen von Störungen lassen sich auch verknüpfen und zwar mithilfe der Diskussion über so genannte „tipping points“ bzw. "thresholds“. Auf Deutsch lassen sich tipping points als Umkipp-Punkte und thresholds als Schwellenwerte bezeichnen. UmkippPunkte und Schwellenwerte spielen in der Forschung zu ökologischen und sozial-ökologischen Systemen eine wichtige Rolle. Auch in der Literatur zu Resilience Engineering im Kontext der Organisationswissenschaft sind sie von Bedeutung. Grundsätzlich handelt es sich bei Umkipp-Punkten und Schwellenwerten um das Phänomen, dass Systeme trotz konstanter Belastung über einen relativ langen Zeitraum weiterhin reibungslos funktionieren. Die Belastungen können durch das System absorbiert werden und nach außen zeigen sich in der Funktionsfähigkeit keine unmittelbar sichtbaren Anzeichen für eine bevorstehende, radikale Veränderung. Allerdings führt die konstante, kumulative Belastung dazu, dass sich das System immer stärker dem Schwellenwert nähert. Der Schwellenwert ist häufig a priori unbekannt, so dass die für das System Verantwortlichen diese kritische Entwicklung nur schwer erkennen können (Connelly et al. 2017: 48, Meadows 1999: 1f). Bewegt sich das System nahe dem Schwellenwert, reicht bereits eine kleine, zusätzliche Belastung aus, um eine radikale und dann häufig abrupte Veränderung in Gang zu setzen, obwohl das System mit einer Störung gleichen Ausmaßes zuvor problemlos umgehen konnte (Wink 2016: 4). Gerade im Hinblick auf kritische Infrastrukturen als einem wichtigen Forschungsgegenstand ingenieurwissenschaftlicher SiFo sind Umkipp-Punkte und Schwellenwerte von Bedeutung. Für unsere Gesellschaft ist es beispielsweise wichtig zu wissen, ob das Energiesystem sicher und zuverlässig funktioniert, auch dann noch, wenn es kurzfristigen 
Störungen ausgesetzt ist. Eine Grundvoraussetzung dafür stellt einerseits Wissen darüber dar, wie nahe das Energiesystem ex ante an einem Schwellenwert operiert. Andererseits muss Wissen darüber vorhanden sein, wie Systeme langfristig davon abgehalten werden können, nahe an Schwellenwerten zu operieren.

Bevor gleich einzelne, besonders wichtige disziplinäre Zugänge zum Resilienz-Konzept vorgestellt werden, muss noch eine grundlegende Unterscheidung zwischen verschiedenen Verständnissen des Begriffs herausgearbeitet werden. Diese Unterscheidung wird in der Literatur nur selten explizit gemacht, sie bildet jedoch einen grundlegenden Bestandteil jeder Resilienz-Diskussion. Das gilt auch für die vorliegende Arbeit. Deren zugrundeliegende Zielsetzung enthält zwei implizite Annahmen, nämlich erstens, dass mithilfe von Resilience Engineering die Resilienz der für die SiFo relevanten Systeme erhöht werden soll. Und zweitens, dass diese Resilienz etwas Wünschenswertes oder Gutes ist. Das entspricht scheinbar einem normativen Verständnis von Resilienz.

In der Literatur lassen sich unterschiedliche disziplinäre Traditionen ausmachen, wenn es darum geht, ob Resilienz stärker normativ oder deskriptiv zu verstehen ist. Während die Psychologie Resilienz primär als wünschenswerte Eigenschaft von Individuen auffasst und zum Beispiel nach Wegen sucht, diese in der psychotherapeutischen Praxis zu erhöhen, herrscht in der Ökologie ein stärker analytisch geprägtes Verständnis vor, mit dessen Hilfe das empirische Vorhandensein oder Nicht-Vorhandensein von Resilienz untersucht werden kann (Folke 2006: 259, Holling 1973: 14, Leach 2008: 5, Vogt 2015: 2, Zander/Roemer 2016: 54). Beide Aussagen sind als Ausdruck einer tendenziellen Schwerpunktsetzung zu verstehen. In beiden Disziplinen werden jeweils auch Fragen untersucht, die mit dem gegensätzlichen Resilienz-Verständnis zusammenhängen (Antonovsky 1997: 103f, Brand/Jax 2007: 8, Duit et al. 2010: 5, Kuhlicke 2010).

Ein stärker normativ geprägter Resilienz-Begriff findet sich vor allen Dingen in Arbeiten, die sich relativ nahe an einer praktischen Umsetzung von Forschung bewegen (Kolliarakis 2013: 109). In der Psychologie geht es darum, Individuen in der Führung eines als positiv oder erfolgreich empfundenen Lebens zu unterstützen und durch Resilienz Potentiale und Ressourcen zur Problembewältigung zur Entfaltung kommen zu lassen. Gerade in einer „Zeit der Krise“, wie Vogt formuliert, könne durch Resilienz erreicht werden, dass nicht länger „angstauslösende Katastrophen und Defizite“ im Mittelpunkt stünden. Gleichzeitig nehme die Resilienz-Diskussion das „Lebensgefühl der Krise“ ernst und gebe dem ganzen Diskurs eine „positive Wendung“ (Vogt 2015: 2). In anderen Disziplinen, in denen 
nicht das Individuum, sondern stärker ganze Gesellschaften als Träger von Resilienz im Zentrum stehen, wird Resilienz häufig als politisches Ziel formuliert. So definiert beispielsweise das United Nations Office for Disaster Risk Reduction (UNDRR) als Teil der Vereinten Nationen Resilienz als wünschenswerten und anzustrebenden Zielzustand. Die Resilienz der Menschheit gegenüber primär natürlich verursachten Extremereignissen zu erhöhen, ist ein wesentlicher Teil der Strategie der Organisation (Fekete et al. 2014: 7). Politische Organisationen wie UNDRR tendieren dazu, Resilienz sehr breit zu verstehen und den Begriff stärker als Metapher denn als konsistentes Konzept zu verwenden. In diesem breiten, metaphorischen Verständnis wird Resilienz inhärent als normativ wünschenswert erfasst (Brand/Jax 2007: 4ff, Duit et al. 2010: 5). Dieses normative Resilienz-Verständnis ist jedoch voraussetzungsreich. Es funktioniert nur dort, wo zumindest implizit der Bezug zu den grundlegenden Werten einer Gesellschaft klar wird. Im Kontext demokratischer Gesellschaften geht es dabei um Werte, die in der jeweiligen Verfassung enthalten sind, also typischerweise Dinge wie Menschenwürde, Freiheit und Gerechtigkeit. Dazu kommt im internationalen Diskurs häufig der Begriff der Nachhaltigkeit, verstanden als intergenerationelle Gerechtigkeit (Vogt 2015: 19). Resilienz kann nur in der positiven Interaktion mit diesen Werten als normativ wünschenswert angesehen werden, indem eine Erhöhung der Resilienz also etwa zur Aufrechterhaltung oder gar Steigerung gesellschaftlicher Freiheiten führt, diese aber zumindest nicht negativ beeinflusst. Gerade in der zivilen Sicherheitsforschung spielt die Diskussion des Zusammenhangs von Sicherheit und Freiheit eine herausragende Rolle. Insofern ist es für die vorliegende Arbeit unerlässlich, den Zusammenhang zwischen „Resilienz und Freibeit" zu diskutieren.

Auf der anderen Seite des Spektrums stehen Ansätze, die Resilienz als deskriptives bzw. analytisches Konzept verstehen. In der Ökologie wird der Begriff verwendet, um Systemverhalten im Angesicht widriger Ereignisse zu beschreiben, ohne das Verhalten als wünschenswert zu charakterisieren. Damit stellt Resilienz eine Theorie dar, die auf der Beobachtung von Systemen beruht. Einige Systeme verhalten sich stabil bzw. bestehen auch gegenüber den widrigsten Ereignissen, andere wiederum brechen zusammen oder hören völlig auf zu existieren. Erstere sind resilient, letztere nicht. Mit diesen Aussagen ist keinerlei Wertung verbunden. Insbesondere lassen sie sich treffen, ohne die eben genannten voraussetzungsreichen Überlegungen dazu, ob das betreffende System an sich normativ wünschenswerte Eigenschaften aufweist. Sowohl "positive“ als auch "negative“ Systeme können gleichermaßen resilient sein (Leach 2008: 5). Resilienz ist also 
keinesfalls immer „gut“. Gerade nicht-wünschenswerte Systeme - nichtwünschenswert aus Sicht eines freiheitlich-demokratischen Gesellschaftsbilds - erweisen sich häufig als resilient gegenüber Veränderungen (Folke 2006: 259). Lukesch nennt beispielhaft die Atomruine von Fukushima und ein mexikanisches Drogenkartell als Systeme, die eine hohe Resilienz aufweisen und damit der Gesellschaft insgesamt schaden. Normativ wäre hier die Frage zu stellen, wie die Resilienz solcher Systeme geschwächt und untergraben werden könnte. Dazu ist es notwendig, mittels eines analytischen Verständnisses herauszufinden, worin die Resilienz derartiger Systeme gründet (Lukesch 2016: 303).

Für die vorliegende Arbeit bedeutet das, dass sie ihre Zielsetzung nicht erreichen kann, ohne eine ausführliche Diskussion darüber zu führen, ob Resilienz im Rahmen der zivilen SiFo und ingenieurwissenschaftlicher Ansätze zur SiFo a priori als wünschenswert angesehen werden kann. Den Ausgangspunkt dieser Diskussion soll dabei die Annahme bilden, dass dies nicht der Fall ist. Resilienz wird in den folgenden Kapiteln primär als analytisches Konzept verstanden. Es wird untersucht, was Resilienz ausmacht und wie sich das Konzept im Verhältnis zu anderen relevanten Begrifflichkeiten auszeichnet. Allerdings wird gleichzeitig akzeptiert, dass Resilienz häufig zwar prinzipiell analytisch zur Beschreibung des Verhaltens von Systemen genutzt wird, viele Autoren aber implizit ein normativ positives Begriffsverständnis zugrunde legen. Das Muster lässt sich in beinahe allen Disziplinen erkennen, die sich mit dem Konzept auseinandersetzen. Eine Ausnahme bildet wie bereits erwähnt die Ökologie, aus der die Tradition eines strikt analytischen Verständnisses stammt. Eine völlig anders gelagerte Ausnahme bilden Teile der Sozialwissenschaften, in denen Resilienz zwar auch normativ verstanden, aber im Gegensatz zum wissenschaftlichen Mainstream als negative Systemeigenschaft charakterisiert wird (Blum et al. 2016: 165, Dombrowsky 2012: 286, 2010: 3). Im weiteren Verlauf der Arbeit, wenn es darum geht, ein erweitertes ResilienzVerständnis und daraus Hypothesen für ingenieurwissenschaftliche SiFo im Sinne eines Resilience Engineering zu entwickeln, wird der Aspekt normatives oder deskriptives/analytisches Konzept erneut zu beleuchten sein.

\subsection{Psychologie}

Nach diesem generellen Überblick über Resilienz als Begriff und wissenschaftliches Konzept, geht es im Folgenden nun darum, einzelne diszipli- 
näre Zugänge näher zu analysieren. Keine andere Disziplin wird mit dem Begriff Resilienz so eng verknüpft wie die Psychologie. Gibt man auf Amazon.de den Suchbegriff „Resilienz“ ein, erscheinen auf den ersten zehn Plätzen die folgenden Werke:

1. Resilienz: Das Geheimnis der psychischen Widerstandskraft. Was uns stark macht gegen Stress, Depressionen und Burn-out

2. Resilienz. Wie man Krisen übersteht und daran wächst

3. Resilienz: Die unentdeckte Fähigkeit der wirklich Erfolgreichen

4. Resilienz trainieren: Wie Sie Schritt für Schritt innere Stärke erlangen und Krisen besser überstehen. Das Ausfüllbuch, das stark macht

5. Das wirft mich nicht um: Mit Resilienz stark durchs Leben gehen

6. Resilienz: 7 Schlüssel für mehr innere Stärke

7. Übungsbuch Resilienz: 50 praktische Übungen, die der Seele helfen, vom Trauma zu heilen

8. Resilienz

9. Resilienz für Anfänger: Das Geheimnis erfolgreicher Menschen. Wie Sie Krisen meistern und Widerstandskraft entwickeln

10. Die 50 besten Spiele zur Resilienzförderung ${ }^{8}$

Jedes einzelne dieser Bücher beschäftigt sich mit Resilienz als wünschenswerter Eigenschaft von Individuen, die sich mit Hilfe bestimmter Maßnahmen optimieren lässt. Mit Ausnahme von Platz acht, einem wissenschaftlichen Überblickswerk über Resilienz als Konzept in der Psychologie, lassen sich sämtliche Bücher dem Bereich der Ratgeberliteratur zuordnen. Zwei davon (Nummer sechs und Nummer zehn) nutzen das Bild des Stehaufmännchens auf dem Cover, um Resilienz zu illustrieren. Die Buchtitel zeigen einen Fakt auf, der auch in der wissenschaftlichen Literatur zu Resilienz gesehen wird: Im Kontext der Psychologie ist das Konzept intuitiv plausibel und eingängig. Allerdings zeigen sie auch den Fakt auf, dass der Begriff Gefahr läuft, trivialisiert und ohne zugrundeliegendes wissenschaftliches Konzept verwendet zu werden (Fooken 2016: 13). In den letzten ca. 30 Jahren ist die Popularität des Begriffs im Bereich der psychologischen Forschung sprunghaft gewachsen. Nach Fooken lässt sich Resilienz sogar als „shooting star“ unter den aktuell verwendeten psychologischen Konzepten bezeichnen (Fooken 2016: 25). Abseits der wissenschaftlichen Literatur ist Resilienz für die meisten jedoch weiterhin ein Fremdwort

8 https://www.amazon.de/s/ref=nb_sb_noss_2?

_mk_de_DE=0C3\%85M\%C3\%85\%C5\%BD\%C3\%95\%C3\%91\&url=search-

alias\%3Daps\&field-keywords=resilienz [Stand: 19.2.2018]. 
(Zander/Roemer 2016: 48). Die zunehmende Attraktivität von Resilienz in moderner Ratgeberliteratur lässt sich hierzulande mutmaßlich wie folgt erklären: Der eigentliche Begriff ist unbekannt und klingt daher nach einer neuartigen und innovativen Herangehensweise, um das gewünschte Ziel zu erreichen. Dieses wird durch den zweiten Teil der meisten Buchtitel qualifiziert - exemplarisch sei „Das Geheimnis erfolgreicher Menschen. Wie Sie Krisen meistern und Widerstandskraft entwickeln" genannt.

Abseits dieser populärwissenschaftlichen Verwendung wurde bereits im vorangegangenen Kapitel festgehalten, dass Resilienz als wissenschaftliches Konzept schon zu Beginn des 20. Jahrhunderts Einzug in die Psychologie gehalten hat (Fooken 2016: 25). Die hauptsächliche Anwendung lag seit den 1950er Jahren im Bereich der Entwicklungspsychologie und der Frage danach, wie sich bestimmte Entwicklungsverläufe von Kindern und Jugendlichen erklären lassen, hauptsächlich mit Blick auf erlittene Traumata (Alexander 2013: 2710, Höfler 2016: 101, Kaufmann/Blum 2012: 237). Den Ausgangspunkt dieser Untersuchungen stellten Menschen dar, die in ihrer Reaktionsweise von den erwarteten Verhaltensmustern abwichen. Das Erleiden eines Traumas stellt das Leben von Menschen häufig buchstäblich auf den Kopf. Der Begriff Trauma kann grundsätzlich weit gefasst werden. $\mathrm{Zu}$ Beginn der entwicklungspsychologischen Resilienzforschung ging es zunächst um schwere Krankheiten wie beispielsweise Schizophrenie, später - und vor allen Dingen in den bahnbrechenden Arbeiten von Emmy Werner und Kollegen - waren es widrige Bedingungen wie Armut, soziale Vernachlässigung, Missbrauch oder sonstige problematische Lebensumstände im Lauf der Kindheit. Mit Trauma können aber auch extreme Erfahrungen von Krieg, sexueller und/oder politischer Gewalt oder das Erleben gravierender Naturkatastrophen gemeint sein. Auch Schicksalsschläge wie Tod und Krankheit von Angehörigen, Scheidung oder Arbeitslosigkeit zählen dazu (Fooken 2016: 36). Erfolgreich mit derartigen Traumata umzugehen, das heißt auch danach in der Lage zu sein, ein erfolgreiches Leben zu führen, ist eine enorme Herausforderung. Diese zu bewältigen, kann keinesfalls als normal angesehen werden. Vielmehr ist es sehr viel wahrscheinlicher, durch Traumata langfristige Schäden zu erleiden. Sich als resilient zu erweisen, ist für Menschen also weder normal noch selbstverständlich (Fooken 2016: 15, 40, Luthar/Cicchetti 2000: 858, von Freyberg 2011: 220, Zander/Roemer 2016: 47). Fooken spricht in diesem Zusammenhang von der Frage „wie und unter welchen Bedingungen trotz Risiko und Schädigung sowie gegen alle Erwartung und Wahrscheinlichkeit ein solcher [erfolgreicher] Lebenszusammenhang hergestellt werden kann“ (Fooken 2016: 28). 
Lange Zeit herrschte in der entwicklungspsychologischen Forschung sogar das Verständnis vor, dass krisenhafte Erfahrungen und das Durchleben von Traumata in der Kindheit zwangsläufig zu schwerwiegenden Problemen im späteren Leben führen. Solche Probleme äußerten sich demnach in psychischen Störungen oder sonstigem, von der Norm abweichendem Verhalten und seien aufgrund der erlittenen Traumata unausweichlich. Dieses Verständnis wurde erst durch die „Entdeckung von Resilienz“ abgelöst: Ein statistisch relevanter Teil von Menschen ist in der Lage, gravierende widrige Ereignisse und Lasten relativ gut und unbeschadet zu überstehen. Teilweise sind Menschen sogar fähig, von derartigen Ereignissen zu profitieren und sich positiv weiterzuentwickeln. Das Potential für derartiges Verhalten besteht relativ unabhängig vom Ausmaß des zu überwindenden Traumas, selbst in scheinbar ausweglosen Situationen tritt es zum Teil auf. Verhaltensweisen dieser Art werden mit dem Begriff der Resilienz assoziiert, teilweise aber auch als „posttraumatische Reifung“ bezeichnet. Das Realisieren der empirisch belegbaren Existenz derartiger Personengruppen und die Forschung zur Frage danach, wie resilientes Verhalten erklärt und vielleicht sogar gefördert werden kann, kann als echter Paradigmenwechsel in der Psychologie bezeichnet werden (Fooken 2016: 13ff, 36, Vogt 2015: 5, Zander/Roemer 2016: 50).

Dieser Paradigmenwechsel basierte unter anderem auf der Arbeit der im hessischen Eltville am Rhein geborenen, amerikanischen Entwicklungspsychologin Emmy Werner und ihrer Kollegen, vor allen Dingen Ruth Smith. Werner war zwar nicht die erste Forscherin, die sich im Bereich der Psychologie mit Resilienz auseinandersetzte. Sie kann aber bis heute als eine der einflussreichsten gelten, deren Ideen immer wieder zitiert und als Ausgangsbasis für die weitere Beschäftigung mit dem Resilienz-Konzept genutzt werden. In ihrer berühmt gewordenen Längsschnittstudie The Children of Kauai untersuchte sie knapp 700 im Jahr 1955 auf Kauai zur Welt gekommene Hawaiianer. Im Alter von 1, 2, 10, 18, 32 und 40 Jahren wurde ihre Entwicklung begutachtet und miteinander verglichen (siehe dazu Werner 1971, Werner/Smith 2001, 1982). Wie von Werner erwartet, konnte ein signifikanter Einfluss der Lebensumstände während der Kindheit auf das individuelle Gelingen im späteren Leben als Erwachsener festgestellt werden. Wer eine einfache, unbelastete Kindheit verbrachte, hatte auch im späteren Leben größere Chancen, erfolgreich zu sein, als jemand, der in instabilen familiären oder finanziellen Verhältnissen aufwuchs oder sonstige schwerwiegende Probleme im engen sozialen Umfeld zu meistern hatte. Überraschend war aber eben der Befund, dass auch ein Drittel der 210 Studienteilnehmer, die unter äußerst schwierigen Bedingungen 
aufwuchsen, eine positive Entwicklung aufwiesen und zu Erwachsenen wurden, die ein geregeltes Einkommen, eine stabile Familiensituation und ein gesundes soziales Umfeld ihr Eigen nennen konnten. Sie hatten sich als „resilient“ erwiesen. Werners Interesse galt nun der Frage, wie diese positive Entwicklung mancher Kinder trotz widriger Umstände erklärt werden konnte. Es ging ihr darum, mögliche Schutzfaktoren zu identifizieren, die Menschen dabei helfen, Resilienz gegenüber widrigen Bedingungen aufzubauen. Diese Schutzfaktoren fand sie in Persönlichkeitsmerkmalen, wie einer gesunden Selbstachtung und den Fähigkeiten, sich emotional von Problemen zu distanzieren und offen auf Andere zugehen zu können, um sich so abseits instabiler Familienverhältnisse Rollenvorbilder zu suchen, beispielsweise in Vereinen oder sozialen Einrichtungen (Antonovsky 1997: 55f, Kaufmann/Blum 2012: 237, Luthar et al. 2000: 544, Ungericht/Wiesner 2011: 188f, Zander/Roemer 2016: 50f).

In Folge der wegbereitenden Arbeiten von Werner entwickelte sich das Konzept der Resilienz in der Entwicklungspsychologie weiter. Während zu Beginn der Forschungsarbeiten derart widerstandsfähige Personen noch als „unverwundbar“ galten, werden sie inzwischen durchgängig als resilient bezeichnet. Das Wort impliziert eine positive Adaption dieser Menschen an veränderte Lebensumstände (Kaufmann/Blum 2012: 237, Ungericht/Wiesner 2011: 188f). Eine „robuste psychische Ausstattung“ ist nützlich, um verschiedene Hürden im Leben zu meistern, darunter auch gravierende Probleme und Traumata, ohne dauerhafte Schäden davonzutragen. Es sind primär mentale Prozesse, die darüber bestimmen, wie Menschen mit widrigen Ereignissen umgehen und ob ein erfolgreiches, gesundes Leben trotz großer psychischer Belastungen möglich ist (Fooken 2016: 21, 26, Höfler 2016: 101). Die Frage lautet dann, wie Menschen zu einer robusten psychischen Ausstattung kommen. In dieser Frage spiegelt sich die Diskussion über zugrundeliegende Mechanismen, die Diskussion darum, wie Resilienz mithilfe bestimmter Faktoren gebildet wird. Bei Werner waren dies zunächst rein interne, individuelle Persönlichkeitsmerkmale. In der weiteren Resilienzforschung wurden die internen um externe Faktoren erweitert, die als kausal für die Bildung von Resilienz angesehen werden können. Die daraus abgeleiteten drei Kategorien von Schutzfaktoren galten Fooken zufolge lange Zeit als „zentraler Ertrag der Resilienzforschung." Sie setzen sich aus den bereits genannten, individuellen Persönlichkeitsmerkmalen und darüber hinaus aus Merkmalen des direkten sozialen Umfelds, also der Intaktheit der Familie, und aus Merkmalen des weiteren sozialen Umfelds, etwa Rollenvorbildern in Schule, Vereinen oder der Nachbarschaft, zusammen (Fooken 2016: 33). Als 
förderlich für Resilienz gelten Faktoren wie Intelligenz und Offenheit gegenüber Neuem, das Vorhandensein positiver Emotionen oder die sogenannte „Selbst-Komplexität“, Religiosität und das Vorhandensein sozialer Unterstützung, der sozioökonomische Status und viele mehr (Höfler 2016: 109).

Schon seit den 1970er Jahren gab es allerdings auch Kritik an Werners Ansätzen zur Erklärung des Auftretens von Resilienz, die sich unter anderem auf eine möglicherweise unterkomplexe Analyse relevanter Schutzfaktoren oder generell missverständliche Erklärungsansätze fokussierte. Infolge dessen ist die Liste an Schutzfaktoren sukzessive erweitert worden, mit dem Ziel, Faktoren zu identifizieren, mit deren Hilfe die Gruppe resilienter Personen durch konkrete politische und praktisch-therapeutische Maßnahmen erhöht werden kann. Moderne entwicklungspsychologische Ansätze beschränken sich zudem nicht auf die Kindheit, sondern gehen davon aus, dass Entwicklung prinzipiell ein Leben lang stattfindet und daher auch Resilienz noch im Erwachsenenalter gebildet werden kann. Allerdings haben sich Listen mit konkreten Schutzfaktoren nicht wirklich bewährt. Dies liegt daran, dass personale Resilienz sich neuen Erkenntnissen zufolge erst in einem komplexen Wechselspiel zwischen Verletzlichkeit, erlittenen Traumata und Risiken sowie vorhandenen Ressourcen, Schutzfaktoren und der individuellen Sensibilität für bestimmte Belastungen spezifisch ausbildet (Fooken 2016: 29ff, Vogt 2015: 6). Nach wie vor ist zudem die Frage nicht eindeutig zu beantworten, wie diese Wechselwirkungen zwischen Risiken auf der einen und Schutzfaktoren auf der anderen Seite biochemisch und psychologisch exakt zu erklären sind. Zusammenfassend hat sich in der modernen Entwicklungspsychologie die Auffassung durchgesetzt, dass Resilienz erstens nicht normal oder selbstverständlich - und damit keine Sache der richtigen Einstellung - ist, zweitens nicht einfach durch die Aufsummierung bestimmter Persönlichkeitsmerkmale entsteht und drittens multikausal und individuell spezifisch zu erklären und verstehen ist (Fooken 2016: 22, Zander/Roemer 2016: 53).

Im Rahmen der zunehmenden Komplexität des Konzepts in der psychologischen Forschung besteht nach Luthar und Cicchetti die Gefahr, Resilienz als Persönlichkeitsmerkmal misszuverstehen und mit bestimmten Verhaltensweisen zu verknüpfen. Treten widrige Ereignisse ein, müssten die Betroffenen lediglich solche Verhaltensweisen an den Tag legen, um erfolgreich damit umzugehen. Wenn Resilienz so verstanden wird, kann es in einem nächsten Schritt dazu führen, betroffene Personen für nicht-resiliente Reaktionen auf krisenhafte Ereignisse verantwortlich zu machen, ihnen sozusagen die Schuld am eigenen Unglück zu geben (Luthar/Cic- 
chetti 2000: 862). „Die Gefahr einer Stigmatisierung von Personen, die psychisch erkranken, ist im Rahmen von Resilienzdebatten" gegeben (Höfler 2016: 115). Im Zuge dessen schlagen Luthar und Cicchetti auch vor, im Englischen den Gebrauch des Worts „resiliency“ zu vermeiden, da er ihnen zufolge sehr viel stärker nach einem Persönlichkeitsmerkmal klinge als das neutralere "resilience“ (Luthar/Cicchetti 2000: 862). Das von Luthar und Cicchetti geschilderte Missbrauchspotential ist vor allem ein nichtwissenschaftliches. Beispielsweise wurde in Gesprächen, die der Autor mit einer im Bereich der Weiterbildung tätigen Organisationspsychologin führen konnte, deutlich, dass Unternehmen immer stärker dazu übergehen, ihren Mitarbeitern Resilienz-Trainings anzubieten. Sie versprechen sich davon Mitarbeiter, die auch unter stressigen Bedingungen zuverlässig gute Ergebnisse liefern und sich von Rückschlägen nicht aus der Bahn werfen lassen. Auf den ersten Blick scheinen von derartigen Trainings beide Seiten profitieren zu können. Die Unternehmen erhalten - verkürzt gesagt - widerstandsfähigere Mitarbeiter, die Mitarbeiter selbst sind in der Lage, ihre eigenen, beruflichen Aufgaben besser zu erfüllen und gewinnen gleichzeitig als Person eine größere Resilienz, die sie auch zur Bewältigung anderweitiger Herausforderungen nutzen können. Mit leicht verändertem Blickwinkel lassen sich jedoch mindestens zwei Punkte ausmachen, die Kritik an Resilienz-Trainings rechtfertigen. Zum einen wird das von Luthar und Cicchetti befürchtete „blame game“ zu einer realen Möglichkeit. Mitarbeiter, die trotz Resilienz-Trainings keine zufriedenstellenden Ergebnisse liefern, sind demnach selbst dafür verantwortlich (Luthar/Cicchetti 2000: 862). Zum anderen erlaubt es Unternehmen ungeeignete Arbeitsbedingungen zu rechtfertigen und beizubehalten, da ihre resilienten Mitarbeiter mit diesen umgehen können. Damit verschiebt sich insgesamt die Verantwortung für Erfolg immer stärker vom Unternehmen auf den einzelnen Mitarbeiter. Ein ähnlich gelagertes Phänomen tritt im Bereich der Sicherheitsforschung auf. Dort wird dem Resilienz-Konzept aus sozialwissenschaftlicher Sicht der Vorwurf entgegengebracht, es leiste einer Verantwortungsverlagerung vom Staat auf den Bürger Vorschub (Fekete et al. 2014: 15). Diese häufig unter dem Stichwort „Resilienz und Neoliberalismus" vorgebrachte Kritik wird im weiteren Verlauf der Arbeit noch intensiver diskutiert, auch mit Verweis auf inhaltlich möglicherweise verwandte Entwicklungen in der Psychologie. Zudem gerät durch Resilienz-Trainings die ursprüngliche Perspektive der psychologischen Resilienzforschung aus dem Blick. Die Trainings sind zumeist nicht dazu gedacht, Mitarbeiter für den Umgang mit außergewöhnlichen Ereignissen wie etwa einer Naturkatastrophe zu schulen. Vielmehr geht es um ein 
besseres Funktionieren im Alltag. Resilienz wurde dagegen „entdeckt“ bei Menschen, die extremen Traumata zum Trotz ihr Leben zu meistern in der Lage waren. Es sollte für den normalen Arbeitsalltag keine Notwendigkeit bestehen, sich als resilient zu erweisen.

Trotz der gerade geschilderten immanenten Missbrauchsgefahr kann das Konzept der Resilienz im Rahmen psychologischer Forschung sehr nützlich sein, insbesondere was seine Anwendbarkeit im Rahmen therapeutischer Ansätze angeht. Das geht einher mit einer Art zweitem Paradigmenwechsel, für den die Einführung von Resilienz in die psychologische Debatte (mit-)verantwortlich war. Während der zuvor geschilderte Paradigmenwechsel darin bestand, Traumata überhaupt als grundsätzlich überwindbar zu verstehen, ist mit dem zweiten die Hinwendung zu einem „Konzept mit einem positiven Menschenbild“ gemeint (Fooken 2016: 13). Ältere therapeutische Ansätze fragten klassischerweise nach den Ursachen und Entstehungsbedingungen für Krankheit und danach, wie diese geheilt werden könnten. Bezieht man Resilienz mit ein, geht es dagegen in erster Linie um die Frage nach Schutzfaktoren, die ein Ausbrechen (psychischer) Krankheiten von vorneherein vermeiden helfen (Zander/Roemer 2016: 70). Der Fokus der Aufmerksamkeit verlagert sich also von den Fällen, in denen Traumata langfristige psychische Schäden ausgelöst haben, zu den Fällen, bei denen Menschen langfristig positiv mit den Auswirkungen der Traumata umzugehen in der Lage waren. In der praktischen, therapeutischen Umsetzung führt das zu einer impliziten Verlagerung hin zu präventiven Maßnahmen und weg von rein reaktiver Betreuung/Behandlung bereits manifest gewordener, gravierender psychischer Erkrankungen. Die damit gegebene Möglichkeit, durch Steigerung der Resilienz in möglichst frühen Entwicklungsstadien, vor allen Dingen schon in der Kindheit und bei besonders gefährdeten Personengruppen, die Zahl derjenigen spürbar zu reduzieren, die im weiteren Verlauf ihres Lebens durch Traumata schwer geschädigt werden, macht das Konzept inhärent attraktiv für die therapeutische Praxis (Luthar/Cicchetti 2000: 857, 861). Es geht um die Frage, wie die Resilienz von Kindern und Erwachsenen durch psychotherapeutische, aber dem vorgelagert vor allem auch durch sozialpädagogische Maßnahmen gestärkt werden kann. In der Betonung auf sozialpädagogischen Maßnahmen lässt sich der beschriebene Paradigmenwechsel prototypisch erkennen, da diese häufig vor Eintreten widriger Ereignisse ansetzen und gefährdete Personengruppen ex ante dazu befähigen wollen, mit herausfordernden Lebensumständen erfolgreich umgehen zu können. Psychotherapeutische Maßnahmen setzen hingegen zum Teil erst da an, wo Erkrankungen bereits manifest geworden sind 
(Zander/Roemer 2016: 54). Für die praktische Umsetzung des ResilienzKonzepts gilt aber zugleich: „Resilienzförderung ist keine Zauberwaffe“ (Zander/Roemer 2016: 68). Der Anwendbarkeit wie auch den Erfolgsaussichten sind Grenzen gesetzt. Menschliche Bewältigungskapazitäten lassen sich nicht beliebig steigern. Gleichzeitig haben moderne theoretische Überlegungen zu Resilienz die Kontextabhängigkeit von Schutzfaktoren und die Komplexität des Wechselspiels zwischen situationsbedingten Risiken und individuell-spezifischer Bewältigungsfähigkeit aufgezeigt. Somit müssen pädagogische und therapeutische Maßnahmen individuell auf einzelne Betroffene abgestimmt sein. Trotzdem besteht keine Garantie, dass sie unter veränderten äußeren Bedingungen funktionieren (Höfler 2016: 109, 115, Zander/Roemer 2016: 68).

Über seine Bedeutung für die praktische Anwendung hinaus, lässt der zweite, durch die „Entdeckung“ von Resilienz ausgelöste Paradigmenwechsel, in der theoretischen Diskussion eine sehr enge Verwandtschaft mit der "Salutogenese“ vermuten. Das Konzept wurde durch den israelisch-amerikanischen Soziologen Aaron Antonovsky in den 1970er Jahren entwickelt. Ihm liegt eine verblüffend ähnliche Beobachtung zugrunde wie dem Resilienz-Ansatz. Antonovsky machte in einer Gruppe von Frauen aus Mitteleuropa, die zwischen 1914 und 1923 geboren wurden und während des Zweiten Weltkriegs in Konzentrationslagern der Nationalsozialisten interniert waren, einen Anteil von knapp einem Drittel aus, die trotz dieser extremen Stressbelastung psychisch relativ gesund zu sein schienen (Antonovsky 1997: 15). Diese empirischen Ergebnisse erinnern stark an Emmy Werners resiliente Kinder von Kauai. Antonovsky verweist in seinem berühmt gewordenen Werk Health, stress, and coping. New perspectives on mental and physical well-being von 1979, in dem er die Grundprinzipien seines Verständnisses von Salutogenese darlegt, auch explizit auf Werners Langzeitstudie und nennt sie "eines der aufregendsten Forschungsprojekte”, die er kannte (Antonovsky 1997: 55). Er sieht Resilienz, so wie Emmy Werner das Konzept versteht, als sehr ähnlich zu seinen Überlegungen zur Salutogenese. Werner und andere Autoren würden demnach die „salutogenetische Frage stellen“ (Antonovsky 1997: 18). Antonovskys Salutogenese-Konzept ist insbesondere dafür bekannt geworden, einen Perspektivwechsel in der Forschung über Gesundheit vorgeschlagen zu haben. Das Konzept geht weg von der "pathogenetischen“ Orientierung auf eine Krankheit und Möglichkeiten der Heilung konkreter Krankheiten, hin zu einem Verständnis von Krankheit und Gesundheit als Kontinuum und der Frage, welche eher generisch wirksamen Merkmale bzw. Maßnahmen Menschen befähigen, auf der gesunden Seite des Kon- 
tinuums zu verbleiben (Antonovsky 1997:15ff). Seine theoretischen Überlegungen bieten eine Vielzahl an Verknüpfungen zum interdisziplinären Resilienz-Diskurs. Insbesondere weil das salutogenetische Konzept einige signifikante Ähnlichkeiten mit den theoretischen Ideen der organisationswissenschaftlichen Resilienzforschung aufweist. Dort geht es grundsätzlich um die Analyse erfolgreicher Organisationen und die Frage, welche resilienzsteigernden Funktionsweisen zu deren Erfolg beitragen und nicht länger, wie in klassischeren Safety-Ansätzen, um Gründe für Scheitern und Unfälle (siehe 2.5).

Bevor der Überblick über disziplinäre Zugänge zu Resilienz aus Sicht der Psychologie mit der Darstellung einer aktuellen Definition des Konzepts abgeschlossen wird, sollen noch kurz zwei Aspekte näher beleuchtet werden, die im Kontext der vorliegenden Arbeit von Interesse sind. Zunächst geht es um eine aus der Gerontologie, also der Wissenschaft des Alterns, stammende Entwicklung, nach der Resilienz in der Psychologie nicht länger nur mit außergewöhnlichen, widrigen Ereignissen und Traumata in Verbindung gebracht wird. Vielmehr gebe es eine Tendenz, Resilienz zu einem „Arbeitsmodell“ weiterzuentwickeln, mit dessen Hilfe auch normale Entwicklungsprozesse in verschiedenen Lebensphasen erklär- und nachvollziehbar gemacht werden können (Wadenpohl 2016: 76f). Ein solches Resilienzverständnis ist eng verknüpft mit den spezifischen Fragestellungen der Gerontologie. Im „klassischen“ Modell soll Resilienz dazu führen, nach und trotz Durchleben eines Traumas wieder zu einer „normalen Funktionsfähigkeit“ im Alltag zurückfinden zu können. Verglichen mit dem Status quo ante entspricht das einem Level von wenigstens annähernd 100 Prozent. In der Gerontologie stellen sich aber auch und vor allem Fragen danach was passiert, wenn eine Rückkehr zur normalen Funktionsfähigkeit nicht länger möglich ist. Bedingt durch das Alter oder nicht heilbare Erkrankungen kann Resilienz auch darin gesehen werden „Fähigkeiten eines Verlustmanagements [zu] entwickeln“ (Wadenpohl 2016: 78). Hier sind durchaus Anknüpfungspunkte zu eher langfristigen Fragen aus dem Bereich der zivilen Sicherheitsforschung vorhanden, etwa wenn es darum geht, kritische Veränderungen wie den Klimawandel und seine Auswirkungen zu akzeptieren und als eine Art „neue Normalität“ und Ausgangspunkt in Lösungsstrategien zu berücksichtigen.

Der zweite Aspekt ist die immer stärkere Betonung von Anpassungsfähigkeit als elementar für Resilienz. Diese findet sich auch und gerade in der ökologischen und der organisationswissenschaftlichen Forschung als das zentrale Merkmal von Resilienz wieder und wird daher im nächsten Kapitel entsprechend breit vorgestellt. Allerdings geht es auch in der 
aktuellen psychologischen Resilienzforschung immer mehr um die „Ausbildung adaptiver Strategien“ und die Frage danach, wie diese auf unterschiedlichen Ebenen in menschlichen Entwicklungsprozessen auftreten bzw. wie sie optimiert werden können (Fooken 2016: 33, Höfler 2016: 105). Resilienz wird als dynamischer Prozess verstanden, innerhalb dessen Individuen es schaffen, sich in positiver Art und Weise anzupassen und so gravierende widrige Ereignisse zu überstehen. Dem Mechanismus der Anpassung wird dabei besondere Beachtung geschenkt als entscheidendem Merkmal von Resilienz (Luthar/Cicchetti 2000: 858, Luthar et al. 2000: 543). Damit ist a priori zunächst unklar, welche spezifischen Schutzfaktoren die Ausbildung adaptiver Verhaltensweisen begünstigen. Allerdings lassen sich Faktoren wie Intelligenz, Offenheit für neues oder ein diverses soziales Umfeld dem zuordnen, weil sie betroffenen Personen einen Möglichkeitsraum öffnen, der im Moment der Krise wirksam werden kann. Ein Zusammenhang zwischen „Resilienz und Anpassungsfähigkeit" wird also auch in der psychologischen Forschung gesehen, wenn er dort auch weniger im Zentrum steht, als in der Ökologie. Nichtsdestotrotz ergibt sich hiermit bereits ein Teil der Motivation, die beiden Begriffe im Rahmen der vorliegenden Arbeit intensiver zu diskutieren.

Die wichtigsten Aspekte des disziplinären Zugangs zu Resilienz aus dem Bereich der Psychologie lassen sich mittels einer ausführlichen Definition von Fooken zusammenfassen. Ihm zufolge ist Resilienz ein „als interaktives Phänomen zu denkendes, breit angelegtes multidimensionales hypothetisches Konstrukt" bei dem es darum geht, wie dynamische Systeme mit gravierenden widrigen Ereignissen umzugehen in der Lage sind. Diesem „systematischen Geschehen“ wohnen nach Fooken drei typische Kennzeichen inne, nämlich erstens als Teil einer „Hoch-Risikogruppe“ gegen alle Erwartung positiv im Angesicht von Traumata zu reagieren indem zweitens eine spezifische Widerstandsfähigkeit entwickelt wird, mit deren Hilfe drittens nach Ablauf einer bestimmten Zeit eine Erholung hin zu einer normalen Funktionsfähigkeit stattfindet (Fooken 2016: 31).

\section{3. Ökologie und sozial-ökologische Forschung}

Neben der Psychologie reüssiert Resilienz als Konzept seit beinahe fünf Jahrzehnten auch in der Ökologie bzw. Ökosystemforschung. Generell bezeichnet Resilienz im ökologischen Kontext die Fähigkeit von Ökosystemen, widrige Bedingungen zu überstehen und ist insofern ein „Name für die Erfolgsgeheimnisse der Natur.“ Es geht um „die Überlebenskunst 
und Anpassungsfähigkeit vieler Pflanzen und Tiere“ (Vogt 2015: 9). Während in der Psychologie, bedingt durch die Ausrichtung der Disziplin, vor allem psychische Erkrankungen als Folge traumatischer Erlebnisse im Fokus stehen, geht es bei ökologischen Systemen sehr viel stärker um eine Dichotomie zwischen Überleben und Aussterben. Allerdings lässt kurzfristiges Überleben einer Spezies noch nicht notwendigerweise auf deren Resilienz schließen, so dass diese als schwierig zu beobachten gilt. Resilienz wird in der Ökologie außerdem häufig in den größeren Kontext der Nachhaltigkeit eingebettet und teilweise als Schlüsselbedingung für letztere gesehen. In diesem Zusammenhang ist auch das Verständnis von Resilienz als förderlich für und gleichzeitig abhängig von Biodiversität zu sehen (Adger 2000: 349, Kaufmann/Blum 2012: 239). Gemein ist allen Ansätzen aus der Ökologie, dass sie sich mehr oder weniger explizit auf Buzz Holling beziehen. Obwohl er nicht der erste war, der den Begriff in die Ökosystemforschung einbrachte, ist der Name von Buzz Holling untrennbar mit der Resilienz ökologischer Systeme verbunden (Alexander 2013: 2711). Auf den Einfluss von Hollings Artikel aus dem Jahr 1973 wurde weiter oben bereits eingegangen. Sein Werk wird als eines der ganz wenigen tatsächlich über die Grenzen der verschiedensten Disziplinen hinaus verwendet und bildet so eine Diskussionsgrundlage, auf deren Basis sich unterschiedliche Forscher austauschen können (Francis/Bekera 2014: 100).

Vor Hollings bahnbrechenden Arbeiten herrschte in der Ökosystemforschung ein lineares Verständnis des Funktionierens von Ökosystemen vor. Direkt aus der klassischen Mechanik und Thermodynamik abgeleitet bedeutete dies, dass sich Systeme nach einer Beeinflussung durch einen externen Schock kontinuierlich, gleichmäßig und reibungslos wieder in ihren Ausgangszustand, das stabile Gleichgewicht, zurückbewegen. In diesem Verständnis gibt es sozusagen eine „balance of nature“, eine unbedingte Fähigkeit zur Selbstreparatur, gibt man dem betroffenen System nur genügend Zeit, um zum Gleichgewichtszustand zurückzukehren (Deppisch 2016: 200, Holling 1973: 17, Walker/Cooper 2011: 145ff). Mit dieser klassischen Grundannahme einher gehen auch typische Forschungsfragen und abgeleitete Aufgaben des Managements von Ökosystemen. Beides fokussiert darauf, Ökosysteme jeweils möglichst nahe an einem spezifisch definierten Optimum zu halten und mithilfe dieses zu bewahrenden Zustands eine möglichst große Funktionsfähigkeit sicherzustellen. Betrachtet durch die Brille menschlichen Managements von Ökosystemen geht es darum, einen möglichst hohen Ertrag über möglichst lange Zeit zu erwirtschaften, das heißt die Effizienz des Produktionssystems zu ma- 
ximieren (Kaufmann/Blum 2012: 238). Gegen dieses effizienz-zentrierte und linear-deterministische Gleichgewichtsverständnis von Ökosystemen setzte Holling ein zu diesem Zeitpunkt völlig neuartiges Konzept. Basierend auf der Beobachtung realer Ökosysteme zweifelte er an der Existenz stabiler Gleichgewichte und kontinuierlicher Entwicklung. Die von ihm beobachteten realen Ökosysteme schienen sich vielmehr deutlich weniger deterministisch und dafür komplexer zu verhalten, als die klassische Theorie erwarten ließ. Hollings Beobachtungen und seine daraus gezogenen Schlüsse waren der Anlass für eine "komplexitätstheoretische Wende“ (complexity turn) in der Ökosystemforschung und sind daher für ein Resilienz-Konzept der zivilen Sicherheitsforschung von entscheidender Bedeutung (Kaufmann/Blum 2012: 238, Kéfi et al. 2013: 641). Zumal Holling sich zur Entwicklung seiner Ideen teilweise bei Theoremen aus der Katastrophentheorie bedient hat (Lovins/Lovins 2001: 411).

Zur besseren Unterscheidbarkeit ging Holling in seinen Ausführungen zunächst von geschlossenen Systemen aus, die eine bestimmte Aufgabe zu erfüllen haben und dabei nur von einer geringen Zahl präzise definierter externer Bedingungen abhängen. In derartigen Systemen sei es natürlich, als Zielgröße möglichst invariable Leistungsmaximierung zu setzen und bereits geringfügige Abweichungen nach unten mittels geeigneter Gegenmaßnahmen zu bekämpfen. Diese abstrakte Art von Systemen lässt sich sinnvoll mit dem klassischen Verständnis erklären. Holling zufolge repräsentieren sie jedoch nicht das Verhalten realer Ökosysteme, die beinahe unausweichlich mit einer Vielzahl an Bedingungen und Fakten umgehen müssen, die im Systemumfeld liegen und sich so der unmittelbaren Kontrolle des Systems entziehen. Vor allen Dingen unerwartete Ereignisse stellen das System vor Herausforderungen, die es unter Beibehaltung einer outputmaximierenden Strategie nicht bewältigen kann. Entscheidend für die Analyse von Ökosystemen sind dann nicht länger stabile Gleichgewichte und invariables Verhalten, sondern die Aufrechterhaltung der systemspezifischen Beziehungsmuster. Prinzipiell geht es nicht mehr um quantitative, sondern um qualitative Fragen der puren Weiterexistenz des Ökosystems. Die Wahrscheinlichkeit des Aussterbens steht im Zentrum von Hollings Überlegungen oder anders gesagt die Bedingungen, die für das Weiterbestehen von ökologischen Systemen relevant sind. Diese können - müssen aber nicht - im direkten Widerspruch zu den Bedingungen stehen, die für maximale Effizienz optimal sind. Mit Verweis auf Fischpopulationen und Wasserversorgung gibt Holling zu bedenken, dass die Maximierung des Ertrags durchaus die Risiken für eine Zerstörung des 
jeweiligen Systems erhöhen könnte (Alexander 2013: 2711f, Holling 1973: 1f).

Reale Ökosysteme sind in den allermeisten Fällen keine geschlossenen Systeme. Sie können demzufolge auch nicht adäquat durch klassische Systemmodelle abgebildet werden. Diese fokussierten sich nach Holling auf Gleichgewichtszustände und die unmittelbare Umgebung um diese Zustände. Gegeben ein genau definiertes Set an Parametern kann so die Existenz stabiler Gleichgewichte nachgewiesen werden. Weichen die Parameter allerdings ab, existieren im Rahmen des Modells keine stabilen $\mathrm{Zu}$ stände und das System verhält sich inhärent instabil. Holling gleicht seine theoretischen Ideen deshalb immer wieder mit Beispielen ab, um sie weiterzuentwickeln. Bezüglich der Repräsentation von Ökosystemen durch Modelle schlägt er verschiedene Möglichkeiten vor, wie diese sich verhalten können. Mithilfe eines Beispiels erklärt er etwa die Existenz einer „domain of attraction“ (Anziehungsbereich) innerhalb derer ein stabiler Gleichgewichtszustand herrscht. Hollings Beispielsystem ist eine Population von Jägern, die von einer Population von Beutetieren abhängt und vice versa. Die domain of attraction ergibt sich nun durch ein Phänomen namens "contagious attack“. Nimmt die Zahl der Jäger übermäßig zu, werden diese überproportional häufig kranke und alte Beutetiere jagen, da sie einfacher zu erbeuten sind. Auf diese Weise kann eine größere Zahl an gesunden Beutetieren entkommen, als bei einer rein zufälligen Verteilung der Opfer zu erwarten wäre. Insgesamt ermöglicht das Phänomen dem Ökosystem aus Jägern und Beutetieren innerhalb eines relativ breiten Anziehungsbereichs stabil zu bleiben. Sobald das System aber aufgrund äußerer Einflüsse aus diesem Bereich gerät, verliert es seine Funktionsfähigkeit - im Beispielfall könnten die Beutepopulation und so im nächsten Schritt auch die Jäger aussterben. Modelliert man Ökosysteme unter Nutzung realitätsnäherer Annahmen als im klassischen Modell, erhält man laut Holling starke Hinweise auf die Existenz mindestens einer domain of attraction. Ökosysteme tendieren nun dazu, begrenzte Veränderungen relativ unbeschadet in ihrer domain of attraction verbleibend überstehen zu können. Dieses stabile Verhalten hat allerdings seine Grenzen. Werden diese überschritten, verändert sich das Ökosystem mit hoher Geschwindigkeit in Richtung eines neuen, andersartigen Zustands (Holling 1973: 5ff). Die interessierenden Fragen lauten dann, wie dieser neue Systemzustand aussieht und auf welche Weise der Übergang zwischen den Zuständen erfolgt.

Holling illustriert die radikale und plötzliche Veränderung von Systemzuständen erneut mit einem realen Beispiel. Er nutzt dazu die Geschichte 
der Fischerei auf den Großen Seen Nordamerikas. Den Ausgangspunkt stellte eine extensive Befischung der Bestände verschiedener Fischarten dar. Über viele Jahre konnte eine gleichbleibend hohe Fangquote erzielt werden, die lediglich geringfügig schwankte. Hier lässt sich ein prototypisches Beispiel einer relativ stabilen domain of attraction ausmachen. Innerhalb weniger Jahre kam es dann jedoch zu dramatischen Veränderungen in der Fangquote, die teilweise ins Bodenlose stürzten. Seit den 1940er Jahren kam es in den Großen Seen zu einigen solchen Vorfällen, bei denen gravierende, plötzliche Veränderungen von Fischpopulationen beobachtet werden konnten. Häufig passierten die Veränderungen Holling zufolge auch im Anschluss an Jahre, in denen die Fangquote ungewöhnlich gut ausgefallen war. Besonders interessant für die theoretische Diskussion war jedoch der Fakt, dass die Fischbestände sich nach dem dramatischen Rückgang nicht wieder erholten. Auch dann nicht, als der Druck auf die Bestände durch verminderte Fischerei bewusst reduziert wurde (Holling 1973: 7f). Die Gründe für den plötzlichen Wechsel des Systemzustands sieht Holling weniger in einzelnen, konkreten Anlässen, als vielmehr in dem generellen Ansatz, Systeme durch Optimierung der Effizienz zur Erzielung eines maximalen Outputs zu triggern. Dies führt ihm zufolge zu einem Operieren des Systems nahe der bereits eingeführten Umkipp-Punkte bzw. Schwellenwerte. Wird ein solcherart operierendes System nur einer kleinen, zusätzlichen Belastung ausgesetzt, kann es zum Kollaps kommen und das System dazu gezwungen sein, seinen Zustand abrupt und radikal zu ändern. An dieser Stelle spricht Holling davon, dass die konstant hohen Fangquoten, obwohl situationsspezifisch keinerlei Probleme erkennbar waren, die „Resilienz" des Ökosystems zunehmend reduziert haben und daher, als das „unvermeidliche unerwartete Ereignis“ (inevitable unexpected event) eingetreten ist, die Population zusammengebrochen ist. Übersetzt in den theoretischen Teil seiner Arbeit erkennt Holling vor allen Dingen die Existenz von mehr als einer domain of attraction als interessant für seine weitere Arbeit. Zentral ist die Erkenntnis eines zweiten Gleichgewichtszustands, der bei einer dramatisch niedrigeren Systemleistung vorliegt, sich jedoch nichtsdestoweniger als (mindestens) ebenso stabil erweist, wie der vorherige, bei hoher Systemleistung vorliegende Gleichgewichtszustand (Holling 1973: 8f).

Reale Ökosysteme können also über mehrere unterschiedliche domains of attraction verfügen, weisen aber kein global stabiles Gleichgewicht auf. Sobald sie die Grenzen einer jeweiligen domain of attraction verlassen, verändern sie sich in radikaler Weise. Entweder das System verliert seine Funktionsfähigkeit völlig, es stirbt aus. Oder aber es ist in der Lage, sich 
in einem veränderten Gleichgewichtszustand zu stabilisieren. Mit einem weiteren Beispiel, dem Zyklus der Populationsdichte einer amerikanischen Schmetterlingsart (spruce budworm), fügt Holling seiner Theorie nun erneut eine realistische Annahme hinzu, indem er das Auftreten zufälliger Ereignisse in dem bisher deterministischen Modell zulässt. Der budworm ist ein Forstschädling, der sich hauptsächlich von verschiedenen Tannenund Fichtenarten ernährt. Er ist Teil eines ausgeklügelten, komplexen Ökosystems, das ihn mit seinen Fressfeinden wie auch seiner Nahrung kausal verbindet, jedoch noch durch ein zufälliges Element, das Auftreten einer Reihe von ungewöhnlich trockenen Jahren, ergänzt wird. In einem dichten, gesunden Tannen- und Fichtenwald, findet der budworm zwar genügend Nahrung, wird jedoch durch eine ausreichende Zahl an Fressfeinden in Schach gehalten, so dass seine Population auf einem niedrigen Niveau verharrt. Die Anzahl der Fressfeinde sinkt bedingt durch langanhaltende Trockenheit, so dass der budworm ideale Voraussetzungen zur sprunghaften Vergrößerung seiner Population findet. Diese führt dazu, dass vor allen Dingen die Dichte an Tannen abnimmt, während Fichten und andere Baumarten wie etwa Birken - weniger anfällig sind. Ab einem gewissen Schädigungsgrad bietet der Wald der gewachsenen budworm-Population nicht mehr genügend Ressourcen und diese bricht zusammen. Bis zur nächsten zufällig auftretenden Periode langanhaltender Trockenheit ist das erneute niedrige Populationsgleichgewicht stabil. Innerhalb seines komplexen Ökosystems erfüllt der budworm gleichzeitig auch die Funktion, die Existenz von Fichten und Birken im Wald sicherzustellen. Denn in Zeiten eines Ausbruchs erweisen sich diese Baumarten als relativ resistent, während sie ohne den budworm gegenüber den konkurrierenden Tannen evolutionär benachteiligt wären. Holling zufolge führt nun die Betrachtung des Ökosystems an sich, ohne Beachtung der zufällig auftretenden klimatischen Veränderungen, zu dem Schluss, dass es sich um ein instabiles System mit stark fluktuierenden Populationen handelt. Erst unter Einbeziehung dieses zufälligen Elements wird klar, dass gerade die Instabilität dem komplexen System das langfristige Überleben überhaupt ermöglicht (Holling 1973: 13f).

Unter Zuhilfenahme der bisher genannten Beispiele sieht sich Holling dann in der Lage, sein zentrales Konzept Resilienz zu definieren. Er tut dies in direkter Abgrenzung zum zweiten Begriff, der im Titel seines Artikels auftaucht, der Stabilität von Ökosystemen. Demnach lässt sich das Verhalten von Ökosystemen durch zwei Eigenschaften beschreiben. Zum einen die Stabilität, verstanden als Fähigkeit zum vorherigen Gleichgewichtszustand zurückzukehren, nachdem das System von einer zeitlich 
begrenzten Störung beeinflusst wurde. Ein Ökosystem ist umso stabiler, je schneller es in das Gleichgewicht zurückkehrt und je geringer die Abweichungen davon ausgefallen sind. Ausmaß und Dauer der Abweichung vom Gleichgewichtszustand sind für Holling ein direktes Ergebnis des Grades an Stabilität, den das System aufweist (Holling 1973: 14, 17). Die zweite Eigenschaft ist die Resilienz des Systems. Holling definiert sie in seinem Artikel an zwei Stellen. Aufgrund der Bedeutung dieser Definitionen, sollen beide zitiert werden:

- Resilience „is a measure of the persistence of systems and of their ability to absorb change and disturbance and still maintain the same relationships between populations or state variables" (Holling 1973: 14).

- „Resilience determines the persistence of relationships within a system and is a measure of the ability of these systems to absorb changes of state variables, driving variables, and parameters, and still persist" (Holling 1973: 17).

Was macht Resilienz nach Holling aus? Resilienz ist eine Systemeigenschaft, deren Ausmaß sich darin zeigt, wie wahrscheinlich „Aussterben“ bzw. Fortbestehen des Systems nach einer Störung sind. Ob die Systemleistung, die in der Ökologie häufig durch die Größe der Population gegeben ist, dabei stabil bleibt, ist kein Kriterium für Resilienz. Entscheidend ist lediglich, dass das Ökosystem überhaupt in der Lage ist, die Störung langfristig zu überleben. Interessant sind in diesem Zusammenhang die Begriffe, mit denen verschiedene Forscher die Bestandteile der betreffenden Systeme bezeichnen, die dank Resilienz auch im Fall einer Störung erhalten bleiben. Sie reichen von „Funktionen“ (functions) über „Kontrollen“ (controls) und „Prozesse“ (processes) bis zu „Beziehungen“ (relationships). Im Gegensatz zur Systemleistung sind sie für Holling und seine Nachfolger in der Ökologie die entscheidenden Elemente von Systemen, die diese auszeichnen und ihre Identität bestimmen (Adger 2000: 349, Carpenter et al. 2001: 766, Gunderson 2000: 426, Holling 1973: 17). Wie auch Stabilität ist Resilienz als Systemeigenschaft darauf bedacht, bestimmte Elemente zu bewahren, mit zwei zentralen Unterschieden. Der erste bezieht sich auf die Art der Störungen und der zweite auf die Art der Bewahrung. Stabilität ist dann eine sinnvolle Strategie, wenn es um bekannte und begrenzte Störungen geht und das System zudem nicht in der Nähe von Schwellenwerten operiert. Letzteres ist allerdings in den meisten Fällen nicht direkt erkennbar. Resilienz wird benötigt, wenn Störungen abrupt und unerwartet auftreten. Passend dazu bewahrt Stabilität durch Invariabilität einen vorherigen Gleichgewichtszustand bzw. führt schnell auf diesen zurück. 
Resilienz hingegen bewahrt das System an sich durch möglichst große Variabilität oder Veränderung - der nach außen sichtbaren Systemleistung (Adger 2000: 349, Holling 1973: 17). Bezogen auf Hollings Beispiel des budworms kann von einem sehr hohen Grad an Instabilität des Systems gesprochen werden bei gleichzeitig sehr hoher Resilienz. Und es ist gerade diese Instabilität, die die Resilienz ermöglicht (Holling 1973: 14f). Voraussetzung dafür ist die Existenz multipler Gleichgewichte bzw. domains of attraction. Es geht bei Resilienz um das Fortbestehen von Systemen im Angesicht beliebiger Störereignisse durch Veränderung und Anpassung, indem das System von einer domain of attraction in eine andere wechselt (Alexander 2013: 2711f, Holling 1973: 15, Wink 2011: 113). Die Fähigkeit zur Anpassung durch Wechsel der domain of attraction ist offensichtlich zentral für Hollings Verständnis von Resilienz. Diese wird durch ein möglichst großes Ausmaß an Variabilität oder "fluctuations" erreicht. Damit begründet er sozusagen die zweite große Denkrichtung der Resilienzforschung - abseits des Fachgebiets der Psychologie. Holling setzt sich sehr deutlich von einem Verständnis des Konzepts als „bounce back“ zu einem früheren Systemzustand ab. In drei Worten zusammengefasst, könnte sein Credo lauten „change to survive“. Noch passender wäre allerdings der Satz „adapt to survive“, denn die Veränderung im Angesicht der Störung muss zielgerichtet erfolgen, um den Übergang in eine neue domain of attraction zu schaffen. Das funktioniert mithilfe einer Anpassung des Systems an die durch die Störung möglicherweise veränderten Umweltbedingungen, in denen es existiert. Diese Fähigkeit zur Anpassung, die adaptive capacity, muss auch für die zivile Sicherheitsforschung dahingehend untersucht werden, ob sie in diesem Bereich eine ähnlich entscheidende Wichtigkeit für sich beanspruchen kann, wie in der Ökosystemforschung. Deshalb wird der Zusammenhang zwischen „Resilienz und Anpassungsfähigkeit" im Rahmen der zivilen SiFo entsprechend ausführlich zu analysieren sein.

Im Anschluss an die Definition führt Holling seine Ideen zu einigen Aspekten des theoretischen Ansatzes noch weiter aus. Dazu zählt der Fokus der Aufmerksamkeit, den eine resilienzzentrierte Systemanalyse im Vergleich zu einer stabilitätszentrierten mit sich bringt. Statt den Fragen nachzugehen, wie Systeme nahe eines Gleichgewichtszustands gehalten werden können und wie sie sich dort verhalten, nimmt erstere die Grenzen und die Form der domain of attraction in den Blick. Auf diese Weise können Forscher und Systemmanager in die Lage versetzt werden, die systemkritischen Umkipp-Punkte oder Schwellenwerte zu definieren, ab deren Erreichen ein abrupter Wechsel des Systemzustands von einer domain of attraction in eine andere erfolgt. Für Holling ist die stabilitätszentrierte 
Analyse einfacher durchzuführen und die empirisch wie theoretisch komplexe Identifikation von Umkipp-Punkten gibt ihm in diesem Punkt recht. Nichtsdestoweniger plädiert er für resilienzzentriertes Arbeiten, da nur so realistischere Annahmen zum Systemverhalten getroffen werden und kontraintuitive Effekte beim Management von Systemen vermieden werden können (Holling 1973: 15). Eine solche Analyse führt beispielweise zu dem Schluss, dass Heterogenität für die Resilienz eines Systems von Vorteil ist. Homogenität wirkt sich zwar günstig auf dessen Stabilität aus, vermindert aber gleichzeitig die Fähigkeit zur Anpassung an abrupte Störereignisse. Inwiefern Ökosysteme eher homogen oder heterogen ausgestaltet sind, ist nur einer der Unterschiede, der sich primär durch ihre evolutionäre Entwicklung bestimmt. Ihre „evolutionäre Geschichte“ (evolutionary history) zeitigt verantwortlich für das spezifische Austarieren zwischen Stabilität und Resilienz, welches das Ökosystem kennzeichnet (Holling 1973: 18). Passend dazu formuliert Holling auch „evolution is like a game“, die Evolution ist also ein Spiel bei dem es einzig darum geht, weiter dabei bleiben zu dürfen, zu überleben. Genau aus diesem Grund kann maximale Effizienz und dadurch Optimierung des Outputs keine sinnvolle Strategie sein. Bleibt man im Bild, so setzen Spieler durch eine solche Strategie alles auf eine Karte. Unter stabilen, unveränderten Bedingungen werden sie als besonders erfolgreich gelten. Sobald sich die Bedingungen durch Auftreten einer Störung jedoch ändern, verlieren sie alles. Holling schlägt deshalb eine alternative Strategie vor: "maintaining flexibility above all else" (Holling 1973: 18, eigene Hervorhebung). Die Strategie basiert auf einer Idee, Anpassungsfähigkeit konkret auszugestalten und daher stellt sich für ein Resilienz-Konzept der zivilen SiFo die Frage nach „Resilienz und Flexibilität."

Warum ist Flexibilität im Umgang mit Störungen nötig, um im „Spiel“ Evolution erfolgreich zu sein? Das liegt nach Holling an der Art der Störungen, mit denen Ökosysteme sich konfrontiert sehen. Statt kleinerer Abweichungen von einem wohldefinierten Gleichgewichtszustand, aber immer noch im Bereich der bekannten domain of attraction, mit denen das System durch möglichst große Starrheit oder Robustheit fertig werden kann, geht es um abrupte, radikale, irreversible und vor allen Dingen seltene und unerwartete, überraschende Störereignisse (Kaufmann/Blum 2012: 239). Im Mittelpunkt von Hollings Theorie steht nicht länger im Gegensatz zum klassischen Ansatz der Ökologie - die Annahme, dass künftige Ereignisse erwartbar sind und ablaufen werden, sondern vielmehr deren inhärente Unerwartbarkeit. Es ist die unerwartete, zufällige und überraschende Natur von Störungen, die Resilienz als Überlebensstrategie 
sinnvoll und notwendig macht. Ein zu großer Fokus auf maximaler Stabilität und Effizienz führt für Holling zwingend zu einer reduzierten Resilienz, die genau in dem Moment fehlt, in dem das Ökosystem von einem derart unerwarteten Störereignis getroffen wird. Konzentriert sich menschliches Management von Ökosystemen dagegen von Beginn an darauf, Resilienz aufzubauen, können auch gravierende Störungen durch Dinge wie Flexibilität oder Heterogenität verarbeitet werden. Resilienz erfordert also keine genaue Kenntnis der Zukunft. Es bedarf keiner quantitativ präzisen Vorhersage darüber, welche Form künftige Ereignisse annehmen, um Resilienz als Überlebensstrategie wirksam werden zu lassen (Holling 1973: 21). Empirisch zeigt sich, dass Ökosysteme sich nicht deterministisch verhalten. Unerwartete und überraschende Ereignisse kommen vor. Um die Reichweite eines Resilienz-Konzepts für die zivile SiFo zu definieren, ist es notwendig, „Resilienz und Unsicherheit" zu diskutieren.

Die nicht-deterministische und nicht linear-kausal verlaufende Welt, die Holling als Ausgangsbasis setzt, führt zu einem besonders wichtigen Bestandteil seiner theoretischen Überlegungen, der bisher noch nicht näher beleuchtet wurde. Zu Beginn des Kapitels war von einer komplexitätstheoretischen Wende die Rede. Die von Holling untersuchten und als Beispiele genutzten Ökosysteme sind komplexe Systeme. Das ist zentral für alle weiteren Ergebnisse seiner Arbeit. Buzz Holling beschäftigt sich mit komplexen Systemen und deren Funktionsweise im Angesicht gravierender, unerwarteter Störereignisse. Dieser Aspekt erscheint erst an einer relativ späten Stelle in der Auseinandersetzung mit seinen Ideen, weil er zugleich den Übergang zu einer Disziplin einleitet, die sich im Anschluss an Holling sehr intensiv seiner Konzepte bedient, sich mit ihnen auseinandergesetzt und sie weiterentwickelt hat. Im Rahmen der sogenannten sozial-ökologischen Forschung spielt die Komplexität der Untersuchungsobjekte, nämlich sozial-ökologischer Systeme, eine noch größere Rolle als in den ursprünglichen, ökologischen Ansätzen. Bevor diese Forschungsrichtung gleich näher vorgestellt wird, erfolgen zunächst noch einige Bemerkungen zum Thema Komplexität in der Ökosystemforschung. Laut Holling führt größere Komplexität ganz generell zu einer geringeren Systemstabilität. Die Variabilität eines komplexen Systems ist unter sonst gleichen Bedingungen immer höher, als die eines weniger komplexen Systems. Je komplexer ein System ist, desto unattraktiver wird demzufolge die Option, einen Gleichgewichtszustand unter möglichst allen Umständen aufrecht zu erhalten. Das heißt gleichzeitig: Je komplexer ein System ist, desto attraktiver und wichtiger wird Resilienz als Strategie bzw. Systemeigenschaft für dessen Überleben - vorausgesetzt das System verfügt über mehr als 
eine domain of attraction (Holling 1973: 19). Um ein Resilienz-Konzept für die zivile SiFo zu entwickeln, ist demnach die Frage nach dem $\mathrm{Zu}$ sammenhang zwischen „Resilienz und Komplexität" konstitutiv. Dazu muss untersucht werden, was Komplexität überhaupt bedeutet, was komplexe Systeme ausmacht und inwiefern die relevanten Systeme im Bereich der zivilen SiFo Komplexität aufweisen. Das erfolgt im Rahmen einer komplexitätstheoretischen Analyse. In Anlehnung an Arbeiten, die auf Holling folgend in der Ökosystemforschung durchgeführt wurden, spielen dafür zum Beispiel vielfältige Rückkopplungen, Feedbackschleifen und multiple, sich kreuzende, räumliche und zeitliche Skalen eine Rolle. Voss und Dittmer sprechen diesbezüglich von einem „systemischen“ Ansatz, der eine sehr viel realistischere Analyse des Verhaltens realer (Öko-)Systeme ermöglicht, als der von Holling kritisierte klassische, deterministische Ansatz in der Ökologie (Voss/Dittmer 2016: 186f).

Die gerade genannten Themen- und Fragestellungen sind sämtlich auch für die sozial-ökologische Forschung von Bedeutung. Holling selbst legte mit seiner Arbeit den Grundstein für einen wichtigen Teilbereich dieser Forschungsrichtung, der sich primär mit der Resilienz gekoppelter ökologischer und sozialer Systeme beschäftigt. Damit bewegen sich Forscher wie Carl Folke, Fikret Berkes oder Brian Walker auf dem Entwicklungspfad weiter, den Holling für die Analyse komplexer Systeme vorgezeichnet hat. Insbesondere im Nachgang seines Artikels von 1973 entwickelte er seine eigenen theoretischen Ideen so weiter, dass ihr Geltungsbereich weit über den Ursprung in der Ökosystemforschung hinausreicht und übergreifend eher den Anspruch hat, ein generelles Modell für den Umgang komplexer Systeme mit evolutionären Veränderungen anzubieten (Kaufmann/Blum 2012: 239). Sozial-ökologische Systeme stellen eine Spielart solcher Systeme dar, bei denen der Fokus auf der komplexen Interaktion zwischen sozialen, von und durch Menschen konstituierten Systemen einerseits und natürlichen Systemen wie etwa dem Klima andererseits liegt. Gerade in Deutschland ist der Begriff der sozial-ökologischen Forschung sehr stark mit den Umwelt- und Nachhaltigkeitswissenschaften verbunden (Deppisch 2016: 203). So postuliert etwa das gemeinnützige Institut für sozial-ökologische Forschung aus Frankfurt am Main gleich im ersten Satz seiner Selbstbeschreibung die Zugehörigkeit zu dieser Forschungsrichtung. Das Institut forscht an Themenstellungen wie Wasserknappheit, Klimawandel, Biodiversitätsverlust und Landdegradation, die sämtlich als sozial verursachte ökologische Herausforderungen mit direkten und vor allem indirekten, räumlich und zeitlich verzögerten Rückwirkungen auf die verursachenden, sozialen Systeme verstanden werden können. Die ei- 
gene Disziplin beschreibt es als „Wissenschaft von den gesellschaftlichen Naturverhältnissen" mit transdisziplinärem, über die Wissenschaft hinaus reichendem Anspruch. ${ }^{9}$

Diese Selbstbeschreibung skizziert das grundsätzliche Forschungsprogramm der sozial-ökologischen Forschung bereits sehr treffend. Wie gerade erwähnt geht es darum, soziale und ökologische Systeme als stark gekoppelt zu begreifen, was wiederum ein Verständnis des Verhaltens sozialer Systeme notwendig vom Verständnis des Verhaltens der gekoppelten ökologischen Systeme abhängig macht - und vice versa. Dass soziale Systeme auf eine Vielzahl an Ökosystemleistungen direkt angewiesen sind, ist unmittelbar einsichtig (Adger 2000: 351). Beginnend bei einem geeigneten Sauerstoffgehalt der Atmosphäre, der durch Photosynthese sichergestellt wird, über das Vorhandensein von ausreichend Süßwasser, reguliert über das Klima, bis hin zu einer zuverlässigen Nahrungsmittelversorgung, die ohne Biodiversität undenkbar wäre, ist menschliches Zusammenleben in sozialen Systemen bzw. menschliches Leben an sich nicht denkbar. Dass auf der anderen Seite soziale Systeme einen entscheidenden Einfluss auf verschiedene Ökosysteme ausüben, ist ebenso eindeutig, hier seien nur die Stichworte Klimawandel und Biodiversitätsverlust genannt. Auch auf einer lokaleren Ebene sind soziale und ökologische Systeme eng miteinander verbunden, beispielsweise durch die Abhängigkeit isoliert lebender Völker von der Nutzbarmachung der sie umgebenden Umwelt oder die Ausnutzung regionaler Spezifika bei Wetter und Untergrund zur Produktion von Wein. Sozial-ökologische Forschung setzt sich durch die explizite Anerkennung derartiger Abhängigkeit von zwei als nichtzutreffend erkannten Annahmen ab. Soziale und ökologische Systeme sind voneinander abhängig, sie können also auch nicht unabhängig voneinander analysiert werden. Die relevanten Mechanismen werden erst durch einen systemischen Ansatz sichtbar. Und Ökosysteme reagieren nicht linear, vorhersagbar und kontrollierbar, mit anderen Worten nicht deterministisch auf Beeinflussung durch menschliche Aktivitäten (Folke et al. 2002: 437). Das von Holling vorgeschlagene Resilienz-Konzept stellt einen solcherart systemischen Ansatz dar, der unter anderem dazu kreiert wurde, Systeme $\mathrm{zu}$ analysieren, die sich nicht-deterministisch verhalten. Insofern war es ein logischer Schritt, aus seinem Resilienz-Verständnis heraus ein Konzept zur Resilienz sozial-ökologischer Systeme zu entwickeln (Berkes 2007: 286, Deppisch 2016: 201).

9 https://www.isoe.de/das-institut/leitbild/ [Stand: 27.6.2019]. 
Als besonders einflussreich für die sozial-ökologische Forschung zu Resilienz kann die 1999 gegründete „Resilience Alliance“ gelten. Deren aktueller Direktor Lance Gunderson zählt neben schon erwähnten Forschern wie Carl Folke und Brian Walker, beide ebenfalls Mitglieder der Resilience Alliance, zu den wichtigsten Akteuren dieser Forschungsrichtung. Nicht zuletzt war auch Buzz Holling Mitglied der Resilience Alliance, die sich selbst als internationale, multidisziplinäre Forschungseinrichtung beschreibt, deren Arbeit sich sowohl auf das theoretische Verständnis von Konzepten wie Resilienz, Anpassungsfähigkeit und Transformation im Kontext sozial-ökologischer Systeme bezieht, als auch auf deren praktische Anwendung. Dazu werden drei Strategien verfolgt. Erstens sollen Beiträge zur Weiterentwicklung der Theorie komplexer adaptiver Systeme geleistet werden. Zweitens sollen die theoretischen Ansätze durch regionale Fallstudien, Modellentwicklung, die Nutzung von Szenario-Techniken und weiteren Methoden umgesetzt und getestet werden. Und drittens sollen Richtlinien und Handlungsempfehlungen für Politik und Management erarbeitet werden, die einen Beitrag zur nachhaltigen Entwicklung leisten können. ${ }^{10}$ Für die vorliegende Arbeit sind vor allem die theoretischen Ideen der Resilience Alliance im Kontext der Diskussionen zu „Resilienz und Systemen“, „Resilienz und Komplexität“, „Resilienz und Unsicherheit" sowie „Resilienz und Anpassungsfähigkeit" von Bedeutung. Eine solche, häufig geäuBerte theoretische Annahme besteht darin, dass küstennahe Ökosysteme im Vergleich zu küstenfernen generell eine höhere Resilienz aufwiesen und deshalb auch küstennahe Kommunen resilienter seien als ihre Pendants im Inland. Allerdings ist das zu kurz gedacht. Küstennahe Ökosysteme mögen als solche relativ gesehen resilient sein. Das bedeutet aber gerade nicht unbedingt, dass auch küstennahe Kommunen eine hohe Resilienz aufweisen. Denn die Wirtschaft küstennaher Kommunen hängt häufig vom Funktionieren des küstennahen Ökosystems ab (Adger 2000: 353). Diese einseitige Abhängigkeit von einem resilienten Ökosystem führt unter Umständen zur Vernachlässigung alternativer Entwicklungspfade. Wenn dann dem küstennahen Ökosystem etwas widerfährt, demgegenüber es nicht resilient ist, führt das in direkter Abhängigkeit zu einem $\mathrm{Zu}$ sammenbruch der küstennahen Wirtschaft. Die starke Kopplung der beiden Systeme kann also einerseits die Resilienz einer küstennahen Kommune gegenüber einer Vielzahl von Störereignissen erhöhen. Andererseits erweist sie sich selbst als systemkritische Schwachstelle, wenn das küstennahe Ökosystem einem Schock gegenübersteht, der seine Bewältigungskapa-

10 https://www.resalliance.org/about [Stand: 27.6.2019]. 
zität übersteigt (Adger 2000: 354). Die Resilienz sozialer Systeme kann ohne die Abhängigkeit von Ökosystemleistungen und Variabilität im Verhalten der verknüpften Ökosysteme zu beachten, nicht definiert werden (Adger 2000: 361).

Im Kontext der sozial-ökologischen Forschung beschreibt Resilienz zunächst grundsätzlich die Eigenschaft eines komplexen adaptiven Systems, seine wesentlichen Funktionen auch dann durch Anpassung aufrechtzuerhalten bzw. schnell wiederherzustellen, wenn das System von unerwarteten, abrupt eintretenden Störereignissen betroffen ist. Die Störereignisse beziehen sich häufig - aber nicht notwendigerweise - auf das Eintreten von Naturgefahren wie etwa Erdbeben, Stürmen oder Überflutungen (Birkmann 2008: 10, Deppisch 2016: 201). Die grundlegenden Prämissen für das Verständnis sozial-ökologischer Resilienz unterscheiden sich nicht von denen im Bereich der Ökosystemforschung. Es geht um das „Ausmaß an Störungen“, dem gegenüber sich ein System als stabil erweist, in seiner ursprünglichen domain of attraction verbleibt und in der der vorherige Gleichgewichtszustand wieder erreicht werden kann. Bei Störungen, die dieses Ausmaß übersteigen ist die „Fähigkeit zur Selbstorganisation“ des Systems dafür entscheidend, ob und in welchem Grad es in der Lage ist, auf die Störung durch adaptive und Lern-Prozesse zu reagieren und so unter Beibehaltung seiner charakteristischen Funktionsbeziehungen in eine neue domain of attraction überzugehen (Deppisch 2016: 202). Aus diesen grundlegenden Prämissen leitet sich die zentrale Definition des Konzepts für die sozial-ökologische Forschung ab: „Resilience is the capacity of a system to absorb disturbance and reorganize while undergoing change so as to still retain essentially the same function, structure, identity, and feedbacks“ (Walker et al. 2004). Äquivalent zu Holling wird aus der Literatur deutlich, dass mit change unerwartete, gravierende und abrupt eintretende Schocks gemeint sind. Darüber hinaus beschäftigt sich die sozial-ökologische Forschung als Teil der Nachhaltigkeitswissenschaften aber auch stärker mit langfristigen, langsam verlaufenden Änderungsprozessen wie dem Klimawandel oder dem Verlust der Artenvielfalt und deren Einfluss auf Gesellschaft und Umwelt (Deppisch 2016: 204).

Ausgehend von den genannten Prämissen und der Definition lassen sich in der sozial-ökologischen Diskussion des Weiteren vier „Hauptkomponenten" von Resilienz ausmachen. Die erste Hauptkomponente ist die Fähigkeit, durch Lernen mit Veränderungen und Unsicherheit umgehen zu können, wobei die Widerstandskraft des Systems gegenüber Veränderungen eine wichtige Rolle spielt. Die zweite bezieht sich darauf, Diversität oder Heterogenität zu erhöhen, um Anpassung möglich zu machen. 
Charakteristisch für die Resilienz sozial-ökologischer Systeme ist das Maß an Veränderung, dem das System unterworfen sein kann, bevor es seine Fähigkeit zur Erholung verliert, also einen kritischen Schwellenwert überschreitet. Zur Analyse gehört demnach immer die Frage danach, wie nah ein System einem kritischen Schwellenwert ist. Die dritte Hauptkomponente besteht in der Fähigkeit, verschiedene Formen von Wissen zu kombinieren. Und schließlich müssen Systeme eine Fähigkeit zur SelbstOrganisation aufweisen. Die beiden letzten Komponenten sind vor allem wegen skalenübergreifender Kopplungen relevant. Damit sind Abhängigkeiten gemeint, die beispielsweise von globalen Veränderungen wie dem Klimawandel hervorgerufen werden, aber lokale Auswirkungen haben (Deppisch 2016: 204f, Kuhlicke 2010, Walker et al. 2004). Insgesamt ergeben sich aus der sozial-ökologischen und der ökologischen Diskussion um Resilienz in der Tradition von Holling eine Vielzahl an Anknüpfungspunkten, die für ein Resilienz-Konzept der zivilen SiFo relevant sind und analysiert werden müssen.

\subsection{Sozialwissenschaften}

Psychologie und Ökologie sind die beiden Disziplinen, in denen Resilienz als wissenschaftliches Konzept „entdeckt“ wurde. Mit Blick auf die Literatur lässt sich feststellen, dass das spezifische Verständnis des Konzepts in relativer Unabhängigkeit von den theoretischen Überlegungen der jeweils anderen Disziplin entstanden ist. Nichtsdestotrotz findet sich eine Vielzahl an inhaltlichen Überlappungen. So geht es in beiden Disziplinen immer darum, mit widrigen Ereignissen fertig zu werden. Obwohl ein Individuum oder ein Ökosystem einer gravierenden Belastung ausgesetzt wird, ist es im Anschluss in der Lage, weiter zu funktionieren und dabei insbesondere den Kern seiner Identität zu wahren (Blum et al. 2016: 171). In beiden Disziplinen ist Resilienz nicht als normal oder selbstverständlich anzusehen. Vielmehr speisen sich Analysen in diesem Themenbereich meistens aus „Beobachtungen überraschender Stabilität bzw. Instabilität“, wie Blum et al. formulieren (Blum et al. 2016: 163). Damit liefern sie zugleich eine prägnante Aussage zu einem wesentlichen Unterschied zwischen der psychologischen und der ökologischen Resilienzforschung. Während erstere die empirische Beobachtung von Stabilität trotz erlittener Traumata als zu erklärendes Phänomen betrachtet, steht für letztere das Fortbestehen von Systemen trotz oder gerade aufgrund großer Instabilität im Fokus. Das breite und äußerst heterogene Feld der Sozialwissenschaften bedient sich 
beider Denkrichtungen und entwickelt das Resilienz-Konzept mit Verweis auf seine Ursprünge sowohl in der Psychologie als auch in der Ökologie weiter. Je nach Untersuchungsgegenstand und theoretischer Ausgangsbasis kombinieren verschiedene Bereiche der Sozialwissenschaften dabei stabilitätszentrierte oder instabilitätszentrierte Ansätze mit einer analytischen oder einer normativen Herangehensweise. Der Graben zwischen mechanischen Ansätzen, die Resilienz stärker mit Stabilität und einer Fähigkeit zum bouncing back in Verbindung bringen und systemischen Ansätzen, für die Resilienz primär mit Veränderung und Anpassungsfähigkeit zu tun hat, ist in den Sozialwissenschaften besonders ausgeprägt. Er bestimmt insbesondere deren Auseinandersetzung mit einem - scheinbar - eindeutig stabilitätszentrierten Resilienz-Verständnis in den Ingenieurwissenschaften (Plodinec 2009: 6, Walker et al. 2004, Wink 2011: 113). In einer normativen Wendung wird einem derartigen Resilienz-Verständnis im Sinne eines engineering resilience nach Holling und damit häufig den Ingenieurwissenschaften an sich dann inhärenter Konservatismus unterstellt, der auf Strukturerhalt, Robustheit und Inflexibilität ziele (Blum et al. 2016: 165, Leach 2008: 11). Den Ingenieurwissenschaften wird ein „Steuerungs- und Kontrolloptimismus" attestiert, der aber bezüglich der "Sicherheit vieler großtechnischer Systeme“ nicht gerechtfertigt sei und dem daher „Grenzen gesetzt werden“ müssten (Blum et al. 2016: 166f, siehe dazu 2.6).

Die scharfe Unterscheidung zwischen mechanischen und systemischen Ansätzen kann in der vorliegenden Arbeit einen geeigneten Ausgangspunkt bilden, um aus dem neu entwickelten Resilienz-Konzept für die zivile SiFo in Abgrenzung zu einem aktuell vorherrschenden Verständnis ingenieurwissenschaftlicher Resilienz Hypothesen für Resilience Engineering zu entwickeln. Dazu bedarf es zunächst der Entwicklung des Resilienz-Konzepts für die zivile SiFo unter Rückgriff auf Ideen und Ansätze aus den „ursprünglichen“ Disziplinen Psychologie und Ökologie, aber auch aus dem breiten Bereich der Sozialwissenschaften. Genau darum geht es an dieser Stelle. Im Folgenden werden verschiedene Ansätze aus den Sozialwissenschaften, vor allem der Soziologie, der Politikwissenschaft, den Wirtschaftswissenschaften sowie der Katastrophenforschung, vorgestellt und die Aspekte identifiziert, die einer detaillierteren Untersuchung im Kontext der zivilen SiFo bedürfen. Zudem wird ein kurzer Exkurs zur Risikoforschung hinzugefügt, der für das Verständnis des innerhalb der Resilienzforschung wichtigen Risikobegriffs bedeutsam ist. Generell kann festgehalten werden, dass in all diesen einzelnen Disziplinen der Resilienz-Begriff nicht originär beheimatet war und insofern keine echte „Tradition“ hat. Wenn sich also sozialwissenschaftliche Forschung 
mit Resilienz beschäftigt, tut sie dies immer im Rekurs auf fachfremde Traditionen, zumeist die Ursprünge des Konzepts in der Ökologie, und entwickelt daraus für die sie interessierenden Untersuchungsgegenstände eigene Ideen (Blum et al. 2016: 151). Im Gegensatz zur Psychologie, deren Untersuchungsgegenstand die individuelle Psyche von Menschen ist, und der Ökologie, die sich mit natürlichen Systemen beschäftigt, geht es in den Sozialwissenschaften offensichtlich immer um soziale Systeme und die Beziehungen, Strukturen und Funktionen in denen Menschen ihr $\mathrm{Zu}-$ sammenleben organisieren und die Gesellschaft konstituieren (Alexander 2013: 172). Die im vorangegangen Kapitel vorgestellten Ideen der sozialökologischen Resilienzforschung haben den Weg für eine Übersetzung des Konzepts in eine „rein“ sozialwissenschaftliche Ideenwelt geebnet. Allerdings lag deren Fokus nach wie vor sehr stark auf ökologischen Aspekten und der Frage danach, wie soziale Systeme mit Ökosystemveränderungen und Naturgefahren umgehen. Eine sozialwissenschaftliche Analyse stellt dagegen sehr viel stärker Begriffe wie Governance, Macht, Demokratie oder Freiheit in den Mittelpunkt, wenn es darum geht, mit dem ResilienzKonzept zu arbeiten. Nach wie vor geht es darum, wie Systeme mit disruptiven Ereignissen umgehen, wobei gerade auch die Definition dessen, was als disruptives Ereignis, was als Störung oder Trauma gelten soll, Gegenstand sozialwissenschaftlicher Diskussionen ist und in starker Abhängigkeit von der „Deutung sozialer Prozesse“ steht. Ob bestimmte Formen von Governance sozialen Systemen zu einer höheren Bewältigungskapazität verhelfen, ob es möglich ist, Institutionen so auszugestalten, dass sie mit komplexen Veränderungsprozessen und abrupten Störereignissen erfolgreich umgehen können und wie Institutionen dafür konkret zu verändern wären, sind ebenfalls Fragen, die im Rahmen sozialwissenschaftlicher Resilienzforschung bearbeitet werden (Blum et al. 2016: 172, Duit et al. 2010: $3 \mathrm{f})$.

Eine der ersten und nach wie vor einflussreichsten - auch aufgrund ihrer Trennschärfe - Definitionen von Resilienz in den Sozialwissenschaften, wurde vom amerikanischen Politikwissenschaftler Aaron Wildavsky entwickelt. Wildavsky bettete Resilienz in seinem 1988 erschienenen Buch Searching for Safety in seine Gesamtbetrachtung der Gesellschaft als Ganzes ein. Ihn interessierte vor allen Dingen das Zustandekommen von (technischen) Innovationen. Dabei ging er - wie zuvor Holling im Feld der Ökologie - einen Schritt über klassische Theorien hinaus. In seinem Fall hieß das, sich der klassischen Theorie von Unsicherheit als im Laufe des technologischen und intellektuellen Fortschritts verschwindendem Problem zu widersetzen. Für Wildavsky sind Risiko und Unsicherheit positiv 
besetzte Faktoren, da ohne das Eingehen von Risiken Innovationen und gesellschaftliche Entwicklungen unmöglich werden. Sicherheit entsteht ihm zufolge aus Antizipation und/oder Resilienz: „Anticipation is a mode of control by a central mind; efforts are made to predict and prevent potential dangers before damage is done. [...] Resilience is the capacity to cope with unanticipated dangers after they have become manifest, learning to bounce back" (Wildavsky 1988: 77, eigene Hervorhebung). Antizipation und Resilienz sind im Theoriegebäude Wildavskys nicht als sich gegenseitig ergänzende Strategien zur Erreichung von Sicherheit, sondern als Gegenpole zu verstehen. Ersteres sieht er nämlich als Unsicherheitsvermeidungsstrategie an. Mit der Vermeidung der Unsicherheit - indem zum Beispiel potentiell hilfreiche Medikamente aufgrund von Sicherheitsbedenken nicht zugelassen werden - geht aber auch eine Verhinderung von Innovation und Fortschritt einher. Weil a priori unklar ist, welche Technologien hilfreich und welche schädigend für die Gesellschaft sein werden, plädiert Wildavsky klar für eine Strategie der Resilienz, also einen Ansatz, bei dem sich über Versuch und Irrtum Lernprozesse einstellen, die langfristig zu mehr Sicherheit führen. Resilienz wird von Wildavsky als Sicherheitsstrategie betrachtet, die sich damit befasst, wie die bestmögliche Abwehr von bereits eingetretenen Schadensereignissen organisiert und durchgeführt werden kann. Resilienz ist hier rein reaktiv zu verstehen. Der Definition von Wildavsky und in seiner Nachfolge einer Großzahl von Forschern zufolge sind Antizipation bzw. Prävention nur im Angesicht erwarteter, bekannter und in ihrer Größe berechenbarer Bedrohungen nützliche Strategien. Eine resiliente Gesellschaft ist dagegen in der Lage, mit unerwarteten Bedrohungen umzugehen und durch Adaption und die Anpassung von Prozessen, Organisationen und Systemen Verwundbarkeiten zu minimieren, Instabilitäten zu überwinden und unvorhersehbare, kritische Situationen gut zu überstehen (Comfort et al. 2001: 144, Hutter 2011, Lorenz 2010, Kaufmann/Blum 2012: 240ff).

Wildavskys theoretische Überlegungen und seine klare Resilienz-Definition sind in mehrfacher Hinsicht relevant für die vorliegende Arbeit. Die Prominenz der Definition verdankt sich auch ihrer Kürze und prägnanten Abgrenzung. Für Wildavsky wird Resilienz erst dann wirksam, wenn ein widriges Ereignis eingetreten ist (Hutter 2011). Darin unterscheidet er sich nicht von Forschern wie Werner oder Holling und der psychologischen wie ökologischen Tradition. Des Weiteren müssen die widrigen Ereignisse eine bestimmte Eigenschaft aufweisen, nämlich unerwartet eingetreten zu sein. Das leitet sich direkt aus seiner Unterscheidung zwischen Antizipation und Resilienz ab. Auf erwartete, bekannte Störungen können 
Systeme sich adäquat vorbereiten, es besteht keine Notwendigkeit für Resilienz (Lorenz 2010). Umso wichtiger wird es, den Zusammenhang zwischen „Resilienz und Unsicherheit“ näher zu analysieren. Mindestens genauso wichtig ist Wildavskys Paraphrasierung der Art, in der Systeme auf unerwartete Störungen reagieren, durch das bereits häufig genannte „bounce back“. Gerade in der sozialwissenschaftlichen Diskussion wird dieser Begriff sehr häufig mit einem mechanischen Verständnis von Resilienz gleichgesetzt und unter Verweis auf Hollings Gedankenwelt als stabilitätszentrierte Sichtweise gekennzeichnet, deren Fokus auf der Rückkehr zu einem vorherigen Gleichgewichtszustand liege. Anpassungsfähigkeit, Lern- und Veränderungsprozesse blieben demnach außen vor. Das widerspricht allerdings zumindest zum Teil Wildavskys eigenem Verständnis der bounce back-Idee. Eine resilienzorientierte Strategie setzt für ihn auf den Nutzen von Versuch und Irrtum und daraus ableitbare Lernprozesse. Gegenüber unbekannten Bedrohungen bedeutet das das Ausnutzen generalisierter und flexibler Ressourcen, um einen bereits eingetretenen Schaden zu minimieren, bevor er gesellschaftsschädigende Ausmaße annimmt (Comfort et al. 2001: 144, Hutter 2011). Er verbindet also „Resilienz und Flexibilität" indem er letztere als eine notwendige Bedingung für erfolgreiches bouncing back definiert.

Entscheidend ist darüber hinaus die Fähigkeit, nach Eintreten eines widrigen Ereignisses möglichst schnell zu handeln. Hier zeigen sich insofern Anknüpfungspunkte an Psychologie und Ökologie, dass der Erfolg einer derartigen Strategie voraussetzungsreich ist. Um auf unerwartete Bedrohungen schnell und adäquat reagieren zu können, benötigen Entscheidungsträger ein großes Erfahrungswissen und die Fähigkeit, dieses situationsspezifisch adaptieren und auf ein bisher unbekanntes Szenario anwenden zu können - was keinesfalls normal oder selbstverständlich ist (Comfort et al. 2001: 144). Neben vielen Parallelen gerade zu Hollings Begriffsverständnis gibt es aus sozialwissenschaftlicher Sicht auch deutliche Unterschiede zwischen Wildavskys Ideen und ökologisch bzw. sozial-ökologisch geprägten Ansätzen. Denn Holling verstand seine Analyse auch als Kritik an der vorherrschenden marktwirtschaftlichen Logik, die vor allen Dingen über möglichst große Effizienz den Output eines Systems zu maximieren sucht und deren erfolgskritische Variablen primär in kurzfristiger Gewinnmaximierung zu finden sind. Die Erfolgsvariable bei Holling ist dagegen das langfristige Überleben des Systems bzw. seiner zugrundeliegenden Struktur und Identität. Wildavsky bringt auf der anderen Seite marktwirtschaftliche Logik und Resilienz insofern in Übereinstimmung, dass er Gesellschaften und deren Ökonomien möglichst wenig reguliert 
sehen möchte. Denn überbordende Regulierung versteht er als innovationshemmend. Fortschritt entstehe demnach durch Fehler. Und um diese Fehler nicht in gesellschaftsschädigende Dimensionen wachsen zu lassen, sieht er Resilienz als geeignete Strategie an. Die dafür notwendigen Lernprozesse und flexiblen Anpassungen finden sich für Wildavsky ebenfalls im freien Spiel der Kräfte am Markt (Kaufmann/Blum 2012: 242).

Im Anschluss an derartige Überlegungen verwundert es nicht, dass auch die Wirtschaftswissenschaften ein Teilbereich der Sozialwissenschaften sind, der sich immer stärker mit dem Resilienz-Konzept auseinandersetzt. Holling folgend und insofern im Unterschied zu Wildavsky wird Resilienz in den Wirtschaftswissenschaften zumeist als "Gegenbegriff" zu Effizienz gesetzt und als „Störungstoleranz" verstanden. Um derart störungstolerant zu sein, müssen Reserven und im Normalfall ungenutzte Spielräume vorgehalten werden, die erst im Ausnahmefall genutzt werden. Solche Reserven sind aus Effizienzgesichtspunkten überflüssig, ja sogar schädlich im Sinne einer Logik der Maximierung des Outputs. Effizienz ist demnach nur in unmittelbarer Nähe von Gleichgewichtszuständen eine sinnvolle Strategie, eine resilienzorientierte ökonomische Strategie betont hingegen Vorsorge und Risikominimierung, unter anderem durch die gerade genannte Störungstoleranz. Die Ähnlichkeiten dieser Konzeption mit dem klassischen Verständnis aus der Ökologie haben auch damit zu tun, dass wirtschaftswissenschaftliche Resilienzforschung sich häufig der traditionellen Konzepte bedient. Denn der Begriff wurde dort bisher nicht in ähnlich systematischer und umfassender Weise konzeptualisiert. Unter Rekurs auf Ökologie und sozial-ökologische Forschung bedeutet Resilienz in den Wirtschaftswissenschaften knapp zusammengefasst also die Fähigkeit zur Anpassung (Plöger/Lang 2016: 359, Vogt 2015: 11f). Im Unterschied zu ökologischen Systemen, bestehen soziale Systeme jedoch aus Akteuren, aus Menschen, die in der Lage sind, bewusste Handlungen vorzunehmen, darüber zu reflektieren und vor allen Dingen ihre Handlungen situationsspezifisch anzupassen, flexibel auf veränderte Umstände zu reagieren und durch kreative Lösungen Problemen zu begegnen, die ihnen vorher unbekannt waren (Strambach/Klement 2016: 269). Mit Blick auf die für die zivile SiFo interessierenden Systeme wird hier unmittelbar einsichtig, warum die Frage nach dem Zusammenhang zwischen „Resilienz und Flexibilität“ oder auch Kreativität für die vorliegende Arbeit von großer Bedeutung ist. Und trotz Hollings beeindruckenden Beispielen aus der Natur, etwa das komplexe Wechselspiel zwischen Schädlings-Population, Waldcharakteristik und externe Umwelteinflüsse betreffend, drängt sich der Eindruck auf, dass sein Konzept von Resilienz für soziale Systeme und die in ihnen 
handelnden Menschen noch geeigneter sein könnte als für Ökosysteme. Das Resilienz-Konzept ist gleich in mehrfacher Hinsicht attraktiv für die Wirtschaftswissenschaften. Denn es erlaubt eine systemische Analyse von Störereignissen verschiedener Art, vor allen Dingen turbulenter, schnell ablaufender Krisen und deren Auswirkungen auf komplexe adaptive Systeme. Zudem erlaubt die Konzentration auf das langfristige Überleben von Systemen auch der Ökonomie den Blick von kurzfristigen, rein auf Nutzenmaximierung fokussierten Analysen weg auf Fragen nachhaltigeren Wachstums zu richten (Strambach/Klement 2016: 275).

Das gilt vor allem für die Betrachtung regionaler ökonomischer Systeme, die im Zentrum des Interesses der Wirtschaftsgeographie stehen. Entsprechend wird dort Resilienz als Fähigkeit einer regionalen Ökonomie verstanden, ihre relevanten Funktionen auch dann noch zu erbringen, wenn sie negativen Umwelteinflüssen ausgesetzt wird (Strambach/Klement 2016: 265). Mit Umwelteinflüssen sind in diesem Fall neben der natürlichen Umwelt sämtliche weiteren systemexternen Faktoren gemeint. Für regionale Ökonomien können das beispielsweise die Lage der Weltwirtschaft, die Preise von Rohstoffen oder die Entwicklung neuer Technologien sein, aber auch der langfristige Klimawandel oder kurzfristige Naturereignisse wie Stürme oder Überschwemmungen. Generell geht es sowohl um gravierende, abrupte Schocks als auch eher langsam verlaufende Veränderungsprozesse. Die relevanten Funktionen bestehen zum Beispiel in der Anzahl und Qualität der bereitgestellten Arbeitsplätze oder der Summe an Steuermitteln, die von den ansässigen Unternehmen entrichtet wird. Eine resiliente regionale Ökonomie ist in der Lage, ihre derart verstandene wirtschaftliche Funktionsfähigkeit auch nach exogenen Schocks aufrechtzuerhalten und zwar mithilfe einer „systemischen Anpassungsfähigkeit.“ Gerade sozioökonomische Krisen, die durch einen technologischen Fortschritt oder Wandel in gesellschaftlichen Prioritäten ausgelöst werden, können regionale Ökonomien, die häufig auf die Produktion bestimmter Güter oder Dienstleistungen spezialisiert sind, vor große Herausforderungen stellen. Um diesen erfolgreich zu begegnen, ist Anpassungsfähigkeit zwingend notwendig, starres, sozusagen „stabiles“ Verharren in den zuvor genutzten Strukturen per definitionem unsinnig (Plöger/Lang 2016: 357, Strambach/Klement 2016: 265f, Wink 2011: 111). Wenn es einer regionalen Ökonomie im Nachgang einer Krise gelingt, beispielsweise das vorherige Beschäftigungsniveau wieder zu erreichen oder gar zu übertreffen, kann von „umfassender Resilienz“ die Rede sein. Dies gilt aber immer nur in Abhängigkeit und in Bezug auf die konkrete Situation. So ist nicht auszuschließen, dass gerade die Lösungsstrategie, die dazu beigetragen hat, 
die akute Krise erfolgreich zu überstehen, langfristig zum Ausgangspunkt neuer Vulnerabilitäten wird (Lintz et al. 2016: 336f, Plöger/Lang 2016: 360). Solche „kumulative[n] Ketten komplexer Wechselwirkungen“ können auch in der zivilen SiFo auftreten und sind im Zusammenhang mit „Resilienz und Komplexität“ zu diskutieren (Vogt 2015: 12).

Ähnlich wie in Psychologie und Ökologie hat sich das Resilienz-Konzept in den Sozialwissenschaften in Folge pionierhafter Arbeiten wie der von Wildavsky weiterentwickelt und sehr stark ausdifferenziert. Die gerade skizzierten Ansätze aus den Wirtschaftswissenschaften sind ein Beispiel dafür. Mit Blick auf die Soziologie kann beispielsweise zwischen „einfacher Resilienz' mit einem Fokus auf der Bewältigung akuter Schadenslagen, und ,reflexiver Resilienz', die auf Veränderung grundlegender Strukturen abstellt, um die Möglichkeit des Eintretens von großräumigen Schadenslagen langfristig zu minimieren" unterschieden werden (Blum et al. 2016: 156). Äquivalent zur Entwicklung in der Psychologie, aufgezeigt durch die kurze Liste an Resilienz-Ratgebern, besteht auch in den Sozialwissenschaften eine starke Tendenz, Resilienz als „buzzword“ zu nutzen und seine theoretischen Hintergründe nicht weiter zu beleuchten. Der Begriff wird $\mathrm{zu}$ einer Art Sammelbecken für Eigenschaften, Fähigkeiten oder Ressourcen, die „allgemeine Lebenskompetenz“ fördert. Gleichzeitig wird Resilienz so zu einem „erstrebenswerten Ziel oder Leitbild“, das durch konkrete Handlungen erreicht werden soll. Ohne theoretisch fundiertes Konzept ist jedoch weder eine nützliche Definition noch eine Abgrenzung von Resilienz gegenüber anderen relevanten Konzepten möglich. Die Sozialwissenschaften sollten daher nach Blum et al. zunächst „Abstand von solchen unmittelbar auf praktische Gestaltung zielenden Fragen" halten (Blum et al. 2016: 152, 159, 163f). Inwiefern Resilienz nur ein Schlagwort ist oder ob hinter dem Begriff sogar ein von vielen Sozialwissenschaftlern als normativ negativ empfundenes politisches Programm steckt, wird unter dem Stichwort „Resilienz und Neoliberalismus“ näher diskutiert.

Ein für die zivile SiFo besonders wichtiger Aspekt aus der sozialwissenschaftlichen Diskussion tangiert einen als „feststehende Kausalkette“ bezeichneten Zusammenhang zwischen zunehmender Vernetzung, größerer Komplexität und wachsender Unsicherheit, der wiederum dazu führe, dass unsere Gesellschaften immer verwundbarer werden und die Gefährdung generell zunehme (Blum et al. 2016: 161). Vermeintlich steigende Verwundbarkeit (Vulnerabilität) spielt in Zusammenhang mit Resilienz tatsächlich eine entscheidend wichtige Rolle. Unter Vulnerabilität wird zumeist eine Kombination aus Anfälligkeit und Bewältigungskapazitäten verstanden. Diese beziehen sich auf unterschiedliche Bereiche der interes- 
sierenden Systeme, etwa physische, soziale, ökonomische und ökologische (Christmann/Ibert 2016: 237). Die beiden Begriffe „Resilienz und Vulnerabilität" werden in der Literatur oft als zwei Enden eines Spektrums betrachtet, als gegenteilige Ausprägung derselben Eigenschaft (Fooken 2016: 1). Inwieweit dies plausibel ist bzw. warum Resilienz gerade im Kontext der zivilen SiFo nicht als Gegenteil von Vulnerabilität verstanden werden kann und sollte, wird im weiteren Verlauf der Arbeit diskutiert. Die genannten Phänomene zunehmender Komplexität und Vernetzung sowie steigender Unsicherheit werden darüber hinaus nicht unhinterfragt als die Vulnerabilität von Gesellschaften erhöhende Faktoren angenommen. Vielmehr erfolgt eine detaillierte Analyse der Frage danach, wie „Resilienz und Komplexität" als Konzepte miteinander interagieren, zusammenhängen, sich voneinander unterscheiden, gegenseitig bedingen und notwendig machen oder sich erschweren. Selbiges gilt für die Frage nach „Resilienz und Unsicherheit", so dass der Anspruch der vorliegenden Arbeit durchaus auch darin besteht, die häufig unreflektierte „Kausalkette“, von der Blum et al. sprechen, zu hinterfragen.

Zumal eine postulierte Unsicherheit noch weitere Fragen aufwirft, etwa die, wie Gesellschaften sich adäquat auf Dinge vorbereiten können, von denen sie nichts wissen. Wenn unbekannt ist, wie schlimm es kommt, scheint es nicht unvernünftig, mit dem Schlimmsten, dem „worst case“ zu rechnen. Eine solche Entwicklung lässt sich sozialwissenschaftlichen Analysen zufolge in aktuellen sicherheitspolitischen Debatten und gesetzgeberischen Tätigkeiten ausmachen (Blum et al. 2016: 156). Der ResilienzDiskurs ist hier insofern inhaltlich beteiligt, als er sich in den Sozialwissenschaften seit einigen Jahren auch mit dem Phänomen des Terrorismus beschäftigt. Ursprünglich - und erklärbar durch die ökologische Tradition - wurde die Resilienz sozialer Systeme vor allem im Hinblick auf Naturgefahren untersucht. Diese Forschungsrichtung ist nach wie vor zum Beispiel in den USA sehr prominent, was unter anderem an der Studie Disaster Resilience. A National Imperative der National Academies sichtbar wird (siehe The National Academies 2012). Nach den Terroranschlägen des 11. September 2001 in New York und Washington, D.C. sowie denen in Madrid am 11. März 2004 und London am 7. Juli 2005 begannen aber immer mehr Sozialwissenschaftler wie beispielsweise Jon Coaffee, Steve Flynn oder Charlie Edwards damit, das Konzept auch im Zusammenhang mit Terrorismus zu diskutieren. Die Gefahr terroristischer Anschläge und die Frage danach, wie Gesellschaften und ihre relevantesten Subsysteme dagegen geschützt werden können, ist auch zentral für die zivile SiFo. Daher muss das Verhältnis von „Resilienz und Terrorismus" näher beleuchtet 
werden, um ein Resilienz-Konzept für die SiFo entwickeln zu können. Gleichzeitig bedarf es einer Generalisierung dieser Diskussion. Resilienz, verkürzt verstanden als Strategie zur Vorbereitung auf das Unerwartete, könnte wie beschrieben einen Impuls zu stärkerer sicherheitspolitischer Gesetzgebung auslösen deren Form dann - zur Verhinderung eines worst case - häufig eher freiheits- und grundrechts-einschränkend zu denken wäre (Blum et al. 2016: 156). In einer derartigen Definition würde mehr Resilienz zu weniger gesellschaftlicher Freiheit führen. Die sozialwissenschaftliche Diskussion kennt aber auch die Gegenposition, nach der gerade das Konzept Resilienz in der Lage sei, die scheinbaren Gegensätze der beiden gesellschaftlichen Zielvorstellungen Freiheit und Sicherheit miteinander zu vereinen (Flynn 2011: ii, Riescher 2013: 1067f). Deshalb bedarf ein Resilienz-Konzept der zivilen SiFo notwendigerweise einer intensiven Diskussion des Begriffspaares „Resilienz und Freiheit".

Stärker die Chancen als die Risiken einer Strategie zur Erhöhung gesellschaftlicher Resilienz betont auch Edwards in seinem 2009 erschienenen Werk Resilient Nation. Edwards beobachtet ein Paradoxon: Individuelles Leben war - in Großbritannien - nie sicherer. Die Menschen haben noch nie gesünder gelebt, waren nie reicher und konnten sich so vieler Hilfsmittel bedienen, um ihr Leben angenehmer zu gestalten. Gleichzeitig führen individuelle Ängste, gesellschaftliche Komplexität und hohe Erwartungen an das Leben dazu, dass in der Gesellschaft das Gefühl herrscht, größeren Risiken als jemals zuvor ausgesetzt zu sein. Gegenüber diesen Risiken und Edwards weist einige Seiten später darauf hin wie oft es trotz eines insgesamt hohen Sicherheitsniveaus zu gravierenden widrigen Ereignissen kommt - gilt es, resilient zu sein (Edwards 2009: 10, 16, 35). Edwards fasst sein Verständnis von Resilienz in der folgenden Definition zusammen: „The capacity of an individual, community or system to adapt in order to sustain an acceptable level of function, structure and identity." Die Definition weist nicht zu übersehende Gemeinsamkeiten mit den traditionellen Ansätzen der Ökologie und der sozial-ökologischen Forschung auf. Noch eindeutiger als sie stellt Edwards die Fähigkeit zur Anpassung in den Mittelpunkt. Hier bestehen Ähnlichkeiten zum weiter oben skizzierten Resilienz-Verständnis der ökonomischen Forschung, in dem ebenfalls die Anpassungsfähigkeit zentral ist. „Resilienz und Anpassungsfähigkeit“ wird also nicht nur in der Psychologie, der Ökologie und der sozial-ökologischen Forschung diskutiert. Auch für verschiedene Bereiche der Sozialwissenschaften lässt sich Resilienz nur verstehen, wenn zeitgleich Anpassungsfähigkeit mitgedacht und analysiert wird. In Abgrenzung zur ökonomischen Forschung, deren Untersuchungsgegenstand im Rahmen der Resilienzfor- 
schung eher ökonomische Systeme sind als einzelne Menschen, misst Edwards der Resilienz von Individuen besondere Bedeutung bei. Seinen eigenen Aussagen zufolge nutzt er hier bewusst eine provokante Zuspitzung, um darauf hinzuweisen, dass Resilienz - spezieller „community resilience“ - zukünftig verstärkt in einem bottom-up-Prozess und von sich selbst befähigenden Individuen ausgehen müsse und nicht mehr als eine Art „Serviceleistung“ staatlicher Behörden angesehen werden könne (Edwards 2009: 17f).

Ausgehend von der Definition des Konzepts Resilienz schlägt Edwards als Ergebnis seiner Studie die Umsetzung verschiedener Maßnahmen vor. Diese fasst er unter den Oberbegriffen „Engagement“, „Education“, „Empowerment“ und „Encouragement“ zusammen. Unter Engagement versteht Edwards eine Weiterentwicklung der Kommunikation zwischen Regierung, lokalen Autoritäten, Katastrophenschützern, Rettungskräften, etc. auf der einen und Bürgern auf der anderen Seite. Es geht nicht um einseitige Informationsweitergabe, sondern um ein wirkungsvolles Einbeziehen der von Katastrophen potentiell direkt Betroffenen vor Ort. Die Anliegen und Kompetenzen von Bürgern sollen durch einen Dialog auf Augenhöhe ernst genommen werden. Im nächsten Schritt ist Bildung (Education) essentieller Bestandteil der Schaffung einer resilienten Gesellschaft. Ein grundlegendes - und je nach persönlicher (z.B. geographischer) Betroffenheit auch spezielleres - Wissen über Bedrohungen und Risiken einerseits sowie das richtige Verhalten im Katastrophenfall andererseits muss möglichst jedem Menschen vermittelt werden. Um nicht Gefahr zu laufen, durch übertriebene Warnungen Panik in der Gesellschaft zu verursachen, müssen die entsprechenden Informationen als Teil des Lehrplans in die alltäglichen Lernaktivitäten von Schülern integriert werden. Durch Bildung und Engagement wird Empowerment möglich. Damit meint Edwards Maßnahmen, die es Betroffenen während einer Katastrophe ermöglichen, selbst tätig zu werden und die negativen Konsequenzen des Ereignisses zu begrenzen. Encouragement entspricht dann einem eher psychologischen Teil von Empowerment. Die Bürger sollen dazu ermutigt werden, selbst aktiv zu werden und so zur Resilienz der Gesellschaft beizutragen (Edwards 2009: 80ff). Insgesamt betrachtet stellt sich bei Edwards Verständnis unmittelbar die Frage nach dem Zusammenhang zwischen „Resilienz und Vertrauen“, sowohl in eigene Fähigkeiten, in die des eigenen sozialen Umfelds und in die des Staates.

Abschließend sollen aus dem Bereich der Sozialwissenschaften noch die Katastrophenforschung sowie die Risikoforschung kurz vorgestellt und auf ihren Umgang mit dem Resilienz-Konzept hin untersucht werden. Begriff- 
lich ist häufig unklar, ob es um Risiko-, Katastrophen- oder Sicherheitsforschung geht, welcher Begriff für welche Inhalte als passender anzusehen ist und vor allen Dingen, wie und ob sie sich voneinander unterscheiden (Fekete et al. 2016: 215). Sicherheitsforschung als dezidiertes Forschungsprogramm ist relativ neu, eine kurze Beschreibung der Entwicklungsgeschichte dieser Forschungsrichtung in Deutschland wurde im Einleitungskapitel dargestellt. Dass die Sicherheitsforschung in Deutschland sich immer stärker mit dem Resilienz-Konzept auseinandersetzt ist evident. Es wird nicht zuletzt durch die vorliegende Arbeit nachgewiesen, aber auch in der Literatur eindeutig so gesehen (Fekete et al. 2016: 218, Thoma 2014). Die Katastrophenforschung hat sehr ähnliche Untersuchungsgegenstände wie die Sicherheitsforschung aufzuweisen. Daher soll an dieser Stelle auch nicht versucht werden, sozusagen mit aller Gewalt nach möglicherweise existierenden wesentlichen Unterscheidungsmerkmalen zu suchen und so zu einer trennscharfen Definition zu kommen. Dies ist aller Wahrscheinlichkeit nach nicht möglich, es ist allerdings auch nicht notwendig. Vielmehr geht es um die Frage, was die Katastrophenforschung ausmacht und wie sie mit dem Resilienz-Konzept umgeht.

Katastrophenforschung im Feld der Soziologie ist zwar als Begriff etwas älter als die dezidierte Sicherheitsforschung, kann nichtsdestotrotz nur einige wenige Jahrzehnte zurückverfolgt werden. In den USA gibt es den Begriff „disaster research“ wiederum schon länger. Mittlerweile hat sich das Thema etabliert und es existieren wissenschaftliche Einrichtungen, die sich dezidiert mit Katastrophenforschung bzw. Katastrophensoziologie beschäftigen, in Deutschland vor allem die Katastrophenforschungsstelle (KFS) am Institut für Sozial- und Kulturanthropologie der Freien Universität Berlin. Die KFS wurde 1987 an der Christian-Albrechts-Universität zu Kiel gegründet und betreibt seitdem sozialwissenschaftliche Forschung zu Entstehungs-, Verlaufs- und Bewältigungsbedingungen von Krisen und Katastrophen (Dombrowsky/Siedschlag 2013: 223). ${ }^{11}$ Katastrophensoziologie wird hierzulande insofern auch primär mit den Namen der bisherigen Leiter der KFS, Lars Clausen, Wolf Dombrowsky und aktuell Martin Voss in Verbindung gebracht. Insbesondere Dombrowsky setzt sich in diesem Kontext sehr kritisch mit dem Resilienz-Konzept auseinander, worauf im Unterkapitel zu „Resilienz und Neoliberalismus" ausführlicher eingegangen wird. Einen ähnlich kritischen Umgang pflegt Dombrowsky mit dem Begriff der Katastrophe bzw. der Katastrophenforschung (Dom-

11 http://www.polsoz.fu-berlin.de/ethnologie/forschung/arbeitsstellen/katastrophenforschung/30-Jahre-KFS/index.html [Stand: 27.6.2019]. 
browsky/Siedschlag 2013: 224). Seine durchgängig polemisch zugespitzte Argumentationsweise erregt instinktiv Widerspruch und Ablehnung, führt jedoch dazu, sich im zweiten Nachdenken näher mit den aufgeworfenen Fragen beschäftigen zu wollen, um deren Relevanz für den wissenschaftlichen Diskurs ergründen zu können. Genau diese Herangehensweise wird für die Diskussion von Resilienz und Neoliberalismus und in diesem $\mathrm{Zu}$ sammenhang die Frage danach, ob Resilienz mehr als ein buzzword ist, genutzt.

Im Rahmen der Katastrophenforschung merken Dombrowsky und Siedschlag zurecht an, dass Katastrophen ein soziales Konstrukt sind (Dombrowsky/Siedschlag 2013: 224). Gravierende widrige Ereignisse sind nicht immer mit einer Katastrophe gleichzusetzen. Bereits die Widrigkeit eines Ereignisses an sich, sowie das Ausmaß der Widrigkeit, also als wie gravierend das Ereignis verstanden wird, sind Ergebnis sozialer Zuschreibungen. Selbiges gilt für den Katastrophen-Begriff, der grundsätzlich nur in Abgrenzung zu einem als normal empfundenen Funktionszustand des Alltags definiert werden kann (Voss/Dittmer 2016: 180). Wesentliches Definitionsmerkmal einer Katastrophe ist dann der radikale Bruch mit dem Alltag. Ein Ereignis, ob natürlichen, technischen oder menschlichen Ursprungs, hat massive und durchweg negative Auswirkungen auf das Leben einer großen Zahl von Menschen. Die beiden entscheidenden Faktoren dieser Aussage, negative Auswirkungen auf das Leben von Menschen und die Zahl der betroffenen Menschen, sind bewusst qualitativ zu verstehen. Es lässt sich weder a priori noch allgemeingültig sagen, wie groß die Zahl der betroffenen Menschen sein muss, um von einer Katastrophe zu sprechen. Und es lässt sich nicht endgültig festlegen, ob notwendigerweise (viele) Menschen sterben müssen, damit ein Ereignis als Katastrophe gelten kann. Voss und Dittmer sprechen davon, dass der Zuschreibung des Begriffs Katastrophe immer ein Machtkampf vorausgehe. Ein Ereignis „Katastrophe“ $\mathrm{zu}$ nennen ist so wenigstens implizit als interessengeleitet zu verstehen (Voss/Dittmer 2016: 181f).

Im Vergleich zur englischsprachigen Forschungsrichtung disaster research fehlt der deutschen Katastrophenforschung zudem eine begriffliche Unterscheidungsmöglichkeit. Katastrophenforschung mit disaster research zu übersetzen, zeigt dieses Fehlen bereits auf. Denn im Englischen existiert neben dem Begriff „disaster" auch der der „catastrophe“, so dass verschiedene widrige Ereignisse auf einem kontinuierlich verlaufenden Spektrum besser platziert und unterschieden werden können. In der Alltagssprache ist disaster deutlich verbreiteter als catastrophe, wird aber im Wesentlichen synonym zu diesem Begriff verwendet (Voss/Dittmer 2016: 180). In der 
wissenschaftlichen Literatur bietet sich dagegen eine Unterscheidung an. Hier wird für das Entstehen eines disaster die Existenz einer Bedrohung oder Gefahr (hazard) vorausgesetzt. Ähnlich wie im Deutschen sind das sowohl nicht-alltägliche, kurzfristig eintretende Schocks wie Erdbeben, Terroranschläge oder Industrieunfälle, als auch eher langfristig wirkende Entwicklungen wie Dürren oder ökonomische Rezessionen. Ein disaster wird genau dann aus dieser Bedrohung, wenn sie gravierende negative Auswirkungen auf die Gesellschaft und die darin lebenden Menschen hat und zwar in Form von Tod oder Verletzungen, ökonomischen Verlusten, der physischen Zerstörung von Werten oder der negativen psychischen Beeinflussung von Menschen (Colten et al. 2008: 3). Äquivalent zum Katastrophenbegriff wird auch disaster ohne explizite quantitative Spezifizierung genutzt und die konkrete Definition ist umstritten. Boin und McConnell halten aber fest: Disaster "tends not to be used in reference to extreme situations where life, property and infrastructure remain intact" (Boin/McConnell 2007: 51, eigene Hervorhebung). Von diesem Begriff noch einmal abgesetzt und sozusagen ganz am Ende einer gedachten Skala gravierender, negativer Ereignisse steht im Englischen die catastrophe. Eine catastrophe ist zu verstehen als disaster, das in einer „eigenen Liga spielt" (Boin/McConnell 2007: 51f). Was damit gemeint ist, wird beim Blick in die englische Wikipedia deutlich. Nach dem Verweis auf disaster listet der Überblicksartikel zu catastrophe eine Reihe bestimmter Katastrophen(arten), von der Nuklearkatastrophe von Tschernobyl, über Klimakatastrophen und kosmische Katastrophen bis hin zur Toba-Katastrophentheorie, die den Einfluss eines Vulkanausbruchs auf die Ausbreitung des Menschen als Spezies zum Inhalt hat. ${ }^{12}$

In jedem Fall übersteigt eine so verstandene Katastrophe definitionsgemäß die Bewältigungskapazität des betrachteten Systems. Allein das macht die mögliche Relevanz des Resilienz-Konzepts für Ereignisse, denen in sozialen Aushandlungsprozessen der Name „Katastrophe“ gegeben wird, deutlich. Katastrophenforschung sucht auch und gerade nach Möglichkeiten, Katastrophen zu verhindern bzw. zumindest die negativen Auswirkungen widriger Ereignisse zu begrenzen. Wenn sie sich mit Resilienz beschäftigt, stellt sich die Frage, ob Katastrophe und Resilienz überhaupt gemeinsam auftreten können. Oder ob Resilienz nicht genau darin besteht, das Entstehen einer Katastrophe - nicht jedoch des ihr zugrundeliegenden widrigen Ereignisses - zu verhindern? Diese Frage kann aufgrund der unpräzisen und vielfach unterschiedlichen Definitionen der beiden Konzepte

12 https://en.wikipedia.org/wiki/Catastrophe [Stand: 27.6.2019]. 
als rein semantischer Natur verstanden werden. Resilienz wird im Kontext der Katastrophenforschung, bzw. genauer gesagt der englischen disaster research bereits seit den 1980er Jahren näher untersucht und als Begriff für die Fähigkeit verwendet, die negativen Auswirkungen widriger Ereignisse absorbieren und sich von ihnen erholen zu können (Plodinec 2009: 1). Basierend auf den ursprünglichen und klassischen Arbeiten zu Resilienz in der Ökologie ging es bei den widrigen Ereignissen zunächst primär um Naturgefahren. Gerade im Bereich der Naturgefahrenforschung und verwandten Gebieten, wie der Forschung zu Klimaschutz und nachhaltiger Entwicklung, wird Resilienz seit langem nicht nur theoretisch-konzeptionell, sondern auch anwendungsorientiert erforscht, Methoden operationalisiert und Lösungsansätze umgesetzt (Fekete et al. 2016: 218). Hier sei stellvertretend erneut auf die Arbeiten der Resilience Alliance verwiesen. Die starke Fokussierung resilienzzentrierter Forschung auf Naturgefahren wurde weiter oben im Zusammenhang mit dem Phänomen des Terrorismus bereits kurz andiskutiert. Für die Katastrophenforschung wird davon ausgegangen, dass Resilienz als Konzept nicht notwendigerweise eine bestimmte Art von Störungen bedingt, um verstanden und genutzt werden zu können (Christmann/Ibert 2016: 234). Damit wird der sogenannte „AllGefahren-Ansatz“ (all hazards approach) in der Katastrophenforschung für die Resilienz-Diskussion genutzt. Dieser aus den USA stammende Ansatz kann grundsätzlich auf zwei verschiedene Weisen verstanden werden. Zum einen im eher klassischen Sinne in der Hinsicht, dass sich Katastrophenforschung zwar mit ganz verschiedenen Gefahren beschäftigt, aber für diese jeweils nach spezifischen Möglichkeiten sucht, bei Eintreten eines konkreten widrigen Ereignisses die Schäden zu minimieren. Oder aber zum anderen als holistischer Ansatz, der nach „universell einsetzbare[n] Methoden" sucht und dabei die Komplexität realer Systeme berücksichtigt (Fekete et al. 2016: 218f, Narzisi et al. 2007: 1f). Der All-Gefahren-Ansatz ist übrigens ein Stück weit - trotz des umfassenderen Anspruchs - ein „all natural hazards approach" geblieben (Birkmann 2008: 7). Insbesondere in den USA wird Resilienz im Bereich der disaster research nach wie vor primär im Kontext von Naturgefahren diskutiert, während das Thema Terrorismus im Bereich der Sicherheitspolitik verortet ist.

Die holistische Verwendung des All-Gefahren-Ansatzes durch Nutzung von Prinzipien des Resilienz-Konzepts wird häufig als der spezifische Mehrwert von Resilienz für die Katastrophenforschung verstanden. Denn im Kontext dieser Forschungsrichtung sieht sich das Resilienz-Konzept, wie es aus Ökologie und sozial-ökologischer Forschung übernommen wurde, der Herausforderung gegenüber, dass viele Überlegungen, die für den 
Resilienz-Diskurs eine Rolle spielen, in leicht veränderter Zuschreibung und mit anderen Begrifflichkeiten bereits Teil der Katastrophenforschung waren. Das gilt zum Beispiel für die Begriffe Risiko, Risikomanagement, Katastrophenschutz, Sicherheit und einige mehr. Um die wissenschaftliche Beschäftigung mit Resilienz inhaltlich rechtfertigen und motivieren zu können, muss klar herausgearbeitet werden, wo das Konzept von diesen Begriffen abweicht und eventuell neue, weitergehende Lösungsvorschläge zur Minimierung der durch widrige Ereignisse verursachten Schäden bereithalten kann. Inwiefern ein solch spezifischer Mehrwert überhaupt gegeben ist, ist in der Katastrophenforschung nicht unumstritten. Nichtsdestotrotz dominiert der Begriff die Diskussion in diesem Bereich seit einigen Jahren in zunehmendem Ausmaß (Fekete et al. 2016: 218f). Das erklärt sich unter anderem auch durch eine interdisziplinäre Auseinandersetzung, die sowohl kennzeichnend für Resilienz- als auch Katastrophenforschung ist und den Begriff somit direkt anschlussfähig für letztere macht. Der Unterschied zwischen einem mechanischen und einem systemischen Verständnis von Resilienz wurde bereits mehrfach erwähnt. Auch innerhalb der Katastrophenforschung ist umstritten, ob sich Resilienz primär auf Widerstandsfähigkeit eines Systems und dessen Vermögen, in einen vorherigen Gleichgewichtszustand zurückzukehren, bezieht oder eher Anpassungsfähigkeit im Angesicht gravierender Veränderungen und den Übergang in eine neue domain of attraction beinhaltet (Fekete et al. 2016: 215). Ähnlich dichotom lässt sich die Katastrophenforschung in einen „mechanisch-ingenieurwissenschaftlichen Ansatz", der Katastrophen ohne den zugehörigen sozialen Kontext betrachte und „auf die Kontrolle von Natur und Gesellschaft ziele" einerseits, und einen stärker soziologisch geprägten Ansatz, der gerade die sozialen Faktoren als ursächlich für Katastrophen ansieht und diese generell als soziales Konstrukt versteht, andererseits teilen (Dombrowsky/Siedschlag 2013: 223f, Voss/Dittmer 2016: 182). Diese Aufteilung ist bewusst polemisch überspitzt und trifft auf den größten Teil ingenieurwissenschaftlich geprägter Katastrophenforschung nicht in derart deterministischer Weise zu. Sie zeigt aber auf, dass in diesem Forschungsbereich - wie in vielen anderen - keine einheitliche Definition von Resilienz zu finden ist und es sich lohnt, das Konzept auch kritisch zu hinterfragen. Zum Beispiel im Hinblick darauf, ob Resilienz „überhaupt das richtige Ziel ist“, also ob es im Rahmen der Katastrophenforschung sinnvoll ist, nach Methoden und „Werkzeugen“ zu suchen, die Gesellschaften resilienter gegenüber widrigen Ereignissen werden lassen (Fekete et al. 2016: 218, 226). Das eben geschilderte holistische Verständnis von Resilienz sucht die gegensätzlichen Positionen ein Stück weit zu vereinen, 
kann aber den theoretischen Widerspruch zwischen mechanischer und systemischer Resilienz nicht ohne weiteres auflösen. In der vorliegenden Arbeit geht es auch darum, genau diesen Widerspruch im Rahmen der zivilen SiFo zu untersuchen und herauszufinden, ob Ingenieurwissenschaften notwendigerweise ein Resilienz-Verständnis der mechanischen Art haben müssen oder ob sie durch ein eher Hollingsches Verständnis nicht neue Erkenntnisse gewinnen könnten.

Neben der Katastrophenforschung gibt es, wie erwähnt, auch die Risikoforschung schon sehr viel länger als die dezidierte zivile Sicherheitsforschung. Wer sich wissenschaftlich und konzeptionell mit Sicherheit und vor allen Dingen mit Resilienz auseinandersetzt, kommt um einen zumindest kurzen Abstecher in diesen Forschungsbereich nicht herum. Der Risikobegriff und die damit verbundene Forschungsrichtung spielt für Resilienz in mehrfacher Hinsicht eine entscheidend wichtige Rolle. Wie Resilienz selbst, ist Risiko ein vielfältiger Begriff, hinter dem sich unterschiedliche wissenschaftliche Konzepte verbergen, die in den unterschiedlichsten Disziplinen erarbeitet und entwickelt wurden und auch weiterhin genutzt werden. Und ebenfalls ähnlich zur Resilienzforschung ist die Risikoforschung durch die Erkenntnis, dass sich ein multidimensionales Konzept wie Risiko kaum disziplinär verstehen lässt, immer stärker zu einem inter- und transdisziplinären Forschungsfeld geworden (Fekete et al. 2016: 217, Renn 2008b: 196). Relevante Disziplinen sind vor allen Dingen die Sozialwissenschaften, insbesondere die Soziologie, auf der einen Seite und die Ingenieur- bzw. Technikwissenschaften auf der anderen Seite. Die wissenschaftliche Beschäftigung mit Risiko begann allerdings in einer anderen Disziplin, nämlich den Wirtschaftswissenschaften und zwar bereits in den 1920er Jahren. Der als Begründer der berühmten Chicagoer Schule geltende, amerikanische Ökonom Frank Knight unterschied in seinem Werk Risk, Uncertainty and Profit von 1921 dezidiert zwischen verschiedenen Arten von Unsicherheit. Risiko ist für ihn eine Art von Unsicherheit (Luhmann 2003: 9, siehe Knight 2009). Die Bedeutung des Begriffs Unsicherheit in der Resilienzdiskussion wurde bereits an einigen Stellen herausgearbeitet und wird in den folgenden Kapiteln als Begriffspaar „Resilienz und Unsicherheit" detailliert untersucht. Diese Analyse bezieht auch den Zusammenhang zwischen Resilienz und Risiko mit ein. Damit wird bewusst auf eine eigenständige Diskussion des Begriffspaars „Resilienz und Risiko“verzichtet, obwohl Resilienz bereits seit den 1970er Jahren in der Risikoforschung existiert (Fekete et al. 2016: 217). Ohne die Bedeutung eines Verständnisses von Risiko für ein Resilienz-Konzept verringern zu wollen, lässt sich diese Entscheidung wie folgt erklären. Risiko 
wird so in den größeren Zusammenhang der Unsicherheit eingeordnet, die fundamental für das Verständnis von Resilienz ist und über Risiko weit hinausgeht. Linkov et al. verknüpfen in ihrem einflussreichen Kommentar Changing the resilience paradigm Resilienz explizit mit Unsicherheit und grenzen den Begriff so von Risiko ab (Linkov et al. 2014: 407). ${ }^{13}$ Die Abgrenzung ist wichtig, da die beiden Konzepte sehr häufig vermischt und ohne jede Trennschärfe verwendet werden. Sie sind jedoch grundlegend verschieden (Linkov et al. 2018: 30). Für ein Resilienz-Konzept der zivilen SiFo gilt es herauszuarbeiten, wo die Unterschiede liegen und wie sich Risiko und Resilienz zueinander verhalten. Das geschieht nicht eigenständig, sondern im größeren Kontext der Unsicherheit. Zunächst soll an dieser Stelle aber eine Zusammenfassung einiger wichtiger sozialwissenschaftlicher Ideen zum Risikobegriff erfolgen, die sowohl eine Einordnung als auch eine Abgrenzung der vorliegenden Arbeit erlaubt. An späterer Stelle wird dann detaillierter auf das technikwissenschaftliche Verständnis von Risiko eingegangen (siehe 2.6).

Das Wort Risiko existiert bereits im Mittelalter, seine Ursprünge sind unbekannt. „Risiken sind keine Erfindung der Neuzeit“ (Beck 2016: 28). Mit dem Buchdruck beginnt seine Verbreitung, wohl in Ländern wie Italien und Spanien. Eine regelrechte begriffsgeschichtliche Forschung gibt es aufgrund der zunächst geringen Bedeutung des Begriffs nicht. Verwendung findet er in Bereichen wie der Seefahrt und dem Handel. In Abgrenzung zu Risiko gab es bereits Begriffe, die inhaltlich eine gewisse Verwandtschaft aufweisen, wie etwa Gefahr, Wagnis, Angst oder Abenteuer und demzufolge muss mit Risiko etwas bezeichnet worden sein, was sich davon grundsätzlich unterscheidet. Mit Blick auf die Seefahrt und historische Persönlichkeiten wie Christoph Kolumbus lässt sich die ursprüngliche Bedeutung von Risiko in einer gewissen Weise als eine Art Glücksspiel verstehen. Ein Risiko eingehen bedeutet dann, etwas Gefährliches und vielleicht Erfolgloses zu tun, weil damit im - ebenfalls nur vielleicht eintretenden - Erfolgsfall ein bestimmter Gewinn verbunden ist (Beck 2016: 28, Luhmann 2003: 17ff). „Dadurch, dass man sich auf Risiken einlässt, gewinnt man Chancen, die einem anderenfalls entgehen würden“ (Luhmann 2003: 80). In diesem Sinne sind Risiko bzw. das Verb riskieren auch Teil der deutschen Alltagssprache. Wer etwas riskiert, unternimmt eine Handlung - oder unterlässt sie gerade - deren Konsequenzen er nur

13 Der Autor der vorliegenden Arbeit war Teil des Autorenteams, das diesen Kommentar verfasst hat und für die Implementierung des Begriffs und der Ideen zu „Resilience Engineering“verantwortlich. 
mit einer gewissen Unsicherheit vorhersehen kann. A priori, also bevor er die Handlung unternimmt, weiß er nicht sicher, ob er am Ende Erfolg haben wird. Unternimmt er sie trotzdem, kann davon ausgegangen werden, dass er entweder vom Erfolgsfall ausgeht oder aber der mögliche Gewinn so groß ist, dass auch eine geringe Erfolgschance die Handlung noch rechtfertigt (Luhmann 2003: 22). In all diesen Überlegungen schwingen die Begriffe Wahrscheinlichkeiten und Konsequenzen bereits mit, die sowohl im wirtschafts- als auch im ingenieurwissenschaftlichen Verständnis von Risiko entscheidend wichtig sind (Baum 2015: 229, siehe 2.6 und 4.3.3). Derart detaillierte Gedanken kommen allerdings den wenigsten Menschen im deutschen Sprachraum in den Sinn, wenn sie das Wort Risiko hören. Die erste Annäherung an den Begriff erfolgt(e) vielmehr bei vielen über das nach wie vor populäre Brettspiel gleichen Namens, das als abstrakte, strategische Kriegssimulation einen relativ hohen Glücksfaktor enthält. ${ }^{14}$ Die meisten Menschen unterscheiden in der Alltagssprache auch nicht zwischen Begriffen wie Gefahr und Risiko, was darauf hindeutet, dass insbesondere der Aspekt der Eintrittswahrscheinlichkeit häufig keine Rolle spielt (Ulbig et al. 2009: 57). Auf der anderen Seite wird Risiko ebenso häufig mit Wahrscheinlichkeiten gleichgesetzt, ohne den Aspekt der Konsequenzen explizit einzubeziehen, allerdings implizit immer verbunden mit als negativ empfundenen Konsequenzen.

In der sozialwissenschaftlichen bzw. soziologischen Diskussion in Deutschland ist der Begriff des Risikos seit den 1980er Jahren untrennbar mit dem Soziologen Ulrich Beck verbunden. Beck veröffentlichte 1986 sein international erfolgreiches Buch Risikogesellschaft. Auf dem Weg in eine andere Moderne (siehe Beck 2016). Dessen Erfolg lässt sich unter anderem bereits daran ablesen, dass für die vorliegende Arbeit mit der 23. Auflage des Buchs gearbeitet wurde. Das Wort „Risikogesellschaft“ ist zu einem feststehenden Begriff geworden, das häufig in den Medien genutzt wird ohne notwendigerweise auf Becks wissenschaftliche Ideen zu rekurrieren (Blum et al. 2016: 154). Die Popularität von Becks Buch verdankt sich wohl unter anderem auch dem beinahe unheimlich passend zu nennenden Zeitpunkt, zu dem es erschienen ist. Während Beck noch im Schreiben begriffen war und sich mit einem „Zeitalter globaler Selbstbedrohungspotentiale von bis dahin unbekanntem Ausmaß" theoretisch beschäftigte, ereignete sich sozusagen parallel im April 1986 die Nuklearkatastrophe von Tschernobyl (Blum et al. 2016: 154). Beck selbst stellt seinem Buch deshalb ein Vorwort vor dem Vorwort voran und nennt es

14 https://de.wikipedia.org/wiki/Risiko_(Spiel) [Stand: 27.6.2019]. 
„Aus gegebenem Anlass“ (Beck 2016: 7). Darin geht er auf Tschernobyl ein, erwähnt, wie er seine Thesen „argumentativ erkämpft“ habe und wie sie nun vielmehr wirkten wie eine "platte Beschreibung der Gegenwart" (Beck 2016: 10). Mehr als alle Unfälle in Kernkraftwerken zuvor, wie etwa der Reaktorunfall im Kernkraftwerk Three Mile Island im März 1979, und danach, bis zur Nuklearkatastrophe von Fukushima im März 2011, versinnbildlicht die Katastrophe von Tschernobyl heute die Gefahren, mit denen sich Becks Risikogesellschaft auseinandersetzen muss. Es geht um nicht-intendierte und häufig - bis zu ihrem tatsächlichen Eintritt auch nicht erwartete Nebenfolgen des (technologischen) Fortschritts, die Gesellschaften an ihre Grenzen bringen oder sogar darüber hinaus (Blum et al. 2016: 154). Die Risiken, um die es Beck geht, sind „globale Gefährdungslagen" und sie unterscheiden sich kategorial von den Risiken, die zuvor für Gesellschaften relevant waren. Wie oben erwähnt, wurde der Begriff ja beispielsweise in der Seefahrt verwendet und hatte „im Kontext dieser Epoche den Beiklang von Mut und Abenteuer", was in einem diametralen Gegensatz zu den von Beck sogenannten „Modernisierungsrisiken“ steht, bei denen es letztlich um die Gefahr einer „möglichen Selbstvernichtung des Lebens auf der Erde“" gehe (Beck 2016: 28f).

Den Grund für die Entwicklung der modernen Gesellschaft hin zur Risikogesellschaft sieht Beck in einem für ihn feststehenden, systematischen Zusammenhang zwischen der "gesellschaftlichen Produktion von Reichtum" und der gleichzeitigen "gesellschaftlichen Produktion von Risiken" (Beck 2016: 25). Vor allen Dingen der technische Fortschritt erlaube eine massive Steigerung der Produktivität, die den Wohlstand von Gesellschaften derart erhöhe, dass Risiken, die in direkter Verknüpfung mit dem Fortschritt entstehen, ignoriert werden (Beck 2016: 80). So kommt es zu Modernisierungsrisiken, die „pauschales Produkt der industriellen Fortschrittsmaschinerie" seien und die sich eben in ihrer Qualität fundamental von zuvor existierenden Risiken unterscheiden. Die neuen Risiken zeichnen sich demzufolge dadurch aus, dass sie zeitlich, räumlich und sachlich entgrenzt auftreten. Sie betreffen nicht (nur) den Verursacher, so dieser überhaupt ermittelbar ist, sondern können prinzipiell jeden Menschen und sogar darüber hinaus das Gesamtsystem Planet Erde betreffen (Beck 2016: 29ff). Beck spricht in diesem Zusammenhang von einer tickenden Zeitbombe und skizziert die Risikogesellschaft als eine Gesellschaft, in der der Ausnahmezustand zum Normalzustand zu werden drohe. Denn es besteht andauernd die Gefahr, dass sich ein Risiko in der Zukunft als Katastrophe materialisiert. Genau das soll in der Risikogesellschaft verhindert werden (Beck 2016: 31, 44). Auf die genannten Vorteile der 
Modernisierung möchte die Gesellschaft nicht verzichten und insofern besteht das „Paradigma der Risikogesellschaft“ Beck zufolge in der Suche nach Antworten darauf, wie diese „systematisch mitproduzierten Risiken und Gefährdungen" verringert und begrenzt werden können. Sie sollen die "Grenzen des Zumutbaren“ in ökologischer, medizinischer, psychologischer und sozialer Hinsicht nicht überschreiten (Beck 2016: 26). Gewünscht wird die Aufrechterhaltung oder Herstellung von Sicherheit, als normativem Gegenentwurf der Risikogesellschaft. Sicherheit wird immer wichtiger, je größer die Risiken werden, weshalb der gesellschaftliche Bedarf nach vermeintlichen oder tatsächlichen Maßnahmen zur Erhöhung der Sicherheit durch „Eingriffe in die technisch-ökonomische Entwicklung"ständig steige (Beck 2016: 26, 65).

Ausgehend von diesen Beobachtungen betreibt Beck im Folgenden in seinem Buch eine umfassende Gesellschaftsanalyse, bei der der Risikobegriff im Prinzip im Wesentlichen der Aufhänger ist, die aber von der Individualisierung, über veränderte Rollen von Mann und Frau sowie Familie, Fragen von Klassen, Schichten und Erwerbsarbeit bis hin zur Zukunft der Wissenschaft sehr weit gespannt ist (siehe Beck 2016). Diese Überlegungen sind für die vorliegende Arbeit und das Resilienz-Konzept der zivilen SiFo nicht weiter relevant, so dass an dieser Stelle nur darauf verwiesen werden soll, wie weitgehend und umfassend eine Beschäftigung mit dem Risikobegriff sein kann. Es gibt aber auch noch einige Aspekte in Becks Werk, die mehr oder weniger direkt auf eine Resilienz-Diskussion übertragbar sind und darauf hinweisen, wie diese sinnvollerweise erfolgen kann. Dazu zählen beispielsweise seine Ideen zum Thema Sicherheit. Sicherheit als Gegenentwurf zur Risikogesellschaft bleibt für Beck „eigentümlich negativ und defensiv“, primär darauf bedacht, „das Schlimmste zu verhindern“ (Beck 2016: 65). In diesem Sinne sieht er in der Risikogesellschaft eine „Tendenz zu einem ,legitimen' Totalitarismus der Gefahrenabwehr.“ Um das gerade skizzierte Schlimmste zu verhindern, was immer damit konkret gemeint sein kann, werden Maßnahmen ergriffen und umgesetzt, die selbst mit negativen Auswirkungen verbunden sind, die Beck als das „Noch-Schlimmere" bezeichnet (Beck 2016: 106). Die Parallelen zum gesellschaftlichen Umgang mit dem Phänomen des Terrorismus sind augenfällig und werden im Begriffspaar „Resilienz und Terrorismus“ ausführlicher beleuchtet. Darüber hinaus spielen derartige Ideen auch immer wieder eine große Rolle, wenn es um die Frage nach der freiheitlichen Verfassung der Gesellschaft geht und inwiefern die Schaffung von Sicherheit durch mehr Resilienz dieser dienlich ist oder sie vielleicht sogar untergräbt. Das wird 
in der vorliegenden Arbeit im Rahmen der Analyse der Zusammenhänge zwischen „Resilienz und Neoliberalismus" sowie Freiheit diskutiert.

Wobei Beck auch die Frage stellt, inwiefern solche akademischen Diskussionen überhaupt von Nutzen sind, wenn er der Wissenschaft aufgrund von Überspezialisierung und Praxisabstinenz unterstellt, auf Risiken nicht reagieren zu können und gleichzeitig sogar ,an deren Entstehen und Wachstum hervorragend beteiligt" zu sein (Beck 2016: 78). Inwiefern Beck diesbezüglich Recht hat, muss notwendig unsicher bleiben. Relativ deutlich lehnt er allerdings einen Rationalitätsanspruch von Wissenschaft ab, wenn es darum geht, Risiken mithilfe von Wahrscheinlichkeiten „sachlich zu ermitteln." Nach Beck lassen sich Wahrscheinlichkeitsaussagen weder widerlegen noch bestätigen. Die Ereignisse, die für ihn im Mittelpunkt seiner Beobachtungen stehen, die „Modernisierungsrisiken“ sind ohnehin kaum quantifizierbar und treffender mithilfe des Konzepts der Unsicherheit zu fassen. Das verweist also erneut auf die Bedeutung der Diskussion von „Resilienz und Unsicherheit“. Gleichzeitig sieht Beck Wahrscheinlichkeitsaussagen als notwendig einer vorgelagerten Wertentscheidung folgend an. Ohne Wertstandpunkt lassen sie sich demnach nicht treffen. Diese Aussage wird in der Analyse von „Resilienz und Freibeit" in ähnlicher Weise aufgegriffen (Beck 2016: 38f). Ein letzter Aspekt, den die vorliegende Arbeit aus Becks Werk ziehen kann, beschäftigt sich mit der durch den technologischen und industriellen Fortschritt bedingten zunehmenden Spezialisierung moderner Gesellschaften. Daraus folgert Beck eine Unmöglichkeit, bestimmte Ereignisse klar „isolierbaren Einzelursachen und Verantwortlichkeiten“ zuzuordnen. Er lehnt demnach einfache, linear-deterministische Erklärungen ab, sieht die Abhängigkeiten vernetzter Systeme untereinander und bewegt sich somit auf einer Diskussionsebene mit der Komplexitätstheorie. Beck weist hier den Weg zur Frage, wie genau sich zunehmende Spezialisierung auf „Resilienz und Komplexität" auswirkt (Beck 2016: 42f).

Neben Becks Risikogesellschaft gibt es in der deutschen Soziologie noch einen zweiten Ansatz, über Risiko nachzudenken, auf den hier noch eingegangen werden soll. Die vorliegende Arbeit zeichnet sich unter anderem dadurch aus, dass sie zur Entwicklung eines eigenständigen Resilienz-Konzepts für die zivile SiFo eine detaillierte systemtheoretische Analyse und Einordnung von Resilienz unternimmt. Grundlagen und Annahmen der Systemtheorie werden dazu an späterer Stelle ausführlicher vorgestellt und im Hinblick auf Resilienz angewendet (4.3.1). Auch in der Risikoforschung spielen systemtheoretische Ansätze eine wichtige Rolle und im Kontext der vorliegenden Arbeit ist der deutsche Soziologe 
Niklas Luhmann zu nennen, dessen Leben und Ideen in 4.3.1 ebenfalls näher beleuchtet werden. Luhmann veröffentlichte 1991 seine Soziologie des Risikos, in der er, aufbauend auf seiner soziologischen Systemtheorie, den Risikobegriff untersucht (siehe Luhmann 2003). Ganz generell stellt er dabei fest, dass die intensive Beschäftigung mit Risiko in unterschiedlichen Disziplinen ein deutliches Zeichen dafür sei, dass Risiko mehr als ein Maß sei, denn „wenn es nur um ein Meßproblem [sic] geht, sieht man nicht recht, weshalb davon so viel Aufhebens gemacht wird" (Luhmann 2003: 16). Luhmann kannte Becks Ideen der Risikogesellschaft beim Verfassen seines Werks und geht auch durchaus an der ein oder anderen Stelle darauf ein. Insgesamt hat er allerdings einen deutlich spezifischeren Risikobegriff, den er aufbauend auf einer kurzen Beschreibung der Historie entwickelt. Nach dem Verweis auf Risiko als etwas, dem sich „einst nur Seefahrer, Pilzsammler oder sonstige, sich selbst einem Wagnis aussetzende Gruppen“ gegenübersahen, fragt Luhmann sich, wie Gesellschaften damit zurechtkommen, dass die Zukunft grundsätzlich ungewiss und Risiken unvermeidbar seien. Während die Vergangenheit bereits feststeht, gilt das für die Zukunft nicht. Es ist lediglich klar, dass auch die „künftige Gegenwart" determiniert sein wird, die Frage ist allerdings, wie sie genau aussehen wird (Kneer/Nassehi 2000: 169f, Luhmann 2003: 3, 23ff). Für den Begriff des Risikos schließt Luhmann darauf basierend auf einen Mangel an Wissen als typisches Charakteristikum (Luhmann 2003: 166). Als besonders problematisch in der Beschäftigung mit Risiko erkennt er eine Tendenz, sich mit „extrem unwahrscheinliche[n], dann aber katastrophale[n] Ereignisse[n]" zu beschäftigen. Das weicht vom normalen Verhalten von Menschen $a b$, die sich sonst stärker um „Wahrscheinlichkeiten mittlerer Häufigkeit kümmern und sehr Unwahrscheinliches außen vorlassen" (Luhmann 2003: 3). Wenn es um Risiken geht, beschäftigen sich Menschen dagegen häufig mit Katastrophen, wobei der Begriff - ähnlich wie in der Katastrophenforschung - als stark dem subjektiven Empfinden ausgesetzt verstanden wird (Luhmann 2003: 5, 159). Das Argumentieren mit Wahrscheinlichkeiten wird nach Luhmann unwichtig, sobald es um Katastrophen geht, sei deren Eintritt auch noch so unwahrscheinlich. In einer Art logischem Zirkelschluss definiert Luhmann Katastrophen dann als eine Art von Ereignis, bei der „die Betroffenen sich weigern, sich von quantitativen Analysen überzeugen zu lassen“ (Luhmann 2003: 11, 159, 243). Katastrophen sind immer einzigartig und insofern „kann eine Organisation kein ausgewogenes Verhältnis“ zu ihnen finden (Luhmann 2003: 211). 
Das Spezifikum seines Risikobegriffs besteht in der Zuschreibung von Verantwortlichkeiten. Um von Risiko sprechen zu können, bedarf es nach Luhmann einer Entscheidung von Menschen, welche kausal für das Ergebnis verantwortlich gemacht werden kann. Das Ergebnis - im Kontext von Risiko ein Schaden - ist kontingent, hätte also durch eine andere Entscheidung vermieden werden können. Es geht darum, etwas zu tun oder zu lassen und darauffolgend verantwortlich für die negativen Konsequenzen zu sein (Kneer/Nassehi 2000: 167f, Luhmann 2003: 25). Dabei kann von Risiko nur die Rede sein, wenn der Risikonehmer zwischen verschiedenen Arten möglicher Konsequenzen unterscheidet im Hinblick darauf, als wie wünschenswert er diese erachtet und er gleichzeitig auch zwischen Eintrittswahrscheinlichkeiten der Konsequenzen unterscheidet. Insofern ist Risiko immer kontextgebunden (Luhmann 2003: 38, 235). In der Unterscheidung von Konsequenzen und Wahrscheinlichkeiten wird eine Verknüpfung zum Risikobegriff der Ingenieurwissenschaften deutlich (siehe 2.6). Allerdings liegt Luhmanns Schwerpunkt klar auf dem Aspekt der Zurechenbarkeit von Risiken zu Entscheidungen. Denn eine Gesellschaft wird ihm zufolge nicht durch die Erfahrung von Leid und Zerstörung zur Risikogesellschaft, sondern durch die Zuschreibung der Verantwortlichkeiten für diese Schäden auf menschliches Handeln - im Vergleich zu früheren Zeiten, in denen häufiger eine übernatürliche Macht wie Gott verantwortlich gemacht wurde (Kneer/Nassehi 2000: 173).Wenn Risiken auf Entscheidungen beruhen, sind Menschen bzw. Organisationen dafür verantwortlich und können auch verantwortlich gemacht, das heißt unter Umständen beschuldigt, werden. Moderne Gesellschaften produzieren Schäden also selbst, können daher im Umkehrschluss aber auch lernen, sie zu verhindern oder zu begrenzen (Kneer/Nassehi 2000: 169, Luhmann 2003: 3).

Bei Luhmann gibt es sogenannte „beobachtungsleitende Unterscheidungen", die sich immer in Dichotomien ausdrücken. Für Risiko ist hier der Gegenbegriff der Sicherheit sehr verbreitet, allerdings laut Luhmann nicht wirklich geeignet (Luhmann 2003: 28). Da risikofreies Entscheiden per se unmöglich ist, kann auch nicht davon ausgegangen werden, dass Risiko durch Sicherheit ersetzt werden kann (Luhmann 2003: 37). Vielmehr muss die Unterscheidung für Luhmann zwischen Risiko und Gefahr erfolgen. Bei Risiken steht die Entscheidung im Mittelpunkt, ein möglicher Schaden ist Resultat dieser Entscheidung. Gefahren hingegen kommen von außen, erleidet man einen Schaden wird dieser nicht als Resultat einer eigenen Entscheidung, sondern als von der Umwelt verursacht verstanden (Kneer/Nassehi 2000: 171, Luhmann 2003: 30f). Oder anders ge- 
sagt: Risiken geht man ein, von Gefahren ist man betroffen bzw. man ist ihnen ausgesetzt (Luhmann 2003: 32). Da Luhmann aber, wie Beck, davon ausgeht, dass bestimmte Entscheidungen nicht nur einen selbst betreffen, können Menschen oder Organisationen Risiken eingehen, die wiederum andere Gruppen von Menschen Gefahren aussetzen (Kneer/Nassehi 2000: 174). Und auch wenn etwa Naturkatastrophen grundsätzlich als Gefahren verstanden werden können, werden sie durch die Möglichkeit, sich mithilfe geeigneter Maßnahmen darauf vorzubereiten, dann zum Risiko, wenn diese Vorbereitung ausbleibt (Luhmann 2003: 40). Von diesem Ausgangspunkt ausgehend lässt sich letzten Endes die gesamte vorliegende Arbeit verstehen und interpretieren, wenn nicht sogar schlechthin Risiko- und Sicherheitsforschung an sich. Denn Luhmanns Verständnis, wonach von Risiko erst dann gesprochen wird, wenn etwas Unheilvolles als Folge menschlichen Handelns charakterisiert wird, gilt eben - strikt in der Luhmannschen Logik bleibend - auch genau anders herum: Auch menschliches Nicht-Handeln ist demnach eine Form von Handeln und wenn in dieser Arbeit von widrigen Ereignissen die Rede ist, dann sind diese an sich zwar teilweise - häufig aber auch nicht - völlig unbeeinflusst von menschlichem Handeln, etwa Erdbeben oder Vulkanausbrüche, ihre Auswirkungen hingegen hängen direkt und massiv von dem ab, wie Menschen vor, während und nach einem derartigen Ereignis handeln (Kneer/ Nassehi 2000: 169f, 173). Zentral ist hier das „Bewusstsein der Gestaltbarkeit, Veränderbarkeit und Steuerbarkeit der Welt“" (Kneer/Nassehi 2000: 173).

Die vorliegende Arbeit folgt Luhmann nicht, wenn es um die Definition von Risiko als Ergebnis von Entscheidungen über Handeln oder NichtHandeln geht. Im weiteren Verlauf wird klar, dass auch bei Ereignissen ohne menschliches Zutun von Risiko gesprochen werden kann und zwar nicht nur im gerade skizzierten Sinne eines Nicht-Handelns etwa zur Vorbereitung auf Naturkatastrophen (siehe 2.6 und 4.3.3). Die Idee, wonach menschliches Handeln oder Nicht-Handeln einen Einfluss auf Ereignisse und deren Auswirkungen hat, wird aber selbstverständlich übernommen, sie erst rechtfertigt angewandte Forschung wie etwa die zivile Sicherheitsforschung. Auch darüber hinaus enthält Luhmanns Soziologie des Risikos einige Ansätze und Überlegungen, auf die ein Resilienz-Konzept der zivilen SiFo rekurrieren kann und die Anregungen für die weiteren Diskussionen bieten. So analysiert er beispielsweise auch den Zusammenhang zwischen Risiko und (Hoch-)Technologie. Beck folgend bezeichnet er die heutige Gesellschaft als Risikogesellschaft und begründet das mit „rasante[n] technologische[n] Entwicklungen" in Disziplinen wie der Physik, 
Chemie und Biologie. Einer wachsenden Skepsis gegenüber durch Technologie bedingtem, scheinbaren Fortschritt begegnet er mit der Aussage, dass a priori unklar sei, ob ein technologisches Eingreifen in die Natur riskanter sei als ein Unterlassen desselben (Luhmann 2003: 93ff). Zumal er gerade bei Schäden an der und in der Umwelt von einer Unmöglichkeit ausgeht, diese einzelnen Entscheidungen kausal zuzuschreiben. Vielmehr gebe es Schwellen, bei deren Überschreiten „eine irreversible Veränderung ökologischer Gleichgewichte oder der Eintritt einer Katastrophe" erfolge (Luhmann 2003: 35). Aber selbstverständlich haben bereits viele vorangegangene Entscheidungen dazu geführt, sich dieser unbekannten Schwelle erst zu nähern. Hier lassen Luhmanns Ausführungen klare Parallelen zu Hollings Analyse von Ökosystemen erkennen. Selbiges gilt für das Phänomen der unintendierten Nebenfolgen, von denen Luhmann spricht und die in der Resilienzforschung eine wichtige Rolle spielen (Luhmann 2003: 76). Diese sind auch die Konsequenzen eines immer weiter zunehmenden Einsatzes neuer Technologien, der durch eine „allmähliche Akkumulation der Effekte" dazu führen kann, dass aufgrund geringfügiger Störungen vermittelt durch „Interferenzen“, gesamte Systeme zusammenbrechen (Luhmann 2003: 99). All diese Überlegungen Luhmanns finden sich so oder so ähnlich in der Komplexitätstheorie wieder und werden daher im weiteren Verlauf der Arbeit bei der Diskussion des Zusammenhangs zwischen „Resilienz und Komplexität" wieder aufgenommen. Wobei er mit der "Interferenz nichttrivialisierter Prozesse“ auch zumindest implizit eine Verknüpfung zum Thema „Resilienz und Anpassungsfähigkeit" macht, nämlich in dem er die Frage danach aufwirft, ob Maschinen also technische Systeme - sich selbst so verändern können, dass sie sich veränderten Anforderungen anpassen können. Er spricht hier von einer Fähigkeit, die „eigentlich nur Menschen“ aufweisen (Luhmann 2003: 103). Technologien, vor allem auch Sicherheitstechnologien, finden nach Luhmann häufig auch in anderer Weise Anwendung, als ursprünglich geplant. Dabei zeitigen sie dann unerwartete Auswirkungen, was in der sonstigen Resilienzforschung häufig im Zusammenhang mit Unsicherheit diskutiert wird (Luhmann 2003: 104f). Denn die in den Ingenieurwissenschaften verbreitete Annahme, man könne durch eindeutige Wenn-Dann-Aussagen präzise Berechnungen der Folgen von Entscheidungen anstellen, negiert Luhmann als etwas, das erst ex post und somit gar nicht funktioniere (Luhmann 2003: 168). Seiner Ansicht nach gibt es zu viele Gründe, ,aus denen etwas auf unwahrscheinliche Weise schief gehen kann, als dass man sie in einer rationalen Kalkulation berücksichtigen könnte" (Luhmann 
2003: 21). Genau darauf basiert letzten Endes auch die Frage danach, wie sich „Resilienz und Unsicherheit" zueinander verhalten.

Insgesamt gesehen liefert Luhmanns Soziologie des Risikos eine ganze Reihe an Anknüpfungspunkten für die Resilienz-Diskussion der vorliegenden Arbeit. Nicht zuletzt finden sich darin auch einige Überlegungen, die eine gewisse Verwandtschaft zu den Arbeiten der organisationswissenschaftlichen Resilienzforschung aufweisen, die im nun folgenden Unterkapitel näher beleuchtet werden. So verweist Luhmann darauf, dass viele Entscheidungen ex post, also nachdem ein Schaden eingetreten ist oder gerade nicht, als unverständlich erscheinen. Es stellt sich die Frage, wieso ein derart riskantes oder aber risikoaverses Verhalten gewählt wurde. Sollte es zu einem Schaden gekommen sein, wird dieser kausal auf die Entscheidungen bestimmter Personen zugeschrieben, denen die „Schuld“ gegeben wird, da es sich um „menschliches Versagen“ gehandelt habe. Luhmann erkennt, dass dabei häufig strukturelle, zugrundeliegende Ursachen der Probleme übersehen werden und eine unzulässige Vereinfachung der vergangenen Entscheidungssituation imaginiert wird (Luhmann 2003: 51, 109f, 210). Hierin stimmt er vollständig mit den Vertretern der organisationswissenschaftlichen Resilienzforschung überein. Interessanterweise kann deren Entstehung und Entwicklung im Wesentlichen auf Erkenntnisse aus der Mitarbeit in einer Kommission zurückgeführt werden, die einen schwerwiegenden Unfall - den Absturz des Space-Shuttles Columbia - untersuchen sollte (Woods 2019: 52). Luhmann führt dazu passend aus: „In gravierenden Fällen setzt man Kommissionen ein, um in dem, was als Zufall erfahren werden musste, Ordnung wiederzufinden" (Luhmann 2003: 208). Die Arbeiten der Organisationswissenschaftler, die in Folge des Columbia-Unglücks das Konzept Resilience Engineering entwickelt haben, lassen sich ohne Weiteres als ein derartiger Versuch verstehen.

\subsection{Organisationswissenschaften - „Resilience Engineering“}

Angaben der Internationalen Arbeitsorganisation zufolge sterben jedes Jahr mehr als 2,7 Millionen Menschen an Arbeitsunfällen oder durch Arbeit verursachten Krankheiten, über 374 Millionen leiden an nicht tödlichen Krankheiten oder werden verletzt (Pillay 2017: 129). Es sind diese furchteinflößenden Zahlen, die als Hauptmotivation für den Strang der Resilienzforschung gelten können, der sich selbst unter der konzeptionellen Überschrift „Resilience Engineering“ versammelt. Dahinter verbergen sich - in kontraintuitiver Weise zum engineering-Begriff stehend - organi- 
sationswissenschaftliche und damit letzten Endes stark sozialwissenschaftliche wie auch psychologische Ansätze. Das Ziel der vorliegenden Arbeit besteht darin, basierend auf einem eigenständigen Resilienz-Konzept der zivilen SiFo Hypothesen für ein ingenieurwissenschaftliches Resilience Engineering aufzustellen. Insofern ist es unumgänglich herauszuarbeiten, wie dieser Begriff bisher in der Literatur Verwendung findet. Dabei stehen zwei Fragen im Vordergrund. Zum einen die ganz generelle danach, was in der Literatur unter Resilience Engineering verstanden wird. Und zum anderen die Frage danach, ob und wenn ja welche Bestandteile dieses Verständnisses für die vorliegende Arbeit genutzt werden können. Hier lohnt es herauszustellen, dass die organisationswissenschaftliche Beschäftigung mit Resilienz - außer den obligatorischen Verweisen auf Holling - relativ losgelöst von sonstigen psychologischen, ökologischen, sozialökologischen und sozialwissenschaftlichen Überlegungen arbeitet. Artikel zu Resilience Engineering zitieren sich im Wesentlichen selbst (Righi et al. 2015: 144). Im Folgenden werden daher die Grundideen dieser Forschungsrichtung skizziert und analysiert, inwiefern ähnliche Begriffe und Konzepte Verwendung finden, wie in anderen Disziplinen. So kann auf konzeptionelle Zugänge verwiesen werden, die im weiteren Verlauf von den einzelnen Forschungsrichtungen und Disziplinen losgelöst dazu genutzt werden, ein Resilienz-Konzept der zivilen SiFo zu entwickeln. Zudem wird am Ende dieses Unterkapitels noch kurz diskutiert, ob „Resilience Engineering“ tatsächlich der geeignete Überbegriff für diese Art der organisationswissenschaftlichen Resilienzforschung ist.

Ganz grundsätzlich geht es um Fragen von (Arbeits-)Sicherheit. Allerdings nicht im Sinne der für die zivile SiFo relevanten security, sondern sehr stark fokussiert auf safety. Es geht um einen „completely new way of thinking about safety" (Woods/Hollnagel 2006: 2). Dieser Ausgangspunkt gilt nach Wissen des Autors im Prinzip für alle Wissenschaftler, die sich mit Resilience Engineering aus einer organisationswissenschaftlichen Perspektive beschäftigen. Dann ist häufig auch die Rede von einem neuen Paradigma des Umgangs mit safety, des Managements von safety in Organisationen. Der Fokus der Forschung liegt auf Unfällen, sowie sicherheitsrelevanten, persönlichen und Prozess-Risiken (Hale/Heijer 2006b: 125, Righi et al. 2015: 144f, Shirali et al. 2013: 88, Tamvakis/Xenidis 2012: 3442). Diese Ideen und der Begriff Resilience Engineering lassen sich mittlerweile gut 15 Jahre zurückverfolgen. Geprägt wurde der Begriff zunächst von Woods. Woods war Mitglied in einer Untersuchungskommission der NASA, die herausfinden sollte, wie es zur Zerstörung des Space Shuttles Columbia beim Wiedereintritt in die Atmosphäre am 1. Februar 2003 
kommen konnte. Um seine - von den in der Öffentlichkeit bekannten, technischen Ursachen abweichenden - Erkenntnisse griffig zusammenfassen und den von ihm beobachteten generellen Fehlermechanismen übergreifende Lösungsmöglichkeiten gegenüberstellen zu können, wählte er den Begriff Resilience Engineering (Pillay 2017: 130, Righi et al. 2015: 143, Woods 2019: 52). Daran anknüpfend fand dann im Jahr 2004 im schwedischen Söderköping das erste Resilience Engineering Symposium statt (Nemeth 2008: 3). Seitdem hat es sieben weitere Symposien gegeben, zuletzt vom 24. bis zum 27. Juni 2019 in Kalmar, ebenfalls in Schweden. ${ }^{15}$ Auf Basis der Ergebnisse des ersten Symposiums erschien im Jahr 2006 der Sammelband Resilience Engineering. Concepts and Precepts unter Herausgeberschaft von Erik Hollnagel, Woods und Nancy Leveson (siehe Hollnagel et al. 2006). Dieser Band erweiterte Woods frühere Überlegungen und gilt als einer der ersten substantiellen Beiträge zum damals sehr jungen Forschungsfeld Resilience Engineering (Ouedraogo et al. 2013: 25). Insbesondere der dänische Psychologe Hollnagel und Woods haben sich in den darauffolgenden Jahren um das hinter dem Begriff stehende Konzept verdient gemacht, unter anderem mit einer Veröffentlichung von 2011, Resilience Engineering in Practice. A Guidebook (siehe Hollnagel et al. 2011). Mittlerweile gibt es eine Vielzahl an Artikeln, wobei der überwiegende Großteil von einer relativ kleinen Gruppe von Wissenschaftlern stammt und im Rahmen der regelmäßig stattfindenden Symposien veröffentlicht wurde. Interessant sind zudem die Domänen, mit denen sich die Artikel auseinandersetzen, nämlich hauptsächlich Luftfahrt, das Gesundheitswesen, die chemische und petrochemische Industrie, Kernkraftwerke und der Schienenverkehr. Diese sind alle als Domänen zu kennzeichnen, in denen für Menschen - ob als Arbeiter, Kunden oder Patienten - zum Teil lebensgefährliche „Risiken“ bestehen (Righi et al. 2015: 143f).

Ausgehend von diesen Domänen entwickeln die Forscher zwei sich ergänzende Komplexe an Argumenten, um die Notwendigkeit eines neuen Verständnisses von safety im Sinne eines Resilience Engineering zu begründen. Dazu muss zunächst festgehalten werden, dass in den genannten Domänen und damit in den Organisationen, die von Interesse sind, in den meisten Fällen wirtschaftliche Interessen ins Spiel kommen. Die beteiligten Organisationen sind zu großen Teilen Unternehmen, die durch ein Maximum an Effizienz versuchen, Gewinne zu erzielen. Die Forscher sprechen nach dem Vorbild von Woods zumeist von Produktionsdruck

15 http://www.resilience-engineering-association.org/symposium/

[Stand: 27.6.2019]. 
(production pressure), der eine wichtige Rolle spielt, wenn es um die Frage geht, ob und wenn ja wieviel Produktivität für mehr Sicherheit geopfert werden soll (Wreathall 2006: 281). ${ }^{16}$ Dieser Produktionsdruck wird als eine der Hauptursachen für Unfälle angesehen, programmatisch hierzu Woods Aussage, wonach häufig „a drift toward failure as defenses erode in the face of production pressure" zu beobachten sei (Woods 2005: 289). Gleichzeitig wird Sicherheit zumeist als oberstes Ziel genannt, nach dem Motto „safety first“ - selbst wenn die Entscheidungen und Handlungen der jeweiligen Organisationen auf etwas Anderes schließen lassen (Huber et al. 2009: 92). Der Grund für ein eher riskantes Verhalten im Hinblick auf Sicherheit liegt darin, dass Sicherheit nicht ohne den Einsatz von Ressourcen zu haben ist, die dann wiederum nicht für andere Zwecke zur Verfügung stehen. Und während mögliche, sicherheitskritische Ereignisse unsicher sind und in der Zukunft liegen, kann mit den gleichen Ressourcen bereits heute der Gewinn vergrößert werden (Woods 2005: 291). Der Konflikt zwischen Produktivität und Sicherheit bedarf demzufolge eines feinen Austarierens, da extreme Ausschläge in beide Richtungen für Organisationen schädlich sein können - ein zuviel an Sicherheit führt zu einem Mangel an Innovation und Effizienz, ein zu wenig wiederum setzt die Organisation der Gefahr schwerwiegender Unfälle aus (Woods 2005: 297). Besonders gefährlich ist nach Leveson et al. die Tatsache, dass sich Einsparungen im Bereich Sicherheit zumeist nicht direkt negativ auswirken und das Ausbleiben von Unfällen als Argument dafür gesehen wird, noch mehr an dieser Stelle zu sparen. Obwohl die Organisation sich gerade ihren - a priori unbekannten - Belastungsgrenzen sehr viel stärker angenähert hat, als zuvor (Leveson et al. 2006: 104). Für Woods laufen all diese Überlegungen in der Erkenntnis zusammen, dass Investitionen in Sicherheit gerade dann am dringendsten benötigt werden, wenn man sie sich am wenigsten leisten kann (Woods 2006b: 319, 2005: 297). Neben diesem wichtigen Punkt identifiziert Woods noch einige andere Mechanismen, die zum Columbia-Unglück beigetragen haben und die für ihn die Notwendigkeit eines neuen Paradigmas für mehr Sicherheit motivieren. Dazu zählt er die Tendenz, Erfolge in der Vergangenheit zu stark als Indiz für Erfolge in der Zukunft zu werten, diffuse Entscheidungsprozesse, die keine Gesamtverantwortlichkeiten kennen, das Versäumnis, Einschätzungen auf Basis neuartiger Entwicklungen zu revidieren sowie Kommunikati-

16 Soweit nicht anders erwähnt, wird in diesem Unterkapitel mit Sicherheit das deutsche Pendant zu safety gemeint und nicht - wie in der restlichen Arbeit - das Pendant zu security. 
onshürden zwischen verschiedenen Bereichen involvierter Organisationen (Dekker/Woods 2010: 135, Woods 2005: 296f).

Die gerade genannten Mechanismen weisen bereits deutlich auf den zweiten Komplex an Argumenten hin, der ins Feld geführt wird, um den Bedarf nach Resilience Engineering zu begründen. Es geht um die Art von Ereignissen, mit denen sich Individuen und Organisationen auseinandersetzen und auf die sie reagieren müssen. Für Woods ist nicht die Möglichkeit, im Anschluss an erwartbare Störungen wieder in einen Normalzustand zurückzukehren, entscheidend, sondern vielmehr die Frage danach, ob und wie Systeme in der Lage sind, mit Überraschungen umzugehen. Systeme sind grundsätzlich darauf ausgelegt, mit bestimmten Arten von Störungen umgehen zu können. Aber alle Systeme haben Belastungsgrenzen und alle Systeme können theoretisch mit Ereignissen konfrontiert werden, die über diese Belastungsgrenzen hinausgehen und das System überraschen (Woods 2019: 54, 2015: 7, Woods/Hollnagel 2006: 3). Es muss zwischen erwart- und planbaren Ereignissen, kleineren Unfällen, außergewöhnlichen Ereignissen und einmaligen, völlig unerwartbaren, gravierenden widrigen Ereignissen unterschieden werden. Gerade letztere verlangen ein "shift in mental framework“, um erfolgreich mit ihnen umzugehen, da sie aufgrund ihrer Unerwartbarkeit und ihrer Ausmaße alle vorher gemachten Pläne über den Haufen werfen (Cook/Nemeth 2006: 218, Westrum 2006: 57). Um mit Überraschungen umgehen zu können und als den genannten Paradigmenwechsel, schlussfolgern die Forscher auf eine Notwendigkeit für mehr Resilienz. All diese Überlegungen finden sich so oder so ähnlich auch in anderen Bereichen der Resilienzforschung, vor allen Dingen der sozial-ökologischen Richtung. Sie zeigen eindrücklich auf, dass das Verhältnis zwischen „Resilienz und Unsicherheit" intensiver diskutiert und eindeutig geklärt werden muss. Dabei können auch Ansätze aus der organisationswissenschaftlichen Resilienzforschung zu Rate gezogen werden.

Die beiden Argumentationslinien dienen zunächst als Bestandsaufnahme. So sieht die Welt den Forschern aus diesem Bereich zufolge aus. Es stellt sich die Frage, ob die aktuell dominierenden Ansätze und Verfahren dazu geeignet sind, um unter diesen Umständen die Sicherheit von Individuen, Organisationen und Systemen zu gewährleisten. Die Antwort auf diese Frage ist eindeutig als negativ zu kennzeichnen. Traditionelle Ansätze zum Sicherheitsmanagement sind demnach zu linear und zu statisch, funktionieren nur, solange sich die betroffenen Systeme innerhalb eng gesteckter Grenzen bewegen, sind zu spezifisch und rigide und versuchen erfolglos, mithilfe eines Mikromanagements jedes noch so kleine Detail 
zu regeln (Fujita 2006b: 327, Hollnagel 2006: 15f, Pariès 2006: 53, Woods/ Hollnagel 2006: 4). Sie gehen von Annahmen aus und weisen typische Muster auf, die als problematisch angesehen werden. Etwa das Unvermögen, zwischen „work as imagined“ und „work as done“ zu unterscheiden (Dekker/Woods 2010: 138). Ersteres beschreibt die festgelegten Regeln und Prozeduren, die vor allem auch in kritischen Situationen einzuhalten sind. Letzteres beschreibt hingegen die tatsächlich geleistete Arbeit, die sich häufig stark von den festgelegten Regeln unterscheidet und den Erfolg in kritischen Situationen eher an der Flexibilität und Anpassungsfähigkeit der Handelnden festmacht (Cook/Nemeth 2006: 214, Hale/Heijer 2006b: 132, McDonald 2006: 164). Die Vorstellung, durch striktes Festhalten an Plänen und Regeln Sicherheit gewährleisten zu können, wird als Kultur der Regeltreue (compliance culture) bezeichnet und gegeben die oben gemachten Beobachtungen als ungeeignet abgelehnt (Hollnagel/Woods 2006: 357, Woods 2019: 59, 2018: 4).

Ein zweiter Aspekt klassischen Sicherheitsmanagements, der gesehen und als kritisch eingeschätzt wird, besteht im sogenannten Rückschaufehler (hindsight bias). Dieser aus der Psychologie bekannte Effekt führt dazu, im Nachhinein bestimmte Situationen und Ereignisse auf unzulässige Weise zu vereinfachen und zu erklären, da das Resultat ja bereits bekannt ist. Aus der komfortablen Situation des Wissenden heraus wird den Handelnden unterstellt, sie hätten Fehler begangen und aufgrund ihrer - unverständlichen - Entscheidungen sei es zum Unfall/Schaden/widrigen Ereignis gekommen. So wird Schuld zugewiesen, indem die Entscheidungen aus ihrem situativen Kontext gelöst werden (Dekker 2004: 90, Woods 2005: 290f). Dabei wird übersehen, dass Menschen grundsätzlich versuchen, in jeder Situation das zu tun, was ihnen sinnvoll erscheint. „[P]eople do not come to work to do a bad job" und "[b]ehaviour is rational within situational contexts" (Dekker 2004: 90). Daher meint Dekker auch, dass die Anwendung derart vereinfachender Rückschaumuster mehr über denjenigen, der sie anwende und dessen Motivation verrate, als über die eigentlich betrachtete Situation (Dekker 2004: 93). Denn die Zuweisung von Schuld auf einzelne handelnde Personen und die - wenn auch unzulässige - Vereinfachung der komplexen Entscheidungssituation ermöglichen es, von den mutmaßlich relevanteren Faktoren, wie etwa ungeeigneten Systemdesigns, abzulenken, deren Anpassung zeitlich und finanziell zumeist sehr viel aufwendiger ist, als der Rausschmiss einzelner Mitarbeiter (Dekker 2004: 89).

Eng verwandt mit dem Rückschaufehler ist ein Phänomen, das als Distanzierung durch Differenzierung (distancing through differencing) be- 
zeichnet wird. Individuen, Organisationen und Systeme tendieren dazu, sich beim Blick auf Unfälle und widrige Ereignisse, die sich an anderen Orten ereignet haben, auf die Unterschiede zur eigenen Situation zu konzentrieren. Es wird Distanz geschaffen, indem zwischen sich selbst und den von Unfällen Betroffenen differenziert wird (Cooks/Woods 2006: 330). Dieser Fokus auf Unterschiede geht teilweise so weit, dass in einem empirischen Beispiel Kraftwerksmitarbeiter einer bestimmten Schicht den Ausbruch eines Feuers in einer anderen Schicht auf deren unzureichende Kompetenzen zurechneten und für sich selbst kein erhöhtes Risiko für den Ausbruch eines Feuers sahen (Cook/Woods 2006: 336). Die Distanzierung führt dazu, mögliche gemeinsame, zugrundeliegende Ursachen zu übersehen und verhindert, aus nur scheinbar unterschiedlichen aber faktisch doch ähnlichen widrigen Ereignissen selbst zu lernen (Cook/Woods 2006: 331, Woods 2005: 298). Um künftig solche Ereignisse, die ja als isolierte, lokale Phänomene verstanden werden, zu verhindern, werden eher spezifische Gegenmaßnahmen ergriffen (Cook/Woods 2006: 336).

Die unterschiedlichen Muster, nach denen traditionelles Sicherheitsmanagement funktioniert, tendieren alle dazu, handelnden Personen in irgendeiner Weise die "Schuld" für Unfälle zuzuschreiben. Der Grund für Unfälle ist dann menschliches Versagen (Dijkstra 2006: 183ff). Ein entschiedener Widerspruch gegenüber dieser Annahme ist die Grundlage der gesamten Forschung zu Resilience Engineering aus organisationswissenschaftlicher Sicht. Dekkers programmatischer Satz, wonach Menschen nicht zur Arbeit gehen, um einen schlechten Job zu machen, steht prototypisch dafür (Dekker 2004: 90). Denn gegeben die beiden oben beschriebenen Argumentationslinien sind sehr viel stärker der zunehmende Effizienzdruck moderner Wirtschaftssysteme kombiniert mit der steigenden Komplexität der betroffenen Organisationen und Systeme die Ursachen für Unfälle und widrige Ereignisse. Und deshalb bedarf es eines neuen Paradigmas für Sicherheitsmanagement, bedarf es eines Resilience Engineering. Ähnlich wie die vorliegende Arbeit das an späterer Stelle tun wird, bedienen sich die Forscher dazu aus der Komplexitäts- und Systemtheorie, um ein Verständnis von Resilienz als Fähigkeit zum erfolgreichen Umgang mit Komplexität (coping with complexity) zu entwickeln (Hollnagel/Woods 2006: 348, Pariès 2006: 53, Shirali et al. 2013, 88, Woods/ Hollnagel 2006: 3). „Resilience Engineering is a paradigm for safety management that focuses on how to help people cope with complexity under pressure to achieve success" (Woods/Hollnagel 2006: 6). Unfälle werden als nichtlineare Phänomene verstanden, die in komplexen Systemen grundsätzlich immer auftreten können, unabhängig davon, wie viele Sicherheits- 
mechanismen das zu verhindern suchen (Hollnagel 2006: 12). Gegeben diese Annahmen, müssen die vielfältigen Erkenntnisse, die innerhalb der organisationswissenschaftlichen Resilienzforschung zum Zusammenhang zwischen „Resilienz und Komplexität" erforscht werden, zur Entwicklung eines Resilienz-Konzepts der zivilen SiFo unbedingt näher beleuchtet werden.

Wie definieren Hollnagel, Woods und ihre Kollegen basierend auf diesen Grundannahmen Resilience Engineering und Resilienz? Wie in allen Bereichen der Resilienzforschung gibt es auch in den Organisationswissenschaften eine ganze Reihe mehr oder weniger unterschiedlicher Definitionen der beiden Konzepte. Die erste Definition stammt dabei wohl von Woods, der - sehr abstrakt - wie folgt formuliert: „Managing resilience, or resilience engineering, then, focuses on what sustains or erodes the adaptive capacities of human-technical systems in a changing environment" (Woods 2005: 302, eigene Hervorhebung). Dieser Definition bleibt Woods treu, wenn er auch in einem aktuellen Artikel von 2019 den wesentlichen Inhalt von Resilience Engineering darin sieht, unterschiedliche Arten von Anpassungsfähigkeiten und wie sich diese entwickeln und verändern, zu verstehen (Woods 2019: 53). Ähnlich generisch versteht Wreathall Resilience Engineering als Managementdisziplin, die neben Sicherheitsmanagement auch noch Aspekte von Prozess- und Finanzmanagement enthalte (Wreathall 2006: 276). Diese Definition betont den Managementaspekt und schließt insofern gut an die sonstige Organisationswissenschaft an. Dekker wiederum konzentriert sich in seiner Definition auf eine Sensibilität gegenüber unterschiedlichen Risikomodellen, die genutzt werden, um Unfälle und Versagen in Systemen zu erklären. Dahinter stehen die gerade skizzierten und eher abgelehnten Mechanismen wie Rückschaufehler und Distanzierung durch Differenzierung. Die Erkenntnis, derartige Modelle zu verwenden, stellt für Dekker bereits einen Weg dar, Resilience Engineering - also eine andere Art von Sicherheitsmanagement - zu betreiben (Dekker 2006: 82). Bereits etwas praktischer orientiert definiert Hollnagel Resilience Engineering als die Methoden und Prinzipien, die dazu genutzt werden sollen, Systeme davon abzuhalten, instabil zu werden (Hollnagel 2006: 16). Eine der aus Sicht der vorliegenden Arbeit im Kontext der gesamten organisationswissenschaftlichen Forschung zutreffendsten Definitionen liefern mit Steen und Aven allerdings interessanterweise zwei Risikoforscher, die selbst einen sehr viel stärker technikwissenschaftlichen Hintergrund haben: „Resilience engineering (management) is all measures and activities carried out to manage resilience (normally increase resilience)” (Steen/Aven 2011: 294). Oder anders gesagt: „The goal of Resilience 
Engineering becomes how to bring about resilience in a system" (Hollnagel 2011: xxvii). Resilience Engineering bezeichnet demzufolge nicht mehr, als alle denkbaren Wege, wie Resilienz hergestellt und beibehalten werden kann (Hollnagel 2011: xxix).

Wenn unter Resilience Engineering alle Maßnahmen und Aktivitäten verstanden werden, mit deren Hilfe die Resilienz von Systemen erhöht werden kann, stellt sich unmittelbar die Frage nach der Definition von Resilienz. Beim Blick auf unterschiedliche Definitionen wird inhaltlich eine relativ enge Verwandtschaft zum Resilienz-Verständnis der sozial-ökologischen Forschung sichtbar, interessanterweise aber ohne dass die jeweiligen Forscher in relevanter Weise aufeinander Bezug nehmen würden. Flin sieht das Wesen von Resilienz jedenfalls in der Fähigkeit von Managern, die oben diskutierten Zielkonflikte zwischen Sicherheit und Produktivität sinnvoll auszutarieren (Flin 2006: 223). Dieser explizite Bezug auf einen der Ausgangspunkte fehlt den meisten anderen Definitionen, wird aber zumindest implizit meist mitgedacht. Beim Blick auf Woods Definition von Resilienz wiederum fällt ein klarer Fokus auf adaptive capacity, auf Anpassungsfähigkeit von Systemen auf. In einem aktuellen Artikel beschreibt er Resilienz als ein Verb "that refers to the capabilities that build and sustain the potential for continuous adaptability" (Woods 2018: 5). Noch direkter definiert er schon 2005 Resilienz als Fähigkeit, sich Störungen und Disruptionen anzupassen oder diese zu absorbieren (Woods 2005: 302). Passend zur Betonung von überraschend auftretenden Ereignissen spezifiziert Woods seine Definition im Hinblick darauf, dass die Resilienz eines Systems sich darin ausdrücke, wie gut es sich an Störungen und Veränderungen anpassen könne, die außerhalb dessen liegen, wofür das System ursprünglich ausgelegt wurde (Woods 2018: 2, 2006: 21). Anpassungsfähigkeit an widrige Umstände und Ereignisse wird auch von anderen Autoren als charakteristisch für Resilienz skizziert (Dekker/Woods 2010: 134, Fujita 2006: 67, Hale/Heijer 2006b: 132, Hollnagel/Sundström 2006: 343, Hollnagel/Woods 2006: 357, Leveson et al. 2006: 95). Das sieht auch McDonald ähnlich, er erweitert seine Definition von Resilienz aber dezidiert um den Aspekt der stabilen Funktionalität, die auch im Angesicht widriger Ereignisse aufrechterhalten werden soll (McDonald 2006: 157). Hollnagel ergänzt dazu die Eigenschaft dynamisch und spricht von Resilienz als einer Fähigkeit, die dynamische Stabilität von Systemen gegenüber Störungen zu garantieren (Hollnagel 2006: 16). Auch derartige Definitionen treten innerhalb der Forschung häufiger auf (Cook/Nemeth 2006: 205, Sundström/Hollnagel 2006: 243, Wreathall 2006: 275). Zusammenfassend stehen also Anpassungsfähigkeit und die dynamische Stabilität 
wichtiger Systemfunktionen im Zentrum des organisationswissenschaftlichen Resilienz-Verständnisses.

Woraus besteht Resilienz dann und wie kommt sie zustande? Der wichtigste Punkt ist offensichtlich die Anpassungsfähigkeit. Allerdings kann Resilienz nicht einfach mit Anpassungsfähigkeit gleichgesetzt werden, da laut Woods grundsätzlich alle Systeme sich anpassen (Woods 2006: 21). Auch Hollnagel sieht das ähnlich, wenn er Anpassungsfähigkeit und Flexibilität als normale Handlungsweisen von Menschen charakterisiert, die es ihnen erlauben, lokalen Bedingungen erfolgreich zu begegnen (Hollnagel 2006: 13). Inwiefern es im Kontext der vorliegenden Arbeit tatsächlich zutrifft, dass alle Systeme über Anpassungsfähigkeit verfügen, kann an dieser Stelle zunächst noch nicht beantwortet werden und wird später wieder aufgegriffen (siehe 4.3.1). Der doppelte Gedanke, wonach Anpassungsfähigkeit zwar zentral für Resilienz, nicht aber mit ihr gleichzusetzen ist, spielt allerdings auch für das Resilienz-Verständnis der zivilen SiFo eine entscheidend wichtige Rolle und wird daher in der Diskussion zu „Resilienz und Anpassungsfähigkeit" system- und komplexitätstheoretisch untersucht. Anpassungsfähigkeit ist für Woods das „potential for adjusting patterns of activities to handle future changes in the kinds of events, opportunities and disruptions experienced" (Woods 2018: 1). Wichtig ist hier, dass Anpassungsfähigkeit schon vor dem Eintreten von Störungen und Disruptionen existiert und dann im tatsächlichen Fall genutzt werden kann. Systeme ändern ihre Vorgehensweise nicht ständig, sondern haben vielmehr das Vermögen darüber zu entscheiden, wann es sinnvoll erscheint, sich an vorgegebene Pläne zu halten und wann die Notwendigkeit besteht, sich aufgrund veränderter Anforderungen entsprechen anzupassen. Dazu verfügen Systeme über unterschiedliche Arten von Anpassungsfähigkeiten. Welche das allerdings konkret sind, lässt Woods hier offen, ein Punkt, der im Rahmen der vorliegenden Arbeit dagegen explizit und intensiv analysiert wird (Woods 2018: 1).

Eng verwandt mit der Anpassungsfähigkeit ist ein weiterer Bestandteil von Resilienz, der immer wieder auftaucht. Äquivalent zu Holling und dessen für Ökosysteme gültige Aussage „maintaining flexibility above all else" geht es hierbei um die Flexibilität von Individuen, Organisationen und Systemen, die diese beim Auftreten von Störungen an den Tag legen (sollen) (Holling 1973: 18). So spricht Woods etwa davon, herausfinden zu wollen, welche grundlegenden Prinzipien Systemen die nötige Flexibilität verleihen, um sich langfristig erfolgreich anpassen zu können (Woods 2019: 56, 2015: 8). Flexibilität ist hier also notwendig für Anpassungsfähigkeit. Teilweise steht Flexibilität auch für sich und kann ein Stück weit mit 
Anpassungsfähigkeit gleichgesetzt werden, wenn darunter die Fähigkeit zum „coping with unexpected and unplanned situations and responding rapidly to events" verstanden wird (Hale et al. 2006: 299, 308, Hale/Heijer 2006: 35). Hale und Heijer wiederum setzen Redundanz in Form von unterschiedlichen Personen, die in der Lage sind, die gleichen Aufgaben zu erfüllen, als Voraussetzung für und Bestandteil von Flexibilität (Hale/Heijer 2006b: 144). McDonald kontrastiert eine angemessene Flexibilität auf der einen Seite mit festgelegten Prozessen und guter Planung auf der anderen Seite und versteht Resilienz als Fähigkeit, diese unterschiedlichen Mechanismen auszutarieren (McDonald 2006: 168). Die Frage nach der Bedeutung von Flexibilität und dem Zusammenhang zwischen „Resilienz und Flexibilität" ist jedenfalls für die organisationswissenschaftliche Resilienzforschung wichtig und wird in Anlehnung daran auch in der vorliegenden Arbeit beachtet. Flexibilität wird teilweise auch in Kombination mit weiteren Prinzipien genannt, die Bestandteil von Resilienz sein sollen, von Pufferkapazitäten über gewisse Toleranzen und Margen bis hin zu skalenübergreifenden Interaktionen, einer gerechten und involvierten Führungskultur und Möglichkeiten, aus Fehlern zu lernen. Auch die Fähigkeiten zum kontinuierlichen Monitoring des Systemzustands, sowie zum Testen, inwiefern Pläne, Regeln und Prozesse nach wie vor zur vorgefundenen Realität passen, stehen bei einigen Forschern im Fokus (Dekker/Woods 2010: 139, Wreathall 2006: 279f, Woods 2006: 24, 2005: 301f).

Einen besonders interessanten Ansatz verfolgen Bergström et al. in einem Artikel von 2009 (siehe Bergström et al. 2009). Darin geht es um die Frage, wie Schiffscrews in simulierten Übungen mit außergewöhnlichen, unerwarteten und unbekannten Situationen umzugehen in der Lage sind. Die Crews wurden in drei Gruppen unterteilt, die sich hinsichtlich ihrer Vorerfahrungen im Bereich maritimer Operationen signifikant unterschieden. Die erste, aus Novizen bestehende Gruppe, hatte keinerlei praktische Vorerfahrung. Die zweite Gruppe, aus Studenten maritimer Fächer bestehend, verfügte über begrenzte praktische Erfahrung. Die dritte Gruppe bestand dagegen aus Seefahrern mit mehreren Jahren praktischer Erfahrung auf großen Schiffen - und extensivem Wissen über Prozesse und Routinen für den Normal- und den Ausnahmefall (Bergström et al. 2009: 77ff). In erwartbarer Weise war die dritte Gruppe grundsätzlich am ehesten in der Lage, die geforderten maritimen Operationen erfolgreich zu bestreiten und die jeweiligen Schiffe sicher in ihren Hafen zu bringen (Bergström et al. 2009: 81-85). Konträr zu einer intuitiven Erwartungshaltung, welche die Vorteile jahrelanger, praktischer Erfahrung hervorzuheben tendiert, war es aber gerade diese dritte, erfahrene Gruppe, die in unerwarteten und von 
etablierten Plänen und Prozessen nicht erfassten Situationen vergleichsweise schlecht abschnitt. Ihre eingeübten Routinen und Hierarchien hinderten sie daran, sich der Situation flexibel anzupassen. Sprichwörtlich sahen sie, die über einen perfekt funktionierenden Hammer verfügten, in jeder Situation die Probleme nur als Nägel (Bergström et al. 2009: 86). Die beiden anderen Gruppen konnten hingegen von einem Mangel an Erfahrung und Routine dergestalt profitieren, dass sie eher bereit waren, sich mithilfe sogenannter generischer Kompetenzen (generic competencies), die ihnen zwischen den verschiedenen Übungen beigebracht wurden, den unbekannten Situationen anzunähern. Es sind gerade diese generischen Kompetenzen, die laut Bergström et al. entscheidend wichtig sind, um in unerwarteten und unbekannten Situationen erfolgreiche Resultate produzieren zu können - und damit das betroffene System als resilient kennzeichnen (Bergström et al. 2009: 89). Generische Kompetenzen weisen eine enge Verbindung zum Konzept der Anpassungsfähigkeit auf und können im Prinzip als eine spezifische Art von Anpassungsfähigkeit verstanden werden. Dieser Gedanke ist für das Resilienz-Konzept der zivilen SiFo fundamental wichtig, so dass er ein entscheidender Bestandteil in der Diskussion zu „Resilienz und Anpassungsfähigkeit“ sein wird.

Bevor gleich auf Probleme des bisherigen Verständnisses, wie sie sich für die vorliegende Arbeit stellen, eingegangen wird, soll der Vollständigkeit halber noch das sogenannte „safety II“-Paradigma kurz Erwähnung finden, das innerhalb der Forschung zu Resilience Engineering große Bedeutung erlangt hat. Die bisher dargelegten Erkenntnisse gelten auch unabhängig von diesem Paradigma, jedoch fokussieren viele Diskussionen innerhalb dieser Forschungsrichtung immer stärker darauf bzw. nehmen es als ihren Ausgangspunkt. Demnach ist safety II eine Art Neuorientierung weg vom Schadens- und hin zum Normalfall. Im Alltag lässt sich feststellen: Normalerweise funktionieren Dinge so wie sie sollen. Es ist ungewöhnlich, wenn etwas - gravierend - schiefläuft. Selbst komplexe Systeme arbeiten im Normalfall erstaunlicherweise relativ reibungslos. Dieser Normalfall ist insofern auch der Ausgangspunkt für Resilience Engineering. Damit ändert sich der Fokus von einer Konzentration auf Unsicherheit, nämlich dem Ausnahmefall einer Störung, hin zu einer Konzentration auf Sicherheit, auf „safe functioning“ von Systemen. Das Verständnis für die Funktionsweise komplexer Systeme ist sowohl notwendige wie hinreichende Bedingung, um mögliche Fehler, Probleme und Risiken für diese Systeme zu identifizieren und zu minimieren. Denn nach Hollnagel gibt es keine „special, error producing' processes that magically begin to work when an accident is going to happen, but which otherwise lie dormant." Er sieht 
keine grundsätzlichen Unterschiede zwischen funktionierenden und versagenden, komplexen Systemen (Hollnagel 2011: xxiv-xxvi, Hollnagel/Fujita 2013: 18, LRF 2015: 28). Wenn diese Annahme Gültigkeit besitzt, kann es folglich nicht darum gehen, Sicherheit durch die Minimierung von Störungen und Fehlern zu erreichen. Vielmehr gilt: „The goal of Resilience Engineering is to increase the number of things that go right rather than to reduce the number of things that go wrong" (Hollnagel 2011: xxvi). Wer das Systemverhalten im Normalfall versteht, ist auch sehr viel besser in der Lage, Systeme im Ausnahmefall erfolgreich zu managen (Connelly et al. 2017: 49). Diese Konzentration auf Dinge, die funktionieren, zieht sich durchaus als eine Art roter Faden durch das Verständnis von Resilience Engineering von Hollnagel, Woods und Kollegen (siehe zum Beispiel Hollnagel 2011, Woods/Hollnagel 2006). In einem Satz zusammengefasst gilt für sie: „Resilience Engineering, however, defines safety as the ability to succeed under varying conditions" (Hollnagel 2011: xxix). Für sie ist es sinnvoller, weil einfacher und effektiver, die Sicherheit eines Systems zu verbessern, indem erfolgreiche und gut funktionierende Prozesse weiter verbessert werden, als Fehler gänzlich vermeiden zu wollen (Hollnagel 2011: xxix). Sicherheit ist dann nicht die Abwesenheit von Störungen und widrigen Ereignissen, sondern vielmehr das Vorhandensein bestimmter Mechanismen, um Risiken erfolgreich zu managen (Dekker/Woods 2010: 125). Damit kristallisiert sich eine Vorgabe heraus, die beachtet und genutzt werden soll, um Systeme resilient zu gestalten und die noch grundlegend im Hinblick auf die Funktionsweise komplexer Systeme unter dem Begriffspaar „Resilienz und Komplexität" diskutiert werden muss.

Beim Blick auf die bisherige Verwendung des Begriffs Resilience Engineering in der Literatur wird deutlich, dass die Forschung sehr dezidiert und explizit ein nicht-technisches Verständnis von Resilienz aufweist. Es handelt sich um sozial- bzw. besser gesagt organisationswissenschaftliche und organisationspsychologische Forschung mit einem Fokus auf menschlichem Verhalten (Attoh-Okine 2016: 22f, Dekker 2006: 86, Huber et al. 2009: 91, McDonald 2006: 160). Die Forscher beschäftigen sich damit, Sicherheitsrisiken in Organisationen zu managen und verstehen Resilience Engineering als neues Paradigma, um zu erklären „how people cope with complexity under pressure“ (Pillay 2017: 134, Woods 2006: 33, eigene Hervorhebung). Ingenieur- und technikwissenschaftliche Ansätze und Inhalte tauchen in keiner relevanten Definition auf. Deutlich wird der sozialwissenschaftliche Fokus auch in Überblicksartikeln wie dem von Pillay, indem ausdrücklich darauf hingewiesen wird, dass von den 123 grob und den 46 detailliert untersuchten Publikationen keine aus dem eigentlichen 
engineering Bereich stammte, da dieser in der Suche außen vorgelassen worden sei (Pillay 2017: 135ff, 147f). Wenn Technologie auftaucht, dann beispielsweise als Herausforderung in Form neuer Technologien, mit denen Menschen in komplexen Organisationen umzugehen lernen müssen und die unter Umständen neue Verwundbarkeiten schaffen (Wreathall 2006: 283). Die sozialwissenschaftliche Ausrichtung führt auch zu einem wahrgenommenen Mangel an quantitativen bzw. quantifizierbaren Ansätzen oder einer formaleren Nutzung des Konzepts, beides Hürden für eine Anwendung in den Ingenieurwissenschaften (Attoh-Okine 2016: 23). Zudem wird zum Teil die ebenfalls mangelnde konzeptionelle Verknüpfung zwischen Resilience Engineering und der stärker ingenieur- bzw. naturwissenschaftlich geprägten Komplexitätstheorie kritisiert (Righi et al. 2015: 149). Wenn bisher unter dem Begriff Resilience Engineering in der Literatur nicht der ingenieurwissenschaftlich geprägte Bereich der Resilienzforschung zu fassen ist, stellt sich die Frage, ob und in welcher Art sich die Ingenieur- und Technikwissenschaften mit Resilienz beschäftigen. Dieser Frage - und der Frage danach, wieso sich daraus eine Forschungslücke ergibt - geht das nächste Unterkapitel nach.

\subsection{Ingenieurwissenschaften - „engineering resilience”}

„Ingenieure entwickeln Lösungen. Sie beobachten Missstände und identifizieren deren Ursachen. Dann entwerfen sie entweder Mechanismen, um die Missstände zu beseitigen oder aber Methoden, um deren negativen Auswirkungen entsprechend positive entgegen zu setzen. Je größer die zu bewältigende Aufgabe ist, desto dringender ist eine Gesellschaft auf den wissenschaftlichen Sachverstand und den kreativen Erfindergeist von Ingenieurinnen und Ingenieuren angewiesen" (Scharte/Thoma 2016: 123). So beginnt ein Artikel zur ingenieurwissenschaftlichen Perspektive auf Resilienz, den der Autor der vorliegenden Arbeit gemeinsam mit Klaus Thoma im Jahr 2016 in einem Sammelband zu multidisziplinären Perspektiven der Resilienzforschung veröffentlicht hat. Dieser Ausgangspunkt und dieses Grundverständnis dessen, was Ingenieure ausmacht, kann auch für die vorliegende Arbeit Gültigkeit beanspruchen. Gegeben diese Annahmen stellt sich dann für Resilienz und besonders für Resilience Engineering die Frage, wie solche Konzepte ausgestaltet sein müssen, damit sie von Ingenieuren bzw. den Ingenieurwissenschaften sinnvoll angewandt und konkret umgesetzt werden können. Dieser Frage geht die vorliegende Arbeit intensiv nach. Damit ordnet sie sich - wenn auch als Besonderheit 
mit einem dezidiert sozialwissenschaftlich-theoretischen Schwerpunkt und einem interdisziplinären Anspruch - in ein stark wachsendes Forschungsfeld ein, das im Folgenden vorgestellt wird. Im Unterkapitel zur Genese des Resilienzbegriffs wurde bereits darauf hingewiesen, dass Resilienz seit dem 19. Jahrhundert im Bereich der Ingenieurwissenschaften als Begriff bekannt ist (siehe 2.1). Als wissenschaftlich fundiertes Konzept ist die Geschichte von Resilienz in den Ingenieurwissenschaften allerdings bedeutend kürzer - auch und gerade im Vergleich zu den beiden „klassischen“ Disziplinen Psychologie und Ökologie (Hosseini et al. 2016: 48). Erst in den letzten 15 bis 20 Jahren hat sich das Konzept - von einer noch näher zu beleuchtenden Ausnahme abgesehen - in den Ingenieurwissenschaften etabliert. Als programmatische Aussage für diesen Forschungsbereich kann dabei ein Punkt von Altherr et al. dienen, den diese in einem aktuellen Artikel formulieren: „Key to achieve or improve resilient system properties is their assessment and quantification" (Altherr et al. 2018: 189).

Resilienzforschung in den Ingenieurwissenschaften beschäftigt sich überproportional häufig mit Wegen das Konzept messbar zu machen. Den Ausgangspunkt bildet dabei zumeist die klassische, ingenieurwissenschaftliche Risikoforschung, an die entweder angeschlossen wird oder von der sich abgehoben werden soll. Wenn es um die Frage nach der Definition von Resilienz geht, gibt es ebenfalls zumeist einen gemeinsamen Ausgangspunkt, das bereits mehrfach erwähnte engineering resilience Verständnis nach C.S. Holling. Eine Ausnahme von all diesen Aspekten bildet ein Werk, das insofern in mehrfacher Hinsicht als Solitär in der ingenieurwissenschaftlichen Resilienzforschung zu sehen ist. Das (damalige) Ehepaar Amory Lovins, amerikanischer Physiker, und L. Hunter Lovins, Juristin und Sozialwissenschaftlerin, veröffentlichte 1982 eine Studie zum amerikanischen Energiesystem mit dem Titel Brittle Power. Energy Strategy for National Security (siehe Lovins/Lovins 2001). Darin nutzen sie ein auf Hollings Ideen basierendes Resilienz-Konzept. Ihre Arbeit bietet für das eigenständige Resilienz-Konzept der zivilen SiFo und vor allem die daraus abzuleitenden Hypothesen für Resilience Engineering viele Anknüpfungspunkte. Die bisherige ingenieurwissenschaftliche Resilienzforschung teilt sich also in unterschiedliche Stränge und weist unterschiedliche Schwerpunkte auf, die für die vorliegende Arbeit systematisch aufbereitet werden müssen. Um diesem Anspruch gerecht werden zu können, teilt sich das folgende Unterkapitel in insgesamt vier Teile. Zunächst wird, wie erläutert, in einem Exkurs das klassische und häufig immer noch vorherrschende Verständnis ingenieurwissenschaftlicher Risikoforschung dargestellt. Davon ausgehend werden die Genese des Begriffs engineering resilience 
bei C.S. Holling und das darunter zu fassende Resilienzverständnis, als das nach wie vor in den Ingenieurwissenschaften am weitesten verbreitete, beschrieben. Danach werden Ansätze zur Quantifizierung von Resilienz vorgestellt, die einen Großteil der ingenieurwissenschaftlichen Literatur zu Resilienz ausmachen. Abschließend folgt ein Exkurs zu Lovins und Lovins Werk Brittle Power, mit Verweis auf ihre Ergebnisse und einer Einschätzung dazu, wie ihre Ideen im Kontext der vorliegenden Arbeit zu sehen sind.

Die ingenieurwissenschaftliche Risikoforschung kann als eine Art Vorläufer zur Resilienzforschung in dieser Disziplin verstanden werden. Stärker sozialwissenschaftliche sowie systemtheoretische Ansätze der Risikoforschung wurden bereits vorgestellt und im Hinblick auf Erkenntnisse, die für ein eigenständiges Resilienz-Konzept der zivilen SiFo nützlich sein könnten, analysiert (siehe 2.4). Generell lässt sich jedoch sagen, dass in der Risikoforschung über Jahrzehnte eine technische Sichtweise vorherrschte und dies an vielen Stellen - trotz vieler, weiterentwickelter theoretischer Ansätze - nach wie vor der Fall ist (Fekete et al. 2016: 221). Dem deutschen Techniksoziologen und Risikoforscher Ortwin Renn zufolge, lässt sich das vor allen Dingen mit dem großen Erfolg erklären, den konventionelles Risikomanagement in den letzten Jahrzehnten aufweisen konnte. Mit anderen Worten half die Verwendung klassischen Risikomanagements dabei, erfolgreich mit widrigen und disruptiven Ereignissen umzugehen (Renn 2016: 29). Denn genau darum geht es in der Risikoforschung technischer Art. Einem intuitiven Verständnis von Risiko als einer Situation, in der etwas für Menschen Wertvolles auf dem Spiel steht, folgend, stellt sich die Frage, wie mehr oder weniger wahrscheinlich es ist, dass ein „unerwünschter Zustand der Realität“ (undesirable state of reality) aufgrund natürlicher oder menschlicher Aktivitäten eintritt (Aven 2010: 626, Renn 2008: 50). Den Argumenten der klassischen Risikoforschung folgend, können Gefahren verschiedener Art erst dann sinnvoll bewältigt werden, wenn sie sich quantifizieren lassen (Renn et al. o.J.: 2). Risikoforschung und Risikomanagement konzentrieren sich demzufolge zumeist stark auf Auswirkungen widriger Ereignisse, die sich leicht messen und quantifizieren lassen. Kulturübergreifend gelten dabei die Anzahl Toter und Verletzter (physical harm) sowie monetäre Schäden als einzige Kategorien, die prinzipiell für alle Gesellschaften unerwünscht sind (Renn 2008: 53).

Um nun Risiken managen zu können, bedarf es in der klassischen Risikoforschung einer Definition des Begriffs, der eine Quantifizierung erlaubt. Gleichzeitig setzt das ein Systemverständnis voraus, in dem die 
grundlegenden Funktionsprinzipien bekannt sind und das System an sich sich nicht verändert während es beschrieben wird (Steen/Aven 2011: 293). Dann wird eine quantitative Risikoanalyse (probabilistic risk assessment, PRA) möglich. Quantitative Risikoanalysen spezifizieren die Dinge, die in Gefahr sind, bestimmen Wahrscheinlichkeiten für unerwünschte Konsequenzen bestimmter Ereignisse und integrieren die unterschiedlichen Bestandteile zumeist zu einer Dimension, zu einer Kennzahl, die dann das Risiko ausdrückt (Renn 2008: 52). Diese Art technischer Risikoanalyse ist dazu gedacht, Entscheidungsträgern eine Einschätzung der zu erwartenden Schäden an die Hand zu geben und vor allem aufzuzeigen, welche Konsequenzen sich mit welcher Wahrscheinlichkeit aus welcher Entscheidung ergeben (Renn 2008: 53). Das ist sehr nahe an Knights ursprünglichen Ideen (siehe 4.3.3). Besonders einflussreich für die klassische Risikoforschung und im Prinzip das Verständnis, auf dem heute noch die meisten Ansätze zur Quantifizierung von Risiko beruhen, ist die Definition von Kaplan und Garrick aus dem Jahr 1981. Diese ist in der ersten Ausgabe der Zeitschrift Risk Analysis erschienen, in einem Artikel, der den ambitionierten Titel On the Quantitative Definition of Risk trägt (siehe Kaplan/Garrick 1981).

Kaplans und Garricks Definition von Risiko enthält drei wesentliche Elemente. Das ist zunächst ein Szenario. Jedem Risiko liegt ein spezifisches Szenario zugrunde. Das zweite Element ist die Wahrscheinlichkeit, dass dieses Szenario eintritt. Und das dritte Element sind die zu erwartenden Konsequenzen für den Fall des Eintritts des spezifischen Szenarios. Aufgrund der grundsätzlich negativen Ausrichtung des Risikobegriffs geht es hier beinahe ausschließlich um das Ausmaß des Schadens. Formalisiert setzt sich nach Kaplan und Garrick das Risiko $(R)$ zusammen aus einem Szenario $(s)$, einer Wahrscheinlichkeit $(p)$ und einer Konsequenz $(x)$. Mathematisch formuliert heißt das: $(\mathrm{R})=\{\langle\mathrm{si}, \mathrm{pi}, \mathrm{xi}\rangle\}, \mathrm{i}=1,2, \ldots, \mathrm{N}(\mathrm{Ka}-$ plan/Garrick 1981: 13). In ihrer praktischen Anwendung wird die Definition meistens umgesetzt als $\mathrm{R}_{\mathrm{i}}=\mathrm{p}_{\mathrm{i}} \times \mathrm{x}_{\mathrm{i}}$, das Risiko $R_{i}$ für den Fall $i$ setzt sich zusammen aus der Wahrscheinlichkeit $p_{i}$, mit der $i$ passiert und den Auswirkungen $x_{i}$, die dann zu erwarten sind (Brühwiler/Romeike 2010: 157). Wahrscheinlichkeiten sind zum Beispiel relative Häufigkeiten für das Auftreten von Ereignissen. Etwa wenn es um Hochwasser geht und alle 100 Jahre mit einem Hochwasser mit einer bestimmten Wasserhöhe zu rechnen ist. Die Wahrscheinlichkeit für diese Art Hochwasser beträgt dann $1 \%$ pro Jahr. Seine Auswirkungen lassen sich zum Beispiel anhand der zu erwartenden finanziellen Schäden quantifizieren (Fekete et al. 2016: 216, Renn 2008: 52). Ausgehend von dieser Definition lassen 
sich dann quantitative Analysen aller Art durchführen, es lassen sich die Risiken unterschiedlicher Szenarien und Ereignisse vergleichen, es können - gesellschaftlich auszuhandelnde - quantitative Grenzen für akzeptable und inakzeptable Risiken festgelegt werden und es kann versucht werden, den Einfluss definierter risikomindernder Maßnahmen auf Eintrittswahrscheinlichkeit und/oder Schadenshöhe eines bestimmten Szenarios abzuschätzen. All das ist Bestandteil klassischer, ingenieurwissenschaftlicher Risikoforschung und muss auch weiterhin - nicht zuletzt aufgrund des erfolgreichen Managements von Systemen, das so über Jahrzehnte möglich war - seine Anwendung finden (Renn 2016: 29).

Für die vorliegende Arbeit hat die bisherige Analyse aber gezeigt, dass es darüber hinaus noch eines weiteren Paradigmas bedarf, um die Sicherheit der für die zivile SiFo relevanten, sozio-technischen Systeme aufrechtzuerhalten bzw. zu erhöhen. Und dieses Paradigma wird in Resilienz gesehen. Allerdings blieb auch die ingenieurwissenschaftliche Risikoforschung nicht bei der quantitativen Risikoanalyse stehen und sieht deren Beschränkungen im Hinblick auf die Analyse realer Systeme (Helbing 2013: 57). Auch und gerade nach Kritik aus den Sozialwissenschaften sowie im Anschluss an die Nuklearkatastrophe in Fukushima, hat sich dieser Forschungsbereich weiterentwickelt und berücksichtigt nun unterschiedliche Erkenntnisse im Hinblick auf Unsicherheit, Komplexität und weitere Phänomene, die auch für die Resilienzforschung von entscheidender Bedeutung sind (Fekete et al. 2016: 228, Park et al. 2013: 357, Renn 2008: 52, 2008b: 202, Renn et al. o.J.: 3ff). Steen und Aven fassen diese Tendenz zusammen, wenn sie Unsicherheit anstelle von Wahrscheinlichkeit als zentrales Element von Risiko beschreiben. Wahrscheinlichkeiten sind demnach nicht mehr als ein Werkzeug, um Unsicherheiten auszudrücken und zwar basierend auf dem verfügbaren Wissen. Dieses Werkzeug habe Grenzen (Stehen/Aven 2011: 293). Auch Francis und Bekera weisen auf die Unterschiede zwischen Wahrscheinlichkeiten und Unsicherheiten hin. Letztere sehen sie als "language for representing uncertainty“ an (Francis/Bekera 2014: 91). Wahrscheinlichkeiten beruhen - ebenso wie die Annahme, man könne Konsequenzen deterministisch vorhersagen auf starken Vereinfachungen und laufen Gefahr, relevante Faktoren zu übersehen (Aven 2010: 629, Steen/Aven 2011: 293). Ein erweitertes Risikoverständnis inkludiert daher auch „uncertainties beyond the probabilities.“ Aven geht sogar noch weiter und erklärt Unsicherheit als das eigentliche Wesensmerkmal von Risiko (Aven 2010: 624ff).

Autoren wie Helbing und Renn qualifizieren ihren Risikobegriff weiter, indem sie von global vernetzten oder systemischen Risiken sprechen. 
Solche Risiken sind komplex, stochastisch und nichtlinear. Sie lassen sich nicht durch einen Blick auf einfache, direkte Kausalketten erklären (Renn 2016: 30f). Aufgrund der zunehmenden Vernetzung realer, soziotechnischer Systeme sind vernetzte bzw. systemische Risiken zudem mit Kaskadeneffekten verknüpft, was zu einer „totality of the threat" führt, der Möglichkeit, dass das gesamte System zusammenbricht. Teilweise werden derartige Risiken deshalb auch als „Hyperrisiken“ (hyper risks) bezeichnet (Helbing 2013: 51, Renn 2016: 29). Die vorliegende Arbeit kann aus diesem Bereich der ingenieurwissenschaftlichen Risikoforschung, der weit über die klassische, quantitative Risikoanalyse hinausgeht, wichtige Erkenntnisse für die Entwicklung ihres Resilienz-Konzepts ziehen. Aufgrund der notwendigen Trennschärfe sowie der nach wie vor ungebrochenen Dominanz des klassischen Verständnisses von Risiko in den Ingenieurwissenschaften, geht sie aber rein begrifflich von diesem klassischen Verständnis aus. Gegeben die vielfältigen Erkenntnisse im Hinblick auf Verknüpfungen zwischen Risiko, Resilienz und Unsicherheit, wird der Begriff zudem, wie an anderer Stelle bereits erwähnt, im größeren Kontext des Zusammenhangs zwischen „Resilienz und Unsicherheit" noch einmal diskutiert und für das Resilienz-Konzept der zivilen SiFo nutzbar gemacht.

Das klassische Risikoverständnis der Ingenieurwissenschaften im Hinterkopf haltend, lohnt nun der Blick auf die vorherrschende Definition von Resilienz innerhalb dieser Disziplin. Der passende Begriff dafür lautet engineering resilience. Dieser wurde allerdings, wie bereits kurz beschrieben, nicht etwa innerhalb der Ingenieurwissenschaften geprägt, sondern im Wesentlichen von Holling in die Diskussion eingeführt. In den 1970er Jahren, als Hollings bahnbrechender Artikel zur Resilienz ökologischer Systeme erschien, arbeitete er am Internationalen Institut für angewandte Systemanalyse (IIASA) in Laxenburg nahe Wien. Dort leitete er eine Gruppe, die sich mit Ökosystemanalyse beschäftigte. Gleichzeitig leitete der deutsche Physiker Wolf Häfele am IIASA das Projekt Energiesysteme. Häfele war unter anderem ein starker Befürworter der Kernkraft und plädierte für ein eher zentralisiertes Energiesystem, indem Kernkraftwerke eine entscheidende Rolle spielen sollten. In Hollings Resilienz-Konzept erkannte er einen wertvollen Beitrag, um seine eigenen Ideen über klassisches Sicherheitsmanagement (safety) und Wahrscheinlichkeitsanalysen hinaus zu erweitern. Da sich Unfälle und widrige Ereignisse nicht sicher ausschließen lassen, müsse es darum gehen, deren Konsequenzen zu minimieren. Hollings Resilienz-Konzept war aber bis dahin rein qualitativ und entzog sich einer Messbarkeit. Daher versuchten Häfele und sein Team, das Konzept mathematisch zu formulieren und es so quantifizierbar zu 
machen. Das führt jedoch Hellige zufolge, der diese Anekdote in einem Artikel von 2019 sehr lesenswert nacherzählt, zu einem wiederum deterministischen Verständnis von Resilienz (Hellige 2019: 40f). Und genau dagegen verwahrte sich Holling, der in einem solchen Resilienz-Verständnis im Wesentlichen das Gegenteil dessen sah, was er mit dem Begriff zu fassen versucht hatte. Daraufhin führte Holling selbst den Begriff engineering resilience in die Debatte ein, sozusagen als Gegenteil der eigentlichen, der ökologischen Resilienz (ecological resilience): „Holling resisted Häfele's appropriation and re-modelling of his theorem. He asserted the basic distinction between stability-orientated engineering resilience and instability-based ecological resilience" (Hellige 2019: 42).

Was versteht Holling dann unter engineering resilience? Das hat er in einem Artikel von 1996 mit dem provokanten Titel Engineering Resilience vs. Ecological Resilience am klarsten dargestellt (siehe Holling 1996). Engineering resilience bestimmt sich durch einen Fokus auf Effizienz, Stabilität und Vorhersagbarkeit. Diese Eigenschaften entsprechen den von Ingenieuren häufig gewünschten Vorgaben für ein „fail-safe design“. Sie sind für Systeme geeignet, die sich nahe eines stabilen Gleichgewichts befinden und in denen die Widerstandsfähigkeit gegenüber Störungen und die Geschwindigkeit, mit der nach einer Störung der Gleichgewichtszustand wieder erreicht wird, im Vordergrund stehen. „That view provides one of the foundations for economic theory as well and may be termed engineering resilience" (Holling 1996: 33, eigene Hervorhebung). Holling sieht zwischen diesem Verständnis von Resilienz und ecological resilience, wie sie in Kapitel 2.3 ausführlich vorgestellt wird, einen fundamentalen Unterschied, sieht sie als alternative und sich quasi ausschließende Paradigmen. Engineering resilience habe demnach einen Fokus auf Effizienz, während ecological resilience die Persistenz, das Fortbestehen des Systems an sich, in den Mittelpunkt des Interesses stelle (Holling 1996: 33). Ein Verständnis im Sinne eines engineering resilience ergibt für Holling nur Sinn, wenn von der Existenz nur eines Gleichgewichtszustands in einem System ausgegangen wird. „And that is certainly consistent with the engineer's desire to make things work, not to make things that break down or suddenly shift their behavior. But nature is different" (Holling 1996: 38, eigene Hervorhebung). Wenn nichtsdestotrotz die beiden unterschiedlichen Arten von Resilienz innerhalb eines Systems beachtet werden sollen, sieht Holling eine negative Verknüpfung zwischen beiden. Hohe Resilienz im Sinne von engineering resilience versteht er als kurzfristige Nutzenmaximierung und das Verhindern von Abweichungen vom Gleichgewichtszustand. Genau das führt aber dazu, dass das System langfristig anfälliger 
gegenüber überraschend auftretenden Störereignissen wird (Holling 1996: 37, Rahimi/Madni 2014: 811). Oder anders gesagt: Maßnahmen, welche die engineering resilience eines Systems erhöhen, sind auf Stabilität und Bewahren ausgelegt, ihnen wohnt nach Hollings Meinung ein „strukturel$\mathrm{le}[\mathrm{r}]$ Konservatismus" inne und sie widersprechen im Prinzip genau dem, was für ihn Resilienz (=ecological resilience) ausmacht (Blum et al. 2016: 165).

Paradoxerweise haben diese Ansichten Hollings jedoch nicht dazu geführt, sein Begriffsverständnis innerhalb der Ingenieurwissenschaften zu diskreditieren. Obwohl viele Forscher gerade aus dem sozial-ökologischen Bereich diese Art der Resilienz-Definition immer wieder wie ein negatives Abziehbild dessen, was sie unter Resilienz verstehen, nutzen, hat das ihrer Prominenz in der ingenieurwissenschaftlichen Resilienzforschung keinen Abbruch getan (Gunderson 2000: 426, Lorenz 2010, Walker et al. 2004). Ganz im Gegenteil. Das auf Hollings Begriff engineering resilience und dessen Definition zurückgehende Verständnis ist mit großem Abstand und teilweise beinahe ausschließlich die dominierende Auslegung von Resilienz in den Ingenieurwissenschaften (Blum et al. 2016: 165). In der überwiegenden Zahl an Artikeln, die sich mit Resilienz aus einer ingenieurwissenschaftlichen Perspektive auseinandersetzen, wird das Konzept zu Beginn in einem mehr oder weniger strikten engineering resilience-Sinne definiert. Beispielhaft kann hier auf eine Definition von Tierney und Bruneau aus dem Jahr 2007 verwiesen werden „Resilience reflects a concern for improving the capacity of physical and human systems to respond to and recover from extreme events" (Tierney/Bruneau 2007:14). Besonders der kanadische Erdbebenforscher und Bauingenieur Michel Bruneau spielt innerhalb der ingenieurwissenschaftlichen Resilienzforschung mit seinen Ansätzen und Ideen eine herausgehobene Rolle, auf die weiter unten noch näher eingegangen wird. Die gerade zitierte Definition enthält als primäre Aspekte von Resilienz die Fähigkeiten, angemessen auf ein disruptives Ereignis zu reagieren und sich davon zu erholen. Als Ziel der Erholung geben die meisten Definitionen die Rückkehr zum Status quo ante an, was einer Rückkehr in den vorherigen, alten, ursprünglichen Gleichgewichtszustand entspricht. Exemplarisch wird das etwa bei Altherr et al. formuliert. „In the community of civil engineering, resilience is defined as the capability to recover from external stresses to an equilibrium state" (Altherr et al. 2018: 188). Zudem wird die Erholung häufig noch weiter spezifiziert und zwar im Hinblick auf ihre Geschwindigkeit. Es geht dann etwa darum, „eine Krise rasch zu bewältigen und die Funktionsund Handlungsfähigkeit schnellstmöglich wieder herzustellen“ (CSS 2009: 
1, eigene Hervorhebung). Sikula et al. kombinieren die beiden Aspekte der Rückkehr in einen vorherigen Gleichgewichtszustand und der Beachtung der Zeitdimension, in dem sie Resilienz als ,associated with the time it takes for a system to recover to equilibrium" verstehen (Sikula et al. 2015: 220). Ganz ähnlich sehen das D'Lima und Medda, für die Resilienz die Geschwindigkeit ist, die ein System aufweist, um nach einer Störung wieder in seinen Gleichgewichtszustand zurückzukehren (D'Lima/Medda 2015: 38). Die Zeitdimension wird auch von vielen anderen Autoren explizit mit ihrer Resilienz-Definition verknüpft (Aven 2011: 517, Birkmann 2008: 10, Brand/Jax 2007: 2, Connelly et al. 2017: 48, Linkov/Kott 2019: 4, Wink 2011: 113). Insgesamt lassen sich mittlerweile prinzipiell beliebig viele Artikel finden, in denen Resilienz aus ingenieurwissenschaftlicher Sicht ähnlich wie bei Zinke und Ummenhofer verstanden und definiert wird. Demnach setzt sich Resilienz zusammen aus zwei Fähigkeiten, der „Fähigkeit, einem Extremereignis zu widerstehen“ und der „Fähigkeit, nach einem Schadenseintritt den ursprünglichen Zustand schnell wiederherstellen zu können“ (Zinke/Ummenhofer 2014: 75). Genau so oder jedenfalls inhaltlich äquivalent finden sich Resilienz-Definitionen beispielsweise bei Altherr et al., Aven, Ayyub, Birkmann, Brand und Jax, Connelly et al., in den CSS-Analysen, bei D'Lima und Medda, Janić, Ji et al., Linkov und Kott, Longstaff, Ouyang, Schulman und Roe, Sikula et al., Steen und Aven, Strambach und Klement, Teodorescu, Voss und Dittmer sowie Wink (Altherr et al. 2018: 188, Aven 2011: 517, Ayyub 2014: 341, Birkmann 2008: 10, Brand/Jax 2007: 2, Connelly et al. 2017: 48, CSS 2009: 1, D'Lima/Medda 2015: 38, Janić 2015: 78, Ji et al. 2017: 1354, Linkov/Kott 2019: 4, Longstaff 2012: 265, Ouyang 2014: 44, Schulman/Roe 2007: 42, Sikula et al. 2015: 220, Steen/Aven 2011: 294, Strambach/Klement 2016: 267, Teodorescu 2015: 279, Voss/Dittmer 2016: 186, Wink 2011: 113).

Vor allem die explizite Berücksichtigung der Zeitdimension ist bereits ein Hinweis darauf, dass die meisten Autoren in den Ingenieurwissenschaften ihre Definition als Ausgangspunkt für eine Quantifizierung nutzen. Zeiträume lassen sich sowohl gut messen, als auch gut vergleichen und eignen sich daher sehr gut dafür, Bestandteil einer Resilienz-Messung zu werden. In diesem Zusammenhang lohnt auch ein Verweis auf eine Aussage von Zinke und Ummenhofer. Ihnen zufolge arbeiten „Resilienzuntersuchungen [...] meist mit vollständiger Quantifizierung, qualitative Ansätze existieren nur teilweise" (Zinke/Ummenhofer 2014: 75). Mit Blick auf die Forschungstradition zu Resilienz in den unterschiedlichen Disziplinen muss diese Aussage als faktisch nicht zutreffend abgelehnt werden. Die Einschätzung von Teodorescu, wonach trotz jahrzehntelanger Beschäf- 
tigung mit dem Resilienz-Konzept bis heute von einer quantitativ eher unspezifischen, einer „somewhat quantitatively inexplicit”, Definition zu sprechen sei, kann eine deutlich stärkere Gültigkeit beanspruchen (Teodorescu 2015: 279). Die Aussage von Zinke und Ummenhofer versinnbildlicht aber eine Tendenz, die in den Ingenieurwissenschaften ebenso anzutreffen ist, wie in den jeweils anderen, bereits betrachteten Disziplinen. Nämlich bei der Beschäftigung mit einem Konzept wie Resilienz im Wesentlichen die Erkenntnisse heranzuziehen, die aus der eigenen Disziplin stammen und möglicherweise verwandte Ideen aus anderen Fachrichtungen nicht zu berücksichtigen. Selbstverständlich gibt es auch eine Reihe von Autoren, die den Blick weiten und so von den Erkenntnissen anderer Forscher profitieren können, Lovins und Lovins sind hier ein augenfälliges und aus Sicht der vorliegenden Arbeit durchaus nachahmenswertes Beispiel. In den Ingenieurwissenschaften wird Resilienz jedoch abgesehen von diesen und einigen wenigen anderen Forschern, größtenteils immer noch als "bounce back“ - und zwar so schnell es geht und dahin, wo das System schon vorher war - verstanden (Ayyub 2014: 343, Cox et al. 2011: 307, Galloway 2013, O'Rourke 2007: 25, Reed et al. 2009: 174).

Zusammengefasst existiert in den Ingenieurwissenschaften in Bezug auf Resilienz eine „narrow engineering perspective“, die sich durch einen Technologie-Fokus und die Vernachlässigung sozialer Aspekte und Dimensionen auszeichnet (Leach 2008: 7). Das sollte aber nicht verwundern, ist es doch gerade die Aufgabe der Ingenieurwissenschaften, mithilfe der (Neu- und Weiter-)Entwicklung von Technologien, gesellschaftliche Problemstellungen zu adressieren und Lösungen zu entwerfen. Deshalb geht es in der ingenieurwissenschaftlichen Resilienzforschung häufig um strukturelle, technische Maßnahmen zur Erhöhung der Sicherheit gegenüber unterschiedlichen Arten von disruptiven Ereignissen (MacAskill/Guthrie 2014: 667). Um sinnvolle und wirksame Maßnahmen entwickeln und einsetzen zu können, und damit Resilienz proaktiv herstellen zu können, bedarf es einer "gewisse[n] Beherrschbarkeit der Einflussbedingungen." Störungen der Stabilität von Systemen werden grundsätzlich als negativ, als „zu vermeidendes Risiko interpretiert“ (Voss/Dittmer 2016: 186). Der Fokus auf technologische Dimensionen von Resilienz betont dann - wie von Holling skizziert - eher diese Stabilität, eine Kontrolle über Systeme und eine Maximierung von deren Effizienz. Er vernachlässigt dagegen Kolliarakis zufolge Aspekte wie Anpassungs- und Lernfähigkeit (Kolliarakis 2013: 108). Ob damit, wie von Holling und weiteren Autoren unterstellt, dem ingenieurwissenschaftlichen Resilienz-Verständnis ein inhärenter Konservatismus zugrunde liegt, kann aus Sicht der vorliegenden Arbeit 
nicht entschieden werden (Blum et al. 2016: 165, Leach 2008: 7). Der Ausgangspunkt ist hier ein anderer. Ein Fokus auf technologische Lösungen für Resilienz muss demzufolge nicht notwendigerweise einhergehen mit einem stabilitätszentrierten, deterministischen Verständnis. Die Frage ist vielmehr, wie die beiden Perspektiven auf Resilienz, eine eher mechanische und eine eher systemische, verknüpft werden können und wie sie voneinander profitieren können (Voss/Dittmer 2016:187). Eine klare Grenze zwischen den beiden Perspektiven lässt sich an vielen Stellen ohnehin nicht (mehr) ziehen. Vielfach nutzen beispielsweise Ingenieure mittlerweile Ansätze und Prinzipien, die sich in ökologischen Systemen finden, um das Design ihrer technischen Systeme zu optimieren (Longstaff 2012: 266). Genau aus diesem Grund, nämlich der zunehmenden Bedeutung eines systemischen Resilienz-Verständnisses auch innerhalb der Ingenieurwissenschaften, unternimmt die vorliegende Arbeit eine detaillierte, systemtheoretische Analyse von Resilienz und erforscht den Zusammenhang zwischen „Resilienz und Systemen“, um ein Resilienz-Konzept der zivilen SiFo zu entwickeln.

Das stabilitätszentrierte Verständnis von Resilienz hat in den Ingenieurwissenschaften zudem noch einen anderen Grund als einen unterstellten strukturellen Konservatismus. Ingenieure müssen Phänomene zunächst messbar machen, um sie erstens verstehen und zweitens zielgerichtet verändern zu können. Das gilt auch für Resilienz. Als metaphorisches Konzept ist es zwar bereits nützlich, für die Ingenieurwissenschaften bedarf es aber sehr viel stärker empirischer Analysen und dazu muss Resilienz zu einem operationalisierbaren und messbaren Konzept werden. Dafür werden objektive und quantitative Indikatoren benötigt (Carpenter et al. 2001: 767, Francis/Bekera 2014: 95, Hickford et al. 2018: 284, Ouedraogo et al. 2013: 27). Bei der Quantifizierung von Resilienz stellt sich generell die Frage, „welche und wie viele Indikatoren mit welcher Gewichtung in einem Gesamtindikator vertreten sein müssten, um zu einer überzeugenden Aussage zu gelangen" (Lintz et al. 2016: 349). Entscheidend ist ein Aspekt, der grundsätzlich für die Messung nicht direkt erfassbarer Phänomene gilt: Messen die genutzten Indikatoren eigentlich das, was gemessen werden soll? Mit anderen Worten, ist die zur Messung von Resilienz notwendige Operationalisierung konsistent mit dem dahinterstehenden theoretischen Konzept (Carpenter et al. 2001: 767)? Gegeben diese Herausforderung zeigt sich sofort der Vorteil eines mechanischen Resilienz-Verständnisses. Dieses eignet sich sehr viel besser zur Messung als sein systemischer Gegenpart. Basierend auf diesem Verständnis sind in den letzten ca. 15 Jahren verschiedene Ideen und Methoden entstan- 
den, mit deren Hilfe Resilienz quantifiziert werden soll. Gemeinsam ist den meisten dabei, dass sie sich mehr oder weniger explizit auf einen Ansatz beziehen, den Michel Bruneau mit Kollegen wie Kathleen Tierney entwickelt hat. Ihr „conceptual framework“ war laut Nan und Sansavini das erste, mit dessen Hilfe Resilienz in den Ingenieurwissenschaften als Konzept messbar gemacht werden sollte (Nan/Sansavini 2017: 36, Sansavini 2016, siehe Bruneau et al. 2003). Von der oben skizzierten, klassischen quantitativen Risikoanalyse unterscheiden sich derartige Resilienz-Messungen primär durch die Messbarmachung unterschiedlicher Phasen - wenn man von einer zyklischen Vorstellung ausgeht (Scharte et al. 2014: 17). Die Risikoanalyse fragt danach, wie groß Risiken für bestimmte Szenarien sind und sucht in einem anschließenden Risikomanagement unter anderem danach, den Eintritt inakzeptabler Szenarien zu verhindern. Dahingegen betrachtet die im Folgenden ebenfalls als „klassisch“ bezeichnete Resilienzanalyse Systeme nachdem ein disruptives Ereignis eingetreten ist und fragt nach der Zeit, die es dauert, das System bzw. seine Leistungsfähigkeit wiederherzustellen.

Wie bereits erwähnt, waren Bruneau et al. 2003 die ersten, die Resilienz in dieser Weise zu quantifizieren suchten. Bis heute gehören insbesondere Bruneau und Tierney zu den einflussreichsten Forschern in diesem Gebiet. Sie gelten als „Erfinder“ des sogenannten „resilience triangle“ (Ayyub 2014: 345, siehe Abbildung 1). Der Ausgangspunkt von Bruneau et al. war ein wahrgenommener Mangel an Ansätzen zur Quantifizierung von Resilienz, wobei der Fokus auf einer spezifischen Art von Resilienz bzw. besser gesagt einer spezifischen Art von Störung lag, nämlich Erdbeben. Um das zu ändern, definieren sie das Ziel ihres Artikels wie folgt: „It is therefore necessary to clearly define resilience, identify its dimensions and find ways of measuring and quantifying those dimensions" (Bruneau et al. 2003: 734). An dieser Stelle ist es wichtig darauf hinzuweisen, dass die Resilienz-Definition von Bruneau et al. gerade nicht dem entspricht, was als mechanisches Verständnis bezeichnet wurde. Die Autoren haben eher ein umfassenderes daher allerdings auch wenig trennscharfes Bild von Resilienz. Ihnen zufolge geht es sowohl um die Fähigkeit, normalen bzw. erwarteten Störungen zu widerstehen, als auch um die Fähigkeit, sich plötzlichen und unerwarteten, außergewöhnlichen disruptiven Ereignissen anpassen zu können. Dafür geeignete Maßnahmen können sowohl präventiv versuchen, die durch widrige Ereignisse verursachten Schäden zu verhindern, als auch reaktiv dazu beitragen, entstandene Schäden zu minimieren (Bruneau et al. 2003: 735). Wie seitdem alle Forscher, die versuchen, Resilienz zu quantifizieren, stehen Bruneau et al. dann vor dem 
Problem, dieses umfassende Konzept messbar zu machen und entscheiden sich an dieser Stelle für einen Weg, der den größten Teil der darauffolgenden ingenieurwissenschaftlichen Resilienzforschung prägt. Sie übersetzen ihr Konzept in ein einfaches Diagramm, indem die Systemleistung (performance) über der Zeit aufgetragen wird. $\mathrm{Zu}$ jedem beliebigen Zeitpunkt erbringt ein beliebiges System eine spezifische Leistung, die gemessen werden kann. Ein Beispiel wäre die Menge an zur Verfügung gestelltem elektrischen Strom. Bruneau et al. abstrahieren noch weiter und geben die Leistung in Prozent an. Im ungestörten Zustand erbringt das System eine Leistung von 100\%. Durch das Eintreten einer Störung verringert sich die Systemleistung bis zu einem bestimmten Punkt und erholt sich danach wieder, bis sie nach einer bestimmten Zeitspanne wieder ihren Ursprungswert erreicht hat. Auch wenn wieder 100\% Systemleistung erreicht werden, ist in der Zwischenzeit ein Leistungsverlust eingetreten. Resilienz trägt nun nach Bruneau et al. dazu bei, diesen Leistungsverlust zu minimieren, indem zum Beispiel die initiale Störung verhindert wird. Dann gibt es keinen Leistungsverlust. Oder, und im Fall von Erdbeben kann das angenommen werden, falls ein Verhindern der Störung nicht möglich ist, indem der initiale Leistungsverlust und/oder die Zeit bis zum Wiedererlangen der vollständigen Systemleistung minimiert werden (Bruneau et al. 2003: 736, siehe Abbildung 1). Bruneau et al. formulieren ihren Ansatz auch noch in mathematischer Weise, indem sie den Resilienz-Verlust $R$ formal wie folgt beschreiben: $\mathrm{R}=\int_{\mathrm{t} 0}^{\mathrm{t1}}[100-\mathrm{Q}(\mathrm{t})] \mathrm{dt}$ (Bruneau et al. 2003: 737).

Abbildung 1: Das resilience triangle

Systemleistung $\mathbf{p}$ (bei Bruneau et al.: Quality of infrastructure in \%)

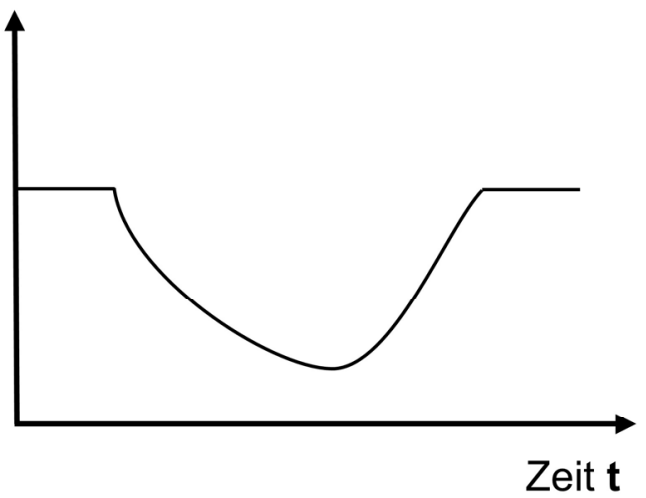

Quelle: eigene Darstellung nach Bruneau et al. 2003. 
Selbst diese scheinbar simple Form der Resilienz-Quantifizierung ist nach Bruneau et al. mit signifikanten Herausforderungen verbunden, wenn es um eine konkrete Messung geht. Darüber hinaus erfordert die Betrachtung konkreter Einzelfälle auch noch die Einbeziehung weiterer, kontextspezifischer Faktoren, welche die Komplexität der Aufgabe noch weiter erhöhen. Aber auch bereits ohne Einzelfall, im konzeptionellen Bereich verbleibend, sehen Bruneau et al. die Grenzen ihres eigenen Ansatzes. Etwa wenn sie von den vier miteinander verknüpften Dimensionen reden, die Resilienz umfasst. Diese sind die technische, organisatorische, soziale sowie die ökonomische Dimension - in der Forschung basierend auf den Anfangsbuchstaben der englischen Adjektive häufig als TOSE bezeichnet, die nicht in adäquater Weise durch eine einfache Messung der Systemleistung mithilfe eines einzelnen Indikators abbildbar sind (Bruneau et al. 2003: 737f). Um das zu adressieren, entwickelt Bruneau gemeinsam mit Reinhorn bereits 2006 ein 3D- und sogar ein 4D-Resilienz-Konzept mit den zusätzlichen Dimensionen Ressourcen und Redundanz (Bruneau/Reinhorn 2006). Nichtsdestotrotz bleiben das ursprüngliche resilience triangle, das Diagramm in dem die Systemleistung über die Zeit und das Verhalten des Systems nach Auftreten einer Störung skizziert sind, und das dahinterstehende, relativ simpel zu erfassende Maß für Resilienz als Minimierung des Leistungsverlusts, der mit Abstand einflussreichste Ausgangspunkt für beinahe sämtliche Ansätze zur Quantifizierung von Resilienz. Und während Bruneau und Reinhorn erkennen, dass sie mit einer Resilienz-Messung nur einen kleinen, aber wichtigen „building block“ zu einem größeren Bild des umfassenderen Resilienz-Konzepts beizutragen in der Lage sind, verselbstständigt sich in der Folge die Idee, dass ingenieurwissenschaftliche Resilienzforschung im Wesentlichen im Entwurf immer neuer Ansätze und Methoden zur Quantifizierung von Resilienz besteht (Bruneau/Reinhorn 2006, Hosseini et al. 2016: 59).

Es gibt mittlerweile eine beinahe unüberschaubare Vielzahl an Versuchen, das Resilienz-Konzept messbar zu machen. Einen Überblick über verschiedene Ansätze gibt beispielsweise Ayyub, der verschiedene Maße für Resilienz mit ihren jeweiligen Stärken, Grenzen und Anwendungsmöglichkeiten beschreibt (Ayyub 2015). Auch Hosseini et al. geben einen guten Überblick über verschiedene Ansätze zur Quantifizierung von Resilienz (Hosseini et al. 2016: 52). Selbiges gilt für Altherr et al., Attoh-Okine und Janić (Altherr et al. 2018: 189ff, Attoh-Okine 2016: 12ff, Janić 2018: 1107f). Ein in dieser Hinsicht besonders interessanter Artikel ist der von Tamvakis und Xenidis, der eine Übersicht über verschiedene Ansätze zur Resilienz-Messung mit einem Resilienz-Verständnis verknüpft, 
das dem von Hollnagel und Woods entspricht (Tamvakis/Xenidis 2013: $341 \mathrm{ff}$ ). Beim Blick auf einige ausgewählte Einzelbeispiele fällt auf, wie unterschiedlich die Ansätze trotz der gemeinsamen Ausgangsbasis häufig sind. Das lässt sich zunächst durch unterschiedliche konkrete Anwendungsgebiete erklären. Sind diese gleich oder ähnlich, wie etwa bei Cox et al., D'Lima und Medda und Janić, die sich alle mit dem Transportsektor bzw. noch konkreter dem Schienenverkehr als kritischer Infrastruktur auseinandersetzen, gibt es deutliche Ähnlichkeiten zwischen den Konzepten. Cox et al. und D'Lima und Medda wählen sogar mit den Terroranschlägen vom Juli 2005 in London und der Reaktion des Londoner U-Bahn-Systems das gleiche Fallbeispiel (siehe Cox et al. 2011, D'Lima/Medda 2015, Janić 2018). Die letzten beiden Artikel haben ein eher klassisches, stabilitätszentriertes Resilienz-Verständnis, während Cox et al. das Konzept durchaus umfassend diskutieren und Überlegungen anstellen, wie auch Aspekte des Konzepts, die eher einem systemischen Verständnis entspringen, quantifiziert werden könnten (Cox et al. 2011. 313ff, D'Lima/Medda 2015: 35, Janić 2018: 1110). Das ist ein augenfälliges Beispiel für die zweite Kategorie, in der sich unterschiedliche Ansätze zur Resilienz-Messung unterscheiden, nämlich konzeptionelle Differenzen, die sich in der Wahl von Indikatoren und auch in der Komplexität (in einem nichtwissenschaftlichen Sinne) der mathematischen Resilienz-Gleichung ausdrückt, die häufig aufgestellt wird.

Und dann unterscheiden sich die verschiedenen Ansätze natürlich auch durch die mathematischen Modelle und Methoden, die zur Anwendung kommen. An dieser Stelle kann die vorliegende Arbeit aufgrund ihrer sozialwissenschaftlichen Verortung keine tiefergehende, bewertende Beschreibung bezüglich unterschiedlicher mathematischer Methoden vornehmen. Es lässt sich aber festhalten, dass beispielsweise die Verwendung probabilistischer Ereignisbaumanalysen, wie Teodorescu sie zur Resilienz-Quantifizierung nutzt, einem klassischen Risiko-Verständnis entspringt (Teodorescu 2015: 280). Dazu passen auch Aussagen wie die, man müsse zunächst alle vernünftigen Gefahren bestimmen, dann deren potentielle Schäden und schließlich die Wahrscheinlichkeit des Auftretens determinieren (Teodorescu 2015: 281ff). Sehr viel stärker dem klassischen Resilienz-Verständnis der Ingenieurwissenschaften entsprechen wiederum Quantifizierungsansätze von Ganin et al. und Reed et al. (siehe Ganin et al. 2016, Reed et al. 2009). Selbiges gilt auch für Ji et al., die zwar verschiedene, aber doch klassische Resilienz-Maße nutzen. Sie sehen allerdings durchaus deren Grenzen und diskutieren relevante Fragen zum Beispiel im Bezug darauf, worin überhaupt die Leistung eines Systems besteht (Ji et al. 2017: 
1355). Ouyang und Wang wiederum gehen zwar vom altbekannten resilience triangle aus, definieren Resilienz aber für bestimmte Zeitspannen, unabhängig davon, ob das System in dieser Zeit bereits wieder seine volle Leistungsfähigkeit zurückerlangt hat oder ob es sogar bereits vorher wieder normal funktioniert (Ouyang/Wang 2015: 74ff). Zum Abschluss dieses kurzen Überblicks über Ideen zur Messbarmachung von Resilienz lohnt es sich, auf einen Artikel von Francis und Bekera einzugehen. Die beiden entwickeln ein ambitioniertes und mit einem systemischen Verständnis von Resilienz durchaus in Einklang zu bringendes Modell. Das gelingt ihnen, indem sie das Konzept der Entropie nutzen. Mit Entropie beschreiben sie "the degree of surprise' in the state of a random variable, multiplied by its probability of occurence" (Francis/Bekera 2014: 97). Ihr Resilienz-Maß enthält dann klassische Angaben, Angaben über Expertenmeinungen, aber auch den durch Entropie vermittelten Unsicherheits- oder Überraschungsfaktor. Überraschend auftretende und extreme Ereignisse werden zudem mathematisch betrachtet stärker gewichtet, als andere Arten von Ereignissen. So gelingt es den Autoren nach eigener Aussage, die probabilistische Risikoanalyse mit dem Resilienz-Konzept zu verknüpfen (Francis/Bekera 2014: 97).

Übergreifend lässt sich festhalten, dass trotz zahlreicher Versuche nach wie vor keine standardisierte Methode zur Quantifizierung von Resilienz existiert (Ferris et al. 2018, Tamvakis/Xenidis 2013: 346). In den meisten Fällen verweisen die Autoren zwar mehr oder weniger explizit auf Bruneaus Ideen und ein Verständnis von Resilienz als Kombination aus Schadensausmaß und Zeit zur Wiederherstellung, entwickeln aber davon ausgehend eigenständige Metriken, die eine Vergleichbarkeit erschweren (Hosseini et al. 2016: 56). Hickford et al. attestieren den meisten Ansätzen, unvollständig und jeweils nur in sehr eng begrenzten Bereichen anwendbar zu sein und führen das auf die starken konzeptionellen Unterschiede zwischen Resilienz in unterschiedlichen Disziplinen zurück (Hickford et al. 2018: 284). Die meisten Definitionen von Resilienz sind in einer Art qualitativ ausgestaltet, dass sie sich nur schwer bis gar nicht dazu eignen, als Grundlage für Metriken zu dienen (Ayyub 2014: 343). Das gilt besonders für die Sozialwissenschaften, die sich wohl „eingestehen [werden] müssen, dass sie ,ihre' Resilienz nicht wissenschaftlich exakt erfassen oder gar messen können" (Zander/Roemer 2016: 49). In zugespitzter Form formuliert Aven das für den Risikobegriff: „[F]irst we define a theoretical quantity that does not exist in the real world, and then we become uncertain what its correct value is" (Aven 2010: 629). Die Aussage kann auch für die Frage nach geeigneten Maßen für Resilienz Gültigkeit beanspruchen. 
Viele der gerade zitierten Ansätze nutzen daher laut Tamvakis und Xenidis etablierte Modelle und Methoden aus anderen Bereichen, um Resilienz zu messen, nehmen dabei aber - bewusst oder unbewusst - in Kauf, dass die Modelle nicht konsistent sind mit der verwendeten Resilienz-Definition (Tamvakis/Xenidis 2013: 346). Diese Ansicht teilen auch andere Autoren (Bocchini/Frangopol 2011: 9, Fekete et al. 2014: 5, Nan/Sansavini 2017:37). Sie gilt jedoch nicht oder nur eingeschränkt für das mechanische Verständnis von Resilienz, was, wie bereits erwähnt, ein Grund für dessen Dominanz in den Ingenieurwissenschaften ist. Zusammenfassend kann für die ingenieurwissenschaftliche Resilienzforschung also noch einmal gesagt werden, dass die Entwicklung von Ansätzen zur Quantifizierung und Messung des Konzepts eine entscheidend wichtige Rolle spielt, aber bisher trotz teilweise sehr ambitionierter Versuche - kein allgemein akzeptiertes Maß gefunden wurde.

Selbstverständlich existieren in den Ingenieurwissenschaften auch Arbeiten, deren Fokus stärker auf Mechanismen und konkreten Ideen liegt, mit deren Hilfe die Resilienz relevanter Systeme erhöht werden könnte. Bereits Bruneau et al. weisen in ihrem Artikel von 2003 auf vier Systemeigenschaften hin, die für Resilienz kennzeichnend bzw. entscheidend sind (Bruneau et al. 2003: 737). Diese sind als „R4 framework of resilience“ in den darauffolgenden Jahren ähnlich wirkmächtig geworden wie das resilience triangle. Die erste dieser Systemeigenschaften ist Robustheit (robustness), verstanden als Fähigkeit, widrigen Ereignissen ohne signifikante Leistungsverluste zu widerstehen (CSS 2009: 2, Flynn 2008: 6f, Tierney/Bruneau 2007: 15). Robustheit gehört zu den wichtigsten, klassischen Elementen ingenieurwissenschaftlicher Sicherheitsforschung. Inwiefern sie Bestandteil von Resilienz sein kann oder sollte, ist durchaus Gegenstand intensiver Debatten (siehe dazu z.B. Woods 2015: 6f). Die zweite Eigenschaft ist Redundanz (redundancy), also die Existenz mehrerer Systembestandteile, die zur Erfüllung der gleichen Aufgabe in der Lage sind und die jeweils füreinander einspringen können, sollten einzelne Bestandteile versagen (CSS 2009: 2, Tierney/Bruneau 2007: 15). Redundanz gehört zu den wichtigsten Prinzipien, wenn es um die Umsetzung von Resilienz geht und ist wohl das am häufigsten genannte. Aus diesem Grund wird das Prinzip sowohl im Zusammenhang mit „Resilienz und Anpassungsfähigkeit“ system- und komplexitätstheoretisch untersucht, als auch an späterer Stelle in den Hypothesen für ein Resilience Engineering auftauchen (siehe 6.5). Die dritte Eigenschaft lässt sich als Einfallsreichtum übersetzen (resourcefulness). Damit ist das Vermögen eines Systems gemeint, Probleme zu erkennen und priorisieren und darauf basierend die geeigneten Lösungsmöglichkei- 
ten durch eine Mobilisierung unterschiedlicher Ressourcen umsetzen zu können (CSS 2009: 2, Flynn 2008: 6f, Tierney/Bruneau 2007: 15). Einfallsreichtum ist eng verknüpft mit Kreativität, einem Aspekt, der unter der Überschrift "Resilienz und Flexibilität" noch eingehender untersucht wird. Die vierte Eigenschaft ist die Schnelligkeit (rapidity), mit der das System auf ein Schadensereignis zu reagieren imstande ist und mit der es seine Funktionsfähigkeit wiederherstellen kann (CSS 2009: 2, Flynn 2008: 6f, Tierney/Bruneau 2007: 15). Cimellaro et al. spezifizieren die vier Eigenschaften noch weiter, wenn sie Redundanz und Einfallsreichtum als Mittel zur Erreichung von Robustheit und Schnelligkeit bezeichnen (Cimellaro et al. 2010: 3641).

Trotz eines Überhangs an Arbeiten zur Quantifizierung von Resilienz, gibt es also in den Ingenieurwissenschaften durchaus auch Versuche, Resilienz konkret umzusetzen. Einer der frühesten aber gleichzeitig umfassendsten und interessanten ist der von Amory und L. Hunter Lovins. In einer 1982 veröffentlichten Studie analysieren sie die Verwundbarkeit des damals existierenden, zentralisierten und von fossilen Energieträgern abhängigen amerikanischen Energiesystems und schlagen als Gegenmaßnahme den Aufbau eines dezentralen, auf Energie aus erneuerbaren Quellen basierenden Systems vor. Das Konzept, das sie zur Beschreibung dieses neuartigen Energiesystems nutzen, ist das der Resilienz. Und zwar explizit das aus der Ökosystemforschung stammende Konzept von Holling, dem Lovins und Lovins bereits im Vorwort danken: „Professor C.S. Holling [...] to whom we owe the whole concept of resilience" (Lovins/Lovins 2001: xi-xii). Im Anschluss an Holling gehen sie beispielsweise davon aus, dass heute noch existierende biologische und ökologische Systeme grundsätzlich „masterpieces of resilience“ sein müssen, da die nicht-resilienten Systeme im Lauf der Zeit bereits ausgestorben seien. Also fragen sie sich, welche Mechanismen und Prinzipien diesen Systemen zum Überleben verholfen haben und ob bzw. wie sich diese auf Energiesysteme (im Wesentlichen das amerikanische Energiesystem) übertragen lassen. Lovins und Lovins Analyse war nach eigener Darstellung die erste derart umfassende Studie zur Verwundbarkeit des amerikanischen Energiesystems. Ihre Zielstellung war es, die Versorgungssicherheit des Energiesystems im Angesicht aller möglichen Arten von disruptiven Ereignissen gewährleisten zu können, sowohl erwarteten als auch unerwarteten (Lovins/Lovins 2001: $3,8,175,190)$.

Grundsätzlich kann ein Energiesystem nur mit den Störungen erfolgreich umgehen, für die es ursprünglich geplant wurde, die im Design mitberücksichtigt wurden (Lovins/Lovins 2001: 31). Das gilt vor allem 
für lineare Systeme, von denen Ingenieure klassischerweise ausgehen und in denen Ereignisse reversibel und erwartbar auftreten. Dann lassen sich Wahrscheinlichkeiten und Konsequenzen voraussagen und mittels klassischen Risikomanagements adressieren. Weil aber das amerikanische Energiesystem eine immer stärkere Vernetzung und damit steigende Komplexität aufweist, treten Fehler auf, die weder erwartbar noch vorhersagbar sind und denen keine Wahrscheinlichkeiten zugewiesen werden können. Dafür sind klassische Herangehensweisen der Ingenieurwissenschaften, die mit einem linearen Systemverständnis arbeiten, nicht anwendbar (Lovins/Lovins $2001: 31,138,177 \mathrm{f}, 190)$. „[It is] simply beyond human ingenuity to think of all possible failure modes" (Lovins/Lovins 2001: 178). Aber genau diesen unerwarteten und gravierenden disruptiven Ereignissen, von Lovins und Lovins als „sudden shocks, rare and of large magnitude“ bezeichnet, gilt ihr Interesse (Lovins/Lovins 2001: 10). Um damit erfolgreich umzugehen, benötigt das System generische Mechanismen, die unabhängig vom konkreten widrigen Ereignis funktionieren, was Lovins und Lovins als „resilience in the design philosophy“ zusammenfassen (Lovins/Lovins 2001: 178). In einer Welt ohne Überraschungen lässt sich eine effizienzmaximierende Strategie der Spezialisierung sinnvoll umsetzen. Die von ihnen beobachtete reale Welt der Energiesysteme wird aber von Unsicherheit, unvollständigem Wissen und andauernden Veränderungen gekennzeichnet. Effizienz kurzfristig zu maximieren, kann dann dazu führen, dass das System nicht mit unerwarteten Störungen umzugehen in der Lage ist. Resilienz hingegen bedeutet die Fähigkeit, "to survive unexpected stress“ (Lovins/Lovins 2001: 188ff). Das bedarf aus Sicht der vorliegenden Arbeit einer tiefergehenden theoretischen Analyse und Begründung, die bei der Diskussion zu „Resilienz und Anpassungsfähigkeit“ verortet werden kann.

Basierend auf diesen von Holling inspirierten Annahmen identifizieren Lovins und Lovins eine Reihe von Prinzipien, die in Energiesystemen umgesetzt werden sollten, um diese möglichst resilient zu gestalten. Diese Prinzipien werden nun der Reihe nach kurz erläutert. Lovins und Lovins geben keine tiefergehenden, theoretischen Begründungen für die Wahl der jeweiligen Prinzipien, zum größten Teil halten sie sie für selbsterklärend und einen logischen Bestandteil von Resilienz. Zudem stand eine detaillierte theoretische Betrachtung nicht im Vordergrund ihres Forschungsinteresses. Ihnen ging es darum, anwendbare und umsetzbare Mechanismen vorzuschlagen, mit deren Hilfe das amerikanische Energiesystem aus ihrer Sicht resilienter gestaltet werden könnte.

Das erste Prinzip ist das der Modularität (fine-grained, modular structure). Laut Lovins und Lovins haben Fehler in modular gestalteten Syste- 
men ceteris paribus - unter sonst gleichen Bedingungen - weniger gravierende Auswirkungen, als in zentralistisch gestalteten. Das zweite Prinzip ist eine frühe Feblerdetektion (early fault detection). Je schneller es gelingt, das Versagen einzelner Bestandteile eines Energienetzes zu bemerken, desto schneller können Gegenmaßnahmen ergriffen und verhindert werden, dass sich das Versagen auf andere Systemteile ausbreitet. Das dritte Prinzip kombiniert die wohlbekannte Redundanz mit Ersetzbarkeit (redundancy and substitutability). Wenn versagende Systemteile durch doppelt vorhandene Bestandteile ersetzt werden können, hat ihr Versagen ebenfalls keine gravierenden Auswirkungen. Lovins und Lovins favorisieren hier primär die sogenannte funktionale Redundanz, verstanden als die Fähigkeit, die gleichen Aufgaben innerhalb eines Systems mithilfe verschiedenartiger Systemkomponenten auszuführen. Funktionale Redundanz ist zu unterscheiden von physischer Redundanz, dem doppelten Vorhandensein identischer und zur Funktionalität des Systems eigentlich nur einfach benötigter Komponenten. Das vierte Prinzip ist eine optionale Vernetzung (optional interconnection). Die verschiedenen Module des Systems sollen im Normalfall durch enge Verknüpfung voneinander profitieren, aber im Versagensfall möglichst schnell und reibungslos entkoppelt werden können. Das fünfte Prinzip, Diversität (diversity), folgt einer ähnlichen Logik wie das Prinzip der funktionalen Redundanz. Je größer die Diversität innerhalb eines Systems, desto geringer ist die Wahrscheinlichkeit, dass einzelne Fehlermechanismen das ganze System lahmzulegen imstande sind. Die Systemkomponenten sollen heterogen ausgestaltet sein. Lovins und Lovins erwähnen das Beispiel redundant vorhandener Dieselgeneratoren als Notstromversorgung für kritische Einrichtungen, etwa Kernkraftwerke. Sie erfüllen zwar das Kriterium der physischen Redundanz, verfügen aber nicht über Diversität und sind so anfällig gegenüber den gleichen Fehlermechanismen - etwa ein Ausbleiben der Versorgung mit Dieselkraftstoff. Lovins und Lovins bezeichnen Diversität auch als „funktionale Flexibilität" (functional flexibility). Der Flexibilitätsbegriff wird an späterer Stelle sowohl system- und komplexitätstheoretisch als auch im Hinblick auf seine Bedeutung für Resilience Engineering untersucht. Bezüglich der Diversität geben sie zu bedenken, dass größere Diversität den Wartungsaufwand in Energiesystemen erheblich erhöht. Deshalb lautet ihr sechstes Prinzip Standardisierung (standardization). Damit ist die Möglichkeit gemeint, einzelne Systembestandteile einfach und schnell ersetzen zu können, da diese immer gleich aufgebaut, eben standardisiert, sind. Das erleichtert die Wartung und Reparatur, widerspricht aber dem Gebot der Diversität. Um dieses Dilemma zumindest teilweise auflösen zu können, schlagen Lovins 
und Lovins vor, nur bestimmte Komponenten austauschbar zu gestalten und Diversität an Stellen einzusetzen, die eine höhere Fehlerwahrscheinlichkeit aufweisen (Lovins/Lovins 2001: 192-198). Allerdings stellt sich die Frage, ob das in komplexen Systemen möglich ist (siehe dazu ausführlich 4.3.2).

Das nächste Prinzip ist das der Streuung (dispersion). Damit ist eine geographisch heterogene Verteilung wichtiger Systemkomponenten gemeint. In einem Energiesystem, das eine gesamte Nation zu versorgen hat, ist es sicher möglich, kritische Bestandteile an unterschiedlichen Orten unterzubringen, die so beispielsweise nicht von derselben Naturkatastrophe getroffen werden können. Das achte Prinzip nennt sich hierarchische Einbettung (hierarchical embedding). Im Vergleich zu Modularität, Diversität oder auch Redundanz wird hier nicht direkt durch den Titel deutlich, was Lovins und Lovins meinen. Der dahinterstehende Gedanke ist der einer bestimmten Netzstruktur, die einzelnen Subsystemen erlaubt zu versagen, ohne dass dadurch das gesamte System betroffen ist. Im Unterschied zur Modularität unterliegen die verschiedenen Systemkomponenten aber einer Hierarchie, es gibt also vor allen Dingen viele, weniger wichtige Subsysteme, deren Versagen das Gesamtsystem abfedern kann. Es gibt aber auch besonders wichtige Hauptsysteme, bei deren Versagen das Gesamtsystem direkt in Gänze betroffen wäre. Inwiefern dieses Prinzip also die Resilienz eines Systems erhöht, bleibt zu diskutieren. Selbiges gilt für das nächste Prinzip, die Stabilität (stability). Stabilität in einem eher klassischen Sinne wird von Holling und den ihm nachfolgenden Resilienzforschern ja gerade als Gegenbild zu Resilienz gesehen. Lovins und Lovins haben jedoch ein Stabilitäts-Verständnis, das sich besser mit ihrer Idee von Resilienz verträgt. Sie meinen mit Stabilität eine Fähigkeit dazu, im Fall des Eintretens einer Störung genügend Pufferzeit zur Reaktion zu haben. Statt eines sofortigen Zusammenbruchs soll ein System nur graduell und "graceful“ versagen. Das zehnte Prinzip kann ebenfalls nicht als unumstritten gelten. Die Forderung nach der Einfachheit (simplicity) von Systemen entspringt laut Lovins und Lovins etablierten Ideen aus den Ingenieurwissenschaften wie dem KISS Prinzip (keep it simple, stupid). Es ist unmittelbar einsichtig, dass einfachere Systeme leichter zu kontrollieren sind, als kompliziertere bzw. komplexere. Insofern scheint es zunächst sinnvoll, Einfachheit als Resilienz-Prinzip zu fordern (Lovins/Lovins 2001: 198-202). Ob das so zutrifft, bedarf aber einer detaillierteren system- und vor allem komplexitätstheoretischen Analyse (siehe dazu ausführlich 4.3.2).

Die bisher aufgeführten Prinzipien weisen einen starken Technikbezug auf. Sie lassen sich im Wesentlichen durch Technologie und ingenieur- 
wissenschaftlich informiertes Systemdesign umsetzen. Darüber hinaus beschreiben Lovins und Lovins noch drei weitere Prinzipien, die sehr viel stärker an der Schnittstelle zwischen technologischen und gesellschaftlichen Fragestellungen angesiedelt sind. Damit nehmen sie ein Stück weit - wenn auch in einer sehr viel weniger detaillierten Ausgestaltung - den Ansatz der vorliegenden Arbeit vorweg, die aber zum einen noch expliziter interdisziplinär ausgerichtet ist und zum anderen trotzdem eine stärker sozialwissenschaftliche Prägung aufweist. Lovins und Lovins deuten in ihren Ausführungen die gesellschaftlichen, teilweise normativ geprägten, Fragestellungen in Bezug auf Resilienz nur an. Ihr elftes Prinzip lautet begrenzte Anforderungen an soziale Stabilität (limited demands on social stability). Die Technologien, die für die Energieversorgung der Bevölkerung genutzt werden, sollen nicht derart gefährlich sein, dass menschliches Versagen unweigerlich zu katastrophalen Schäden führt. Menschen seien nun mal keine Engel oder Roboter, die keine Fehler begehen. Wenn Technologien zudem nur durch eine quasi-militärisch strenge Überwachung oder gar durch Einschränkung gesellschaftlicher Freiheiten zu sichern seien, sollte von ihrer Verwendung abgesehen werden. Die Formulierungen richten sich primär, wenn auch nicht ausschließlich, gegen die Verwendung der Kernkraft. Zu Zeiten des Kalten Krieges sprechen Lovins und Lovins von der nuklearen Abschreckung, die dazu diene, die Freiheit der Vereinigten Staaten aufrechtzuerhalten und kontrastieren diese mit der zivilen Nutzung der Atomenergie als latenter Gefahr für ebendiese Freiheit. Resilienz heißt für sie dann die Sicherstellung der „sozialen Kompatibilität“ (social compatibility) der eingesetzten Technologien. Während das elfte Prinzip primär gesellschaftlichen Aushandlungsprozessen unterliegt, stellt das zwölfte, die Zugänglichkeit (accesibility), sozusagen eine Forderung an die Ingenieurwissenschaften, wie ihre Technologien und Systeme ausgestaltet werden sollen, damit sie überhaupt gesellschaftlich verhandelt werden können. Demnach sollen auch Laien in der Lage sein, grundsätzlich $\mathrm{zu}$ verstehen, worum es geht und wie die Technologie bzw. das System funktioniert. Selbstverständlich müssen Laien die Systeme nicht selbst gestalten und bedienen können. Sie sollen aber die zugrundeliegende Logik verstehen können, um etwa über ihren Einsatz in gesellschaftlichen Aushandlungsprozessen entscheiden zu können. Die beiden Prinzipien elf und zwölf finden sich in ähnlicher Weise in der normativen Diskussion des Resilienz-Konzepts im weiteren Verlauf der vorliegenden Arbeit wieder (siehe 4.2.2). Das finale, dreizehnte Prinzip der Reproduzierbarkeit (reproducibility) meint dann sozusagen eine technische Umsetzung des Zugänglichkeit-Prinzips. Die im Energiesystem genutzten Technologien 
sollen auch ohne exklusives Spezialwissen und außergewöhnliche Ressourcen wart- und reparierbar sein. Nur so können sie nach einem disruptiven Ereignis schnell wiederhergestellt werden. Wenn das Energiesystem dagegen auf wenige, hochspezialisierte Experten angewiesen ist, erhöht das seine Verwundbarkeit (Lovins/Lovins 2001: 204ff).

Auf Seite 213 ihrer Studie fassen Lovins und Lovins alle Prinzipien noch einmal übersichtlich in neun Aufzählungspunkten zusammen. Die Modularität steht dabei weiter an erster Stelle der Aspekte, die ein Energiesystem ihnen zufolge resilient machen. Die gerade diskutierten stärker gesellschaftlich wirkenden Prinzipien werden hingegen im letzten Aufzählungspunkt nur sehr knapp zusammengefasst. Das zeigt den Fokus auf Technologie bzw. Systemdesign, den die Beiden haben (Lovins/Lovins 2001: 213). Mithilfe ihrer Prinzipien wollen sie helfen, ein Energiesystem zu gestalten, das mit einem hohen Grad an inhärenter Resilienz gegenüber prinzipiell beliebigen disruptiven Ereignissen versehen ist (Lovins/Lovins 2001: 266). Aus heutiger Sicht entsprechen viele der konkreten Vorschläge, die sie basierend auf den Prinzipien im weiteren Verlauf der Studie machen, beinahe eins zu eins denen, die im 21. Jahrhundert im Kontext einer nachhaltigen Energieversorgung diskutiert werden. Lovins und Lovins nehmen Idee und Inhalt der deutschen Energiewende knapp 30 Jahre vorher vorweg und werben mit ihrer Studie vehement für den Ausbau erneuerbarer Energieträger wie Wind- und Solarenergie. Ihre Begründung dafür ist aber interessanterweise eben gerade nicht die Nachhaltigkeit, sondern vielmehr die immanente Verwundbarkeit eines zentralisierten, auf fossilen Energieträgern basierenden Systems, die sie zu überwinden suchen. Und das Resilienz-Konzept - im Hollingschen Sinne - ist ihr Vehikel dazu.

Mit diesem abschließenden Beispiel einer zum damaligen Zeitpunkt sehr innovativen und außergewöhnlichen Nutzung des Resilienz-Konzepts, das gleichzeitig sehr viele wertvolle Hinweise und Anknüpfungspunkte für die vorliegende Arbeit enthält, endet dieses Kapitel zum Überblick über den Stand der Forschung in den relevantesten Disziplinen der Resilienzforschung. Wie im ersten Kapitel der Arbeit angekündigt, war neben der Beschreibung der existierenden Ansätze auch bereits deren Analyse Teil des Kapitels. Das diente zwei Zielsetzungen. Zum einen wurden die disziplinären Zugänge genutzt, um insgesamt zehn besonders relevante und mit Resilienz auf die ein oder andere Art verknüpfte Konzepte zu identifizieren, mit deren Hilfe im Folgenden ein eigenständiges Resilienz-Konzept für die zivile Sicherheitsforschung entwickelt werden kann. Zum anderen haben besonders die letzten beiden Unterkapitel zur 
unter dem Titel Resilience Engineering firmierenden organisationswissenschaftlichen, sowie zur häufig als engineering resilience bezeichneten ingenieurwissenschaftlichen Resilienzforschung gezeigt, warum ein derartiges, eigenständiges Resilienz-Konzept notwendig ist. Und warum es notwendig ist, darauf aufbauend Hypothesen für ein Resilience Engineering im ingenieurwissenschaftlichen Sinne für die zivile SiFo zu entwickeln. Mit anderen Worten wurden die existierenden Forschungslücken offenbar, auf die nun im nächsten Kapitel näher eingegangen wird. 


\section{Forschungslücken und wissenschaftliche Fragestellung}

Resilienz ist ein Begriff mit vielen Bedeutungen. Mittlerweile kann sogar gesagt werden, dass Resilienz gerade aufgrund seiner vielen unterschiedlichen Bedeutungen Gefahr läuft, bedeutungslos zu werden. Der Begriff ist als semantisch überladen zu kennzeichnen (Lundberg/Johansson 2015: 23, Madni/Jackson 2009: 186). Es ließe sich also polemisch formuliert fragen, ob es wirklich noch einer Arbeit zur Bedeutung von Resilienz bedarf? Nach dem Motto, es wurde alles schon gesagt, aber noch nicht von jedem, erscheint es auf den ersten Blick nicht unbedingt ersichtlich, wo der wissenschaftliche Mehrwert liegen könnte. Gerade auch gegeben die ungewöhnliche Länge des Kapitels zum Stand der Forschung, ist die Frage durchaus statthaft. Beim genaueren Blick auf die vorangegangene Analyse wird jedoch unmittelbar deutlich, dass in Bezug auf Resilienz und Resilience Engineering in der zivilen Sicherheitsforschung noch eine Reihe an Forschungslücken bestehen. Insgesamt konnten fünf, zum Teil miteinander verknüpfte, Forschungslücken identifiziert werden, zu deren Schließung die vorliegende Arbeit beitragen möchte.

Erstens gibt es nach wie vor kein eigenständiges, theoretisch fundiertes Resilienz-Konzept für die zivile Sicherheitsforschung. Zumeist wird das Resilienz-Verständnis anderer Disziplinen und Forschungsrichtungen aufgenommen und, ohne auf die Spezifika der zivilen SiFo zu achten, übertragen. Es sind aber gerade diese Spezifika, nämlich die inhärente Interdisziplinarität, die Anwendungsnähe und vor allem auch der normative Anspruch, die für ein Resilienz-Konzept eine Rolle spielen müssen. Gleichzeitig gilt auch über die zivile SiFo hinaus, dass Resilienz zwar häufig in den Kontext der System- und/oder Komplexitätstheorie eingeordnet wird, aber eine tatsächliche Analyse unter Zuhilfenahme dieser Theoriegebäude unterbleibt. Hier kann die vorliegende Arbeit insofern Pionierarbeit leisten, die über ihr eigentliches Anwendungsgebiet hinausreicht. Ebenso wenig wie es die beiden genannten Aspekte, ein auf den normativen Anspruch der zivilen SiFo verweisendes Resilienz-Konzept einerseits und eine system- und komplexitätstheoretische Analyse von Resilienz andererseits, einzeln gibt, tauchen sie bisher gemeinsam auf. Auch darin drückt sich die geschilderte Forschungslücke aus.

Zweitens heißt Anwendungsnähe in der zivilen SiFo immer die Frage danach, wie Sicherheit verbessert oder aufrechterhalten werden kann. Die 


\section{Forschungslücken und wissenschaftliche Fragestellung}

Erhöhung der Resilienz soll als eine Möglichkeit dazu verstanden werden. Und die Ingenieurwissenschaften können dazu beitragen, indem sie Resilienz ingenieurwissenschaftlich umsetzen. Die Frage ist dann, wie sie das tun. In der bisherigen Literatur herrscht ganz überwiegend ein klassisches, tendenziell stabilitätszentriertes Verständnis vor, zusammengefasst unter dem Stichwort engineering resilience. Die Notwendigkeit einer Resilienzorientierung wird gesehen, da die klassische, quantitative Risikoanalyse aufgrund verschiedener Entwicklungen nicht mehr ausreiche (GoesslingReisemann/Thier 2019: 120f, IRGC 2018: 45, Kröger 2011: 69, 75, 2008: 1786, Linkov/Kott 2019: 2, Park et al. 2013: 359, Sikula et al. 2015: 220, Wachsmuth 2014: 5, Wreathall 2006: 276ff, Zio 2007: 489, 505). Nichtsdestotrotz ist der Großteil der ingenieurwissenschaftlichen Resilienzforschung bisher nach wie vor geprägt von Annahmen, die eher denen der klassischen Risikoforschung entsprechen (Sikula et al. 2015: 221). Die Faktoren und Entwicklungen, die von anderen Disziplinen als entscheidend im Kontext von Resilienz angesehen werden, namentlich vor allen Dingen Komplexität und Unsicherheit, stehen nur selten im Mittelpunkt des Interesses. Gegeben den Fokus der Resilienzforschung in Disziplinen wie Ökologie, Sozial- und Organisationswissenschaften ergibt sich hier eine weitere, konzeptionelle Forschungslücke.

Drittens, und darin liegt unter anderem auch ein Grund für das bisher unzureichende Resilienz-Verständnis der Ingenieurwissenschaften im Sinne eines engineering resilience, überwiegen in der ingenieurwissenschaftlichen Forschung Ansätze zur Quantifizierung von Resilienz bei weitem solche, die konkrete Ideen und Mechanismen zur Erhöhung von Resilienz entwickeln. Es ist ohne jeden Zweifel von großer Wichtigkeit, theoretische und häufig eher schwammige Konzepte operationalisierbar und damit messbar zu machen. Die Messung von Phänomenen wie Resilienz erlaubt eine Bewertung - die zumeist nicht von den Ingenieurwissenschaften selbst vorgenommen werden kann, sondern durch die Gesellschaft zu erfolgen hat - oder auch einen Vergleich zwischen verschiedenen Systemen. Sie stellt einen zum Teil unabdingbaren Ausgangspunkt für die Beantwortung der Frage dar, an welchen Stellen interveniert werden sollte, um die Resilienz - oder was immer gemessen wird - zu verändern. Dieser zweite Schritt, die Frage nach Ideen und Mechanismen zur Veränderung von Resilienz, kommt allerdings in den meisten Fällen in der ingenieurwissenschaftlichen Resilienzforschung zu kurz (Hosseini et al. 2016: 59, Sansavini 2016). Damit existiert hier eine Forschungslücke, besonders im Bereich der zivilen SiFo, für die ja gerade die Entwicklung konkreter, sicherheitserhöhender Maßnahmen entscheidend ist. 
Viertens existieren zwar auch dazu Ansätze, wie etwa Lovins und Lovins inspirierende Arbeiten, diese werden aber bisher zum einen nur unzureichend rezipiert und zum anderen sind sie wiederum selbst theoretisch nicht hinreichend fundiert. Die Prinzipien für mehr Resilienz entstammen bei Lovins und Lovins einer stärker empirischen Betrachtung unterschiedlicher Systeme und weniger der Resilienz-Theorie von Holling. Ähnliches gilt beispielsweise für den Überblick über ingenieurwissenschaftliche Resilienzforschung, wie er von der Lloyd's Register Foundation in einem Band von 2015 gegeben wird (siehe LRF 2015). Eine ausführliche Verortung, etwa in System- und Komplexitätstheorie, und eine darauf basierende Untersuchung bzw. Entwicklung relevanter Prinzipien findet nicht statt.

Und fünftens existiert eine ganze Forschungsrichtung, die ihre Ideen unter dem Label Resilience Engineering verortet. Aber diese Forschungsrichtung ist sozialwissenschaftlich orientiert, lehnt zum Teil technologische Lösungen für Resilienz sogar mehr oder minder explizit ab. Wer bisher den Begriff Resilience Engineering wissenschaftlich recherchiert, stößt nur auf einzelne Artikel, in denen er in einem ingenieurwissenschaftlichen Sinne Verwendung findet (siehe z.B. Attoh-Okine 2016, ENISA 2011, Ouyang/Wang 2015, Rahimi/Madni 2014, Sansavini 2016). Dagegen finden sich buchstäblich hunderte Artikel, die Resilience Engineering rein organisations- und damit letztlich sozialwissenschaftlich verstehen und deren Inhalt sich primär um die Frage dreht, wie Menschen in komplexen Organisationen Sicherheit im Sinne von safety schaffen können (Pillay 2017: 132). Nichtsdestoweniger scheint der Begriff - gerade vor dem Hintergrund der Bedeutung von engineering resilience - dazu geeignet, als Überschrift über ingenieurwissenschaftliche Ansätze zur Umsetzung von Resilienz zu dienen. Und in diesem Sinne soll er in der vorliegenden Arbeit auch verwendet werden.

Trotz umfassender Beschäftigung mit Resilienz in einer Vielzahl an Disziplinen, gilt also nach wie vor Lovins und Lovins Aussage von 1982, wonach noch kein "comprehensive applicable body of theory“ existiere, der Ingenieure im Hinblick auf die Umsetzung von Resilienz anleiten könne (Lovins/Lovins 2001: 139). Auf die Qualität ingenieurwissenschaftlicher Lösungen und inwiefern diese den Herausforderungen von Komplexität und Unsicherheit gewachsen sind, kommt es aber an, wenn es darum geht, disruptive Ereignisse gut zu überstehen (Madni/Jackson 2009: 183). Woods formuliert - wenn auch mit anderer Zielrichtung - programmatisch: „The end story remains to be written of how to engineer in [resilience]" (Woods 2015: 9). Dazu möchte die vorliegende Arbeit einen Beitrag leisten. Basierend auf den fünf Forschungslücken und der allgemeinen Zielsetzung, wie 
im einleitenden Kapitel dargestellt, lässt sich nun eine wissenschaftliche Fragestellung formulieren:

Was bedeutet Resilienz als Konzept in der zivilen Sicherheitsforschung und welche Hypothesen ergeben sich daraus für ein ingenieurwissenschaftliches Resilience Engineering?

Diese Frage versuchen die folgenden Kapitel, unter Berücksichtigung der bereits erfolgten Analyse von Resilienz und Resilience Engineering, zu beantworten, indem Resilienz anhand konzeptioneller Zugänge untersucht wird, daraus ein eigenständiges Resilienz-Konzept der zivilen SiFo entwickelt wird und dann wiederum dieses genutzt wird, um Hypothesen für Resilience Engineering in der zivilen SiFo aufzustellen. 


\section{Resilienz - Normative sowie system- und komplexitätstheoretische Analyse}

\subsection{Diskussion von Resilienz anhand konzeptioneller Zugänge}

Im Sommer 2014 wurde an der Johannes Gutenberg-Universität Mainz das „Deutsche Resilienz Zentrum“ (DRZ) gegründet. Dessen Selbstbeschreibung behauptet selbstbewusst, das „europaweite erste Zentrum dieser Art“ geschaffen zu haben, in dem Neurowissenschaftler, Mediziner, Psychologen und Sozialwissenschaftler interdisziplinär zum Thema Resilienz arbeiten. ${ }^{17}$ In seiner exakten Ausrichtung und mit der Fokussierung auf die Neurowissenschaften mag diese Aussage zutreffen. Deutschland- oder europaweite Sichtbarkeit in der Resilienzforschung können aber sicher auch andere Standorte für sich in Anspruch nehmen. Dazu zählt das Stockholm Resilience Centre der Universität Stockholm, das im Bereich sozial-ökologischer Resilienzforschung zu den weltweiten Vorreitern gehört. ${ }^{18}$ Dazu zählt zum Beispiel auch die Universität Bremen mit ihrem interdisziplinären Ansatz zur Erforschung der Resilienz des Energiesystems. ${ }^{19}$ In den Sozialwissenschaften wird Resilienz beispielsweise im Forschungsforum Öffentliche Sicherheit schwerpunktmäßig erforscht. ${ }^{20}$ Und die ETH Zürich steht mit ihrem 2011 gegründeten Risk Center für führende Forschung zur Resilienz kritischer Infrastrukturen. ${ }^{21}$ Übergreifend kann also zutreffend von multiplen Zentren der Resilienzforschung in Deutschland und Europa gesprochen werden. All diese Zentren lassen sich, obschon sie intern interdisziplinär aufgestellt sind, jeweils einer spezifischen disziplinären Tradition der Resilienzforschung zuordnen. Innerhalb der entsprechenden Traditionen wird tatsächlich sehr stark interdisziplinär geforscht. Zwischen den Traditionen herrschte hingegen lange Zeit wenig Austausch. So bemerkt Alexander in seiner Überblicksarbeit zu Resilienz treffend: „[I]t is striking how the term is used in different disciplines without any reference

17 https://www.drz-mainz.de/ [Stand: 27.6.2019].

18 http://www.stockholmresilience.org/about-us.html [Stand: 27.6.2019].

19 https://www.uni-bremen.de/res/ [Stand: 27.6.2019].

$20 \mathrm{http}: / / \mathrm{www} \cdot$.sicherheit-forschung.de/forschungsforum/index.html

[Stand: 27.6.2019].

21 http://www.riskcenter.ethz.ch/ [Stand: 27.6.2019]. 
to how it is employed in other fields, as if there were nothing to learn or transfer from one branch of science to another" (Alexander 2013: 2713).

Das widerspricht ein Stück weit der zu Beginn der vorliegenden Arbeit postulierten Behauptung, bei Resilienz handele es sich um ein Brückenkonzept, mit dessen Hilfe interdisziplinäre Gräben sinnvoll überwunden werden könnten. Aktuellere Forschungsanstrengungen versuchen daher, Resilienz aus multidisziplinärer Perspektive zu betrachten, wie etwa der Leipziger Wirtschaftswissenschaftler Rüdiger Wink in einem Sammelband aus dem Jahr 2016 (siehe Wink 2016). Die darin versammelten Überblicksartikel - unter anderem zur Ingenieurwissenschaftlichen Perspektive vom Autor dieser Arbeit (siehe Scharte/Thoma 2016) - bleiben allerdings relativ unverbunden nebeneinanderstehen. Gleichzeitig finden Veranstaltungen statt, auf denen bewusst Vertreter unterschiedlicher Forschungstraditionen miteinander ins Gespräch kommen sollen, wie etwa ein Kolloquium des „Sonderforschungsbereichs 805 - Beherrschung von Unsicherheit in lasttragenden Systemen des Maschinenbaus" der Technischen Universität Darmstadt im Dezember 2017, bei dem neben Ingenieurwissenschaftlern auch Vertreter des DRZ aus den Neurowissenschaften und der Volkswirtschaft (Rüdiger Wink) vertreten waren. ${ }^{22}$ Auch auf der „Infrastructure Resilience"-Konferenz des Risk Centers der ETH Zürich im Februar 2018 stand der interdisziplinäre Austausch zwischen den verschiedenen Denkschulen der Resilienzforschung mit im Mittelpunkt. Dort waren neben einer Vielzahl an Ingenieurwissenschaftlern mit Brian Walker und Kathleen Sutcliffe zwei prominente Vertreter der sozial-ökologischen bzw. psychologischen Resilienzforschung als Keynote-Speaker Teil der Veranstaltung. ${ }^{23}$ Trotz immer noch bestehender starker Unterschiede im Verständnis des Konzepts nähern sich die verschiedenen Disziplinen also zumindest insofern an, dass sie die Diskussion über Resilienz dazu nutzen, das jeweils eigene, disziplinspezifische Verständnis weiterzuentwickeln.

Auch in der vorliegenden Arbeit wurden im Kapitel zum Stand der Forschung disziplinäre Zugänge zu Resilienz untersucht und voneinander getrennt analysiert. Teilweise wurde dabei bereits der Versuch unternommen, die verschiedenen Denkschulen miteinander zu verknüpfen, Ähnlichkeiten aufzuzeigen und auf Unterschiede zumindest hinzuweisen. Im weiteren Verlauf der Arbeit wird nun der disziplinäre Zugang völlig

22 http://www.sfb805.tu-darmstadt.de/sfb805/resilienz_kolloquium/Index.de.jsp [Stand: 27.6.2019].

23 https://frs.ethz.ch/News/frs-news-channel/2018/03/infrastructure-resilience-international-conference-2018.html [Stand: 27.6.2019]. 
aufgelöst und durch einen „konzeptionellen Zugang“ über relevante Begrifflichkeiten ersetzt. Resilienz hängt als wissenschaftliches Konzept mit einer Reihe unterschiedlicher Begriffe und den jeweils dahinterstehenden Konzepten eng zusammen, angefangen vom Systembegriff über Komplexität und Anpassungsfähigkeit bis zu Freiheit und Vertrauen. $\mathrm{Zu}$ jedem dieser Begriffe existieren im Resilienz-Diskurs und darüber hinaus bereits Theorien, Ansätze und Untersuchungen, deren zentrale Charakteristika für ein Resilienz-Konzept der zivilen SiFo herausgearbeitet und weiterentwickelt werden müssen (Galloway 2013). An dieser Stelle setzt sich die vorliegende Arbeit bewusst von einem Argument Alexanders ab, der für die Verwendung einfacherer Sprache in der Resilienzforschung plädiert und meint, so einen Großteil der Diskussionen um unterschiedliche Begriffsverständnisse und konzeptionelle Zugänge überflüssig machen zu können (Alexander 2013: 2713). Hier wird vielmehr davon ausgegangen, dass Resilienz gerade nicht in wenigen Worten oder unter Zuhilfenahme eines Bildes wie dem vom Stehaufmännchen zutreffend und umfassend erläutert und beschrieben werden kann. Solche Bilder sind im besten Fall als Ausgangspunkt einer Diskussion zu gebrauchen, im schlechtesten Fall verengen sie den Blick auf Resilienz auf einen oder wenige Aspekte, wie etwa den des bounce back, und schließen so einen Großteil der eigentlich interessierenden Ideen aus. Der konzeptionelle Zugang mithilfe relevanter Begrifflichkeiten erlaubt hingegen für jede Begriffspaardiskussion „Resilienz und X" den Zugriff auf innovative Ansätze aus den unterschiedlichsten Disziplinen der Resilienzforschung. Diese können miteinander verglichen und gemeinsam dahingehend analysiert werden, wie sie für ein ResilienzKonzept der zivilen SiFo nutzbar gemacht werden können. Damit möchte die vorliegende Arbeit dazu beitragen, die konzeptionell-theoretische Diskussion innerhalb der Resilienzforschung voranzubringen.

Seit von einigen Forschern vor beinahe 20 Jahren die Notwendigkeit konstatiert wurde, Resilienzforschung mit größerer wissenschaftlicher Sorgfalt zu betreiben, wurde das Konzept bereits signifikant weiterentwickelt und insbesondere sein Anwendungsbereich über die „klassischen“ Bereiche Psychologie und Ökologie hinaus erweitert (Luthar et al. 2000: 556). Allerdings gilt dies nur bedingt für die zivile Sicherheitsforschung in Deutschland, deren Beschäftigung mit Resilienz bisher zwei unterschiedliche Muster aufweist. Zum einen eine Anlehnung an ein „holistisches“ Begriffsverständnis im Sinne der amerikanischen Tradition des disaster research. Daraus resultiert ein theoretisch unterkomplexes Vorgehen, dessen Ergebnis sich eher in politischen Handlungsempfehlungen als einem wissenschaftlichen Konzept ausdrückt (siehe beispielhaft Thoma 2014). 
Zum anderen eine kritisch-soziologische Auseinandersetzung, die stärker die gesellschaftlichen Auswirkungen der zunehmenden Attraktivität von Resilienz in den Blick nimmt, als dessen theoretischen Unterbau für den Bereich der zivilen SiFo zu adaptieren (siehe beispielhaft Blum et al. 2016, Dombrowsky 2012, 2010, Kaufmann/Blum 2012). Aus diesem Grund wird im Folgenden versucht, das Resilienz-Konzept mittels zehn unterschiedlicher konzeptioneller Zugänge zu analysieren, die aus der oben erfolgten Diskussion der wichtigsten disziplinären Zugänge heraus identifiziert werden konnten. Das Ziel der folgenden Unterkapitel besteht darin, für jeden der konzeptionellen Zugänge dessen spezifischen Beitrag zu einem Resilienz-Konzept für die zivile $\mathrm{SiFo}$ auszumachen.

Die zehn disziplinären Zugänge werden zudem noch einmal in zwei unterschiedliche Bereiche oder Arten eingeteilt. Das rekurriert auf den bereits mehrfach erwähnten Unterschied zwischen einerseits normativen und andererseits deskriptiv-analytischen Ansätzen zu Resilienz. Für ein Resilienz-Konzept der zivilen SiFo spielen beide Bereiche eine wichtige Rolle. Zunächst wird es daher im Folgenden darum gehen, übergreifende gesellschaftliche und primär normative Bedingungen für und Aspekte von Resilienz aus der Diskussion besonders relevanter konzeptioneller Zugänge in diesem Bereich zu identifizieren. Der Überblick über den Stand der Forschung hat gezeigt, dass das Konzept des Neoliberalismus in mehrfacher Hinsicht mit Resilienz verknüpft ist und daher entsprechend analysiert werden muss. Selbiges gilt für das Thema Freiheit und die Frage danach, wie sich Freiheit zu Resilienz verhält. Als eine Art Spezialfall, der aber aufgrund seiner Bedeutung für den Resilienz-Diskurs einzeln aufgeführt wird, kann der Zusammenhang zwischen Resilienz und Terrorismus gelten. Und schließlich taucht vor allem in der sozialwissenschaftlichen Resilienzforschung mindestens implizit immer wieder die Frage auf, was Resilienz mit Vertrauen zu tun hat. Danach geht es um die deskriptiv-analytische Herleitung von Bestandteilen eines Resilienz-Konzepts der zivilen SiFo. Dazu nutzt die vorliegende Arbeit eine Kombination aus Systemund Komplexitätstheorie und interpretiert die identifizierten konzeptionellen Zugänge unter dieser Maßgabe. Eine solche Analyse gibt es nach Kenntnisstand des Autors an keiner anderen Stelle in der Literatur. Sie stellt insofern eine originäre Leistung der vorliegenden Arbeit dar. Die system- und komplexitätstheoretische Analyse startet logischerweise mit der Verortung der beiden Konzepte Systeme und Komplexität im Resilienz-Konzept der zivilen SiFo. Anschließend stehen mit Unsicherheit und Vulnerabilität Entwicklungen bzw. Umweltbedingungen im Fokus, die als Herausforderungen verstanden werden können und eine Notwendigkeit 
für Resilienz motivieren. Mit Anpassungsfähigkeit und Flexibilität werden dann Eigenschaften system- und komplexitätstheoretisch diskutiert, die aus verschiedenen Disziplinen heraus eine zentrale Bedeutung für Resilienz in Anspruch nehmen können. In jedem der folgenden Unterkapitel werden jeweils aus der Analyse heraus besonders wichtige Merksätze identifiziert, die am Ende des jeweiligen Unterkapitels noch einmal zusammenfassend aufgeführt werden. Diese ergeben gemeinsam in einer systematisch aufbereiteten Art und Weise das eigenständige Resilienz-Konzept der zivilen SiFo. Gleichzeitig dienen sie als Grundlage zur Identifikation von Hypothesen für ein Resilience Engineering.

\subsection{Normative Analyse von Resilienz}

\subsubsection{Resilienz und Neoliberalismus}

Resilienz wird in den meisten Fällen als etwas Wünschenswertes angesehen. Es wäre gut für den Menschen / die Gesellschaft / das Stadtviertel / die Infrastruktur / das System, wenn er / sie / es sich gegenüber den Widrigkeiten dieser Welt als „resilient“ erweisen würde - was auch immer das im Einzelfall dann konkret heißt. Eine solche Deutung bleibt, auch wenn sie sozusagen den Mainstream ausmacht, jedoch bei weitem nicht unwidersprochen. Im Bereich der Erziehungswissenschaften fordert beispielsweise Thomas von Freyberg in geradezu emotionaler Art dazu auf, über die Erwünschtheit einer Zielvorstellung von resilienten Kindern nachzudenken: „Das resiliente Kind - ein Stehaufmännchen! Kann man sich ein grusligeres Erziehungsziel vorstellen: unberührbar und unerschütterlich zu sein, komme was da wolle" (von Freyberg 2011: 225)? Paradoxerweise sind es gerade stärker normativ arbeitende wissenschaftliche Betrachtungen, die den Konsens über Resilienz als etwas inhärent Gutes und Erstrebenswertes in Frage stellen und sich mit den möglichen "Schattenseiten“ des Konzepts auseinandersetzen (Blum et al. 2016, Dombrowsky 2012, 2010, Fekete et al. 2014, von Freyberg 2011). Daher liegt die Einschätzung nahe, dass Forscher, die Resilienz deskriptiv-analytisch untersuchen, das Konzept tendenziell normativ positiv besetzen und mithilfe ihrer Analysen dazu beitragen wollen, durch ein fundierteres Verständnis darüber, was Resilienz ausmacht und wie sie zustande kommt, die Resilienz der von ihnen untersuchten Systeme zu erhöhen. Das gilt insbesondere für die Disziplinen, die sich mit Menschen und/oder menschgemachten Systemen beschäftigen, also etwa die Psychologie oder die Ökonomie. Auch in der sozial-ökologi- 
schen Forschung wird - im Gegensatz zur reinen Ökosystemforschung häufig mit einem implizit normativ positiven Resilienz-Verständnis gearbeitet (siehe 2.3).

Diesem Vorwurf kann auch die vorliegende Arbeit ganz generell ausgesetzt werden. Obwohl beispielsweise das folgende Unterkapitel (siehe 4.3) prinzipiell rein deskriptiv-analytisch unter Zuhilfenahme system- und komplexitätstheoretischer Untersuchungen Annahmen für ein eigenständiges Resilienz-Konzept der zivilen Sicherheitsforschung entwickelt, liegt der Analyse ein normativ positives Resilienz-Bild zugrunde. Denn die Gesamtzielstellung der Arbeit besteht unter anderem darin, Hypothesen für Resilience Engineering zu entwickeln. Und Resilience Engineering soll dazu dienen, die Resilienz der für die zivile SiFo relevanten sozio-technischen Systeme zu erhöhen. Damit wird Resilienz mindestens implizit als wünschens- und erstrebenswert charakterisiert. Nichtsdestotrotz kann festgehalten werden: die Erkenntnisse aus 4.3 gelten völlig unabhängig davon, ob Resilienz „gut“ oder „schlecht“ ist. Sie sind normativ neutral. Um sie nachvollziehen zu können, bedarf es keines positiven Resilienz-Bildes. Allerdings reicht die rein deskriptiv-analytische Betrachtung des Konzepts aufgrund der Zielstellung der Arbeit nicht aus. Die Diskussion der disziplinären Zugänge hat gezeigt, wie unterschiedlich Resilienz bewertet werden kann. Etwa als sehr negativ, wenn es um die Resilienz allgemein als wenig wünschenswert anerkannter Strukturen, Zustände und Verhaltensweisen geht - von diktatorischen Regierungsformen über Umweltverschmutzung bis hin zu mexikanischen Drogenkartellen (Lukesch 2016: 303). Oder als sehr positiv, wenn es um die Überwindung extremer traumatischer Erlebnisse wie etwa die Inhaftierung in Konzentrationslagern geht (Antonovsky 1997: 15). Im Rahmen der Diskussion über disziplinäre Zugänge konnten verschiedene Begrifflichkeiten identifiziert werden, die in stärker normativ arbeitenden Bereichen der Resilienzforschung eine wichtige Rolle spielen. Um also dem möglichen Vorwurf eines implizit normativ positiven Resilienz-Verständnisses, das kritische Aspekte verschweigt, begegnen zu können, wird das Thema in den nächsten vier Unterkapiteln proaktiv aufgegriffen. Dabei steht nicht das jeweilige Konzept, etwa Neoliberalismus an sich, und seine unterschiedlichen Definitionsmöglichkeiten im Mittelpunkt des Interesses. Es geht vielmehr um eine jeweils knappe Diskussion des Zusammenhangs zwischen Resilienz und Neoliberalismus, Freiheit, Terrorismus sowie Vertrauen. Auf diese Weise werden die folgenden, systemtheoretischen konzeptionellen Annahmen um normative Aspekte ergänzt und zu einem übergreifenden Resilienz-Konzept für die zivile SiFo zusammengeführt. 
Den Anfang macht dabei die Diskussion des Zusammenhangs zwischen Resilienz und Neoliberalismus. Darunter werden drei unterschiedliche inhaltliche Punkte zusammengefasst, die im Einzelnen näher beleuchtet werden sollen. Zunächst geht es um Resilienz als neoliberales Paradigma und die Frage danach, wessen Aufgabe es ist, Vorsorge für Katastrophen bzw. widrige Ereignisse zu treffen - so dass letztere gerade nicht zu Katastrophen werden. Der zweite Punkt beschäftigt sich mit Resilienz als Modeerscheinung. Lohnt es überhaupt, ein „neues“ Konzept auszuarbeiten, wenn damit möglicherweise doch nur „alte“ Inhalte neu verpackt werden sollen? Und der dritte Punkt wiederum ist ein Verständnis von Resilienz als post-neoliberaler Governance-Ansatz, mit dessen Hilfe die generelle Komplexität moderner Gesellschaften adressiert werden kann. Mit den ersten beiden Fragen beschäftigen sich vor allen Dingen Sozialwissenschaftler aus Deutschland. Im internationalen Resilienz-Diskurs sind beide Aspekte weniger stark vertreten bzw. werden inhaltlich eher gegensätzlich gefasst. In der deutschen Diskussion sind sie jedoch sehr präsent und werfen Überlegungen auf, denen im Kontext der zivilen SiFo nachgegangen werden muss. Gerade deshalb, weil die vorliegende Arbeit mithilfe der vorgenommenen theoretischen Analyse eine Schnittstelle zwischen sozialwissenschaftlicher und ingenieurwissenschaftlicher Resilienz- und Sicherheitsforschung sein möchte. Die dritte Frage wiederum entstammt einer politikwissenschaftlichen Deutung von Resilienz und kann durch ihre Verbindung von Resilienz-Konzept, Politikwissenschaft und Komplexitätstheorie möglicherweise dazu beitragen, weitere Erkenntnisse zu gewinnen.

Inwiefern kann Resilienz also als „neoliberales Paradigma“ verstanden werden? Das ist gerade nicht unmittelbar einsichtig, da es vor allem im internationalen Kontext, wie an späterer Stelle noch gezeigt wird, eher gegen einen scheinbaren „Neoliberalismus“ gesetzt wird. Gemein ist jedenfalls der deutschen wie auch der internationalen, normativ argumentierenden Perspektive ein stark negatives Verständnis des Begriffs Neoliberalismus. Während zum Beispiel Resilienz als Strategie verstanden wird, deren Funktionieren auf Konsens basierende Gesellschaftsformen voraussetzt, wird dem ein „neoliberales kapitalistisches System“ entgegengesetzt, das auf Enteignung und Ausbeutung beruhe und gerade deshalb dem Aufbau einer gegenüber Gefahren sicheren und robusten Gesellschaft entgegenstehe (Alexander 2013: 2714). Auch in der klassischen, ökologischen Resilienzforschung wurde Resilienz eher als Gegenbegriff zu (neo-)liberalen Ansätzen gesehen, da letztere sich nur auf eine durch möglichst große Effizienz erreichbare Maximierung des Outputs bestimmter Systeme konzentrierten. Diese an kurzfristiger Gewinnmaximierung orientierte Strategie 
stand nach Holling der Resilienz von Systemen - verstanden als langfristiges Überleben der zugrundeliegenden Strukturen bzw. Beziehungsmuster seiner Elemente zueinander - entgegen. Nicht zuletzt wirtschaftswissenschaftliche Ansätze definieren Resilienz in Abgrenzung von Effizienz und werden damit im Widerspruch zu einem (neo-)liberalen Verständnis des Funktionierens der Welt gesehen. Dagegen verknüpfte ein weiterer, klassischer Resilienzforscher marktwirtschaftliche Logik mit dem Resilienz-Konzept. Allerdings unter buchstäblich umgedrehten Vorzeichen. Für Wildavsky ist Resilienz ein (neo)liberales Paradigma, das er für geeignet hält, sich positiv auf Gesellschaften auszuwirken. Wildavsky definiert überbordende Regulierung und ein auf Antizipation bzw. Verhinderung möglicher Schäden ausgelegtes System als innovationshemmend. Resilienz besteht hingegen in der Umsetzung flexibler Anpassungen mithilfe notwendiger Lernprozesse. Und dazu ist nach Wildavsky das freie Spiel der Kräfte am Markt gut geeignet (Kaufmann/Blum 2012: 242).

Im Anschluss an derartige Überlegungen, aber mit völlig anderen Ergebnissen, beschäftigen sich die deutschen Sozialwissenschaften und insbesondere die Soziologie bzw. spezifisch die soziologische Katastrophenforschung mit dem Zusammenhang zwischen Resilienz und Neoliberalismus. Die polemisch zugespitzten Formulierungen von Dombrowsky, der den gesamten Resilienz-Diskurs in wenigen Sätzen beinahe abkanzelt, bilden einen guten Ausgangspunkt für diese Diskussion: „Many words for the simple insight that the burden of disasters and relief will be shifted onto the citizens' shoulders" (Dombrowsky 2010: 3). Inhaltlich ähnlich, aber im Ton neutraler, analysieren Kaufmann und Blum die Bedeutung des Resilienz-Begriffes im Kontext aktueller gesellschaftspolitischer Sicherheitsstrategien. Ihnen zufolge ist mit Resilienz immer ein Fokus auf eine Verantwortlichkeit der Bürger für ihre eigene Sicherheit gemeint. Resilienz-Strategien versuchen demzufolge Bürger zu befähigen, erfolgreich selbst mit widrigen Ereignissen umzugehen (Kaufmann/Blum 2012: 237). Das wird insofern als „liberale Form von Governance“ gesehen, als es den Bürgern ein großes $\mathrm{Ma}$ an Autonomie und Eigenverantwortlichkeit gestattet. Bürger sind selbst soziale Akteure, die dazu in der Lage sein sollen, sich individuell auf das Eintreten widriger Ereignisse vorzubereiten und im Ernstfall mit deren Auswirkungen zurechtzukommen. Die Autonomie und Eigenverantwortlichkeit ist aber gleichzeitig mit einer deutlichen Verantwortungszuschreibung verbunden (Kaufmann/Blum 2012: 249). Aufgrund ihrer individuellen Fähigkeiten und Handlungen überstehen Bürger widrige Ereignisse besser oder weniger gut. Gelingt es ihnen nicht, mit den widrigen Ereignissen zurechtzukommen, sind sie sozusagen selbst 
schuld. In der Einführung des Resilienz-Gedankens in Katastrophenschutz und Vorsorgemaßnahmen könnte sich demnach ein „hidden transfer of responsibility from public authorities to citizens" verbergen (Fekete et al. 2014: 15). Auch wenn etwa sozial-ökologische Resilienz-Ansätze immer wieder betonten, dass sie rein deskriptiv-analytisch arbeiteten, seien auch in diesen Konzepten implizit immer normative Wertungen enthalten, die bestimmte Verhaltensweisen nahelegen und andere tendenziell eher delegitimieren. Das gilt besonders für die Betonung von Fähigkeiten zur Selbst-Organisation und individuellen Anpassungsfähigkeit an veränderte Bedingungen, die in der sozial-ökologischen Resilienzforschung im Zentrum stehen. Genau darin könne eben auch ein „Rückzug des Staates aus seiner Verantwortung und seinen Aufgaben in der Gesellschaft" gesehen bzw. motiviert werden, meint Deppisch (Deppisch 2016: 203). Für die zivile Sicherheitsforschung in Deutschland hat ein derartiges Resilienz-Verständnis unmittelbare Folgen. Wenn individuelle Resilienz es ermöglicht, dass sowohl einzelne Bürger als auch - in kumulierter Form - ganze Gesellschaften widrige Ereignisse möglichst unbeschadet überstehen können, wird gleichzeitig individuell „richtiges“ Handeln zu einer moralischen Verpflichtung. Oder anders gesagt, „sich nicht auf Probleme, Bedrohungen, Katastrophen einzustellen, keine Vorsorge dafür zu treffen, dass der Strom ausfallen könnte, nicht aktiv für seine Gesundheit zu sorgen, und nicht aktiv an der positiven Verarbeitung persönlicher Problemlagen zu arbeiten" ist dann gesellschaftlich eigentlich nicht mehr akzeptabel (Blum et al. 2016: 165).

Warum wird ein solches Resilienz-Verständnis nun teilweise als „neoliberales Paradigma“ bezeichnet? Das resultiert aus der dahinterstehenden Interpretation von Neoliberalismus. Im Prinzip ist damit im vorliegenden Kontext nicht mehr gemeint, als ein in allen Bereichen möglichst schlanker Staat, der seinen Bürgern möglichst viele Freiheiten lässt, ihnen aber gleichzeitig die Verantwortung für alles aufbürdet, was daraus an Konsequenzen erwächst. Während sich in Bereichen wie der Wirtschaft noch trefflich wissenschaftlich diskutieren lässt, ob und wie weit der Staat regulierend und kontrollierend in das Wirtschaftsgeschehen eingreifen sollte, ist ein solches Staatsverständnis im Bereich der Katastrophenforschung nach Dombrowsky geradezu „zynisch“ zu nennen. Denn hier gehe es um Ereignisse eines Ausmaßes, die Individuen generell nicht zu schultern in der Lage seien (Dombrowsky 2012: 286). Für Dombrowsky entspringt das Resilienz-Konzept einer „weltanschauung of individuality“, die er insgesamt für „brutal“ hält. Der Hintergrund seiner Kritik am Staatsverhalten in diesem Bereich scheint in einer wahrgenommenen Entwicklung hin zu 
immer größeren - gewollten (?!) - Defiziten im staatlichen System des Katastrophenschutzes zu liegen (Dombrowsky 2010: 3). Neoliberalismus so zu verstehen, wird diesem umfassenden Theoriegebäude, das neben eher radikal liberal denkenden Forschern wie Friedrich August von Hayek und Milton Friedman gerade auch in Deutschland die Freiburger Schule des Ordoliberalismus nach Walter Eucken umfasst, natürlich in keinster Weise gerecht. Dombrowsky gelingt es aber, einen pointierten Gegenentwurf zu einer von ihm konstatierten disziplinübergreifenden Begeisterung zu entwickeln, der als Ausgangspunkt für weitere Diskussionen dienen kann. Er versteht Resilienz demnach wie folgt: „The things that went wrong are the excreted matter of our cultural metabolism, the externalities of human error - and they become not only shifted off to third parties, but transformed into their responsibility to make them willing to internalize what should have been prevented at its origins. This internalization is more and more called ,resilience"” (Dombrowsky 2010: 4).

Im Bereich der Katastrophenforschung bzw. der zivilen Sicherheit in Deutschland gibt es also Forscher, für die das Resilienz-Konzept von einem Instrument zur Optimierung menschlicher Entwicklung zu einer Ideologie mutiert ist, die Menschen davon zu überzeugen sucht, die Schuld an durch widrige Ereignisse verursachten Schäden bei sich selbst und ihrem Mangel an Resilienz zu sehen (Dombrowsky 2010: 4). Weiter oben wurde bereits deutlich, dass eine solche Ansicht nicht exklusiv dieser Forschungsrichtung vorbehalten ist. Auch in der Psychologie spielt eine sehr ähnlich gelagerte Problematik eine Rolle (siehe 2.2). Je nach konkreter Ausgestaltung verschiedener Definitionen von Resilienz besteht die Gefahr, Resilienz als Attribut oder Eigenschaft von Individuen misszuverstehen. Ähnlich wie in der Katastrophenforschung in Bezug auf gesellschaftlich wirksame widrige Ereignisse wie etwa Naturkatastrophen kann das in der Psychologie in Bezug auf Traumata wie schwere Erkrankungen, den Tod naher Angehöriger oder andere Dinge dazu führen, den Individuen die Schuld daran zu geben, wenn sie es nicht schaffen, leistungserhaltend mit den Traumata umzugehen. Luthar und Cicchetti umschreiben das sehr passend, wenn sie sagen „if only they displayed particular behaviors, then they could withstand adversities. Such perspectives can inadvertently pave the way for blaming the individual for not possessing characteristics needed to function well" (Luthar/Cicchetti 2000: 862). Die Angst vor einem Blame Game, einer Schuldzuschreibung vonseiten des Staates oder interessierter Öffentlichkeiten in Richtung betroffener Gruppen von Menschen bzw. in Richtung des betroffenen Individuums verbindet hier Katastro- 
phenforschung und Psychologie. Es ist unmittelbar einsichtig, dass eine solche Verwendung des Resilienz-Begriffs normativ negativ belegt ist.

Daher stellt sich die Frage, was das für das in der vorliegenden Arbeit zu entwickelnde Resilienz-Konzept der zivilen SiFo bedeutet. Dazu muss zunächst festgehalten werden, dass das Verständnis von Resilienz als normativ negativ zu fassendes "neoliberales Paradigma“ durchaus auf einer sehr verbreiteten Sichtweise auf das Thema Resilienz basiert. Coaffee und Rogers fassen diese sehr treffend zusammen: „[I]ncreased attention is now being paid to how individuals and a broad range of local communities might become more responsible for their own risk management. The aim here is to develop ,community resilience' which might reinforce broader institutional security strategies" (Coaffee/Rogers 2008: 102). So oder so ähnlich wird community resilience oder soziale Resilienz von einer Vielzahl von Autoren immer wieder definiert (Coaffee 2010, 2008, Coaffee/Bosher 2008, Coaffee/Rogers 2008, Colten et al. 2008, CSS 2009, Edwards 2009, Elran 2012, Fekete et al. 2014, Flynn 2011, 2011b, 2008, Flynn/Burke 2012, Lovins/Lovins 2001, Thoma 2014). Aber zieht sich der Staat damit automatisch und unweigerlich aus der Verantwortung? Ist die Befähigung nichtstaatlicher Akteure zum effektiven Katastrophenschutz eine zu vermeidende Entwicklung? Und ist Resilienz so verstanden per se normativ negativ zu sehen? Alle drei Fragen werden von den meisten in diesem Bereich aktiven Forschern mindestens implizit eindeutig verneint. Die Gefahr für einen Missbrauch dieser Art besteht durchaus. Die mit dem Resilienz-Begriff verbundenen Chancen und Möglichkeiten werden aber als durchweg größer eingeschätzt. So führt eine Verlagerung der Kompetenzen für Katastrophenschutz und -vorsorge auf dazu fähige Bürger zum Beispiel dazu, dass sich staatliche Maßnahmen stärker auf die Bevölkerungsgruppen konzentrieren können, die aus verschiedenen Gründen besonders anfällig gegenüber widrigen Ereignissen sind (Kaufmann/Blum 2012: 252).

Allein aufgrund der Begrenztheit der ihnen zur Verfügung stehenden Ressourcen ist es nicht sinnvoll, staatliche Akteure als „primary guarantors of resilience“ zu sehen. Sie können aber als „important facilitator[s]" dienen (Longstaff 2012: 273). So hat beispielsweise das Bundesamt für Bevölkerungsschutz und Katastrophenhilfe, eine Bundesoberbehörde im Geschäftsbereich des Bundesministeriums des Innern, für Bau und Heimat, im April 2017 die vierte Auflage seiner Broschüre Katastrophenalarm. Ratgeber für Notfallvorsorge und richtiges Handeln in Notsituationen herausgegeben. Darin werden Bürgern Tipps zu Vorsorgemaßnahmen und Verhaltensweisen gegeben, die ihnen dabei helfen sollen, extreme widrige Ereig- 
nisse möglichst unbeschadet zu überstehen (siehe BBK 2017). Auf Seite 7 heißt es etwa: „WICHTIG! In dieser Broschüre finden Sie Hinweise darauf, wie Sie sich auf Notfälle vorbereiten können und wie Sie sich in einer Notsituation richtig verhalten. Lesen Sie die Handlungsempfehlungen und treffen Sie die notwendigen Vorbereitungen" (BBK 2017: 7)! Aus einer eher radikal-kritischen Position heraus, die an dieser Stelle in ebenso bewusst polemischer Zuspitzung als Dombrowsky-Haltung bezeichnet werden kann, dient eine solche Broschüre dem Staat nur dazu, im tatsächlichen Katastrophenfall auf eine mangelnde Umsetzung der Empfehlungen auf Seiten der Bürger hinzuweisen und diesen so die Schuld an eingetretenen Schäden zu geben. Es ist jedoch wohl deutlich zutreffender, hier von einem Angebot des Staates zu sprechen, das dieser seinen Bürgern unterbreitet und das als - freiwillig anzunehmende - Hilfestellung zu verstehen ist. In Gesprächen mit Verantwortlichen sowohl des BBK als auch anderer staatlicher Akteure aus dem Bereich des Zivil- und Katastrophenschutzes, die der Autor im Rahmen seiner beruflichen Tätigkeit über mehrere Jahre hinweg führen konnte, wurde immer wieder deutlich, dass hauptamtliche Katastrophenschützer gerade nicht mit einer großflächigen Umsetzung solcher Empfehlungen rechnen und ihre Aufgaben auch weiterhin darin sehen, die Bevölkerung im Katastrophenfall wirksam zu schützen. Die Einführung von die individuelle Resilienz Einzelner fördernder Maßnahmen sehen sie eher als Chance, zusätzlich - und nicht alternativ - zu dem, was ihre eigenen Aufgaben sind, den Schutz der Bevölkerung zu verbessern. Zumal sich Menschen immer wieder - trotz mangelnder Vorbereitung - als deutlich resilienter erweisen als gedacht. Das trifft selbst in extremen Ausnahmesituationen, wenn sämtliche staatlichen Hilfsmaßnahmen versagen, zu. Beispielhaft kann hier die Situation in New Orleans nach dem Hurrikan Katrina genannt werden, in der neben vielen negativ einzuschätzenden Ereignissen auch beobachtet wurde, wie durch „locals taking care of themselves" Einzelne und Gruppen von Menschen der Katastrophe zu trotzen in der Lage waren (Colten et al. 2008: 15). Aus diesen Überlegungen lassen sich nun drei Annahmen für das Resilienz-Konzept der zivilen SiFo ableiten. Zunächst gilt: Eine unreflektierte Übernahme des Resilienz-Begriffs birgt die Gefahr, diesen als neoliberales Paradigma einer Verantwortungsverlagerung vom Staat auf den einzelnen Bürger misszuverstehen. Deshalb gilt: Die Verwendung des Resilienz-Konzepts in der zivilen Sicherheitsforschung darf nicht dazu fübren, dass von widrigen Ereignissen betroffenen Personen und Gruppen die Verantwortung für erlittene Schäden zugeschrieben wird. Und das heißt: Strategien zur Erhöhung der Resilienz der für die zivile SiFo relevanten sozio-technischen Systeme müssen als Angebote formuliert wer- 
den, die für die Gesellschaft einen klaren, über ohnehin erfolgende Maßnahmen des Bevölkerungsschutzes und der Katastrophenhilfe hinausgehenden, Mehrwert baben.

Im Kontext der Diskussion um Resilienz als neoliberales Paradigma wird von verschiedenen Autoren - unter anderem wieder Dombrowsky der Mehrwert und das genuin Neuartige an der Verwendung des Begriffs bzw. eines sich dahinter verbergenden wissenschaftlichen Konzepts wenn nicht bestritten, so doch sehr stark bezweifelt. Diese in den Sozialwissenschaften verortete Ansicht wurde im Kapitel zu den disziplinären Zugängen bereits kurz adressiert. Häufig erfolgt die Verwendung des Begriffs Resilienz eben ohne theoretische Fundierung wie sie beispielsweise in der vorliegenden Arbeit in aller Ausführlichkeit für die zivile Sicherheitsforschung vorgenommen wird. Dann ist Resilienz nicht mehr als ein Begriff, der zu einer Art Sammelbecken für positive Eigenschaften, Fähigkeiten oder Ressourcen wird. Dabei ist eines klar: Resilienz ist definitiv ein „Modewort" in verschiedenen wissenschaftlichen Disziplinen, aber auch und gerade in der Populärwissenschaft. Das wurde etwa an der unterschiedlichen Ratgeberliteratur deutlich, die es zum Thema Resilienz zu erwerben gibt (siehe 2.2). Oder daran, dass sich die Zahl der Studien zu Resilienz über verschiedene Disziplinen hinweg zwischen 1995 und 2013 verdoppelt hat (Righi et al. 2015: 146). Im Rahmen von Sicherheitsforschung und Sicherheitspolitik wurde Resilienz im Anschluss an die Terroranschläge vom 11. September 2001 nach und nach zu einem "Schlagwort" (buzzword) (Elran 2012: 294). Dabei ist mit Schlagwort zunächst keine Wertung verbunden. Vielmehr vereinen solche Schlagwörter grundsätzlich nur unterschiedliche Diskussionsstränge zu einem bestimmten Thema unter einem begrifflichen Hut. Fekete et al. formulieren hierzu elegant, dass Resilienz als Begriff ein „hohes Potential [...] als Kommunikationsmotor" habe (Fekete et al. 2016: 220). Dem kann sicher zugestimmt werden.

Aber ist Resilienz tatsächlich nicht mehr als ein Trend oder eine Modeerscheinung? Ähnlich wie in der breiteren, medialen Öffentlichkeit an sich, gibt es auch in der Wissenschaft, bzw. in einzelnen wissenschaftlichen Disziplinen, immer wieder solche Trends, während derer bestimmte Schlagwörter die Diskussion bestimmen und lenken. Ob sich dahinter etwas tatsächlich Neues verbirgt oder es vielmehr darum geht, mithilfe bestimmter Begrifflichkeiten etablierte Inhalte gegenüber spezifischen Zielgruppen anders oder besser kommunizieren zu können, ist a priori unklar. Ein aktuelles Beispiel kann hier im Begriff der „Biologischen Transformation" gesehen werden, mit dessen Hilfe die Fraunhofer-Gesellschaft verschiedene wissenschaftliche Entwicklungen unter einem Dach zu vereinen 
sucht. In einem Whitepaper zu Biologische Transformation und Bioökonomie schreibt Wiltrud Treffenfeldt als Mitglied des Senats der Fraunhofer-Gesellschaft dazu passend: „Mit Biologisierung und Digitalisierung in die Zukunft! Deutsche Produkte, Verfahren und Anlagen sind weltweit gefragt und wettbewerbsfähig. Die Fraunhofer-Gesellschaft als die führende anwendungsorientierte Forschungsorganisation trägt in vielen Bereichen der Technik zu diesem Erfolg bei. Die Bedeutung von Nachhaltigkeit hat hierbei eine immer wichtigere Rolle eingenommen. [...] Inspiriert werden die Forscherinnen und Forscher durch biologische Prinzipien und in der Natur etablierte stoffliche Kreislaufsysteme“ (Fraunhofer 2018: 5). Das Zitat erklärt den Begriff der biologischen Transformation mittels weiterer, immer wieder auftauchender Schlagwörter wie Biologisierung, Digitalisierung und dem in der vorliegenden Arbeit bereits andiskutierten Begriff der Nachhaltigkeit. Innerhalb des Whitepapers stellt Fraunhofer kurz und bündig aktuelle Forschungsinhalte aus verschiedenen Bereichen vor, deren übergreifende Gemeinsamkeit darin besteht, dass sie - sehr abstrakt "Dinge" aus der Natur in technische Anwendungen transferieren. Dabei sind die einzelnen Forschungsinhalte bei weitem nicht alle als neu und nie vorher gesehen zu kennzeichnen (siehe Fraunhofer 2018). Der „neue“ Begriff biologische Transformation erlaubt es aber, die verschiedenen Forschungsideen im Rahmen einer konsistenten Storyline zusammenzufassen, die sich gegenüber der allgemeinen Öffentlichkeit und vor allen Dingen auch der interessierten Forschungspolitik nutzen lässt, um die gesellschaftliche Relevanz der von der Fraunhofer-Gesellschaft durchgeführten Forschung besser zu begründen.

Einen prinzipiell äquivalenten Mechanismus sehen Forscher wie Dombrowsky bezüglich des Resilienz-Begriffs am Werk (Fekete et al. 2016: 227). Für sie ist Resilienz „one of these shimmering slogans from the wording-mint" (Dombrowsky 2010: 2). Demzufolge wäre Resilienz tatsächlich nicht mehr als eine vorübergehende Modeerscheinung, dessen Attraktivität spätestens dann nachlässt, wenn der nächste, „neue” Begriff aufkommt. Dann ließe sich mit Dombrowsky prägnant formulieren: „Yes, ,resilience' is a buzz-word, a shibboleth in the beginning" (Dombrowsky 2010: 2). Wobei damit ja, wie oben beschrieben wurde, noch keine Wertung verbunden ist. Die Wertung liegt vielmehr darin, dass Dombrowsky Resilienz nicht als eigenständiges wissenschaftliches Konzept versteht, sondern als alten Wein in neuen Schläuchen, mit dessen Hilfe eigentlich längst bekannte Inhalte in verschiedenen Disziplinen besser verkauft werden können. In dieser Hinsicht habe der Resilienz-Begriff perfekt funktioniert, so Dombrowsky und sei ein gemeinsamer Diskussionspunkt für so un- 
terschiedliche Disziplinen wie Ökologie, Ökonomie, Biologie, Geologie, Ingenieurwissenschaften und seine eigene Richtung der Katastrophenforschung (Dombrowsky 2010: 2). Mit dieser Einschätzung ist er nicht alleine. Alexander zufolge gibt es einige Autoren, die in Resilienz nicht mehr sehen als ein „fashionable buzz-word“ (Alexander 2013: 2713). Forscher, die sich des Resilienz-Begriffs bedienen, seien demnach davon überzeugt, die Realität mit dessen Hilfe besser zu verstehen und erklären zu können, als andere Forscher und als das in der Vergangenheit der Fall gewesen sei (Dombrowsky 2010: 2). Und es ist exakt dieser unterstellte Anspruch, gegen den sich Autoren wie Dombrowsky oder beispielsweise auch von Freyberg wehren. Letzterer fasst die gesamte Resilienzforschung innerhalb seiner Disziplin der Pädagogik vor diesem Hintergrund mit einer starken Polemik wie folgt zusammen: „Ein erstes Resümee mit bösem Blick legt nahe: Alle Fragen offen, alle Kontroversen unentschieden, alle Probleme ungelöst - jedoch: mal wieder ein feines, weil wissenschaftlich klingendes, Sahnehäubchen der bekannten theoretischen und praktischen Misere der Pädagogik aufgesetzt“" (von Freyberg 2011: 224).

Welche Konsequenzen ergeben sich aus dieser Diskussion für das Resilienz-Konzept der zivilen SiFo? Nimmt man Dombrowskys Aussagen ernst, bewegt sich die vorliegende Arbeit inmitten des von ihm konstatierten Trends zur Verwendung des Resilienz-Begriffs für alle möglichen Phänomene, Eigenschaften und Entwicklungen, die bereits seit langem bekannt sind und die bisher mithilfe anderer Begrifflichkeiten diskutiert wurden. Zumal die zivile Sicherheitsforschung als solche auch selbst als Modeerscheinung skizziert werden könnte, die das Ergebnis politischer Aushandlungsprozesse ist, aber inhaltlich letztlich nichts Anderes als eine - zumindest zu Beginn - stärker technikwissenschaftlich orientierte Variante der Katastrophenforschung darstellt. Wird dieser Gedanke radikal zu Ende gedacht, wäre ein eigenständiges Resilienz-Konzept der zivilen SiFo in mehrfacher Hinsicht weder notwendig noch möglich. Allerdings wird diese Ansicht den vielfältigen Ansätzen und Diskussionen zu Resilienz, die in unterschiedlichen Disziplinen zum Teil seit vielen Jahrzehnten geführt werden, in keiner Weise gerecht. Forscher wie Dombrowsky haben durchaus überzeugende Argumente vorzuweisen und ihnen ist grundsätzlich zuzustimmen: Ja, Resilienz ist ein wissenschaftliches Schlagwort, dessen aktuelle Popularität über Disziplingrenzen hinweg es zu einem Modewort hat werden lassen. Das bedeutet aber im Umkehrschluss nicht notwendigerweise, dass sich hinter Resilienz kein inhaltlich relevantes wissenschaftliches Konzept verbergen kann. Wenn Resilienz in der zivilen Sicherheitsforschung als Modewort Verwendung findet, ist es umso wichtiger, die 
Frage zu beantworten, was hinter dem Modewort steckt. Und dazu bedarf es zum einen der sorgfältigen Analyse existierender Forschungsstränge anderer Disziplinen. So wird bereits in frühen psychologischen Ansätzen deutlich, dass es bei Resilienz um ein Phänomen geht, mit dessen Auftreten nicht gerechnet wurde - Resilienz ist eine Nicht-Selbstverständlichkeit - und für das aus diesem Grund eine neue Bezeichnung gewählt wurde (Fooken 2016: 15, 28, 40, Luthar/Cicchetti 2000: 858, von Freyberg 2011: 220, Zander/Roemer 2016: 47). Und zum anderen bedarf es einer konsistenten theoretischen Einordnung von Resilienz in ein übergreifendes Theoriegebäude. Denn nur so lassen sich zugrundeliegende Mechanismen identifizieren und mit Resilienz in Verbindung bringen. Dazu eignen sich, wie noch detailliert dargestellt wird, System- und Komplexitätstheorie. In der Verknüpfung dieser Vorgehensweisen liegt die Chance herauszuarbeiten, worin Resilienz genau besteht, was das Konzept ausmacht und auch, was nicht Bestandteil von Resilienz sein muss und kann. Für die vorliegende Arbeit gilt also: Resilienz ist ein Modewort. Das führt aber nicht dazu, dass Resilienz nicht zu einem fundierten wissenschaftlichen Konzept ausgearbeitet werden kann. Um den Begriff innerhalb der zivilen SiFo konzeptionell zu fundieren, ist sowohl eine system- und komplexitätstheoretische Einordnung als auch der Rückgriff auf Erkenntnisse aus Disziplinen mit einem etablierten Resilienz-Verständnis notwendig.

Damit führt die Analyse an dieser Stelle in einer Art Zirkelschluss auf ihren eigenen Ausgangspunkt zurück. Denn die Diskussion der Frage danach, ob Resilienz mehr sein kann, als nur ein Modewort, wurde weiter oben als eine der relevanten Fragen aus dem disziplinären Resilienz-Diskurs herausgefiltert. Und die Antwort auf die Frage besteht in der Analyse selbst: Ja, Resilienz kann mehr sein als ein Modewort und zwar genau dann, wenn der Begriff mittels einer ausführlichen theoriegeleiteten Analyse untersucht und zu einem konsistenten Konzept ausgearbeitet wird.

Ein weiterer Bestandteil der konkreten Analyse des Zusammenhangs zwischen Resilienz und Neoliberalismus besteht wiederum in der Frage, wie weit Resilienz sich als post-neoliberales Paradigma staatlichen Handelns zum Umgang mit genereller Komplexität eignet. Die Frage wird von David Chandler gestellt, einem englischen Politikwissenschaftler. Und sie enthält selbst bereits einige erklärungswürdige Bestandteile, nämlich erstens was Chandler unter Komplexität versteht, zweitens was Neoliberalismus für ihn bedeutet und drittens wie er Resilienz definiert. Diese Fragen sollen im Folgenden kurz adressiert werden, um anschließend diskutieren zu können, inwiefern die von Chandler damit aufgeworfene Behauptung, wonach Resilienz in der Politikwissenschaft sozusagen die 
nächste Evolutionsstufe staatlichen Handelns darstelle, sich auf die zivile SiFo übertragen lässt.

Grundsätzlich unterscheiden sich für Chandler komplexe Systeme von einfachen, reduktionistisch erklärbaren durch die Interaktion der Systemelemente, also durch die Existenz von Feedbackschleifen. Diese Annahme ist konsistent mit der Theorie komplexer adaptiver Systeme und liegt damit im Theorieverständnis der vorliegenden Arbeit (siehe 4.3.2). Weiterhin unterscheidet Chandler aber noch zwischen verschiedenen Arten von Komplexität. Zum einen nennt er „einfache“ (simple) Komplexität, bei der Systeme zwar in unvorhersehbarer Weise auf initiale Ereignisse reagieren, die Welt generell aber trotzdem bestimmten zugrundeliegenden Mechanismen gehorcht. Dass diese Mechanismen nicht erkannt werden können, liegt nicht an einer prinzipiellen Unmöglichkeit, sondern vielmehr an der Limitierung, der zur Verfügung stehenden Ressourcen. Um ein vollständiges Bild aller unabhängigen, miteinander interagierenden Variablen zu bekommen, wären unüberschaubar viele Ressourcen notwendig. Nichtsdestotrotz basiert Chandlers Definition von einfacher Komplexität auf der Annahme, dass es theoretisch möglich ist, die zugrundeliegenden Mechanismen zu identifizieren (Chandler 2014: 49). Dagegen stehen Systeme, die sich durch "generelle“ (general) oder emergente Komplexität auszeichnen. In derartigen Systemen lassen sich nicht nur keine deterministischen Zusammenhänge ausmachen, auch Kausalitäten an sich sind a priori nicht erkennbar. „Systems of general or emergent complexity are not observable from the outside" (Chandler 2014: 49). Es geht daher nicht mehr um die Frage, wie sich zugrundeliegende Mechanismen erkennen lassen, sondern vielmehr darum, ob es überhaupt derartige Mechanismen gibt: „[The] problem exists at the level of what is to be known (it is not linear and law-bound) rather than at the level of how we might know the underlying reality" (Chandler 2014: 50).

Gegeben diese Definitionen interessiert sich Chandler nun dafür, welche Arten staatlichen Handelns (Governance) jeweils passende Strategien für unterschiedliche Arten von Systemen darstellen. Und er nennt klassische liberale Ansätze, Neoliberalismus sowie Resilienz als drei unterschiedliche Governance-Ansätze, die jeweils für unterschiedlich komplexe Arten von Systemen geeignet seien (Chandler 2014: 52). Um die Verknüpfung zwischen der Komplexität von Systemen und dem jeweils passenden Governance-Ansatz herstellen zu können, bedient er sich den weiter unten ausführlicher beschriebenen Begriffspaaren der known knowns, known unknowns und der unknown unknowns (siehe 4.3.3). Demnach besteht liberale Governance darin, staatliches Handeln auf einem linearen System- 
verständnis beruhen zu lassen. Durch kontinuierlichen Wissenszuwachs über die Zeit können Akteure verstehen, welche deterministischen Regeln dem System zugrunde liegen und ihr Handeln entsprechend darauf ausrichten. Sie handeln in der Annahme, die Folgen ihres Handelns sicher zu kennen. Demgegenüber akzeptiert neoliberale Governance die grundsätzliche Komplexität der Systeme und sieht ein deterministisches Verständnis als eher kontraproduktiv an. Dieser Ansatz geht nach wie vor davon aus, dass es zugrundeliegende Regeln gibt, diese allerdings unbekannt sind. Resilienz wiederum geht noch einen Schritt weiter und nimmt als Governance-Ansatz die Existenz genereller Komplexität als gegeben an. Aufgrund der emergenten Eigenschaften der interessierenden Systeme sind Ereignisse kontingent, um eine Begrifflichkeit von Luhmann zu verwenden. Wie ein bestimmtes Ereignis aussieht wird immer erst in dem Moment klar, in dem es sich realisiert. Vorher ist es rein theoretisch nicht möglich zu wissen, was passieren wird - es könnte eben immer auch anders kommen. Die konkreten Kausalitäten werden erst im Nachgang des Ereignisses als solche sichtbar (Chandler 2014: 50). Diese unterschiedlichen Sichtweisen haben dann natürlich auch einen direkten Einfluss darauf, wie staatliches Handeln aussehen sollte. Der klassische liberale Ansatz funktioniert in simpler, deterministischer Weise: Weil die UrsacheWirkungs-Zusammenhänge generell bekannt sind, ist ein aktives staatliches, lenkend von außen eingreifendes und steuerndes Handeln ohne weiteres möglich. Es handelt sich um einen traditionellen top-down-Ansatz. Solcherart reduktionistische Formen von Governance „may work well when there is the need for a ,technical, mechanical solution which can be employed everywhere' but do not work in an age of complexity when they need flexibly to respond to local particularities, when they need to act nimbly or with nuance" (Chandler 2014: 60). Neoliberale GovernanceAnsätze tragen dieser „age of complexity” Rechnung und funktionieren nach Chandler in einer bottom-up-Richtung. Staatliche Akteure handeln unter der Maßgabe, dass sie wissen, dass sie zu wenig wissen, um aktiv steuernd in gesellschaftliche - und vor allem wirtschaftliche - Prozesse einzugreifen. Die zugrundeliegenden Kausalitäten können durch den Markt als rationalen Akteur besser gesteuert werden, als durch Regierungen (Chandler 2014: 62). Der Mechanismus, über den dies funktioniert, ist der Preis. Daraus wurde nach Chandler die Schlussfolgerung gezogen, dass staatliches Handeln sich im Wesentlichen darauf beschränken sollte, den freien Markt und seine regulierenden Mechanismen möglichst wenig zu behindern (Chandler 2014: 53f). 
Hier ist es wichtig, zwischen Neoliberalismus als Theorie und Neoliberalismus als Governance-Ansatz zu unterscheiden. Denn die Theorie impliziert bereits die generelle Komplexität von Systemen. Die Theorie prägnant zusammenfassbar mit dem Satz „Wir wissen nichts, also sollten wir nicht intervenieren" - kann deshalb aber gleichzeitig nicht wirklich herangezogen werden, wenn staatliches Handeln aufgrund konkreter situationsspezifischer Charakteristika als notwendig erachtet wird (Chandler 2014: 54). Nachdem neoliberale Ansätze spätestens in den 1980er Jahren zu einer dominanten Governance-Form wurden, stellte sich die Frage, wie sich die theoretischen Erkenntnisse auf staatliches Handeln anwenden ließen. Und daraus wurde dann - reichlich verkürzt dargestellt - die oben bereits erwähnte Erkenntnis abgeleitet, dass der Staat sich möglichst schlank machen sollte, um keine ungewollten, komplexitätsbedingten Ergebnisse staatlicher Interventionen zu produzieren, die sich durch ein freies Spiel der Kräfte am Markt hätten verhindern lassen (Chandler 2014: 53f). Empirisch scheint jedoch auch diese Form von Governance nicht zu zufriedenstellenden Ergebnissen zu führen. Demnach unterscheiden sich die Ergebnisse derart neoliberalen staatlichen Handelns nicht wesentlich von den Ergebnissen klassischer, stärker interventionistischer Ansätze. Beide Arten von Governance gehen nicht von der generellen Komplexität der relevanten Systeme aus (Chandler 2014: 60). Daraus folgt: „The limitations of neoliberal frameworks [...] is at the heart of the rise of resilience-thinking" (Chandler 2014: 55f). Chandler führt den Begriff der Resilienz also als eine neue, post-neoliberale Art von Governance ein, die sich grundlegend von den bisherigen Formen unterscheidet. Damit arbeitet er sich, wie gesagt, an neoliberaler Governance ab, nicht an der Theorie an sich, die konsistent ist mit genereller Komplexität und seinem Resilienz-Verständnis (Chandler 2014: 63).

Ein grundsätzlicher Unterschied zwischen Liberalismus und Neoliberalismus auf der einen und Resilienz auf der anderen Seite ist die SubjektObjekt-Beziehung des Governance-Verständnisses. In (neo-)liberalen Ansätzen gibt es eine klare Trennung zwischen Subjekt (staatliche Akteure) und Objekt (zu regulierendes System, etwa die Wirtschaft). Dieses deterministische Verständnis von Komplexität greift aber nach Chandler zu kurz. Für ihn ist ein Ansatz, in dem das Subjekt selbst Teil des Objekts ist, sozusagen „embedded or entangled“, sehr viel näher an den realen Gegebenheiten. Governance im Sinne von Resilienz ,is no longer a matter of intervening in an external problematic but [is about] self-reflexive understandings of entanglement" (Chandler 2014: 51). Sämtliches staatliches Handeln findet sozusagen innerhalb des Systems statt, das sich durch generelle Kom- 
plexität auszeichnet und dessen wichtigste Eigenschaft Emergenz ist. Eine proaktive, zielgerichtete Kontrolle eines derartigen Systems ist unmöglich und zwar nicht aufgrund der Unbekanntheit der zugrundeliegenden Mechanismen, sondern weil diese sich erst ex post zeigen und Systeme mit genereller Komplexität ständig Kontingenz aufweisen (Chandler 2014: 49f). Entscheidend für Resilienz-Ansätze sind deshalb die unknown unknowns, Probleme, die erst durch ihre Realisierung erkannt werden können. Konkretes staatliches Handeln muss sich auf deren Bewältigung konzentrieren und funktioniert in dieser Hinsicht "rückwärts“ (backwards), vom Problem ausgehend, und nicht vorwärts, in dem versucht wird, ein bestimmtes politisches Ziel mithilfe konkreter Maßnahmen zu erreichen (Chandler 2014: 62). Eine resiliente Strategie besteht für Chandler in der Betonung von Flexibilität, Anpassungsfähigkeit und Systemdenken. Und darin, staatliches Handeln, das zu Problemen führt, nicht als Staatsversagen zu verstehen, sondern als Gelegenheit zu Lernen und systemische Prozesse, die in komplexen Systemen zu unintendierten Konsequenzen und Ereignissen führen, besser zu verstehen. Insofern darf sich staatliches Handeln nicht als systemexterne, einmalige Entscheidung konstituieren, sondern muss als kontinuierlicher Prozess verstanden werden, der in selbst-reflexiver Weise das eigene Handeln immer wieder hinterfragt und anhand der auftretenden Konsequenzen dynamisch und andauernd anpasst. Im englischen Originaltext unterscheidet Chandler daher zwischen "policy failure“ auf der einen und "governance failure" auf der anderen Seite. Ersteres ist unbedingt notwendig in einem Governance-Ansatz, der nach den Prinzipien von Resilienz arbeitet. Nur durch kontinuierliches Begehen von Fehlern ist es möglich zu lernen und das System durch Anpassung weiterzuentwickeln. Governance failure besteht dann darin, sich diesen reflexiven Lernprozessen zu verweigern und weiterhin von einer reduktionistisch erklärbaren - und damit deterministisch steuerbaren - Welt auszugehen (Chandler 2014: 56f).

Die Überschneidungen zwischen Chandlers Ideen und den Bestandteilen des in der vorliegenden Arbeit entwickelten Resilienz-Konzepts für die zivile SiFo sind mehr als augenfällig. Ganz übergreifend lässt sich eine Notwendigkeit für Resilienz konstatieren, die sich aus der zunehmenden Komplexität der Systeme ergibt, die für menschliches Zusammenund Überleben relevant sind. Das gilt sowohl für Gesellschaftssysteme und politische Systeme, denen Chandlers primäres Interesse gilt, als auch für die sozio-technischen Systeme - etwa kritische Infrastrukturen - mit denen sich die zivile Sicherheitsforschung auseinandersetzt. Zunehmende Komplexität, und zwar das, was Chandler generelle Komplexität nennt, ist 
sowohl empirisch beobachtbar als auch aus den Annahmen der Theorie komplexer adaptiver Systeme theoretisch ableitbar (siehe 4.3.2). Diese generelle Komplexität macht Systeme unsteuerbar, das heißt ihr Verhalten lässt sich nicht deterministisch mithilfe spezifischer Maßnahmen festlegen. Und aus diesem Grund müssen wiederum Vorgehensweisen und Charakteristika entwickelt werden, die genau das miteinbeziehen. Chandler nennt Reflexivität, Flexibilität und Anpassungsfähigkeit als entscheidende Bestandteile einer resilienten Governance, die dynamisch auf Probleme zu reagieren in der Lage ist und sich durch ex post-Analysen ständig selbst weiterentwickelt (Chandler 2014: 56).

Damit ist er sowohl begrifflich als auch inhaltlich sehr nahe am hier entwickelten Resilienz-Konzept. Die Gemeinsamkeiten entspringen einer dezidiert deskriptiv-analytischen Diskussion von Chandlers Ideen. Bezieht man sich stärker auf deren normative Aspekte wird deutlich, dass er den neoliberalen wie auch den klassisch liberalen Ansatz von Governance für ungeeignet hält und sein eigenes Verständnis von Resilienz dagegensetzt. Chandler sieht Resilienz als einen neuartigen und normativ wünschenswerten Ansatz, der die reale Komplexität der relevanten gesellschaftlichen Systeme akzeptiert. Für ihn ist Resilienz ein „radically distinctive approach to governing complexity [...] through reposing complexity as an ontological rather than an epistemological problem" (Chandler 2014: 56). Daraus ergeben sich in direkter Ableitung aus Chandlers politikwissenschaftlichen Ideen und in Anlehnung an die oben bereits geführte, auf normative Aspekte zentrierte Diskussion folgende Annahmen für das Resilienz-Konzept der zivilen SiFo: Resilienz ist kein neoliberales Paradigma. Und weiterhin: Resilienz ist damit mehr als "alter Wein in neuen Schläuchen. "

Diese Annahmen sind auch konsistent zu den weiter oben getroffenen. Chandlers Definition von Neoliberalismus lässt sich mit einem Verständnis, das den Fokus auf eine Verlagerung der Verantwortung vom Staat auf das Individuum legt, in Übereinstimmung bringen. Ihm zufolge - bzw. den von ihm zitierten Autoren zufolge - sollte sich der Staat möglichst wenig in das gesellschaftliche Geschehen einmischen, um „den Markt“ nicht in der Entfaltung seiner wohlstandsmaximierenden Kräfte zu behindern (sehr vereinfacht ausgedrückt). Dieses Staatsverständnis lässt sich als „Steuern durch Nicht-Steuern“ umschreiben und es führt unmittelbar zu einer sehr deutlichen Verschiebung der Verantwortung vom Staat auf das jeweilige Individuum. Das ist aber gerade nicht das, was Chandler unter Resilienz versteht. Vielmehr steht die Frage nach den Möglichkeiten zielgerichteten staatlichen Handelns - oder auch Nicht-Handelns, das prinzipiell auch eine Form von Handeln ist - an sich unter der Bedingung 
genereller Komplexität im Mittelpunkt. Und zwar mitsamt der Einsicht, dass mit kontinuierlicher Reflexivität und Anpassungsfähigkeit geeignete Mechanismen für derartige, post-neoliberale Governance zur Verfügung stehen (Chandler 2014: 62). Warum ist Resilienz also mehr als alter Wein in neuen Schläuchen? Für die vorliegende Arbeit lässt sich festhalten: Resilienz ist ein post-neoliberales Paradigma. Denn Resilienz geht aufgrund der Komplexität von Systemen von der grundsätzlichen Unvorhersebbarkeit der Auswirkungen zielgerichteter, proaktiver Interventionen im System aus. Daher muss der Fokus auf der Maximierung von Reflexivität und (generischer) Anpassungsfähigkeit liegen, die dem System eine kontinuierliche und dynamische Weiterentwicklung - auch durch staatliches Handeln - im Fall des Eintretens von Problemen ermöglichen.

Zusammenfassend ergeben sich für ein eigenständiges Resilienz-Konzept der zivilen Sicherheitsforschung aus der Diskussion des Zusammenhangs zwischen Resilienz und Neoliberalismus folgende Annahmen:

- Resilienz ist ein Modewort.

- Das führt aber nicht dazu, dass Resilienz nicht zu einem fundierten wissenschaftlichen Konzept ausgearbeitet werden kann.

- Denn Resilienz ist mehr als „alter Wein in neuen Schläuchen.“

- Um den Begriff innerhalb der zivilen SiFo konzeptionell zu fundieren, ist sowohl eine system- und komplexitätstheoretische Einordnung als auch der Rückgriff auf Erkenntnisse aus Disziplinen mit einem etablierten Resilienz-Verständnis notwendig.

- Neoliberalismus lässt sich - stark vereinfacht ausgedrückt und unter bewusster Verkürzung der wissenschaftlichen Diskussion dieses Konzepts - mithilfe folgender Annahmen beschreiben: Aufgrund der Komplexität von Systemen sind diese grundsätzlich nicht steuerbar, weshalb auf regulierende Eingriffe vonseiten des Staates verzichtet werden sollte. Allerdings existieren zugrundeliegende Mechanismen, die dazu führen, dass sich Systeme selbst regulieren. Individuen innerhalb dieser Systeme sind damit selbst für sich verantwortlich, auch im Fall des Eintretens gravierender Störungen.

- Damit ist klar: Resilienz ist kein neoliberales Paradigma.

- Resilienz ist vielmehr ein post-neoliberales Paradigma. Denn Resilienz geht aufgrund der Komplexität von Systemen von der grundsätzlichen Unvorhersehbarkeit der Auswirkungen zielgerichteter, proaktiver Interventionen im System aus. Daher muss der Fokus auf der Maximierung von Reflexivität und (generischer) Anpassungsfähigkeit liegen, die dem System eine kontinuierliche und dynamische Weiterentwicklung - 
auch durch staatliches Handeln - im Fall des Eintretens von Problemen ermöglichen.

- Diese Definition von resilienter Governance kann bei oberflächlicher Betrachtung mit einem neoliberalen Konzept verwechselt werden. Deshalb gilt: Eine unreflektierte Übernahme des Resilienz-Begriffs birgt die Gefahr, diesen als neoliberales Paradigma einer Verantwortungsverlagerung vom Staat auf den einzelnen Bürger misszuverstehen.

- Die Verwendung des Resilienz-Konzepts in der zivilen Sicherheitsforschung darf nicht dazu führen, dass von widrigen Ereignissen betroffenen Personen und Gruppen die Verantwortung für erlittene Schäden zugeschrieben wird.

- Strategien zur Erhöhung der Resilienz der für die zivile SiFo relevanten sozio-technischen Systeme müssen als Angebote formuliert werden, die für die Gesellschaft einen klaren, über ohnehin erfolgende Maßnahmen des Bevölkerungsschutzes und der Katastrophenhilfe hinausgehenden, Mehrwert haben.

\subsubsection{Resilienz und Freiheit}

Die zivile Sicherheitsforschung ist keine grundlagenorientierte wissenschaftliche Disziplin, in der es darum geht, eine wie auch immer geartete natur- oder sozialwissenschaftliche Wahrheit zu ergründen. Vielmehr besteht ihre Zielstellung darin, „Lösungen, die den Schutz der Bevölkerung und der kritischen Infrastrukturen vor Bedrohungen durch Terrorismus, Sabotage, organisierte Kriminalität, Piraterie, aber auch vor den Folgen von Naturkatastrophen und Großunfällen gewährleisten und einen Beitrag zum Schutz unseres freiheitlichen Lebensstils leisten" zu entwickeln (BMBF 2016: 3). Es geht um die Sicherheit der gesamten Bevölkerung, also der Gesellschaft und die Frage danach, wie diese erhalten oder je nach Situation sogar erhöht werden kann. Auch ingenieurwissenschaftliche Sicherheitsforschung ist eindeutig und klar diesem Ziel verpflichtet. Allerdings geht es gerade nicht darum, die Sicherheit der Gesellschaft unter allen Umständen und mit allen Mitteln zu maximieren, sozusagen ohne Rücksicht auf Verluste. Schon bevor die eben genannten Ziele aufgeführt werden verweist das BMBF im (noch) aktuellen Sicherheitsforschungsprogramm darauf, dass es in der SiFo ganz zentral auch darum geht, „eine ausgewogene Balance zwischen Freiheit und Sicherheit zu bewahren" (BMBF 
2016: 3, eigene Hervorhebung). ${ }^{24}$ Auch in der Aufzählung der Ziele kommt diese Zielstellung vor, als „Beitrag zum Schutz unseres freiheitlichen Lebensstils." Die Kombination aus Freiheit und Sicherheit zieht sich wie ein roter Faden durch das Sicherheitsforschungsprogramm der deutschen Bundesregierung, wenn es etwa heißt, die SiFo strebe danach „die zivile Sicherheit der Menschen zu erhöhen, ohne den Schutz bürgerlicher Grundwerte wie Freiheit und Selbstbestimmung zu beeinträchtigen“ (BMBF 2016: 4). Oder wenn nach der Zusammenfassung am Anfang das eigentliche SiFo-Programm mit der Überschrift „Sicherheit als Basis eines freien Lebens“ beginnt und explizit herausgestellt wird, dass „Sicherheit und Freiheit kein Gegensatz“ seien. Weiter wird dazu ausgeführt: „Sie stehen aber in einem Spannungsverhältnis. Zu wenig Sicherheit bedroht unseren freiheitlichen Lebensstil. Zu viel Sicherheit kann unsere persönliche Freiheit und das Recht auf informationelle Selbstbestimmung gefährden. Sicherheitsforschung, wie wir sie verstehen, hat dieses Spannungsfeld immer im Blick. Sie wird dazu beitragen, eine ausgewogene Balance zwischen Freiheit und Sicherheit zu bewahren" (BMBF 2016: 5). Deshalb steht ein durch die SiFo erreichbarer „Beitrag zum Schutz unseres freiheitlichen Lebensstils“ auch an der ersten Stelle der ausführlicher präsentierten Ziele des Rahmenprogramms (BMBF 2016: 8).

Gerade aus politikwissenschaftlicher Sicht überrascht diese häufige und explizite Nennung der Notwendigkeit einer Ausgewogenheit zwischen Freiheit auf der einen und Sicherheit auf der anderen Seite oder sogar die kausale Verknüpfung einer Aufrechterhaltung der Freiheit durch Sicherheit nicht. In der Politikwissenschaft bzw. in den Sozialwissenschaften generell, spielte und spielt die Analyse und Diskussion des Zusammenhangs zwischen Freiheit und Sicherheit immer wieder eine herausragende Rolle, auch wenn Riescher 2010 konstatiert, dass sich die politische Theorie lange nicht mehr mit dem Thema Sicherheit beschäftigt habe (Riescher 2010b: 11). Das wahrgenommene Spannungsverhältnis der beiden Begriffe zueinander reicht teilweise so weit, dass sie als „gegensätzliche Pole auf einem Kontinuum“ verstanden werden, „an dessen einem Ende die grenzenlose, aber unsichere Freiheit, an dessen anderem Ende die Herstellung von

24 Mittlerweile [Stand: 22.5.2019] wurde das neue Sicherheitsforschungsprogramm der deutschen Bundesregierung veröffentlicht. Darauf wird in Kapitel 1 (siehe 1.2) eingegangen. Dessen Ausrichtung unterscheidet sich in Bezug auf das Thema Sicherheit und Freiheit aber inhaltlich nicht von den bisherigen Sicherheitsforschungsprogrammen, so dass die hier stehenden Ausführungen weiterhin Gültigkeit beanspruchen können. 
Sicherheit steht, die individuelle Formen von Freiheit unmöglich macht" (Riescher 2010: 4). Gerade im Anschluss an Entwicklungen, die mit dem Auftreten islamistisch motivierter Terroranschläge seit Beginn des 21. Jahrhunderts in Zusammenhang stehen, stellen sich Riescher zufolge alte Fragen neu und ganz neue Fragen kommen auf. Dazu zählt sie beispielsweise: „Wie viel Freiheit darf der Sicherheit geopfert werden? Wo sind die Grenzen des liberalen Staates, die nicht hintergangen werden dürfen? Was ist das Maß an Grundsicherheit, das menschliche Freiheit erst ermöglicht? Gibt es so etwas wie ein anthropologisches Grundbedürfnis nach Sicherheit und ist es die Politik, die gefordert ist, diese Sicherheit herzustellen" (Riescher 2010: 4)? Insbesondere die Frage nach der Rolle des Staates bei der Herstellung von Sicherheit und der gleichzeitigen Aufrechterhaltung von Freiheit ist hier von Interesse (Riescher 2010b: 19f). Das Ziel der vorliegenden Arbeit besteht nicht darin, diese Fragen zu beantworten. Für einen guten Überblick über politikwissenschaftliche, juristische und philosophische Ansätze dazu, soll hier auf einen von Riescher herausgegebenen Sammelband verwiesen werden (siehe Riescher 2010). Es geht auch nicht darum, eine politik- bzw. sozialwissenschaftlich umfassende Analyse des Begriffs und Konzepts der Freiheit vorzunehmen.

Vielmehr steht die Entwicklung eines eigenständigen Resilienz-Konzepts und darauf basierend von Hypothesen für Resilience Engineering im Mittelpunkt des Interesses. Beides jedoch jeweils für die zivile Sicherheitsforschung. Im Rahmen der zivilen SiFo bezieht sich der Begriff der Freiheit laut BMBF auf den „freiheitlichen Lebensstil» in den Gesellschaften, deren Schutz Ziel der zivilen SiFo ist. Daher wird der Begriff in der vorliegenden Arbeit bewusst breit verstanden und umfasst prinzipiell die Aufrechterhaltung der Grund- bzw. Menschenrechte und der liberalen und rechtsstaatlichen Demokratie. Damit steht unmittelbar fest: Die zivile Sicherheitsforschung bewegt sich nicht in einem normativ neutralen Raum. Wenn Resilienz in einer deskriptiv-analytischen Weise so verstanden wird, wie das in den folgenden Unterkapiteln der Fall sein wird, bedeutet größere Resilienz eine stärker ausgeprägte Fähigkeit komplexer adaptiver Systeme mit unerwarteten, disruptiven Ereignissen erfolgreich umzugehen. Das lässt sich im Rahmen der zivilen SiFo als größere bzw. mehr Sicherheit verstehen. Mehr Resilienz führt also unter sonst gleichen Bedingungen zu mehr Sicherheit. Diese Einschätzung hängt natürlich auch direkt davon $\mathrm{ab}$, was unter Sicherheit verstanden wird. Der Begriff ist selbst ein wissenschaftlich stark diskutiertes Konzept, unter dem sich, je nach spezifischer Definition, durchaus stark unterschiedliche Inhalte fassen lassen. Für die vorliegende Arbeit wird Sicherheit bereits in Kapitel 1 definiert und auf 
diese Definition rekurriert der hier formulierte Zusammenhang (siehe 1.2). Ob allerdings mehr Sicherheit durch größere Resilienz gleichzeitig immer normativ wünschenswert ist, hängt auch und vor allen Dingen davon ab, welchen Einfluss ein Zuwachs an Resilienz auf die in der Gesellschaft vorhandene Freiheit hat. In der Resilienzforschung, primär aus dem Bereich der Sozialwissenschaften und hier spezifisch Soziologie sowie Politikwissenschaft, wird der Zusammenhang zwischen Resilienz und Freiheit durchaus kontrovers diskutiert. Für das Resilienz-Konzept der zivilen SiFo sind insbesondere zwei Diskussionsstränge relevant. Zum einen die Frage danach, inwiefern auch normativ als negativ empfundene Strukturen Resilienz aufweisen können bzw. ob nicht gerade einige derartige Strukturen besonders resilient sind. Hier geht es häufig um Strukturen, die einem freiheitlichen Verständnis von Gesellschaft diametral gegenüberstehen. Und zum anderen wird diskutiert, ob mehr Resilienz eher zu mehr oder zu weniger Freiheit führt. Dabei sehen einige Autoren in Resilienz sogar eine Möglichkeit, den (scheinbaren) Gegensatz zwischen Freiheit und Sicherheit zu überwinden (Flynn 2011: ii, Riescher 2013: 1067).

Forscher, die sich mit der ersten der beiden gerade aufgeworfenen Fragen auseinandersetzen, verweisen mitunter auf einen inhärenten Konservatismus, der mit der Verwendung des Resilienz-Begriffs einhergehe. Sie beziehen sich auf die "begriffliche Herkunft“ von Resilienz bzw. die hauptsächliche Verwendung, die auf eine "persistence of a system“ abziele (Leach 2008: 11, Vogt 2015: 3). Damit unterstellen sie, dass diejenigen, die die Resilienz eines bestimmten Systems erhöhen möchten, mindestens implizit von der Erwünschtheit des Erhalts des bestehenden Systems und seiner Strukturen ausgehen. Ob das System in seiner existierenden Ausgestaltung allerdings tatsächlich normativ überhaupt wünschenswert ist - indem es beispielsweise bürgerliche Freiheiten zuverlässig garantiert - werde dann im Rahmen der Resilienzforschung nicht weiter untersucht (Blum et al. 2016: 169, Kuhlicke 2010). Einer ähnlichen Kritik sieht sich die Systemtheorie Luhmanns immer wieder ausgesetzt. Auch ihr wird vorgeworfen, „dass sie einem heimlichen Konservatismus das Wort rede“ (Kneer/Nassehi 2000: 36). Die Kritik kommt dabei beispielsweise aus Richtung der Soziologen der Frankfurter Schule, der zufolge es bei Luhmanns Theorie „primär um eine Verwaltung der Menschen, aber nicht um einen Abbau von Herrschaft und Ungerechtigkeit“ gehe (Kneer/Nassehi 2000: 45). Da Luhmann allerdings eine universalistische Theorie sozialer Systeme formulieren möchte, die allein deskriptiv-analytischer Natur ist und keine normativen Aussagen darüber trifft, wie wünschenswert bestimmte Gesellschaftsformen sind, trifft ihn diese Kritik nur sehr bedingt (Kneer/Nassehi 
2000: 45, siehe 4.3.1). Selbiges gilt auch für die rein deskriptiv-analytische Erarbeitung von Bestandteilen eines eigenständigen Resilienz-Konzepts für die zivile SiFo aus system- und komplexitätstheoretischen Überlegungen heraus (siehe 4.3). Für dieses Konzept müssen generell aber auch stärker normativ orientierte Bestandteile beachtet und in den Gesamtkontext eingeordnet werden.

Auch wenn das Resilienz-Verständnis der vorliegenden Arbeit nicht darin besteht, den Begriff als „persistence of a system“ zu verstehen, geht es doch primär um die Frage, wie ein System seine grundlegende Identität und/oder die Leistung, die es erbringt, trotz Eintretens eines disruptiven Ereignisses möglichst aufrechterhalten kann. Das System muss sich dazu zwar grundsätzlich anpassen und weiterentwickeln, aber nicht völlig neu erfinden. Und hier lässt sich im Rahmen der Resilienzforschung ein breiter, disziplinübergreifender Konsens dahingehend ausmachen, dass auch Systeme, die normativ als wenig oder gar nicht wünschenswert gesehen werden, sich als durchaus sehr resilient erweisen können (Carpenter et al. 2001: 766, Duit et al. 2010: 3, Folke 2006: 259, Leach 2008: 5, Walker et al. 2004). Inwiefern ein System als normativ wünschenswert verstanden wird, hängt natürlich von der individuellen Perspektive desjenigen ab, der darüber jeweils spezifisch urteilt. Oder anders formuliert - ohne damit eine Kontinuität zwischen Resilienz auf der einen und Vulnerabilität auf der anderen Seite unterstellen zu wollen: „[S]omeone's resilience may be someone else's vulnerability, or resilience at one scale may compromise that at another" (Leach 2008: 15). Gerade die Subjektivität der Beurteilung macht allerdings den Wahrheitsgehalt der oben getroffenen Aussage noch deutlicher. Ein offensichtliches Beispiel verdeutlicht diesen Sachverhalt: Resilienz gegenüber terroristischen Anschlägen wird von den meisten Menschen mutmaßlich als normativ stark wünschenswert verstanden werden. Dies gilt allerdings nicht für den Terroristen, der sich zudem selbst in den meisten Fällen auch nicht als „Terrorist“ versteht, und für den eine resiliente Gesellschaft, die sich in ihrem Verhalten und ihren Einstellungen durch Anschläge nicht langfristig verunsichern und beeinflussen lässt, ein gewichtiges Problem darstellt.

Insofern gilt: „Unlike sustainability, resilience can be desirable or undesirable" (Carpenter et al. 2001: 766). Wobei selbst diese Aussage in Zweifel zu ziehen wäre, da je nach beobachteter Zeitspanne auch eine auf Nachhaltigkeit ausgerichtete Handlungsweise für Akteure, die einer Strategie kurzfristiger Nutzenoptimierung folgen, schädlich wäre. Jedenfalls finden sich empirisch eine große Menge an Systemen, die sich auch gegenüber - für sie - gravierenden und disruptiven Ereignissen als äußerst resilient 
erwiesen haben und weiterhin erweisen und die aus Sicht eines breiten gesellschaftlichen Konsens - aus einer „westlichen“ Warte gesehen - normativ alles andere als wünschenswert sind. Diese reichen von der Verbrennung fossiler Rohstoffe zur Energiegewinnung über soziale Phänomene wie Sklaverei, terroristische Gruppierungen und aktuell wieder verstärkt Rechtspopulismus und Rechtsextremismus bis hin zu politischen Systemen wie autokratischen oder diktatorischen Herrschaftsformen (Carpenter et al. 2001: 766, Leach 2008: 11). Hier gilt zusammenfassend: „[R]esilience is not always a good thing” (Walker et al. 2004). Denn es erweist sich empirisch als zum Teil äußerst herausfordernd, die gegenwärtige Identität der betreffenden Systeme so stark zu verändern, dass sie als normativ wünschenswert gelten können. Teilweise, etwa wenn es um Sklaverei geht, ist es dazu sogar notwendig, das System an sich zu zerstören. Dazu schlägt etwa die sozial-ökologische Forschung vor, beim Management von Systemen gerade nicht nur die Mikroebene zu betrachten, sondern auf größeren Skalen auch zu bewerten, ob aus normativer Sicht ein Überwinden der Resilienz des betreffenden Systems notwendig sein könnte (Folke 2006: 259, Leach 2008: 5, Walker et al. 2004).

Wenn Resilienz als Konzept für die zivile Sicherheitsforschung Verwendung finden soll, ergeben sich im Zusammenspiel mit den generellen Charakteristika dieser Forschungsrichtung einige Implikationen, die beachtet werden müssen. Zunächst lässt sich sehr allgemein festhalten: Resilienz ist nicht notwendigerweise normativ wünschenswert. Ob Resilienz wünschenswert ist, hängt von der zugrundeliegenden Identität des konkreten Systems $\mathrm{ab}$, dessen Resilienz bewertet und im Zweifel durch Maßnahmen der Sicherheitsforschung erhöht werden soll: , $[\mathrm{N}]$ otions of resilience $[. .$.$] may$ have wholly different meanings, depending on how the social system was configured to begin with and from whose viewpoint resilience is assessed" (Duit et al. 2010: 3). Wenn die zugrundeliegende Identität des Systems normativ wünschenswert ist, dann ist auch eine Erhöhung seiner Resilienz wünschenswert. Daraus folgt: Die Erhöhung der Resilienz eines Systems ist genau dann normativ wünschenswert, wenn die zugrundeliegende Identität des Systems normativ wünschenswert ist. Allerdings kann die Frage, ob die zugrundeliegende Identität eines Systems normativ wünschenswert ist, nicht allgemeingültig objektiv beantwortet werden. Das wurde weiter oben anhand eines offensichtlichen Beispiels aufgezeigt. Inwiefern bestimmte, gesellschaftlich und für die zivile SiFo relevante Systeme als ihrer Identität nach normativ wünschenswert verstanden werden, ist das Ergebnis politischer und gesellschaftlicher Aushandlungsprozesse. Die Analyse dieser Aushandlungsprozesse und die Entwicklung möglicher Empfehlun- 
gen zur konkreten Ausgestaltung der relevanten Systeme ist Aufgabe verschiedener Bereiche der Politikwissenschaft bzw. weiterer Disziplinen aus den Sozialwissenschaften. Kuhlicke zufolge wurde die Diskussion von Fragen zu Themen wie Interessen, Macht und sozialen Konflikten im Zusammenhang mit Resilienz bisher stark vernachlässigt und sollte künftig verstärkt erfolgen (Kuhlicke 2010). Die Aushandlungsprozesse selbst und auch die Frage danach, was ein normativ wünschenswertes System konkret ausmacht, stehen allerdings nicht im Fokus des Interesses der vorliegenden Arbeit. Nichtsdestotrotz kann ein eigenständiges Resilienz-Konzept der zivilen SiFo nur vollständig sein, wenn es auf diese vorgelagerten Prozesse mindestens kurz rekurriert. Gerade wenn die normative Erwünschtheit der Identität der zu schützenden Systeme in der zivilen SiFo ein Stück weit politisch gesetzt zu sein scheint, da deren primäres Ziel ja darin besteht, „Sicherheit als Basis eines freien Lebens“ zu ermöglichen, wird eine politikwissenschaftliche Hinterfragung dieser Setzung notwendig (BMBF 2016: 5). Daraus folgt für das Resilienz-Konzept der zivilen SiFo: Ob Lösungen zur Erhöhung der Resilienz eines Systems erforscht und entwickelt werden sollten, hängt von vorgelagerten gesellschaftlichen und politischen Aushandlungsprozessen zur normativen Erwünschtheit des Systems ab.

Wenn es um die Resilienz normativ negativer Strukturen oder als normativ nicht wünschenswert empfundener Systeme geht, muss zudem ein weiterer Aspekt beachtet werden. Neben der Resilienz des unerwünschten Systems gegenüber radikaler Veränderung in Richtung einer größeren Erwünschtheit, können auch solche Systeme Resilienz gegenüber den Arten von disruptiven Ereignissen aufweisen, mit denen sich die zivile SiFo beschäftigt. Mit anderen Worten können beispielsweise auch Diktaturen gravierende Naturkatastrophen oder Terroranschläge in verschiedener Art und Weise verarbeiten und dazu unter anderem bestrebt sein, ihre eigene Resilienz zu erhöhen. Im Bereich der Arbeitspsychologie erweisen sich zum Teil normativ negative Strukturen - etwa Arbeitsbedingungen - gerade deshalb als resilient, weil Mitarbeiter in Resilienz geschult werden, um mit eigentlich ihre Belastungsgrenzen übersteigenden Arbeitsumfängen umgehen zu können. So wird die prinzipiell als normativ wünschenswert anzusehende individuelle Resilienz von Personen in gewisser Weise missbraucht, um ein System zu perpetuieren, welches selbst normativ nicht wünschenswert ist - zumindest aus Sicht der betroffenen Personen (Vogt 2015: 7). Für die zivile SiFo gilt dieser grundsätzliche Zusammenhang in einer ähnlichen Ausgestaltung zum Teil auch. Sowohl Blum et al. als auch bereits Tobin in einem Artikel aus dem Jahr 1999 stellen eine Tendenz dahingehend fest, dass Strategien zur Erhöhung der Resilienz gegenüber 
extremen disruptiven Ereignissen auch in demokratischen Systemen nicht selten dazu genutzt werden, bestehende, als ungerecht und damit normativ nicht wünschenswert charakterisierte Strukturen zu erhalten. Demnach kämen Resilienz-steigernde Maßnahmen etwa in Städten häufig spezifischen Eliten zu Gute und würden ohnehin bereits benachteiligten Teilen der Bevölkerung sogar noch weiter schaden (Blum et al. 2016: 169). Nach Tobin werden Katastrophenschutz und der auf Extremereignisse folgende Wiederaufbau häufig dazu genutzt, existierende soziale Strukturen beizubehalten, was gerade aus sozialer Sicht die Wahrscheinlichkeit weiterer Katastrophen erhöht, weil die zugrundeliegenden Probleme, die erst dazu führen, dass Extremereignisse zu Katastrophen werden, und die beispielsweise in sozialer Ungerechtigkeit bestehen, nicht gelöst werden (Tobin 1999: 23).

Ein eindrückliches Beispiel dafür bietet der Wiederaufbau der Stadt New Orleans im Anschluss an die verheerenden Schäden, die Hurrikan Katrina 2005 verursachte. Obwohl immer wieder von einem sehr erfolgreichen Wiederaufbau die Rede ist und die Stadt sich wirtschaftlich sehr gut entwickelt, sind Tendenzen erkennbar, die ethisch mindestens diskussionswürdig erscheinen. So änderte sich die Verteilung der Bevölkerung im Anschluss an den Hurrikan. Der Anteil an weißen sowie relativ wohlhabenden Personen ist tendenziell gestiegen, während eine Vielzahl an sozial schlechter gestellten Personen, auch und vor allem schwarzer Hautfarbe, nach dem Hurrikan nicht wieder zurückgekehrt sind und insofern kein Teil des „neuen“ New Orleans mehr sind (siehe Flaherty 2016, Jakob/ Schorb 2008). Diese Erkenntnis konnte der Autor bei einem Besuch anhand einiger Unterhaltungen, auf der einen Seite mit offiziellen Vertretern der Stadt und auf der anderen Seite mit einem Taxifahrer auf der Fahrt vorbei an sozial extrem benachteiligten Stadtgebieten, zumindest subjektiv deutlich bestätigen. Insofern scheint die von Tobin aufgeworfene Frage, ob Gesellschaften, in denen massive soziale Ungerechtigkeiten existieren, überhaupt resilient gestaltet werden sollten - auch und gerade gegenüber den in der zivilen SiFo relevanten Extremereignissen - durchaus berechtigt und relevant. Er beantwortet sie eindeutig mit Blick darauf, was er unter Resilienz versteht: „[R]esilience, of course, mean[s] much more than this, and planning with such goals in mind usually requires significant changes in the structure of society" (Tobin 1999: 23). Ihm zufolge besteht Resilienz also auch darin, normative nicht wünschenswerte gesellschaftliche Strukturen zu überwinden. Für Blum et al. liegt jedoch die „Crux von Resilienzstrategien" gerade darin, dass sie solche Fragen nicht stellen und somit die „Notwendigkeit von Resilienz als Fähigkeit zu permanenter 
flexibler Anpassung an und Bewältigung von dauerhaft unsicheren und instabilen Lebensbedingungen als unhinterfragte[n] Konsens" etablieren. Sie stellen das Resilienz-Konzept deshalb unter "generellen Ideologieverdacht“ (Blum et al. 2016: 169). Beide Positionen vereint jedoch die normativ begründete Ablehnung von als ungerecht skizzierten sozialen Strukturen. Insofern stellt sich die Frage, wie das Resilienz-Konzept der vorliegenden Arbeit mit diesen konfliktären Positionen bei gleichlautender Zielsetzung umgehen sollte. Es geht zudem weniger um Freiheit, als um den in der Politik- und sonstigen Sozialwissenschaft ebenfalls intensiv diskutierten Begriff der Gerechtigkeit. Als solcher steht dieser nicht im Fokus der vorliegenden Arbeit und daher soll an dieser Stelle der Verweis auf den vorletzten Abschnitt genügen, in dem bereits davon die Sprache war, dass vor der Entwicklung von Lösungen zur Erhöhung der Resilienz immer vorgelagerte gesellschaftliche und politische Aushandlungsprozesse stehen müssen. Und selbiges gilt an dieser Stelle: Selbst, wenn ein System grundsätzlich normativ wünschenswert ist, gilt es bei der Entwicklung von Strategien zur Erhöhung seiner Resilienz darauf zu achten, dass möglicherweise bestehende Ungerechtigkeiten nicht perpetuiert oder sogar verstärkt werden. Um das sicherstellen zu können, bedarf es sozialwissenschaftlicher Analysen.

Bevor im nächsten Schritt die Frage beantwortet wird, ob nicht auch die Erhöhung von Resilienz selbst sich negativ auf Freiheit auswirken könnte - im Vergleich zum bisherigen Fokus, der fragte, inwiefern Resilienz dazu ge-/missbraucht werden kann, um Unfreiheit oder Ungerechtigkeit zu festigen - soll noch kurz ein Aspekt beleuchtet werden, der bei Antonovsky auftaucht. Nämlich ein kausal umgedrehter Mechanismus, nach dem gerade Unfreiheit, bzw. unfreie, als normativ nicht wünschenswert angesehene Strukturen zu mehr Resilienz führen können. Antonovsky geht generell der Frage nach, wie Menschen trotz Stressoren gesund bleiben können. Entscheidend sind für ihn hier ,generalisierte Widerstandsressourcen“, da diese „es leichter machen, den zahllosen Stressoren, von denen wir fortwährend bombardiert werden, einen Sinn zu geben" (Antonovsky 1997: 16). Mit Hilfe dieser generalisierten Widerstandsressourcen können Menschen ein starkes Kohärenzgefühl (sense of coherence SOC) ausbilden (Antonovsky 1997: 16, 93). Diese Konzeption weist einige Ähnlichkeiten zum Resilienz-Verständnis der vorliegenden Arbeit auf. Gegeben sein Verständnis des Kohärenzgefühls fragt Antonovsky allerdings auch, ob beispielsweise der Glaube an fundamentalistische Führer, die eine apokalyptische aber konsistente Weltsicht vertreten, zu einem hohen SOC führen kann. Und obwohl er dies normativ strikt ablehnt und selbst hofft, ein derart entstehendes SOC sei aufgrund seiner „Rigidität“ eher 
zerbrechlich und unecht, deutet seine wissenschaftliche Analyse auf etwas Anderes hin. Demzufolge kann auch im Befolgen einer fundamentalistischen Ideologie eine Grundlage für ein starkes SOC liegen, welches es Individuen ermöglicht, dem „gesunden“ Ende des Kontinuums Krankheit - Gesundheit näher zu sein und auch im Angesicht von Herausforderungen näher zu bleiben (Antonovsky 1997: 103f). Übersetzt man ein hohes SOC mit einem hohen Maß an Resilienz ließe sich also sagen, dass hier Resilienz mithilfe normativ negativer Strukturen geschaffen werden kann. Antonovsky zufolge kann zum Beispiel die Bevölkerung in Diktaturen, wenn sie die Realität mithilfe einer Ideologie als sinnhaft, handhabbar und bedeutsam begreift, durchaus ein hohes SOC aufweisen (Antonovsky 1997: 104). Um die Anknüpfungspunkte zum Resilienz-Konzept für die zivile SiFo klar zu machen, ließe sich feststellen: wer die Welt mithilfe einer Ideologie als sinnhaft, handhabbar und bedeutsam begreift, benötigt zur Ordnung seines Alltags tendenziell weniger Ressourcen und verfügt beim Eintritt eines disruptiven Ereignisses somit eher über ungenutzte Spielräume, die zu mehr Resilienz führen. Gleichzeitig ließe sich aber auch die Frage stellen, ob Menschen, die einer derart strikten Ideologie unterworfen sind, nicht über generell weniger Ressourcen und Flexibilität verfügen, da ihr Erfahrungsschatz vergleichsweise begrenzter ist und ihnen somit insgesamt eventuell weniger Optionen zur Verfügung stehen. Das würde zu einer geringeren Resilienz führen. An dieser Stelle ergeben sich aus der Verknüpfung von Antonovskys wissenschaftlicher Arbeit und dem Resilienz-Konzept der zivilen SiFo einige sehr spannende Forschungsfragen, die im Rahmen politikwissenschaftlicher Analysen detaillierter und intensiver untersucht werden sollten. Für die vorliegende Arbeit reicht es aus, folgendes festzuhalten: Auch Systeme, deren zugrundeliegende Identität sich durch unfreie Strukturen auszeichnet, scheinen in der Lage zu sein, Resilienz gegenüber disruptiven Ereignissen auszubilden.

Weiter oben wurde festgehalten, dass die Aushandlungsprozesse, die über die normative Erwünschtheit eines Systems entscheiden, nicht im Fokus der vorliegenden Arbeit stehen. In gewissem Sinne muss diese Aussage präzisiert werden und zwar insofern es um den Zusammenhang zwischen Freiheit und Sicherheit bzw. Resilienz geht, wie er in einem normativ bereits als wünschenswert anerkannten System definiert werden kann. Hier gibt es zwar eine grundsätzliche Übereinkunft über die normative Erwünschtheit des Systems an sich. Nichtsdestotrotz ist die Frage zu untersuchen, welchen normativen Einfluss eine Erhöhung der Resilienz auf die im System vorhandene Freiheit hat. Das hängt natürlich primär davon ab, was genau unter Resilienz verstanden wird. Im Kontext der zivilen SiFo 
haben Blum et al. verschiedene Ansätze diskutiert, mit deren Hilfe sie den Zusammenhang zwischen Resilienz und Freiheit besser zu verstehen suchen. Diese sollen hier kurz nachgezeichnet werden, um daraus für das Resilienz-Konzept der zivilen SiFo ableiten zu können, wo Gemeinsamkeiten und Unterschiede bestehen und wie sich daher der Zusammenhang zwischen Resilienz und Freiheit in der vorliegenden Arbeit fassen lässt.

Nach Blum et al. habe im 20. Jahrhundert generell das „Versicherungsund Präventionsparadigma" geherrscht und im Gegensatz dazu entspreche das Konzept der Resilienz eher einer „neuartigen ,Logik der Vorbeugung““ (Blum et al. 2016: 156). Prävention geht davon aus, dass sich künftige disruptive Ereignisse und ihre Auswirkungen prognostizieren lassen und es sich demzufolge im Wesentlichen um Risiken handelt, die einer „wissenschaftlich-technischen Beherrschbarkeit" unterliegen. Dazu müssen die Quellen und Dynamiken von Bedrohungen möglichst deterministisch bekannt sein (Boin/McConnell 2007: 52). Bei der ausführlichen Diskussion des Zusammenhangs zwischen Resilienz und Unsicherheit wird dazu passend auf die Unvollständigkeit dieser Annahme hingewiesen. Auch und gerade die zunehmende Komplexität relevanter sozio-technischer Systeme sowie der (Um-)Welt, in der wir leben, führen zu neuen und vor allen Dingen nicht deterministisch beherrschbaren Unsicherheiten (siehe 4.3.3). Eine mögliche Konsequenz dieser Sichtweise ist es, davon auszugehen, dass prinzipiell alles passieren kann, damit auch ein schlimmstdenkbarer Fall möglich ist und auch für diesen Fall entsprechend vorgebeugt werden sollte: „Der prinzipiell mögliche worst case wird zum Ausgangspunkt zeitgenössischer Sicherheitspolitiken und -planungen. Gegenwärtiges Regieren steht im Bann möglicher zukünftiger Katastrophen“ (Blum et al. 2016: 156). Blum et al. identifizieren daraufhin drei unterschiedliche Modi, um mit der gerade konstatierten Unsicherheit im Sinne einer Logik der Vorbeugung strategisch und praktisch umzugehen: „precaution, preemption und preparedness“ (Blum et al. 2016: 156). „Precaution“ übersetzen sie als Vorsorge. Allerdings scheint eine wörtliche Übersetzung als Vorsicht eher dem Verständnis zu entsprechen, das Blum et al. von diesem Begriff haben. Nach dem Motto „better safe than sorry“ würde eine derartige Strategie darin bestehen, potentiell schädliches Handeln konsequent zu unterlassen, da nicht sicher auszuschließen ist, dass es künftig zu katastrophalen Konsequenzen führt (Blum et al. 2016: 156). Blum et al. reden in Anlehnung an Ewald von einem „inhärent konservativen Zug des Vorsorgeprinzips“ (Blum et al. 2016: 157). Konsequent umgesetzt bedeutet Resilienz-Erhöhung in diesem Sinne die Einführung einer ganzen Reihe möglicherweise freiheitsbegrenzender Vorschriften und Gesetze (Blum et 
al. 2016: 158, Riescher 2010b: 17). Hier fällt sofort der starke Gegensatz auf, der zwischen einer Resilienz-Definition, die eine Vorsichts-Logik einschließt einerseits und klassischen Verständnissen des Begriffs, etwa bei Wildavsky herrscht. Für letzteren besteht Resilienz ja gerade im Gegenteil von Vorsicht. Auch für die vorliegende Arbeit scheint ein derartiges Verständnis von Resilienz erstens ungeeignet, weil es insgesamt im Resilienz-Diskurs nicht anschlussfähig ist, und zweitens schlechterdings ausgeschlossen, gegeben die bisherigen Argumentationsketten. Das bedeutet also: Resilienz besteht nicht darin, aufgrund der Unsicherheit künftiger Ereignisse eine Strategie der Vorsicht im Sinne vorbeugender Freiheitseinschränkung anzuwenden.

Selbiges gilt für den zweiten Modus, den Blum et al. identifizieren, nämlich den der „preemption“. Mit Präemption ist das „aktivistische Gegenstück“ der passiven Vorsicht gemeint, „etwa in Form von Präemptivschlägen gegen mutmaßliche Terroristen. Präemption gehorcht der Maxime, der möglichen Bedrohung - wie ungewiss sie auch immer sei um jeden Preis zuvorzukommen" (Blum et al. 2016: 157). Die Logik dahinter geht wieder vom schlimmstdenkbaren Fall aus, nimmt diesen sozusagen als "Quasi-Gewissheit" und rechtfertigt damit die Umsetzung aller denkbaren Maßnahmen. Blum et al. bezeichnen das als „entfesselten Hyperaktivismus" und nennen den war on terror als Beispiel (Blum et al. 2016: 157f). Dank des plakativen Beispiels werden die ethisch problematischen Auswirkungen einer derartigen Strategie augenblicklich offensichtlich: „Die inhärente Logik von Präemption [...] liegt darin, das, was sie zu bekämpfen sucht, permanent selbst (mit) hervorzubringen. So etwa werden terrorgeeignete Bioagenzien produziert, um dem Bioterrorismus vorzubeugen, Terror und Folter im Dienste der Terrorbekämpfung gerechtfertigt" (Blum et al. 2016: 157). Daher lässt sich sehr deutlich sagen, dass Resilienz, wenn sie eine solche Logik der Präemption enthält, in Bezug auf den Zusammenhang mit Freiheit normativ klar nicht wünschenswert ist. Oder präziser: je höher die Resilienz einer Gesellschaft, denn um Gesellschaften geht es an dieser Stelle, desto geringer ist ihre Freiheit. Blum et al. fassen das prägnant zusammen: „Präemption droht damit zu einem selbstzerstörerischen Modus des Umgangs mit Ungewissheit zu werden, der letztlich die Ordnung, die er zu schützen verspricht, selbst aushöhlt und demontiert" (Blum et al. 2016: 157). Aus normativer Sicht ist also klar, dass das Resilienz-Konzept der zivilen SiFo die Logik der Präemption ablehnen muss. Denn die Aufrechterhaltung der freiheitlichen Gesellschaft ist ein unverhandelbares Grundziel der Sicherheitsforschung, wie weiter oben eindrücklich dargelegt wurde. Und Präemption - wie 
auch Vorsicht - sind Modi, die eine „in Illiberalität zu kippen drohende Ordnungs- und Sicherheitsfixierung" aufweisen (Blum et al. 2016: 158). Deshalb gilt: Resilienz besteht nicht darin, aufgrund der Unsicherheit künftiger Ereignisse eine Strategie der Präemption im Sinne der Aushöhlung von Freibeit durch aktivistische Maßnahmen anzuwenden.

Der dritte von Blum et al. konstatierte Modus des Umgangs mit Unsicherheit ist „preparedness“, also Vorbereitung. Darunter verstehen sie zunächst vor allen Dingen auch - ähnlich wie bei den beiden anderen Modi - das explizite Akzeptieren, dass präventive Maßnahmen keinen Erfolg haben und es immer wieder zu disruptiven Ereignissen kommen wird. Der entscheidende Unterschied besteht nun darin, nicht die als solche wahrgenommenen Ursachen solcher Ereignisse bekämpfen zu wollen, sondern sich auf die Abmilderung von deren Folgen zu konzentrieren und zu verhindern, dass sie zu Katastrophen werden. Dazu sollen beispielsweise auch die ,zentralen Funktionen von ,vital systems' [...] schnellstmöglich“ wiederhergestellt werden (Blum et al. 2016: 158f). Der Modus der Vorbereitung enthält dabei auch die Idee, sich auf „Diskontinuitäten“ einstellen zu müssen und diese in „kontinuitätserhaltender“ Weise zu bewältigen. Weil Störungen gerade nicht verhindert werden können - oder nur durch eine letztlich selbstzerstörerische Aufgabe von Freiheit zugunsten einer falsch verstandenen Sicherheit - geht es darum, sich diesen gegenüber so gut es geht zu ,immunisieren“ und aus ihnen zu lernen (Blum et al. 2016: 158). Im Vergleich mit den beiden anderen Modi und insbesondere mit dem Resilienz-Verständnis der vorliegenden Arbeit wird sofort eine deutlich größere Übereinstimmung zwischen letzterem und dem Modus der Vorbereitung deutlich. Unsicherheit ist eine Realität, mit der komplexe adaptive Systeme umgehen können müssen und Resilienz im Sinne einer Vorbereitung eine mögliche Umsetzung dieser Tatsache. Blum et al. analysieren Resilienz hier in einem gouvernementalitätsperspektivischen Sinn und verstehen darunter verschiedene Formen von „Störungsverarbeitungskapazitäten“. Dann lässt sich Resilienz als „eine Eigenschaft und ein Telos des Regierens, Ungewissheit resp. Unsicherheit nicht auszuschalten, sondern sich ihr auszusetzen, von als objektive Möglichkeiten vorgestellten Bedrohungsszenarien zu lernen und damit vorausschauend umzugehen" begreifen (Blum et al. 2016: 158). Diese Annahmen und Ideen sind konsistent mit dem hier entwickelten Resilienz-Konzept der zivilen SiFo, und zwar sowohl in deskriptiv-analytischer Hinsicht als auch normativ gesprochen. Wenn Blum et al. davon sprechen, Resilienz könne „mithin als liberaler Modus des Regierens interpretiert werden“, kann diese Aussage auch über die Perspektive des Regierens hinaus erweitert werden (Blum et al. 2016: 
158). Für das Resilienz-Konzept der zivilen SiFo bedeutet das: Resilienz besteht darin, aufgrund der Unsicherheit künftiger Ereignisse eine Strategie der Vorbereitung im Sinne der Maximierung von Störungsverarbeitungskapazitäten anzuwenden, unter gleichzeitiger Beibehaltung gesellschaftlicher Freiheiten.

Ein derart formulierter Zusammenhang zwischen Resilienz und Freiheit findet sich auch, wie weiter oben bereits angedeutet, bei Forschern wie Riescher und Flynn wieder. Gerade Riescher analysiert mithilfe politikwissenschaftlicher Begrifflichkeiten die Frage, wie Sicherheit und Freiheit sich zueinander verhalten, wenn das (zumindest für die Politikwissenschaft) neuartige Konzept Resilienz miteinbezogen wird. Den Ausgangspunkt ihrer Überlegungen bilden - wie in der Sicherheitsforschung sehr häufig der Fall - die Terroranschläge des 11. September 2001. In und nach derartigen Ausnahmesituationen agiert nach Riescher vor allen Dingen die Exekutive, weil eine rasche Entscheidungsfindung und unmittelbares Handeln notwendig sind, oder zumindest von der Bevölkerung als notwendig empfunden werden. Schnelle Entscheidungen wiederum folgen „nicht dem ,Normalverfahren“ des Policy-making“ (Riescher 2012: 96). Daraus folgt die Frage, ob und wie schnelle Entscheidungen demokratisch legitimiert sind und welche Konsequenzen solche Entscheidungen zeitigen. Riescher setzt Begriffe wie Kommunikation mit Betroffenen, Vertrauen und Akzeptanz in die Politik und von ihr getroffene Maßnahmen und eine Abwägung zwischen Nutzen und Schaden ein, um die Frage zu beantworten. Letztlich bedürfe es eines „policy-making für demokratische Sicherheit", um Freiheit und Sicherheit miteinander zu vereinen (Riescher 2012: 98). Auf der Suche nach dieser „demokratischen Sicherheit“, mit deren Hilfe Bedrohungen wie der Terrorismus, die aufgrund ihrer spezifischen Charakteristika häufig zu freiheits-einschränkendem policy-making führen, „aus der Mitte der Gesellschaft heraus bewältigt werden“ können, führt Riescher das Konzept der Resilienz ein. Resilienz beinhaltet für sie sowohl Aspekte der Vorbereitung auf disruptive Ereignisse als auch der adäquaten Reaktion - ermöglicht durch geeignete Vorbereitung - bei deren tatsächlichem Eintreten (Riescher 2013: 1067). Entscheidend ist dabei die Definition dessen, was als adäquate Reaktion basierend auf geeigneter Vorbereitung verstanden werden soll. Nach Riescher ist zwar der Begriff der Resilienz in der Politikwissenschaft nicht geläufig, dass aus ihrer Sicht dahinterstehende Konzept jedoch werde mittels bestimmter demokratietheoretischer Überlegungen bereits intensiv diskutiert. Für sie stimmt der Gedanke hinter Resilienz im Wesentlichen mit Ideen überein, wie sie von sogenannten „input-orientierten“ Demokratietheorien stark gemacht werden (Riescher 2013: 1067f). 
Der mit Abstand prominenteste Vertreter dieser input-orientierten Demokratietheorien war der amerikanische Politikwissenschaftler Benjamin Barber, dessen Konzept der „starken Demokratie“, erstmals komplett ausformuliert in seinem 1984 erschienenen, gleichnamigen Werk Strong Democracy. Participatory Politics for A New Age sehr stark auf kommunikative und partizipative Formen der politischen Teilhabe setzte (siehe dazu Barber 1984). Riescher nutzt für ihre Diskussion der Frage nach dem Zusammenhang zwischen Resilienz, Sicherheit und Freiheit Barbers Ideen und stellt eine große Ähnlichkeit zwischen einer starken, input-orientierten Demokratie auf der einen Seite und einer - in ihrem Verständnis - resilienten Gesellschaft auf der anderen Seite fest. Barbers Demokratietheorie ist dabei eindeutig sehr stark normativ konnotiert, er stellt seine Vorstellung einer starken, input-orientierten Demokratie diametral der „mageren“, outputorientierten Demokratie gegenüber. Erstere orientiert sich gerade nicht primär an den Ergebnissen politischer Aushandlungsprozesse, sondern vielmehr an der normativen Erwünschtheit der Strukturen der Aushandlungsprozesse an sich. Es geht ihm um möglichst vielfältige und umfassende Möglichkeiten der Beteiligung von Bürgern am politischen Prozess, um Transparenz in der Entscheidungsfindung, um die Möglichkeit der Artikulation unterschiedlicher Interessen. Je stärker die Bürger in fairer, transparenter und wirkungsvoller Weise an politischen Entscheidungen partizipieren können, desto „besser“ für die Demokratie. Demgegenüber stellt Barber die magere Demokratie, der es lediglich um die Effektivität und Effizienz der politischen Entscheidungsfindung gehe. Hier stehen also nicht der Prozess und die vorgelagerten Aushandlungsprozesse im Mittelpunkt, sondern rein das resultierende Ergebnis (Riescher 2013: 1068f). Nach Riescher zeichnen sich output-orientierte Demokratien durch eine Fokussierung auf „Werte wie Individualismus, Freiheit als Selbstbestimmung, das Menschenbild des homo oeconomicus, Gewinnstreben, fehlende Gemeinschaftsorientierung, Repräsentations- statt Partizipationsbeziehungen und die Politik einer paternalistischen Verwaltung" aus (Riescher 2013: 1068).

Die Verbindung zur Resilienz-Diskussion lässt sich dann erneut über das vielzitierte Beispiel der Terroranschläge des 11. September 2001 herstellen. Insofern wird an dieser Stelle deutlich, wie einflussreich ein einzelnes Ereignis für die wissenschaftliche Diskussion bestimmter Konzepte mitunter sein kann, bzw. wie stark ein einzelnes Ereignis die wissenschaftliche Diskussion eigentlich bekannter Konzepte auch in neue Richtungen lenken kann. Dazu werden im Folgenden Unterkapitel noch weitere Überlegungen angestellt (siehe 4.2.3). Barber jedenfalls verwendete seine 
politikwissenschaftlich-theoretischen Überlegungen im Anschluss an den 11. September 2001 im politischen Raum, um gegen genau die Entwicklungen zu argumentieren, die letztlich stattfanden, nämlich eine massive Verschärfung der Sicherheitsgesetzgebung verbunden mit einer deutlichen Einschränkung von Freiheitsrechten (Riescher 2013: 1070). Der in direkter Folge der Anschläge verabschiedete USA PATRIOT Act, mit dessen Hilfe Bürgerrechte eingeschränkt und die Kompetenzen der Exekutive bzw. der ihr unterstehenden Geheimdienste und Strafverfolgungsbehörden ausgeweitet wurden, war nach Barbers Verständnis exakt die Reaktion, welche die Terroristen mit ihren Aktionen hervorrufen wollten. Damit - und nicht durch das Gelingen der Anschläge an sich - hätten die Terroristen ihre Ziele erreichen können. Denn auf diese Weise schwäche sich das Land selbst und „lasse eine ängstliche und passive Bevölkerung zurück“ (Riescher 2013: 1072). Barber dagegen empfahl der Regierung eine exakt gegenteilige Vorgehensweise und Strategie zur Bekämpfung der Effektivität des Terrorismus. Ihm zufolge hätten die USA starkdemokratische Elemente ihres politischen Systems ausbauen müssen, um ihre Bürger dazu zu befähigen, selbst als „aktive, partizipative und mündige Staatsbürger" bei der Aufrechterhaltung des "demokratische[n] Gemeinwesen[s]" mitzuwirken. Durch eine derartige Aktivierung und Befähigung von Bürgern hin zu direkter, demokratischer Beteiligung sah Barber eine Chance, dass die zugrundeliegende Strategie terroristischer Anschläge, die in der Verbreitung von Angst und Unsicherheit besteht, überwunden werden könnte. „Aktive Bürger, so Barbers Argumentation, lassen sich nicht durch Terror verängstigen oder aus Angst instrumentalisieren“" (Riescher 2013: 1071). Hier wird erneut die Verknüpfung zur im nächsten Unterkapitel durchgeführten Diskussion des Zusammenhangs zwischen Resilienz und Terrorismus deutlich. An dieser Stelle geht es aber zunächst um die direkten Implikationen, die eine solche Strategie der Fokussierung auf starkdemokratische Eigenschaften von Gesellschaften auf deren Ausgestaltung im Sinne von Freiheitsrechten hat. Und darum, inwiefern die Idee der starken Demokratie mit dem Resilienz-Konzept verknüpft ist.

Dazu können, wie von Riescher umgesetzt, zwei Begriffe aus der Resilienz-Diskussion, nämlich „response“ und „recovery“ genutzt und in einem starkdemokratischen Sinne gedeutet werden. Dann lässt sich die response, also die unmittelbare Reaktion auf das Eintreten disruptiver Ereignisse insgesamt als Phase deuten, in der sich die Resilienz der Gesellschaft essentiell darin ausdrückt, Freiheitsrechte und andere relevante Werte der Demokratie zu sichern und deren möglicher Aushöhlung durch unmittelbare, exekutive ad hoc-Entscheidungen planvoll entgegenzutreten (Rie- 
scher 2013: 1072). Als in diesem Sinne negatives Paradebeispiel lässt sich die Reaktion des damaligen französischen Präsidenten Francois Hollande auf die verheerenden Terroranschläge in Paris am 13. November 2015 verstehen. Hollande verkündete öffentlich, Frankreich befinde sich „im Krieg" und die unter ihm agierende Regierung rief den Ausnahmezustand aus. ${ }^{25}$ Ohne näher auf diese Begrifflichkeiten eingehen zu wollen wird unmittelbar deutlich, wie sehr Hollande und seine Regierung damit der Logik der Terroristen folgen und wie stark sie - rein begrifflich - die demokratische Grundlage der französischen Gesellschaft zur Disposition stellen. Der „Ausnahmezustand“ ist schon qua Begriff denkbar weit vom Normalzustand entfernt. Er weitet klassischerweise die Befugnisse der Exekutive aus und richtet den Fokus sehr stark auf den Output, das Resultat bestimmter Handlungen und weniger auf den Input, die zum Resultat führenden Handlungsweisen. Das steht dem Gedanken der input-orientierten Demokratie diametral entgegen. Riescher folgend besteht Resilienz aber gerade darin, durch Partizipation, Kommunikation und Transparenz Bürger so zu stärken, dass sie terroristischen Angriffen ohne Angst und Unsicherheit zu begegnen in der Lage sind (Riescher 2013: 1072). Der zweite Begriff, die recovery, also die eher längerfristig gedachte Erholung von den negativen Folgen eines disruptiven Ereignisses, lässt sich ebenfalls starkdemokratisch verstehen. Dazu kann zudem auf die Diskussion des Zusammenhangs zwischen Resilienz und Neoliberalismus aus dem vorangegangenen Unterkapitel rekurriert werden. Dort konnte festgehalten werden, dass eine unreflektierte Übernahme des Resilienz-Begriffs die Gefahr birgt, diesen als neoliberales Paradigma einer Verantwortungsverlagerung vom Staat auf den einzelnen Bürger misszuverstehen. Insofern darf die Verwendung des Resilienz-Konzepts in der zivilen Sicherheitsforschung nicht dazu führen, dass von widrigen Ereignissen betroffenen Personen und Gruppen die Verantwortung für erlittene Schäden zugeschrieben wird. Barber bzw. seinen Gedanken folgend Riescher dreht diesen Gedanken nun um. Statt der Gefahr einer Verantwortungsverlagerung betont sie die Chancen einer bewussten Verantwortungsannahme durch die Bürger. Der Recovery-Prozess kann genau dann als resilient bezeichnet werden, wenn „bürgerschaftliche Teilhabe“ zugelassen und gefördert wird, „sodass das Vertrauen in die eigenen Stärken dominiert“ (Riescher 2013: 1073).

Was bedeuten diese Überlegungen nun für das Resilienz-Konzept der zivilen SiFo? Die weiter oben postulierte Behauptung, wonach Resilienz da-

25 http://www.spiegel.de/politik/ausland/francois-hollande-nach-terror-in-parisfrankreich-ist-im-krieg-a-1063145.html [Stand: 27.6.2019]. 
rin bestehe, aufgrund der Unsicherheit künftiger Ereignisse eine Strategie der Vorbereitung im Sinne der Maximierung von Störungsverarbeitungskapazitäten anzuwenden, unter gleichzeitiger Beibehaltung gesellschaftlicher Freiheiten, lässt sich weiter präzisieren und ein Stück weit demokratietheoretisch ausdehnen. Das Ziel der starkdemokratischen Perspektive besteht in einer durchgängigen Stützung partizipationsermöglichender Strukturen und Prozesse im politischen Geschehen. Die politischen Aushandlungsprozesse zur normativen Erwünschtheit bestimmter Systeme, die für die zivile SiFo essentiell sind im Hinblick auf die Beantwortung der Frage nach der Sinnhaftigkeit des Einsatzes von Strategien zur Erhöhung der Resilienz der betreffenden Systeme, müssen also unter angemessener Beteiligung aktiver Bürger in fairer und transparenter Weise ausgestaltet werden. Darin - und nicht erst in den aus diesen Aushandlungsprozessen resultierenden Ergebnissen - liegt bereits ein normativer Wert, der über die Erwünschtheit des zugrundeliegenden gesellschaftlichen Systems entscheidet (Riescher 2013: 1073). Für Riescher sind deshalb input-orientierte Demokratietheorien ,nahezu ,natürliche' Anschlusstheorien für die Resilienz-Konzepte der zivilen Sicherheitsforschung" (Riescher 2013: 1076f). Resilienz lässt sich Riescher zufolge insofern als „starkdemokratische Sicherheit" verstehen, mit einem Fokus auf gesellschaftlicher Teilhabe und die durch aktive Beteiligung von Bürgern möglich werdende Aufrechterhaltung essentieller Freiheitsrechte auch und gerade im Angesicht gravierender, disruptiver Ereignisse wie etwa den Terroranschlägen des 11. September 2001 (Riescher 2013: 1077). Für das Resilienz-Konzept der zivilen SiFo gilt also: Resilienz lässt sich normativ als starkdemokratische Sicherheit verstehen.

Genau darin sieht Riescher das Potential des Konzepts, „die politischen Spannungsfelder von Freiheit und Sicherheit miteinander versöhnen zu können" (Riescher 2013: 1067). In sehr ähnlicher Weise formuliert auch der amerikanische Politikwissenschaftler Steve Flynn den Zusammenhang zwischen Resilienz und Freiheit. Ihm zufolge ist die weithin anerkannte Sicht eines spannungsgeladenen Verhältnisses zwischen Freiheit und Sicherheit "flawed“ und könne durch einen Fokus auf das Konzept der Resilienz aufgelöst werden. Passend zu Riescher und ihrer Verwendung von Barbers Ideen definiert Flynn ein Erfolgsmaß für Resilienz nicht etwa als die Anzahl verhinderter Terroranschläge, sondern als die Aufrechterhaltung gesellschaftlicher Freiheitsrechte trotz einer anhaltenden Bedrohung durch mögliche terroristische Anschläge (Flynn 2011: ii). Und in der Einschränkung solcher Rechte, wie sie beispielsweise durch den USA PATRIOT Act vorgenommen wurden, sieht er eher das Gegenteil von Resilienz, 
da auf diese Weise „means with ends“ verwechselt würden. Das lässt sich eins zu eins auf Rieschers Ideen übertragen. Wenn zur angeblichen Erhöhung der Sicherheit als Zielstellung (end) Mittel (means) zum Einsatz kommen, welche gesellschaftliche Freiheiten einschränken, bedienen sich Regierungen in ihrem policy-making einer output-orientierten Logik (Flynn 2011: ii, Riescher 2013: 1067ff). Resilienz besteht aber gerade darin, auch bei den Mitteln - durch Verwendung einer input-orientierten Logik - auf demokratische Passung zu achten und „auf die Stärken einer offenen Gesellschaft, auf ihre politischen Werte und auf ihre Selbstheilungskräfte“ zu setzen (Riescher 2013: 1067).

Der entscheidende Begriff an dieser Stelle ist dann die weiter oben bereits kurz erwähnte Verantwortungsannahme als aktive Handlung der durch ihre Partizipation die Demokratie erst konstituierenden Bürger. Das steht bewusst im direkten Gegensatz zu der aus der Soziologie kommenden Annahme der passiven Verantwortungsverlagerung vom Staat auf die Bürger, bei der den Bürgern ohne deren Zutun etwas aufgezwungen wird, was sie nicht zu leisten imstande sind. Nämlich der durch zunehmende Komplexität und Unsicherheit gekennzeichneten Welt resilient gegenüberzutreten (siehe 4.2.1). Mit der Umdrehung dieser Logik unter Zuhilfenahme demokratietheoretischer Überlegungen, wie sie von Riescher angestellt werden, kann das Resilienz-Konzept der zivilen SiFo also um den normativ entscheidend wichtigen Begriff der "Verantwortungsannahme" erweitert werden. Das ändert nichts an den deskriptiv-analytischen Annahmen bezüglich des Konzepts. Es ermöglicht aber eine sozialwissenschaftlich fundiertere Einordnung und Aussage darüber zu treffen, inwiefern bestimmte Maßnahmen und Entwicklungen innerhalb der Sicherheitsforschung tatsächlich in ein starkdemokratisch unterfüttertes Resilienz-Konzept passen bzw. wann das gerade nicht der Fall ist. Damit wird eine Analyseebene hinzugefügt, die aus der rein deskriptiv-analytischen Beschreibung des Konzepts nicht ableitbar ist, die jedoch aufgrund der der zivilen SiFo zugrundeliegenden Zielstellung, wie sie zu Beginn dieses Unterkapitels dargelegt wurde, unabdingbar ist. Nämlich der Erhöhung der Sicherheit bei gleichzeitiger unbedingter Aufrechterhaltung der Freiheit. Es geht an dieser Stelle nicht um eine ausführliche Beurteilung input-orientierter Demokratietheorien und der Frage danach, wie eindeutig diese Form der Demokratie als per se normativ wünschenswert anzusehen ist. So spricht Riescher selbst durchaus kritisch von den ,gelegentlich plakative[n] Begrifflichkeiten“ Barbers (Riescher 2013: 1073). Nichtsdestotrotz trifft die vorliegende Arbeit die Annahme, dass partizipative und transparente Bürgerbeteiligung im Sinne einer Verantwortungsannahme min- 
destens implizit gleichzeitig freiheits-erhaltend wie sicherheits-erhöhend und damit zusammenfassend resilienzerhöhend wirkt. Oder kürzer gefasst: Politikwissenschaftlich gesprochen besteht Resilienz normativ in einer durch Verantwortungsannahme aktiver Bürger möglich werdenden Aufrechterhaltung gesellschaftlicher Freiheiten bei gleichzeitiger Erhöhung der Sicherheit.

Damit schließt sich insgesamt der Kreis der Betrachtung des Zusammenhangs zwischen Resilienz und Freiheit, an dessen Beginn ja die Aussage stand, dass Resilienz nicht notwendigerweise normativ wünschenswert ist. Die Analyse hat diese Aussage präzisiert und gleichzeitig Bedingungen herausgearbeitet unter denen die Erhöhung von Resilienz auch demokratietheoretisch als normativ wünschenswert angesehen werden kann. Für das Resilienz-Konzept der zivilen SiFo ergeben sich so zusammenfassend folgende Annahmen:

- Resilienz ist nicht notwendigerweise normativ wünschenswert.

- Auch Systeme, deren zugrundeliegende Identität sich durch unfreie Strukturen auszeichnet, scheinen in der Lage zu sein, Resilienz gegenüber disruptiven Ereignissen auszubilden.

- Die Erhöhung der Resilienz eines Systems ist genau dann normativ wünschenswert, wenn die zugrundeliegende Identität des Systems normativ wünschenswert ist.

- Ob Lösungen zur Erhöhung der Resilienz eines Systems erforscht und entwickelt werden sollten, hängt von vorgelagerten gesellschaftlichen und politischen Aushandlungsprozessen zur normativen Erwünschtheit des Systems ab.

- Selbst, wenn ein System grundsätzlich normativ wünschenswert ist, gilt es bei der Entwicklung von Strategien zur Erhöhung seiner Resilienz darauf zu achten, dass möglicherweise bestehende Ungerechtigkeiten nicht perpetuiert oder sogar verstärkt werden.

- Resilienz besteht nicht darin, aufgrund der Unsicherheit künftiger Ereignisse eine Strategie der Vorsicht im Sinne vorbeugender Freiheitseinschränkung anzuwenden.

- Resilienz besteht nicht darin, aufgrund der Unsicherheit künftiger Ereignisse eine Strategie der Präemption im Sinne der Aushöhlung von Freiheit durch aktivistische Maßnahmen anzuwenden.

- Resilienz besteht darin, aufgrund der Unsicherheit künftiger Ereignisse eine Strategie der Vorbereitung im Sinne der Maximierung von Störungsverarbeitungskapazitäten anzuwenden, unter gleichzeitiger Beibehaltung gesellschaftlicher Freiheiten.

- Resilienz lässt sich normativ als starkdemokratische Sicherheit verstehen. Mit „starkdemokratisch“ sind dabei nach Barber Strukturen ge- 
meint, die eine aktive, transparente und partizipative Rolle von Bürgern bei gesellschaftlichen und politischen Aushandlungsprozessen bzgl. der normativen Erwünschtheit relevanter Systeme ermöglichen.

- Politikwissenschaftlich gesprochen besteht Resilienz dann normativ in einer durch Verantwortungsannahme aktiver Bürger möglich werdenden Aufrechterhaltung gesellschaftlicher Freiheiten bei gleichzeitiger Erhöhung der Sicherheit.

\subsubsection{Resilienz und Terrorismus}

Wie kam der Resilienz-Begriff eigentlich ursprünglich in die zivile Sicherheitsforschung? Die vorliegende Arbeit beruht darauf zu untersuchen, was Resilienz für die zivile SiFo bedeutet und wie sich daraus Hypothesen für Resilience Engineering, verstanden als ingenieurwissenschaftliche Wege zur Erhöhung gesellschaftlicher Resilienz, ableiten lassen. Dabei kann sie einen breiten Diskurs über Resilienz als Konzept innerhalb der zivilen Sicherheitsforschung als gegeben annehmen. Dieser Diskurs bezieht mittlerweile auch immer stärker die zum Teil jahrzehntelange Forschung zu Resilienz aus Disziplinen wie der Ökosystemforschung und den Sozialwissenschaften mit ein. Darauf basiert auch die vorliegende Arbeit, die dies in einer systemischen Breite wie Tiefe vornimmt, wie es bisher für die zivile SiFo noch nicht erfolgt ist. Den Ausgangspunkt der Beschäftigung mit Resilienz bildete allerdings ein Ereignis, in dessen Anschluss die Debatte zunächst sehr viel stärker politisch geführt wurde. Und resultierend aus der politischen Diskussion wurde Resilienz im Wesentlichen als ein Modewort verwendet, als wünschenswerte Eigenschaft von Individuen und Gesellschaften im Angesicht einer neuartigen, gravierenden Bedrohung nämlich der Bedrohung durch den organisierten, internationalen islamistisch motivierten „Terrorismus“. Als Terrorismus bezeichnet man nach der völkerrechtlich bindenden Definition der Resolution 1566 des UN-Sicherheitsrats von 2004 „Straftaten, namentlich auch gegen Zivilpersonen, die mit der Absicht begangen werden, den Tod oder schwere Körperverletzungen zu verursachen, oder Geiselnahmen, die mit dem Ziel begangen werden, die ganze Bevölkerung, eine Gruppe von Personen oder einzelne Personen in Angst und Schrecken zu versetzen, eine Bevölkerung einzu- 
schüchtern oder eine Regierung oder eine internationale Organisation zu einem Tun oder Unterlassen zu nötigen“ (S.C. Res. 1566 (2004), para. 3). ${ }^{26}$

Das auslösende Ereignis waren die Terroranschläge des 11. September 2001. Durch diese in ihren Auswirkungen mit Abstand gravierendsten Anschläge, die jemals ein „westliches“ Land erleiden musste, wurden insbesondere die Vereinigten Staaten von Amerika, aber darüber hinaus auch die europäischen Staaten schwer getroffen. Ein zufällig gewähltes Beispiel macht die enorme Bedeutung dieses einzelnen Ereignisses deutlich: gibt man das Stichwort „11. September 2001“ bei Google ein, führt eines der ersten Ergebnisse auf die Webseite der Tagesschau, die einen Rückblick zehn Jahre nach den Ereignissen mit der Überschrift „Wie der 11. September die Welt verändert hat" betitelt. ${ }^{27}$ Der „11. September” oder „Nine-Eleven" (9/11) sind zu feststehenden Begrifflichkeiten geworden, mit denen die meisten Menschen sofort etwas anfangen können. Und mit denen ein mindestens unterschwelliges Gefühl der Unsicherheit und Bedrohung verknüpft ist. Der englische Politikwissenschaftler und Resilienzforscher Jon Coaffee ordnet den 11. September im Kontext der Sicherheitsforschung wie folgt ein: „If nuclear accidents and climate change represented acts one and two of the ,world risk society' play, the events of September 11 became the third act, signaling the ,universalising' of the fear of terrorist attacks" (Coaffee 2008: 4634).

An dieser Stelle muss nun zwischen der nationalen, deutschen und der internationalen Perspektive der Sicherheitsforschung unterschieden werden. Bereits zu Beginn der vorliegenden Arbeit wurde die Genese der zivilen Sicherheitsforschung kurz nachgezeichnet. Auch für diese war der 11. September ein entscheidender Ausgangspunkt. Während jedoch in Deutschland zunächst nicht mit dem Begriff der Resilienz gearbeitet wurde und dieser erst nach und nach immer stärkere Verwendung fand, wurde in den USA und vor allem auch Großbritannien unter dem Stichwort „Resilienz“ als möglicher Antwort auf Terroranschläge, u.a. auch die Anschläge vom Juli 2005 in London, längst intensiv geforscht (siehe z.B. Coaffee 2008, Coaffee/Bosher 2008, Coaffee/Rogers 2008, Flynn 2011,

26 Der Begriff bzw. das Konzept des Terrorismus ist wissenschaftlich stark umstritten und wird gerade in der Soziologie, der Politikwissenschaft und der Rechtswissenschaft unter anderem im Hinblick darauf diskutiert, wo und wie sich legitimer Widerstand gegen staatliche Strukturen von Terrorismus abgrenzt. Diese Diskussionen sind nicht Bestandteil der vorliegenden Arbeit. Siehe dazu unter anderem Golder/Williams 2004, Hoffmann 2008, Marsavelski 2013, Tilly 2004, Waldmann 2003.

27 https:/www.tagesschau.de/ausland/meldung486936.html [Stand: 27.6.2019]. 
2011b, 2008, Thoma 2014). Insofern scheint es bereits rein aus der Genese des Begriffs in der Sicherheitsforschung geboten, den Zusammenhang zwischen Resilienz und dem Phänomen des Terrorismus näher zu untersuchen. Allerdings kann dieser Zusammenhang gleichzeitig als Spezialfall oder Anwendungsfall der allgemeineren Diskussion aus dem vorangegangenen Unterkapitel zu Resilienz und Freiheit verstanden werden. Sowohl die Ausführungen von Blum et al. als auch Riescher nutzen das Beispiel des Terrorismus, um ihre generelleren, gesellschafts- und demokratietheoretischen Überlegungen zu motivieren. Die Annahmen, die im vorangegangenen Unterkapitel herausgearbeitet wurden, gelten daher auch für den spezifischen Fall des Terrorismus. Flynn fasst die zugrundeliegende Logik prägnant zusammen: „Terrorism is fueled by the confidence that Americans will react to it by embracing draconian measures" (Flynn 2011b: 136). Wenn das geschieht, lässt sich der Terrorismus als erfolgreich charakterisieren. Denn, wie Kaufmann und Blum treffend skizzieren, „the very goal of terrorism is to weaken the resilience of the targeted society" (Kaufmann/Blum 2012: 238). Und eine Freiheitsrechte einschränkende Reaktion auf terroristische Anschläge verringert per definitionem die Resilienz einer Gesellschaft - das kann als zentrale Erkenntnis der Überlegungen des vorangegangenen Unterkapitels festgehalten werden.

Wie muss demnach eine resiliente Reaktion auf die Bedrohung durch Terrorismus, ob islamistisch oder anderweitig motiviert, aussehen? Um das Resilienz-Konzept der zivilen SiFo bezüglich dieses eminent wichtigen Spezialfalls zu vervollständigen, sollen im Folgenden kurz die wichtigsten Ideen dazu nachgezeichnet und daraus weitere Annahmen für das betreffende Konzept abgeleitet werden. Dabei gilt es, einige Kernherausforderungen zu beachten, denen sich sowohl Forscher als auch und vor allen Dingen Praktiker aus Politik, Geheimdiensten, Strafverfolgungsbehörden sowie die Gesellschaft generell gegenübersehen. So lassen sich zwar umfassend geplante und mit sehr viel Koordinationsaufwand verbundene Anschläge wie die des 11. September prinzipiell verhindern. Das gilt aber nicht für individuell geplante Anschläge, die sich zudem auch mit sogenannten „Alltagswaffen“, etwa Messern, Autos oder LKWs durchführen lassen und letzten Endes auch ohne jede Vorbereitung von entsprechend motivierten Einzeltätern jeder Zeit und an jedem Ort durchgeführt werden können. Solche Angriffe sind beinahe unmöglich zu verhindern. Gerade durch ihre Unvorhersehbarkeit und die Tatsache, dass sie sich letztlich überall ereignen könnten, müssen Angriffe dieser Art auch nicht notwendigerweise vergleichbar spektakulär sein wie die Ereignisse des 11. September, um ihr Ziel - das primär in der Verunsicherung der Bevölkerung 
besteht - zu erreichen (Flynn/Burke 2011: 5). Und da jeder einzelne, „erfolgreiche“ Terroranschlag von der Gesellschaft generell als inakzeptables Ereignis angesehen wird, sehen sich die politisch Verantwortlichen sowie die Sicherheitsbehörden einem beständigen Erfolgsdruck ausgesetzt, dem sie beinahe zwingend nicht gerecht werden können. Es gilt: „[T]errorists have to be right only once, whereas [...] officials have to be right 100 percent of the time" (Flynn 2011b: 132). So wenig es eine vollständige, hundertprozentige Sicherheit geben kann, so wenig können Sicherheitsbehörden realistischerweise alle Terroranschläge verhindern. Nichtsdestotrotz ist es für ihre eigene Legitimation, sowie für das Sicherheitsgefühl der Gesellschaft entscheidend wichtig, zumindest eine gefühlte Kontrolle über die Gesamtsituation zu behalten bzw. (wieder) zu erlangen (Coaffee 2010: 945).

Genau aus diesem Grund begann nach den Terroranschlägen vom 11. September 2001 sowie denen vom 7. Juli 2005 in London gerade in Großbritannien eine Diskussion darüber, wie sich mithilfe mehr oder weniger sichtbarer, physischer und technologischer Schutzmaßnahmen die Resilienz gegenüber Terroranschlägen steigern ließe und wie solche Schutzmaßnahmen dazu am besten auszugestalten seien (Coaffee 2010: 945). Es stellt sich an dieser Stelle die Frage, ob Resilienz hier der richtige Begriff für die Phänomene ist, die von Autoren wie Coaffee betrachtet werden. Coaffee geht mit einem stark anwendungsorientierten Blick an die Themen Sicherheit und Resilienz im Kontext von Terrorismus heran. So bezeichnet er etwa Resilienz als eine „integrated policy“, die sowohl Nachhaltigkeits- als auch Sicherheitsaspekte abdecke (Coaffee 2008: 4636). Einigkeit besteht jedenfalls darin, dass Terroranschläge wie die oben genannten dazu führten, klassische Sicherheitsmaßnahmen, wie sie in Großbritannien etwa gegenüber den Anschlägen vonseiten der IRA umgesetzt wurden, als nutzlos anzusehen (Coaffee 2010: 943). So eigne sich beispielsweise der sogenannte „ring of steel“, bestehend aus tatsächlichen physischen Schutzmaßnahmen, die verhindern sollen, dass Terroristen überhaupt ihr Zielgebiet erreichen, nicht zur Abwehr von islamistisch motivierten Selbstmordattentätern, die ihre Bomben inmitten belebter Plätze oder im öffentlichen Nahverkehr zünden. Mithilfe solcher Maßnahmen ist es nicht möglich, „to ,design-out' such threats“ und deshalb stellen sie eine neuartige Herausforderung für Sicherheitsbehörden dar (Coaffee 2010: 943, Coaffee/Rogers 2008: 102).

Und dieser Herausforderung soll mithilfe von mehr Resilienz begegnet werden. Coaffee zufolge zeichnet sich eine resiliente physische Infrastruktur durch verschiedene Eigenschaften aus. Die Infrastruktur selbst und 
damit auch die Menschen und weitere technische Systeme, auf die sie einen Einfluss hat, können dank der Resilienz der Infrastruktur extreme Ereignisse, ob Terroranschläge oder Naturkatastrophen, adäquat verarbeiten, in dem sie widerstandsfähig sind, sich schnell erholen und erfolgreich anpassen. Dazu muss die Infrastruktur bereits resilient gebaut und designt werden, aber auch im späteren Verlauf ihres Lebenszyklus in resilienter Weise betrieben und gewartet werden (Coaffee 2010: 947f). Hier tritt ein wichtiger Aspekt zum Vorschein, der in der Resilienz-Diskussion immer wieder auftaucht. Um gegenüber disruptiven Ereignissen gerüstet sein zu können, müsse Resilienz bei der Systemkonstruktion bereits in der Design-Phase beachtet werden (Coaffee/Bosher 2008: 78). Diese Forderung erfolgt vor dem Hintergrund einer wahrgenommenen ex post-Beachtung von Sicherheit/Resilienz. Systeme werden demzufolge primär im Hinblick auf Effizienz gestaltet, verbunden mit einem Ansatz, der Kostenminimierung als Entscheidungsprinzip beinhaltet. Sicherheit werde immer erst dann beachtet bzw. hinzugefügt, nachdem durch einen Schadensfall eine direkte Notwendigkeit dafür erkennbar werde. Dagegen setzen einige Forscher bewusst den Begriff des „Security-by-design“, also der Beachtung von sicherheitsrelevanten Fragestellungen bereits vor der Konstruktionsphase von Systemen, im Design desselben. Dieser Gedanke lässt sich noch einen Schritt weiterführen und es kann von „Resilience-by-design“ gesprochen werden, wie das auch im Projekt Resilien-Tech der Fall war (siehe Thoma 2014). Genau darin erkennen Forscher wie Coaffee und Thoma bereits einen wichtigen Aspekt von Resilienz: im Vergleich zu einer erst nachträglichen Implementierung von Maßnahmen zur Erhöhung der Sicherheit von Systemen, bestehe eine auf Resilienz basierende Strategie unter anderem darin, diese Maßnahmen schon „by design“ mitzudenken. Dem ist grundsätzlich zuzustimmen. Auch wenn die Einsicht trivial erscheint und damit noch keinerlei Aussage darüber getroffen wurde, worin die zu beachtende Resilienz eigentlich genau besteht, kann auch für das Resilienz-Konzept der zivilen SiFo festgehalten werden: Um die für die zivile SiFo relevanten, komplexen adaptiven Systeme resilient gestalten zu können, sollten resilienzerhöhende Maßnahmen und Gestaltungsprinzipien bereits in der Designphase Beachtung finden. Was im Umkehrschluss aber nicht bedeutet, dass Systeme ausschließlich in der Designphase resilient gestaltet werden können. Einer nachträglichen Veränderung des Systems hin zu größerer Resilienz steht prinzipiell nichts im Wege.

Im konkreten Anwendungsfall des Terrorismus stellt sich aber auch weiterhin die Frage, welche Maßnahmen und Gestaltungsprinzipien mit Resilienz verbunden werden. Coaffee und Bosher zufolge geht es um eine 
gleichzeitige Beachtung von Fragen der Proportionalität, der Akzeptanz, der Kosten und der Ästhetik. Nur wenn diese zufriedenstellend beantwortet werden können, dienen bestimmte Maßnahmen dazu, die Resilienz gegenüber Terroranschlägen zu erhöhen. Der Vorteil, diese Fragen bereits im Design von Systemen beantworten zu können, besteht übrigens darin, einer Reaktionslogik zu entkommen, die im Anschluss an tatsächliche disruptive Ereignisse möglichst schnelle und drastische Maßnahmen verlangt und dabei gerade Aspekte wie etwa Proportionalität und Ästhetik übersieht (Coaffee 2010: 953, Coaffee/Bosher 2008: 79). Resiliente Schutzmaßnahmen sollten dagegen so unauffällig wie möglich sein. Coaffee und Bosher sprechen von „softer, more subtle and ,landscaped“ security.“ Derartig gestaltete Maßnahmen sind nicht direkt als mit Sicherheit verbunden zu erkennen: „Provide security in the context of streetscape enhancement and public realm beautification, rather than as a separate or redundant system of components, the only purpose of which is security." So kann zum Beispiel eine Bepflanzung mit Bäumen in ähnlicher Weise als Sicherheitsmaßnahme wirken, wie das Aufstellen von Betonblöcken (Coaffee/Bosher 2008: 78). Nach Coaffee und Bosher sind es solche und ähnliche Ideen, die gerade im politischen Bereich nach den Anschlägen von 2001 und 2005 diskutiert und auch großflächig umgesetzt wurden, um die Resilienz gegenüber Terroranschlägen zu erhöhen. Mit Blick auf das deskriptiv-analytische Verständnis von Resilienz in der vorliegenden Arbeit muss jedoch festgehalten werden: Die gerade diskutierten Ideen sind nicht dazu geeignet, die Resilienz der betroffenen Systeme zu erhöhen. Das trifft gleich in mehrfacher Hinsicht zu. Zum einen geht es um Ideen und Maßnahmen, die sehr spezifisch gegenüber einer bestimmten Art von disruptivem Ereignis zu helfen in der Lage sind - nämlich mithilfe von Sprengstoff durchgeführten terroristischen Anschlägen. Derart spezifische Maßnahmen lassen sich logisch dem Bereich spezialisierter Anpassung zuordnen und damit nicht dem Resilienz-Bereich. Und zum anderen ist die Wirkweise der Ideen und Maßnahmen unvereinbar mit dem Resilienz-Verständnis der vorliegenden Arbeit, das wesentlich auf Anpassungsfähigkeit beruht, mit deren Hilfe komplexe Systeme auch Ereignisse zu meistern in der Lage sind, die ihre eigentlichen Belastungsgrenzen übersteigen (siehe 4.3). Physische Schutzmaßnahmen haben jedoch per definitionem festgelegte Belastungsgrenzen. Das alles heißt keinesfalls, dass derartige Ideen und Schutzmaßnahmen ungeeignet oder normativ negativ zu sehen sind (Elran 2012: 291). Sie lassen sich im Rahmen des Resilienz-Verständnisses für die zivile SiFo der vorliegenden Arbeit rein deskriptiv-analytisch lediglich nicht als Beitrag zur Erhöhung der Resilienz der betreffenden Systeme be- 
zeichnen. Deshalb gilt: Die Resilienz der Gesellschaft gegenüber der Bedrohung durch das Phänomen des Terrorismus lässt sich nicht mithilfe rein physischer Schutzmaßnahmen erhöhen.

Das behaupten allerdings Forscher wie Bosher, Burke, Coaffee, Elran, Flynn und Rogers auch nicht. Sie nutzen Analysen der politisch gewollten physischen Schutzmaßnahmen eher als Ausgangspunkt ihrer Betrachtung von Resilienz, die sie stark als community resilience und ein Stück weit auch in Ergänzung zu bzw. sogar Abgrenzung von den gerade skizzierten Ideen verstehen (Coaffee/Bosher 2008: 75, Coaffee/Rogers 2008: 102, Elran 2012: 291ff, 299, Flynn/Burke 2011: 4). Denn Terrorismus ist trotzdem ein Resilienz-Thema, da sich im Wesentlichen weder verlässliche Angaben über Wahrscheinlichkeiten noch über die durch Anschläge verursachten Schäden, bzw. die diese Schäden verursachende Art von Anschlägen treffen lassen. In der Unsicherheit über ihre konkrete Ausprägung und gegeben die Tatsache, dass Terroristen immer wieder neue Strategien und Anschlagspläne entwerfen und umsetzen, wird eine Notwendigkeit erkennbar, diesen - über sicherlich weiterhin notwendige und an vielen Stellen sehr sinnvolle, physische Schutzmaßnahmen hinaus - mittels Resilienz zu begegnen (Elran 2012: 291f). Von dieser deskriptiv-analytischen Argumentation soll nun der Weg wieder zurück zur normativ geprägten Analyse des Konzepts führen. Dazu lassen sich einige Aspekte des vorangegangenen Abschnitts nutzen. Laut Kaufmann und Blum basieren zahlreiche Elemente der sogenannten Resilienz-Strategie Großbritanniens auf einem verkürzten Resilienz-Verständnis im Sinne des bereits dargestellten engineering resilience. Davon zeugen auch die relevanten Stichworte wie „rebordering", „militarization of urban design" oder "defensible space“ (Kaufmann/Blum 2012: 244). Hier besteht die Gefahr, eine „architecture of terror" zu produzieren, die sich immer stärker verselbstständigt - strikt der Logik folgend, dass einmal etablierte Sicherheitsstandards nicht mehr aufgegeben werden können, ohne die Bevölkerung unverantwortlichen Risiken auszusetzen (Coaffee/Bosher 2008: 80). Eine derartige Architektur wirkt dann in ähnlicher Weise freiheitseinschränkend, wie die im vorangegangenen Kapitel beschriebenen Prinzipien der Vorsicht und der Präemption nach Blum et al. (siehe 4.2.2). Für das Resilienz-Konzept der zivilen SiFo lässt sich also auch aus normativer Sicht sagen: Resilienz besteht nicht darin, aufgrund der Unsicherheit künftiger Ereignisse eine Strategie der maximalen physischen Robustheit der betreffenden Systeme anzuwenden.

Gerade wenn es um das konkrete Beispiel des Terrorismus geht, lässt sich auch darüber hinaus noch sehr viel generischer feststellen: „[N]o technical measures will stop terrorism altogether" (Elran 2012: 299). Also 
rein durch Technologie ist dem Phänomen des Terrorismus nicht beizukommen. Das heißt aber im Umkehrschluss keinesfalls, dass technologische Maßnahmen nicht dazu beitragen könnten, die Resilienz relevanter Systeme auch und gerade gegenüber terroristischen Angriffen zu erhöhen. Für die vorliegende Arbeit und den Begriff des Resilience Engineering wäre eine solche Erkenntnis auch sowohl unerwartet als auch äußerst problematisch, würde aus letzterem doch quasi ein Widerspruch in sich selbst. Es geht lediglich, aus der sozusagen entgegengesetzten Richtung argumentierend, darum, dass Resilienz nicht rein technisch gedacht werden kann. Coaffee und Bosher plädieren beispielsweise dafür, für ein Resilienz-Konzept im Bereich der kritischen Infrastrukturen neben deren physikalischen Eigenschaften und Bedingungen auch sozio-politische Aspekte zu beachten. Das sei entscheidend wichtig, denn „resilient engineering also demands a more resilient infrastructural context with regard to the professions and the structures and processes which govern construction activity" (Coaffee/Bosher 2008: 75). Noch einen Schritt weiter gehen zum Beispiel Elran, Flynn, Kaufmann und Blum oder auch Coaffee selbst gemeinsam mit Rogers, die jeweils die Bedeutung von gesellschaftlicher Resilienz oder community resilience betonen. So sieht Elran darin sogar eine „strategische Waffe“ im Krieg gegen den Terror (Elran 2012: 299) - wobei der Kriegsbegriff im Zusammenhang mit dem Phänomen des Terrorismus sicher bereits an sich ein Teil des Problems darstellt (siehe dazu zum Beispiel Holzinger 2011). Für Kaufmann und Blum drückt sich gesellschaftliche Resilienz in einer „emotionalen Stärke“ aus, mit der auf Terroranschläge reagiert werden kann (Kaufmann/Blum 2012: 238). Flynn betont die Möglichkeit, durch adäquate Information der Bevölkerung dieser dabei zu helfen, terroristische Angriffe möglichst unbeschadet zu überstehen, sich schnell von ihnen zu erholen und an ihre Folgen anzupassen (Flynn 2011b: 134). Wenn es gelingt, gesellschaftliche Resilienz in diesem Sinne aufzubauen, wird dem Terrorismus ein großer Teil seiner Wirksamkeit genommen. Denn je weniger sich eine Gesellschaft von terroristischen Angriffen verunsichern lässt und je weniger sie sich darauf einlässt, mit freiheitseinschränkenden Maßnahmen zu antworten, je mehr sie gleichzeitig bürgerschaftliches Engagement stützt, desto weniger attraktiv sind Strategien, die auf die Tötung und Verletzung Unschuldiger zielen (Elran 2012: 292f, Flynn/Burke 2011: 4ff). Dazu muss die Bevölkerung allerdings ein Stück weit selbst Verantwortung übernehmen, so dass unter dem Stichwort community resilience, wie es von Coaffee und Rogers verwendet wird, die in den vorangegangenen Unterkapiteln geführten Diskussionen zu Resilienz, Freiheit und Neoliberalismus wieder Beachtung finden müs- 
sen (Coaffee/Rogers 2008: 102). Hier wird deutlich, wieso Resilienz und Terrorismus sozusagen einen Anwendungsfall dieser generischen Diskussionen darstellt. Aufgrund der Bedeutung, welche das Thema Terrorismus im Rahmen des Resilienz-Diskurses in der Sicherheitsforschung einnimmt, musste dieser Zusammenhang nichtsdestotrotz explizit untersucht werden. Zumal sich aus der gerade erfolgten normativen Analyse noch eine wichtige Erkenntnis ergibt. Egal um welche Art von Maßnahmen, die zur Erhöhung der Resilienz relevanter Systeme dienen sollen, es sich dreht: diese dürfen keine zu starken negativen Auswirkungen haben. Sie dürfen also weder unverhältnismäßig teuer sein, noch die eigentliche Funktionalität des Systems über Gebühr beeinträchtigen, noch - und das wurde bereits ausführlich dargestellt - negativ auf gesellschaftliche Freiheiten wirken. Insgesamt geht es hier um Fragen der angemessenen Proportionalität. In welchem Verhältnis Kosten - nicht nur monetärer Art - und Nutzen stehen sollen, lässt sich nicht aus der Theorie heraus beantworten, sondern muss Ergebnis gesellschaftlicher Aushandlungsprozesse sein. Für das Resilienz-Konzept der zivilen SiFo heißt das: Lösungen zur Erhöhung der Resilienz eines Systems müssen einer Proportionalität im Hinblick auf das angemessene Verhältnis zwischen Kosten und Nutzen entsprechen, über deren Ausgestaltung in vorgelagerten gesellschaftlichen und politischen Aushandlungsprozessen entschieden werden muss.

Zusammenfassend ergeben sich für ein eigenständiges Resilienz-Konzept der zivilen Sicherheitsforschung aus der Diskussion des Zusammenhangs zwischen Resilienz und Terrorismus folgende Annahmen:

- Um die für die zivile SiFo relevanten, komplexen adaptiven Systeme resilient gestalten zu können, sollten resilienzerhöhende Maßnahmen und Gestaltungsprinzipien bereits in der Designphase Beachtung finden.

- Die Resilienz der Gesellschaft gegenüber der Bedrohung durch das Phänomen des Terrorismus lässt sich nicht mithilfe rein physischer Schutzmaßnahmen erhöhen.

- Resilienz besteht nicht darin, aufgrund der Unsicherheit künftiger Ereignisse eine Strategie der maximalen physischen Robustheit der betreffenden Systeme anzuwenden.

- Lösungen zur Erhöhung der Resilienz eines Systems müssen einer Proportionalität im Hinblick auf das angemessene Verhältnis zwischen Kosten und Nutzen entsprechen, über deren Ausgestaltung in vorgelagerten gesellschaftlichen und politischen Aushandlungsprozessen entschieden werden muss. 


\subsubsection{Resilienz und Vertrauen}

Gravierende und überraschend auftretende disruptive Ereignisse fordern die Funktionsfähigkeit sowohl der betroffenen Einzelpersonen, als auch der sozialen Gemeinschaften heraus, in denen diese sich bewegen. Es ist nicht ohne weiteres damit zu rechnen, dass derartige Ereignisse erfolgreich überstanden werden können, zumal dazu zunächst definiert werden muss, worin erfolgreiches Überstehen sich ausdrückt. In der medialen wie der künstlerischen Rezeption wird häufig das Bild einer im Anschluss an gravierende disruptive Ereignisse notwendig zusammenbrechenden sozialen Ordnung gezeichnet. Demnach führen solche Ereignisse zu panischem und irrationalem Verhalten bei den meisten Menschen, was die negativen Auswirkungen des Ereignisses weiter verstärkt. Wissenschaftlich ist dieses Bild nicht unbedingt als zutreffend zu bezeichnen. So betonen beispielsweise Prior und Roth, dass die soziale Ordnung im Anschluss an gravierende disruptive Ereignisse eben nicht in jedem Fall versagt (Prior/Roth 2013: 68). Gegeben die Analysen der vorliegenden Arbeit kann das Vorhandensein von Resilienz als (mindestens mit)kausal dafür gesehen werden, wenn eine Gesellschaft erfolgreich mit gravierenden und überraschend auftretenden disruptiven Ereignissen umzugehen in der Lage ist und also die soziale Ordnung gerade nicht versagt. Um die Entwicklung eines eigenständigen Resilienz-Konzepts für die zivile SiFo normativ abschließen zu können, soll nun noch ein letzter Begriff in seinem Verhältnis zu Resilienz analysiert werden. Denn mit Blick auf die Aussage von Prior und Roth lässt sich direkt fragen, inwiefern ein Vertrauen in eigene Fähigkeiten sowie Fähigkeiten des Staates, mit disruptiven Ereignissen umgehen zu können, genau diese Fähigkeiten erst hervorruft bzw. verstärkt. Die Frage nach dem Zusammenhang zwischen Resilienz und Vertrauen wurde vor allen Dingen aus den Ideen von Edwards abgeleitet, der zur Erhöhung gesellschaftlicher Resilienz verschiedene Maßnahmen vorschlägt. Diese reichen von Bildung und Engagement bis zu Empowerment und Encouragement, wobei gerade letzteres als eine Art Ermutigung von Bürgern zu verstehen ist, selbst aktiv zu werden und so zur Resilienz der Gesellschaft beizutragen (Edwards 2009: 80ff).

Um untersuchen zu können, wie Resilienz und Vertrauen zusammenhängen, bedarf es einer Definition dessen, was mit „Vertrauen“ gemeint ist. Vertrauen ist ein wissenschaftliches Konzept, das in vielen verschiedenen Disziplinen Verwendung findet, von der Psychologie über die Organisationstheorie und Betriebswirtschaftslehre bis hin zur Politikwissenschaft (siehe z.B. Brugger et al. 2013, Osterloh/Weibel 2006, Schweer 1997, 
Wiens 2013). Vertrauen ist darüber hinaus ein soziologisches Konzept, das von Niklas Luhmann mithilfe seiner systemtheoretischen Ideen untersucht und definiert wird. Gegeben die systemtheoretische Einbettung des Resilienz-Konzepts der vorliegenden Arbeit ergibt es deshalb Sinn, auch den Vertrauensbegriff aus einem systemtheoretischen Blickwinkel heraus zu untersuchen. ${ }^{28}$ Zumal mit Blick auf Luhmanns detailliertere Untersuchung des Begriffs, die den Titel Vertrauen. Ein Mechanismus der Reduktion sozialer Komplexität trägt (siehe Luhmann 2014). Diese Überlegungen Luhmanns stammen noch aus den 1960er Jahren, sind also zeitlich vor der autopoietischen Wende in seinem Werk zu verorten. Nichtsdestotrotz können sie auch heute noch wertvolle Hinweise darauf liefern, was unter Vertrauen verstanden werden kann. Insofern bilden sie den Ausgangspunkt der folgenden Ausführungen. Dabei stehen die Anknüpfungspunkte zwischen Luhmanns Vertrauensbegriff und dem Resilienz-Konzept der vorliegenden Arbeit im Vordergrund, so dass ersterer nur insoweit vorgestellt wird, wie zum Verständnis der Anknüpfungspunkte notwendig. Zudem wird Luhmanns rein deskriptiv-analytisches Verständnis von Vertrauen genutzt, um die stärker normativ konnotierte Diskussion des Zusammenhangs zwischen Resilienz und Vertrauen definitorisch anzuleiten.

Für Luhmann ist Vertrauen von zentraler Bedeutung für die Konstitution sozialer Systeme. Demnach ist Vertrauen zwar nicht der einzig relevante Mechanismus zur Reduktion von Komplexität, aber nichtsdestotrotz hält er das Vorhandensein von Vertrauen für essentiell wichtig, um das Zustandekommen komplexer Gesellschaften erklären zu können (Luhmann 2014: 126). Mit Blick auf die Bedeutung von Vertrauen aus Sicht von Individuen geht er sogar noch einen Schritt weiter. Bereits zu Beginn seines Buchs über Vertrauen beschreibt er, wie sehr Menschen auf das Vorhandensein eines Mindestmaßes an Vertrauen angewiesen sind. Luhmann geht sogar so weit zu postulieren, dass Menschen ganz ohne Vertrauen „morgens [nicht einmal ihr] Bett [...] verlassen“ könnten. Denn ohne Vertrauen wäre ihm zufolge alles möglich und „[s]olch eine unvermittelte Konfrontierung mit der äußersten Komplexität der Welt hält kein Mensch aus“ (Luhmann 2014: 1). Komplexität ist bei Luhmann hier, abweichend vom ausführlich erarbeiteten Komplexitäts-Konzept der vorliegenden Arbeit, die Anzahl der Zustände, die etwas annehmen kann (siehe 4.3.1 und 4.3.2). Die Welt, in der wir uns bewegen, zeichnet sich

28 Eine ausführlichere Einleitung zu Luhmann und seinen systemtheoretischen Ideen sowie die systemtheoretischen Analysen von Resilienz finden sich in 4.3 und insbesondere in 4.3.1. 
durch „unfassbare Komplexität“ aus, sie ist „übermäßig komplex“, das heißt „sie enthält mehr Möglichkeiten als die, auf die [ein] System sich erhaltend reagieren kann" (Luhmann 2014: 5). Deshalb ist die Zukunft ungewiss bzw. unsicher, was Luhmann noch generischer ausdrückt indem er von der „elementareren Tatsache, dass nicht alle Zukunft Gegenwart und damit Vergangenheit werden kann" spricht (Luhmann 2014: 14). Gegeben die unfassbare Komplexität der Umwelt und die Unsicherheit der Zukunft sind Menschen darauf angewiesen, Mechanismen zur Reduktion von Komplexität zu entwickeln (Luhmann 2014: 8, siehe ausführlicher dazu auch 4.3.1). Vertrauen stellt für Luhmann nun einen denkbaren Mechanismus dar, um genau das zu bewerkstelligen. Er bezeichnet Vertrauen als „soziale Beziehung“ mit spezifischen Eigenschaften. Wenn Menschen einander vertrauen, bieten sich ihnen demnach „mehr Möglichkeiten des Erlebens und Handelns" und das führt wiederum dazu, dass die Komplexität des sozialen Systems, dessen Teil sie sind, zunimmt. Damit kann dieses System im nächsten Schritt auf eine größere Zahl an aus der Umwelt stammenden Ereignissen reagieren, oder anders gesagt, die Komplexität der Welt effektiver reduzieren (Luhmann 2014: 4ff). Für Luhmann kann Vertrauen zudem nur in der Gegenwart bestehen. Basierend auf der „jeweils gegenwärtigen Gegenwart" bezieht sich Vertrauen auf den „Zukunftshorizont“, der sich aus dieser Gegenwart ergibt. Bei Vertrauen geht es darum „Zukunft zu vergegenwärtigen und nicht etwa, künftige Gegenwarten zu verwirklichen" (Luhmann 2014: 13ff). Deshalb bezeichnet Luhmann Vertrauen auch als eine „Form der Sicherheit“ (Luhmann 2014: 13).

Was hat es damit genau auf sich? Was versteht Luhmann nun genau unter Vertrauen? Um das zu verstehen muss zwischen Vertrauen und „Vertrautheit" unterschieden werden. Mit Vertrautheit meint Luhmann das Vorhandensein bekannter Umstände, einer alltäglichen und bekannten Wirklichkeit, in der Dinge im Normalfall in erwartbaren Bahnen verlaufen. Vertrautheit bezieht sich insofern relativ stark auf die Vergangenheit, in der Komplexität bereits reduziert wurde. Vertrautheit ist insofern ein Stück weit eine „Orientierung am Gewesenen“ und sie kann „die Welt vereinfachen und verharmlosen" (Luhmann 2014: 22f). Vertrautheit ist dabei weder notwendig normativ positiv noch negativ besetzt, sie kann sich sowohl auf erwünschte wie unerwünschte Bedingungen wie Ereignisse beziehen. Aber sie ermöglicht „relativ sicheres Erwarten und damit auch ein Absorbieren verbleibender Risiken." Luhmann bezeichnet Vertrautheit deshalb als „Voraussetzung für Vertrauen wie für Misstrauen“ (Luhmann 2014: 22ff). Er geht davon aus, dass Menschen sich im Alltag im Wesentlichen in einer vertrauten Lebenswelt bewegen, die erwartbar verläuft und 
in der es keine „spezifische[n] Vertrauens- oder Misstrauensprobleme“ gibt (Luhmann 2014: 26). Diese treten erst dann auf, wenn durch steigende Komplexität auch die Ungewissheit zunimmt und sich deshalb der Bezug auf die Vergangenheit als handlungsleitendes Motiv nicht länger eignet. Der "Charakter der Selbstverständlichkeit" geht verloren, weshalb nach Luhmann Vertrautheit und Vertrauen eine „neues Verhältnis wechselseitiger Stabilisierung“ eingehen müssen (Luhmann 2014: 24).

Mit Bezug auf die bereits durchgeführten Überlegungen für ein Resilienz-Konzept der zivilen SiFo, lassen sich diese Gedanken zu einer ersten Erkenntnis über den Zusammenhang zwischen Resilienz und Vertrauen zusammenführen. Demzufolge tritt in komplexen Systemen - hier wieder unter Verwendung der noch darzustellenden Komplexitätsdefinition der vorliegenden Arbeit (siehe 4.3.2) - im Vergleich zu einfachen Systemen das Motiv der Ungewissheit bzw. passender der Unsicherheit in den Vordergrund. Ereignisse, ihr Eintreten und ihre Auswirkungen sind a priori unsicher. Resilienz ist für spezifische Arten von Unsicherheit sowie spezifische Arten von Ereignissen von Bedeutung, nämlich ungewisse und echt unsichere, zusammengefasst als unerwartete Ereignisse sowie Ereignisse mit sehr geringen Eintrittswahrscheinlichkeiten, die jeweils die eigentlichen Belastungsgrenzen des betroffenen Systems übersteigen (siehe 4.3.3). Damit ist unmittelbar einsichtig: Vertrautheit als Mechanismus ist nicht dazu geeignet, die Resilienz eines Systems zu erhöhen. Denn bei Resilienz geht es gerade nicht um die „gemäßigte Zone“, in der Menschen sich „alltäglich" aufhalten, sondern um den existenzbedrohenden Ausnahmefall und die Frage, welche Mechanismen auch in diesem Fall zum Systemerhalt beitragen können. Ob Vertrauen ein solcher Mechanismus sein kann, ist noch zu klären. Für Vertrautheit gilt aber: Vertrautheit als Orientierung am Gewesenen und Bekannten ist kein Mechanismus zur Erhöhung der Resilienz der für die zivile SiFo relevanten, komplexen adaptiven sozio-technischen Systeme.

Im Gegensatz zu Vertrautheit ist Vertrauen im Luhmannschen Sinne ein zukunftsorientierter Mechanismus. Allerdings beruht Vertrauen immer auf einer gewissen Vertrautheit. Luhmann bezeichnet das als „Hintergrundsicherung." Er geht davon aus, dass es nicht möglich, zumindest aber nicht sinnvoll ist, ohne vorher gemachte Erfahrungen sozusagen blind zu vertrauen. Vertrauen hängt insofern durchaus mit Vertrautheit zusammen und Vertrautheit ist notwendig für Vertrauen. Damit gilt: Vertrauen ist ohne ein Mindestmaß an Vertrautheit nicht möglich. Vertrauen geht aber über Vertrautheit hinaus. Luhmann beschreibt Vertrauen als „überzieh[en] [der] Informationen, die [...] aus der Vergangenheit" bekannt sind. Wer 
vertraut, der „riskiert eine Bestimmung der Zukunft“ (Luhmann 2014: 23f). Denn wer vertraut nimmt sozusagen an, die Zukunft zu kennen. Er handelt in einer Weise, die genau dann Sinn ergibt, wenn sich die Dinge in einer ganz spezifischen Weise entwickeln. In Luhmanns Worten legt, wer vertraut, „seine gegenwärtige Zukunft auf eine künftige Gegenwart fest“ (Luhmann 2014: 24). Diese Aussage präzisiert die weiter oben getroffene Feststellung, wonach Vertrauen nur in der Gegenwart bestehen kann. Basierend auf dieser Gegenwart geht ein Vertrauender davon aus, die $\mathrm{Zu}-$ kunft zu „kennen“. Für den Vertrauenden ergibt sich so eine größere Sicherheit bzgl. der konkreten Wahl, die er unter allen ihm zur Verfügung stehenden Handlungsoptionen treffen sollte. Genau in diesem Sinne ist Vertrauen ein Mechanismus zur Reduktion sozialer Komplexität.

Vertrauen ist allerdings auch problematisch, da es sich um eine „riskante Vorleistung" handelt. Durch Vertrauen ergibt sich ein Vorteil in der Entscheidungsfindung. Vertrauen kann aber auch gebrochen werden. Und der mögliche Nachteil, wenn das Vertrauen gebrochen wird, ist nach Luhmann größer als der daraus ziehbare Nutzen. Wer sich trotzdem bewusst für ein solches Verhalten entscheidet, weil er erwartet, dass seine Annahmen über die Zukunft korrekt sind, der vertraut. Das unterscheidet Luhmann von einer „bloße[n] Hoffnung“, die nicht auf derart „vertrauensvolle[r] Erwartung" basiert, sondern letzten Endes ein Glücksspiel ist (Luhmann 2014: 27f). In ähnlicher Weise spricht er außerdem von „unbedacht $[\mathrm{em}]$, leichtsinnig[em], routinemäßig[em] “ Vertrauen, das gerade in alltäglichen Situationen häufig gewährt wird, wenn sich die Vertrauenden quasi sicher sind, vertrauen zu können. Trotzdem lässt sich prinzipiell immer erst im Anschluss an gegenwärtiges Vertrauen in einer Art Rückschau beurteilen, ob es sinnvoll und richtig war, zu vertrauen. Zum Zeitpunkt des Vertrauens, in der gegenwärtigen Gegenwart, ist Vertrauen als riskante Vorleistung immer mit Unsicherheiten verknüpft. Und diese Unsicherheiten sind charakteristischerweise keine Risiken, sondern ungewiss bzw. echt unsicher, es lässt sich also nicht quantifizieren, wie wahrscheinlich Vertrauen die richtige Alternative darstellt (Luhmann 2014: 29). Warum kann Vertrauen trotzdem sinnvoll sein? Das liegt nach Luhmann eben an der vielfach beschriebenen unüberschaubaren Komplexität der Umwelt, in der Menschen agieren. Denn ihre Mitmenschen können „zu jedem beliebigen Zeitpunkt sehr verschiedene Handlungen frei wählen.“ Jeder einzelne Mensch muss aber in spezifischen Zeitpunkten (für ihn „jetzt") konkret handeln. Dabei bleibt ihm ganz einfach nicht die Zeit, alle - oder auch nur eine irgendwie ausreichende Zahl an - Alternativen sinnvoll zu überblicken. Ein auf bestimmten Erwartungen basierendes Vertrauen hilft 
Menschen, sinnvolle Handlungsalternativen zu wählen, weil sie auf diese Weise deren Konsequenzen für sich festlegen (Luhmann 2014: 27f).

Die Festlegung der Konsequenzen erfolgt nicht durch eine detaillierte Analyse aller in einer spezifischen Situation verfügbarer Informationen und Fakten (Luhmann 2014: 37). Auch darin kann ein Vorgehen bestehen, mit dessen Hilfe Entscheidungen getroffen werden. Gegeben die Komplexität der meisten Entscheidungssituationen und die Knappheit der zur Verfügung stehenden Zeit, ist dies häufig nicht umsetzbar. Vertrauen basiert daher auf einem anderen Prinzip, nämlich dem bereits erwähnten „Überziehen der vorhandenen Information.“ Es ist somit „letztlich immer unbegründbar" (Luhmann 2014: 31). Luhmann formuliert das sehr treffend: „Vertrauen beruht auf Täuschung. Eigentlich ist nicht so viel Information gegeben, wie man braucht, um erfolgssicher handeln zu können" (Luhmann 2014: 38). Und genau deshalb beruht Vertrauen auf einer Generalisierung von Erwartungen. Indem bereits gemachte Erfahrungen aus der Vergangenheit bei hinreichender Ähnlichkeit auf die in der jeweiligen Gegenwart möglichen Zukünfte übertragen werden, wird eine gegenwärtige Zukunft bestimmt, aus der sich die konkrete Wahl der Handlungsoption ableitet. Wenn sich das Vertrauen wiederholt als gerechtfertigt erweist, entsteht so auch eine Art „Indifferenz gegen Unterschiede" (Luhmann 2014: 31). Das heißt, auch wenn Situationen bzw. Ereignisse sich relativ stark voneinander unterscheiden, können Menschen aufgrund des generisch funktionierenden Mechanismus des Vertrauens mit diesen erfolgreich umgehen. Eine Vertrauensbeziehung ist grundsätzlich „latent", sie besteht also „als Sicherheitsüberlegung im Verborgenen“ und wird gerade nicht explizit gemacht (Luhmann 2014: 45).

Selbiges gilt übrigens auch für Misstrauen, das an manchen Stellen ebenso angebracht sein kann. Luhmann versteht Misstrauen nicht als normativ negativ - so wenig wie er Vertrauen als normativ positiv sieht, sondern als einen weiteren Mechanismus, Komplexität zu reduzieren. Nur das dieser Mechanismus mit der entgegengesetzten Wirkrichtung funktioniert, wie der Mechanismus des Vertrauens (Luhmann 2014: 112). Vertrauen und Misstrauen sind auch nicht die einzigen Mechanismen zur Reduktion von Komplexität. Darauf wurde bereits kurz hingewiesen. Luhmann nennt beispielsweise auf Wahrscheinlichkeiten basierende Prognosen und „Kalkülmodelle“ als funktionale Äquivalente von Vertrauen. Sobald diese zum Einsatz kommen, ist es nicht länger notwendig, zu vertrauen. Ihm zufolge reichen diese Mechanismen aber nicht immer aus, um Ungewissheit zu absorbieren. Gerade in unbestimmten Situationen, in denen nicht genügend Informationen zur Verfügung stehen, um wahrscheinlichkeitsbasiert vali- 
de die Zukunft prognostizieren zu können, werden generisch funktionierende Komplexitätsreduktionsmechanismen wie Vertrauen wichtig (Luhmann 2014: 116). Mithilfe der bisherigen Ausführungen kann nun für die vorliegende Arbeit ein Verständnis von Vertrauen präzisiert werden, das im Hinblick auf sein Verhältnis zum Resilienz-Konzept näher untersucht werden kann. Demzufolge gilt: Vertrauen ist ein Mechanismus zur Reduktion von Komplexität, der darin besteht, die Auswahl von Handlungsentscheidungen in der Gegenwart aufgrund generalisierter Erwartungen über die gegenwärtige Zukunft zu treffen. Die generalisierten Erwartungen konstituieren sich mithilfe ähnlicher Erfahrungen aus der Vergangenheit.

Wie hängt derart verstandenes Vertrauen nun mit Resilienz zusammen? Grundsätzlich stellt Vertrauen für Luhmann etwas dar, was zwischen Menschen funktioniert. Ein Mensch vertraut einem anderen Menschen, er kann ihm aber auch misstrauen. Trifft weder das eine noch das andere $\mathrm{zu}$, benötigt der betreffende Mensch entsprechend andere Mechanismen, um die Komplexität in der sozialen Interaktion entsprechend reduzieren zu können (Luhmann 2014: 49). Neben diesem Vertrauen zwischen Menschen führt Luhmann aber auch eine weitere Art des Vertrauens ein, die für den Bereich der zivilen SiFo ein Stück weit relevanter ist, nämlich das sogenannte „Systemvertrauen“. Systemvertrauen bezieht sich auf die sichergestellte Funktionalität des betreffenden Systems. Statt einer bestimmten Person zu vertrauen, die dazu zunächst eingeschätzt werden muss, was Zeit und Ressourcen kostet, besteht Systemvertrauen im Vertrauen auf das Funktionieren des Systems. Also darauf, dass das System die von ihm erwarteten Leistungen zuverlässig erfüllt. Luhmanns Beispiel ist hier das Geld und das Vertrauen in eine Stabilität des Geldwertes, die es Menschen überhaupt erst ermöglicht, Geld zu verwenden (Luhmann 2014: 64).

Für die vorliegende Arbeit fällt der Blick unmittelbar auf die kritischen Infrastrukturen der Gesellschaft, deren Funktionsfähigkeit entscheidend für das tägliche Leben von Menschen ist. Auch hier ist Systemvertrauen insofern notwendig, dass beispielsweise nur wenige Bürger über eine eigene, autonome Wasserversorgung oder die Möglichkeit verfügen, sich autark mit Nahrungsmitteln zu versorgen. Wenn das Vertrauen in das zuverlässige Funktionieren von Wasser- oder Nahrungsmittelversorgung verloren ginge, wären die Menschen dazu gezwungen, ihre gesamte Lebensweise auf die Sicherstellung dieser überlebensnotwendigen Versorgung zu fokussieren. Dieses Systemvertrauen ist nach Luhmann allerdings relativ leicht erlernbar, jedenfalls sehr viel leichter, als das Vertrauen in konkrete Personen. Obwohl die meisten Menschen nicht wissen, wie Wasser- und Nahrungsmittelversorgung funktionieren, vertrauen sie doch darauf, dass sie 
funktionieren, auch und gerade weil sie täglich eine entsprechende Erfahrung machen können. Im Gegensatz zum Vertrauen in einzelne Personen, ist Systemvertrauen auch nicht einfach zu erschüttern. Denn im Prinzip bleibt den meisten Menschen nichts Anderes übrig, als den entsprechenden Systemen zu vertrauen. Eine wirksame Kontrolle können sie nicht oder kaum ausüben. Deshalb führen auch einzelne Vertrauensbrüche wenn also beispielsweise einen Tag lang kein Wasser mehr aus der Leitung käme - nicht direkt zu einer Zerstörung des Systemvertrauens (Luhmann 2014: 64, 75). Anders sieht es allerdings aus, wenn es zu wiederholten Vertrauensbrüchen kommt, sich das System also langfristig als unzuverlässig herausstellt. Denn Systemvertrauen ist gerade keine "naiv' erlebte [...] Vertrautheit der alltäglichen Welt", sondern ein bewusstes Vertrauen in ein von Menschen geschaffenes System „mit ausdrücklichen Prozessen der Reduktion von Komplexität“ (Luhmann 2014: 78). Dann gilt: Systemvertrauen besteht im Vertrauen darin, dass das betreffende System seine Funktion, die in einer spezifischen Form der Reduktion von Umweltkomplexität besteht, zuverlässig und ohne größere Störungen erfüllt.

Resilienz ist eine Nicht-Selbstverständlichkeit und von daher gehen Menschen tendenziell eher davon aus, dass betroffene Systeme nach Eintritt eines gravierenden disruptiven Ereignisses ihre Funktionalität - etwa zuverlässige Wasserversorgung - einbüßen. Ihr Systemvertrauen weist hier eine Art Sollbruchstelle auf. Wenn ein Ereignis eintritt, das die eigentlichen Belastungsgrenzen des Systems übersteigt, ist davon auszugehen, dass dieses zusammenbricht. Wenn allerdings bekannt ist, dass dieses System über große Resilienz verfügt - inwieweit dies a priori überhaupt feststellbar ist, soll an dieser Stelle nicht näher beleuchtet werden - erhöht sich das Systemvertrauen ceteris paribus, weil die gerade skizzierte Sollbruchstelle verschwindet. Für den Zusammenhang zwischen Resilienz und Systemvertrauen lässt sich also festhalten: Je größer die Resilienz eines komplexen adaptiven Systems ist, desto größer ist das ihm entgegengebrachte Systemvertrauen.

Luhmann analysiert seinen Begriff des Systemvertrauens noch weiter mithilfe des Beispiels Geld. Darauf soll hier ebenfalls noch eingegangen werden, da sich einige Überschneidungen zum Resilienz-Konzept der zivilen SiFo ergeben. Denn Luhmann bezeichnet Geld als ,generelles Problemlösungsmittel“. Wer über Geld verfügt, kann auf eine „Voraussicht spezifischer Problemsituationen verzichten“ (Luhmann 2014: 64, Hervorhebung im Original). Um ein generelles Problemlösungsmittel sein zu können, bedarf es des Systemvertrauens in die Stabilität des Geldwertes. Das Systemvertrauen ist insofern eine Art vorgelagerte Bedingung dafür, 
dass Geld diese Funktion erfüllen kann. Wenn das Systemvertrauen in die Stabilität des Geldwerts über den Einzelnen hinaus gegeben ist, kann die Verfügung über Geldmittel für diesen Einzelnen durchaus als Erhöhung seiner Resilienz verstanden werden. Im Kontext der zivilen SiFo sind dem zwar enge Grenzen gesetzt, da es hier häufig um disruptive Ereignisse geht, die großflächig wirken und die zu einer physischen Nicht-Verfügbarkeit lebensnotwendiger Güter wie Wasser, Nahrungsmittel, Medikamente und Strom führen. Fallbeispiele wie der Hurrikan Katrina zeigen aber, dass auch in solchen Fällen die Verarbeitung des Ereignisses durchaus zu großen Teilen davon abhängt, über wieviel Geld bzw. Vermögen jemand verfügt. So formuliert wäre Systemvertrauen in die Stabilität des Geldwerts eine Vorbedingung für die Resilienz Einzelner über den Mechanismus der Verfügbarkeit von Geldmitteln. Natürlich kann ihre Resilienz auch auf anderen Wegen erhöht werden, so dass ein derartiges Systemvertrauen keine notwendige Bedingung für Resilienz darstellt. Zudem ist a priori unklar, ob mehr Geld im System notwendigerweise dessen Resilienz erhöht. Das kommt stark auf die Verteilung des Geldes an, wie das Fallbeispiel des Hurrikans Katrina gezeigt hat, in dessen Anschluss sich die Sozialstruktur der Stadt New Orleans verändert hat. Es ist zumindest zweifelhaft, dass diese Änderung, die mit einem im Schnitt größeren Wohlstand einhergeht, zu einer Erhöhung der Resilienz des Gesamtsystems New Orleans geführt hat (siehe 4.2.2). Weiter oben wurde eine kausale Verknüpfung der Wirkrichtung "mehr Resilienz führt zu größerem Systemvertrauen“ begründet. Die umgekehrte Wirkrichtung „mehr Systemvertrauen führt zu größerer Resilienz" lässt sich allein über den Mechanismus des Geldes nicht ableiten.

Luhmann abstrahiert daher auch von der Verfügung über liquide Geldmittel und nennt weitere Mechanismen wie Macht und Wahrheit, die er alle als „vertrauensabhängige Mechanismen“ charakterisiert. An dieser Stelle soll darauf verzichtet werden, Luhmanns Verständnis der Begriffe Macht und Wahrheit näher auszuführen. Spannend ist hier vielmehr die Rolle, die er dem vorgelagerten Mechanismus des Vertrauens zuschreibt. Ihm zufolge ermöglicht Vertrauen „eine Indifferenz des Systems gegenüber zahllosen Umweltereignissen und damit Gewinn an Reaktionszeit" (Luhmann 2014: 117). Ohne Vertrauen ist laut Luhmann nur eine sehr rudimentäre Form menschlicher Kooperation möglich. Ausgeweitet auf Systemvertrauen wäre komplett ohne Systemvertrauen auch nur eine sehr archaische Form von Gesellschaft denkbar, da jeder Einzelne ständig damit beschäftigt wäre, sein eigenes Überleben durch Versorgung mit lebensnotwendigen Gütern sicherzustellen. Mit der Hilfe von Vertrauen gewinnen 
Systeme dagegen Zeit, um komplexere Strukturen aufzubauen (Luhmann 2014: 117). Es geht dabei um die Eigenkomplexität der Systeme. Je stärker Vertrauen innerhalb eines Systems und in ein System ausgeprägt ist, desto mehr Zeit bleibt zur Erfüllung systemrelevanter Aufgaben, auch und gerade im Fall des Eintretens disruptiver Ereignisse. Denn je größer das Vertrauen, desto weniger leicht lässt es sich durch einzelne, kurzfristige Enttäuschungen stören. Vertrauen, ob in Personen oder Systeme, ist ein Mechanismus, der nur von Menschen angewandt werden kann. Es „erfordert zahlreiche Hilfsmechanismen des Lernens, Symbolisierens, Kontrollierens, Sanktionierens, und [es] strukturiert die Weise der Erlebnisverarbeitung in einer Form, die Kraft und Aufmerksamkeit kostet" (Luhmann 2014: 117). Im Kontext der zivilen SiFo führt größeres Vertrauen in die relevanten komplexen adaptiven sozio-technischen Systeme dazu, dass betroffene Menschen mit vorübergehenden Funktionalitätseinbußen des Systems besser fertig werden. Damit steigt insgesamt die Resilienz des Systems. Dieses Vertrauen wird allerdings nicht naiv gewährt. Luhmann betont beispielsweise auch die Bedeutung von Kontrollen durch institutionalisierte Misstrauensmechanismen. Mit Blick auf das Systemvertrauen sieht er aber gerade im Vorhandensein solcher "Misstrauensgebote“ an kritischen Stellen einen Garanten für "Vertrauen in Systeme als Ganzes“ (Luhmann 2014: 124). Deshalb kann für das Resilienz-Konzept der zivilen SiFo insgesamt festgehalten werden: Je stärker das Systemvertrauen ausgeprägt ist, desto größer ist die Resilienz des Systems.

Die bisherige Analyse des Vertrauensbegriffs war mit Bezug zu Luhmann primär deskriptiv-analytisch zu deuten. Da das Resilienz-Konzept der zivilen SiFo aber auch dezidiert normative Aspekte beinhaltet und gerade der Vertrauensbegriff im Kontext der Sozialwissenschaften stark normativ diskutiert wird, soll dieser Blickwinkel ebenfalls noch kurz beleuchtet werden. Im Unterkapitel zu Resilienz und Neoliberalismus ging es stark um die Frage, ob Resilienz mit einer Verantwortungsverlagerung des Staats auf die Bürger verbunden ist und wie diese normativ zu deuten wäre (Blum et al. 2016: 165, Fekete et al. 2014: 15, Kaufmann/Blum 2012: 237, 249 und zugespitzt Dombrowsky 2012: 286, 2010: 4). Teilweise wird von einem „Rückzug des Staates aus seiner Verantwortung und seinen Aufgaben in der Gesellschaft" gesprochen (Deppisch 2016: 203). Wenn das zutrifft, würde es einen automatischen Vertrauensverlust der Bürger implizieren. Normativ wünschenswert - wenn das zugrundeliegende System selbst normativ wünschenswert ist - sind allerdings sowohl hohes Systemvertrauen als auch hohe Resilienz. 
Resilienz darf also nicht unreflektiert als Begriff übernommen werden und so einer Verantwortungsverlagerung vom Staat auf den einzelnen Bürger Vorschub leisten. Denn das führt zu einem doppelten Vertrauensverlust. Zum einen des Bürgers in den Staat, da dieser seinen Aufgaben des Bevölkerungsschutzes und der Katastrophenhilfe nicht mehr gerecht wird und so die Funktionalität dieses Systems erodiert. Zum anderen der Bürger in sich selbst, da sie notwendigerweise von den disruptiven Ereignissen, um die es in der vorliegenden Arbeit geht, überfordert sein müssen und daher dem Versagen ihrer eigenen Fähigkeiten gegenüberstehen (Dombrowsky 2012: 286). Zwar spricht Luhmann davon, dass Vertrauen in andere Personen und Systeme ganz generell erst möglich wird durch „strukturell nicht gebundene innere Ressourcen [...], die im Falle einer Enttäuschung des Vertrauens eingesetzt [werden] und die Last der Komplexitätsreduktion und Problemlösung übernehmen können“ (Luhmann 2014: 105). Dieser von Luhmann als „Selbstsicherheit“ bezeichneten Fähigkeit sind jedoch Grenzen gesetzt. Normativ gesprochen verstärkt der gegenseitig positive Wirkmechanismus zwischen Resilienz und Systemvertrauen also die Notwendigkeit, Resilienz konzeptionell sehr klar von einem „neoliberalen Paradigma“ zu trennen. Gleichzeitig erweitert es das Resilienz-Konzept der zivilen SiFo im Hinblick auf normativ zu wertende Bestandteile wie folgt: Strategien zur Erhöhung der Resilienz der für die zivile SiFo relevanten sozio-technischen Systeme müssen so ausgestaltet sein, dass sie sich positiv zumindest aber nicht negativ auf das Vertrauen in die zuverlässige Funktionalität der jeweiligen Systeme auch und gerade im Fall des Eintretens extrem unwahrscheinlicher und unerwarteter, die eigentlichen Belastungsgrenzen der Systeme übersteigender disruptiver Ereignisse, auswirken.

Die vorliegende Arbeit möchte das Resilienz-Konzept der zivilen SiFo dazu nutzen, Hypothesen für Resilience Engineering zu entwickeln, die sich aus den generelleren Überlegungen zu Resilienz ergeben. Daher soll an dieser Stelle noch auf einen letzten Aspekt von Luhmanns Analyse des Vertrauensbegriffs eingegangen werden, der sich ein Stück weit bereits auf technisch-wissenschaftliche Aspekte bezieht. Nach Luhmann ist Vertrauen dazu geeignet die „Toleranz für Mehrdeutigkeit“ zu stärken, was er als Bestandteil einer „bekannte[n] psychologische[n] Theorie“ bezeichnet. Von dieser Toleranz für Mehrdeutigkeit unterscheidet er die sogenannte ,instrumentelle Ereignisbeherrschung“. Eine „Toleranz für Mehrdeutigkeit“ ist in einer komplexen und immer komplexer werdenden Umwelt die Grundvoraussetzung dafür, mit unerwarteten, gravierenden widrigen Ereignissen umgehen zu können, diese sinnvoll - oder „sinnhaft“, um in Luhmanns Duktus zu bleiben - in die eigene Lebenswelt einordnen zu 
können. Wenn Ereignisse dagegen „instrumentell“ beherrscht werden, ist Vertrauen nicht nötig. Denn die Ereignisbeherrschung ist selbst ein Mechanismus zur Komplexitätsreduktion. Nichtsdestotrotz sieht Luhmann in Vertrauen und Ereignisbeherrschung nicht nur funktionale Äquivalente, die beliebig substituierbar sind. Vielmehr müssen beide bei zunehmender Komplexität „komplementär und nebeneinander stärker beansprucht werden“ (Luhmann 2014: 18f). Für die vorliegende Arbeit ist dann vor allen Dingen folgende Aussage von Luhmann bedenkenswert, zumal sie angesichts der ausführlichen system- und komplexitätstheoretischen Analysen als zutreffend anerkannt werden muss: „Demnach ist nicht zu erwarten, dass das Fortschreiten der technik-wissenschaftlichen Zivilisation die Ereignisse unter Kontrolle bringen und Vertrauen als sozialen Mechanismus durch Sachbeherrschung ersetzen und so erübrigen werde. Eher wird man damit rechnen müssen, dass Vertrauen mehr und mehr in Anspruch genommen werden muss, damit technisch erzeugte Komplexität der Zukunft ertragen werden kann" (Luhmann 2014: 19f, eigene Hervorhebung). Damit wird sehr deutlich, dass Resilience Engineering als primär ingenieurwissenschaftlich geprägte Sicherheitsforschung eben niemals rein ingenieurwissenschaftlich funktionieren kann, wenn das in der vorliegenden Arbeit entwickelte Resilienz-Konzept der zivilen SiFo als Grundlage genommen wird. Diese Erkenntnis wird im weiteren Verlauf noch ausführlicher zu analysieren und diskutieren sein.

Für den Zusammenhang zwischen Resilienz und Vertrauen ergeben sich jedenfalls mit Blick auf das eigenständige Resilienz-Konzept der zivilen SiFo folgende Annahmen:

- Vertrautheit als Orientierung am Gewesenen und Bekannten ist kein Mechanismus zur Erhöhung der Resilienz der für die zivile SiFo relevanten, komplexen adaptiven sozio-technischen Systeme.

- Vertrauen ist ohne ein Mindestmaß an Vertrautheit nicht möglich.

- Vertrauen ist ein Mechanismus zur Reduktion von Komplexität, der darin besteht, die Auswahl von Handlungsentscheidungen in der Gegenwart aufgrund generalisierter Erwartungen über die gegenwärtige Zukunft zu treffen. Die generalisierten Erwartungen konstituieren sich mithilfe ähnlicher Erfahrungen aus der Vergangenheit.

- Systemvertrauen besteht im Vertrauen darin, dass das betreffende System seine Funktion, die in einer spezifischen Form der Reduktion von Umweltkomplexität besteht, zuverlässig und ohne größere Störungen erfüllt.

- Je größer die Resilienz eines komplexen adaptiven Systems ist, desto größer ist das ihm entgegengebrachte Systemvertrauen. 
- Je stärker das Systemvertrauen ausgeprägt ist, desto größer ist die Resilienz des Systems.

- Strategien zur Erhöhung der Resilienz der für die zivile SiFo relevanten sozio-technischen Systeme müssen so ausgestaltet sein, dass sie sich positiv zumindest aber nicht negativ auf das Vertrauen in die zuverlässige Funktionalität der jeweiligen Systeme auch und gerade im Fall des Eintretens extrem unwahrscheinlicher und unerwarteter, die eigentlichen Belastungsgrenzen der Systeme übersteigender disruptiver Ereignisse, auswirken.

\subsection{System- und komplexitätstheoretische Analyse von Resilienz}

\subsubsection{Resilienz und Systeme}

Neben „Was ist Resilienz?“ ist „Wer ist resilient?" eine der wichtigsten Fragen für die Analyse. Die vorliegende Arbeit geht mit Wink von einem abstrakten Begriff "System“ als Träger von Resilienz aus (Wink 2016: 2f). Damit wird es notwendig, „System“ zu definieren. Hier bietet sich die Systemtheorie als Analyseinstrument an. Das Resilienz-Konzept wird von verschiedenen Forschern explizit in diesem Bereich verortet. Wink selbst spricht zum Beispiel von Resilienz als Begriff, der in der Systemtheorie dazu genutzt werde, die Fähigkeit „nach äußeren Störungen die Form und Funktion [des Systems] elastisch zu erhalten" zu beschreiben (Wink 2011: 111). Alexander bezeichnet Resilienz als „Systemkonzept“ (Alexander 2013: 2712). Strambach und Klement diskutieren Möglichkeiten und Schwierigkeiten einer systemtheoretischen Einbettung des Konzepts für den Bereich der Wirtschaftsgeographie unter Einbeziehung von Aspekten wie der Komplexitätstheorie oder der Eignung von sogenannten agentenbasierten Simulationsmethoden für die Analyse der interessierenden Systeme (Strambach/Klement 2016: 276f). Andere Autoren, wie beispielsweise Chandler, Duit et al. und Lorenz greifen in ihren Publikationen ebenfalls auf Ideen aus der Systemtheorie zurück und analysieren das Resilienz-Konzept in deren Rahmen (Chandler 2014: 49, Duit et al. 2010: 2, Lorenz 2010). Von derartigen Ausnahmen abgesehen, wird ein Zusammenhang zwischen Systemtheorie und Resilienz in der Literatur in den meisten Fällen zwar gesehen, aber nicht näher untersucht. Selbst die Ökosystemforschung und die sozial-ökologische Forschung, die ihre Ideen im Bereich der Theorie komplexer adaptiver Systeme verorten, setzen ihren Schwerpunkt deutlich auf die beiden qualifizierenden Adjektive 
komplex und adaptiv und weniger auf den Systembegriff. Komplexität und adaptive capacity spielen auch für ein Resilienz-Konzept der zivilen SiFo entscheidende Rollen. Bevor allerdings darauf näher eingegangen werden kann, sollte eine allgemeinere Definition von „System“ erfolgen und die Frage beantwortet werden, wie Resilienz systemtheoretisch verortet werden kann. Die größte Anschlussfähigkeit zwischen Resilienzforschung und Systemtheorie ist dabei durch Hollings Arbeit gegeben. Er operiert explizit mit systemtheoretischem Vokabular.

Dazu wird zunächst auf die Geschichte der Systemtheorie verwiesen, bevor mit Niklas Luhmanns Theorie sozialer Systeme eines der einflussreichsten soziologischen Theoriegebäude des 20. Jahrhunderts kurz vorgestellt wird. Luhmanns Systemtheorie steht nicht im Zentrum der vorliegenden Arbeit, sondern soll lediglich dazu dienen, eine systemtheoretische Einordnung von Resilienz zu ermöglichen. Die Erklärung seiner Theorie wird daher eher knapp gehalten und fokussiert auf Aspekte, die für den Resilienz-Diskurs von Bedeutung sind. Dazu zählt zum Beispiel Luhmanns ursprüngliches Verständnis der Aufgabe sozialer Systeme, die er in der Reduktion von Komplexität sieht. In Folge der sogenannten „autopoietischen Wende" in seinem Werk - diese wird weiter unten dargestellt - verliert diese Aussage zwar einiges von ihrer Zentralität, wird aber nicht ungültig (Kneer/Nassehi 2000 40, 46). Noch stärkeren Bezug zu Resilienz hat Luhmanns Beschreibung der Fähigkeit sozialer Systeme, durch Änderung ihrer Struktur und Bedürfnisse auf veränderte Bedingungen zu reagieren und so den eigenen Fortbestand zu sichern. Auch dieser Gedanke wurde vor der autopoietischen Wende formuliert. Er umfasst die Schwierigkeit, Prozesse der Anpassung eines bestehenden Systems an veränderte Bedingungen von solchen der Transformation in ein neues System eindeutig zu unterscheiden (Kneer/Nassehi 2000: 38). Luhmann veröffentlichte diese Überlegungen bereits Anfang der 1970er Jahre und damit zeitgleich bzw. sogar vor und mutmaßlich in Unkenntnis von Hollings Resilienz-Theorie. Die Ähnlichkeiten sind jedoch augenfällig im Hinblick auf die postulierte Fähigkeit der untersuchten Systeme (soziale Systeme bei Luhmann und ökologische Systeme bei Holling) zur Anpassung an veränderte Bedingungen in der Systemumwelt. Bereits dieser kurze Verweis auf zwei Aspekte aus Luhmanns Theorien zeigt signifikante Überschneidungen mit Überlegungen, die auch in der und für die Resilienzforschung relevant sind. Im vorangegangenen Unterkapitel wurde zudem der konzeptionelle $\mathrm{Zu}-$ sammenhang zwischen Resilienz und Vertrauen im Sinne von Luhmann analysiert und aufgezeigt, dass der Vertrauensbegriff für Resilienz und vor allem für eine resiliente Gesellschaft durchaus zentral ist. 
Allgemein dient der Systembegriff ganz ähnlich wie der Resilienz-Begriff als Label, unter dem eine Vielzahl verschiedener Disziplinen ihre jeweils eigenen, auf den interessierenden Untersuchungsgegenstand spezifizierten Theorien entwickelt haben. „Systemtheoretische Denkweisen finden sich in den unterschiedlichsten wissenschaftlichen Disziplinen wie Ökonomie, Soziologie, Pädagogik, Politikwissenschaft, Psychologie, Medizin, Neurologie, Psychiatrie bis hin zu Meteorologie und Astronomie“ (Kneer/Nassehi 2000: 20). Deren einzige übergreifende Gemeinsamkeit besteht in der Verwendung des Systembegriffs. Laut Kneer und Nassehi, auf deren Überblicksarbeit zu Niklas Luhmanns Systemtheorie sich die folgenden Ausführungen im Wesentlichen berufen, ist mit einem System ganz ursprünglich immer „etwas Zusammengesetztes“ gemeint, das aus einzelnen Teilen oder konkreter Systembestandteilen besteht (Kneer/Nassehi 2000: 17). Entstanden ist die Systemtheorie nach Kneer und Nassehi als Reaktion auf ein klassisches, Newtonsches Wissenschaftsverständnis, wonach die Welt mit Hilfe deduktiver Beschreibungen mathematisch fassbar sei. Das Newtonsche Wissenschaftsverständnis besteht dann darin, Gesetzmäßigkeiten in Natur - und im Prinzip auch der Gesellschaft - durch die Beobachtung einzelner Phänomene isoliert vom Gesamtsystem identifizieren zu können. Anschließend können die Phänomene im Rahmen standardisierter, wiederholbarer Laborexperimente verifiziert und wiederum zur Erklärung für Vorgänge in der realen Welt herangezogen werden. Dieses wissenschaftliche Prinzip hat sich vor allen Dingen in Physik und Technikwissenschaften bewährt. Zur Erklärung von Phänomenen, die zum Beispiel in biologischen Systemen auftreten, scheint es dagegen weniger geeignet, weshalb der als „Nestor“ der allgemeinen Systemtheorie geltende Zoophysiologe Ludwig von Bertalanffy einen neuen Erklärungsansatz suchte (Kneer/Nassehi 2000: 18f). In Abgrenzung zur der Fokussierung des Newtonschen Verständnisses auf die deduktive Herleitung des Systemverhaltens aus dem Verhalten einzelner Bestandteile, wurde der Systembegriff „im Sinne einer Einheit, die mehr sei als die bloße Summe ihrer Teile“ verstanden. Unter System wird dann nicht nur eine bestimmte Menge an Elementen verstanden, sondern sind immer zugleich auch deren kausale Beziehungen untereinander gemeint (Kneer/Nassehi 2000: 16f). Um Systeme abgrenzen zu können, ist in der Systemtheorie der Begriff der Umwelt geläufig. Demzufolge sind Elemente entweder Bestandteil eines Systems oder von dessen Umwelt. Die Grenze zwischen einem System und seiner jeweiligen Umwelt ist eindeutig. Mit Luhmann gesprochen ist es gerade diese „Unterscheidung von Innen und Außen“, die Systeme charakterisiert (Kneer/Nassehi 2000: 20, Luhmann 2014: 120). Auch Holling grenzt die 
ihn interessierenden Systeme eindeutig von ihrer Umwelt ab. Als Beispiel kann hier seine Beschreibung der budworm-Population dienen, die erst im Zusammenspiel mit den ihr als Nahrung dienenden Baumarten sowie ihren Fressfeinden zum für Holling relevanten Ökosystem wird. Die zufällig auftretenden klimatischen Veränderungen sieht Holling dagegen als systemextern, sie sind Teil der Umwelt (Holling 1973: 13f). Von Bertalanffy formalisiert diese grundlegenden Ideen in folgender Definition von System: Ein System ist "eine Anzahl von in Wechselwirkung stehenden Elementen $\mathrm{p}_{1}, \mathrm{p}_{2} \ldots \mathrm{p}_{\mathrm{n}}$ charakterisiert durch quantitative Maße $\mathrm{Q}_{1}, \mathrm{Q}_{2}$ $\ldots \mathrm{Q}_{\mathrm{n}}$. Ein solches kann durch ein beliebiges System von Gleichungen bestimmt sein“" (v. Bertalanffy 1951: 127, nach Kneer/Nassehi 2000: 20 und Sundström/Hollnagel 2006: 236).

In der Systemtheorie wird des Weiteren generell zwischen offenen und geschlossenen Systemen unterschieden. Geschlossene Systeme interagieren nicht mit ihrer Umwelt. Der fehlende Austausch mit der Umwelt führt dazu, das geschlossene Systeme sich langfristig in einem unveränderlichen Gleichgewichtszustand befinden. Ohne eine Verbindung zur Umwelt, besteht weder die Möglichkeit noch die Notwendigkeit für das System, sich zu verändern. Offene Systeme zeichnen sich im Gegensatz dazu gerade durch ihre Interaktion mit der Umwelt aus. Sobald sie aus ihrer Umwelt einen Reiz erfahren, verarbeiten sie diesen Input zu einem bestimmten Output. Wie dieser Output aussehen kann, wird durch die internen Mechanismen des Systems bestimmt, die in aller Regel nicht durch einfache, lineare Kausalitäten gegeben, sondern im Sinne einer „black box“ unklar sind. Ändern sich die Umweltbedingungen sind offene Systeme in der Lage, durch Veränderung der systeminternen kausalen Abhängigkeiten der Elemente zueinander, ihre Zustände anzupassen und gleichzeitig ihre Struktur beizubehalten - ein Gedanke, der auch bei Luhmann wieder wichtig wird. Selbiges gilt für das Prinzip der Selbstorganisation von Systemen, welches für offene Systeme gilt und ihnen zubilligt, sich selbst durch dynamische Steuerung ihrer inneren Zustände organisieren zu können (Kneer/Nassehi 2000: 22ff). Hier wird erneut deutlich, wie sehr Hollings Resilienz-Konzept in der Systemtheorie zu verorten ist. Resilienz wird demnach erst möglich, aber auch erst relevant, wenn Systeme als offen gegenüber ihrer Umwelt konzipiert werden. Hollings Verständnis von geschlossenen Systemen ist zwar weniger strikt als das der klassischen Systemtheorie, aber trotzdem kann nur durch ein Verständnis von Systemen als offen gegenüber Input aus der Umwelt und fähig zu Output in diese Umwelt eine Analyse des Verhaltens realer Ökosysteme sinnvoll sein (Holling 1973: 1f). 
Die auch für Holling zentrale Unterscheidung zwischen offenen und geschlossenen Systemen und die damit einhergehende Fokussierung des Erkenntnisinteresses auf holistische anstelle von reduktionistischen Erklärungsmustern, stellt von Bertalanffys zentralen Beitrag zur Systemtheorie dar - natürlich neben der Einführung des Begriffs an sich. Von Bertalanffy grenzte sich so von älteren, deterministischen Systemverständnissen ab. Das Zusammendenken der Begriffe System und Komplexität bestimmt insofern seine Konzeptionierung der Systemtheorie (Alexander 2013: 2711). Systeme sind nicht nur Elemente, die in einer bestimmten Beziehung zueinanderstehen. Diese Beziehung aller Elemente untereinander wird darüber hinaus dergestalt ausdifferenziert, dass sie nur in ihrer Gesamtheit, also unter Einbeziehung aller Elemente und aller Relationen, erkennbar wird (Kneer/Nassehi 2000: 20). „Organisierte Komplexität ist dann gegeben, wenn Einzelphänomene nicht schlicht linear logisch miteinander gekoppelt sind, sondern wenn Wechselwirkungen zwischen ihnen bestehen" (Kneer/Nassehi 2000: 21). Die Komplexität von Systemen ist dann nach von Bertalanffy der zentrale Untersuchungsgegenstand der Systemtheorie. Ein derart konzipiertes Systemverständnis läuft auf eine weitgehende Gleichsetzung der Begriffe System und Komplexität hinaus. Es stellt sich unmittelbar die Frage, ob damit Systeme stets und immer auch komplex sind, also ob jedes System inhärent Komplexität aufweist. Dies wird im Folgenden Unterkapitel diskutiert. Im Vorgriff darauf kann jedoch bereits festgehalten werden, dass der dieser Arbeit zugrundeliegende Systembegriff explizit nicht davon ausgeht, dass jedes System notwendig komplex ist. Ein System stellt zunächst lediglich eine Menge an Elementen dar, die miteinander in kausaler Beziehung stehen, wobei diese Kausalität in nicht-komplexen Systemen durchaus mithilfe einfacher, reduktionistischer Erklärungsmuster aufgedeckt und beschrieben werden kann. Inwiefern die für die zivile SiFo relevanten Systeme komplex sind oder nicht, ist damit noch nicht gesagt. Ein derartig konzeptionierter Systembegriff erlaubt lediglich eine Differenzierung zwischen komplexen und nicht-komplexen Systemen. Diese Differenzierungsmöglichkeit ist gerade vor dem Hintergrund der Frage nach ingenieurwissenschaftlicher SiFo im Sinne eines Resilience Engineering wichtig, da sie mindestens indirekt in die Diskussion zwischen einem mechanischen und einem systemischen Verständnis von Resilienz eingreift.

Auch bei Luhmann spielt die Komplexität von Systemen zumindest in der älteren Version seiner Systemtheorie eine entscheidende Rolle. Er versteht Komplexität aber anders, als gerade dargestellt. Ideengeschichtlich basiert Luhmanns Theoriegebäude auf den Arbeiten von Talcott Parsons, 
der mit seiner strukturfunktionalistischen Systemtheorie zu den einflussreichsten Soziologen des 20. Jahrhunderts gezählt werden kann. Sein berühmt gewordenes „AGIL“ Schema, das für Anpassung (adaptation), Zielerreichung (goal attainment), Integration (integration) und Strukturerhaltung (latent structure maintenance) steht, beschreibt die Funktionen, die ein System zur Selbsterhaltung erfüllen können muss. Entscheidend für die Identität eines Systems ist dessen Struktur, oder die Systemelemente, die nicht von kurzfristigen Veränderungen in der Beziehung zwischen System und Umwelt betroffen sind. Davon zu unterscheiden sind die Funktionen des Systems, die Parsons mithilfe des AGIL-Schemas beschreibt, und die den Strukturerhalt im Angesicht sich verändernder Umweltbedingungen ermöglichen sollen (Kneer/Nassehi 2000: 29f). Auf den ersten Blick scheinen zwischen Parsons Systemverständnis und Hollings Resilienz-Konzept einige Parallelen zu bestehen. Allerdings steht bei Holling nicht der Erhalt einer bestimmten Systemstruktur im Vordergrund, sondern lediglich der Erhalt des Systems an sich bzw. der dessen Identität ausmachenden Bestandteile. Diese werden sehr viel stärker in Funktionen oder Prozessen gesehen, als in spezifischen Strukturen (Adger 2000: 349, Carpenter et al. 2001: 766, Gunderson 2000: 426, Holling 1973: 17). Und damit rückt direkt Niklas Luhmanns soziologische Systemtheorie ins Blickfeld. Luhmann, der nach einem rechtswissenschaftlichen Studium in Freiburg und längerer Tätigkeit als Verwaltungsbeamter von 1968 bis zu seiner Emeritierung im Jahr 1993 Professor der Soziologie an der neu gegründeten Universität Bielefeld war, hatte Talcott Parsons während eines Forschungsaufenthalts an der Harvard Universität zu Beginn der 1960er Jahre kennengelernt. ${ }^{29}$ Im Anschluss an Parsons entwirft Luhmann eine eigene, funktional-strukturelle Systemtheorie (Kneer/Nassehi 2000: 34).

Sein Ziel besteht darin, an die aus der allgemeinen Systemtheorie stammenden Überlegungen anknüpfend eine „Theorie von universalistischer Reichweite" für soziale Systeme zu entwickeln - was für ihn auch der Grund war, den Ruf auf einen Lehrstuhl für Soziologie anzunehmen, da diese sich im Unterschied zu anderen sozialwissenschaftlichen Disziplinen wie den Rechtswissenschaften oder der Politikwissenschaft nicht mit spezifischen Themenbereichen beschäftige, sondern allgemein mit sozialen Zusammenhängen (Kneer/Nassehi 2007: 7f, 33). Seine Theorie ist allein deskriptiv-analytischer Natur. Luhmann ist in Abgrenzung zu Vertretern der Kritischen Theorie nicht daran interessiert, Theorien einer Gesellschaft so zu formulieren, wie sie normativ sein sollte und mithilfe

29 http://agso.uni-graz.at/lexikon/klassiker/luhmann/26bio.htm [Stand: 27.6.2019]. 
seiner wissenschaftlichen Arbeit dazu beizutragen, die Entwicklung hin zu einer normativ wünschenswerteren Gesellschaftsform zu unterstützen (Kneer/Nassehi 2000: 11). Sein Theorieansatz wurde daher immer wieder scharf von Soziologen der Frankfurter Schule wie etwa Jürgen Habermas kritisiert. Diesem zufolge gehe es bei Luhmanns Theorie „primär um eine Verwaltung der Menschen, aber nicht um einen Abbau von Herrschaft und Ungerechtigkeit“" (Kneer/Nassehi 2000: 45). Die Kritik ist laut Luhmann aber insofern nicht relevant für seine Theorie, da Habermas „eine wissenschaftliche Theorie mit politischen Begriffen" kritisiere (Kneer/Nassehi 2000: 45, Hervorhebung im Original). In der Resilienzforschung gibt es, wie bereits im vorangegangenen Kapitel skizziert, eine sehr ähnlich gelagerte Diskussion. Verschiedenen Resilienz-Ansätzen wird von Seiten der kritischen Soziologie immer wieder vorgeworfen, dass sie nicht nur nicht zu einem Abbau von Ungerechtigkeiten beitragen, sondern diese vielmehr sogar durch Identifizierung und Stärkung systemerhaltender und -stabilisierender Maßnahmen weiter verstetigen helfen. Sobald Resilienz unhinterfragt als normativ positiv besetzter Begriff gesetzt und somit eher als Zielvorstellung denn als wissenschaftliches Konzept genutzt wird, trifft diese Kritik zu und muss etwa zur Entwicklung von Hypothesen für Resilience Engineering, bei der dezidiert nach Ansätzen zur Erhöhung von Resilienz mit ingenieurwissenschaftlichen Mitteln gesucht wird, entsprechend berücksichtigt werden. Solange Resilienz allerdings deskriptiv-analytisch untersucht wird geht die Kritik, ebenso wie die politisch motivierte Kritik der Kritischen Theorie an Luhmanns wissenschaftlicher Theorie, fehl.

Luhmann entwickelt seine Theorie seit ca. Mitte der 1960er Jahre und in expliziter Abgrenzung von Parsons strukturfunktionalistischem Ansatz. Während dieser nur erklären kann, welche Funktionen verantwortlich dafür sind, dass in sozialen Systemen bestimmte Strukturen - und damit das System an sich - aufrechterhalten werden können, soll Luhmanns Theorie generischer einsetzbar sein und so von ihm identifizierte Schwachstellen der Systemtheorie Parsons überwinden (Kneer/Nassehi 2000: 34ff). Luhmann möchte nicht nur die Aufrechterhaltung von Strukturen erklären können, sondern sowohl „Integration als auch Konflikt, sowohl Ordnung als auch Wandel, sowohl Struktur als auch Prozess" und entwickelt daher eine „funktional-strukturelle Systemtheorie“ (Kneer/Nassehi 2000: 37). Die Entwicklung seines Theoriegebäudes vollzieht sich in zwei Schritten. Vor der sogenannten „autopoietischen Wende“, die zeitlich ungefähr in der Mitte der 1980er Jahre zu verorten ist, kann der Begriff der Komplexität als zentral für sein Werk gelten. Die Aufgabe von sozialen Systemen besteht in der Reduktion von Komplexität. Im Anschluss an die Wende steht dage- 
gen die Selbstorganisation von Systemen im Fokus und daraus resultierend Kommunikationen als Elemente sozialer Systeme (Kneer/Nassehi 2000: 34f, 40, 65). Für die vorliegende Arbeit und die Einordnung des ResilienzKonzepts in systemtheoretische Ansätze bieten beide Entwicklungsphasen von Luhmanns Theorie interessante Anknüpfungspunkte.

In der ersten Entwicklungsphase versteht Luhmann unter einem sozialen System „den Zusammenhang von aufeinander verweisenden sozialen Handlungen." Von der klassischen Systemtheorie übernimmt er die Unterscheidung zwischen System und Umwelt. Soziale Systeme bezeichnet er auch als „Handlungssysteme“, weil sie diejenigen Systeme sind, die die Handlungen von Menschen miteinander verknüpfen. Alles, was außerhalb dieser miteinander verknüpften Handlungen steht, ist für das betreffende soziale System Umwelt (Kneer/Nassehi 2000: 38). Im Vergleich zu Parsons strukturfunktionalistischer Systemtheorie nimmt Luhmann nun zwei Anpassungen vor. Zunächst müssen derart konstituierte soziale Systeme nicht notwendigerweise über verbindende Normen und Werte (ausgedrückt in Form von Strukturen) verfügen. Das sieht er angesichts gesellschaftlicher Realitäten als theoretisch sehr viel zutreffenderes Verständnis sozialer Systeme. Menschen können auch dann interagierend handeln, wenn sie keine gemeinsamen Werte teilen oder sich nicht an die gleichen Normen gebunden fühlen. Die zweite Anpassung erscheint im Kontext der Resilienzforschung besonders relevant. Denn Luhmann grenzt sich von Parsons Annahme ab, dass es "spezifische, nicht substituierbare Leistungen“ gibt, die soziale Systeme zum Überleben benötigen. Vielmehr sind Systeme ihm zufolge in der Lage, ausgefallene Leistungen durch Alternativen zu ersetzen. Parsons hatte hier noch einen „Kausalfunktionalismus“ angenommen, wonach sich bestimmte Systembeiträge gerade nicht ersetzen lassen. Für Luhmanns funktional-strukturelle Systemtheorie gilt hingegen, dass soziale Systeme ihre Struktur unter dem Einfluss veränderter Umweltbedingungen zu ändern in der Lage sind, um ihr eigenes Überleben sicherstellen zu können. Diese Anpassung an sich verändernde Bedingungen is aufgrund eines Mechanismus möglich, den Luhmann als „Äquivalenzfunktionalismus" bezeichnet. Statt danach zu fragen, welche Funktionen für einen Strukturerhalt des Systems notwendig sind, geht es darum, welche Funktionen von bestimmten Systemleistungen erfüllt werden und wie diese - wenn aufgrund systemexterner Einflüsse die Notwendigkeit entsteht - durch funktional äquivalente Systemleistungen ersetzt werden können. Luhmann formuliert also einen „Möglichkeitsspielraum alternativer Lösungen des Ausgangsproblems, die miteinander verglichen werden können“ (Kneer/Nassehi 2000: 38f). Hier wird eine enge Verknüpfung zwi- 
schen Luhmanns ursprünglichen, theoretischen Ideen und einem in der Tradition von Holling stehenden Resilienz-Verständnis für soziale Systeme sichtbar. Bewusst zugespitzt ließe sich mit Blick auf Hollings Definition von Resilienz und Luhmanns Verständnis von sozialen Systemen sagen, dass Resilienz die konstitutive Eigenschaft aller sozialen Systeme darstellt.

Allerdings fehlte in einem derartigen Verständnis eine Möglichkeit der Differenzierung zwischen resilienten und nicht resilienten sozialen Systemen. Dazu bedarf es einer näheren Betrachtung der Aufgabe, die Luhmann sozialen Systemen im Rahmen seiner ursprünglichen soziologischen Systemtheorie zuschreibt. An dieser Stelle kommt der Komplexitäts-Begriff ins Spiel. Luhmann unterscheidet wie bereits geschildert zwischen System und Umwelt. Die Summe aller Systeme und ihrer jeweiligen Umwelten bildet für ihn die „Welt.“ Diese kann selbst nicht als System verstanden werden, weil sie keine Umwelt besitzt. Sie ist aber gleichfalls nicht einfach Umwelt, weil in ihr auch alle Systeme enthalten sind. Die Welt hat keine eindeutigen Grenzen und ist daher nach Luhmann "nicht bedrohbar“ (Kneer/Nassehi 2000: 39f, Luhmann 2014: 4). Im Umkehrschluss sind dagegen Systeme immer durch ihre Umwelt in ihrer Existenz bedroht. Im „globalen“ Maßstab stellt sich nun für Luhmann die Komplexität der Welt als das zentrale Problem, die zentrale Bedrohung für Systeme dar. Er spricht in diesem Zusammenhang sogar von „unfassbarer Komplexität“ als „Innenansicht der Welt" und „Problemaspekt" von Systemen „die sich in der Welt erhalten wollen“ (Luhmann 2014: 4). Was meint er mit Komplexität? Luhmanns Definition von Komplexität ist tatsächlich relativ einfach. Komplexität drückt sich demnach in der Anzahl an Möglichkeiten oder Zuständen aus, die etwas aufweisen kann. Es gilt eine Mindestzahl von zwei Zuständen, ab der etwas „komplex“ genannt werden kann. Bei zwei möglichen Zuständen, bzw. generell bei einer derart geringen Zahl, ist die Komplexität entsprechend gering und stellt für Systeme nicht notwendigerweise ein Problem dar. Reale Systeme sind laut Luhmann aber in der Welt verortet, die sich gerade durch „unfassbare“ Komplexität auszeichnet, weil sie prinzipiell eine beinahe unendliche Anzahl von Möglichkeiten aufweist und damit in jedem Fall mehr ,als die, auf die das System sich erhaltend reagieren kann." Menschen sind als Individuen in der überkomplexen Welt nicht in der Lage, alle Möglichkeiten oder Zustände zu erfassen. Sie leiden an Überforderung, da ihr Bewusstsein die Komplexität der Welt nicht verarbeiten kann. Hier kommen nun für Luhmann soziale Systeme ins Spiel. Sie werden sozusagen zwischengeschaltet und reduzieren die umfassende Komplexität der Umwelt bzw. Welt so weit, dass einzelne 
Menschen damit umgehen können (Kneer/Nassehi 2000: 40f, Luhmann 2014: 5).

Soziale Systeme treten in drei verschiedenen Arten auf. Zunächst als Interaktionssysteme, die durch die Handlungen anwesender Personen ad hoc zustande kommen und sich nach Ende des Zusammentreffens dieser Personen auch wieder auflösen. Somit ist beispielsweise ein gemeinsamer Spieleabend mit Freunden als soziales Interaktionssystem zu charakterisieren. Die zweite Art von sozialen Systemen sind Organisationssysteme, die aus Mitgliedern bestehen und bei denen eine Mitgliedschaft bestimmten formalen Bedingungen unterliegt. Ihre Existenz ist im Gegensatz zu der von Interaktionssystemen als dauerhaft zu kennzeichnen, sie hängt nicht von der Anwesenheit der Mitglieder des Systems ab. Organisationssysteme dienen dazu, Verhaltensweisen, die nicht spontan zu erwarten sind, mithilfe formaler Regeln berechenbar und für die Systemmitglieder sowie die Umwelt des Systems verlässlich zu machen. Sportvereine können damit ebenso gut als Organisationssystem verstanden werden wie zum Beispiel Unternehmen. Und die dritte Art von sozialen Systemen sind Gesellschaftssysteme, die aus der Gesamtheit aller Interaktions- und Organisationssysteme bestehen, ohne allerdings auf diese reduziert werden zu können (Kneer/Nassehi 2000: 42f). In der zivilen Sicherheitsforschung geht es letztlich darum, wie im einleitenden Kapitel bereits skizziert wurde, die Gesellschaft vor den negativen Auswirkungen widriger Ereignisse bestmöglich zu schützen. Häufig sind primär Teilbereiche der Gesamtgesellschaft von solchen Ereignissen betroffen. Diese lassen sich zum Teil als Interaktions- und zum Teil als Organisationssysteme verstehen. Neben sozialen Aspekten umfassen sie vor allen Dingen auch technische Aspekte, so dass sich die Bezeichnung der sozio-technischen Systeme etwa für kritische Infrastrukturen etabliert hat (zur näheren Beschreibung der für die zivile SiFo relevanten Systeme siehe 4.3.2). Das Einbeziehen von technischen Aspekten ist mit der „reinen Lehre“ Luhmanns zunächst nicht abdeckbar, es ist in seiner älteren Theorie sozialer Systeme nicht enthalten - genauso wenig wie in den neueren Entwicklungen. Wobei zu beachten ist, dass technische Aspekte in Luhmanns Verständnis von sozialen Systemen als Handlungssystemen schlicht nicht notwendig eigenständig zu behandeln sind. Für die vorliegende Arbeit sind sie dagegen von besonderem Interesse, da Resilience Engineering gerade darauf zielt, durch Beachtung der technischen Aspekte sozio-technischer Systeme deren Resilienz zu erhöhen. Luhmanns weitere Überlegungen zum Zusammenhang zwischen sozialen Systemen und Komplexität werden insofern ein Stück weit erweitert verstanden und technische Aspekte zumindest implizit immer mitgedacht. 
Es gilt, dass ein System - auch die Gesellschaft - niemals so komplex sein kann wie seine Umwelt, da es ansonsten seiner Aufgabe der Reduktion von Komplexität nicht nachkommen könnte (Kneer/Nassehi 2000: 41). Gleichzeitig bedarf das soziale System einer gewissen Eigenkomplexität, um mit Veränderungen in der Umwelt umgehen zu können. Je größer die Eigenkomplexität eines sozialen Systems, desto besser kann es die Komplexität der Umwelt für die in ihm handelnden Menschen erfassen und reduzieren. Der zugrundeliegende Mechanismus ist unmittelbar einleuchtend: je mehr Möglichkeiten innerhalb eines sozialen Systems, desto anpassungsfähiger ist es gegenüber Einwirkungen aus seiner Umwelt, weil es flexibel darauf reagieren kann und nicht an eine bestimmte Reaktionsweise gebunden ist (Kneer/Nassehi 2000: 41f). Komplexität ist also zugleich Herausforderung, wenn sie in der Umwelt des Systems bzw. der Welt auftritt, und Ressource, wenn sie innerhalb des Systems genutzt werden kann, um auf sich verändernde Umweltbedingungen zu reagieren. Mit Blick auf die weiter oben beschriebene zugespitzte Formulierung, wonach Resilienz die konstitutive Eigenschaft aller sozialen Systeme darstelle, lässt sich nun nach Einführung des für Luhmanns ältere systemtheoretische Ansätze zentralen Grundbegriffs der Komplexität präziser sagen: soziale Systeme sind genau dann auch resiliente Systeme, wenn sie hohe Eigenkomplexität aufweisen. Bezieht man die obige Diskussion zu den für die zivile SiFo relevanten Systemen mit ein, ist hohe Eigenkomplexität demzufolge konstitutiv für die Resilienz sozio-technischer Systeme. Inwiefern der Grad an Eigenkomplexität auch zu groß werden kann, wird im nächsten Unterkapitel näher analysiert. Insgesamt wird deutlich, dass eine Einordnung des Resilienz-Konzepts in systemtheoretische Ansätze voraussetzungsreich ist. Denn es bedarf dafür eines Resilienz-Verständnisses, das Anpassungsfähigkeit als mindestens eine zentrale Komponente, wenn nicht sogar als das Charakteristikum für Resilienz überhaupt enthält. Die Einordnung eines Resilienz-Konzepts für die zivile SiFo in systemtheoretische Ansätze ist aber auch sinnvoll und notwendig. Denn Resilienz kann in diesem Rahmen nur als „Systemkonzept“" verstanden werden. Der Rückgriff auf Luhmanns ältere systemtheoretische Ansätze erlaubt es, ein Hollingsches Resilienz-Verständnis umzuformulieren und auf sozio-technische Systeme anzuwenden. Für die zivile SiFo bleibt festzuhalten: Resilienz erfordert hohe Eigenkomplexität, um Anpassungsfähigkeit bei sich verändernden Umweltbedingungen zu ermöglichen.

Ausgehend von dieser Erkenntnis stellt sich die Frage, welche Bedeutung die neueren Entwicklungen in Luhmanns systemtheoretischem Verständnis seit Mitte der 1980er Jahre für ein Resilienz-Konzept der zivilen 
SiFo haben. Im Rahmen der „autopoietischen Wende“ in seinem Werk verliert die Idee der Reduktion von Komplexität als Aufgabe sozialer Systeme ihre zentrale Stellung. Luhmann spricht von drei Entwicklungen in der Allgemeinen Systemtheorie, von Systemen als Einheit von Elementen, über offene, mit ihrer Umwelt interagierende Systeme hin zu „autopoietischen“ Systemen (Kneer/Nassehi 2000: 46f). Der Begriff „Autopoiesis“ wurde bereits einige Male ohne nähere Erklärung genutzt. Für Luhmanns Theorie sozialer Systeme wird er spätestens mit seinem 1984 erschienen Werk Soziale Systeme entscheidend wichtig. Dabei stammt er nicht von ihm selbst, sondern wurde von ihm in einer Art interdisziplinärem Manöver aus der biologischen Forschung entlehnt. Autopoiesis ist ein Kunstwort, das ein von den chilenischen Biologen Humberto R. Maturana und Francisco Varela entwickeltes Konzept bezeichnet. Es setzt sich aus den griechischen Worten „autos“ und „poiein“ zusammen und bedeutet so viel wie „selbst machen“ oder weniger wörtlich übersetzt Selbsterzeugung (Kneer/Nassehi 2000: 24, 47f). Ein autopoietisches System ist für Maturana und Varela ein System, das sich selbst herstellt und erhält. Es produziert die Elemente, aus denen es besteht - und damit sich selbst - fortlaufend aus sich selbst heraus. Für seine Erfinder Maturana und Varela sind Lebewesen autopoietische Systeme. Nicht-lebende Systeme, beispielsweise technische Maschinen aber auch soziale Systeme, sind ihnen zufolge nicht autopoietisch, sondern allopoietisch organisiert. Sie benötigen Eingriffe aus der Systemumwelt, um sich selbst erhalten zu können (Kneer/Nassehi 2000: 48f). Maturana und Varela unterscheiden zwischen Autopoiesis und Selbstreferentialität. Sie definieren beispielsweise das Nervensystem als ein selbstreferentielles System. Das Nervensystem konstruiert demnach lediglich ein Bild der umgebenden Welt, indem es Sinneswahrnehmungen in neuronale Aktivitäten transformiert. Eine eindeutige Korrelation zwischen der äußeren Welt und den inneren, den neuronalen Aktivitäten, lässt sich nicht feststellen. Die menschliche Wahrnehmung ist keine „adäquate Widerspiegelung“ der eigentlichen Welt, sondern eine „systeminterne Konstruktion einer systemexternen Welt" (Kneer/Nassehi 2000: 52ff). Laut Maturana und Varela wird das Nervensystem dadurch aber nicht zum autopoietischen System. Es stellt ein geschlossenes System dar, das externe Einflüsse nur mithilfe seiner Selbstbezüglichkeit verarbeiten kann. Autopoietisch sind Systeme nur dann, wenn sie sich selbst erzeugen und erhalten. Das Nervensystem stellt nur einen Teil eines autopoietischen Systems, etwa eines Menschen, dar (Kneer/Nassehi 2000: 54f).

Luhmann nutzt für seine neueren systemtheoretischen Ansätze den Grundgedanken der Autopoiesis von Systemen, überträgt ihn aber explizit 
von Lebewesen auch auf andere Systeme. Er „generalisiert“ das Konzept. Da sein Interesse nach wie vor sozialen Systemen gilt, konzipiert er diese als „selbstreferentiell-geschlossene, autopoietische Systeme“ (Kneer/Nassehi 2000: 57). Im Gegensatz zu Maturana und Varela versteht Luhmann beispielsweise auch psychische oder neuronale Systeme als autopoietisch. Damit wäre auch das Nervensystem autopoietisch organisiert und die Unterscheidung zwischen Selbstreferentialität und Autopoiesis ist nicht länger relevant. Für Luhmann sind autopoietische Systeme generell selbstreferentiell organisiert. Obwohl er den Anwendungsbereich der Idee der Autopoiesis stark erweitert, bleibt die inhaltliche Grundidee dieselbe. Wie Maturana und Varela meint Luhmann mit Autopoiesis die Fähigkeit von Systemen, „die Elemente, aus denen sie bestehen, durch die Elemente, aus denen sie bestehen, selbst [zu] produzieren und reproduzieren" (Kneer/ Nassehi 2000: 59). Autopoietische Systeme existieren aber nicht völlig losgelöst von ihrer Umwelt. Kneer und Nassehi nutzen Luhmanns Beispiel des Bewusstseins als autopoietischem System, um diesen Sachverhalt zu erklären. Die Elemente des Systems Bewusstsein sind Gedanken. Das Bewusstsein kann nur denken, wenn entsprechende neuronale Prozesse im Gehirn ablaufen. Allerdings lassen sich spezifische Gedanken nicht anhand der konkreten neuronalen Prozesse ableiten.

Luhmann führt hier den Begriff der „Emergenz“ ein, der im Kontext der Diskussion zu Resilienz und Komplexität im nächsten Unterkapitel wieder auftauchen wird. Er meint damit das „Auftreten eines neuen Ordnungsniveaus, das aus den Eigenschaften des materiellen und energetischen Unterbaus nicht erklärt werden kann." Gedanken benötigen zwar, sie entstehen aber nicht durch neuronale Aktivitäten im Gehirn, sondern als „nicht weiter auflösbare Letzteinheiten des Bewusstseinssystems" (Kneer/Nassehi 61f). Ob die Unterscheidung zwischen Bewusstsein und Gedanken auf der einen Seite und Gehirn und neuronalen Aktivitäten als jeweils autopoietische Systeme angesichts neuer Entwicklungen in der Hirnforschung noch als gültig anzusehen ist, ist eine berechtige Frage, die aber nicht Gegenstand dieser Beschreibung von Luhmanns Ideen ist. Autopoietische Systempaare wie zum Beispiel Gehirn und Bewusstsein bezeichnet Luhmann jedenfalls als strukturell gekoppelte Systeme. Diese stehen in einem spezifischen Abhängigkeitsverhältnis voneinander. Mindestens eines der Systeme ist zur Aufrechterhaltung seiner Funktionsfähigkeit, seiner Autopoiesis, auf das jeweils andere System angewiesen. Nichtsdestotrotz sind auch strukturell gekoppelte Systeme weiterhin füreinander Umwelt. Im Beispiel kann das Bewusstsein ohne Gehirnaktivitäten nicht existieren, es geht aber nicht in den Gehirnaktivitäten auf. Strukturell gekoppelte 
Systeme operieren also bezogen aufeinander autonom, jedoch nicht autark (Kneer/Nassehi 2000: 62f).

Ausgestattet mit dem Konzept autopoietischer Systeme entwirft Luhmann dann eine aktualisierte Version seiner Theorie sozialer Systeme. Standen bis dahin Handlungen zur Reduktion der Komplexität der realen Welt im Fokus, fragt Luhmann nun mit Blick auf den angenommenen autopoietischen Charakter sozialer Systeme nach deren „nicht weiter auflösbaren Letzteinheiten“, den Elementen, aus denen sie bestehen. Seine Antwort kann als radikaler Bruch mit traditionellen Auffassungen in Philosophie und Soziologie verstanden werden. Für Luhmann bestehen soziale Elemente aus „Kommunikationen.“ Das widerspricht klassischen subjektphilosophischen Ansätzen, in denen immer der Mensch dergestalt im Zentrum der Überlegungen steht, dass er die „kleinste Einheit des Sozialen" bildet: Menschen kommunizieren miteinander und konstituieren auf diese Weise soziale Systeme. Aufgrund der Einführung des Autopoiesis-Konzepts in seine Theorie lehnt Luhmann diese subjektphilosophische Herangehensweise ab. Kommunikation versteht er als systemisch unabhängig vom Menschen und als Produkt sozialer Systeme (Kneer/Nassehi 2000: 65f). Zumal er "Menschen“ nicht als Systeme charakterisiert, sondern als eine Menge strukturell gekoppelter aber autopoietisch operierender Systeme, die gemeinsam einen Menschen ausmachen, aber füreinander Umwelt bleiben. Eines dieser Systeme ist das Bewusstsein als psychisches System, dessen Elemente wie beschrieben Gedanken sind. Menschen können also nicht kommunizieren. Ihr jeweiliges Bewusstsein kann lediglich denken, dass sie dies tun. Etwas weniger abstrakt gesagt, kann das Bewusstsein eines Menschen nicht wissen, ob das von ihm gesagte im Bewusstsein eines anderen Menschen so ankommt, wie von ihm intendiert oder aber in gänzlich anderer Weise. Als selbstreferentielles System kann ein Bewusstsein nur denken, dass das gesagte entsprechend angekommen ist (Kneer/Nassehi 2000: 66f): „Es ist für uns unmöglich, in die Köpfe unserer Kommunikationspartner hineinzusehen, wir werden niemals - auch mit der Hilfe von Kommunikation nicht - erfahren, was sie denken“ (Kneer/ Nassehi 2000: 72).

Äquivalent zur Verbindung zwischen Gehirn und Bewusstsein, stellt Luhmann auch eine strukturelle Kopplung zwischen Bewusstsein, bzw. psychischen Systemen, und sozialen Systemen fest (Kneer/Nassehi 2000: 71). Soziale Systeme sind für ihre Existenz notwendig auf das Bestehen von mindestens zwei psychischen Systemen angewiesen. Ein psychisches System allein kann demnach kein soziales System konstituieren. Mehr noch, Kommunikationen als Elemente sozialer Systeme können nur durch 
Bewusstsein „irritiert“ oder „gereizt“, also ausgelöst werden. Trotzdem bilden sie eine neue emergente Ordnungsebene im Vergleich zu psychischen Systemen (Kneer/Nassehi 2000: 68ff). Gemeinsam ist sozialen und psychischen Systemen zudem die Verwendung von „Sinn“. Darin unterscheiden sie sich von allen anderen Systemtypen. Psychische und soziale Systeme bilden ständig Sinn indem sie zwischen "Aktualität und Möglichkeit“ unterscheiden. Sinn wird von Luhmann verstanden als „das fortlaufende Aktualisieren von Möglichkeiten“ (Kneer/Nassehi 2000: 75). Sinn verweist bei Luhmann immer wieder auf Sinn. Es geht darum, dass Systeme immer aus einer Reihe von Möglichkeiten auswählen müssen, die dann aktuell werden, ohne aber damit die anderen Möglichkeiten für alle Zeit auszuschließen. Diese können später noch aktuell werden. Die Elemente sinnhafter Systeme, Gedanken und Kommunikationen, sind intentional zu verstehen. Im Moment der Kommunikation wird eine vorherige Möglichkeit aktualisiert, sie wird realisiert. Gleichzeitig bestehen sofort Anschlussmöglichkeiten, neue Kommunikationen, die sich aus der aktuell und den zuvor realisierten Kommunikationen ergeben und die im nächsten Moment aktualisiert werden. So entsteht Kommunikation aus Kommunikation und das System operiert im Luhmannschen Sinne autopoietisch (Kneer/Nassehi 2000: 75ff). Durch das Aktualisieren von Möglichkeiten sind soziale Systeme als sinnhaft operierende Systeme in der Lage, Komplexität im Augenblick zu reduzieren und sie gleichzeitig für die Zukunft zu erhalten. Hier ist Luhmanns neue Systemtheorie anschlussfähig zu seinen älteren Ideen, mit dem Unterschied, dass soziale Systeme Komplexität nun nicht mehr durch die Reduktion der Zahl möglicher Handlungsalternativen zu reduzieren in der Lage sind, sondern durch das fortwährende Aktualisieren von Kommunikations-Möglichkeiten (Kneer/Nassehi 2000: 77f).

Kommunikation im Luhmannschen Sinne kann dann nicht in der Weitergabe einer Information von einem Sender an einen Empfänger bestehen. Vielmehr beinhaltet jede Kommunikation einen dreiteiligen Prozess der Selektion einer Information, der Art einer Mitteilung und schließlich dem Prozess des Verstehens. In der Alltagssprache wird Kommunikation häufig mit dem Prozess des Mitteilens gleichgesetzt, was systemtheoretisch für Kommunikation nicht ausreicht. Die Mitteilung einer Information, ob schriftlich mündlich oder auf andere Weise, kann erst erfolgen, wenn aus der unüberschaubaren Vielzahl theoretisch mitteilbarer Informationen eine ausgewählt wurde. Eine Kommunikation wird dann und nur dann daraus, wenn die mitgeteilte Information verstanden wurde. Verstehen ist ebenfalls anders als in der Alltagssprache zu verstehen, nämlich als Auftreten einer Anschlusskommunikation (Kneer/Nassehi 2000: 81ff). Kommu- 
nikationen sind generell nur von kurzer Dauer, sie sind „Ereignisse“, die im nächsten Moment bereits wieder durch das nächste Ereignis abgelöst werden müssen, um das System erhalten zu können. Wie in der strukturfunktionalistischen Systemtheorie nach Parsons steht also der Systemerhalt im Mittelpunkt. Aber im Unterschied zum Strukturfunktionalismus sind spezifische Strukturen für den Systemerhalt irrelevant. Es geht um die ständige Reproduktion der Systemelemente bzw. Ereignisse, in sozialen Systemen eben Kommunikationen. Obwohl auch soziale Systeme im Sinne Luhmanns Strukturen besitzen, werden die möglichen Ereignisse von diesen Strukturen nicht bestimmt - zumindest nicht vollständig. Strukturen sind im Luhmannschen Sinne eine Einschränkung der vorhandenen Anschlussmöglichkeiten. Sie machen die Wahl bestimmter Möglichkeiten wahrscheinlicher und anderer wiederum unwahrscheinlicher. In sozialen Systemen bestehen Strukturen aus Erwartungen darüber, wie die jeweilige Anschlusskommunikation aussehen wird bzw. auszusehen hat. Von diesen Strukturen sind Prozesse zu unterscheiden, die einzelne Ereignisse miteinander verknüpfen. „Strukturen treffen eine Vor-Auswahl somit über Exklusion, Prozesse leisten das gleiche durch Anschlusssuche“ (Kneer/Nassehi 2000: 92ff).

Bevor nun im Folgenden auf die aus diesen Überlegungen Luhmanns resultierende Theorie der Gesellschaft eingegangen und untersucht wird, inwieweit eine derart verstandene Systemtheorie zum besseren Verständnis des Resilienz-Konzepts genutzt werden kann, muss noch kurz auf Luhmanns Begriff des „Beobachtens“ eingegangen werden. Eine Beobachtung ist für ihn eine „Bezeichnung-anhand-einer-Unterscheidung." Beobachten heißt etwas zu benennen und damit gleichzeitig eine andere mögliche Benennung auszuschließen. Die dafür notwendigen Schritte, erstens eine Unterscheidung zu formulieren und zweitens anhand dieser Unterscheidung den interessierenden Gegenstand konkret zu bezeichnen, sind für eine Beobachtung beide zwingend. Beispielhaft nennen Kneer und Nassehi die Unterscheidungen Mann/Frau oder an späterer Stelle Lüge/keine Lüge, zwischen denen gewählt werden muss, um zu einer Beobachtung zu kommen. Die Fähigkeit zur Beobachtung ist nicht notwendigerweise nur mit autopoietischen Systemen verbunden. Bezogen auf ein soziales System heißt sie aber, dass dieses seine Umwelt beobachtet, indem es über sie kommuniziert (Kneer/Nassehi 2000: 96ff). Luhmanns Verständnis von Beobachten impliziert eine Reihe von Annahmen, die seine neuere Systemtheorie im Kontext konstruktivistischer Ansätze verorten. Dazu zählt etwa der „blinde Fleck“ der sich durch Beobachten ergibt. Jede Beobachtung schränkt die Sicht der Welt auf eine dichotome Unterscheidung ein und 
bezeichnet eine der beiden Seiten. Alles was außerhalb dieser Unterscheidung liegt bleibt unsichtbar. Um das zu verdeutlichen führt Luhmann das Konzept des „Beobachters zweiter Ordnung“ ein. Dieser beobachtet den Beobachter erster Ordnung, indem er dessen Beobachtung mithilfe einer eigenen Unterscheidung bezeichnet und erkennt so dessen blinden Fleck. Seinen eigenen blinden Fleck kann er zwar nicht erkennen, aber zumindest wird ihm bewusst, dass es Dinge gibt, die er nicht sehen kann (Kneer/Nassehi 2000: 99ff). „Damit entfällt die absolut ,richtige“ Sicht der Dinge. Was auch immer behauptet wird, es wird von einem Beobachter behauptet, der sich gefallen lassen muss, dass er kritisiert und über seine eigenen blinden Flecke aufgeklärt wird" (Kneer/Nassehi 2000: 103). Luhmanns eigene Theorie sozialer Systeme versteht er als „autologisch“, da sie ein Gegenstand ihrer selbst ist. Zum Beispiel bezeichnet die Theorie sozialer Systeme Kommunikationen als Elemente dieser Systeme in Unterscheidung von anderen Theorien, die Menschen als Elemente sozialer Systeme bezeichnen (Kneer/Nassehi 2000: 108).

Aus diesem stark modernisierten systemtheoretischen Vokabular bedient sich Luhmann dann, um eine Theorie der Gesellschaft zu entwickeln. Die Gesellschaft besteht nach wie vor aus der Gesamtheit aller Interaktions- und Organisationssysteme. Im Vergleich zu Luhmanns älteren Ansätzen ist ein Grundbegriff für eine Theorie der Gesellschaft ebenfalls gleichgeblieben, nämlich der der Komplexität. Dazu kommt als zweiter Grundbegriff die sogenannte „Systemdifferenzierung“. Ersteres bezeichnet immer noch das Vorhandensein von mindestens zwei Anschlussmöglichkeiten, in sozialen Systemen also mindestens zwei Anschlusskommunikationen. Eine nicht-komplexe soziale Situation, eine, in der nur genau eine Anschlusskommunikation möglich ist, ist schlechterdings kaum vorstellbar. Mit Systemdifferenzierung ist die Art gemeint, in der die Gesellschaft sich „intern in Sub- und Teilsysteme differenziert“, die untereinander für sich dann wieder in einer System-Umwelt-Beziehung stehen (Kneer/Nassehi 2000: 112f). Differenzierung kann als Möglichkeit verstanden werden, Komplexität zu reduzieren. Der Zusammenhang zwischen beiden Konzepten ist aber nicht deterministisch zu verstehen. Es gilt also nicht: Je stärker die Binnendifferenzierung eines Systems, desto geringer die Komplexität. Vielmehr kann Binnendifferenzierung auch zu einer detaillierteren Problemorientierung führen, da sich spezialisierte Subsysteme nun mit ihrer gesamten Kapazität einem Teilaspekt eines Gesamtproblems widmen können und daraus unmittelbar wieder mehr Optionen - also größere Komplexität - zum Umgang mit diesem Problem erwachsen (Kneer/Nassehi 2000: 113f). Die entscheidende Frage für eine Theorie der Gesellschaft 
lautet dann, „wie sich Gesellschaftssysteme differenzieren, also welche Form der inneren Differenzierung sie zur Verarbeitung ihrer Eigenkomplexität nutzen" (Kneer/Nassehi 2000: 114, Hervorhebung im Original). Luhmann versteht das Prinzip der Binnendifferenzierung als Ausbildung bestimmter Beobachterpositionen, da Beobachtungen ja gerade auf dem Prinzip der Unterscheidung - oder Differenzierung - und Bezeichnung dieser Unterscheidung bestehen. Differenzierungen sind damit nicht exklusiv, sondern „kontingent.“ Mit „Kontingenz" führen Kneer und Nassehi einen weiteren wichtigen Begriff von Luhmanns Systemtheorie ein. Etwas ist kontingent, wenn es ist wie es ist, aber auch anders sein könnte (Kneer/ Nassehi 2000: 115). Bezogen auf die verschiedenen Möglichkeiten der Ausbildung differenzierter Beobachtungsverhältnisse in einer Gesellschaft lässt sich der Begriff beispielhaft erklären: Ferienorte in Ägypten bieten ganzjährig gutes Wetter und sind im Vergleich zu europäischen Reisezielen günstig. Nimmt ein Beobachter also eine Position ein, in der er rein nach Klima und Preis unterscheidet, wie geeignet eine Feriendestination ist, erscheint die Bezeichnung „geeignet" für Ägypten als gerechtfertigt. Gleichzeitig könnte ein anderer Beobachter mit der Unterscheidung funktionierende Demokratie/keine funktionierende Demokratie, Ägypten mit einigem Recht als nicht-funktionierende Demokratie bezeichnen. Keiner der Beobachter kann von sich behaupten, seine Wahrnehmung sei die einzig mögliche und richtige. Und aufgrund der bestehenden Kontingenz kann keiner sicher voraussehen, wie der andere den Beobachtungsgegenstand wahrnehmen wird. Luhmanns Theorie der Gesellschaft möchte ja die Frage beantworten, wie und in welcher Form sich Gesellschaftssysteme differenzieren. Gegeben das Phänomen der Kontingenz ist es dazu notwendig, die gegenseitigen Beobachtungsverhältnisse der unterschiedlichen Teilsysteme, also ihre Beziehungen untereinander, mit sich selbst sowie mit der gesamten Gesellschaft zu analysieren. „An der Differenzierungsform und an den Beziehungsmöglichkeiten der gesellschaftlichen Teilsysteme lässt sich die Struktur eines Gesellschaftssystems ablesen“ (Kneer/ Nassehi 2000: 115).

Hier kommt wieder der Begriff der Struktur eines Systems ins Spiel. Luhmann nutzt diesen ja bereits in seiner aktualisierten Theorie sozialer Systeme. Auch für die Theorie der Gesellschaft wird er - in einem ähnlichen, nicht-deterministischen Verständnis - wichtig. Demnach entwickeln komplexe Systeme Strukturen, um die theoretisch unbegrenzte Zahl möglicher Anschlusshandlungen bzw. im Fall von sozialen Systemen Anschlusskommunikationen praktisch zu reduzieren. Diese Unterscheidung ist insofern relevant, dass die Reduktion von Anschlussmöglichkeiten auf 
ein „erwartbares Maß" tatsächlich rein praktisch erfolgt und theoretisch weiterhin alle Möglichkeiten offen sind, da es sich um Kontingenz-aufweisende Systeme handelt. Die Möglichkeiten werden durch Strukturen nicht statisch, sozusagen a priori, festgelegt, sondern entwickeln sich dynamisch mit den tatsächlich aktualisierten Optionen weiter. Selbiges muss dann auch für die Strukturen selbst gelten, weshalb Luhmann autopoietischen Systemen dieser Art eine „dynamische Stabilität“ zuschreibt. Die Strukturen dienen einerseits der Kontingenzeinschränkung und ermöglichen andererseits durch ihre eigene Weiterentwicklung ein Fortbestehen der Kontingenz (Kneer/Nassehi 2000: 116f). Für die Theorie der Gesellschaft folgt daraus ebenfalls eine Sichtweise, in der Gesellschaftsstrukturen sich dynamisch weiterentwickeln, allerdings nicht zufällig und chaotisch, sondern evolutionär. Unterschiedliche Anschlusskommunikationen weisen unterschiedliche Wahrscheinlichkeiten auf, so dass sich langfristig bestimmte Entwicklungspfade nachzeichnen lassen und spezifische gesellschaftliche Strukturen ausgebildet werden, die wiederum die Wahrscheinlichkeiten weiterer Anschlusskommunikationen beeinflussen. Zugleich ermöglicht aber die nicht-deterministische Sichtweise auf diesen soziokulturellen Evolutionsprozess auch den Fall, dass sehr unwahrscheinliche Anschlusskommunikationen gewählt werden. Dazu bedarf es rein mathematisch gesprochen lediglich einer hinreichend großen Menge an Möglichkeiten und vor allem einer hinreichend langen Zeitspanne, so dass entsprechend viele Ereignisse stattfinden können. Soziokulturelle Evolution wird so als zufälliger Prozess charakterisiert, bei dem evolutionäre Schübe, also die gravierende Veränderung der als Selektionsmechanismen für Anschlusskommunikationen dienenden gesellschaftlichen Strukturen, dann auftreten, wenn extrem unerwartbare Ereignisse realisiert werden (Kneer/Nassehi 2000: $117 \mathrm{f})$.

Um unwahrscheinliche, gravierende „Ereignisse“ geht es auch im Kontext der Resilienz-Diskussion. Der Ereignis-Begriff ist dort deutlich anders konzeptualisiert als bei Luhmann. Nichtsdestotrotz können auch dessen aktualisierte systemtheoretische Überlegungen genutzt werden, um das Resilienz-Konzept zu analysieren. Im Anschluss an Luhmanns ursprüngliche Ideen konnte ein hoher Grad an Eigenkomplexität in sozio-technischen Systemen als konstitutiv für deren Resilienz identifiziert werden, da nur so Anpassungsfähigkeit bei sich verändernden Umweltbedingungen möglich wird. Dieser Ansatz behält nun für die vorliegende Arbeit - und bei einer Luhmannschen Definition von Komplexität - zunächst seine Gültigkeit. Er lässt sich aber sinnvoll mit Luhmanns neueren Ideen ergänzen. Die verschiedenen Traditionen der Resilienzforschung stimmen wei- 
testgehend darin überein, dass Resilienz sich dann zeigen kann, wenn ein System von einem disruptiven, extern getriggerten, gravierenden und häufig extrem unerwarteten „Reiz“ getroffen wird. Für das Resilienz-Konzept der zivilen SiFo gilt also: Das Vorhandensein oder Nicht-Vorhandensein von Resilienz lässt sich nur beobachten, wenn Systeme von einem disruptiven, in der Systemumwelt verorteten, gravierenden und häufig extrem unwabrscheinlichen Reiz getroffen werden.

Von „Reiz“ ist hier bewusst die Sprache, um den Ereignisbegriff systemtheoretisch verwenden zu können. Obwohl es sich um autopoietische Systeme handelt, sind derartige Reize von außen nicht ausgeschlossen, da autopoietische Systeme wie gezeigt zwar autonom nicht jedoch autark agieren. Übersetzt in eine systemtheoretische Sprache wird das System durch einen solchen Reiz von seiner Umwelt gezwungen, ein extrem unwahrscheinliches Anschlussereignis zu realisieren. Das kann dem System entweder gelingen, es erweitert damit den Möglichkeitsraum für die folgenden Anschlussereignisse und entwickelt seine Strukturen in dieser Hinsicht weiter. Dann kann das soziale System als resilient bezeichnet werden. Oder aber es ist nicht in der Lage, eine solche, extrem unwahrscheinliche Anschlussmöglichkeit zu realisieren und das System hört zu existieren auf. Resilienz derart systemtheoretisch zu fassen, entspricht einem Hollingschen Verständnis, bei dem Resilienz sich durch Persistenz, durch Überleben des Systems ausdrückt. Es lässt sich festhalten: Resilienz ist ein systemtheoretisches Konzept. Sozio-technische Systeme sind die Träger von Resilienz.

Für ein Resilienz-Konzept der zivilen SiFo reicht das nicht aus, weshalb darüber hinaus das Resilienz-Verständnis der Psychologie ergänzend systemtheoretisch genutzt wird. In der Psychologie geht es bei Resilienz um die erfolgreiche Bewältigung von Traumata. Traumata können als sehr unwahrscheinliche, extern verursachte Reize für ein psychisches System, für das Bewusstsein formuliert werden. Entscheidend für die Unterscheidung zwischen Resilienz, als erfolgreichem Umgang mit dem Trauma, und nicht resilientem Systemverhalten, verstanden etwa als Ausbildung psychischer Erkrankungen, ist also nicht mehr das Realisieren einer unwahrscheinlichen Anschlussmöglichkeit im Vergleich zu Realisierung gar keiner Anschlussmöglichkeit. Es kommt vielmehr darauf an, welche spezifischen Ereignisse realisiert werden. Resilienz wird in der Psychologie wie auch in der vorliegenden Arbeit nicht als etwas Selbstverständliches oder Normales - oder systemtheoretisch „Erwartbares“ - verstanden. Demzufolge bedeutet Resilienz auch hier wieder zum einen die Realisierung eines extrem unwahrscheinlichen, eines unerwartbaren, Anschlussereignisses. 
An dieser Stelle bleibt das System aber nicht stehen. Es werden fortlaufend weitere Anschlussmöglichkeiten realisiert. Resilienz erfordert deshalb zum anderen auch die Rückkehr in einen Prozess der Realisierung erwartbarer Anschlussmöglichkeiten. Und zwar erwartbar in Bezug auf das bereits realisierte, unerwartbare Anschlussereignis. Nicht-resilientes Systemverhalten besteht hingegen in der direkten Realisierung eines erwartbaren durch die existierenden Systemstrukturen wahrscheinlich gemachten Anschlussereignisses. Strenggenommen ist Resilienz nach Luhmann „unsinnig“, da Sinn in psychischen und sozialen Systemen darin besteht festzulegen, wenn auch nicht deterministisch, „was in einem System wann von wem erwartet bzw. nicht erwartet werden kann" (Kneer/Nassehi 2000: 120, Hervorhebung im Original). Und Resilienz kann nicht erwartet werden. Der Aspekt des erfolgreichen Umgangs mit einem unwahrscheinlichen externen Reiz wird in der vorliegenden Arbeit in analoger Weise aus der Psychologie auf die für die zivile SiFo relevanten sozio-technischen Systeme übertragen. Auch für diese gilt also systemtheoretisch gesprochen, dass Resilienz dann vorliegt, wenn ein System auf einen sehr unwahrscheinlichen Reiz aus der Umwelt, der ein unerwartbares Ereignis im System auslöst, mit der Realisierung einer wiederum extrem unerwartbaren Anschlussmöglichkeit reagiert, um anschließend auf einen so realisierbar gewordenen, neuen Entwicklungspfad erwartbarer Anschlussmöglichkeiten einzuschwenken. Die folgende Abbildung veranschaulicht die Idee (siehe Abbildung 2).

\section{Abbildung 2: Resilienz in systemtheoretischer Sichtweise}

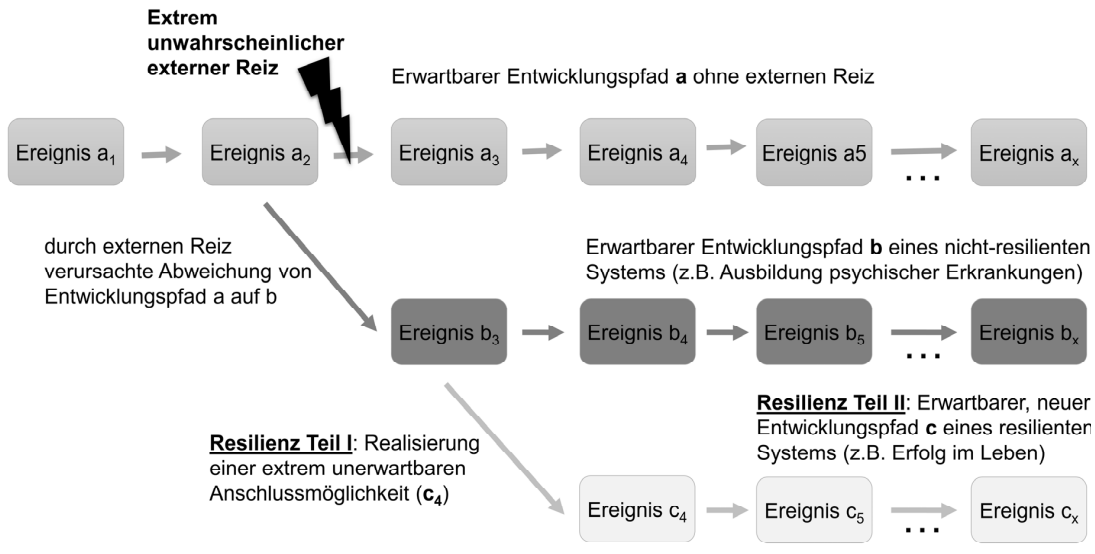

Quelle: eigene Darstellung. 
Die Abbildung verdeutlicht gleichzeitig einen weiteren Aspekt einer derartigen systemtheoretischen Analyse des Resilienz-Konzepts: Im Rahmen der zivilen SiFo kann Resilienz nicht als „bouncing back“ zu einem vorherigen Entwicklungspfad und dem damit verbundenen Erhalt der vorherigen Systemstrukturen verstanden werden. Denn nach der Realisierung der „resilienten Anschlussmöglichkeit" eröffnet sich dem System ein erweiterter Möglichkeitsraum, seine Strukturen haben sich geändert und es betritt einen neuen, als evolutionär weiterentwickelt zu bezeichnenden Entwicklungspfad. Eine evolutionäre Weiterentwicklung an sich ist nicht notwendigerweise normativ positiv zu sehen. Auch bei nicht-resilientem Systemverhalten ändern sich dessen Strukturen durch das bereits eingetretene, extern getriggerte unerwartbare Ereignis und eine evolutionäre Weiterentwicklung findet statt. Darüber, ob resilientes Systemverhalten normativ wünschenswert ist, sagt ein derart konzipiertes, systemtheoretisches Resilienz-Verständnis a priori ebenfalls nichts aus. Da es mit Wahrscheinlichkeiten operiert, ist die Ausbildung von Resilienz vom Zufall abhängig. Sie ist kontingent, zwar unwahrscheinlich, aber nicht unmöglich. Und sie ist nicht dichotom zu verstehen. In der Systemtheorie sind die Anschlussmöglichkeiten für komplexe Systeme prinzipiell unbegrenzt und unterscheiden sich nur in der Wahrscheinlichkeit ihrer Realisierung, die wiederum Systemstrukturen konstituieren. Ein System kann sich also als mehr oder weniger resilient erweisen, je nachdem, wie wahrscheinlich oder unwahrscheinlich die realisierte Anschlussmöglichkeit war. Oder präziser gesagt: je nachdem wie wahrscheinlich oder unwahrscheinlich die realisierten Anschlussmöglichkeiten, im Plural, waren. Anschlussmöglichkeiten materialisieren sich in Form von Ereignissen, die in Luhmanns Systemtheorie immer nur momentan zu verstehen sind. Resilienz erfordert aber mehr als ein einmaliges, instantanes Abweichen vom erwartbaren Entwicklungspfad. Die Darstellung in Abbildung 2 diente nur zur Veranschaulichung der grundsätzlichen Idee. Um Resilienz systemtheoretisch treffender fassen zu können, kann der an vorheriger Stelle bereits eingeführte Begriff des Prozesses, verstanden als aneinander anknüpfende Anschlussereignisse, genutzt werden (Kneer/Nassehi 2000: 94). Für Resilienz im Rahmen der zivilen SiFo gilt dann weiterhin, dass eine möglichst hohe Eigenkomplexität des Systems im Angesicht der überkomplexen Umwelt notwendig ist, um die Zahl der überhaupt theoretisch zur Verfügung stehenden Anschlussmöglichkeiten zu maximieren. Resilienz erfordert notwendig bohe Eigenkomplexität des betroffenen Systems, um Anpassungsfähigkeit in Form der Verfügbarkeit einer Vielzabl alternativer Handlungsoptionen bei Eintreten eines gravierenden und unwahrscheinlichen Reizes überhaupt zu ermöglichen. 
Hohe Eigenkomplexität ist aber keine hinreichende Bedingung für Resilienz. Gleichzeitig darf die Wahl des tatsächlich realisierten Prozesses an Anschlussmöglichkeiten gerade nicht dem evolutionären Spiel des Zufalls überlassen werden. Vielmehr geht es darum, die Wahrscheinlichkeit für die Realisierung eigentlich extrem unerwartbarer Prozesse von Anschlussmöglichkeiten zu erhöhen. Die oben getroffene Aussage, wonach Resilienz sich in der Realisierung einer extrem unerwartbaren Anschlussmöglichkeit und dem anschließenden Einschwenken auf einen neuen Entwicklungspfad erwartbarer Anschlussmöglichkeiten zeige, muss daher wie folgt präzisiert werden. Resilienz liegt dann vor, wenn ein System auf einen sehr unwahrscheinlichen Reiz aus der Umwelt, der ein unerwartbares Ereignis im System auslöst, mit der Realisierung eines Prozesses eigentlich extrem unerwartbarer Anschlussmöglichkeiten reagiert, um anschließend auf einen so realisierbar gewordenen, neuen Entwicklungspfad erwartbarer Anschlussmöglichkeiten einzuschwenken. Die Aufgabe der zivilen Sicherheitsforschung besteht darin, die Wahrscheinlichkeit zur Realisierung solcher Prozesse eigentlich extrem unerwartbarer Anschlussmöglichkeiten zu erhöhen.

Zusammenfassend lässt sich für den Zusammenhang zwischen Resilienz und System festhalten: Resilienz ist tatsächlich, wie von Alexander skizziert, ein Systemkonzept (Alexander 2013: 2712). Resilienz lässt sich als Konzept systemtheoretisch im Sinne der klassischen Systemtheorie und vor allen Dingen im Sinne von Niklas Luhmanns verschiedenen Entwicklungsstufen seiner Theorie sozialer Systeme fassen und analysieren. Es kann grundsätzlich als systemtheoretisches Konzept verstanden werden und in den größeren Zusammenhang systemtheoretischen Denkens eingeordnet werden. Im Rahmen der zivilen Sicherheitsforschung ergeben sich für ein eigenständiges Resilienz-Konzept damit folgende Annahmen:

- Resilienz ist ein systemtheoretisches Konzept.

- Sozio-technische Systeme sind die Träger von Resilienz.

- Der Grad an Komplexität drückt sich in der Zahl der zur Verfügung stehenden Möglichkeiten aus. Systeme sind stets weniger komplex als ihre Umwelt und dienen ihren Mitgliedern zur Reduktion umfassender Komplexität. Zur Reduktion externer Komplexität sind Systeme intern auf das Vorhandensein vieler Handlungsoptionen angewiesen. Die Zahl der systeminternen Handlungsmöglichkeiten wird als Ausmaß der Eigenkomplexität des Systems verstanden.

- Das Vorhandensein oder Nicht-Vorhandensein von Resilienz lässt sich nur beobachten, wenn Systeme von einem disruptiven, in der Systemumwelt verorteten, gravierenden und häufig extrem unwahrscheinlichen Reiz getroffen werden. 
- Resilienz erfordert notwendig hohe Eigenkomplexität des betroffenen Systems, um Anpassungsfähigkeit in Form der Verfügbarkeit einer Vielzahl alternativer Handlungsoptionen bei Eintreten eines gravierenden und unwahrscheinlichen Reizes überhaupt zu ermöglichen.

- Hohe Eigenkomplexität ist notwendig, aber nicht hinreichend zur Ausbildung von Resilienz. Denn Resilienz ist eine Nicht-Selbstverständlichkeit, sie ist nicht erwartbar.

- Resilienz liegt dann vor, wenn ein System auf einen sehr unwahrscheinlichen Reiz aus der Umwelt, der ein unerwartbares Ereignis im System auslöst, mit der Realisierung eines Prozesses eigentlich extrem unerwartbarer Anschlussmöglichkeiten reagiert, um anschließend auf einen so realisierbar gewordenen, neuen Entwicklungspfad erwartbarer Anschlussmöglichkeiten einzuschwenken.

- Die Aufgabe der zivilen Sicherheitsforschung besteht darin, die Wahrscheinlichkeit zur Realisierung solcher Prozesse eigentlich extrem unerwartbarer Anschlussmöglichkeiten zu erhöhen.

\subsubsection{Resilienz und Komplexität}

Komplexität ist sowohl ein zentraler Begriff der Systemtheorie, wie im vorangegangenen Unterkapitel deutlich wurde, als auch der Resilienzforschung, insbesondere in der Tradition von Holling. Nach Kaufmann und Blum sind die Wurzeln des Hollingschen Resilienz-Konzepts in der Komplexitätstheorie zu finden. Und dieser Fakt ist von entscheidender Wichtigkeit für den Transfer des Konzepts von der Ökologie in den Bereich der zivilen SiFo (Kaufmann/Blum 2012: 238). Bisher hat sich die vorliegende Arbeit an Luhmanns Definition von Komplexität orientiert. Demzufolge tritt Komplexität immer auf, wenn etwas mindestens zwei Zustände annehmen kann. Je mehr Zustände in einem System möglich sind, desto komplexer ist es. Die Summe aller Systeme und ihrer jeweiligen Umwelten bildet die Welt, die durch „unfassbare“, prinzipiell unendliche Komplexität gekennzeichnet ist (Kneer/Nassehi 2000: 40, Luhmann 2014: 4f). Diese Definition von Komplexität führte für das zu entwickelnde Resilienz-Konzept der zivilen SiFo zu einer Reihe von Annahmen, die sich am Ende des vorangegangenen Unterkapitels finden. Dazu zählt unter anderem eine Unterscheidung zwischen Eigenkomplexität und Komplexität der (Um-)Welt. Während letztere durchgängig als Herausforderung oder gar „oberstes Bezugsproblem“ gekennzeichnet wurde, trug erstere als quasi wünschenswerte Eigenschaft von Systemen zur Ausbildung von Resilienz 
bei (Kneer/Nassehi 2000: 40). Ein solches Verständnis von Eigen- und Weltkomplexität ergab sich unmittelbar aus der Definition von Komplexität unter Zuhilfenahme der Anzahl möglicher System- bzw. Weltzustände. Die Welt kann beinahe unendlich viele Zustände annehmen, auf die das System sich selbst erhaltend zu reagieren in der Lage sein muss. Das kann es umso besser, je mehr Zustände es annehmen kann. Für das folgende Unterkapitel wird, dem Verständnis von Resilienz als systemtheoretischen Konzept gemäß, weiterhin eine Unterscheidung in Eigenkomplexität eines Systems und (Um-) Weltkomplexität getroffen. Inwiefern hohe Eigenkomplexität förderlich für Resilienz ist oder nicht, muss dagegen untersucht werden. Die Analyse von Resilienz unter Zuhilfenahme disziplinärer $\mathrm{Zu}$ gänge im letzten Kapitel hat bereits gezeigt, dass unter Komplexität in der Resilienzforschung zumeist mehr und etwas Anderes verstanden wird, als die Anzahl möglicher Zustände in einem System. Deshalb wird im Folgenden mithilfe von Ansätzen aus der Komplexitätstheorie der Zusammenhang zwischen "Resilienz und Komplexität“ ausführlich diskutiert und daraus Annahmen für ein Resilienz-Konzept der zivilen SiFo abgeleitet.

Die wissenschaftliche Beschäftigung mit dem Phänomen der Komplexität blickt mittlerweile auf eine knapp hundertjährige Geschichte in den Natur- und Sozialwissenschaften zurück, in spezifischeren Bereichen, wie etwa der Management-Theorie, reicht die Forschung auch schon mindestens 50 Jahre zurück (Chandler 2014: 48, Longstaff 2012: 262). Im Gegensatz zu Resilienz und dem Adjektiv resilient sind die Begriffe Komplexität und komplex Teil der Alltagssprache im Deutschen und werden zumeist ohne dahinterliegendes theoretisches Konzept verwendet. Wenn etwas als „komplex“ bezeichnet wird, geht es häufig um etwas Schwieriges, nicht einfach zu Durchschauendes, ein Phänomen mit vielen, miteinander verbundenen Bestandteilen. Der Begriff „kompliziert“ wird nicht selten synonym zu „komplex“ gebraucht. Damit enthält der alltägliche Komplexitäts-Begriff schon einige Elemente, die sich auch im wissenschaftlichen Komplexitäts-Begriff wiederfinden werden. Vor allen Dingen wird auch ein Unterschied zum Luhmannschen Komplexitäts-Begriff sichtbar. Sprechen Menschen von Komplexität, meinen sie - mindestens implizit mehr, als nur die Anzahl möglicher Zustände. Ohne der wissenschaftlichen Definition vorgreifen zu wollen, lässt sich feststellen: Komplexität ist ein Phänomen, von dem Menschen täglich und ständig umgeben sind. Soziale und ökologische Systeme sind in der Regel komplexe Systeme. Einige augenfällige und bekannte Beispiele dafür sind etwa das Wetter oder gar das Klimasystem, die Aktienmärkte oder der Straßenverkehr. Ihre Komplexität drückt sich darin aus, dass wir niemals alles über sie wissen 
können und unsere Fähigkeit, ihre künftige Entwicklung und mögliche Veränderungen zu prognostizieren und vorherzusagen, selbst mit den Erkenntnissen der modernen Wissenschaft notwendig unvollständig bleibt. Die individuell wahrgenommene Unzuverlässigkeit der täglichen Wettervorhersagen kann dafür beispielhaft ins Feld geführt werden (Berkes 2007: 285, Smith 2002).

Für die Resilienzforschung bzw. ein zu entwickelndes Resilienz-Konzept für die zivile SiFo geht es neben dem Verständnis darüber, was Komplexität überhaupt bedeutet, immer auch um die Frage, wie sinnvoll mit Komplexität umgegangen werden kann. Also grundsätzlich erst einmal danach, ob im Kontext von Resilienz Komplexität eher Problem, Möglichkeit, Chance oder all das zugleich darstellt. ${ }^{30} \mathrm{Um}$ mit Komplexität

30 Wie sich die - zunächst nur behauptete - zunehmende Komplexität der modernen Welt auf deren Sicherheit auswirkt, insbesondere im Bereich der Hochtechnologie, ist Gegenstand einer intensiven und mittlerweile schon einige Jahrzehnte andauernden Debatte in den Sozialwissenschaften, die durchaus Ähnlichkeiten zur Resilienz-Diskussion aufweist (Hale et al. 2006: 290). Dabei stehen sich Verfechter der sogenannten "normal accidents" Theorie nach Charles Perrow auf der einen Seite und Verfechter der Idee von sogenannten „High Reliability Organizations" (HRO) auf der anderen Seite gegenüber (Boin/McConnell 2007: 52). Der amerikanische Soziologe Perrow argumentierte in seinem sehr einflussreichen Werk dahingehend, dass unsere technischen Systeme - wie etwa Kernkraftwerke - mit steigender Komplexität automatisch verletzlicher werden (siehe Perrow 1999). Zentral ist hier der Begriff der "tight couplings“ aufgrund derer bereits kleine Fehler im System propagieren und zu einem Totalausfall führen können. Nach Perrow ist das in komplexen System letztlich unvermeidlich, deshalb spricht er von „normalen Unfällen“, die nicht zu verhindern seien (Rijpma 1997: 16, Wachsmuth 2014: 8). Als Lösungsstrategien schlägt er „unelegantes“ und „robustes“ Design vor, das von Anfang an in Systemen vorhanden sein muss und nicht als add-on (Perrow 1999b: 150ff). Im Gegensatz dazu sind Vertreter der HRO-Theorie der Ansicht, dass gerade Komplexität in Organisationen dazu beitragen kann, Zuverlässigkeit (reliability) - und ein Stück weit auch Resilienz - herzustellen (Fekete et al. 2014: 14, Schulman et al. 2004: 15). Etwa, indem in HROs bewusst die Binnen-Komplexität durch die Aufnahme von Mitarbeitern mit unterschiedlichen Charakteristika und Qualifikationen gefördert wird oder auch die regelmäßige Rotation von Aufgaben, indem Meinungsvielfalt gefördert wird, etc. All das kann dazu führen, eine größere Zahl potentieller Probleme schneller zu erkennen und über die geeigneten Mechanismen zu deren Behebung zu verfügen (Schulman et al. 2004: 26, Ungericht/Wiesner 2011: 192). Auch wenn die skizzierte Debatte durchaus Anknüpfungspunkte zur vorliegenden $\mathrm{Ar}$ beit aufweist, steht sie nicht im Mittelpunkt des Forschungsinteresses da sie, ähnlich wie die Forschung zu Resilience Engineering nach Woods und Hollnagel, primär mit komplexen Organisationen beschäftigt ist und nicht mit ziviler Sicherheit. 
umgehen zu können, muss diese zunächst als Faktum realisiert und als Herausforderung akzeptiert werden. Gerade traditionelle Management-Ansätze, wie sie auch dem klassischen Risikomanagement zugrunde liegen, negieren Komplexität zwar nicht explizit, beziehen sie in ihre Analysen jedoch nicht mit ein (Lorenz 2010, siehe 2.6). Das liegt an einer Reihe von Eigenschaften, die Komplexität mit sich bringt, beispielsweise der Nichtlinearität von Prozessen und Ereignissen, dem langfristigen Zeithorizont, der zu beachten ist, der Unmöglichkeit soziale Phänomene eindeutig den Handlungen spezifischer Akteure zuzuschreiben und einigen mehr. Derartige Eigenschaften unterscheiden komplexe von nicht-komplexen Systemen, sie sind jedoch empirisch häufig zu beobachten, mindestens in den für die zivile SiFo relevanten sozio-technischen Systemen (Duit et al. 2010: 2). Gleichzeitig schließt Komplexität von Systemen deren Kontrollierbarkeit oder die Möglichkeit, sie zu managen, nicht notwendig aus. Jedoch bedarf es nichttraditioneller Methoden und der Verwendung empirisch zutreffender Annahmen, um komplexe Systeme managen zu können. Dazu zählen zum Beispiel nichtlineare und nichtreduktionistische, man könnte auch sagen systemische Ansätze (Chandler 2014: 48, Longstaff 2012: 261). Die Diskussion der verschiedenen disziplinären Zugänge hat gezeigt, dass Resilienz vor allen Dingen in der Ökosystemforschung, der sozial-ökologischen Forschung und den Sozialwissenschaften als ein dergestalt systemischer Ansatz verstanden wird. Brand und Jax bringen das mit einer knappen und zugespitzten Definition von Resilienz auf den Punkt: „, $[\mathrm{R}]$ esilience is better described as a collection of ideas about how to interpret complex systems“ (Brand/Jax 2007: 8). Diese Aussage, wonach Resilienz eine Sammlung von Ideen zur Interpretation komplexer Systeme darstelle, soll den Ausgangspunkt für die folgenden Diskussionen bilden (IRGC 2018: 53f). Dabei kommen der Resilienzforschung zwei Aspekte zugute, die sie mit der Komplexitätstheorie gemein hat. Zum einen können beide nicht für sich in Anspruch nehmen, Teil des Mainstreams sozialwissenschaftlicher Forschung zu sein. Trotz der stark zunehmenden Beschäftigung mit Resilienz stehen nach wie vor andere Konzepte, wie etwa Macht, Demokratie oder auch System, in deren primären Fokus. Das erlaubt eine gemeinsame Analyse beider Konzepte ohne Gefahr zu laufen, zu stark „quer" zu etablierten fachspezifischen Traditionen zu laufen. Zum anderen - und dazu passend - sind weder Resilienz noch Komplexität eineindeutige Konzepte mit nur einer „richtigen“ bzw. allgemein akzeptierten Definition. Vielmehr sind beide vielschichtige konzeptionelle Überbauten, die von verschiedenen Disziplinen und für verschiedene Untersuchungsgegenstände herangezogen werden (Duit et al. 2010: 1f, IRGC 2018: 53f). 
In der zivilen SiFo geht es um die Frage, wie relevante sozio-technische Systeme mit widrigen Ereignissen umgehen. Das steht - für die jeweils relevanten Systeme - auch im Mittelpunkt verschiedener disziplinärer Ansätze der Resilienzforschung. Und auch für komplexe Systeme gilt, dass es für sie eine eminent wichtige Rolle spielt, wie und ob sie in der Lage dazu sind, mit disruptiven Ereignissen erfolgreich umzugehen, die durch Diskontinuität, Konfusion, Unordnung oder andere Effekte die normale Funktionsfähigkeit der Systeme stören. Komplexe Systeme sehen sich häufig mit „wicked problems“ konfrontiert, Problemstellungen, die diffus und nicht abgrenzbar sind und durch ihre vielfältig miteinander vernetzten Bestandteile und Ebenen keine einfache, linearkausale Erklärung möglich machen. Das trifft auch und gerade zu, weil komplexe Systeme in den meisten Fällen aus einer sehr großen Zahl an Elementen bestehen (Christmann/Ibert 2016: 253, Rahimi/Madni 2014: 810, Smith 2002). Für Smith ist deshalb die Entwicklung des Computers und die exponentielle Steigerung von dessen Rechenkraft in den letzten Jahrzehnten entscheidend für die Forschung zu komplexen Systemen gewesen. Denn Computer sind in der Lage, die Interaktionen einer großen Zahl an Elementen zu simulieren, eine Aufgabe, der das menschliche Gehirn so nicht gewachsen ist (Smith 2002). Für die in der vorliegenden Arbeit aufgeworfene Fragestellung sowie die Beschäftigung mit Komplexitätstheorie aus einer stärker sozialwissenschaftlichen Sicht gilt Smith Einschätzung so nicht. Rechenleistung mag für die Untersuchung realer komplexer Systeme und deren Verhalten, wenn sie von einem disruptiven Ereignis getroffen werden, äußerst hilfreich sein. Für die konzeptionelle Arbeit zu Komplexitätstheorie und deren Verbindungen zur Resilienzforschung braucht es sie dagegen nicht. Hier stehen vielmehr spezifische theoretische Ansätze im Mittelpunkt. Wie bereits erwähnt ist die Komplexitätstheorie ein diverses, interdisziplinäres Forschungsfeld mit unterschiedlichen Schwerpunkten und uneinheitlichen Definitionen von Komplexität. In der Resilienzforschung, vor allen Dingen dort, wo sie sich in der Tradition von Hollings Arbeiten bewegt, wird immer wieder auf Modelle und Theorien zu „komplexen adaptiven Systemen" und die Ähnlichkeiten zwischen einem Hollingschen Resilienz-Verständnis und den Annahmen dieses Zweigs der Komplexitätstheorie verwiesen (Berkes 2007: 286, Folke 2006: 257, Vogt 2015: 9). Daher bietet es sich an, für die Beschäftigung mit dem Zusammenhang zwischen Resilienz und Komplexität auf die Ansätze und Ideen der Theorie zu komplexen adaptiven Systemen zurückzugreifen.

Grundsätzlich geht die vorliegende Arbeit davon aus, dass ein System nicht notwendigerweise die Eigenschaft Komplexität aufweisen muss. Da- 
mit bewegt sie sich eindeutig im Einklang mit der Komplexitätstheorie. Es ergibt erst dann Sinn, von komplexen adaptiven Systemen zu sprechen, wenn es auch nicht-komplexe nicht-adaptive Systeme gibt. Ansonsten wären derart qualifizierende Adjektive tautologisch und schlicht überflüssig. Entwickelt wurde die Theorie komplexer adaptiver Systeme am Santa Fe Institute in New Mexiko. Dieses 1984 gegründete, interdisziplinäre Forschungsinstitut befasst sich mit Grundlagenforschung im Bereich der Komplexitätstheorie und bezeichnet sich selbst als „world headquarters for complexity science. “31 Die Gründungsmitglieder, wie zum Beispiel der Chemiker George Cowan, der bis 1991 als erster Präsident des Instituts agierte, waren zum großen Teil Wissenschaftler des für die Entwicklung der Atombombe im Rahmen des Manhattan-Projekts bekannt gewordenen Los Alamos National Laboratory. Als hauptsächliche „Väter“ der im Santa Fe Institute entwickelten Theorie komplexer adaptiver Systeme zählen vor allem der Informatiker John Holland und der Physiker und Physik-Nobelpreisträger Murray Gell-Mann (Dillon 2001: 5ff).32 Laut Holland kann zwar noch keine Rede davon sein, dass eine übergreifende Theorie zu Komplexität existiere, aber nichtsdestotrotz sind die Ansätze und Ideen des Santa Fe Institute mittlerweile stark ausgearbeitet und werden in verschiedenen wissenschaftlichen Disziplinen verwendet (Holland 2014: 90). Systeme als komplex zu beschreiben bzw. verstehen geschieht dabei nicht zum Selbstzweck. In komplexen Systemen lässt sich beispielsweise nicht einfach zwischen top-down- und bottom-up-Effekten unterscheiden, die beiden Wirkrichtungen treten sehr häufig kombiniert auf. Wie gleich deutlich werden wird, erschweren solche Eigenschaften die Systemanalyse und machen bekannte, traditionelle Analysewerkzeuge, allen voran reduktionistische Herangehensweisen, unbrauchbar. Dafür werden aber Fragen, die sich mit Hilfe der traditionellen Werkzeuge nicht beantworten lassen, zugänglich und können mindestens diskutiert, wenn nicht gelöst werden (Holland 2014: 5).

Wie definieren Komplexitätsforscher Komplexität? Holland zufolge gibt es für das Konzept keine strikte oder strenge Definition. Um sich dem Ganzen zu nähern, schlägt er eine Unterscheidung zwischen komplexen und komplizierten (complicated) Systemen vor. Smith bringt darüber hi-

31 https://www.santafe.edu/ [Stand: 27.6.2019].

32 Stärker populärwissenschaftlich geschrieben, aber nichtsdestoweniger eine Lektüre wert, ist Gell-Manns Werk „The Quark and the Jaguar“, in dem er die Theorie komplexer adaptiver Systeme mit vielen Erkenntnissen aus seiner wissenschaftlichen Arbeit im Bereich der Teilchenphysik verknüpft (siehe Gell-Mann 1994). 
naus noch die Kategorie der einfachen (simple), weder komplizierten noch komplexen Systeme ins Spiel. Ein einfaches System besteht demnach aus einzelnen Elementen und sein Verhalten kann erklärt werden, indem das Verhalten der einzelnen Elemente erklärt und zum Gesamtsystemverhalten aggregiert wird. Einfache Systeme lassen sich demzufolge reduktionistisch erklären. Ihre Bestandteile stehen in einem kausal-deterministischen Zusammenhang zueinander und es reicht aus, diese Zusammenhänge und die Eigenschaften der Systemelemente zu kennen, um das System zu beschreiben. Kausal-deterministisch heißt in diesem Fall, dass der Zusammenhang „a führt zu b“ für das System ceteris paribus, also unter sonst gleichen Umweltbedingungen, immer gilt. Komplizierte Systeme sind im Prinzip ebenfalls noch einfache Systeme, die typischerweise aus einer unüberschaubaren Vielzahl an Elementen und Kausalbeziehungen zwischen diesen Elementen bestehen, deren Verhalten sich aber nach wie deterministisch erklären und vorhersagen lässt, wenn alle Systembestandteile und Kausalbeziehungen zwischen ihnen bekannt sind. Kompliziert sind sie daher im Sinne von „unübersichtlich“ oder auch „praktisch schwer zu verstehen." Theoretisch ist ein kompliziertes System bei vollständiger Information aber reduktionistisch erklärbar. Damit kommt es prinzipiell nur auf die verfügbare Rechenkraft an, ob und wie schnell Systemverhalten korrekt vorhergesagt werden kann (Holland 2014: 3ff, Smith 2002). Holland bedient sich des sehr eingängigen Beispiels einer mechanischen Uhr, um die Eigenheiten komplizierter Systeme zu erklären. Eine mechanische Uhr besteht aus vielen verschiedenen Teilen, von Zahnrädern, Bolzen, Pendeln, einer Unruh, Schwingsystemen und einem Antrieb bis hin zu Zeigern und Zifferblatt. Durch das Zusammenspiel der einzelnen Teile und das dafür notwendige präzise Ineinandergreifen der Rädchen des Uhrwerks ist sie in der Lage, die Uhrzeit anzuzeigen. Für die meisten Menschen, denen die exakte Funktionsweise mechanischer Uhrwerke fremd ist, ist nicht unmittelbar nachvollziehbar, warum und wie die Uhr funktioniert. Nichtdestotrotz verrichten die einzelnen Bestandteile des komplizierten Systems Uhr rein deterministisch ihre Funktion. Und die gesamte Funktionsweise der Uhr lässt sich exakt bis auf ihre einzelnen Teile zurückverfolgen. Um zu wissen, was ein einzelner Bestandteil des Systems tut, reicht die Beobachtung dieses Bestandteils aus. Insgesamt sind die Kausalitäten in der Uhr linear, das heißt aufgrund des Ineinandergreifens der Rädchen bewegen sich die Uhrzeiger (Holland 2014: 5). Mithilfe dieser Definition lassen sich weitere, komplizierte Systeme ausmachen. Etwa Flugzeuge, die zwar beliebig kompliziert aufgebaut sein können, aber - solange sie nicht als sozio-technisches System aufgefasst und Piloten, Besatzung, War- 
tungsmannschaft und Management der betreffenden Fluglinien als Teil des Systems verstanden werden - prinzipiell bekannten und eindeutigen natur- und technikwissenschaftlichen Gesetzmäßigkeiten und Prinzipien zu gehorchen haben. Selbiges gilt beispielsweise für Kraftwerke (Kröger 2011: 73).

Auf der anderen Seite des Spektrums stehen, wie bereits erwähnt, Systeme wie die Aktienmärkte, das Internet, das Energienetz und andere, die üblicherweise als komplex verstanden werden (Kröger 2011: 73). Die Unterscheidung zwischen komplizierten und komplexen Systemen ist dabei empirisch nicht derart trennscharf zu treffen, wie es durch die gerade erfolgte Aufzählung scheint. Ob ein System als kompliziert oder komplex definiert wird, hängt immer auch vom Beobachter (hier nicht im Luhmannschen Sinne), vom Detailgrad der Beobachtung und vom dahinterstehenden Erkenntnisinteresse ab. Das wird auch deutlich, wenn wir auf das Beispiel der Uhr als scheinbar eindeutig kompliziertem - und nicht komplexem - System zurückkommen. Holland sinniert darüber, ob das „Messen der Zeit" eine „emergente“ Eigenschaft des Systems Uhr darstelle, was die Uhr zu einem komplexen System machen würde. Sein Ergebnis: diese Interpretation des Systems ist möglich, aber nicht hilfreich und daher unnötig. Mit dem Verständnis einer Uhr als komplexem System ließen sich keine Fragen beantworten, die nicht bereits durch das Verständnis der Uhr als kompliziertem System klarwerden (Holland 2014: 3ff, Smith 2002). Was also unterscheidet nun komplizierte von komplexen Systemen? Hier kommt der zentrale Grundbegriff der Komplexitätstheorie ins Spiel. Komplexe Systeme weisen emergente Eigenschaften auf. Es ist das Auftreten von Emergenz, das komplexe Systeme von nicht-komplexen Systemen scheidet und sie nach Holland zu einer eigenständigen und wichtigen Teilmenge komplizierter Systeme macht. Umgangssprachlich gefasst lässt sich Emergenz und damit die zentrale Eigenschaft komplexer Systeme beschreiben als die Tatsache, dass das Ganze mehr ist als die Summe seiner Teile. Wenn das zutrifft, schließen sich reduktionistische Erklärungsansätze rein logisch aus. Diese bestehen ja gerade darin, Systeme durch Analyse ihrer Bestandteile und deren Wechselwirkungen untereinander, sozusagen durch „Aufsummierung“ erklären zu können. Sobald Emergenz auftritt, also Eigenschaften, die nur im Gesamtsystem aber eben nicht in seinen einzelnen Elementen beobachtet werden können und die sich durch nichtlineare Interaktionen auszeichnen, reicht das nicht länger aus. Komplexe Systeme können nur systemisch, als Ganzes, sinnvoll analysiert und verstanden werden (Ahmed et al. 2005: 1f, Holland 2014: 2ff, 85, Smith 2002). Zur Verdeutlichung des Prinzips der Emergenz soll ein Beispiel 
Verwendung finden. Für Holland ist die Eigenschaft „nass“ eine emergente Eigenschaft von Wasser, da sie nur auf Ebene des Systems (Wasser), nicht aber auf Ebene der einzelnen Systembestandteile (Wassermoleküle) auftauche. „Nass“ lässt sich nicht aufsummieren und unterscheidet sich somit als Eigenschaft von der Masse des Wassers, die sich aus der Summe der Massen der einzelnen Bestandteile deterministisch ergibt (Holland 2014: 4, 49). Komplexe Systeme weisen selbstverständlich nicht nur emergente Eigenschaften auf. Viele Eigenschaften auch komplexer Systeme lassen sich durch Aufsummierung bzw. deterministische Erklärungsmuster herleiten, nicht jedoch die emergenten. Insgesamt gilt, wie Holland knapp zusammenfasst: "[E]mergent behavior is an essential requirement for calling a system ,complex"” (Holland 2014: 6).

Innerhalb der am Santa Fe Institute entwickelten Komplexitätstheorie wird zwischen zwei verschiedenen Arten komplexer Systeme unterschieden, nämlich komplexen physischen Systemen (CPS) auf der einen Seite und komplexen adaptiven Systemen (CAS) auf der anderen Seite. Komplexe physische Systeme bestehen aus fixen, unveränderlichen Einheiten, die im Zusammenspiel nichtsdestotrotz emergente Eigenschaften in das System einbringen. Das macht sie einer mathematischen Modellierung unter Zuhilfenahme partieller Differentialgleichungen zugänglich. Die Analyse komplexer physische Systeme konzentriert sich auf Zustandsveränderungen und die dahinterliegenden komplexen Dynamiken bestimmter Flussbewegungen. Das komplexe System und seine Strukturen und Regeln an sich bleiben unverändert (Holland 2014: 7ff). Um das Prinzip hinter komplexen physischen Systemen zu verdeutlichen, greift Holland auf das Beispiel des Schmetterlings zurück, dessen Flügelschlag am einen Ende der Welt einen Hurrikan am anderen Ende auslösen könne. Das Verhalten solcher Systeme über die Zeit erscheint also eher chaotisch als deterministisch (Holland 2014: 13). Für bestimmte Systemzustände S(t) zum Zeitpunkt $\mathrm{t}$ lässt sicher aber für komplexe physische Systeme modellieren, welche Handlungsmöglichkeiten dem System als nächstes offenstehen. Denn in komplexen physischen Systemen sind sowohl die Elemente fix und unveränderlich als auch die Regeln, nach denen sie funktionieren, bekannt. Als Beispiel dient Holland hier Schach. Völlig unabhängig davon, wie eine spezifische Situation auf dem Spielbrett zustande gekommen ist, reicht ein Blick auf diese spezifische Situation aufgrund der bekannten Schachregeln aus, um zu wissen, welche Züge ab diesem Zeitpunkt möglich sind (Holland 2014: 19).

Komplexe physische Systeme versteht Holland durchaus im Wortsinn als physikalisch auftretende, natürliche Systeme. Sie unterscheiden sich 
damit von künstlichen einfachen Systemen, wie etwa Maschinen. Und sie unterscheiden sich von „erzeugten“ (generated) komplexen Systemen, die in Form komplexer adaptiver Systeme auftreten. Erzeugt heißt nicht notwendigerweise künstlich erzeugt, auch biologische Systeme können komplex und adaptiv sein. Im Gegensatz zu komplexen physischen Systemen bestehen komplexe adaptive Systeme jedenfalls gerade nicht aus unveränderlichen Elementen, sondern aus „Agenten“, welche die Fähigkeit zur Anpassung haben und diese in der Interaktion mit anderen Agenten sowie der Systemumwelt zu nutzen in der Lage sind. Anpassungsfähigkeit bzw. adaptive capacity wird verschiedentlich auch als Lernfähigkeit bezeichnet. Die Agenten in komplexen adaptiven Systemen sind zudem nicht homogen, sondern heterogen zu verstehen, sie unterscheiden sich also voneinander durch verschiedene Rollen, Attribute, Fähigkeiten, etc. Prinzipiell können Agenten sehr einfach konzeptualisiert werden, was jedoch den Mehrwert der Nutzung von Agenten statt fixen Systemelementen beträchtlich schmälert. Wie auch die Elemente in komplexen physischen Systemen unterliegen die Agenten in komplexen adaptiven Systemen bestimmten Regeln, nach denen sie „handeln“ und die ihre Anpassungsfähigkeit begrenzen. Selbst wenn die Verhaltensregeln einzelner Agenten einfach sind, entsteht aus der Kombination einer Vielzahl autonom entscheidender Agenten schnell ein sehr komplexes System. Das liegt primär an sogenannten „Feedbackschleifen“, also der ständigen Anpassung des Verhaltens eines Agenten an das wahrgenommene Verhalten anderer, die wiederum auf die Veränderungen des Verhaltens des ersten Agenten reagieren, usw. Darüber hinaus reagieren Agenten - und damit das System - auch auf Veränderungen in der Systemumwelt. Bedingt durch das Konzept des Feedbacks erfolgen die Interaktionen der Agenten untereinander in nichtlinearer und häufig auch in nicht-intuitiver Weise. Das macht eine Erklärung und vor allem eine Vorhersage des Verhaltens komplexer adaptiver Systeme extrem voraussetzungsreich (Ahmed et al. 2005: 1, Holland 2014: 9ff, 24, 32, Narzisi et al. 2007: 2, Smith 2002). Gegeben diese Charakterisierung, konzentriert sich eine Analyse komplexer adaptiver Systeme daher auch sehr viel stärker auf vergleichsweise langsam verlaufende Prozesse der Anpassung durch Veränderung der Interaktionsmuster zwischen den Agenten, als auf vergleichsweise schnelle Zustandsveränderungen (Holland 2014: 23). Zumal komplexe adaptive Systeme im Normalfall nicht dazu tendieren, in einem Gleichgewichtszustand zu verharren. Ihr Kennzeichen liegt in der ständigen Veränderung und Anpassung, ermöglicht durch die anpassungsfähigen Agenten aus denen sie bestehen (Holland 2014: 9). 
Diese grundsätzlichen Ideen über komplexe adaptive Systeme und die Agenten, aus denen sie bestehen, können in Verbindung mit den systemtheoretischen Überlegungen des vorangegangenen Unterkapitels dazu genutzt werden, den Zusammenhang zwischen Resilienz und Komplexität im Rahmen eines Resilienz-Konzepts für die zivile SiFo zu untersuchen. Bevor allerdings darauf näher eingegangen wird, sollen noch einige weitere Grundannahmen und Begrifflichkeiten, mit denen die Wissenschaftler des Santa Fe Instituts innerhalb ihrer Komplexitätstheorie arbeiten, vorgestellt werden. Dazu muss angemerkt werden, dass das Ziel der genannten Wissenschaftler primär darin besteht, mathematisch formalisierbare Regeln zur Modellierung und Simulation komplexer adaptiver Systeme zu identifizieren. Dieses Ziel unterscheidet sich von dem der vorliegenden Arbeit, der es stärker darum geht, einen sozialwissenschaftlichen Handlungsrahmen für ingenieurwissenschaftliche zivile SiFo im Sinne eines Resilience Engineering aufzuspannen und die dazu zunächst das ResilienzKonzept an sich für diesen Bereich zu spezifizieren sucht. Deshalb werden im Folgenden ausschnittartig Elemente der Theorie komplexer adaptiver Systeme erläutert, die in diesem Kontext einen Mehrwert versprechen.

Holland selbst bezeichnet die theoretischen Ansätze des Santa Fe Instituts auch als frühe Versionen einer noch weiter auszuarbeitenden Theorie, hält diese Ausarbeitung aber prinzipiell für möglich, da es Eigenschaften gebe, die allen komplexen adaptiven Systemen gemein seien. Dazu zählt er eine hierarchische Organisation. Demzufolge sind die Regeln, nach denen komplexe adaptive Systeme funktionieren auf höheren Ebenen immer konditional von denen auf niedrigeren Ebenen abhängig. Diese macht es laut Holland etwas einfacher, eine (mathematische) Theorie zu entwickeln. Die hierarchische Organisation ergibt sich aus zwei Tatsachen. Erstens sind komplexe adaptive Systeme „erzeugte“ Systeme, die sich von einem Ausgangspunkt aus entwickeln und nicht als sozusagen "fertiges“ System entworfen werden. Bestimmten hierarchischen Regeln zu folgen, ist zweitens vorteilhaft für die Evolution des Systems. Holland erklärt das am Beispiel der Beschreibung einer aus verschiedenen Bauklötzen bestehenden Ritterburg. Wenn diese Beschreibung für jedes Bauklötzchen dessen Position relativ zu allen anderen Bauklötzchen enthält, wird sie sehr kompliziert und unverständlich. Wenn die Beschreibung dagegen zwischen Bereichen der Burg (etwa Türme, Tore, Mauern, etc.) unterscheidet und die Position der Bauklötzchen relativ zu diesen größeren Bereichen angibt, sowie deren Position wiederum relativ zueinander, wird sie insgesamt einfacher. Die Einführung unterschiedlicher Bereiche entspricht dabei der Einführung einer Hierarchieebene. Die Ritterburg aus Bauklötz- 
chen ist zwar ein Beispiel eines einfachen erzeugten Systems, das Prinzip gilt jedoch äquivalent auch für komplexe adaptive Systeme. Hierarchische Organisation ergibt sich in solchen Systemen, weil sie evolutionär vorteilhaft ist (Holland 2014: 52f).

$\mathrm{Zu}$ den Eigenschaften, die allen komplexen adaptiven Systemen gemeinsam seien, zählt Holland ebenfalls die Existenz sogenannter „lever points“, Hebelpunkte in der direkten Übersetzung, an denen kleine, zielgerichtete Handlungen zu gravierenden aber vorhersagbaren Veränderungen im Systemverhalten führen. Holland zufolge funktioniert die Suche nach solchen Hebelpunkten aktuell nach dem Prinzip Versuch und Irrtum. Wenn Hebelpunkte eine systemische Eigenschaft komplexer adaptiver Systeme sind, würde eine entsprechende Theorie dagegen dabei helfen, die Mechanismen zu identifizieren, die für das Entstehen von Hebelpunkten verantwortlich sind und somit eine systematische Identifikation ermöglichen (Holland 2014: 25, 32ff). Hollands Hebelpunkte erinnern nicht nur zufällig an die im vorangegangenen Kapitel schon mehrfach erwähnten Umkipp-Punkte oder Schwellenwerte. Sie beschreiben denselben Mechanismus, enthalten allerdings einen entscheidenden Unterschied. Hebelpunkte sind nur in Bezug auf ihre Lokalisierung Unsicherheiten unterworfen, nicht aber was ihre Auswirkungen angeht. Umkipp-Punkte oder Schwellenwerte sind dagegen in doppelter Hinsicht unsicher, sowohl was ihre Lokalisierung, als auch was ihre Auswirkungen auf das System betrifft (Wink 2016: 4). Hebelpunkte, Umkipp-Punkte oder Schwellenwerte in komplexen adaptiven System zuverlässig - und idealerweise a priori identifizieren zu können, wäre zur Erhöhung der Resilienz der für die zivile SiFo relevanten sozio-technischen Systeme äußerst hilfreich. Das legt zumindest nahe, die Verwendung der vom Santa Fe Institut entwickelten Theorie komplexer adaptiver Systeme als möglichen Bestandteil von Resilience Engineering in Betracht zu ziehen (siehe 6.2 und 6.3).

Innerhalb der Theorie ergibt es nach Holland keinen Sinn, von vollständig rational handelnden Agenten auszugehen. Vollständige Rationalität erfordert vollständige Information und diese wiederum setzt vollständiges Wissen über alle vergangenen, aktuellen und zukünftigen Handlungen aller Agenten des Systems und seiner Umwelt voraus - eine selbst in einfachen Systemen kaum jemals zu erfüllende Bedingung. Gegeben die Existenz von Feedbackschleifen im System, muss die Annahme vollständig rational handelnder Agenten verworfen werden. Holland setzt für die Theorie komplexer adaptiver Systeme auf „begrenzte Rationalität“ (bounded rationality), nach der die Agenten in Systemen versuchen, unter den gegebenen Informationen optimal zu handeln. Im Lauf der Zeit erfahren 
sie, wie andere Agenten und die Umwelt handeln bzw. auf ihr eigenes Handeln reagieren und passen ihre Handlungen entsprechend an, um ihr Ziel möglichst gut erreichen zu können. Die ständige Anpassung führt zu Systemen, innerhalb derer große Diversität herrscht, da nicht davon auszugehen ist, dass alle Agenten sich immer in der gleichen Weise anpassen. Und da Agenten ihre jeweils eigenen Ziele verfolgen, resultiert das Verhalten des Gesamtsystems in den meisten Fällen nicht aus den Vorgaben einer zentralen Autorität, sondern aus den komplexen Abhängigkeiten der Handlungen der einzelnen Agenten (Holland 2014: 24f). Um Agenten überhaupt die Interaktion mit anderen Agenten innerhalb des Systems sowie der Systemumwelt zu ermöglichen, stattet Holland sie mit Detektoren und Effektoren aus. Detektoren sind dazu da, externe Signale intern so zu verarbeiten, dass der Agent damit etwas anfangen kann, Holland nennt Geruchs- und Tastsinn als Beispiele. Die interne Verarbeitung externer Signale erlaubt prinzipiell ein Einbeziehen beliebiger Signale in das System. Effektoren wiederum ermöglichen eine direkte Beeinflussung der Umwelt, beispielsweise durch Muskeln (Holland 2014: 27).

Die Handlungen von Agenten konzeptualisiert Holland mithilfe dreier Arten von Aktivitäten. Zunächst stehen einem Agenten bestimmte „Leistungen“ (performance) zur Verfügung. Zu jedem bestimmten Zeitpunkt kann er eine Reihe von Handlungen ausführen, formalisiert durch bedingte WENN/DANN-Regeln. Zur Veranschaulichung nimmt Holland einen einfachen, evolutionär allerdings durchaus relevanten Fall: WENN (etwas in dein Blickfeld kommt) DANN (flieh). Diese bedingten Regeln treten im Normalfall nicht isoliert auf. Sie bilden bestimmte Regelketten, von denen es laut Holland nur eine relativ geringe Zahl benötigt, um jedes denkbare Verhalten abzubilden. Wie schon erwähnt, geht es dem Santa Fe Institut darum, eine formalisierbare Theorie zu entwickeln, die zum Beispiel durch Computersimulationen angewendet werden kann. Dazu beschreibt Holland den Austausch von Signalen zwischen Agenten in Form von Netzwerken, die aus Kanten und Knoten bestehen. Die dahinterstehende mathematische Graphentheorie wird generell häufig zur Analyse von Komplexität genutzt. Bestimmte WENN/DANN-Regeln legen fest, wie Signale von einem Knoten zum nächsten transportiert werden, also welche Kanten (Verbindungen zwischen zwei Knoten) im Netzwerk existieren. Komplexität kommt auf zwei Arten in diese zunächst linearkausal und deterministisch wirkende Beschreibung. Zum einen existieren typischerweise viele Feedbackschleifen in den Netzwerken, so dass bestimmte Regeln wieder auf sich selbst verweisen. Zum anderen passen die Agenten ihr Handeln über die Zeit an, in dem sie die handlungsleitenden WENN/ 
DANN-Regeln verändern (Holland 2014: 17, 26ff). Dazu nutzen Agenten eine Aktivität, die als „Kreditvergabe“ (credit-assignment) bezeichnet wird. Gerade war bereits die Sprache davon, dass Agenten Erfahrungen sammeln und ihr Handeln entsprechend ihrer Erfahrungen anpassen. Dazu gehört es auch, die bisher genutzten Regeln zur Signalverarbeitung im Hinblick auf ihre Nützlichkeit zu überprüfen. Manche Regeln sind nützlicher als andere, einige vielleicht sogar schädlich für die Erreichung der Ziele des Agenten. Um das beurteilen zu können, werden die Regeln untereinander qualifiziert und mit einem Attribut namens Stärke (strength) versehen. Unmittelbare Bedürfnisbefriedigung lässt sich sehr leicht mit großer Stärke in Verbindung bringen. In komplexen adaptiven Systemen kann es aber sein, dass bestimmte Handlungen erst im späteren Zeitverlauf zu einem höheren Nutzen führen und unmittelbar vielleicht sogar Kosten verursachen. Dann ist es sinnvoll, die Stärke einzelner Regeln miteinander in Verbindung zu bringen, so dass ein späterer, höherer Nutzen sich bereits in der Stärke zeitlich vorgelagerter Regeln ausdrückt (Holland 2014: 28f). Sobald Agenten in der Lage sind, Regeln in dieser Art zu bewerten, werden sich nützliche, bessere und weniger nützliche, oder gar schädliche Regeln voneinander scheiden lassen. Die Agenten haben ein Interesse daran, die nützlichen Regeln zu bewahren und weiter zur Erreichung ihrer Ziele zu nutzen. Gleichzeitig haben sie ein Interesse daran, weniger nützliche, Holland nennt sie „schwache“ (weak) Regeln möglichst durch neue zu ersetzen. Hier kommt nun die Anpassungsfähigkeit, die adaptive capacity, der Agenten zum Tragen. Die dritte Art von Aktivitäten, die Agenten in komplexen adaptiven Systemen zur Verfügung steht, ist die „Regelerkennung“" (rule-discovery). Es geht um die Frage, wie Agenten die Wahrscheinlichkeit maximieren können, dass neue Regeln, mit denen sie die alten, schwachen ersetzen, tatsächlich zu besseren Ergebnissen führen. Eine Möglichkeit, schwache Regeln zu ersetzen, besteht in der Wahl einer neuen, zufällig generierten Regel. Die Wahrscheinlichkeit, auf diese Weise zu einer besseren Regel zu kommen, ist allerdings verschwindend gering. Demzufolge muss die Wahl einer neuen Regel zielgerichtet erfolgen. Dazu können Agenten ihre bis zum relevanten Zeitpunkt gesammelten Erfahrungen nutzen. Holland führt den Begriff der „Bausteine“ (building blocks) ein, die Agenten bei der Auswahl sinnvoller neuer Regeln nutzen können. Ein Baustein besteht aus Handlungsweisen oder Ergebnissen, die sich durch das Befolgen starker Regeln in verschiedenen Fällen schon als nützlich erwiesen haben. Empirisch entstehe Innovation in komplexen adaptiven Systemen zumeist durch eine kombinierte Verwendung bekannter Bausteine in neuer Art und Zusammensetzung. Anpassungsfähigkeit zeigt 
sich demnach darin, verschiedene, bekannte Bausteine so miteinander zu kombinieren, dass starke Regeln resultieren (Holland 2014: 30f).

Verwendet man erneut die Netzwerk-Analogie, können einzelne Agenten als Knoten verstanden werden, die zu jedem Zeitpunkt eine bestimmte Anzahl an Möglichkeiten zur Interaktion mit anderen Agenten haben, repräsentiert durch die Zahl der mit dem Knoten verbundenen Kanten. Holland zufolge liege diese Zahl bei Maschinen typischerweise im niedrigen zweistelligen Bereich, während komplexe adaptive Systeme durchaus einen "Grad“ (degree) von 1.000 oder mehr erreichen könnten. In solchen Systemen existieren typischerweise "Gemeinschaften" (communities), in denen die Verknüpfungen der einzelnen, beinhalteten Knoten untereinander hauptsächlich auf andere Knoten innerhalb der Gemeinschaft verweisen. Je größer die Prozentzahl der innerhalb einer Gemeinschaft aufeinander zurückführenden Verknüpfungen, desto „enger“ (tight) ist diese. Zudem zeichnen sich komplexe adaptive Systeme durch Feedbackschleifen (loops) aus, die eine zirkuläre Signalverarbeitung ermöglichen. Durch Schleifen können Signale sowohl verstärkt als auch abgeschwächt werden, je nach Wirkrichtung. In Gemeinschaften existieren häufig mehr solcher Schleifen als zwischen oder außerhalb von Gemeinschaften (Holland 2014: 38f). Die Existenz von Gemeinschaften ist konstitutiv für die Ausbildung von Hierarchien in komplexen adaptiven Systemen. Sie begünstigt auch die Entwicklung spezialisierter Interaktionsmuster und Agenten. Holland argumentiert hier mit Adam Smith, wonach Arbeitsteilung innerhalb eines Systems dessen Produktivität erhöht, weil Spezialisten ihre spezifische, kleinteilige Aufgabe besser zu erfüllen in der Lage seien als Generalisten und durch das Zusammenwirken vieler Spezialisten ein größerer Output, eine höhere Systemleistung, erreicht werden kann, als durch die Zusammenarbeit vieler Generalisten (Holland 2014: 42f). Ein Trend hin zu zunehmender Spezialisierung ist für Holland basierend auf empirischen Beobachtungen als immanentes Merkmal komplexer adaptiver Systeme zu sehen. Spezialisierung geht einher mit größerer Diversität, da die Zahl unterschiedlicher Agenten und Signalverarbeitungsmuster zunimmt. Die Signale können selbst kompliziert werden. Allerdings existieren in komplexen adaptiven Systemen sogenannte „Label“ (tags), verstanden als relativ simple Bestandteile von Signalen, die darüber bestimmen, wie diese verarbeitet werden, welche Signalverarbeitungsmuster zur Anwendung kommen. Weiter oben wurde Kreditvergabe über mehrere Regeln, also Signalverarbeitungsmuster, hinweg angesprochen. Label ermöglichen es, schneller über die Stärke von Regeln zu urteilen, als eine Beachtung des gesamten Signals. Die so gewonnene Zeit nutzen komplexe adaptive 
Systeme zur Prüfung weiterer Kombinationen an verschiedenen Signalverarbeitungsmustern, was letztlich in einer weiteren Spezialisierung und Erhöhung der Diversität resultiert (Holland 2014: 44ff). Die Art der Signalverarbeitung ist für Holland zentral für komplexe Systeme. Nachdem er die beiden Begriffe Bausteine und Label eingeführt hat, bringt er sie dann logisch zusammen. Etablierte Label können als Bausteine dienen, um die Produktivität von Systemen, bzw. generell ihre Funktionsfähigkeit, zu erhöhen. Verschiedene, etablierte Label in neuer Weise zu kombinieren führt zu Emergenz, der charakteristischen Eigenschaft komplexer Systeme. Auf Ebene der Systemanalyse wiederum bestehen Agenten gerade aus spezifischen Kombinationen von Bausteinen, so dass durch deren zielgerichtete Neu-Anordnung zugleich neue Agenten entstehen, aus denen das komplexe adaptive System besteht. Anpassungsfähigkeit heißt - abstrakt gesprochen - damit gleichzeitig Entstehung neuer Agenten (Holland 2014: $56,76)$.

Den Zusammenhang zwischen Bausteinen und Emergenz versucht er anhand eines sehr konkreten Beispiels, nämlich des menschlichen Gesichts, näher zu erläutern. Um ein derart komplexes Objekt charakterisieren zu können, wird nach unterscheidbaren Merkmalen gesucht, etwa die Form der Nase, Farbe der Augen, Haarfarbe und viele mehr. Jedes dieser Merkmale kann spezifische Ausprägungen annehmen, wobei jede Ausprägung eines jeden Merkmals einen eigenen Baustein im Sinne Hollands ausmacht. Ein Gesicht besteht nun aus einer spezifischen Kombination von Bausteinen. Angenommen die Zahl der relevanten Merkmale eines Gesichts beträgt lediglich zehn und jedes Merkmal besitzt zehn unterschiedliche Ausprägungen, resultieren daraus 100 Bausteine, die zu zehn Milliarden unterschiedlichen Gesichtern zusammengesetzt werden können. Die Zusammensetzung erfolgt aber nicht willkürlich. Es gibt bestimmte Regeln, die eingehalten werden müssen und legitime Kombinationen der verschiedenen Merkmale darstellen. Holland nutzt das Gesichts-Beispiel hier sehr plastisch, indem er die Regel „Der Mund gehört unter die Nase“ erwähnt. Zur Unterscheidung verschiedener Gesichter voneinander müssen die Bausteine bekannt sein. Hier wird die Verknüpfung zwischen Labels und Bausteinen deutlich. Nicht alle denkbaren Bestandteile eines Gesichts, abstrakter formuliert als „Signale“, sind dazu geeignet, diese voneinander zu unterscheiden. Bausteine - oder Labels - sind diejenigen Bestandteile - oder Signale - eines Gesichts, die eine Unterscheidbarkeit ermöglichen. Für die Analyse komplexer adaptiver Systeme lässt sich das Beispiel wieder abstrahieren und formulieren, dass durch den Vergleich verschiedener komplexer adaptiver Systeme Phänomene, die in einem Sys- 
tem nur versteckt stattfinden und nicht zu erkennen sind, unter Umständen durch ähnliche Phänomene in anderen Systemen, die dort offen zu Tage treten, besser erklärt werden können (Holland 2014: 50ff). Worin besteht nun nach Holland die Komplexität des Objekts Gesicht? Wo sieht er Emergenz am Werk? Komplex ist ein Gesicht deshalb, weil seine einzelnen Bestandteile erst im Zusammenspiel ein Gesicht ergeben und nicht einfach „aufaddiert“ werden können. An dieser Stelle ist die Verknüpfung der Theorie komplexer adaptiver Systeme zu mathematischen Formalismen erneut sichtbar. Mathematisch gesprochen ist es nicht möglich, die einzelnen Bausteine zu einem Gesicht zu summieren, das Gesicht als solches ist also etwas Neues, das erst auf Systemebene entsteht. Entscheidend ist die Nichtlinearität der kausalen Zusammenhänge (Holland 2014: 54).

In komplexen adaptiven Systemen sind häufig co-evolutionäre Prozesse zwischen den verschiedenen Agenten verantwortlich für diese Nichtlinearität. Unter Co-Evolution wird eine Weiterentwicklung von Agenten in gegenseitiger Abhängigkeit verstanden. Wenn zwei Agenten zur Erreichung ihrer jeweiligen Ziele aufeinander angewiesen sind, führt eine Entwicklung eines der beiden Agenten, die ihn im Vergleich zum zweiten Agenten bevorteilt, dazu, dass dieser in einem evolutionären Prozess seine Regeln durch Neuanordnung seiner Bausteine anpasst. Das wiederum wird auch den ersten Agenten dazu veranlassen, seine Regeln zur Signalverarbeitung wieder anzupassen, usw. Im Verhältnis der beiden Agenten ändert sich durch diese Co-Evolution auf lange Sicht zwar nichts, im Verhältnis zu ihrer Umwelt haben sich die Agenten jedoch gemeinsam weiterentwickelt. Co-Evolution wird häufig durch Änderungen der relevanten Labels erreicht. Aufgrund ihrer Bedeutung für die Wahl der geeigneten Regeln, können bereits kleine Änderungen in den verwendeten Labels zu großen Änderungen in den Signalverarbeitungsmustern führen. Komplexe adaptive Systeme bestehen allerdings aus einer Vielzahl an Agenten, so dass das System an sich auch dann relativ unverändert bleibt, wenn einzelne Agenten stark abweichende Regeln ausprobieren. Holland fasst das treffend zusammen: „a complex system can explore alternatives while exploiting what it already finds useful" (Holland 2014: 54f). Co-Evolution führt dann häufig zur Ausbildung sogenannter „Nischen”, in denen aufeinander angepasste und voneinander abhängige Agenten in einem relativ stabilen Gleichgewicht miteinander koexistieren können. Nischen entstehen durch wiederholte Verwendung der immer gleichen Bausteine und sie führen dazu, dass sich die in ihnen handelnden Agenten immer weiter spezialisieren. Diese hochspezialisierten Agenten sind in der Lage, die vorhandenen, begrenzten Ressourcen möglichst effizient einzusetzen. 
Das macht die Ausbildung derartiger Nischen evolutionär sinnvoll. Anders formuliert: Regeln, die einen effizienten Umgang mit Ressourcen ermöglichen, setzen sich in komplexen Systemen durch, in dem die Label, mit deren Hilfe die Anwendung der Regeln getriggert werden, langfristig zu Bausteinen des Systems werden. Allerdings sind Nischen auch generell anfällig für das Auftreten überraschender oder unerwarteter Ereignisse, was Holland interessanterweise am Beispiel einer Nische im übergreifenden Ökosystem, bestehend aus einer Beute- und einer Jäger-Population, verdeutlicht. Bedingt durch das Aufkommen einer dritten Spezies im System, ebenfalls ein Jäger, geht die Beute-Population derart zurück, dass die hochspezialisierte ursprüngliche Jäger-Population zusammenbricht. Eine nischen-spezifische Anpassungsfähigkeit, die den evolutionären Erfolg der beiden Populationen in ihrer gegenseitigen Abhängigkeit voneinander erst möglich gemacht hat, führt also im Moment des Auftretens gravierender, überraschender, externer Störereignisse zu Problemen (Holland 2014: 59ff, 67ff, 73).

Nicht nur an diesem letzten Punkt wird deutlich, dass die Anknüpfungspunkte zwischen der Theorie komplexer adaptiver Systeme und der Resilienzforschung unübersehbar sind. Holland verwendet den ResilienzBegriff in seinem Einführungswerk zum Thema Komplexität auch selbst an zwei Stellen. Zunächst bei einer Beschreibung co-evolutionärer Entwicklungen zwischen dem Menschen und ihn besiedelnden, gutartigen Bakterien. Die gutartigen Bakterien leben mit den körpereigenen Zellen des Menschen in einem grundsätzlich stabilen Gleichgewicht. Sie nutzen Ressourcen, die ansonsten von bösartigen Bakterien zum Schaden des Körpers genutzt werden könnten, um selbst zu überleben: „They have co-evolved with the body to greatly enhance the overall resilience of the whole system, through cross-species exchange of genes (horizontal transfer) some genes of bacterial origin even appear in the human chromosomes" (Holland 2014: 62, eigene Hervorhebung). Holland verwendet Resilienz hier als nicht weiter erklärungsbedürftigen Begriff. Sinngemäß ließe er sich an dieser Stelle relativ treffend mit „Widerstandsfähigkeit“ übersetzen. Die zweite Erwähnung des Resilienz-Begriffs erinnert unmittelbar an Hollings Definition. Holland diskutiert die Frage, was den Menschen als Spezies derart erfolgreich macht, was es ihm erlaubt in der "human niche“ zu überleben. Er stellt diese Frage als Frage nach den Charakteristika, die von Generation zu Generation weitergegeben werden und fortbestehen: „What is preserved and how?" Er verallgemeinert die Frage dann weg vom Menschen hin zu komplexen adaptiven Systemen per se und kommt zu folgender Antwort: „Indeed, the resilience of a CAS when confronted with 
,shocks' (invasive species, new trade conventions, or the like) generally depends upon inherited persistent characteristics" (Holland 2014: 79, eigene Hervorhebung des Worts resilience + Hervorhebung im Original der Worte persistent characteristics). Die in diesem Satz enthaltene Definition von Resilienz ist beinahe wortgleich zu der von Holling, der bei Resilienz ja von der Fähigkeit eines Systems sprach, mit extern verursachten, gravierenden und disruptiven Veränderungen der Umwelt fertig werden zu können. Bei Holling ist „persistence“, das Fortbestehen des Systems, oder wie er auch sagt "to stay in the game“, das Ziel, in dessen Erreichen sich Resilienz ausdrückt (Holling 1973: 14ff). Für Holland sind bestimmte, immer weiter vererbte „persistent characteristics“ entscheidend für Resilienz. Das klingt zunächst wie eine Tautologie: „persistent characteristics“ ermöglichen Resilienz ermöglicht „persistence“. Allerdings schließt Holland mit seiner Formulierung die Vererbung weiterer, die Identität des Systems bestimmender Charakteristika, die nicht direkt mit Resilienz in Verbindung stehen, nicht aus. Und exakt das Fortbestehen der identitätsstiftenden Charakteristika von Systemen meint Holling mit seinem „persistence“-Begriff. Die Identifikation von Resilienz-begünstigenden „persistent characteristics" in den für die zivile SiFo relevanten sozio-technischen Systemen, könnte unter Umständen mithilfe der Anwendung der Theorie komplexer adaptiver Systeme sowie verwandter Konzepte, wie etwa dem von Holland entwickelten mathematischen Optimierungsprinzip der genetischen Algorithmen, ermöglicht werden (Holland 2014: 79).

Dass Hollings Resilienz-Konzept immer wieder im Spektrum der Komplexitätstheorie bzw. konkret der Theorie komplexer adaptiver Systeme verortet wird, verwundert nicht mehr. Die meisten Arbeiten im Bereich der ökologischen, sozial-ökologischen und sozialwissenschaftlichen Resilienzforschung nutzen die Theorie allerdings nur als relativ grobe Orientierung. Fasst man die Ansätze des Santa Fe Instituts nach der Beschreibung von Holland zusammen, bieten sich insgesamt drei Diskussionsstränge, denen im Einzelnen gleich näher nachgegangen wird. Holland zufolge unterscheiden sich komplexe von nicht-komplexen Systemen durch die Existenz emergenter Eigenschaften. Eine emergente Eigenschaft wird erst auf Systemebene wirkmächtig und lässt sich daher nicht durch eine reduktionistische Analyse einzelner Systemelemente erklären. Es existieren mindestens zwei Arten komplexer Systeme. Komplexe physische Systeme einerseits, die aus fixen, unveränderlichen Elementen bestehen. Und komplexe adaptive Systeme andererseits, die in der vorliegenden Arbeit als die „eigentlichen“ komplexen Systeme verstanden werden. Die Elemente komplexer adaptiver Systeme sind Agenten. Agenten versuchen im Rah- 
men ihrer begrenzten Rationalität bestimmte Ziele zu erreichen, indem sie Signale verarbeiten. Die Regeln oder Muster, nach denen das geschieht werden dabei von den Agenten nach ihrer Nützlichkeit zur Zielerreichung bewertet und gegebenenfalls ersetzt. In der Ersetzung ungeeigneter Signalverarbeitungsmuster besteht die Anpassungsfähigkeit von Agenten. Diese werden nicht durch zufällige neue Muster ersetzt, sondern durch eine Rekombination bekannter und im vorherigen Zeitverlauf als nützlich erkannter Bausteine. Das neue Muster bzw. die neue Regel besteht in der neuen Art der Anordnung bekannter Bausteine. Um komplizierte Signale besser verarbeiten zu können, nutzen die Signalverarbeitungsmuster sogenannte Labels, also kleine Teile der Signale, deren Aufgabe spezifisch darin besteht, die Verarbeitung in einer bestimmten Weise zu triggern. Im Lauf der Zeit entwickeln sich die Agenten in komplexen adaptiven Systemen zu Spezialisten weiter, da diese die knappen zur Verfügung stehenden Ressourcen effizienter zu nutzen in der Lage sind, als Generalisten. Dieses Prinzip der Arbeitsteilung ist möglich, weil Agenten in komplexen adaptiven Systemen in ihrem Verhalten vom Verhalten anderer Agenten abhängen. Das führt zur Bildung von Feedbackschleifen und ist verantwortlich für das nichtlineare Verhalten komplexer adaptiver Systeme.

Vor diesem Hintergrund stellt sich zunächst die Frage, ob die für die zivile SiFo relevanten sozio-technischen Systeme als komplexe adaptive Systeme zu charakterisieren sind. Sollte dies nicht zutreffen, wäre der $\mathrm{Zu}$ sammenhang zwischen Resilienz und Komplexität für ein Resilienz-Konzept der zivilen SiFo im Prinzip irrelevant. Der nächste relevante Aspekt besteht dann im konkreten Verhältnis von Resilienz und Komplexität zueinander, auch unter Rückgriff auf die systemtheoretischen Überlegungen des vorangegangenen Unterkapitels. Macht Komplexität Resilienz notwendig oder ermöglicht Komplexität Resilienz, treffen diese Aussagen womöglich beide gleichzeitig zu oder ist genau das nicht der Fall? Der dritte interessante Aspekt beschäftigt sich mit Hollands Aussagen zur evolutionären Entwicklung komplexer adaptiver Systeme, zur Co-Evolution, zunehmender Spezialisierung und zur Herausbildung immer effizienterer Nischen. Hier liegt der Fokus auch vor allem darauf, wie Anpassungsfähigkeit im Rahmen der Theorie komplexer adaptiver Systeme verstanden wird.

Die zivile Sicherheitsforschung in Deutschland zielt, wie bereits im Eingangskapitel dargestellt, darauf ab, „Lösungen, die den Schutz der Bevölkerung und der kritischen Infrastrukturen vor Bedrohungen durch Terrorismus, Sabotage, organisierte Kriminalität, Piraterie, aber auch vor den Folgen von Naturkatastrophen und Großunfällen gewährleisten und einen Beitrag zum Schutz unseres freiheitlichen Lebensstils leisten“ zu ent- 
wickeln (BMBF 2016: 3). Damit beschäftigt sich zivile SiFo grundsätzlich mit zwei Themenkomplexen. Zum einen dem Schutz der Bevölkerung vor den genannten Bedrohungen. Und zum anderen der Sicherheit kritischer Infrastrukturen, wobei für letztere ihre Kritikalität immer in Abhängigkeit der Funktionen zu verstehen ist, die sie für die Bevölkerung erfüllen. Eine Schädigung bzw. ein Ausfall kritischer Infrastrukturen wirkt sich immer auch negativ auf die Sicherheit der Bevölkerung aus, unter Umständen versehen mit einer zeitlichen Verzögerung (siehe 1.3). Im von 2012 bis 2017 laufenden zweiten Rahmenprogramm der deutschen Bundesregierung zur Forschung für zivile Sicherheit wurden die zwei Themenkomplexe in vier verschiedenen Bereichen adressiert. Im ersten Bereich ging es um Innovationen für die Sicherheit kritischer Infrastrukturen. Die Forschung sollte darauf abzielen, Verwundbarkeiten der kritischen Infrastrukturen frühzeitig zu realisieren und deren Robustheit entsprechend zu stärken. Der zweite Bereich war mit "Sicherheit der Wirtschaft“ überschrieben und befasste sich mit Forschung zum Schutz explizit mittelständischer Unternehmen sowie Betreiber kritischer Infrastrukturen vor Bedrohungen durch Naturgefahren und organisierte Wirtschaftskriminalität. Im dritten Bereich stand eine spezifische kritische Infrastruktur, nämlich der durch den Cyberraum dargestellte Teil der Informationsinfrastrukturen im Mittelpunkt. Dieser sollte aufgrund der immer dominanteren Rolle, die er im Alltagsund Wirtschaftsleben spielt, durch innovative Forschungsideen besser vor Angriffen geschützt werden, ohne dabei Datenschutz und Persönlichkeitsrechte zu kompromittieren. ${ }^{33}$ Und der vierte Bereich war generell der "Sicherheit der Bürgerinnen und Bürger" gewidmet. Sicherheitsforschung sollte sich an gesellschaftlichen Fragestellungen ausrichten, „die Bürgerinnen und Bürger in ihrem unmittelbaren Lebensumfeld betreffen." Ziel aller Anstrengungen der zivilen SiFo war - und ist - ja gerade, den „Schutz der Bevölkerung und ihrer Lebensgrundlagen sicherzustellen.“ Dieser Bereich des Rahmenprogramms sollte zudem dazu dienen, Sicherheitsforschung bzw. das Thema Sicherheit stärker im Alltag zu verankern und „einen breiten gesellschaftlichen Dialog zur Ausgestaltung der zivilen Sicherheit in Deutschland" anzustoßen (BMBF 2016: 3f). Das Bundesministerium für Bildung und Forschung erarbeitet gerade das dritte Rahmenprogramm zur Forschung für zivile Sicherheit, so dass die Fokussierungen des vergangenen Programms unter Umständen ein Stück weit revidiert

33 Siehe zum Thema Digitalisierung sowie Sicherheit/Resilienz und Digitalisierung u.a. Neugebauer 2018 und darin die Beiträge von Waidner sowie Hiermaier/Scharte (siehe Neugebauer 2018). 
werden könnten. ${ }^{34}$ Erste Hinweise darauf, wie das nächste Rahmenprogramm ausgestaltet sein könnte, können Positionspapieren entnommen werden, die von relevanten Gremien im Bereich der zivilen SiFo erarbeitet wurden. ${ }^{35}$ So hat der Wissenschaftliche Programmausschuss für zivile Sicherheitsforschung (WPA), ein unabhängiges Expertengremium, dessen Aufgabe in der Beratung des BMBF zu Fragen der SiFo besteht, für dieses dritte Rahmenprogramm eine Reihe von Empfehlungen veröffentlicht. Darin identifiziert der WPA insgesamt fünf „Themencluster“ und zwei sogenannte „Überschneidungsthemen“. Die Themencluster I „Sicherheit kritischer Infrastruktur" und II "Sicherheit und Digitalisierung - neue Chancen, neue Vulnerabilitäten" werden durch das Überschneidungsthema „Kommunikationsinfrastrukturen“ miteinander verbunden, die Themencluster III „Sicherheitsverantwortung im Kontext gesellschaftlicher Veränderungen“ und IV „Verteilung von Sicherheitsverantwortung - Staat, Wirtschaft, Bevölkerung“ durch das Überschneidungsthema „Organisierte Kriminalität“. Der Themencluster V „Globale Veränderungen“ ist generell als Querschnittsthema zu sehen. Relevante Stichwörter innerhalb dieses Clusters sind grenzüberschreitende Themen wie Ressourcensicherheit, der Klimawandel oder Migration (WPA 2017: 7ff). Unabhängig davon, ob das nächste Rahmenprogramm der Bundesregierung die vorgeschlagenen Themencluster übernehmen wird, werden aus der Übersicht in Verbindung mit den vier Bereichen des zweiten Rahmenprogramms die relevanten Fragestellungen der zivilen SiFo und damit die relevanten Systeme deutlich. Es geht um die Sicherheit der gesamten Bevölkerung, also der Gesellschaft. Je nach Bedrohung wird das übergreifende System Gesellschaft räumlich konkretisiert, zum Beispiel, wenn der Schutz bestimmter Kommunen vor Extremwettereignissen erhöht werden soll. Oder bei der Frage danach, wie für Terroristen potentiell attraktive Zielorte, von Weihnachtsmärkten über Volksfeste bis hin zu anderen Großveranstaltungen jeder Art, geschützt werden können. Daneben stehen die kritischen Infrastrukturen der Gesellschaft explizit im Fokus der zivilen SiFo. Diese können aufgrund der Interaktion ihrer technischen Bestandteile mit Betreibern, Verbrauchern, Nutzern, etc. nicht als rein technische Systeme konzipiert bzw. verstanden werden. Vielmehr sind kritische Infrastrukturen sozio-technische Systeme. Selbiges gilt auch dann, wenn die Konkretisierung der interessierenden

34 Stand: 5.3.2018.

35 Mittlerweile [Stand: 27.6.2019] wurde das neue Sicherheitsforschungsprogramm veröffentlicht. Es entspricht im Wesentlichen den hier genannten Inhalten. Daher kann die Analyse so stehenbleiben. 
Systeme nicht über ihre Zuordnung zu den kritischen Infrastrukturen, sondern über eine räumliche Eingrenzung erfolgt. Auch in Kommunen oder im Spezialfall bedrohter Veranstaltungsorte sind neben den sozialen Aspekten immer auch technisch-physikalische oder technisch-virtuell/digitale Aspekte zu beachten, um die Bevölkerung schützen zu können.

Sind diese Systeme komplex? Oder spezifischer gefragt: Handelt es sich um komplexe adaptive Systeme? In diesem Fall müssten die gerade identifizierten sozio-technischen Systeme eine Reihe von Eigenschaften aufweisen. Zunächst müssten sie aus veränderbaren und veränderungsfähigen Agenten bestehen und nicht aus fixen, unveränderlichen Elementen. Da in der zivilen SiFo unter anderem Menschen die entscheidenden Bestandteile der relevanten Systeme sind, kann diese Eigenschaften als gegeben angesehen werden. Neben Menschen können aber auch die technischen Bestandteile der sozio-technischen Systeme als Agenten aufgefasst und modelliert werden. Die Agenten in komplexen adaptiven Systemen verfolgen Ziele unter der Nebenbedingung unvollständiger Information. Auch das gilt für die in der zivilen SiFo relevanten sozio-technischen Systeme, wobei diese Ziele vom Besuch eines Weihnachtsmarkts zur Erhöhung des persönlichen Glücksgefühls, über die Ausrichtung von Veranstaltungen zur Erzielung eines ökonomischen Gewinns bis zur effektiven Rettung von Menschenleben im Katastrophenfall oder der zuverlässigen Bereitstellung von Energie reichen können. Wenn die Agenten in diesen sozio-technischen Systemen ihre Ziele - aus welchen Gründen auch immer - nicht mehr erreichen können, werden sie versuchen, die Regeln oder Muster ihres Handelns so zu verändern, dass die Zielerreichung wieder möglich wird. Sie passen sich also veränderten Umweltbedingungen an. Das gilt ohne jeden Zweifel für die Menschen als Agenten der sozio-technischen Systeme. Je nach Ausmaß der Veränderung gilt es auch für die technischen Bestandteile der Systeme. Inwiefern die Anpassung hauptsächlich durch Rückgriff auf in der Vergangenheit als nützlich erkannte Verhaltensweisen und deren Neu-Anordnung im Lichte der veränderten Umweltbedingungen erfolgt, lässt sich nicht ohne Weiteres feststellen. Für technische Bestandteile des Systems erscheint es allerdings sehr plausibel. Was Menschen angeht, resultiert aus der Psychologie die Erkenntnis, dass diese mit Veränderungen dann gut umgehen können, wenn sie ein „durchdringendes, andauerndes, aber dynamisches Gefühl des Vertrauens [haben], dass die eigene interne und externe Umwelt vorhersagbar ist und dass es eine hohe Wahrscheinlichkeit gibt, dass sich die Dinge so entwickeln werden, wie vernünftigerweise erwartet werden kann“ (Antonovsky 1997: 16). Das spricht dafür, dass auch die Menschen als Agenten in den für die zivile SiFo relevanten sozio- 
technischen Systemen Anpassungsfähigkeit primär durch neue Signalverarbeitungsmuster bestehend aus Rekombinationen bekannter Handlungsweisen umsetzen. Insofern spricht auch diese Eigenschaft dafür, in der SiFo von komplexen adaptiven Systemen als Untersuchungsgegenstand auszugehen. Ein weiterer, entscheidender Aspekt unterstützt diese Annahme. Die Agenten in den oben skizzierten sozio-technischen Systemen interagieren miteinander, das gilt sowohl für die menschlichen als auch die technischen Bestandteile der Systeme. Ihre Interaktion erfolgt zudem in retrospektiver Weise wie auch in prospektiver Weise. Sie richten ihr Handeln also sowohl an ihren vergangenen Erfahrungen aus, als auch an dem, was sie für die Zukunft erwarten. In ihre Erfahrungen aus der Vergangenheit und die Erwartungen über die Zukunft fließen Informationen über das erlebte und erwartete Verhalten der anderen Agenten im System ein. Es existieren also Feedbackschleifen und daraus resultierend stark nichtlineare Verhaltensmuster des Systems. Aus der Betrachtung einzelner Agenten in solchen sozio-technischen Systemen lassen sich demzufolge keine Rückschlüsse auf das Gesamtsystem ziehen, dieses weist emergente Eigenschaften auf und ist somit eindeutig als komplex zu charakterisieren. Diese strikt der Theorie komplexer adaptiver Systeme folgende Herleitung ist konsistent mit der sonstigen Resilienzforschung, die ebenfalls von der Komplexität der für die zivile SiFo relevanten, sozio-technischen Systeme ausgeht (Boin/McConnell 2007: 50, Brunner/Giroux 2009: 9, Deppisch 2016: 205, Dueñas-Osorio/Vemuru 2009: 157, ENISA 2011: 12, Helbing 2013: 52ff, Lovins/Lovins 2001: 1, 19, 22, Schaffer/Schneider 2019: 9, 13, Voss/Dittmer 2016: 193). Interessant ist etwa das Beispiel der Kommunikationsinfrastruktur, die für einen bestimmten Zweck - direkte Gespräche zwischen zwei Menschen über das Telefon - geschaffen wurde, aber durch die Entwicklung des Internets zur Grundlage eines beliebig komplexen Systems wurde, mitsamt neuartiger Herausforderungen - sozusagen emergenten Eigenschaften, an die bei der ursprünglichen Installation des Systems niemand denken konnte (ENISA 2011: 9). Ein weiteres, einfaches Beispiel aus dem Bereich der Energieversorgung verdeutlicht diese Tatsache: Die Installation von Photovoltaik-Anlagen auf privaten Hausdächern ist für Hausbesitzer nicht nur ökonomisch reizvoll, sondern erhöht auch deren Versorgungssicherheit im Fall eines großflächigen Stromausfalls zumindest insofern sie auch über genügend Speichermöglichkeiten verfügen. Ob die Installation von Photovoltaik-Anlagen generell aber auch die Versorgungssicherheit im Gesamtsystem erhöht, lässt sich beim Blick auf einzelne Hausbesitzer nicht sagen. Es lässt sich auch nicht sagen, wenn man alle Häuser mit Photovoltaik-Anlagen betrachtet und ihre mögliche 
Energieproduktion aufsummiert. Erst bei Betrachtung des gesamten Energiesystems und seiner Abhängigkeiten lässt sich unter Umständen sagen, ob mehr Photovoltaik-Anlagen unter sonst gleichen Bedingungen zu größerer Versorgungssicherheit führen oder nicht. Hier handelt es sich um ein geradezu prototypisches Beispiel von Emergenz. Deshalb gilt zusammenfassend: Die in der zivilen SiFo relevanten sozio-technischen Systeme sind komplexe adaptive Systeme.

Unmittelbar an diese Annahme schließt sich im Kontext der Sicherheitsforschung eine weitere Frage an: Werden diese Systeme immer komplexer? Blum et al. problematisieren eine angeblich unhinterfragt postulierte Zunahme von Komplexität als Rechtfertigung eines Nachdenkens über neue Formen der zivilen SiFo (Blum et al. 2016: 161). Nachdem bereits festgestellt wurde, dass es in der zivilen SiFo um komplexe adaptive Systeme geht, lässt sich die Frage mit einem Blick auf die von Holland und seinen Kollegen am Santa Fe Institut skizzierten Eigenschaften komplexer adaptiver Systeme beantworten. Denn die Agenten in derartigen Systemen tendieren dazu, sich über die Zeit zu Spezialisten weiterzuentwickeln und gemeinsam mit anderen Spezialisten Nischen im System auszubilden, mit deren Hilfe die begrenzten Ressourcen, über die jeder Agent und jedes System per definitionem nur verfügt, effizienter eingesetzt werden können. Ceteris paribus führt größere Spezialisierung zu einer steigenden Notwendigkeit, sich mit anderen Agenten im System zu vernetzen. Mit Vernetzung wird an dieser Stelle ein weiterer, von Blum et al. problematisierter Begriff eingeführt. Aus der Theorie komplexer adaptiver Systeme ergibt sich, dass Spezialisierung für Agenten eine sinnvolle Strategie ist. Spezialisierung heißt aber gleichzeitig, dass ein „Produkt“ nur hergestellt werden kann, oder anders gesagt Agenten ihr Ziel nur erreichen können, wenn sie zusammenarbeiten. Je größer die Spezialisierung, desto mehr Zusammenarbeit wird erforderlich. Zusammenarbeit kann im Sinne der Theorie komplexer adaptiver Systeme auch als Vernetzung bezeichnet werden. Unter Bezugnahme auf die gesellschaftlichen Entwicklungen der vergangenen Jahrhunderte kann die Annahme immer weiter steigender Spezialisierung als empirisch bestätigt angesehen werden. Daraus folgt, wendet man die Theorie komplexer adaptiver Systeme an, zwingend auch eine immer stärkere Vernetzung der Agenten untereinander. Damit ist die These von Blum et al. aus der Theorie direkt ableitbar. Das ist gleichzeitig ebenfalls konsistent mit der sonstigen Resilienzforschung. Auch dort wird, wenn auch zumeist theoretisch unreflektiert, von einer steigenden Vernetzung der kritischen Infrastruktursysteme der Gesellschaft untereinander ausgegangen (Al-Khudhairy et al. 2012: 572, Chang 2009: 1ff, Flynn/Burke 
2012: 20, Galland 2012: 304, Rogers et al. 2012: 74, Woods 2015: 5). Detaillierter wird das Thema Vernetzung von Autoren wie Attoh-Okine, Kröger und Ouyang betrachtet. Demnach gibt es vier verschiedene Arten von Vernetzung. Zunächst physikalische Vernetzung, etwa Gaspipelines, die zur Versorgung von Kraftwerken verwendet werden, selbst aber wiederum auf die Versorgung mit Energie angewiesen sind. Dann geographische Vernetzung, die durch geographische Nähe bedingt ist. Informationelle Vernetzung resultiert aus der informationstechnischen Steuerung kritischer Infrastruktursysteme. Und logische Vernetzung umfasst alle anderen Möglichkeiten, die nicht in die ersten drei Kategorien fallen (Attoh-Okine 2016: 36, Kröger 2011: 71f, Ouyang 2014: 45). Die Autoren sind sich darin einig, dass die Vernetzung zwischen den verschiedenen Systemen immer weiter steigt. Und aus der steigenden Vernetzung folgt aus der Theorie der komplexen adaptiven Systeme heraus eine immer weiter zunehmende Komplexität. Denn durch die Vernetzung nehmen die gegenseitigen Abhängigkeiten der Agenten voneinander zu, die Zahl der Feedbackschleifen und der dadurch ausgelösten Rückkopplungseffekte steigt. Die Systeme weisen einen immer größeren Grad an Emergenz auf. Insofern lässt sich festhalten: Der Grad an Vernetzung und die Komplexität der für die zivile SiFo relevanten komplexen adaptiven sozio-technischen Systeme nimmt kontinuierlich weiter $z u$.

Komplexität und steigende Komplexität sind also Phänomene, die nicht nur in der Resilienzforschung eine herausgehobene Rolle spielen, sondern auch in der zivilen Sicherheitsforschung berücksichtigt werden müssen. Wie verhalten sich Komplexität und Resilienz zueinander? Longstaff formuliert dazu eine prägnante Beschreibung, die den Ausgangspunkt der folgenden Diskussion bilden soll: „Resilience capacity is often an emergent property of the system“ (Longstaff 2012: 264). Die Aussage kann weiter zugespitzt werden: Resilienz ist eine emergente Eigenschaft von Systemen und kann daher nur in komplexen Systemen auftreten. Komplexität ist notwendig für Resilienz. Vogt fasst den Zusammenhang etwas anders. Er postuliert: „Resilienz modelliert komplexe Systemabläufe“ (Vogt 2015: 22). Auch in dieser Formulierung hängen aber Resilienz und Komplexität zusammen. Rein begriffsgeschichtlich gibt es keinen notwendigen $\mathrm{Zu}$ sammenhang zwischen Resilienz und Komplexität. Der nach Alexander bis mindestens zu Beginn des 20. Jahrhunderts vorherrschenden Begriffsbedeutung eines bounce back liegt keine Spezifizierung des Systems zugrunde. Egal, um was für eine Art System es sich handelt, nach einer Belastung kehrt es wieder in seinen Ursprungszustand zurück und „federt“ die negativen Auswirkungen widriger Ereignisse sozusagen ab. Gelingt 
dem System das, ist es resilient und vice versa. Da es in den Sozialwissenschaften keine „richtigen“ oder „falschen“, sondern lediglich mehr oder weniger zweckmäßige Definitionen gibt, kann ein derartiges Resilienz-Verständnis selbstverständlich legitim genutzt werden. Der Verweis auf die zunehmende Komplexität der für die zivile SiFo relevanten sozio-technischen Systeme erübrigt sich dann. In der Tradition von Holling handelt es sich hier um ein mechanisches Resilienz-Verständnis bzw. sogar eher ein stabilitäts- als ein resilienzzentriertes Denken, bei dem die Rückkehr in einen vormaligen Gleichgewichtszustand im Mittelpunkt steht (Brand/Jax 2007: 2, Gunderson 2000: 426f, Lorenz 2010, Voss/Dittmer 2016: 186). Für die vorliegende Arbeit greift ein Resilienz-Verständnis, das ohne den Bezug auf Komplexität auskommt, jedoch zu kurz. Denn in der zivilen SiFo geht es - wie gerade nachgewiesen wurde - um komplexe adaptive sozio-technische Systeme. Und solche Systeme kehren nach gravierenden Belastungen gerade nicht in ihren Ausgangszustand zurück. Sie entwickeln sich kontinuierlich durch Anpassung der Agenten, aus denen sie bestehen, an interne und externe Veränderungen, weiter. Das gilt auch, aber nicht nur, für gravierende, schockartige Belastungen (Holland 2014: 5f). Für die Analyse komplexer adaptiver Systeme ist es insofern weder zweckmäßig noch sinnvoll, Resilienz als Rückkehr in einen Ausgangszustand zu definieren. Mechanische Resilienz „does not, however, capture the complexity, permanent change, and dynamic of systems" (Lorenz 2010). Diese Erkenntnis geht im Prinzip auf Holling und die gesamte auf ihn folgende ökologische, sozial-ökologische und sozialwissenschaftliche Tradition der Resilienzforschung zurück. Das Resilienz-Konzept für die zivile SiFo muss entsprechend ein systemisches Konzept sein, das Komplexität und Anpassungsfähigkeit als entscheidende Komponenten mitberücksichtigt: „Der systemische Ansatz bietet einen guten Ausgangspunkt, um katastrophale Prozesse soziologisch in ihrer realen Komplexität zu denken“" (Voss/Dittmer 2016: 187). Zudem ist die Verwendung eines systemischen Ansatzes auch anschlussfähig an die im vorangegangenen Unterkapitel vorgenommene systemtheoretische Einordnung von Resilienz als Konzept für die zivile SiFo, in der Komplexität bereits eine entscheidende Rolle für Resilienz gespielt hat.

Allerdings wurde der Begriff dort noch anders definiert, nämlich als Anzahl der zur Verfügung stehenden Anschlussmöglichkeiten. Für die Welt ist diese Zahl beliebig groß, für ein System jedoch begrenzt. Um mit der Komplexität der (Um-)Welt umgehen zu können, benötigt ein System hohe Eigenkomplexität, die somit notwendig - aber nicht hinreichend - für Resilienz ist. Gilt dieser Zusammenhang auch noch für ein 
aus der Komplexitätstheorie heraus spezifiziertes Verständnis von Komplexität? Für Holland und generell Vertreter der Theorie komplexer adaptiver Systeme zeichnen diese sich ja durch spezifische Eigenschaften und Verhaltensweisen aus, allen voran das Auftreten von Emergenz und die Tatsache, dass ihre Elemente interagierende, heterogene und anpassungsfähige Agenten sind. Komplexe Systeme organisieren sich selbst nach bestimmten Mustern, etwa in Form spezialisierter Nischen. Sie weisen ein scheinbar „chaotisches" Verhalten auf, bei dem kleine Veränderungen drastische Auswirkungen haben können, die außerdem häufig erst mit zeitlicher Verzögerung auftreten. Auch sogenanntes „fat-tail behavior" ist charakteristisch für komplexe adaptive Systeme, also das relativ häufigere Auftreten bezogen auf statistische Erwartungen in einer sogenannten Normalverteilung - eigentlich sehr unwahrscheinlicher Ereignisse. Komplexe Systeme sind dynamisch und anpassungsfähig und somit ständiger Veränderung ausgesetzt (Folke 2006: 257, Holland 2014: 5f, Rahimi/Madni 2014: 810, Smith 2002, Voss/Dittmer 2016: 186, Walker et al. 2004). Aus der Systemtheorie heraus wurde das Auftreten von Resilienz an einen gravierenden, häufig extrem unwahrscheinlichen und in der Systemumwelt verorteten Reiz gebunden. Die Komplexitätstheorie hat nun gezeigt, dass die Komplexität komplexer adaptiver Systeme - und damit auch der jeweiligen Systemumwelt, da für ein spezifisches System alle anderen jeweils Umwelt sind - tendenziell immer weiter steigt. Je komplexer die Systemumwelt ist, desto wahrscheinlicher wird das gerade beschriebene „fat-tail behavior", also das Auftreten eigentlich extrem unwahrscheinlicher, disruptiver Ereignisse (oder in systemtheoretischer Sprache „Reize“), mit denen das betroffene System umgehen muss (Dekker 2014: 33, Helbing 2013: 53, IRGC 2018: 9, Lovins/Lovins 2001: 29, Park et al. 2013: 359). Resilienz liegt nach der Systemtheorie dann vor, wenn Systeme auf solche Reize mit der Realisierung eigentlich extrem unerwartbarer Anschluss-Prozesse reagieren und so dem System das Einschwenken auf einen neuen Entwicklungspfad ermöglichen. Aufgrund der Nicht-Selbstverständlichkeit von Resilienz führt resilientes Verhalten nach dieser Definition ceteris paribus zu einer langfristig höheren Systemleistung, als nicht-resilientes Verhalten des Systems. Weil die Systemumwelt der für die zivile SiFo relevanten sozio-technischen Systeme immer komplexer wird und damit eigentlich extrem unwahrscheinliche disruptive Ereignisse immer häufiger auftreten, wird Resilienz zur Aufrechterhaltung der Systemleistung der betroffenen Systeme immer wichtiger. Oder kürzer gesagt: Die steigende Komplexität der Welt macht Resilienz als Eigenschaft komplexer adaptiver Systeme immer notwendiger. 
Die Frage nach dem Zusammenhang zwischen Resilienz und der Eigenkomplexität komplexer adaptiver Systeme lässt sich nicht derartig eindeutig beantworten. In der Literatur, gerade im Bereich der zivilen Sicherheitsforschung, wird Komplexität häufig implizit auf die Systemumwelt bezogen und entsprechend als Herausforderung charakterisiert (Hiermaier/Scharte 2018: 297, Scharte/Thoma 2016: 124, Thoma et al. 2016: 5). Eine explizite Untersuchung der Frage nach der Rolle von Eigenkomplexität in diesem Kontext ist relativ selten. Es gibt jedoch eine ganze Reihe von Autoren, die in der durch steigende Komplexität von Systemen gleichzeitig steigenden Vernetzung der Agenten innerhalb eines Systems wie auch zwischen verschiedenen Systemen einen Fakt sehen, der diese Systeme bei Auftreten einer externen Störung schneller zusammenbrechen lässt. Wenn Eigenkomplexität - wobei der Begriff so nicht fällt, sondern generell von der zunehmenden Komplexität von Systemen die Rede ist - genannt wird, dann als mindestens indirekter Grund für die stetige Zunahme der Verwundbarkeit oder Vulnerabilität von Systemen. Die meisten Autoren beziehen sich bei der Rede von der zunehmenden Komplexität und Vernetzung vor allen Dingen auf die kritischen Infrastrukturen einer Gesellschaft und deren immer größere Abhängigkeit voneinander. Gegeben eine stark vernetzte Infrastruktur, können bereits harmlos wirkende, kleine Störereignisse durch Auslösung sogenannter „Kaskadeneffekte“ zu großflächigen und langanhaltenden Ausfällen der Infrastruktur führen. Kaskadeneffekte lösen eine Art „Dominoeffekt“ im System aus. Oder anders gesagt: „One damned thing leads to another” (Lovins/Lovins 2001: 18). Nichtsdestotrotz ist es unzureichend, Kaskadeneffekte mit dem Dominoeffekt gleichzusetzen, da erstere weitere Probleme in einer probabilistischen Weise auslösen und zudem gleichzeitig mehrere neue Probleme auslösen können, während der klassische Dominoeffekt einer stärker deterministischen Abfolge einzeln nacheinander fallender Dominosteine entspricht. Da Kaskadeneffekte in komplexen adaptiven Systemen auftreten, ist ein nichtlineares Verhalten kennzeichnend für sie (Al-Khudhairy et al. 2012: 571-574, Chang 2009: 1, Edwards 2009: 28, Galland 2012: 304, Kröger 2011: 67, Pursiainen 2009: 724, Rogers et al. 2012: 74, Wink 2016: 4). Dass immer stärkere Vernetzung in komplexen adaptiven Systemen mit immer stärkerer Abhängigkeit der Agenten voneinander einhergeht, lässt sich direkt aus der Theorie ableiten. Und auch wenn sich komplexe adaptive Systeme einer deterministisch, linear-kausalen Analyse entziehen, machen stärkere Vernetzung und stärkere innere Abhängigkeit das Auftreten von Kaskadeneffekten nach einem initialen Störereignis zumindest sehr viel wahrscheinlicher. Gegeben diese Annahmen, lässt sich der Zusammenhang zwischen 
Eigenkomplexität und Resilienz ähnlich formulieren, wie der zwischen (Um-)Weltkomplexität und Resilienz. Je höher die Eigenkomplexität eines Systems, desto wahrscheinlicher führt das Eintreten widriger Ereignisse zu Kaskadeneffekte und so zu langfristigen Schäden am System. Um diese verhindern zu können, benötigt das System Resilienz. Das lässt sich aus der systemtheoretischen Analyse des Konzepts ableiten. Es kann insofern festgehalten werden: Die steigende Eigenkomplexität komplexer adaptiver Systeme macht Resilienz als Systemeigenschaft immer notwendiger.

Eigenkomplexität und (Um-)Weltkomplexität bzw. deren kontinuierliches Anwachsen stellen für die zivile SiFo Herausforderungen dar, denen mithilfe einer Steigerung der Resilienz der relevanten komplexen adaptiven sozio-technischen Systeme begegnet werden kann. Das erweitert das aus dem vorangegangenen Unterkapitel abgeleitete systemtheoretische Resilienz-Verständnis. Nicht mehr die prinzipiell unbegrenzte (Um-)Weltkomplexität macht Resilienz notwendig. Vielmehr ist es die Zunahme beider Arten von Komplexität, Eigen- und (Um-)Weltkomplexität, die Resilienz aufgrund des vermehrten Auftretens eigentlich extrem unwahrscheinlicher Ereignisse in der Umwelt sowie der höheren Wahrscheinlichkeit von Kaskadeneffekten immer notwendiger macht. In der systemtheoretischen Analyse wurde darüber hinaus noch ein weiterer Zusammenhang festgestellt. Demnach ist hohe Eigenkomplexität auch notwendig für Resilienz. Der Unterschied ist eindeutig: Während steigende Komplexität komplexitätstheoretisch dazu führt, dass Resilienz immer stärker benötigt wird, ist hohe Eigenkomplexität systemtheoretisch notwendig, damit Resilienz überhaupt möglich ist. Denn nur durch hohe Eigenkomplexität, verstanden als große Zahl an Anschlussmöglichkeiten, stehen auch sehr unwahrscheinliche Anschlussmöglichkeiten zur Verfügung, die zur Realisierung von Resilienz benötigt werden. Die Frage ist, ob diese Annahme auch aus Sicht der Komplexitätstheorie - zusätzlich zum Verständnis von Komplexität als Herausforderung - ihre Gültigkeit behält. Um sie zu beantworten zu können, müssen zunächst komplexe und einfache Systeme miteinander verglichen werden. Einfache Systeme reagieren deterministisch, in eindeutiger und linear-kausal vorhersagbarer Weise auf Beeinflussung von außen. Tritt also ein widriges Ereignis ein, lässt sich die Reaktion eines einfachen Systems daran festmachen, ob das Ereignis die spezifizierten Grenzen der Belastbarkeit des Systems übersteigt oder nicht. Wenn das der Fall ist, bricht das System zusammen, verliert seine Funktionsfähigkeit und kann ohne Unterstützung aus der Systemumwelt nicht weiter existieren. Wenn die Grenzen der Belastbarkeit nicht überschritten werden, operiert das System weiter und der Grad an Funktionsfähigkeit hängt 
von der Robustheit und Elastizität des Systems ab. Das erinnert nicht zufällig an das schon verschiedentlich beschriebene mechanische Verständnis von Resilienz. Innerhalb der Grenzen der Belastbarkeit, die in einfachen Systemen theoretisch identifizierbar sind, ist ein "bounce back" in den vorherigen Gleichgewichtszustand möglich, bzw. das System operiert mit niedrigerer Funktionalität weiter. Sobald die Grenzen der Belastbarkeit allerdings überschritten werden, muss das einfache System - ohne Hilfe von außen - notwendig versagen. Systemtheoretisch ließe sich auch formulieren: Einfache Systeme sind nach Ereignissen, welche die Grenzen ihrer Belastbarkeit überschreiten, nicht länger in der Lage einen Entwicklungspfad zu realisieren, der mit einer Systemleistung größer null verknüpft ist. So wie Resilienz in der vorliegenden Arbeit als systemisches Konzept für die zivile SiFo verstanden wird, erfordert sie aber die Realisierung eigentlich unerwartbarer neuer Entwicklungspfade im Angesicht extrem unwahrscheinlicher, disruptiver widriger Ereignisse, welche sich gerade darin auszeichnen, dass sie die eigentlichen Grenzen der Belastbarkeit des Systems übersteigen. Dazu bedarf es einer hohen Zahl verschiedener Anschlussmöglichkeiten, die dem System eine adäquate, leistungserhaltende Anpassung ermöglichen. Über Anpassungsfähigkeit können einfache Systeme nicht verfügen, da ihnen die dafür notwendigen Elemente - adaptive Agenten - und Mechanismen - neue Signalverarbeitungsmuster durch Neuanordnung nützlicher Bausteine zu kreieren sowie in Interaktion mit anderen Agenten zu treten und so Feedback und Rückkopplungen zu produzieren - fehlen. Komplexe adaptive Systeme zeichnen sich dagegen gerade durch ihre Anpassungsfähigkeit aus. In komplexen adaptiven Systemen lassen sich zwar die Grenzen der Belastbarkeit a priori nicht feststellen. Das Auftreten emergenter Eigenschaften und die Nichtlinearität der kausalen Zusammenhänge in solchen Systemen verhindern eine eindeutige Definition der Grenzen. Nichtsdestotrotz wird deutlich, dass Resilienz, so wie das Konzept in der vorliegenden Arbeit definiert wird, nur in komplexen adaptiven Systemen möglich ist. In Anlehnung an Longstaff lässt sich Resilienz als emergente Eigenschaft komplexer Systeme verstehen und formulieren: Komplexität von Systemen ist eine notwendige Bedingung für Resilienz.

Führt höhere bzw. steigende Eigenkomplexität damit direkt zu höherer bzw. steigender Resilienz? In der Resilienzforschung gibt es einige Autoren, die das so sehen. Prior und Roth konstatieren etwa eine Stärkung der Robustheit von Systemen durch höhere Eigenkomplexität, da diese zu stärkerer Vernetzung führe und stärkere Vernetzung wiederum das Auftreten von Redundanz fördere. Robustheit ist hier als ein Teil eines breiteren 
Resilienz-Verständnisses zu sehen (Prior/Roth 2013: 60). Ob Vernetzung tatsächlich zu Redundanz führt, ist im Rahmen der Theorie komplexer adaptiver Systeme eher fraglich, wie weiter unten noch gezeigt wird. Für das Resilienz-Verständnis der zivilen SiFo interessanter ist insofern auch ein Gedanke, der sich im Rahmen der HRO-Theorie findet und dort relativ prominent ist (siehe Fußnote 30). Demzufolge erhöhen besonders zuverlässig arbeitende Organisationen ganz bewusst ihre Eigenkomplexität, indem sie die Diversität ihrer Mitarbeiter möglichst maximieren und diese durch Fortbildungen, freie Diskussionen innerhalb der Organisation und immer wieder veränderte Rollen und Zuständigkeiten fordern und fördern. Das gibt solchen Organisationen ein „breites Spektrum an Handlungsoptionen" mit deren Hilfe sie herausfordernden Situationen begegnen können (Ungericht/Wiesner 2011: 192). Das breite Spektrum an Handlungsoptionen ist aus der Systemtheorie bekannt und von dort kommend als notwendig aber nicht hinreichend für Resilienz konzipiert worden. In der HRO-Theorie, die als Organisationstheorie sehr eng mit psychologischen Ansätzen der Resilienzforschung verknüpft ist, scheint der Zusammenhang zwischen Resilienz und Eigenkomplexität ähnlich aufgefasst zu werden. Aber auch darüber hinaus, in anderen Bereichen der Resilienzforschung, etwa stärker sozialwissenschaftlich geprägten Ansätzen, wird die Beziehung zwischen höherer Resilienz und steigender Eigenkomplexität teilweise so gesehen. Komplexität wird dann im Hinblick auf ihre Verbindung zu Diversität zur „Problemlösungsstrategie“. Je diversifizierter ein System ist, desto mehr alternative Möglichkeiten besitzt es, um mit einem Problem fertig werden zu können, also resilient zu sein. Daraus folgt: „, $[\mathrm{R}]$ esilience is characterized by a positive correlation between complexity/ diversity and adaptability" (Brunner/Giroux 2009: 7). Steigende Eigenkomplexität führt scheinbar aufgrund steigender Diversität und der damit einhergehenden Erhöhung der Zahl alternativer Handlungsmöglichkeiten dazu, dass die Resilienz des Systems größer wird. Im Rahmen einer komplexitätstheoretischen Analyse bedeutet steigende Eigenkomplexität von Systemen aber mehr, als nur eine Erhöhung der Anschlussmöglichkeiten. Sie geht auch einher mit stärkeren Rückkopplungseffekten und Feedbackschleifen, sie drückt sich - wie gezeigt - in größerer Vernetzung und einer stärkeren Spezialisierung der Agenten aus. Diese Agenten reagieren auf neue, unbekannte Reize von außen, indem sie ungeeignete Signalverarbeitungsmuster durch neue, mit größerer Wahrscheinlichkeit geeignete Muster ersetzen. Neue Muster bestehen zumeist in einer Neuanordnung bekannter und in der Vergangenheit als nützlich erkannter Bausteine. Die Neuanordnung bekannter Bausteine erlaubt den Agen- 
ten also, neue Reize zu verarbeiten. Je komplexer das System, desto mehr unterschiedliche, spezialisierte Agenten gibt es. Holland schreibt Agenten in evolutionär weit entwickelten komplexen adaptiven System sogar Prognosefähigkeiten zu. Sie sind demnach in der Lage, die Auswirkungen der Anwendung neuer Signalverarbeitungsmuster intern zu simulieren und so auf eine reale Umsetzung potentiell ungeeigneter Anpassungsoptionen zu verzichten (Holland 2014: 86). Die unterschiedlichen Agenten verfügen aufgrund ihrer Erfahrungen im Zeitverlauf, die sich in nichtlinearer Weise aus der Interaktion mit den anderen Agenten des Systems sowie der Umwelt ergeben haben, über sehr unterschiedliche Bausteine, die sie zu neuen Signalverarbeitungsmustern zusammenfügen können. Je mehr spezialisierte Agenten es gibt, desto mehr unterschiedliche Bausteine existieren insgesamt im System. Das erhöht ceteris paribus die Wahrscheinlichkeit, dass mindestens einige Agenten über geeignete Bausteine verfügen, um auch auf extrem unwahrscheinliche, disruptive Ereignisse von außen adäquat, also in leistungserhaltender Weise reagieren zu können. Durch Interaktion mit den anderen Agenten im System können diese sich erfolgreich anpassenden Agenten ihre eigenen Verhaltensweisen weiter übertragen und so $\mathrm{zu}$ einer emergenten Eigenschaft des Systems werden lassen. Das System erweist sich dann aufgrund seiner hohen Eigenkomplexität und des gerade geschilderten, damit verbundenen Mechanismus, als resilient gegenüber dem erlebten disruptiven Ereignis. Basierend auf dieser Analyse lässt sich sagen: Je komplexer ein System ist, desto höher ist seine Resilienz gegenüber extrem unwahrscheinlichen, disruptiven Ereignissen.

Allerdings lässt ein näherer Blick auf Hollands Aussagen zur evolutionären Entwicklung komplexer adaptiver Systeme daran zweifeln, dass der oben genannte Zusammenhang derart eineindeutig formuliert werden kann. Hier kommt der dritte Aspekt der Theorie komplexer adaptiver Systeme ins Spiel, der für ein Resilienz-Konzept der zivilen SiFo von Interesse ist, nämlich die Frage danach, wie Anpassungsfähigkeit innerhalb der Theorie verstanden wird. Die Agenten in komplexen adaptiven Systemen versuchen in begrenzt rationaler Weise ihre jeweiligen Ziele zu erreichen. Dafür steht ihnen nur eine bestimmte Menge an Ressourcen zur Verfügung. Um ihre Ziele besser erreichen zu können, bietet sich Arbeitsteilung und damit verbunden Spezialisierung als Mechanismus an. Die Agenten entwickeln sich evolutionär weiter, sie passen sich an, indem sie sich immer stärker spezialisieren. Je größer ihre Spezialisierung, desto besser werden sie in der Erfüllung einer spezifischen Aufgabe, die im Gesamtkontext des Systems allen Agenten bei der besseren Erreichung ihrer Ziele dient. Der Grad an Zielerreichung wird also durch steigende Komplexität bei 
gegebenen Ressourcen maximiert. Oder anders formuliert: Die Effizienz des Systems wird größer. Der Effizienzbegriff wurde bereits von Holling problematisiert. Er versteht eine auf maximale Effizienz des Systems zielende Strategie als resilienzmindernd (Alexander 2013: 271f, Holling 1973: 8f, 18, Kaufmann/Blum 2012: 238). Auch in den Wirtschaftswissenschaften, in denen Effizienz für den Großteil der Forschung nach wie vor eine der wichtigsten Zielgrößen darstellt, wird Resilienz tendenziell als Gegenbegriff zu Effizienz gesetzt. Um möglichst effizient zu arbeiten, sind Reserven und ungenutzte Spielräume - oder anders gesagt Möglichkeiten zur Anpassung an sich radikal ändernde Umweltbedingungen - schädlich. Solche Reserven und ungenutzten Spielräume werden für Resilienz aber benötigt, da nur so eine Anpassung an extrem unwahrscheinliche, disruptive Ereignisse möglich ist (Plöger/Lang 2016: 359, Vogt 2015: 11f). Aus Sicht der Theorie komplexer adaptiver Systeme lässt sich das wie folgt verstehen. Die Agenten passen ihre Signalverarbeitungsmuster den Erfordernissen ihrer jeweiligen Spezialisierung an, so dass sie langfristig nur noch über Bausteine verfügen, die einer möglichst effizienten Erfüllung ihrer spezifischen Aufgabe dienlich sind. Wird das System nun von einem extrem unwahrscheinlichen, disruptiven Ereignis getroffen, verfügen die meisten spezialisierten Agenten nicht über die notwendigen Bausteine, um sich dem Ereignis erfolgreich anzupassen. Ihre Anpassungsfähigkeit hat sich ebenfalls spezialisiert. In komplexen adaptiven Systemen verlieren Agenten ihre Anpassungsfähigkeit durch zunehmende Spezialisierung nicht. Die Art der Anpassungsfähigkeit ändert sich aber. Verändern sich die Anforderungen, die sie von anderen Agenten oder aus der Systemumwelt erhalten, in inkrementeller Weise, reagieren sie sehr schnell darauf und passen ihre Signalverarbeitungsmuster entsprechend an. Dazu ist ihre spezialisierte Anpassungsfähigkeit" in idealer Weise geeignet. Sind die Veränderungen, auf die das System reagieren muss, aber abrupt, gravierend und unwahrscheinlich, das heißt unterscheiden sie sich von der von den Agenten prognostizierten Entwicklung, nutzt die spezialisierte Anpassungsfähigkeit den Agenten nicht weiter. Um sich auch an derartige Veränderungen anpassen zu können, benötigen sie eher ungenutzte Spielräume, wie weiter oben beschrieben. Ungenutzte Spielräume können auch als generische Anpassungsfähigkeit bezeichnet werden. Sie sind in Agenten zu finden, die als „Generalisten“ beschrieben werden können. Spezialisierte Agenten können unter gravierend veränderten Umweltbedingungen ihre Aufgabe nicht länger erfüllen. Aufgrund ihrer engen Verknüpfung über Feedbackschleifen mit den anderen Agenten des Systems, führt der Ausfall einzelner Agenten sehr schnell zum kaskadierenden Ausfall weiterer 
Agenten, weil diese zur Erfüllung ihrer Aufgaben notwendig auf die $\mathrm{Zu}-$ sammenarbeit mit den bereits ausgefallenen Agenten angewiesen sind. Die ausgefallenen Agenten können aufgrund ihrer Spezialisierung auch nicht einfach durch andere Agenten ersetzt werden, denn ähnlich spezialisierte Agenten fallen ebenfalls aus. Damit führt höhere Komplexität, vermittelt durch höhere Effizienz und Vernachlässigung einer generischen zugunsten einer spezialisierten Anpassungsfähigkeit zu einer Verminderung der Resilienz des Systems. Oder prägnanter: Je komplexer ein System ist, desto geringer ist seine Resilienz gegenüber extrem unwahrscheinlichen, disruptiven Ereignissen.

Mithilfe der Theorie komplexer adaptiver Systeme lässt sich der Zusammenhang zwischen Komplexität und Resilienz also sowohl als kausal positiv als auch kausal negativ verknüpft plausibel erklären. Die Analyse erfolgt sogar unter Zuhilfenahme derselben Annahmen und Wirkungsweisen. Beide Male wird die Zunahme der Komplexität durch immer stärkere Spezialisierung und darauffolgende Vernetzung ausgelöst. Spezialisierung lohnt sich, da sie das System insgesamt effizienter arbeiten lässt. Und sie führt dazu, dass sich eine Vielzahl unterschiedlicher Agenten ausbilden, die jeweils über ihre individuell eigenen Bausteine verfügen, mit deren Hilfe sie neue Signalverarbeitungsmuster bilden können, wenn die Notwendigkeit dazu entsteht. Das stark unterschiedliche Ergebnis der Analyse resultiert dann aus der Interpretation dieses so geschilderten Verständnisses der Funktionsweise komplexer adaptiver Systeme. Wenn der Fokus auf den einzelnen spezialisierten Agenten und ihrer spezialisierten Anpassungsfähigkeit liegt, scheint größere Komplexität zu geringerer Resilienz zu führen. Wenn der Fokus aber auf der durch Spezialisierung erreichten Diversität und der Vielzahl an im System vorhandenen unterschiedlichen Anschlussmöglichkeiten liegt, scheint größere Komplexität sich positiv auf die Resilienz des Systems auszuwirken. Komplexität von Systemen ist zwar an sich generell notwendig, um überhaupt erst Resilienz zu ermöglichen. Aber zunehmende Komplexität kann sich sowohl positiv als auch negativ auf Resilienz auswirken. Um das zu verdeutlichen, ist es hilfreich, die Unterscheidung zwischen spezialisierter und generischer Anpassungsfähigkeit auf Systemebene zu transportieren. Bisher war von der unterschiedlichen Art von Anpassungsfähigkeit bei Agenten die Rede. Ein Agent ist umso besser in der Lage, sich veränderten Umweltbedingungen anzupassen, je generischer seine individuelle Anpassungsfähigkeit ausgeprägt ist. Selbiges gilt auch für das System. Je ausgeprägter dessen generische Anpassungsfähigkeit ist, desto größer ist die Zahl an disruptiven Ereignissen, mit denen das System erfolgreich umzugehen in der Lage ist, desto größer ist 
also seine Resilienz. Umgekehrt gilt genauso, dass größere spezialisierte Anpassungsfähigkeit das System insgesamt weniger resilient werden lässt. Die Anpassungsfähigkeit eines komplexen adaptiven Systems setzt sich zusammen aus der individuellen Anpassungsfähigkeit seiner Agenten - aber in nichtlinearer Weise. Hier zeigt sich das wesentliche Charakteristikum komplexer Systeme, das Auftreten von Emergenz, als Hindernis für eine eindeutige Analyse. Selbst wenn die individuelle Anpassungsfähigkeit aller Agenten des betreffenden Systems bekannt wäre - eine empirisch wahrscheinlich ohnehin unerfüllbare Bedingung, zumal Agenten prinzipiell selbst als komplexe adaptive Systeme modelliert werden könnten - ließe sich daraus nicht auf die genaue Art der Anpassungsfähigkeit des Systems schließen. Ob die Kombination spezialisierter Anpassungsfähigkeiten der Agenten eher zu einem System mit stärker spezialisierter oder stärker generischer Anpassungsfähigkeit führt, lässt sich nicht eindeutig festlegen. A priori ist unklar, welcher Effekt überwiegt, der durch Diversität hervorgerufene große Möglichkeitsraum von Bausteinen zur Erarbeitung neuer Signalverarbeitungsmuster, die durch Feedbackschleifen vermittelt dem System eine Anpassung auch an gravierende, disruptive Ereignisse ermöglichen. Oder der durch die Maximierung der spezialisierten Anpassungsfähigkeit einzelner Agenten möglicherweise insgesamt reduzierte Rahmen generischer Anpassungsfähigkeit des Systems. Holland selbst formuliert noch eine weitere Beobachtung, die in diesem Zusammenhang von Bedeutung ist. Obwohl komplexe adaptive Systeme generell zu immer größer werdender Diversität und Spezialisierung ihrer Agenten tendieren, bilden sie zum Teil auch Nischen aus, in denen Generalisten die Agenten sind, die sich durchsetzen. Die Gründe dafür sieht er in dem größeren Möglichkeitsraum, eben der größeren generischen Anpassungsfähigkeit, die solchen Generalisten im Vergleich mit Spezialisten zur Verfügung steht. Sie sind zwar schlechter dazu in der Lage, bestimmte Ressourcen in nutzenmaximierender Weise zu verarbeiten - oder anders formuliert ihre spezialisierte Anpassungsfähigkeit ist geringer, sie gleichen diesen evolutionären Nachteil aber dadurch aus, dass sie nicht nur bestimmte, sondern viele Ressourcen verarbeiten können (Holland 2014: 80). Eine größere Zahl an Generalisten lässt eine höhere generische Anpassungsfähigkeit des Systems plausibel erscheinen. Deterministisch ist aber auch dieser Zusammenhang aufgrund der Komplexität der untersuchten Systeme nicht. Gegeben die Ergebnisse dieser Analyse gilt: In komplexen adaptiven Systemen kann steigende Komplexität sowohl zu mehr als auch zu weniger Resilienz führen. Die Resilienz eines komplexen adaptiven Systems nimmt bei steigender Komplexität genau dann zu, wenn die generische Anpassungsfähigkeit des Systems steigt. 
Zusammenfassend ergeben sich für ein eigenständiges Resilienz-Konzept der zivilen Sicherheitsforschung aus der Theorie komplexer adaptiver Systeme folgende Annahmen:

- Die für die zivile SiFo relevanten sozio-technischen Systeme sind komplexe adaptive Systeme, weil sie die dafür relevanten Elemente - interagierende, heterogene, anpassungsfähige Agenten - und Eigenschaften - Emergenz, Feedbackschleifen, Selbstorganisation - aufweisen.

- Der Theorie komplexer adaptiver Systeme folgend, nimmt daher der Grad an Vernetzung und die Komplexität der für die zivile SiFo relevanten sozio-technischen Systeme kontinuierlich weiter zu.

- Die (Um-)Welt komplexer adaptiver sozio-technischer Systeme besteht wiederum aus anderen Systemen, so dass auch ihre Komplexität stetig zunimmt. Je komplexer die (Um-)Welt, desto wahrscheinlicher wird das Auftreten extrem unwahrscheinlicher, disruptiver Ereignisse. Die systemtheoretische Analyse des vorangegangenen Unterkapitels hat gezeigt, dass Resilienz benötigt wird, um mit solchen Ereignissen erfolgreich umgehen zu können. Die steigende Komplexität der Welt macht Resilienz als Eigenschaft komplexer adaptiver Systeme also immer notwendiger.

- Immer größer werdende Eigenkomplexität der Systeme erhöht die Wahrscheinlichkeit für das Auftreten von Kaskadeneffekten, die beim Eintreten widriger Ereignisse zu großflächigen und häufig langfristigen Schäden am System führen. Um diese verhindern zu können, benötigt das System Resilienz. Auch die steigende Eigenkomplexität komplexer adaptiver Systeme macht Resilienz als Systemeigenschaft also immer notwendiger.

- Einfache Systeme reagieren in deterministischer Weise auf Belastungen. Übersteigen diese die Grenzen ihrer Belastbarkeit, bricht das System zusammen. Es kann nicht mehr auf einen neuen Entwicklungspfad umschwenken, auch nicht auf einen mit verminderter Systemleistung. Dazu sind nur komplexe Systeme aufgrund ihrer Anpassungsfähigkeit in der Lage. Resilienz drückt sich durch das Betreten neuer Entwicklungspfade aus, obwohl die eigentlichen Belastungsgrenzen des Systems überstiegen werden. Die Komplexität von Systemen ist also eine notwendige Bedingung für Resilienz.

- A priori ist unklar, welchen Effekt steigende Komplexität auf das Ausmaß der im System vorhandenen bzw. auftretenden Resilienz hat. Steigende Komplexität kann sowohl zu mehr als auch zu weniger Resilienz führen. In komplexen adaptiven Systemen muss deshalb zwischen spezialisierter und generischer Anpassungsfähigkeit unterschieden wer- 
den. Die Resilienz eines komplexen adaptiven Systems nimmt bei steigender Komplexität genau dann zu, wenn die generische Anpassungsfähigkeit des Systems steigt.

\subsubsection{Resilienz und Unsicherheit}

Mit der Aussage, dass es manchmal besser kommt, als man denkt, setzt die vorliegende Arbeit bereits zu Beginn ein kontraintuitives Statement als Ausgangspunkt aller weiteren Diskussionen (Zander/Roemer 2016: 47). Wenn es besser kommt, als man denkt, geht damit unmittelbar der Fakt einher, dass man vorher nicht sicher wusste, wie es kommen würde. Die Zukunft ist nicht deterministisch vorausbestimmt, sondern hängt in probabilistischer zum Teil aber auch in stochastischer Weise von den Entwicklungen in der Gegenwart und der Vergangenheit ab. In den vorangegangenen beiden Unterkapiteln spielte das Thema probabilistische Zusammenhänge bzw. mehr oder weniger wahrscheinliche Ereignisse für Systemtheorie und Komplexitätstheorie eine wichtige Rolle. Die bisherigen Annahmen für ein Resilienz-Konzept der zivilen SiFo beruhen daher auch zum Teil auf Aussagen über wahrscheinliche oder weniger wahrscheinliche Reize, Ereignisse und Anschlussmöglichkeiten. Resilienz wird weiterhin als systemtheoretisches und komplexitätstheoretisches Konzept verstanden. Die in den beiden vorangegangenen Unterkapiteln herausgearbeiteten Annahmen gelten sowohl für dieses, als auch für alle kommenden Unterkapitel als grundlegende theoretische Fundierung des Resilienz-Konzepts der zivilen SiFo. Sie werden sukzessive durch weitere Annahmen ergänzt bzw. überarbeitet. Das zusammenfassende und analyseleitende Begriffspaar für dieses Unterkapitel lautet „Resilienz und Unsicherheit.“ Für die Resilienzforschung steht die Frage, welche widrigen Ereignisse sich in Zukunft materialisieren werden, in mehrfacher Hinsicht im Mittelpunkt. Zunächst geht es darum, wie zukünftige Ereignisse konkret aussehen werden. Dann ist die Wahrscheinlichkeit von Interesse, mit der sich bestimmte Dinge ereignen werden oder nicht. Vielfach wird auch die Frage gestellt, ob solche Wahrscheinlichkeiten überhaupt erkannt oder festgelegt werden können. Und nicht zuletzt geht es darum, bei Eintritt welcher Art Ereignisse Resilienz überhaupt notwendig und/oder möglich ist. Um das Resilienz-Konzept für die zivile SiFo präziser fassen zu können, werden im Folgenden Annahmen und Antworten auf diese Fragen aus den verschiedenen Disziplinen analysiert und weiterentwickelt. 
Unter Unsicherheit wird dabei nicht das Gegenteil von Sicherheit im Sinne der zivilen SiFo verstanden. Deren Ziel besteht im Schutz der Bevölkerung und in der Sicherheit der kritischen Infrastrukturen. Eine „klassische“ Herausforderung in der SiFo besteht nun gerade darin, Sicherheit zu definieren. Die Abwesenheit von Sicherheit, charakterisiert durch nichtfunktionierende Infrastrukturen oder Schäden an Leib und Leben einer großen Zahl von Menschen, lässt sich leichter erkennen. Diese Art von "Unsicherheit" ist in der nun folgenden Diskussion explizit nicht gemeint. Vielmehr geht es um Unsicherheit darüber, wie die für die zivile SiFo relevanten komplexen adaptiven sozio-technischen Systeme und ihre jeweiligen Systemumwelten sich in Zukunft entwickeln werden, welche widrigen Ereignisse sie treffen könnten und wie sie darauf reagieren werden. Um ein solches Verständnis von Unsicherheit - und Sicherheit besser abgrenzen zu können, lohnt ein Blick auf die englische Sprache. Dort gibt es die Unterscheidung zwischen „Certainty“ und „Security." Beide Begriffe lassen sich mit Sicherheit übersetzen. Security kann verstanden werden als Sicherheit gegenüber extern verursachten, disruptiven Ereignissen, etwa Terroranschlägen. „Secure“ bzw. sicher - oder besser: geschützt - sind Menschen dann, wenn Terroranschläge verhindert werden können oder sie aufgrund von Schutzmaßnahmen den Anschlag unverletzt überstehen können. „Certainty“ bzw. der Gegenbegriff „Uncertainty“ beziehen sich dagegen auf das Ausmaß an zur Verfügung stehendem Wissen über bestimmte Sachverhalte. Die Reflektion über Sicherheit (Certainty) und Unsicherheit (Uncertainty) stammt ursprünglich aus der Philosophie. Besonders bekannt in diesem Zusammenhang ist das aus dem Jahr 1641 stammende Werk Meditationes de prima philosophia, in qua Dei existentia et animae immortalitas demonstratur des französischen Philosophen René Descartes (siehe Descartes 1986).

Für die vorliegende Arbeit ist vor allen Dingen das im 20. Jahrhundert etablierte Verständnis von Unsicherheit in den Wirtschaftswissenschaften von Bedeutung. Im Rahmen der sogenannten Entscheidungstheorie geht es um die Frage, wie rational handelnde Akteure Entscheidungen treffen (können). Die deskriptive Entscheidungstheorie sucht nach Erklärungen für empirisch beobachtete Handlungen von Individuen und Gruppen. Die normative Entscheidungstheorie versucht, möglichst allgemeingültige Regeln zu entwickeln, mit deren Hilfe rational handelnde Akteure möglichst "gute“ - in den Wirtschaftswissenschaften zumeist nutzenmaximierende - Entscheidungen treffen können (Laux et al. 2014: 3f). Grundsätzlich ist zwischen den beiden Möglichkeiten Entscheidung unter Sicherheit und Entscheidung unter Unsicherheit zu trennen. Wenn eine Entschei- 
dung unter Sicherheit getroffen werden kann, sind ihre Auswirkungen deterministisch festgelegt und damit a priori vollständig bekannt. Die Wahrscheinlichkeit ihres Eintretens liegt bei 100\%. Präzise gefasst gilt dies nicht nur für die Auswirkungen der Entscheidungen. Die gesamte Entscheidungssituation ist bei Entscheidungen unter Sicherheit deterministisch bestimmt, alle Auswirkungen aller potentiellen Handlungen sind bekannt. In einem solchen Fall können die Konsequenzen alternativer Handlungsoptionen miteinander verglichen und ohne Probleme in eine nach ihrem Nutzen geordnete, eindeutige Reihenfolge gebracht werden. Um Entscheidungen unter Sicherheit treffen zu können, müssen ökonomische Akteure theoretisch über vollständige Information verfügen. Empirisch ist diese Annahme nur äußerst selten erfüllt. Das theoretische Modell der Entscheidung unter Sicherheit wird in den Wirtschaftswissenschaften nichtsdestotrotz als vereinfachende Annäherung häufig genutzt (Laux et al. 2014: 57ff). Mit Blick auf die Diskussion in den vorangegangenen Unterkapiteln fällt sofort eine mögliche Verbindung zwischen Entscheidung unter Sicherheit und einfachen Systemen auf. Einfache Systeme reagieren deterministisch, in eindeutiger und linear-kausal vorhersagbarer Weise auf Beeinflussung von außen. Die Auswirkungen der Beeinflussung von außen sind daher ebenfalls eindeutig vorherbestimmt. Für einfache Systeme scheint die Anwendung der theoretischen Annahmen von Entscheidung unter Sicherheit also plausibel und sinnvoll. In der zivilen SiFo geht es aber um komplexe adaptive Systeme. Bei der Definition dieser Systemart wurde immer wieder darauf verwiesen, dass deterministische Annahmen und reduktionistische Erklärungsversuche nicht geeignet sind, um deren Verhalten zu bestimmen. Die nicht-deterministische Welt erschließt sich im Rahmen der Entscheidungstheorie durch den Begriff der Unsicherheit. Für die vorliegende Arbeit soll dieser entscheidungstheoretische Begriff genutzt und im Hinblick auf seinen Zusammenhang mit dem Resilienz-Konzept analysiert werden. Dazu muss er zunächst erläutert werden. Grundsätzlich heißt Unsicherheit zunächst nur, dass nicht sicher bekannt ist, welche künftige Situation eintreten wird. Das Ziel der oben genannten normativ orientierten Entscheidungstheorie besteht dann darin, trotz dieser Unsicherheit zu Regeln zu kommen, nach denen (begrenzt) rational handelnde Akteure ihren jeweiligen Nutzen maximieren können (Laux et al. 2014: 3f). Die aus diesem Erkenntnisinteresse resultierenden, mathematisch formalisierten Regeln sind für die vorliegende Arbeit nicht von Interesse, da hier der Fokus auf der Erarbeitung eines theoretisch gehaltvollen Resilienz-Konzepts für die zivile SiFo liegt. Das Resilienz-Konzept wird keine formalisierten Entscheidungsregeln beinhalten, sondern aus 
Annahmen darüber bestehen, was Resilienz auf Basis system- und komplexitätstheoretischer Überlegungen bedeutet und welche spezifischen Beziehungen zu relevanten Konzepten, wie Unsicherheit, bestehen.

Was also ist mit Unsicherheit im Rahmen der vorliegenden Arbeit gemeint? Basierend auf der Arbeit von Knight lassen sich in der Entscheidungstheorie drei Arten von Unsicherheit unterscheiden (siehe Knight 2009). Knight unterscheidet zwischen Risiko, Ungewissheit und der eigentlichen oder echten Unsicherheit (Aven 2010: 624). Aufgrund des großen Einflusses, den sein Werk in den Wirtschaftswissenschaften genießt, wird diese eigentliche Unsicherheit auch als „Knightsche Unsicherheit“ (Knightian Uncertainty) bezeichnet. Laut Aven findet diese auf Knight zurückgehende Einteilung in der Praxis nur wenig Verwendung, da die konkrete Verteilung von Wahrscheinlichkeiten nur selten bekannt sei (Aven 2010: 624). Nichtsdestotrotz kann sie aufgrund der Prominenz des hier zugrundeliegenden Risikobegriffs in der zivilen SiFo hilfreich für die vorliegende Arbeit sein (siehe unten). Eine Entscheidung wird genau dann unter Risiko getroffen, wenn ihre Auswirkungen zwar nicht deterministisch aber probabilistisch vollständig bekannt sind. Demzufolge können Entscheidungen verschiedene Auswirkungen haben und es ist a priori unklar, welche dieser Auswirkungen realisiert wird. Bekannt sind bei Entscheidung unter Risiko aber alle möglichen Auswirkungen sowie die Wahrscheinlichkeiten für deren Eintreten. Insgesamt addieren sich alle Wahrscheinlichkeiten zu 100\% auf. Die Wahrscheinlichkeiten können sowohl objektiv bekannt sein, als auch von den Akteuren subjektiv geschätzt werden (Knight 2009: 101ff, Laux et al. 2014: 83ff). Glücksspiele wie zum Beispiel Roulette sind gute Beispiele, um das Prinzip der Entscheidung unter Risiko bei objektiven Wahrscheinlichkeiten zu veranschaulichen. Im Roulette sind alle möglichen Ergebnisse a priori bekannt und die Wahrscheinlichkeit ihres Eintretens ebenfalls. Sobald sich der Spieler für einen Einsatz entscheidet, gibt es für ihn noch die beiden möglichen Auswirkungen Gewinn oder Verlust, deren Wahrscheinlichkeiten sich ebenfalls eindeutig zuordnen lassen. Dieser Risikobegriff entspricht, wenigstens was die Betrachtung objektiver Wahrscheinlichkeiten betrifft, im Prinzip dem der „klassischen“, ingenieurwissenschaftlich dominierten Risikoforschung (siehe 2.6). Gegeben die Zielrichtung der vorliegenden Arbeit wird er daher im Folgenden für das eigenständige Resilienz-Konzept der zivilen SiFo verwendet, ohne deshalb die umfassendere Tradition der Risikoforschung an sich inhaltlich zu negieren (siehe 2.4 und 2.6). Davon zu unterscheiden ist das Phänomen der Ungewissheit. Bei Entscheidungen unter Ungewissheit sind die möglichen Auswirkungen von Entscheidun- 
gen immer noch bekannt. Oder anders gesagt, die möglichen Ergebnisse zum Zeitpunkt b sind zum Zeitpunkt a, der vor b liegt, bekannt und es ist ebenfalls klar, zwischen welchen alternativen Handlungsmöglichkeiten entschieden werden kann. Die Eintrittswahrscheinlichkeiten sind jedoch unklar. Es ist nicht klar, ob die Wahl einer bestimmten Handlungsmöglichkeit zum gewünschten Ergebnis führt oder zu einem gänzlich anderen. Und selbiges gilt für die Realisierung einer anderen Handlungsmöglichkeit. Erst nachdem eine Entscheidung getroffen wurde lässt sich sagen, zu welchen Auswirkungen sie geführt hat. Das gilt auch für Entscheidungen unter echter oder Knightscher Unsicherheit. In solchen Situationen ist ebenfalls a priori unklar, zu welchen Ergebnissen die Auswahl bestimmter Handlungsmöglichkeiten führen wird. Darüber hinaus ist aber zusätzlich noch unklar, welche Ergebnisse oder Auswirkungen von Entscheidungen überhaupt möglich sind (Knight 2009: 101ff, Laux et al. 2014: 83ff). Entscheidungen unter echter Unsicherheit finden sich damit an einem Ende des Spektrums, dessen andere Seite von Entscheidungen unter Sicherheit dargestellt wird. Empirisch ist es häufig sehr schwer oder sogar unmöglich herauszufinden, in welcher Art Entscheidungssituation man sich befindet. Das trifft vor allen Dingen auch deshalb zu, weil die subjektive Zuordnung möglicher Ergebnisse bestimmter Handlungsoptionen und eine ebenfalls subjektive Zuschreibung von Eintrittswahrscheinlichkeiten für diese Ergebnisse nicht notwendigerweise mit objektiven Gegebenheiten übereinstimmen muss. Sogar die Tatsache, ob es diese objektiven Gegebenheiten überhaupt gibt, lässt sich - wenn man eine Luhmannsche, systemtheoretische Position einnimmt - zumindest für soziale „Tatsachen“ in Zweifel ziehen (Kneer/Nassehi 2000: 42, 103, 146). Davon abgesehen können die drei vorgestellten, voneinander zumindest theoretisch unterscheidbaren Arten von Entscheidungen unter Unsicherheit sehr gut dazu genutzt werden, mehr Klarheit in den Umgang der Resilienzforschung mit verschiedenen Arten von Ereignissen zu bringen.

In der Resilienzforschung werden die drei Begriffe Risiko, Ungewissheit und Unsicherheit ubiquitär verwendet, allerdings häufig ohne eine genauere Spezifizierung. Meistens geht es um eine Abgrenzung älterer, risikoorientierter Handlungsweisen zu einem „neuen“ Ansatz, der auch Ungewissheit/Unsicherheit mit einbezieht (Allenby/Fink 2005: 1034, Berkes 2007: 285, Blum et al. 2016: 155, Lorenz 2010). Generell ist das Verständnis der Begriffe anders als in der Entscheidungstheorie. Der dort zentrale Gegenstand der Entscheidung fällt zunächst weg und steht erst im weiteren Verlauf der Analysen wieder im Mittelpunkt. Es geht primär um verschiedenartige Ereignisse (im nicht-systemtheoretischen Sinn), die 
mit bestimmten (oder unbestimmten) Wahrscheinlichkeiten eintreten und mit einer gewissen (oder ungewissen) Wahrscheinlichkeit zu bestimmten Auswirkungen führen. Dazu zählen auch - und in einigen Bereichen der Resilienzforschung vor allem - besonders überraschende, seltene und unerwartete Ereignisse im Sinne der gerade erläuterten Knightschen Unsicherheit. Die Ereignisse resultieren in den meisten Fällen aus der Systemumwelt, systemtheoretisch gesprochen handelt es sich zum Beispiel um extrem unwahrscheinliche externe Reize mit denen ein System sich selbst erhaltend umgehen können soll, etwa ein extremes Wetter-, Klima- oder geologisches Ereignis, um bewusst den Begriff der „Naturkatastrophe“ zu vermeiden. Sie lassen sich aber auch als Ergebnisse bewusster Entscheidungen des betrachteten Systems bzw. seiner Elemente (Agenten) mit unintendierten oder intendierten Konsequenzen begreifen. Insofern sie systemintern getriggert werden - was im Rahmen der zivilen SiFo beispielsweise bei gravierenden Unfallereignissen in kritischen Infrastrukturen oder Industrieanlagen der Fall sein könnte - sind sie entweder das unintendierte Ergebnis der adaptiven Interaktion von Agenten in komplexen Systemen, oder anders gesagt Ergebnis der Emergenz des Systems. Oder sie kommen durch bewusste Manipulation durch einzelne Agenten zustande. Im Fall von Unfällen sind die Konsequenzen des Handelns sowohl auf Ebene der Agenten wie auf Systemebene unintendiert. Dagegen verfolgen manipulative Agenten bewusst Zielstellungen, die in der (Zer-)Störung des Gesamtsystems bestehen, die möglicherweise stark negativen Konsequenzen ihrer Handlungen sind also von ihnen gewollt. Das gilt jedoch nicht für das System, für das auch negative Auswirkungen infolge der Handlungen manipulativer Agenten eine unintendierte Konsequenz der System-Operation darstellen (Longstaff 2012: 259). Für das Resilienz-Konzept der zivilen SiFo lässt sich dann ergänzend zu den Annahmen aus System- und Komplexitätstheorie festhalten, dass die gravierenden disruptiven Ereignisse, die Resilienz notwendig machen, nicht nur aus der Systemumwelt, sondern auch aus dem System selbst stammen können. Systemtheoretisch handelt es sich immer noch um die Realisierung eines unerwartbaren Ereignisses, der auslösende externe Reiz entfällt jedoch. Komplexitätstheoretisch werden sowohl Unfälle als auch Manipulationen mit steigender Eigenkomplexität wahrscheinlicher. Für Unfälle gilt das, weil steigende Eigenkomplexität sich unter anderem durch eine größere Zahl an Feedback- und Rückkopplungseffekten ausdrückt, damit die Nichtlinearität des Systems steigt und die Agenten so die Konsequenzen ihres eigenen Handelns weniger gut abschätzen können. Je schlechter Agenten die Auswirkungen von Handlungen voraussehen können, desto wahrscheinlicher wird unter sonst glei- 
chen Bedingungen die Wahl einer Alternative, die zu einem Unfall führt. Und für Manipulationen gilt es, weil zunehmende Komplexität sich auch in immer stärkerer Diversifizierung der Agenten ausdrückt, so dass die Wahrscheinlichkeit für das Auftauchen von Agenten zunimmt, deren optimale Handlungsweise zur individuellen Nutzenmaximierung konträr zu den Zielen des Gesamtsystems läuft. Zusammenfassend gilt: Die für die zivile SiFo relevanten komplexen adaptiven sozio-technischen Systeme müssen mit dem Auftreten interner, durch die Entscheidungen von Agenten ausgelösten, von diesen unintendierten oder intendierten Störungen umgehen können. Die Wahrscheinlichkeit des Auftretens solcher Störungen steigt mit der Eigenkomplexität der Systeme.

Die vorliegende Arbeit ist bisher implizit bereits davon ausgegangen, dass in den empirisch meisten Fällen, wenn es um Resilienz geht, die verschiedenen Arten von Unsicherheit betrachtet werden müssen. Sicherheit im Sinne vollständig determinierter Ereignisse und damit auch einer vollständig determinierten Zukunft ist eine unrealistische und empirisch wie theoretisch ungeeignete Annahme für die Resilienzforschung. Insbesondere die Verortung in der Theorie komplexer adaptiver Systeme und die aus System- und Komplexitätstheorie hergeleitete Annahme der Notwendigkeit von komplexen Systemen für Resilienz zeigt dies auf. In gewissem Sinne kann die Resilienzforschung als Abrücken von einer „Standardposition der Moderne" verstanden werden, wie Blum et al. formulieren. Mit Bezug zu Parsons beziehen sie sich auf eine klassische, modernisierungstheoretische Weltsicht. Aus der modernisierungstheoretischen Perspektive sei demnach der immer weiter voranschreitende Fortschritt von Wissenschaft und Technik grundsätzlich dazu geeignet, Unsicherheit langfristig zu überwinden. Unsicherheit, oder Nicht-(Sicher-)Wissen, könne modernisierungstheoretisch eher ein „Noch-Nicht-Wissen“ sein als ein Nicht-Wissen-Können. So verstanden wird Unsicherheit ein durch Fortschritt überwindbares, „beseitigbares Ärgernis“ (Blum et al. 2016: 155). Das wird in der Resilienzforschung gänzlich anders gesehen. Diese geht von einer Welt aus, in der Unsicherheit sich weder theoretisch noch praktisch vermeiden lässt und in der sie auch durch gesellschaftliche Anstrengungen in Wissenschaft, Technik oder anderen Bereichen nicht überwunden werden kann. Gegeben die (steigende) Komplexität der Welt ist es schlicht unmöglich, alle Szenarien darüber, wie sich die Zukunft entwickeln könnte, zu bedenken. Es ist nicht einmal möglich, nur alle „worst case“-Szenarien zu beachten (Blum et al. 2016: 155, Boin/McConnell 2007: 52). In der Ökosystemforschung, die sich stark mit dem menschlichen Management komplexer Ökosysteme auseinandersetzt, hat sich in diesem Zusammen- 
hang zunehmend die Erkenntnis von der prinzipiellen Unvorhersehbarkeit und Unkontrollierbarkeit solcher Systeme durchgesetzt. Die in komplexen Ökosystemen ablaufenden und sich materialisierenden Ereignisse sind „inherently unpredictable“ (Berkes 2007: 286). Das gilt nicht nur für Ökosysteme, sondern für komplexe Systeme an sich. Unsicherheit kann in komplexen Systemen auch als die konzeptionell-theoretische Fassung der begrenzten Möglichkeiten zum umfassenden Verständnis von nichtlinearen Interaktionsprozessen definiert werden, die eine sichere Vorhersage der Systemleistung zu einem bestimmten Zeitpunkt erschweren oder sogar verunmöglichen (Chandler 2014: 48). Diese systeminterne Argumentation wird durch eine Betrachtung systemexterner Faktoren ergänzt. David Woods gibt zu bedenken, dass alle Systeme, unabhängig davon wie erfolgreich sie mit kleineren und größeren Störungen umzugehen in der Lage sind, Belastungsgrenzen haben. Auch wenn die Identifikation dieser Belastungsgrenzen in komplexen adaptiven Systemen mindestens sehr schwer, wenn nicht sogar a priori unmöglich ist, gibt es laut Woods per definitionem immer wieder Ereignisse, welche die Belastungsgrenzen überschreiten, unter anderem deshalb, weil das System nicht mit ihnen gerechnet hat. Solche Ereignisse bezeichnet Woods als Überraschungen (surprises) (Woods 2015: 7, siehe auch Lovins/Lovins 2001: 29, 269). Unsicherheit und Überraschungen sind also „part of the game”, komplexe Systeme sollen grundsätzlich auch mit dem Eintreten von derart konzipierten Überraschungen rechnen, sich darauf vorbereiten und damit umgehen können (Folke 2006: 255). Für die in der zivilen SiFo relevanten widrigen Ereignisse lassen sich Überraschungen nicht nur als das Ereignis an sich verstehen, sondern auch auf die, diesem widrigen Ereignis folgenden Ereignisse, oder systemtheoretisch gesprochen die realisierten Prozesse an Anschlussmöglichkeiten, übertragen. Für Colten et al. zeichnen sich derartige widrige Ereignisse gerade durch das Entstehen von überraschenden Effekten und Auswirkungen aus, jedem „hazard“ und noch mehr jedem "disaster" wohnten Überraschungen inne, sei es durch die Realisierung des unerwarteten Ereignisses an sich oder zum Beispiel durch eine verfehlte Reaktion des betroffenen Systems (Colten et al. 2008: 20). Zumal das weiter oben analysierte Auftreten systemintern verursachter Störungen ebenfalls für Überraschungen sorgen kann. Steen und Aven zufolge werden viele widrige Ereignisse in komplexen Systemen nicht durch den Zusammenbruch oder Fehlfunktionen bestimmter Systemelemente verursacht, sondern durch die unintendierten Folgen der für das normale Funktionieren des Systems verantwortlichen Interaktion seiner Agenten (Steen/Aven 2011: 292). Weil es um komplexe adaptive Systeme geht, ist 
dieses „law of unintended consequences“ typisch für die in der zivilen SiFo relevanten widrigen Ereignisse (Longstaff 2012: 259). Um Überraschungen im Sinne Woods handelt es sich, weil auch diese systemintern getriggerten Ereignisse die Belastungsgrenzen des Systems übersteigen und daher zu katastrophalen Auswirkungen führen können.

In der Resilienzforschung spielt das Auftreten von Überraschungen, von unerwarteten, die Belastungsgrenzen der Systeme übersteigenden, widrigen Ereignissen eine zentrale Rolle. Das gilt vor allen Dingen für die Disziplinen, die in ihrem Verständnis des Konzepts zumindest ein Stück weit der Tradition Hollings folgen. Mit Blick auf das Resilienz-Verständnis der Psychologie lässt sich festhalten: Auch dort sind Ereignisse, die die Belastungsgrenzen des Systems, in diesem Fall des komplexen adaptiven Systems Mensch oder im Luhmannschen Sinne des menschlichen Bewusstseins, übersteigen die Resilienz erforderlich machenden Phänomene. Klassischerweise werden sie als Traumata beschrieben. Für die Betroffenen sind sie insofern überraschend, als sie ihre gewohnte Routine des täglichen Lebens massiv unterbrechen oder sogar verunmöglichen. Die wenigsten Menschen erwarten derart gravierende Einschnitte. Und das obwohl sie auf einer übergeordneten Systemebene, beim Blick auf die Gesellschaft, durchaus erwartbar sind. Rein statistisch lässt sich zum Beispiel für Deutschland sicher sagen, dass eine bestimmte Anzahl an Menschen pro Jahr vom Unfalltod eines Partners oder einer Partnerin betroffen sein wird. Selbiges gilt für den Ausbruch schwerer Krankheiten, den Verlust des Arbeitsplatzes, usw. Das ändert aber nichts am individuellen Blick auf das Erleben eines Traumas als überraschendes, also unerwartetes und die eigenen Belastungsgrenzen übersteigendes Ereignis. Insofern ist es nicht nur dem Hollingschen Resilienz-Verständnis nach folgerichtig, dass die sozialwissenschaftliche Resilienzforschung sich vorrangig solcherart verstandenen Überraschungen zuwendet. An dieser Stelle muss nun zwischen den verschiedenen Arten von Unsicherheit auch explizit unterschieden werden. Die Diskussion in den Sozialwissenschaften dreht sich meist um die Frage, worin ein originärer Beitrag der Resilienz-Perspektive liegen könne, der nicht bereits durch ältere, klassische Risikoforschung abgedeckt werde. Eine häufig gegebene Antwort fokussiert auf das Entstehen und Aufkommen (scheinbar) neuer, „zeitlich, sachlich und sozial entgrenzte[r]" widriger Ereignisse. Mit Blick auf den aus der Entscheidungstheorie entliehenen Risikobegriff ist unmittelbar einsichtig, warum dieser für derartige Ereignisse keine geeignete Handlungsgrundlage bietet. Die beschriebenen widrigen Ereignisse sind bzgl. der Wahrscheinlichkeit ihres Eintretens nicht quantifizierbar, sie sind also ungewiss. Zum Teil ist darüber hinaus 
a priori auch unbekannt, welche Ereignisse überhaupt eintreten können. Es muss demzufolge auch nach Wegen gesucht werden, erfolgreich mit echter, Knightscher Unsicherheit umzugehen (Blum et al. 2016: 155). Die Bewältigung unvorhersehbarer Ereignisse, zu der existierende, etablierte Strategien nicht länger genutzt werden können, steht im Mittelpunkt. Denn diese „failure of expectations“, realisiert in der Manifestation völlig unerwarteter Ereignisse, führt zwar in vielen Systemen zu katastrophalen Auswirkungen, aber eben nicht in allen, nicht in resilienten Systemen (Bach et al. 2011: 4, Berkes 2007: 285, Kaufmann/Blum 2012: 235, Lorenz 2010). Oder wie Blum et al. formulieren: „Die neue Maßgabe lautet, sich auf das forcierte Bewusstsein ungewisser Zukünfte einzustellen und sich auf das Nicht-Vorbereitbare vorzubereiten“ (Blum et al. 2016: 155).

$\mathrm{Zu}$ besonderer Prominenz ist im Zusammenhang mit dem Eintreten unerwarteter, gravierender und die Belastungsgrenzen des Systems übersteigender Ereignisse der Begriff der „schwarzen Schwäne“ (black swans) gekommen. In seiner diesbezüglichen Verwendung geht der Begriff, bzw. die dahinterstehende Metapher, auf den libanesisch-amerikanischen Essayisten und populärwissenschaftlichen Autor Nassim Nicholas Taleb zurück. In seinem 2007 erschienenen Werk The Black Swan: The Impact of the Highly Improbable beschäftigt er sich mit der Macht höchst unwahrscheinlicher Ereignisse, wie der Untertitel der deutschen Ausgabe des Buchs lautet (siehe Taleb 2007). Allerdings war er damit bei weitem nicht der erste, der sich (mehr oder weniger) wissenschaftlich mit dem Sujet der schwarzen Schwäne auseinandersetzte. Schon John Stuart Mill und vor allen Dingen Karl Popper nutzten den schwarzen Schwan, um bestimmte Sachverhalte bzw. theoretische Annahmen zu verdeutlichen. Dafür muss zunächst festgehalten werden: Es gibt schwarze Schwäne. Die Gattung Cygnus Atratus, der Trauerschwan, weist eine schwarze Färbung der Federn auf. Er ist in Australien heimisch und war in Europa bis zum Ende des Mittelalters und in die Neuzeit hinein unbekannt. In der antiken römischen Philosophie wurde daher die Aussage „Alle Schwäne sind weiß“ als „Binsenweisheit“ und nicht weiter zu hinterfragende "Wahrheit“ gesehen (Frankel 2010). Das lieferte Karl Popper den idealen Ausgangspunkt, um mithilfe der Metapher von den schwarzen Schwänen die Grundprinzipien der von ihm gegründeten Denkrichtung des kritischen Rationalismus zu veranschaulichen. Grob zusammengefasst lassen sich bestimmte Aussagen grundsätzlich nicht beweisen, sondern lediglich sicher widerlegen. Deshalb muss das Ziel wissenschaftlicher Forschung in der Falsifikation von Theorien oder einzelner Annahmen bestimmter Theorien bestehen. Gelingt dies über längere Zeiträume nicht, erscheinen die Annahmen plausibel, letztgültig 
als richtig nachweisen lassen sie sich aber nicht (siehe Popper 1973). Diese Tatsache kann mithilfe der schwarzen Schwäne erläutert werden. Für einen in Europa lebenden Menschen ist es höchst unwahrscheinlich, so er sich nicht in Australien aufhält oder einen Zoo besucht, einen schwarzen Schwan zu sehen. Man kann im Laufe seines Lebens durchaus hunderte weiße und keinen einzigen schwarzen Schwan sehen. Aufgrund dieser Beobachtung liegt dann der Schluss nahe, dass alle Schwäne weiß sind. Genau deshalb galt die Aussage „Alle Schwäne sind weiß“ in Europa sehr lange Zeit als wahr. Popper argumentiert nun, dass sich aus der rein empirischen Beobachtung nur weißer Schwäne gerade nicht darauf schließen lasse, dass alle Schwäne notwendigerweise weiß seien. Denn es bedarf nur eines einzigen schwarzen Schwans, um die Aussage zu falsifizieren. Und deren Existenz wurde bereits Ende des 17. Jahrhunderts von Europäern entdeckt, auch wenn sie den meisten zunächst noch über viele Jahre unbekannt blieb (Frankel 2010). Wieso greift nun Taleb das Bild vom schwarzen Schwan wieder auf? Ihm geht es darum zu zeigen, wie Ereignisse oder Entwicklungen, die von der Gesellschaft als höchst unwahrscheinlich empfunden oder deren mögliches Eintreten sogar negiert wird, gerade doch passieren und damit diese Gesellschaft vor Herausforderungen stellen. A priori ist bei Taleb unklar, ob ein schwarzer Schwan sich in Form eines für die Gesellschaft mit primär negativen oder positiven Auswirkungen verbundenen Ereignisses manifestiert. Ihm geht es um die - vermeintliche - Unvorhersehbarkeit und den Aspekt der Überraschung. Die sozialwissenschaftliche Resilienzforschung hat die Metapher im Anschluss an Taleb übernommen, wendet sie aber primär für negativ besetzte Ereignisse an (Vogt 2015: 16).

Taleb versteht unter einem schwarzen Schwan ein a priori unerwartetes, großflächiges und mit extremen Auswirkungen einhergehendes, sowie darüber hinaus seltenes Ereignis. Mit Blick auf bekannte Wahrscheinlichkeitsverteilungen, bei denen ein Großteil der zu erwartenden Ereignisse sich nahe des statistischen Mittelwerts der Verteilung abspielt - im Rahmen einer oder weniger Standardabweichungen - sind schwarze Schwäne an den Rändern der Verteilung zu finden und als extreme Ausreißer zu charakterisieren. Schwarze Schwäne zeichnen sich auch dadurch aus, dass sie unter Rückgriff auf etablierte Methoden zur Prognose künftiger Ereignisse nicht vorhergesagt werden können, da die dafür genutzten Modelle normalerweise durch Nutzung retrospektiver, also in der Vergangenheit beobachteter Daten gekennzeichnet bzw. darauf angewiesen sind. Die grundsätzliche Existenz solcher „black swan events“ ist nicht von zentralem Interesse für Taleb. Er beschäftigt sich vielmehr mit dem nach seiner 
Analyse sehr viel häufigeren Auftreten von schwarzen Schwänen. Sehr viel häufiger im Vergleich zu allgemeinen gesellschaftlichen Erwartungen. Für Taleb handelt es sich bei schwarzen Schwänen eher um die Regel, sozusagen um den Normalfall, als um die Ausnahme (Frankel 2010). Er greift somit eine Annahme der Theorie komplexer adaptiver Systeme auf, nämlich deren „fat-tail behavior", das statistisch häufigere Auftreten von Ereignissen, die stark vom Erwartungswert abweichen, als in einer Standardnormalverteilung von Ereignissen zu erwarten wäre. Insofern können schwarze Schwäne auch als Ereignisse verstanden werden, die in komplexen adaptiven System häufiger vorkommen, als in einfachen Systemen (Holland 2014: 5f). Taleb führt dann - durchaus in der Tradition Poppers - weiter aus: Die Zukunft lasse sich aus mehreren Gründen nicht sicher vorhersagen. Denn was immer sich heute als zutreffend erweise - oder besser: zutreffend scheine - könne sich in Zukunft aufgrund veränderter Bedingungen oder eines Zuwachses an Wissen als falsch herausstellen. Umgekehrt könnten aber auch Sachverhalte, die heute als eindeutig falsch zu charakterisieren seien, in Zukunft richtig werden, ebenfalls im Zuge neuer Entwicklungen und Entdeckungen. Und auch die zukünftigen Zuschreibungen seien wiederum nicht fixiert und für alle Zeit sicher, sondern von noch weiter in der Zukunft liegenden Dingen zu beeinflussen (Frankel 2010). Taleb fragt sich, wie Gesellschaften mit schwarzen Schwänen am besten umgehen, also negative unbeschadet überstehen und von positiven profitieren können. In einem späteren Werk nennt er Gesellschaften oder Individuen, die ersteres schaffen „antifragil“. Resilienz assoziiert er als Eigenschaft hingegen stärker mit Robustheit. Ohne auf Talebs Konzept der Antifragilität näher eingehen zu wollen, lassen sich darin sehr viele Ähnlichkeiten zu einem systemischen Resilienz-Verständnis ausmachen (Lukesch 2016: 296, 313).

Sind schwarze Schwäne ungewisse oder unsichere Ereignisse? Taleb versteht sie sicherlich eher als letztere, also Ereignisse, bei denen nicht nur die Eintrittswahrscheinlichkeit, sondern auch die Art des Ereignisses an sich unbekannt ist. Mit Blick auf Ereignisse, die sehr häufig als mittlerweile fast „klassische“ Beispiele für schwarze Schwäne herangezogen werden, ist diese Zuordnung zumindest theoretisch fragwürdig. Sie zeigt aber auf, dass eine Einordnung von Ereignissen in die Kategorie schwarzer Schwan sehr stark von den subjektiven Einschätzungen des Einordnenden abhängt. Auch der zeitliche und räumliche Kontext spielen eine Rolle. Häufig schränkt ein zeitlich und räumlich zu fokussierter Blick die Wahrnehmung ein und lässt ein Ereignis als schwarzen Schwan erscheinen, das in anderen Regionen oder zu früheren Zeiten sehr wohl bekannt war 
(Frankel 2010). Inwiefern das auf die folgenden Beispiele jeweils zutrifft oder nicht, ob es sich also um „echte" schwarze Schwäne handelt, lässt sich nicht ohne Weiteres feststellen. Sowohl die Terroranschläge vom 11. September 2001 als auch die globale Finanzkrise der Jahre 2007 und 2008 und die durch Kaskadeneffekte verursachte Nuklearkatastrophe von Fukushima im März 2011 werden jedenfalls immer wieder als prototypische schwarze Schwäne bezeichnet. Im Rahmen der Resilienzforschung wird neben der Metapher der schwarzen Schwäne auch von den „unknown unknowns" gesprochen, in Anlehnung an eine Aussage des damaligen amerikanischen Verteidigungsministers Donald Rumsfeld, die dieser auf einer Pressekonferenz im Februar 2002 tätigte. ${ }^{36}$ Unabhängig vom politischen Kontext der Aussage kann eine matrixhafte Unterscheidung zwischen „known knowns“, „known unknowns“ und „unknown unknowns“ getroffen werden. Die erste Kategorie bilden bekannte Ereignisse, deren Auswirkungen und Eintrittswahrscheinlichkeiten klar sind. Die zweite Kategorie könnte dann als ungewisse Ereignisse verstanden werden, also Ereignisse, die vorstellbar sind, deren Eintrittswahrscheinlichkeiten aber unklar sind. Und die dritte Kategorie bilden wiederum die unsicheren Ereignisse, oder schwarzen Schwäne, von denen im Prinzip nichts bekannt ist, bis sie sich manifestieren. Analytisch wäre zudem noch die Kombination von „unknown knowns“ möglich, die allerdings nur politisch, etwa durch den Philosophen Slavoj Žižek, interpretiert wurde als Dinge, „die wir nicht wissen wollen" und für das Erkenntnisinteresse der vorliegenden Arbeit nicht von zentralem Interesse ist. ${ }^{37}$ Eine weitere mögliche Umschreibung von Talebs schwarzen Schwänen liefert Erik Hollnagel. Hollnagel spricht im Zusammenhang mit der Nuklearkatastrophe von Fukushima und dem dieser vorausgehenden Tōhoku-Erdbeben von „unexampled events“, von beispiellosen Ereignissen. Diese seien für das betroffene System a priori quasi unvorstellbar und gehen weit über alles hinaus, mit dem es zuvor konfrontiert wurde (Hollnagel/Fujita 2013: 16). Den Begriff verwendet auch Ron Westrum so ähnlich (Westrum 2006: 57f). Ein weiterer Begriff in diesem Zusammenhang ist der der „wild card“, von dem Amanatidou et al. sprechen (Amanatidou et al. 2012: 210). Viele Autoren sehen in beispiellosen Ereignissen, in schwarzen Schwänen, oder Ereignissen, deren Auftreten im Knightschen Sinne unsicher ist und die bei ihrem Auftreten die Grenzen der Belastbarkeit des Systems weit übersteigen den eigentlichen Anwendungsbereich von Resilienz (Allenby/Fink

36 https:/www.youtube.com/watch?v=GiPe1OiKQukv [Stand: 27.6.2019].

37 http://www.lacan.com/zizekrumsfeld.htm [Stand: 27.6.2019]. 
2005: 1034, Folke et al. 2002: 440, Kuhlicke 2010, Lorenz 2010). Um komplexen adaptiven Systemen zu ermöglichen, auf solche Ereignisse ihre eigene Identität und Leistungsfähigkeit erhaltend reagieren zu können, müssen sie resilient sein.

Die Frage ist dann, wie weit Resilienz gehen kann und ob Systeme prinzipiell, bei genügend ausgeprägter generischer Anpassungsfähigkeit, mit beinahe allen Widrigkeiten umzugehen in der Lage sind. Nach kurzer Überlegung lassen sich bereits einige Beispiele finden, die eine eindeutig negative Antwort auf die eben formulierte Frage nahelegen. Streng genommen sind die folgenden Beispiele nicht als schwarze Schwäne oder unsichere Ereignisse zu verstehen, schon allein aus dem Grund, dass sie in der Aufzählung enthalten sind. Beispiellos im Sinne von nie dagewesen sind sie auch nur zum Teil. Nichtsdestotrotz scheint ihr Auftreten zwar denkbar, aber jeweils extrem unwahrscheinlich und gleichzeitig wäre bei einem Auftreten beinahe sicher davon auszugehen, dass sie die Belastungsgrenzen beinahe aller für die zivile SiFo relevanten sozio-technischen Systeme weit übersteigen würden. Derartige Ereignisse könnten im Ausbruch eines Super-Vulkans oder dem Einschlag eines Meteoriten auf der Erde und anschließenden dramatischen klimatischen Veränderungen bestehen. Oder in der Mutation hoch-ansteckender und hoch-tödlicher Krankheitserreger und dem Ausbruch einer weltweiten Pandemie. Oder einem nuklear geführten dritten Weltkrieg. Oder der versehentlichen Kreierung eines schwarzen Lochs im Rahmen teilchenphysikalischer Experimente. Oder der Invasion einer außerirdischen, technisch weit überlegenen Spezies. In solchen Fällen ist nach Baum auch ein resilientes System hilflos: „If humanity loses control of the planet, then traditional means of increasing resilience - such as creating redundant networks, stockpiling resources, or planning to adapt and recover - do not help humanity retain its critical functionality. [...] For all such species, resilience does not help" (Baum 2015: 231). Dieser Argumentation schließt sich die vorliegende Arbeit grundsätzlich an. Völlig unabhängig davon, wie das Resilienz-Konzept konkret verstanden wird, lässt sich festhalten: Auch resiliente Systeme sind nicht in der Lage, mit allen beliebigen, denkbaren und undenkbaren widrigen Ereignissen fertig zu werden. Allerdings beziehen sich die Grenzen von Resilienz für das in der vorliegenden Arbeit entwickelte Konzept primär auf das Ausmaß der widrigen Ereignisse und nicht auf den Grad an Unsicherheit. Baum argumentiert hier anders. Für ihn lässt sich das Resilienz-Konzept gerade nicht auf echt unsichere Ereignisse anwenden. Um die Resilienz eines Systems erhöhen zu können, müsse der Betreiber oder Manager des Systems wissen, auf welche Weise ein potentielles widri- 
ges Ereignis auf das System wirke. Rein empirisch erwiesen sich häufig auch scheinbar im Knightschen Sinne unsichere Ereignisse als gar nicht so unbekannt und es ließen sich zumindest einige wenige Informationen dazu finden und dann für resilientes Management nutzen (Baum 2015: 230). Das wird in zweifacher Hinsicht im Rahmen der vorliegenden Arbeit anders verstanden. Zum einen geht es zunächst um ein analytisches Resilienz-Konzept und die Frage, was Resilienz an sich ausmacht. Inwiefern Resilienz durch welche Handlungen in Systemen erhöht werden kann, steht erst an späterer Stelle der Arbeit im Fokus. Und zum anderen konnte auch rein analytisch deskriptiv in der system- und vor allem in der komplexitätstheoretischen Diskussion gezeigt werden, dass mithilfe generischer Anpassungsfähigkeit grundsätzlich auch eine erfolgreiche Reaktion auf das Eintreten echt unsicherer Ereignisse denkbar ist.

Der in der Resilienzforschung immer wieder erhobene Anspruch, das Unerwartete erwartbar machen zu wollen, ist im Prinzip ein Oxymoron (Berkes 2007: 288f). Entweder ein Ereignis wird erwartet oder nicht, beides zugleich ist logisch unmöglich (Goessling-Reisemann/Thier 2019: 124). Dieser Widerspruch lässt sich allerdings auflösen, wenn die Aussage spezifiziert wird. Wer die Welt aus einer komplexitätstheoretischen Sicht sieht, erkennt, dass sie nicht-deterministisch und nicht linear-kausal funktioniert. $\mathrm{Zu}$ einem beliebigen Zeitpunkt lässt sich nicht sicher sagen, wie die weitere Entwicklung eines komplexen adaptiven Systems und seiner Umwelt vonstattengehen wird. Die Zukunft ist unsicher. Resilienzforschung nimmt - zumindest implizit - genau diese Perspektive ein. Erneut hilft der Rückgriff auf Holling, um das zu verdeutlichen. Er war es, der die wissenschaftliche Diskussion über das Resilienz-Konzept zu einer „science of surprise“ machte (Folke 2006: 255). Und die Überzeugung, Überraschungen müssten erwartet werden, hat sich im Anschluss an Holling in der Resilienzforschung etabliert (Colten et al. 2008: 20, Lovins/Lovins 2001: 29, 269). Wer Überraschungen erwartet, der richtet sich auf das Eintreten unerwarteter Ereignisse, die die Belastungsgrenzen des relevanten Systems übersteigen, ein. Er erwartet also sozusagen das Unerwartete indem er mit etwas rechnet, das ihn überraschen und potentiell überfordern wird. Um damit erfolgreich umgehen zu können, benötigt er Resilienz in Form geeigneter Werkzeuge, die unabhängig von der konkreten Form des widrigen Ereignisses - denn dieses ist ja überraschend aufgetreten - hilfreich sind (Berkes 2007: 288f). Resilienz besteht also zum einen darin, mit dem Eintreten unerwarteter, oder präziser ungewisser und unsicherer, Ereignisse zu rechnen und dafür zu planen und deshalb zum anderen die Fähigkeiten zu entwickeln, mit prinzipiell beliebigen, 
die Belastungsgrenzen des Systems sprengenden, Ereignissen umgehen zu können. So oder so ähnlich wird Resilienz vor allen Dingen in der sozialökologischen und sozialwissenschaftlichen Forschung immer wieder definiert. Berkes spricht zum Beispiel von Resilienz als effektiver Möglichkeit, um mit Veränderungen umzugehen, die durch künftige Überraschungen oder unerkennbare Risiken charakterisiert seien (Berkes 2007: 284). Folke geht ebenfalls von einer unvorhersehbaren Zukunft aus, in der Überraschungen wahrscheinlich seien. Resilienz-zentriertes Management erhöhe dann die Chancen „of sustaining desirable pathways for development in changing environments” (Folke 2006: 254). Schon 2002 erklärt Folke zusammen mit Kollegen Resilienz in sozial-ökologischen Systemen als Fähigkeit, Überraschungen zu bewältigen (Folke et al. 2002: 440). Für Allenby und Fink stellt die Erhöhung von Resilienz eine rationale Strategie dar, wenn die Wahrscheinlichkeit für das Auftreten und die genaue Art widriger Ereignisse nur schwer definiert werden können (Allenby/Fink 2005: 1034). Die Beschäftigung mit Resilienz als Konzept lenkt die Aufmerksamkeit ein Stück weit weg von kalkulierbaren Risiken zu Fähigkeiten für den Umgang mit Unsicherheiten, meint Leach (Leach 2008: 11). Und Kuhlicke sieht ebenfalls eine Häufung von Resilienz-Definitionen, welche die Fähigkeit eines Systems beinhalten, sich „singular, unique and most often radically surprising events" anzupassen, „to come to terms with new and unexpected events" (Kuhlicke 2010). Kaufmann und Blum wiederum verorten Resilienz in der zivilen SiFo als neues Sicherheits-Paradigma, das immer häufiger Anwendung findet, da eine Notwendigkeit gesehen wird, sich an ständig und unvorhersehbar ändernde Bedingungen anzupassen (Kaufmann/Blum 2012: 243).

Der Zusammenhang zwischen Resilienz und Unsicherheit scheint also klar. Weil die Möglichkeit besteht, das unerwartete Ereignisse eintreten und die Belastungsgrenzen des Systems übersteigen, benötigen Systeme die Eigenschaft Resilienz. Als unerwartet wurden Ereignisse weiter oben dann beschrieben, wenn sie ungewiss oder im Knightschen Sinne unsicher sind. Ereignisse, denen ein spezifisches Risiko zugeschrieben werden kann, sind dagegen erwartbar. Denn das Ereignis an sich, die Wahrscheinlichkeit seines Auftretens und seine Auswirkungen auf das System sind bekannt. Gegeben dieses Wissen können Systeme sich auf spezifische Risiken auch mithilfe spezifischer Maßnahmen, in komplexitätstheoretischer Diktion mittels spezialisierter Anpassungsfähigkeit vorbereiten. Es lässt sich festhalten: Um Risiken zu bewältigen, benötigen Systeme keine Resilienz. Je nach Größe des Risikos, das sich aus der Multiplikation von Eintrittswahrscheinlichkeit und Ausmaß der Auswirkungen ergibt, kann ein System 
einen Teil seiner Ressourcen dazu nutzen, das Risiko zu verarbeiten. Das System kann aber auch entscheiden, dies nicht zu tun und ein Risiko bewusst einzugehen, sich nicht spezifisch darauf vorbereiten.

Mit Blick auf die Theorie komplexer adaptiver Systeme lässt sich fragen, ob überhaupt Situationen denkbar sind, in denen spezifische Risiken genau bekannt sind. Aufgrund der emergenten Eigenschaften komplexer adaptiver Systeme wäre die Antwort rein aus der Theorie gesprochen negativ. Prinzipiell lassen sich die Auswirkungen bestimmter systeminterner wie -externer Ereignisse auf ein komplexes adaptives System nicht umfassend und mit bestimmten Wahrscheinlichkeiten versehen vorhersagen. Empirisch weisen komplexe adaptive Systeme Holland zufolge jedoch häufig wiederkehrende Muster auf, anhand derer eine Analyse durchgeführt werden kann. Sie verhalten sich normalerweise nicht (völlig) zufällig (Holland 2014: 9). Das macht die Verwendung risikobasierter Strategien zumindest näherungsweise möglich und den Zusammenhang zwischen Risiko als einer Art von Unsicherheit und Resilienz auch für die in der zivilen SiFo relevanten komplexen adaptiven sozio-technischen Systeme interessant. Gerade wurde die Annahme getroffen, dass Resilienz zur Bewältigung von Risiken nicht notwendig ist. Für Folke et al. kann die Vorbereitung auf spezifische widrige Ereignisse, also eine risikobasierte Strategie, sogar dazu führen, dass Systeme an Resilienz einbüßen. Systeme, die eine hohe Robustheit angesichts häufig auftretender Störungen aufweisen, sind demnach besonders anfällig für seltene und überraschend auftretende Ereignisse (Folke et al. 2010). Im vorangegangenen Unterkapitel wurde dieser Aspekt bereits unter dem Stichwort der spezialisierten Anpassungsfähigkeit diskutiert. Die Vorbereitung auf bestimmte Risiken lässt sich komplexitätstheoretisch als Spezialisierung des Systems verstehen. Komplexe adaptive Systeme bzw. die Agenten, aus denen sie bestehen, verfügen insgesamt nur über begrenzte Mittel. Diese versuchen sie in nutzenmaximierender Weise zu verwenden. Ergänzend lässt sich nun für die zivile SiFo eine Tendenz feststellen, die begrenzten Ressourcen dazu aufzuwenden, das System gegen Risiken zu schützen, da diese quantifizierbar sind und den benötigten Ressourcen damit direkt ein ebenso quantifizierbarer Nutzen gegenübersteht (Cox et al. 2011: 308). Bezogen auf Menschen formuliert Edwards sehr treffend: „[P]eople make good choices in contexts in which they have experience, good information and prompt feedback" (Edwards 2009: 42). Die Wahrscheinlichkeiten und Auswirkungen ungewisser und unsicherer Ereignisse lassen sich gerade nicht quantifizieren, so dass es rational handelnden Agenten in komplexen adaptiven Systemen schwerfällt, entsprechende Ressourcen zu allokieren, die einer Erhöhung der generi- 
schen Anpassungsfähigkeit dienlich wären. Für das Resilienz-Konzept der vorliegenden Arbeit ergibt sich daraus: Die Quantifizierbarkeit von Risiken kann in komplexen adaptiven Systemen dazu führen, dass Ressourcen zur Erhöhung spezialisierter Anpassungsfähigkeit an Risiken genutzt werden. Aufgrund der Endlichkeit der Ressourcen führt dies ceteris paribus zu einer Verringerung generischer Anpassungsfähigkeiten und damit geringerer Resilienz.

Es gibt aber auch Risiken, auf die komplexe adaptive Systeme sich bewusst nicht vorbereiten. Das ist vor allen Dingen bei Risiken der Fall, deren Eintrittswahrscheinlichkeiten extrem niedrig sind, deren negative Auswirkungen auf das System dagegen extrem groß wären (Lundberg/Johansson 2015: 26f). Ereignisse, die mit solchen Risiken verbunden sind, übersteigen die Belastungsgrenzen des Systems. Sie stellen zwar keine Überraschung im Woodschen Sinne dar. Edwards zufolge sind es aber insbesondere derartige Ereignisse, die in Gesellschaften die größten Schäden verursachen: „The most destructive single disasters are usually the least surprising" (Edwards 2009: 35). Er plädiert dafür, sich weniger mit schwarzen Schwänen zu beschäftigen, als vielmehr mit diesen Ereignissen. Falls die Kosten zur Vorbereitung auf sie vertretbar seien, solle die Gesellschaft sie zahlen. Sehr viel häufiger zeichnen sich die gerade skizzierten Risiken aber durch extrem hohe Kosten aus, die zur Bewältigung aufgewendet werden müssten. Das erweist sich aufgrund der extrem geringen Eintrittswahrscheinlichkeit als unrentabel. Edwards spricht in solchen Fällen davon, die Ressourcen lieber zur Bewältigung anderer Risiken einzusetzen und Risiken mit extrem geringer Eintrittswahrscheinlichkeit aber extrem negativen Auswirkungen bewusst einzugehen (Edwards 2009: 41, Park et al. 2013: 359). Risiken einzugehen schließt jedoch nicht aus, tatsächlich eintretende widrige Ereignisse, deren Wahrscheinlichkeit und Auswirkungen im Vorhinein bekannt waren, dank hoher Resilienz im System zu bewältigen. Weiter oben wurde festgehalten, dass Resilienz nicht notwendig ist, um erfolgreich mit Risiken umzugehen. Dies gilt nur, wenn es sich um Risiken handelt, auf die ein komplexes adaptives System sich bewusst spezifisch vorbereitet und die innerhalb seiner Belastungsgrenzen liegen. Sobald keine spezifische Vorbereitung erfolgt, keine spezialisierte Anpassungsfähigkeit entwickelt wird, und gleichzeitig die Auswirkungen des potentiellen Ereignisses die Belastungsgrenzen des Systems übersteigen, ist das System bei Eintreten des Ereignisses auf das Vorhandensein generischer Anpassungsfähigkeit und damit auf Resilienz angewiesen. Denn „resilience increases the ability of systems to handle disturbances in general" (Baum 2015: 230). Für das Resilienz-Konzept der vorliegenden Arbeit heißt das: Zur Bewältigung von Risiken, für die komplexe adaptive Systeme kei- 
ne spezialisierte Anpassungsfähigkeit entwickeln und die ibre Belastungsgrenzen übersteigen, ist Resilienz notwendig.

In komplexen adaptiven Systemen ist das Eintreten bestimmter Ereignisse generell theoretisch mindestens ungewiss oder sogar echt unsicher. Das gilt auch für die mit den Ereignissen verbundenen Auswirkungen, die sich im System zeigen. Deshalb ist die gerade vorgenommene Diskussion von näherungsweise definierten Risiken ausgegangen. Sicher ist in komplexen adaptiven Systemen und ihrer Umwelt hingegen die Möglichkeit des Auftretens von unerwarteten Ereignissen. Die Frage ist, ob Resilienz immer nötig ist, wenn ein unerwartetes Ereignis eintritt. Mit Blick auf die Resilienzforschung und unter Rückgriff auf die Ergebnisse der beiden vorangegangenen Unterkapitel lässt sich das verneinen. Unerwartete Ereignisse können prinzipiell jede denkbare Form annehmen, bei ungewissen Ereignissen ist lediglich unbekannt, wie wahrscheinlich ihr Auftreten ist und bei echt unsicheren Ereignissen zudem noch, wie sie sich auswirken würden. Sie müssen jedoch nicht notwendigerweise mit gravierend negativen, mit „katastrophalen“ Auswirkungen für das System verbunden sein. Sie können sich ebenso gut als Chance für das betroffene System offenbaren oder das System nur wenig tangieren. Echte Unsicherheit und Ungewissheit heißt aber auch, dass es Ereignisse geben könnte, die die Belastungsgrenzen des betroffenen Systems übersteigen. Solche Ereignisse, Überraschungen im Woodschen Sinn, schwarze Schwäne in Talebs Verständnis oder beispiellose Ereignisse wie sie Hollnagel definiert, lassen sich nur mithilfe von Resilienz erfolgreich bewältigen. Die Ausbildung spezialisierter Anpassungsfähigkeit, wie sie eine risikobasierte Strategie nahelegen würde, ist dafür logisch unmöglich, da nicht klar ist, was passieren und wie es sich im System auswirken könnte. Inwiefern ein Ereignis in die genannten Kategorien fällt, ist stark kontextabhängig. Komplexe adaptive Systeme erleben einen gravierenden „failure of expectations“ und müssen darauf in Form einer sich selbst und ihre eigene Leistungsfähigkeit erhaltenden Veränderung reagieren. Dazu bedarf es nicht unbedingt der weiter oben beschriebenen Invasion durch eine außerirdische Spezies. Krisen oder Notfälle, welche die normale Funktionsfähigkeit eines Systems übersteigen und daher Resilienz notwendig machen, können auch rein für das System unerwartet und gravierend sein (Lorenz 2010). Mit ihren „normalen" Mitteln, die sich in komplexen adaptiven Systemen insbesondere in stark spezialisierten Signalverarbeitungsmustern ausdrücken, können sie mit diesen Ereignissen nicht fertigwerden. Also gilt: Zur Bewältigung von ungewissen und echt unsicheren Ereignissen, welche die Belastungsgrenzen komplexer adaptiver Systeme übersteigen, benötigen diese Resilienz. 
In diesem und den beiden vorangegangenen Unterkapiteln wurde die Notwendigkeit für Resilienz am Auftreten extrem unwahrscheinlicher oder unerwarteter Ereignisse, welche die Belastungsfähigkeit von komplexen adaptiven Systemen übersteigen, festgemacht. Die englischsprachige Resilienzforschung fasst solche Ereignisse mit dem Begriff der "shocks" zusammen. Neben shocks finden sich in der Literatur immer wieder auch „stresses“, also langsam ablaufende externe (Veränderungs-)Prozesse, denen gegenüber Systeme resilient sein können, oder nicht. So untersucht zum Beispiel die Psychologie neben Schock-Resilienz gegenüber plötzlichen Ereignissen wie dem Tod eines Angehörigen auch Stress-Resilienz etwa gegenüber langanhaltenden Bedingungen wie Armut in der Kindheit, einer eigenen chronischen Erkrankung oder beispielsweise in der Gerontologie gegenüber einer Demenzerkrankung des Partners (siehe 2.2). In der Psychologie, aber beispielsweise auch in der wirtschaftsgeographischen Forschung, wenn es darum geht, einen langfristigen Strukturwandel in einer Region erfolgreich zu gestalten, ergibt es Sinn, das Resilienz-Konzept auch auf stresses auszuweiten. Basierend auf der system- und komplexitätstheoretischen Analyse und der Konzeptualisierung des Zusammenhangs zwischen Resilienz und Unsicherheit gilt dies allerdings nicht für das Resilienz-Konzept der zivilen SiFo, das in der vorliegenden Arbeit entwickelt wird. Hier ist Resilienz nur notwendig, wenn ein schockartiges Ereignis eintritt, für das das betroffene komplexe adaptive System aufgrund begrenzter Ressourcen oder der Unbekanntheit des Ereignisses a priori nicht die Möglichkeit hatte, eine spezialisierte Anpassungsfähigkeit auszubilden. Bei langsam ablaufenden, systemextern auftretenden Prozessen bleibt dem System hingegen genügend Zeit, sich auf die Charakteristika des Prozesses spezifisch einzustellen und seine Signalverarbeitungsmuster entsprechend zu spezialisieren. Resilienz weiter zu fassen und auch eine derart spezialisierte Anpassung an langfristig ablaufende Prozesse miteinzubeziehen, ergibt wie gesagt für Disziplinen wie die Psychologie oder die Wirtschaftsgeographie Sinn. Ein Resilienz-Konzept der zivilen SiFo, bei dem explizit auch der Mehrwert gegenüber bekannten Konzepten und Strategien wie vor allen Dingen dem risikobasierten Ansatz im Mittelpunkt steht, sollte jedoch präziser gefasst werden. Mit Blick auf die Ziele des noch aktuellen zweiten Rahmenprogramms der zivilen Sicherheitsforschung ist eine derartige Konzeptualisierung von Resilienz zudem konsistent mit dem grundsätzlichen Erkenntnisinteresse des relevanten Anwendungsbereichs (BMBF 2016: 3).

Schockartig heißt dabei nicht innerhalb von Sekunden, Minuten oder auch Tagen. Die relevante Zeitspanne lässt sich nicht a priori festlegen. 
Es geht aber immer um relativ schnelle, radikale und abrupte Veränderungen, bei denen das betroffene System nicht genügend Zeit hat, sich mithilfe einer evolutionären Weiterentwicklung darauf einzustellen. Ein solches Schockereignis kann beispielsweise auch in einem pandemischen Ausbruch ansteckender Infektionskrankheiten bestehen, der wahrscheinlich einige Wochen in Anspruch nehmen würde (BMBF 2016: 24f). Mit langfristigen Entwicklungen wären dagegen eher Phänomene wie der Klimawandel, der gerade genannte wirtschaftliche Strukturwandel in Regionen oder die Alterung der Gesellschaft im Zuge des demographischen Wandels gemeint. Die relevanten Zeitspannen für diese Entwicklungen bemessen sich in Jahren oder sogar Jahrzehnten. Für das zu entwickelnde Resilienz-Konzept der zivilen SiFo sind sie allerdings in einer Hinsicht doch von Interesse. Und zwar dann, wenn sich ein langfristiger Prozess durch Erreichen eines Umkipp-Punktes bzw. Schwellenwertes plötzlich als kurzfristiges, abruptes und gravierend widriges Ereignis materialisiert. Schwellenwerte sind a priori unbekannt. Gleichzeitig kann das Erreichen eines Schwellenwertes konzeptionell als Überschreiten der Belastungsgrenze des betroffenen komplexen adaptiven Systems verstanden werden. Es handelt sich also um ein im Woodschen Sinne überraschendes Ereignis, einen schwarzen Schwan. Indirekt trägt Resilienz dann doch dazu bei, dass das betroffene komplexe adaptive System erfolgreich mit den negativen Auswirkungen langfristiger Entwicklungen umgehen kann. Für die Art der für Resilienz relevanten Störungen gilt daher: Um mit langfristigen Veränderungsprozessen erfolgreich umgehen zu können, benötigen komplexe adaptive Systeme keine Resilienz. Erst wenn die langfristigen Prozesse durch Erreichen eines Schwellenwertes ein unerwartetes und die Belastungsgrenzen des Systems überschreitendes Ereignis auslösen, wird Resilienz notwendig.

Zusammenfassend ergeben sich für ein eigenständiges Resilienz-Konzept der zivilen Sicherheitsforschung aus der Diskussion des Zusammenhangs zwischen Resilienz und Unsicherheit folgende Annahmen:

- In den für die zivile SiFo relevanten komplexen adaptiven sozio-technischen Systemen sind Ereignisse, ihr Eintreten und ihre Auswirkungen a priori unsicher.

- Neben Ereignissen, die aus der Systemumwelt resultieren, müssen komplexe adaptive Systeme auch mit dem Auftreten unsicherer interner, durch die Entscheidungen von Agenten ausgelöster, von diesen unintendierter oder intendierter Störungen umgehen können. Die Wahrscheinlichkeit des Auftretens solcher Störungen steigt mit der Eigenkomplexität der Systeme. 
- Es gibt drei Arten von Unsicherheit. Die erste Art kann als Risiko bezeichnet werden. Die Wahrscheinlichkeit für das Auftreten von Ereignissen, die in diese Kategorie fallen, ist ebenso bekannt wie ihre Auswirkungen. Die zweite Art kann als Ungewissheit bezeichnet werden. Bei ungewissen Ereignissen sind die möglichen Auswirkungen bekannt aber nicht die Wahrscheinlichkeit, mit der das Ereignis eintritt. Die dritte Art ist echte Unsicherheit. Echt unsichere Ereignisse sind nicht nur ihrer Wahrscheinlichkeit nach, sondern auch im Hinblick auf ihre Auswirkungen unbekannt. Ungewisse und unsichere können zusammenfassend als unerwartete Ereignisse bezeichnet werden.

- Um Risiken zu bewältigen, benötigen Systeme keine Resilienz. Das Ereignis an sich, die Wahrscheinlichkeit seines Auftretens und seine Auswirkungen auf das System sind bekannt, so dass spezifische Maßnahmen zum Umgang mit dem Risiko ausreichen.

- Die Quantifizierbarkeit von Risiken (Eintrittswahrscheinlichkeit multipliziert mit den Auswirkungen bestimmter Ereignisse) kann in komplexen adaptiven Systemen dazu führen, dass Ressourcen zur Erhöhung spezialisierter Anpassungsfähigkeit an Risiken genutzt werden. Aufgrund von deren Endlichkeit führt dies ceteris paribus zu einer Verringerung generischer Anpassungsfähigkeiten und damit geringerer Resilienz.

- Es gibt Risiken, die komplexe adaptive Systeme ganz bewusst eingehen, weil ihre Eintrittswahrscheinlichkeit extrem gering ist, aber gleichzeitig die Auswirkungen so groß, dass die Ausbildung spezialisierter Anpassungsfähigkeit sehr viele Ressourcen in Anspruch nehmen würde. Zur Bewältigung von Risiken, für die komplexe adaptive Systeme keine spezialisierte Anpassungsfähigkeit entwickeln und die ihre Belastungsgrenzen übersteigen, ist Resilienz notwendig.

- In komplexen adaptiven Systemen besteht die Möglichkeit, dass unerwartete Ereignisse mit gravierenden Auswirkungen, sogenannte schwarze Schwäne bzw. beispiellose Ereignisse auftreten. Zur Bewältigung solcher ungewissen und echt unsicheren Ereignisse, welche die Belastungsgrenzen komplexer adaptiver Systeme übersteigen, benötigen diese Resilienz.

- Um mit langfristigen Veränderungsprozessen erfolgreich umgehen zu können, benötigen komplexe adaptive Systeme keine Resilienz. Erst wenn die langfristigen Prozesse durch Erreichen eines Schwellenwertes ein unerwartetes und die Belastungsgrenzen des Systems überschreitendes Ereignis auslösen, wird Resilienz notwendig. 
- Resilienz hat Grenzen. Auch resiliente Systeme sind nicht in der Lage, mit allen beliebigen, denkbaren und undenkbaren widrigen Ereignissen fertig zu werden.

\subsubsection{Resilienz und Vulnerabilität}

Komplexe adaptive Systeme „leben“ in einer unsicheren Welt. Aufgrund ihrer Komplexität und der Komplexität ihrer Umwelt lässt sich die $\mathrm{Zu}$ kunft, also künftige systemexterne wie systeminterne Ereignisse und Reize sowie die Reaktion des Systems auf diese Ereignisse und Reize, nicht sicher voraussagen. Es besteht systemintern wie -extern die Möglichkeit, dass Ereignisse auftreten, welche eine als „normal“ empfundene Funktionsfähigkeit der Systeme herausfordern. Solche „Störungen“ wirken sich vielfach unterschiedlich auf die betroffenen Systeme aus. Aus der psychologischen Forschung kommt beispielsweise die Idee einer inhärenten Notwendigkeit des Auftretens von Störungen, um komplexen Systemen, wie dem Menschen oder von Menschen gebildeten Gesellschaften, die Möglichkeit zu geben, sich selbst durch einen erfolgreichen Umgang mit der Störung weiterzuentwickeln (Vogt 2015: 4). Ob eine Störung einem komplexen System eher schadet oder nutzt, wie groß der Schaden ist und welche - unterschiedlichen - Auswirkungen sie kurz- und langfristig im System zeitigt, hängt demzufolge nicht allein von der Störung an sich ab. Mindestens genauso wichtig sind Eigenschaften oder Fähigkeiten, die das betroffene System aufweist - oder nicht aufweist. Die Nähe zur Diskussion des ResilienzKonzepts ist bei diesen Gedanken offensichtlich. In den vorangegangenen Unterkapiteln wurde Resilienz dann als notwendig definiert, wenn komplexe adaptive Systeme sich unerwarteten oder extrem unwahrscheinlichen Ereignissen gegenübersehen, die ihre Belastungsgrenzen übersteigen. Je größer ihre Resilienz, desto besser können sie mit solchen Ereignissen fertigwerden. Gegeben eine spezifische Störung unterscheiden sich die Reaktionen zweier komplexer adaptiver Systeme darauf, weil sie unterschiedliche Grade an Resilienz aufweisen. Der so formulierte Gedanke liegt auch einer weiteren, mit der Resilienzforschung verwandten Richtung der Katastrophenforschung, der sogenannten Verwundbarkeitsforschung zugrunde. Die amerikanische Geographin und Verwundbarkeitsforscherin Susan Cutter, deren einflussreiche Arbeiten zum spezifischen Aspekt der sozialen Verwundbarkeit weiter unten vorgestellt werden, formuliert dazu treffend: „Vulnerability helps us to understand why the same hazard event can 
produce different consequences across the natural and human landscape" (Cutter 2013).

Die beiden Begriffe „Verwundbarkeit“ und „Vulnerabilität“ werden in der vorliegenden Arbeit synonym verwendet. Je stärker sie ausgeprägt sind, je vulnerabler etwa eine Gesellschaft gegenüber einer Störung ist, desto größer sind die Schäden, die diese bei Auftreten der Störung zu erwarten hat. Vulnerabilität lässt sich nach Cutter grob als „potential for loss" verstehen (Cutter 2013, Cutter et al. 2003: 242). Auf den ersten Blick scheint es daher mehr als plausibel, Vulnerabilität einfach als Gegenbegriff zu Resilienz zu nutzen. Je größer die Vulnerabilität eines Systems, desto geringer seine Resilienz und vice versa (Fooken 2016: 1). Bei einem derartigen Begriffsverständnis wäre allerdings einer der beiden Begriffe verzichtbar, seine Verwendung rein deskriptiver, jedoch nicht analytischer Natur. Aufgrund der Bedeutung, die die beiden Konzepte in der jeweils anderen Forschungsrichtung innehaben, lohnt ein näherer Blick auf den Zusammenhang zwischen Resilienz und Vulnerabilität. Ähnlich wie der Resilienz-Begriff wird auch Vulnerabilität bereits seit Jahrzehnten erforscht. Und ebenfalls in großer Ähnlichkeit zur wissenschaftlichen Diskussion über Resilienz, besteht auch bei Vulnerabilität keine disziplinoder fachübergreifende Einigkeit darüber, was mit dem Begriff oder dem zugrundeliegenden Konzept genau gemeint ist. Weder zu Resilienz noch zu Vulnerabilität existiert eine präzise und allgemein anerkannte Definition (Fekete et al. 2014: 4). Vielmehr wird auch Vulnerabilität, wie Resilienz, je nach disziplinärem Zugang und vorliegendem Erkenntnisinteresse sehr unterschiedlich verstanden und konzeptualisiert. Vulnerabilität wird zudem sowohl deskriptiv als auch normativ verwendet, im Vergleich zu Resilienz bei einem letzteren Verständnis aber als normativ negativ gewertet und als etwas angesehen, dass möglichst überwunden oder verringert werden sollte (Bara 2010: 7ff). Im Gegensatz zu Resilienz gibt es für das Vulnerabilitäts-Konzept nach Fekete et al. bereits deutlich mehr Ansätze zur Operationalisierung und damit zur Anwendung im Rahmen empirischer Fallstudien. Vulnerabilität sei methodisch als Konzept weiter fortgeschritten (Fekete et al. 2014: 14). Der eben angekündigte, nähere Blick auf den Zusammenhang zwischen Resilienz und Vulnerabilität sollte dem entsprechend Rechnung tragen.

Wissenschaftliche Forschung zum Thema Vulnerabilität existiert seit vielen Jahrzehnten. Galland zufolge hat sie ihre Ursprünge in Ansätzen aus der Geographie, die zwischen den beiden Weltkriegen, also in den 1920er und 1930er Jahren, an der Universität von Chicago entwickelt wurden. Der Fokus dieser Ansätze - und der Verwundbarkeitsforschung insgesamt 
- lag auf der Untersuchung von Naturgefahren. In ihrem ursprünglichen Verständnis ging die Verwundbarkeitsforschung davon aus, dass die Vulnerabilität bestimmter Orte davon abhängt, wie wahrscheinlich das Auftreten bestimmter Naturgefahren an diesen Orten ist (Galland 2012: 311). Dieser Gedanke ist nach wie vor naheliegend. Auch in einem moderneren Begriffsverständnis von Vulnerabilität spielt die Ausgesetztheit gegenüber Naturgefahren eine wichtige Rolle. Unter sonst gleichen Bedingungen also, wenn zwei komplexe adaptive Systeme denkbar wären, die sich nur in ihrer räumlichen Verortung unterscheiden und ansonsten exakt gleich sind - ist die Vulnerabilität einer an einer Küste liegenden Kommune größer als die einer Kommune im erhöhten Hinterland. Denn erstere sieht sich der - zusätzlichen - Gefahr von Überflutungen ausgesetzt. Das ursprüngliche Verständnis von Vulnerabilität geht mit der Annahme einher, dass die Realisierung bestimmter Naturgefahren zu ganz bestimmten Auswirkungen in Gesellschaften führt und zwar unabhängig von der spezifischen Gesellschaft. Das primäre Forschungsinteresse liegt dann im Verständnis der Naturgefahren, in Versuchen, ihre Ursachen herauszufinden und darin, die Wahrscheinlichkeit für ihr Auftreten quantifizieren zu können. Die Beantwortung dieser Fragen ist bis heute Forschungsgegenstand verschiedener Disziplinen und zur Untersuchung der Vulnerabilität von Gesellschaften und ihren kritischen Infrastrukturen von entscheidender Bedeutung. Empirisch lässt sich die Annahme, wonach der Schaden, den eine Gesellschaft durch ein widriges Ereignis erleidet, nur von diesem Ereignis abhängt, allerdings nicht halten (Bara 2010: 2f, Fekete et al. 2016: 216).

Seit den 1970er Jahren hat sich daher als eine Art kritischer „Gegenbewegung zu reinen Gefahrenansätzen“ die eigentliche Verwundbarkeitsforschung entwickelt. Für diese sind nicht mehr länger nur die Naturgefahren an sich von zentralem Interesse, sondern auch die - möglicherweise aufgrund anderer Faktoren unterschiedlich ausgeprägte - Vulnerabilität verschiedener komplexer, adaptiver Systeme. Im klassischen Sinne war der Schaden eine „absolute Größe“. In einem moderneren Verständnis von Vulnerabilität hängt er hingegen von ganz verschiedenen Faktoren $\mathrm{ab}$, die nicht notwendigerweise mit der eigentlichen Gefahr, mit dem eigentlichen, widrigen oder disruptiven Ereignis in Verbindung stehen müssen (Fekete et al. 2016: 216, Galland 2012: 311). Die Verwundbarkeitsforschung bewegt sich hier im Kontext der Katastrophenforschung, in der es unter anderem auch darum geht, zu erklären, wie und warum bestimmte widrige Ereignisse zu einer Katastrophe werden - oder gerade nicht (Voss/Dittmer 2016: 181f). Ein in seinem Ausmaß gravierendes 
widriges Ereignis, zum Beispiel ein schwerer Sturm oder Hurrikan, muss nicht notwendigerweise auch zu schweren negativen Auswirkungen für das betroffene System führen. Auf der anderen Seite kann ein scheinbar harmloses Ereignis, wie etwa anhaltende Regenfälle, das System vor unlösbare Herausforderungen stellen. Wie groß die durch widrige Ereignisse verursachten Schäden sind, hängt also nur indirekt mit dem eigentlichen Ereignis zusammen, ist aber direkt zurückzuführen auf die Vulnerabilität des betroffenen Systems (Al-Khudhairy et al. 2012: 576). Und diese setzt sich wie erwähnt aus verschiedenen Faktoren zusammen. Die Faktoren können physischer, sozialer, ökonomischer oder auch politischer Art sein und hängen häufig eben gerade nicht mit dem widrigen Ereignis zusammen. Es sind vielmehr generelle gesellschaftliche Umstände, Bedingungen oder häufig Ungleichheiten, die zusammengenommen die Vulnerabilität eines Systems, seine Anfälligkeit für Schäden, ausmachen (Bara 2010: 2). Eine zusammenfassende Definition könnte dann wie folgt lauten: Vulnerabilität beschreibt die „Zustände und Prozesse, die die Ausgesetztheit, Anfälligkeit sowie die Reaktionskapazitäten eines Systems oder Objekts hinsichtlich des Umgangs mit Gefahren (...) bedingen. Dabei spielen physische, soziale, ökonomische und umweltbezogene Faktoren eine Rolle“ (Christmann/Ibert 2016: 237, siehe ähnlich auch Cutter 2013). Inwiefern tatsächlich Reaktionskapazitäten sinnvollerweise in ein Vulnerabilitätsverständnis miteinbezogen werden sollten, lässt sich diskutieren. Im Verlauf dieses Unterkapitels wird im Hinblick auf ein theoretisch-konzeptionell stringent zu fassendes Verständnis von Vulnerabilität im Vergleich zu Resilienz weiter darauf eingegangen.

Die Definition von Christmann und Ibert, die diese nach Jörn Birkmann zitieren, zeigt die Vielschichtigkeit des Vulnerabilitäts-Konzepts auf. Bara verdeutlicht diese anhand eines Beispiels. Hurrikan Katrina, der im Jahr 2005 zur bis dato schwersten Naturkatastrophe in der Geschichte der Vereinigten Staaten von Amerika wurde, traf New Orleans und Louisiana gegen Ende des Monats August. Zu diesem Zeitpunkt, bevor die monatlichen Lohnzahlungen und Sozialleistungen überwiesen wurden, fehlten vielen ärmeren Bewohnern schlicht die finanziellen Mittel, um sich vor dem heranziehenden Hurrikan in Sicherheit bringen zu können (Bara 2010: 3). Katrina kann auch darüber hinaus generell als „gutes“ oder besser gesagt dramatisches Beispiel dafür verstanden werden, wie ein erwartbares, ein erwartetes Ereignis aufgrund hoher Vulnerabilität unerwartet schwerwiegende, negative Auswirkungen zeitigen konnte. Die Anfälligkeit des Mississippi-Deltas und insbesondere der Stadt New Orleans gegenüber Stürmen, Hurrikans und Überschwemmungen war empirisch bereits lange 
vor Eintreten des Hurrikans Katrina bekannt. Im Verlauf der vergangenen 300 Jahre sah sich beispielsweise New Orleans insgesamt 27 schwerwiegenden Flut-Ereignissen gegenüber. Die Lage der Stadt, die nicht nur im Deltabereich eines der größten Flüsse der Welt liegt, sondern zudem noch direkt am Ufer des Lake Pontchartrain und in großen Teilen unterhalb des Meeresspiegels, führt dazu, dass besonders großflächige Überschwemmungen auch heute, mehr als ein Jahrzehnt nach Katrina, weiterhin als größte Bedrohung für New Orleans angesehen werden (Colten et al. 2008: 1, Renn et al. o.J.: 1). In den Jahren vor Hurrikan Katrina gab es vielfältige Hinweise darauf, welche Auswirkungen ein Hurrikan haben könnte. Neben populärwissenschaftlichen Arbeiten und Artikeln in lokalen Zeitungen, beschäftigten sich auch wissenschaftliche Publikationen und Katastrophenschutzübungen mit vergleichbaren Szenarien (Colten et al. 2008: 6, Renn et al. o.J.: 1).

Nichtsdestotrotz führte Katrina zu nicht anders als katastrophal zu nennenden Schäden in der Stadt und für die in ihr lebenden Menschen. Woran lag das? Vor Katrina war „Betsy“ der Hurrikan, der in New Orleans die schwersten Schäden verursacht hatte. Hurrikan Betsy traf die Stadt im Jahr 1965, setzte über 40 Prozent ihrer Fläche unter Wasser und verursachte schwere Schäden an über 14.000 Häusern. Dank der schnellen und koordinierten Reaktion der Behörden funktionierte die Stadt jedoch bereits einen Monat nach Ende des Hurrikans wieder in fast normalem Umfang, das heißt die Schulen waren geöffnet, Unternehmen konnten ihrer Tätigkeit wieder nachgehen und die öffentliche Infrastruktur lieferte die benötigten Leistungen, wie etwa Strom und Wasser. Darüber hinaus wurden großzügige Mittel bereitgestellt, um die Dämme und Deiche, die New Orleans vor Überflutungen schützen sollen, auszubauen. Als „rule of thumb“ galt dabei laut Colten et al., die Dämme im Anschluss an eine Überflutung ca. einen Fuß, gut 30 Zentimeter, höher zu bauen, als zuvor. Zudem wurden Gesetze erlassen, die den Abschluss von Versicherungen gegen Hochwasserschäden forcieren sollten (Colten et al. 2008: 7f). Diese im Anschluss an Betsy durchgeführten Maßnahmen und die vergleichsweise erfolgreiche unmittelbare Reaktion auf den Hurrikan halfen vierzig Jahre später allerdings nicht dabei, mit Katrina fertigzuwerden. Colten et al. sehen sogar eher einen gegenteiligen Effekt, nach dem die Bevölkerung und insbesondere die Verantwortlichen in New Orleans sich zu stark auf die Wirkung der existierenden, strukturellen Schutzmechanismen verlassen hätten und dadurch in eine Abhängigkeit von diesen notwendigerweise unzureichenden Vorkehrungen geraten seien. Sie formulieren dies zugespitzt wie folgt: „Recovery following Betsy has not produced a more 
resilient population within the city" (Colten et al. 2008: 7). Inwiefern Resilienz oder ein Mangel daran während und nach Hurrikan Katrina erkennbar wurde, ist an dieser Stelle aber zunächst weniger interessant als verschiedene andere Faktoren, die den Hurrikan zu einer Katastrophe werden ließen. So lässt sich beispielsweise festhalten, dass vor Hurrikan Betsy nur ein kleiner Teil der Bevölkerung in besonders tiefliegenden und damit flutanfälligen Gegenden der Stadt lebte. Nach 1965 - und im Gefühl der Existenz schutzbietender Dämme - siedelten sich dagegen immer mehr Menschen dort an. Vor allen Dingen Menschen afroamerikanischer Herkunft und/oder ärmere Menschen nutzten das vergleichsweise günstige Land. Hurrikan Katrina führte dann dazu, dass gerade die Wohngegenden, in denen ärmere Menschen lebten, stärker überflutet wurden, gerade weil sie ja eher tiefliegend - und damit günstig - waren. Unterschiedliche sozioökonomische Voraussetzungen führten demzufolge zu einer unterschiedlichen Betroffenheit von den Auswirkungen des Hurrikans. Gleichzeitig spielte zum Beispiel auch das Alter eine Rolle dabei, wie gravierend die Auswirkungen von Katrina waren. Unter den Todesopfern des Hurrikans waren ältere Menschen sehr stark überrepräsentiert (Colten et al. 2008: 1, 21).

Das Alter und der sozioökonomische Status sind zwei soziale Faktoren, die offensichtlich einen Einfluss darauf haben, wie sich widrige Ereignisse auf eine Gesellschaft auswirken. Je älter und je ärmer jemand ist, desto schwerer wird er von einer Störung getroffen. Das gilt nicht nur auf individueller Ebene, sondern vor allem auch bezogen auf soziale Gruppen. Widrige Ereignisse treffen „marginalisierte Sub-Kulturen“ normalerweise härter, als reichere Teile der Gesellschaft (Tobin 1999: 15). Diese sozialen Aspekte von Vulnerabilität, oder kürzer gesagt „soziale Vulnerabilität“, waren, wie weiter oben argumentiert wurde, lange Zeit unterrepräsentiert in der Verwundbarkeitsforschung (Cutter et al. 2003: 243). Es ist unter anderem Susan Cutter zu verdanken, dass dies heute so nicht länger gilt. Cutter nutzt ein sogenanntes „hazards-of-place model“ zur Definition von Vulnerabilität. Demnach setzt sich die Vulnerabilität spezifischer Orte aus verschiedenen Faktoren zusammen, die sich mit den Begriffen biophysikalische und soziale Vulnerabilität zusammenfassen lassen (Cutter et al. 2003: 243f). Die Forschung zu sozialer Vulnerabilität beinhaltet also den Ausschnitt eines umfassenderen Vulnerabilitäts-Konzepts, der sich mit „pre-existing social and demographic characteristics of people and places that influence the distribution of the social burdens of risk" beschäftigt (Cutter 2013). Für die vorliegende Arbeit dient die folgende Vorstellung der Ideen und Konzepte Cutters zur sozialen Vulnerabilität als Beispiel 
eines Verständnisses von Vulnerabilität, mit dessen Hilfe sich der Zusammenhang des Konzepts zu Resilienz in geeigneter Form analysieren lässt. Ausgehend von der Beobachtung, dass Risiken in Gesellschaften nicht gleich verteilt sind und selbiges auch für "social patterns“, also soziale Randbedingungen oder Muster gilt, stellt Cutter die These auf, dass eine Verbindung zwischen der Anfälligkeit für Schäden, also der Vulnerabilität, und der Ausprägung verschiedener sozialer Faktoren besteht. Dabei spielt laut ihr kein einzelner Faktor wie zum Beispiel die oben erwähnte Armut oder das Alter eine allein entscheidende Rolle. Soziale Vulnerabilität ist für Cutter ein multidimensionales Konzept, das erst in der Interaktion verschiedener sozialer Faktoren wie Alter, Armut, Ethnizität, Geschlecht, sozioökonomischer Status und vieler weiterer, wirksam wird und dazu führt, dass bestimmte Gruppen von Menschen an spezifischen Plätzen besonders verwundbar sind im Vergleich zu anderen (Cutter 2013). Die Multidimensionalität des Konzepts und die Betonung auf der Interaktion verschiedener Faktoren miteinander verortet Cutters Ideen in komplexitätsund systemtheoretischen Überlegungen. Auch aus diesem Grund ist die Beschäftigung mit sozialer Vulnerabilität anschlussfähig zu den bisherigen Erkenntnissen. Cutters Untersuchungsgegenstand sind letztlich nichts Anderes als komplexe adaptive Systeme, so dass die in den vorangegangenen Unterkapiteln herausgearbeiteten Annahmen gültig sind und die folgende Diskussion direkt zur weiteren Erarbeitung des Resilienz-Konzepts für die zivile SiFo genutzt werden kann.

Nach Cutter herrscht in den Sozialwissenschaften ein grundsätzlicher Konsens über eine ganze Reihe der wesentlichen Einflussfaktoren für den Grad an sozialer Vulnerabilität, den ein Individuum bzw. eine soziale Gemeinschaft aufweist. Zu diesen Einflussfaktoren zählen der Zugang zu Ressourcen, politischer Macht und adäquater Repräsentation, das Maß an Sozialkapital, Überzeugungen und Bräuchen, die körperliche und geistige Fitness von Menschen, aber auch die Art und Dichte der Bebauung und der kritischen Infrastrukturen an einem bestimmten Ort (Cutter et al. 2003: 245). Die eigentliche Gefahr, das widrige Ereignis an sich kommt in dieser Liste nicht vor. Genau darin sieht Bara einen wesentlichen Mehrwert in der Diskussion um das Thema soziale Vulnerabilität. „The social vulnerability perspective thus marks a shift away from a focus on the hazard towards a focus on the people" (Bara 2010: 4). Soziale Vulnerabilität ist demnach eine Eigenschaft von Menschen oder Gruppen von Menschen, die Unterschiede innerhalb von Gesellschaften verdeutlicht und daher auch immer nur relativ zu anderen Menschen oder Gruppen verstanden bzw. gemessen werden kann. Zudem rückt dank einer Betrachtung mit 
Fokus auf der sozialen Vulnerabilität nicht nur das Überleben von Menschen als Zielsetzung von Sicherheits- und Katastrophenforschung in den Blick. Der durch widrige Ereignisse verursachte Schaden wird nicht nur in der Zahl der Toten oder ökonomischen Verlusten ausgedrückt, sondern auch darin, wie sehr die Lebensweise und -wirklichkeit betroffener Personen bzw. Gruppen von Personen beeinflusst wird (Bara 2010: 4). Neben der relativen Einigkeit bezüglich des theoretischen Verständnisses von sozialer Vulnerabilität weist Cutter aber auch darauf hin, dass kaum Einigkeit dahingehend bestehe, wie sich die oben genannten Einflussfaktoren auf geeignete Art und Weise operationalisieren und messen lassen (Cutter et al. 2003: 245). Um das multidimensionale Konzept der sozialen Vulnerabilität besser messbar zu machen, entwickelte Cutter deshalb gemeinsam mit Kollegen den sogenannten „Social Vulnerability Index (SoVI)“ (Cutter 2013).

Wirft man, diesen SoVI verwendend, einen Blick auf das oben beschriebene Fallbeispiel des Hurrikans Katrina und seiner Auswirkungen in New Orleans, lassen sich negative Auswirkungen hoher sozialer Vulnerabilität vor allem auch in der Zeit nach dem Hurrikan erkennen. Demnach besteht ein statistisch relevanter aber schwacher negativer Zusammenhang zwischen sozialer Vulnerabilität und der Rückkehrrate evakuierter Bewohner nach New Orleans. Je höher deren soziale Vulnerabilität, desto geringer die Wahrscheinlichkeit, dass sie zwei Jahre nach Katrina bereits in die Stadt zurückgekehrt waren (Finch et al. 2010: 198). An Ergebnissen wie diesem lässt sich Cutter zufolge die Bedeutung von Maßen in der Art des SoVI erkennen. Durch ihn lässt sich Entscheidungsträgern in leicht eingängiger Weise erklären, wie etwa Mittel für den Katastrophenschutz am sinnvollsten eingesetzt werden sollten (Cutter 2013). Das fügt der weiter oben bereits beschriebenen Erkenntnis, dass unterschiedliche sozioökonomische Voraussetzungen zu einer unterschiedlichen Betroffenheit von Auswirkungen widriger Ereignisse führen können, eine empirisch belegte theoretische Fundierung hinzu.

Gleichzeitig lassen sich am Fallbeispiel Katrina auch einige Phänomene ausmachen, bei denen sich die Frage stellt, wie gut sie in einer Analyse mit Fokus auf der Vulnerabilität von New Orleans und seinen Bewohnern verortet werden können. Colten et al. schreiben von zwar insgesamt mangelnder Resilienz gegenüber dem Hurrikan, aber doch zumindest einem „resilient spirit“, der sich in vielfachen Anstrengungen zur möglichst vollständigen Wiederherstellung der Stadt widerspiegele (Colten et al. 2008: 2). Oder von der Tatsache, dass das Rote Kreuz zwar als Hilfsorganisation zur Verfügung stand, aber aufgrund der massiven Überflutungen nicht in 
die Stadt kam - ein von den Katastrophenschützern und Ersthelfern so nicht erwartetes Ereignis. Ebenso unerwartet war die weitverbreitete Sorge um Haustiere, die häufig eine Evakuierung der Bewohner erschwerte bzw. verzögerte. Colten et al. zufolge wurden einige vor Katrina gehegte Erwartungen durch die reale Erfahrung widerlegt. Obwohl Pläne für die Reaktion auf derartige Ereignisse vorlagen, führten die extremen Bedingungen - Bedingungen, welche die Belastungsgrenzen der betroffenen, komplexen, adaptiven Systeme weit überstiegen - zu einem Versagen verantwortlicher Institutionen in verschiedenen Bereichen. Außerdem führte darüber hinaus der Einsatz von Truppen der Nationalgarde von Louisiana im Irakkrieg dazu, dass diese dringend benötigten Kräfte mitsamt ihrer modernen Ausrüstung nicht zur Verfügung standen (Colten et al. 2008: 10f, 19f). Legt man der Analyse ein Vulnerabilitäts-Verständnis zugrunde, wie es etwa von Christmann und Ibert bzw. Birkmann vertreten wird, lassen sich Phänomene wie die Schwierigkeiten beim Einsatz des Roten Kreuzes oder das Nicht-Vorhandensein ausreichender Ressourcen der Nationalgarde problemlos darin verorten. Sie gehören zum Bereich der Reaktionskapazitäten eines Systems (Christmann/Ibert 2016: 237). Gleichzeitig wären sie relevante Aspekte einer systemtheoretischen Resilienz-Diskussion des Fallbeispiels Hurrikan Katrina. Dabei gilt: je besser das Rote Kreuz darauf eingestellt ist, dass im Katastrophenfall unerwartete Hindernisse auftreten, desto größer sind die Reaktionskapazitäten und desto geringer ist die Vulnerabilität des betroffenen Systems. Ebenso hätte eine voll zur Verfügung stehende Nationalgarde die unmittelbaren Reaktionskapazitäten des Systems erhöht und damit ebenfalls seine Vulnerabilität verringert. Im Sinne der vorangegangenen Analyse gilt aber auch: ein auf unerwartete Ereignisse eingestelltes Rotes Kreuz und flexibel einsetzbare Ressourcen in Form der Nationalgarde erhöhen ceteris paribus die generische Anpassungsfähigkeit des komplexen adaptiven Systems New Orleans - sie erhöhen seine Resilienz. Resilienz und Vulnerabilität scheinen bei einem derartigen Vulnerabilitätsverständnis eng zusammenzuhängen. Ohne tiefergehende Analyse ließe sich sowohl formulieren „Mehr Resilienz führt zu weniger Vulnerabilität“ als auch „Mehr Vulnerabilität führt zu weniger Resilienz“ - oder auch „Resilience increases the capacity to cope with stress and is hence a loose antonym for vulnerability“ (Adger 2000: 348).

Den Zusammenhang zwischen Resilienz und Vulnerabilität so zu formulieren, reicht für die vorliegende Arbeit nicht aus. Mit Blick auf die Literatur findet sich eine ganze Bandbreite unterschiedlicher Deutungen dieses Zusammenhangs. Es gibt Ansätze, die Resilienz und Vulnerabilität als zwei Seiten einer Medaille beschreiben, wie es die gerade formulierten 
Ausführungen nahelegen. Es gibt aber auch Ansätze, die zum Beispiel Resilienz als verwundbarkeitsreduzierende Komponente von Vulnerabilität fassen oder solche, die beides, Resilienz und Vulnerabilität, als Komponenten anderer Konzepte, etwa eines erweiterten Risikobegriffs, verstehen (Bara 2010: 6f, Fekete et al. 2014). Gerade erstere Ansätze, wonach die beiden Konzepte sich antonym zueinander verhalten, sind in der Literatur stark umstritten. Autoren wie Cutter, Fooken, Leach oder Lorenz lehnen sie $\mathrm{ab}$ und betonen immer wieder, dass Resilienz gerade nicht das Gegenteil von Vulnerabilität sei (Cutter 2013, Fooken 2016: 1, Leach 2008: 5, Lorenz 2010). Obschon es Überlappungen zwischen ihnen gibt, handele es sich um zwei voneinander unabhängige Konzepte. Die exemplarische Darstellung des SoVI als einer Möglichkeit, soziale Vulnerabilität zu operationalisieren und zu messen, unterstützt Cutters Aussage, wonach hohe soziale Vulnerabilität nicht unbedingt mit mangelnder Resilienz gleichzusetzen sei (Cutter 2013). Für das Resilienz-Konzept der zivilen SiFo stellt sich unmittelbar die Frage: Wo liegt der Unterschied zwischen diesen beiden verwandten Konzepten? Hier liefert Fooken eine überzeugende Antwort, die sich sehr gut in die vorliegende system- und komplexitätstheoretische Analyse von Resilienz einordnen lässt und daher entsprechend übernommen werden soll. Für Fooken geht es bei der Analyse von Vulnerabilität hauptsächlich um „Risikofaktoren“, während Resilienz primär mit „Schutzfaktoren“ in Verbindung zu bringen ist. Die beiden grundsätzlich unabhängigen Konzepte sind in „komplexer und nichtlinearer Weise wechselseitig aufeinander bezogen." Sie können demzufolge theoretisch wie empirisch in jeder denkbaren Kombination auftreten. Komplexe adaptive Systeme können gleichzeitig sehr vulnerabel und hoch resilient sein, sie können vulnerabel und nicht resilient sein, usw. (Fooken 2016: 1).

In der vorliegenden Arbeit geht es nicht darum, das Konzept der Vulnerabilität im Bereich der zivilen SiFo neu zu deuten und zu definieren. Um den Zusammenhang zwischen Vulnerabilität und Resilienz fassen zu können und eine Abgrenzung zwischen den beiden Konzepten vornehmen zu können, ist es nichtsdestotrotz notwendig, eine Arbeitsdefinition von Vulnerabilität zu nutzen. Vulnerabilität kann im Rahmen dieser Arbeit - im Gegensatz zum Verständnis von Christmann und Ibert, für die Vulnerabilität auch bei „hoher Exposition“ aufgrund potentiell vorhandener Reaktionskapazitäten niedrig sein kann - den Aspekt der Schutzfaktoren bzw. Reaktionskapazitäten gerade nicht enthalten (Christmann/Ibert 2016: 237). Nur so lässt sich eine sinnvolle Unterscheidung zu Resilienz treffen. An anderer Stelle formulieren Christmann und Ibert diesbezüglich sehr passend: „Wir begreifen Vulnerabilität als ein Konzept, dem im so- 
zialen Handeln unter unsicheren Bedingungen die Funktion zukommt, Gefährdungslagen einzuschätzen. [...]. Resilienz hingegen betont die Möglichkeiten der Aktion und Reaktion" (Christmann/Ibert 2016: 249). Ähnlich wie von Cutter soll Vulnerabilität auch hier als multidimensionales Konzept verstanden werden, allerdings mit einem klaren Fokus auf die Ausgesetztheit und die in verschiedener Weise auftretende Anfälligkeit eines komplexen adaptiven Systems gegenüber widrigen Ereignissen. Ohne Anspruch auf umfassende theoretische Fundierung lautet die Arbeitsdefinition von Vulnerabilität in Abwandlung von Christmann und Ibert bzw. Birkmann dann: Vulnerabilität bezeichnet die physische, soziale, ökonomische und umweltbezogene Ausgesetztheit und Anfälligkeit eines Systems gegenüber widrigen Ereignissen. Dann lässt sich für ein Resilienz-Konzept der zivilen SiFo ebenso festhalten: Resilienz ist nicht das Gegenteil von Vulnerabilität.

Nichtsdestotrotz sind die beiden Konzepte eng miteinander verknüpft, nicht zuletzt in der Zielsetzung derjenigen, die sich wissenschaftlich damit beschäftigen. Sowohl Resilienz- als auch Vulnerabilitätsforschung versuchen, eine wissenschaftliche Basis für wirkungsvolle politische und sonstige Maßnahmen zur Reduktion der negativen Auswirkungen widriger Ereignisse auf Gesellschaften zu schaffen (Cutter 2013). Die Verknüpfung der Konzepte lässt sich aber auch system- und komplexitätstheoretisch analysieren. Dazu stellt sich zunächst die Frage, inwiefern Vulnerabilität als systemtheoretisches Konzept verstanden werden kann. Äquivalent zur Resilienz-Diskussion wird auch in der Vulnerabilitätsforschung immer wieder darüber gestritten, wessen Verwundbarkeit im Fokus der Analyse stehen sollte. Geht es um die Vulnerabilität von Individuen oder Gruppen, von sozialen, technischen, geografischen oder anderen Einheiten. Ebenfalls äquivalent zur Resilienz-Diskussion bietet es sich daher an, abstrakt den Begriff des Systems als Träger von Vulnerabilität zu setzen. Wie passt Vulnerabilität in die in den vorangegangenen Unterkapiteln vorgenommene systemtheoretische Analyse von Resilienz? Systemtheoretisch liegt Resilienz dann vor, wenn ein System auf einen sehr unwahrscheinlichen Reiz aus der Umwelt, der ein unerwartbares Ereignis im System auslöst, mit der Realisierung eines Prozesses eigentlich extrem unerwartbarer Anschlussmöglichkeiten reagiert, um anschließend auf einen so realisierbar gewordenen, neuen Entwicklungspfad erwartbarer Anschlussmöglichkeiten einzuschwenken. Vulnerabilität wiederum wurde als Ausgesetztheit und Anfälligkeit definiert. Daraus ergibt sich direkt ein systemtheoretisches Verständnis von Vulnerabilität. Diese ist eine Bezeichnung dafür, wie sich das Auftreten eines sehr unwahrscheinlichen Reizes aus der Umwelt auf die Realisierung des initialen, unerwartbaren Ereignisses im 
System auswirkt. Größere Vulnerabilität lässt sich übersetzen in eine größere, initiale Abweichung vom eigentlich - ohne Umweltreiz - erwartbaren Entwicklungspfad. Damit ist gleichzeitig klar, dass derart verstandene Systeme immer einen gewissen Grad an Vulnerabilität aufweisen. Auf einen sehr unwahrscheinlichen externen Reiz reagieren Systeme im Verständnis der Systemtheorie immer durch die Realisierung eines eigentlich unerwartbaren Ereignisses, sie können nicht auf ihrem ursprünglichen Entwicklungspfad verbleiben. Variabel ist allerdings die konkrete Art des unerwartbaren Ereignisses, die im Anschluss an den Umweltreiz realisiert wird. Je stärker sich dieses vom eigentlich erwartbaren Ereignis unterscheidet, desto höher ist die Vulnerabilität des Systems ausgeprägt. Bezogen auf zivile SiFo kann also postuliert werden: Vulnerabilität ist - wie Resilienz - ein systemtheoretisches Konzept. Und: Je höher die Vulnerabilität eines Systems ist, desto stärker weicht das von einem unwahrscheinlichen externen Reiz verursachte, unerwartbare initiale Anschlussereignis vom obne externen Reiz eigentlich erwartbaren Entwicklungspfad ab.

Resilienz tritt systemtheoretisch erst einen Schritt später auf den Plan. Nach der Realisierung des initialen, unerwartbaren Ereignisses stellt sich die Frage, wie das System reagiert. Dieser Punkt wurde ausführlich diskutiert. Resilienz ist aber auch systemtheoretisch nicht gänzlich unabhängig von Vulnerabilität zu sehen. Denn je höher die Vulnerabilität ausgeprägt ist, also je weiter der externe Reiz das System von seinem ursprünglichen Entwicklungspfad entfernt, desto mehr benötigt das System Resilienz, um von dem nun eigentlich erwartbaren, neuen und aufgrund der theoretischen Annahmen als „negativ“ einzustufenden Entwicklungspfad wieder in „positiver“ Richtung abzuweichen. Den Zusammenhang zwischen Resilienz und Vulnerabilität so zu fassen, macht die systemtheoretische Analyse anschlussfähig an Ansätze der Vulnerabilitätsforschung, in denen Resilienz zum Teil so verstanden wird, dass sie „sich im Wechselspiel zwischen Störeinwirkung und Vulnerabilität [manifestiert]“ (Lukesch 2016: 310). Gleichzeitig treten auch deutliche Unterschiede zu klassischen Begriffsund Konzeptdiskussionen auf. Colten et al. zum Beispiel verstehen die Reduktion von Vulnerabilität, definiert als „potential for harm and social disruption from multi-hazard threats“, als entscheidend zur Erhöhung von Resilienz (Colten et al. 2008: 5). Systemtheoretisch beeinflusst höhere oder niedrigere Vulnerabilität das Ausmaß an Resilienz in einem System ceteris paribus jedoch überhaupt nicht. Lediglich die Notwendigkeit für Resilienz. Oder präziser: In einem systemtheoretischen Verständnis führt höhere Vulnerabilität zu einer größeren Notwendigkeit für Resilienz. 
Im Kontext der Verwundbarkeitsforschung lässt sich systemtheoretisch noch ein weiterer interessanter Punkt für das Resilienz-Konzept der vorliegenden Arbeit kurz diskutieren: Resilienz wird immer noch häufig als „bounce back“, also die Rückkehr in einen wie auch immer gearteten Ausgangs- oder Ursprungszustand nach Eintreten eines widrigen Ereignisses (bzw. systemtheoretisch eines sehr unwahrscheinlichen externen Reizes) verstanden. Dieser Ausgangszustand würde ceteris paribus die gleichen Vulnerabilitäten enthalten wie vor Eintritt des externen Reizes, dem System wäre jegliche Weiterentwicklung diesbezüglich unmöglich. Bara formuliert prägnant: „Returning to ,normal' also means reproducing and reinforcing the vulnerabilities that existed prior to the disaster" (Bara 2010: 13). Allerdings wurde bereits in den vorangegangenen Unterkapiteln nachgewiesen, dass es theoretisch nicht möglich ist, nach Eintritt eines widrigen Ereignisses wieder in einen Ursprungszustand - bzw. auf den eigentlich erwartbaren Entwicklungspfad - zurückzukehren. Das System entwickelt sich notwendig weiter, die für die Resilienz-Diskussion entscheidende Frage ist die nach dem „Wie?“ der Weiterentwicklung. An dieser Stelle muss in die theoretische Analyse der Fall des wiederholten Eintretens sehr unwahrscheinlicher externer Reize eingeführt werden. Ein System, welches hohe Vulnerabilität aufweist, benötigt ein großes Maß an Resilienz, wenn ein solcher Reiz eintritt. Weist das System genügend Resilienz auf, schwenkt es auf einen neuen, dann wieder erwartbaren Entwicklungspfad ein, der mit einer mindestens äquivalenten - im Vergleich zum Status vor Eintritt des Reizes - Systemleistung einhergeht. Das Ausmaß der Vulnerabilität des weiterentwickelten Systems sinkt allerdings nicht notwendigerweise. Zwar ist zu erwarten, dass das System im Falle des Eintretens eines sehr ähnlichen externen Reizes ein weniger unerwartbares initiales Anschlussereignis realisiert - zumal der Reiz an sich, wenn er wiederholt auftritt, vom System als weniger unwahrscheinlich charakterisiert wird. Das deutet auf eine geringere Vulnerabilität hin. Aber im weiterentwickelten, sich auf dem neuen Entwicklungspfad befindenden System, sind entsprechend neue, sehr unwahrscheinliche Reize möglich, von denen sich rein theoretisch nicht a priori sagen lässt, wie stark das realisierte initiale Anschlussereignis vom eigentlich erwartbaren Entwicklungspfad abweichen wird. Dieser Zusammenhang wurde weiter oben im Prinzip bereits formuliert und wird nur durch den Aspekt der zeitlichen Zuordnung ergänzt: Größere Resilienz führt nicht notwendigerweise zu im Zeitverlauf sinkender Vulnerabilität.

In der zivilen Sicherheitsforschung geht es um komplexe adaptive Systeme. Insofern ist neben den gerade bereits dargestellten Verbindungen 
zwischen Resilienz und Vulnerabilität auch von Interesse, wie der Zusammenhang sich bei Beachtung von Umwelt- und Eigenkomplexität der relevanten Systeme analysieren lässt. Der Theorie komplexer adaptiver Systeme zufolge, tendieren komplexe adaptive Systeme dazu, durch zunehmende Spezialisierung und die so notwendig werdende zunehmende Vernetzung immer komplexer zu werden. Das gilt sowohl für die Eigenkomplexität der Systeme, als auch für die Umweltkomplexität, da die Umwelt spezifischer Systeme wiederum aus anderen komplexen adaptiven Systemen besteht. Aus der Systemtheorie heraus wurde Vulnerabilität aber unabhängig von systemexternen Faktoren definiert. Insofern spielt nur die Eigenkomplexität eine Rolle. Diese steigt in komplexen adaptiven Systemen. Nimmt damit auch die Vulnerabilität derartiger Systeme beständig zu? In 4.3.2 wurde auf das Phänomen der Kaskadeneffekte verwiesen, welche bei steigender Eigenkomplexität zunehmend wahrscheinlicher werden und gleichzeitig als Ausweis steigender Vulnerabilität gelten können. Die Annahme behält weiterhin ihre Gültigkeit. Je spezialisierter und vernetzter ein System, desto größer ist die Wahrscheinlichkeit für das Auftreten von kaskadierenden Fehlern nach Eintritt eines externen Reizes/eines widrigen Ereignisses. Solche Kaskadeneffekte sind ein Ausweis hoher Anfälligkeit und im Verständnis der vorliegenden Arbeit hoher Vulnerabilität. Die Vulnerabilität eines komplexen adaptiven Systems besteht aber selbstverständlich nicht nur aus der Wahrscheinlichkeit des Auftretens von Kaskadeneffekten. Sie setzt sich als multidimensionales Konzept aus verschiedenen Faktoren zusammen, wie die Darstellung der sozialen Vulnerabilität nach Cutter exemplarisch verdeutlicht hat. Colten et al. geben diesbezüglich zum Beispiel zu bedenken, dass insbesondere soziale Ungleichheit und Ungerechtigkeit dazu führen, dass Faktoren wie Alter, Ethnizität, Geschlecht, Behinderungen oder soziale Klasse zu inhärenten Vulnerabilitäten führen. Eine von ihnen skizzierte fiktiven Welt, in der alle - unabhängig von der Ausprägung dieser Faktoren - einen sicheren Job, ein ausreichendes Einkommen, Zugang zu medizinischer Versorgung, gute Bildung etc. haben, könnte mit widrigen Ereignissen viel besser umgehen (Colten et al. 2008: 25f). Colten et al. liefern hier sehr brauchbare Hinweise darauf, wie sich Eigenkomplexität und Vulnerabilität zueinander verhalten. Aufgrund der Multidimensionalität des Konzepts Vulnerabilität lässt sich a priori nicht eindeutig bestimmen, ob steigende Eigenkomplexität zu mehr oder weniger Vulnerabilität führt. Zunehmende gesellschaftliche Spezialisierung und Vernetzung können einerseits zu stärkeren sozialen Ungleichheiten, andererseits aber auch zu einem für alle steigenden generellen Wohlstandsniveau führen. Welcher 
Effekt überwiegt, oder ob noch ganz andere Effekte oder auch Feedbacks im System auftreten, ist theoretisch nicht bestimmbar. Bedingt durch den Teilaspekt der zunehmenden Kaskadeneffekte gilt weiterhin, dass zunehmende Eigenkomplexität Resilienz immer notwendiger macht. Ob aber Vulnerabilität durch Eigenkomplexität steigt, ist unklar. Komplexität und Vulnerabilität sind somit ebenfalls zwei verwandte aber doch eigenständige Konzepte. Davon unberührt bleibt die systemtheoretische Erkenntnis, dass steigende Vulnerabilität Resilienz immer notwendiger macht. Mit Blick auf die Komplexitätstheorie und das im vorangegangenen Unterkapitel analysierte Thema Unsicherheit kann dieser Zusammenhang aber noch weiter präzisiert werden. Demnach benötigen komplexe adaptive Systeme genau dann Resilienz, wenn widrige Ereignisse extrem unwahrscheinlich oder unerwartet sind und sie gleichzeitig die Grenzen der Belastbarkeit des betroffenen Systems übersteigen. Je größer die initiale Abweichung vom ursprünglichen Entwicklungspfad ist, desto wahrscheinlicher übersteigt ein externer Reiz diese Belastungsgrenze. Die Größe der Abweichung wurde weiter oben gerade als kennzeichnend für Vulnerabilität verstanden. Deshalb lässt sich formulieren: Je höher die Vulnerabilität eines komplexen adaptiven Systems, desto wahrscheinlicher und stärker übersteigen widrige Ereignisse die eigentlichen Belastungsgrenzen des Systems und desto größer muss ceteris paribus seine Resilienz sein, um erfolgreich mit dem Ereignis umgehen zu können.

Zusammenfassend ergeben sich für ein eigenständiges Resilienz-Konzept der zivilen Sicherheitsforschung aus der Diskussion des Zusammenhangs zwischen Resilienz und Vulnerabilität folgende Annahmen:

- Vulnerabilität bezeichnet die physische, soziale, ökonomische und umweltbezogene Ausgesetztheit und Anfälligkeit eines Systems gegenüber widrigen Ereignissen.

- Vulnerabilität ist damit ein Ausmaß für Risikofaktoren, denen ein System ausgesetzt ist. Bewältigungskapazitäten und Schutzfaktoren sind nicht Teil von Vulnerabilität. Sie spielen dafür eine entscheidende Rolle für das Resilienz-Konzept.

- Resilienz ist deshalb nicht das Gegenteil von Vulnerabilität. Die beiden Konzepte sind unabhängig voneinander

- Vulnerabilität ist - wie Resilienz - ein systemtheoretisches Konzept.

- Je höher die Vulnerabilität eines Systems ist, desto stärker weicht das von einem unwahrscheinlichen externen Reiz verursachte, unerwartbare initiale Anschlussereignis vom ohne externen Reiz eigentlich erwartbaren Entwicklungspfad ab. In einem systemtheoretischen Verständnis 
führt deshalb höhere Vulnerabilität zu einer größeren Notwendigkeit für Resilienz.

- Je höher die Vulnerabilität eines komplexen adaptiven Systems, desto wahrscheinlicher und stärker übersteigen widrige Ereignisse die eigentlichen Belastungsgrenzen des Systems und desto größer muss ceteris paribus seine Resilienz sein, um erfolgreich mit dem Ereignis umgehen zu können.

- Vulnerabilität ist ein multidimensionales Konzept. Deshalb ist a priori unklar, ob die in komplexen adaptiven Systemen inhärent steigende Eigenkomplexität auch zu steigender Vulnerabilität führt.

- Größere Resilienz führt nicht notwendigerweise zu im Zeitverlauf sinkender Vulnerabilität.

\subsubsection{Resilienz und Anpassungsfähigkeit}

„Resilience is largely about learning how to change in order not to be changed." So fasste Brian Walker die zentrale Erkenntnis seiner Keynote zusammen, die er während der „Infrastructure Resilience“-Konferenz des Risk Centers der ETH Zürich im Februar 2018 hielt. Ein solches Resilienz-Verständnis lässt sich direkt aus den ursprünglichen Ideen von Holling ableiten, dessen Credo in der vorliegenden Arbeit mit den Worten "change to survive" umschrieben wurde. Noch passender als die Betonung auf Veränderung an sich zu legen, war es aber im Rahmen ökologischer und sozial-ökologischer Forschung von „adapt to survive“ zu sprechen, also einer zielgerichteten Veränderung im Angesicht widriger Ereignisse. „Adaptive capacity“ bzw. im Deutschen Anpassungsfähigkeit scheint von zentraler Bedeutung zu sein für Resilienz. Das wurde einerseits bereits in der Analyse des Resilienz-Begriffs mithilfe disziplinärer Zugänge im vorangegangenen Kapitel deutlich. Besonders in der Ökosystemforschung und der sozial-ökologischen Forschung steht Anpassungsfähigkeit im Zentrum des Interesses. Auch die Sozialwissenschaften betonen den unmittelbaren Zusammenhang zwischen Resilienz und Anpassungsfähigkeit. Selbiges gilt für Ansätze aus der psychologischen Resilienzforschung, in der es immer stärker um die „Ausbildung adaptiver Strategien“ geht. Und auch in der organisationswissenschaftlichen Resilienzforschung steht Anpassungsfähigkeit im Fokus (siehe zum Beispiel Alexander 2013: 2711f, Edwards 2009: 18, Fooken 2016: 33, Höfler 2016: 105, Holling 1973: 15, Hollnagel/Sundström 2006: 343, Hollnagel/Woods 2006: 357, Luthar/Cicchetti 2000: 858, Luthar et al. 2000: 543, Wink 2011: 113, Woods 2018: 
5, 2006: 21, 2005: 302). Andererseits haben die vorangegangenen Unterkapitel mit ihrem Fokus auf System- und Komplexitätstheorie, Unsicherheit und Vulnerabilität ebenfalls gezeigt, dass Anpassungsfähigkeit essentiell für Resilienz ist. Daher ist es unabdingbar, diesen Zusammenhang näher zu untersuchen. Die folgende Analyse bewegt sich weiterhin im Rahmen der system- und komplexitätstheoretischen Annahmen, mit deren Hilfe das Resilienz-Konzept für die zivile SiFo schon deutlich konkretisiert werden konnte. Um Anpassungsfähigkeit in diesem Kontext zu verorten, wird zunächst noch einmal detaillierter auf die Art und Weise eingegangen, in der der Begriff in der Resilienzforschung bisher gebraucht wird. Ganz ähnlich zu Begriffen wie Komplexität, Unsicherheit und Vulnerabilität wird auch Anpassungsfähigkeit häufig als eher „schwammiger“ (fuzzy) Ausdruck innerhalb der Resilienz-Diskussion verwendet, gemeinsam mit ähnlichen Begriffen wie Fähigkeit (capability), Kompetenz (competence), Improvisation (improvisation) und Lernfähigkeit (learning) (Hutter 2011). Teilweise geht die Diskussion sogar noch darüber hinaus und Resilienz wird auch mit der Fähigkeit zur Transformation eines Systems in etwas völlig Neuartiges gleichgesetzt (Folke et al. 2010, Folke 2006: 262, Lorenz 2010). In jedem Fall kann das Resilienz-Konzept für die zivile SiFo nur dann sinnvoll um den Aspekt der Anpassungsfähigkeit erweitert werden, wenn der Begriff im Folgenden aus der bisherigen Resilienzforschung heraus definiert, sein Verhältnis zu Resilienz charakterisiert und beides anschließend system- und komplexitätstheoretisch analysiert und weiterentwickelt wird.

Zunächst stellt sich die Frage, was unter Anpassungsfähigkeit zu verstehen ist. Im Englischen geht es um „adaptive capacity“ oder "adaptation“. Insbesondere der zweite Begriff ist vor allen Dingen in Debatten um das Thema Klimawandel präsent. Dort wird darunter eine Veränderung bzw. Anpassung des Verhaltens (meist von Gesellschaften) verstanden, um dadurch die durch den Klimawandel hervorgerufenen Schäden zu minimieren oder sogar möglicherweise vorteilhafte Entwicklungen zu nutzen. Mit adaptation ist also eine Modifizierung der relevanten sozialen und ökologischen Systeme, auf die wir angewiesen sind, gemeint, so dass diese den durch den Klimawandel bedingten Veränderungen, wie etwa einem Ansteigen des Meeresspiegels, erfolgreich begegnen können, „so that these systems can persist over time“ (Jabareen 2013: 225). Sozusagen als Gegenbegriff zu adaptation wird von „mitigation“ gesprochen, worunter alle Maßnahmen fallen, die den Klimawandel an sich verhindern sollen, vor allen Dingen durch eine Reduktion der Emission von Treibhausgasen. Strategien zur mitigation sind bisher in der politischen 
Diskussion um den Klimawandel bei weitem verbreiteter als Fragen bzgl. geeigneter Maßnahmen zur adaptation, zur Anpassung an die Folgen des Klimawandels (Jabareen 2013: 225). Selbst wenn der Begriff der Resilienz in derartigen Diskussionen nicht immer fällt, ist die enge Verknüpfung zu zentralen Fragestellungen der Resilienzforschung offensichtlich, nicht zuletzt durch Formulierungen wie die von Jabareen. Demzufolge ist das Ziel von adaptation bzw. Anpassungsfähigkeit das langfristige Weiterbestehen des Systems, was sich beinahe wortgleich mit Hollings ursprünglicher Resilienz-Definition deckt (Holling 1973: 14). Insofern lässt sich Anpassungsfähigkeit auch über den Anwendungsbereich des Klimawandels bzw. Klimaschutzes hinaus in Anlehnung an die obige Definition verstehen. Anpassungsfähigkeit ist nur dann notwendig, wenn sich etwas verändert, wie zum Beispiel das Klima. Die Veränderung muss nicht unbedingt negative Folgen für das betroffene System zeitigen und sie kann sowohl von außen kommen als auch systemintern getriggert sein. Ein Beispiel für letzteres wäre etwa der Ausbruch einer genetisch bedingten Krankheit, die eine Verhaltensänderung des Betroffenen notwendig macht und daher Anpassungsfähigkeit erfordert. Bei Anpassungsfähigkeit geht es damit im Prinzip um eine Veränderung in Folge einer Veränderung. Entscheidend ist der Charakter der Veränderung. Diese erfolgt nicht zufällig, sondern im Hinblick auf ein klar definiertes Ziel, im abstraktesten Sinne die Weiterexistenz des betroffenen Systems - im gerade genannten Beispiel etwa das Überleben trotz Krankheit. Die Veränderung ist also auf ein bestimmtes Ziel gerichtet und geschieht nicht um ihrer selbst willen (Francis/Bekera 2014: 94, IRGC 2018: 16). Es lässt sich festhalten: Anpassungsfähigkeit meint das Vermögen, sich selbst angesichts von Veränderungen zielgerichtet weiterzuentwickeln, um die eigene Weiterexistenz sicherzustellen. Diese erste grobe Annäherung an Anpassungsfähigkeit dient als Ausgangspunkt der folgenden Diskussion. Sie wird an späterer Stelle erneut aufgegriffen und vor dem dann bereits dargestellten Hintergrund der Diskussion zum Thema Anpassungsfähigkeit in der Resilienzforschung system- und komplexitätstheoretisch analysiert und weiterentwickelt.

Die gerade skizzierte Arbeitsdefinition von Anpassungsfähigkeit führt unmittelbar zur Diskussion über die Frage, was Resilienz eigentlich bedeutet. Plodinec gibt dazu in seiner Übersichtsarbeit einige gute Hinweise. Er differenziert die unterschiedlichen Definitionen von Resilienz anhand der Unterscheidung zwischen Anpassungsfähigkeit (adaptation) auf der einen und Widerstandsfähigkeit (resistance) auf der anderen Seite. Ihm zufolge definieren die meisten Resilienz anhand der „idea of adaptation to cope with adversity“ (Plodinec 2009: 6). Um resilient sein zu können, 
verändern Systeme ihre Funktionsweise oder nutzen ihre Ressourcen in neuartiger, kreativer und innovativer Weise. Resilienz wird von einer ganzen Reihe von Autoren aber auch in der Fähigkeit gesehen, negativen Veränderungen zu widerstehen und die vorhandenen Ressourcen gerade dazu zu nutzen, eine Veränderung des Systems möglichst zu vermeiden. Dann ist Resilienz ein Maß für das Ausmaß an Widrigkeiten, denen ein System standhalten kann, ohne sich selbst zu verändern (Plodinec 2009: 6). Die beiden Positionen stehen sich auf den ersten Blick diametral und unvereinbar gegenüber. Sie reflektieren zudem Hollings ursprünglichen Ausgangspunkt. Eine Betonung auf Widerstandsfähigkeit wäre aus seiner Sicht einer stabilitätszentrierten Sichtweise zuzuordnen und insofern kein Teil von Resilienz. Diese Unterscheidung entspricht auch der bereits breit diskutierten Trennung zwischen ecological resilience und engineering resilience, wobei letzteres mit Widerstandsfähigkeit bzw. Stabilität gleichgesetzt wird und von großen Teilen der Resilienzforschung im Bereich der Ökologie und der Sozial-Ökologie skeptisch gesehen bzw. als unzureichend empfunden wird. Denn streng genommen schließt engineering resilience Anpassungsfähigkeit als Teil von Resilienz aus. Gerade mit Blick auf Wildavsky wurde aber bereits deutlich, dass eine strikte Trennung der beiden Perspektiven nicht notwendigerweise sinnvoll ist (siehe 2.4).

Um Resilienz zu verstehen und zu definieren, ist es jedenfalls unbedingt notwendig, sich eingehender mit Anpassungsfähigkeit auseinanderzusetzen. Dabei gehen Autoren teilweise so weit, den Zusammenhang zwischen beiden Konzepten umzudrehen und Resilienz als einen Teil eines umfassenderen adaptation-Begriffs zu deuten (Alexander 2013: 2713). Eher im Mainstream der - mindestens sozial-ökologischen - Resilienzforschung bewegt sich Brian Walker mit seiner prägnanten Beschreibung: "Adaptability is the capacity of actors in a system to influence resilience“" (Walker et al. 2004). Diese oder ähnliche Formulierungen finden sich in allen Bereichen der Resilienzforschung. Resilienz wird häufig gänzlich oder zumindest zu großen Teilen mit Anpassungsfähigkeit gleichgesetzt. Folke spricht zum Beispiel davon, dass ein Verlust an Resilienz direkt einhergeht mit einem Verlust an Anpassungsfähigkeit (Folke 2006: 262). Rogers et al. setzen in ihrem Überblick über Resilienz-Definitionen aus verschiedenen Disziplinen die Begriffe sogar direkt gleich, wenn es um ökologische Resilienz geht. Ein System, heißt es dort, kann über „'adaptive capacity', or resilience“ verfügen (Rogers et al. 2012: 76). Ähnlich abstrakt beschreibt Vogt Resilienz als „die Fähigkeit eines Systems, sich Veränderungen anzupassen, ohne kritische Schwellen (Kipppunkte, tipping points) zu überschreiten" (Vogt 2015: 10). Diese Definition enthält mehr, 
als nur den Verweis auf Anpassungsfähigkeit. Die Bedeutung von Schwellenwerten im Kontext von Resilienz wurde weiter oben bereits diskutiert. Hier wird der Begriff etwas anders verwendet. Das Resilienz-Konzept der vorliegenden Arbeit geht davon aus, dass Resilienz benötigt wird, wenn langfristige Veränderungsprozesse durch Erreichen eines Schwellenwertes ein unerwartetes und die Belastungsgrenzen des Systems überschreitendes Ereignis auslösen. Im obigen Zitat hingegen besteht Resilienz gerade darin, sich anzupassen, um ein Überschreiten kritischer Schwellen zu verhindern. Derartiges Verhalten bzw. derartige Fähigkeiten sind von großer Relevanz für komplexe adaptive Systeme. Die Herausforderung besteht darin, die Schwellenwerte rechtzeitig zu identifizieren. Gelingt dies, kann durch eine spezifische Anpassung ein Überschreiten verhindert werden. So verstandene, spezifische Anpassungsfähigkeit ist jedoch, der system- und komplexitätstheoretischen Analyse dieser Arbeit folgend, kein Teil von Resilienz, wie weiter oben bereits dargelegt wurde.

Im Bereich der Katastrophenforschung und sozialwissenschaftlicher Ansätze wird Resilienz ebenfalls häufig mit Anpassungsfähigkeit gleichgesetzt. Es geht um die Frage, ob und wie es Systemen bzw. der Gesellschaft an sich gelingt, nach und während des Eintretens gravierender widriger Ereignisse aus den Ereignissen zu lernen und sich ihnen kurz- und langfristig anzupassen (Fekete et al. 2016: 229). Dieses Verständnis bewegt sich wieder näher an den bisherigen Erkenntnissen der vorliegenden Arbeit. Auch Al-Khudairy et al. formulieren ähnlich und identifizieren soziale Resilienz mit dem „potential for adaptation and self-organization“ (Al-Khudhairy et al. 2012: 587). Christmann und Ibert wiederum verstehen Resilienz als „erfolgreiche Anpassung von Gesellschaften an Naturrisiken“ und betonen die dahinterliegende Zielstellung, Schäden am System entweder zu vermeiden, oder aber möglichst gut auszugleichen (Christmann/Ibert 2016: 236). Dass es nicht nur um Anpassungen an Naturrisiken geht, zeigt Elran auf, für den sich Resilienz darin ausdrückt „to contain a disaster or a series of catastrophes in an adaptive manner and to react to them flexibly (by bending rather than breaking)" und dessen Forschungsschwerpunkt auf dem Thema gesellschaftlicher Resilienz gegenüber terroristischen Anschlägen liegt (Elran 2012: 294). Und auch in der Wirtschaftsgeographie wird Resilienz als „Fähigkeit des aktiven Agierens (adapt) im Gegensatz zu einem passiven Reagieren (respond)" verstanden und damit direkt mit Anpassungsfähigkeit gleichgesetzt (Plöger/Lang 2016: 357). Etwas weniger direkt sieht Longstaff Resilienz als abhängig von einer Fähigkeit von Individuen oder auch Technologien, eine große Bandbreite unterschiedlicher Bedingungen tolerieren zu können. Empirisch seien vor allen Dingen die 
Organisationen als resilient zu bezeichnen, die über Erfahrungen im Umgang mit Überraschungen verfügen und sich diesen anpassen konnten, um zu überleben (Longstaff 2012: 268). Höfler stellt fest, dass das ResilienzKonzept sich durch drei wesentliche Merkmale auszeichnet. Ihre Aussagen fußen auf einer ausführlichen Konzeptanalyse von Windle, die insgesamt 271 Studien zum Thema Resilienz untersucht hat (siehe Windle 2011). Und obwohl beide Autorinnen, sowohl Höfler als auch Windle, aus dem Kontext der Psychologie bzw. genauer gesagt der Gerontologie stammen, stimmen ihre Ergebnisse im Wesentlichen mit den Ansätzen anderer Forschungsbereiche wie Ökosystemforschung, sozial-ökologischer Forschung und Sozialwissenschaften überein. Demnach spiegelt sich Resilienz in „effektive[n] adaptive[n] Prozess[en]“, die zur „Realisierung eines positiven Ergebnisses“ führen (Höfler 2016:108).

Wo kommt diese herausragende Bedeutung von Anpassungsfähigkeit im Resilienz-Diskurs her? Es wurde bereits mehrfach auf die Ursprünge in der Ökosystemforschung verwiesen. Hollings zentrale Erkenntnis, wonach eine stabilitätszentrierte Sichtweise auf Ökosysteme empirisch nicht haltbar ist, verwies gleichzeitig direkt auf eine Notwendigkeit zur Veränderung, eine Notwendigkeit zur Anpassung an „untenable situations.“ Diese Veränderung von Ökosystemen wird von ihm nicht länger als katastrophal und unerwünscht definiert, sondern vielmehr als nicht zu verhindernde und oftmals notwendige Anpassung an sich verändernde äußere Umstände, durch die ein langfristiges Überleben des Ökosystems (persistence) erst möglich wird (Lorenz 2010). Folke zufolge wurden die Erkenntnisse von Holling - wenn man so will ein "complexity change“ in der Ökologie zunächst vom Mainstream der Ökosystemforschung ignoriert. Es wurde weiter von einem stabilen Gleichgewicht in Ökosystemen ausgegangen, die Existenz multipler Gleichgewichtszustände wurde als empirisch nicht nachweisbar bezeichnet. Laut Holling lag das allerdings an falschen (zu kurzen) Beobachtungszeiträumen, nicht an einer fehlerhaften Theorie (Folke 2006: 256). Mittlerweile sind seine Ideen und Theorien sehr viel stärker akzeptiert, wobei es insbesondere die sozial-ökologische Forschung und weniger die reine Ökosystemforschung ist, die sich auf Holling beruft (siehe 2.3). Um von einem Gleichgewichtszustand in einen anderen zu gelangen, müssen Systeme sich in jedem Fall verändern. Wie sie das konkret tun, wie ihre Anpassungsfähigkeit ausgestaltet ist, hängt stark vom jeweiligen Kontext und den spezifischen Ereignissen ab, an die sich Systeme anpassen müssen (Lorenz 2010). Die Verknüpfung zwischen externen Veränderungen, notwendiger Anpassungsfähigkeit und langfristiger Persistenz des Systems unter dem Begriff der Resilienz wurde jedenfalls erstmals 
von Holling in theoretisch stringenter Weise umgesetzt. Um die Resilienz von Ökosystemen erhöhen zu können, entwickelte Holling den Ansatz des adaptiven Managements. Dieser geht - im Vergleich zu herkömmlichen Management-Ansätzen, welche Komplexität und Unsicherheit in Systemen durch Reduktionismus zu minimieren suchen und damit nur ein fragmentiertes und unvollständiges Systemverständnis erlangen - von einem holistischen Systemverständnis aus. Das Wissen über komplexe Systeme ist notwendig unvollständig, Überraschungen damit zwangsläufig und die Systeme entwickeln sich evolutionär ständig weiter. Adaptives Management versucht, die Anpassungsfähigkeit komplexer Ökosysteme durch Identifikation und Nutzung zugrundeliegender Strukturen und Beziehungsmuster zu erhöhen - und damit ihre Resilienz gegenüber abrupt und überraschend auftretenden Störereignissen (Gunderson 2000: 433). Anpassungsfähigkeit ist also nicht nur zum Verständnis von Resilienz zentral, sondern auch eine entscheidende Stellschraube bei Bemühungen zur Erhöhung der Resilienz realer komplexer Systeme. Letzteres kann ohne die Diskussion über Resilienz als normatives Konzept wiederholen zu wollen (siehe 2.1 und 4.2) - als eine der wichtigsten Aufgaben aktueller ziviler Sicherheitsforschung verstanden werden.

Im Kontext Resilienz und Anpassungsfähigkeit ist ein weiteres Modell von Holling bedeutsam und soll daher an dieser Stelle kurz vorgestellt werden. Vor allen Dingen spätere, einflussreiche Überlegungen von Carl Folke und anderen Autoren aus der sozial-ökologischen Forschung basieren zum Teil auf dem Modell des sogenannten „adaptive cycle“. Holling hat diesen Begriff und das dahinterstehende Konzept 1986 in einem Kapitel für das Buch Sustainable Development of the Biosphere von Clark und Munn eingeführt (siehe Holling 1986). Das geht aus einem Briefwechsel zwischen ihm und Folke hervor, den letzterer in einem Artikel von 2006 ausführlich zitiert. Holling schreibt ihm dazu folgendes: „Nonlinearities were essential. Multi-stable states were inevitable. Surprise was the consequence. It was the place where the ,Adaptive Cycle' was first described and presented" (Folke 2006: 255, zitiert aus Brief von Holling). Gemeinsam mit Lance Gunderson baute Holling diese Ideen später zu einem zusammenhängenden Theoriegebäude weiter aus und entwickelte das Konzept der Panarchie (siehe Holling/Gunderson 2002). Diese weitergehenden Ideen von Holling und Gunderson stehen nicht im Zentrum des Interesses der vorliegenden Arbeit. Der adaptive cycle bietet jedoch wertvolle Anknüpfungspunkte zur Diskussion des Zusammenhangs zwischen Resilienz und Anpassungsfähigkeit und wird daher im Folgenden basierend auf einer Beschreibung von Lukesch, die dieser im Sammelband von Wink 
2016 publiziert hat, skizziert. Lukesch übersetzt adaptive cycle mit „Wandlungszyklus" und begründet dies mit einem Unbehagen gegenüber dem deutschen Begriff „Anpassung.“ In der vorliegenden Arbeit wird für adaptive cycle auf eine Übersetzung verzichtet. Generell gilt aber, dass mit „Anpassungsfähigkeit“ das englische „adaptive capacity“ gemeint ist und damit - wie Lukesch prägnant ausführt - auch ein Stück weit der Begriff des Wandels mitgedacht wird (Lukesch 2016: 298).

Der adaptive cycle nach Holling ist ein heuristisches Modell, das auf abstrakte Weise das Verhalten komplexer adaptiver Systeme über den Zeitverlauf und ihren jeweiligen Umgang mit Veränderungen in unterschiedlichen Phasen beschreibt. Er besteht generell aus vier Phasen, die im Rahmen des Modells aufeinanderfolgen und in einer zweidimensionalen Grafik veranschaulicht werden können (siehe Abbildung 3). Die vier Phasen werden mit griechischen bzw. lateinischen Buchstaben bezeichnet und gliedern sich in eine Entfaltungsphase $r$ (exploitation phase), eine Erhaltungsphase K (conservation phase), eine Auflösungsphase $\Omega$ (release phase) sowie eine Erneuerungsphase $\alpha$ (renewal bzw. re-organization phase). Die Entfaltungsphase ist geprägt von einem Wachstum des Systems und der fortwährenden, inkrementellen Steigerung der Systemleistung, der Grad an Vernetzung im System nimmt zu und damit auch seine Komplexität. Irgendwann geht das System in die Erhaltungsphase über, in dem es sich durch hohe Stabilität, geringe Variabilität aber eine sehr hohe und konstante Systemleistung auszeichnet. Gleichzeitig ist das System in dieser Phase gekennzeichnet durch eine in Form sehr starker Vernetzung vorliegende hohe Eigenkomplexität. Erfährt ein derartiges System einen starken externen Reiz, zum Beispiel in Form eines unerwarteten, widrigen Ereignisses, geht es sehr wahrscheinlich in die Auflösungsphase über, die gekennzeichnet ist durch eine rapide und drastisch sinkende Systemleistung. Gelingt es dem System durch Anpassung seiner Funktionalitäten weiterzubestehen, tritt es in eine Erneuerungsphase ein. In dieser passt sich das System den veränderten Umweltbedingungen durch seinerseits rapide und umfassende Veränderungen an und die Systemleistung beginnt sich zu erholen. Je besser die Anpassung gelingt, desto schneller und stärker steigt die Systemleistung an, bis das System wieder in eine nächste Entfaltungsphase eintritt, in welcher der adaptive cycle sozusagen von vorn beginnt (Folke 2006: 258, Lukesch 2016: 298f).

Die verschiedenen Phasen lassen sich auch anhand der beiden Dimensionen Verbundenheit (connectedness) und Entwicklungspotential (capital) unterscheiden. Die Verbundenheit - die auch als Vernetzung bezeichnet werden kann - ist vor allen Dingen in der Erhaltungs- und am Beginn 
der Auflösungsphase sehr groß. Die Elemente des Systems sind sehr stark miteinander gekoppelt. Das sorgt für eine hohe Stabilität des Systems, das sich gegenüber bekannten und eingeplanten Störungen als sehr robust erweist. Im Laufe der Auflösungsphase und der Erneuerungsphase nimmt sie rapide $a b$, bevor sie in der Entfaltungsphase wieder ansteigt. Das Entwicklungspotential ist zwar in der Erhaltungsphase am größten, allerdings sind in dieser die vorhandenen Ressourcen auch stark gebunden und daher nicht flexibel und innovativ (zur Anpassung des Systems an externe Veränderungen) einsetzbar. Die größte Freiheit zum Einsatz seiner Ressourcen in innovativer Weise hat das System in der Erneuerungsphase sowie am Übergang von der Entfaltungs- in die Erhaltungsphase. Die Resilienz wiederum ist in der Erneuerungs- und der Entfaltungsphase tendenziell größer als in den beiden anderen Phasen. Denn in diesen Phasen sind die zur Verfügung stehenden Ressourcen des Systems weniger stark gebunden sowie seine durch Vernetzung bedingte Rigidität weniger stark ausgeprägt als in der Erhaltungsphase. In der Auflösungsphase wiederum stehen kaum noch Ressourcen zur Verfügung. Die Auflösungs- und die Erneuerungsphase sind durch abrupte und schnelle Veränderungsprozesse verbunden, die durch externe oder interne Störungen getriggert werden, während Entfaltungs- und Erhaltungsphase eher durch graduelle, langsame Anpassungen gekennzeichnet sind. Damit ist das heuristische Modell des adaptive cycle auch konsistent zu Hollings Denken über Resilienz und Stabilität in komplexen Systemen. Das Auftreten von Störungen und von ihnen ausgelöste radikale Veränderungsprozesse des Systems gehören genauso zum Lebenszyklus komplexer Systeme wie Perioden weitgehender Stabilität mit höchstens langsam und graduell ablaufenden Veränderungen (Folke 2006: 258, Lukesch 2016: 300ff). 
Abbildung 3: Der adaptive cycle

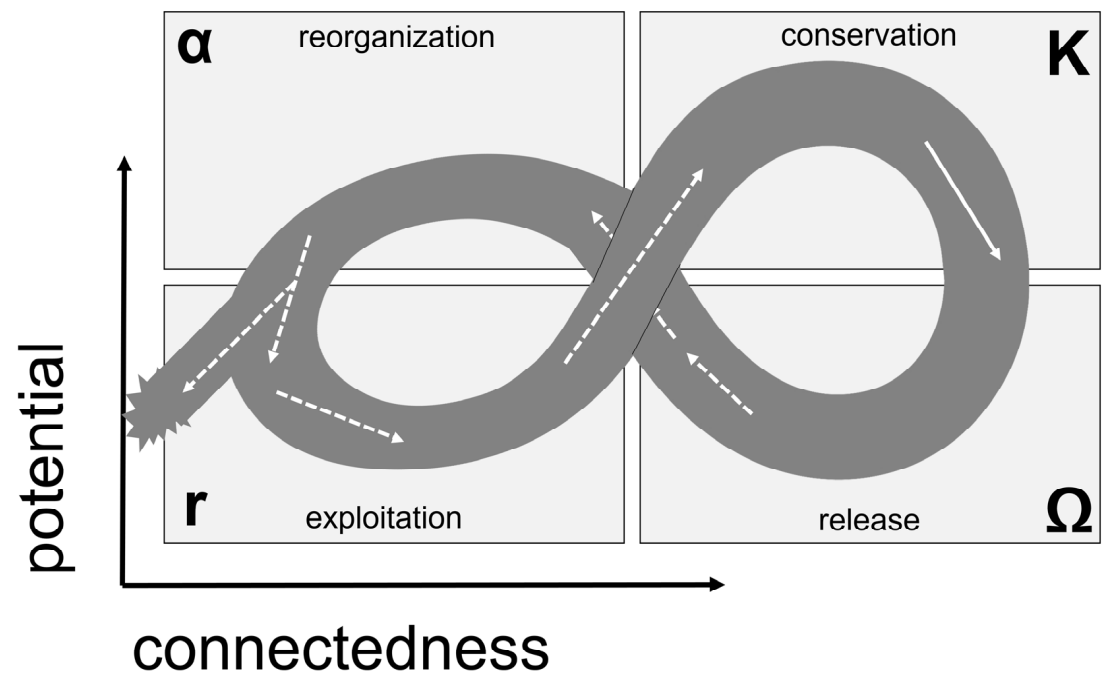

Quelle: eigene Darstellung nach Lukesch 2016.

Gegeben die oben dargelegten Annahmen lässt sich der adaptive cycle auch im Rahmen der system- und komplexitätstheoretischen Analyse von Resilienz interpretieren. Dazu muss er zunächst um einen weiteren möglichen Entwicklungspfad eines komplexen adaptiven Systems ergänzt werden. In der Erneuerungsphase besteht auch die Möglichkeit, dass das System es gerade nicht schafft, in eine neue Entfaltungsphase überzugehen und stattdessen zu existieren aufhört. Abbildung 3 stellt diesen „Ausgang“ aus der beständigen Weiterentwicklung eines komplexen adaptiven Systems dar. Neben dem Erlöschen seiner Existenz, kann der Ausgang auch den Übergang in einen neuen Systemzustand mit langfristig deutlich verringerter Leistung bedeuten (Rogers et al. 2012: 76). In der vorliegenden Arbeit gilt: das Vorhandensein oder Nicht-Vorhandensein von Resilienz lässt sich nur beobachten, wenn Systeme von einem disruptiven, entweder extern getriggerten oder aus dem System selbst stammenden, gravierenden und extrem unwahrscheinlichen Reiz getroffen werden. Dies wurde sowohl systemtheoretisch hergeleitet, als auch in der Diskussion über das Konzept der Unsicherheit bestätigt. Demnach benötigen komplexe adaptive Systeme Resilienz zur Bewältigung von Risiken, für die sie keine spezialisierte Anpassungsfähigkeit entwickeln, sowie zur Bewältigung ungewisser und echt unsicherer Ereignisse, wenn diese jeweils ihre 
Belastungsgrenzen übersteigen. Im Modell des adaptive cycle werden die Belastungsgrenzen des Systems am offensichtlichsten in der Auflösungsphase überschritten. Gleichzeitig steigt die Wahrscheinlichkeit, in eine Auflösungsphase einzutreten immer weiter an, je länger sich das System in der Erhaltungsphase befindet. Denn in dieser wird das Auftreten extrem unwahrscheinlicher bzw. unsicherer, die Belastungsgrenzen des Systems übersteigender Ereignisse immer größer. Dies ist der Fall, weil die Komplexität des Systems in der Erhaltungsphase immer weiter ansteigt, der Grad an Vernetzung zunimmt und die Wahrscheinlichkeit für das Auftreten von Kaskadeneffekten steigt. In der Erhaltungsphase nimmt auch das Ausmaß an Spezialisierung der Agenten - und damit auch ihre jeweilige spezialisierte Anpassungsfähigkeit - immer weiter zu. Das System ist insgesamt hervorragend dazu in der Lage, mit kleineren, innerhalb seiner Belastungsgrenzen und erwartet auftretenden Veränderungen umzugehen. Sobald es jedoch in eine Auflösungsphase eintritt, im Rahmen der zivilen Sicherheitsforschung zum Beispiel ausgelöst durch ein Extremwetterereignis, das zu kaskadierenden Ausfällen komplexer, vernetzter Infrastruktursysteme führt, reichen diese Fähigkeiten nicht länger aus, um das System langfristig zu erhalten. Die Auflösungsphase - ob intern oder extern getriggert - zeichnet sich gerade durch den Fakt aus, dass die eingeübten Routinen und bekannten Maßnahmen und Handlungsweisen des Systems nicht geeignet sind, die durch einen gravierenden Reiz gestellten Herausforderungen zu bewältigen. Im Bild des adaptive cycle benötigt das System nun einen möglichst schnellen Übergang in eine Erneuerungsphase, aus der es wiederum dank grundlegender Anpassungsschritte, Lukesch spricht von „radikale[n] Innovationen“, relativ schnell in eine Entfaltungsphase übergehen kann (Lukesch 2016: 300). Genau darin drückt sich das Vorhandensein von Resilienz aus. Resilienz wurde in der vorliegenden Arbeit als Nicht-Selbstverständlichkeit charakterisiert. Zu erwarten wäre also nach der Auflösungsphase der Übergang in eine nicht-erfolgreiche Erneuerungsphase und dann ein Übergang in eine Phase verminderter Systemleistung oder sogar das Erlöschen des Systems. Systemtheoretisch gesprochen geht es hier um die Frage, auf welchen neuen Entwicklungspfad komplexe adaptive Systeme nach der Auflösungsphase einschwenken. Das hängt im adaptive cycle von ihrer Anpassungsfähigkeit ab. Ohne Anpassung an die extern oder intern getriggerten Veränderungen und eventuell sogar Wandel im Angesicht neuartiger Herausforderungen, kann kein Übergang von Auflösungs- in Erneuerungs- und dann in Entfaltungsphase gelingen. Damit ist gleichzeitig auch klar, dass Resilienz auf Anpassungsfähigkeit an- 
gewiesen ist. Oder anders gesagt: Das Vorhandensein von Anpassungsfähigkeit ist eine zwingende Grundvoraussetzung für Resilienz.

Anpassungsfähigkeit per se ist nach der Analyse des adaptive cycle aber nicht gleichbedeutend mit Resilienz. Weiter oben wurde Anpassungsfähigkeit definiert als das Vermögen, sich selbst angesichts von Veränderungen zielgerichtet weiterzuentwickeln, um die eigene Weiterexistenz sicherzustellen. Aus der Komplexitätstheorie kommend lässt sich Anpassungsfähigkeit als eine konstitutive Eigenschaft komplexer adaptiver Systeme beschreiben. Die notwendige Verbindung zwischen Komplexität und Resilienz wird also über die komplexen Systemen eigene Fähigkeit zur Anpassung bzw. Weiterentwicklung vermittelt. Komplexe adaptive Systeme sind jedoch nicht automatisch auch resiliente Systeme. Im adaptive cycle können Systeme auch weiterexistieren, wenn sie den in Abbildung 3 links unten dargestellten Ausgang aus ihrem Entwicklungszyklus nehmen müssen. Auch das entspricht einer Anpassung, einer Weiterentwicklung, des Systems als Reaktion auf einen gravierenden Reiz. Systemtheoretisch wäre diese Entwicklung sogar die wahrscheinlichere, die erwartbare. Resilienz weist das System aber erst auf, wenn es im adaptive cycle gesprochen in eine neue Entfaltungsphase übergeht. Die Realisierung dieses unerwartbaren Entwicklungspfads ist systemtheoretisch mit einer vergleichsweise hohen Systemleistung gleichzusetzen, die mittel- bis langfristig mindestens so hoch ausfallen wird, wie im ursprünglichen, ungestörten System - also in der vorangegangenen Erhaltungsphase. Für den Zusammenhang zwischen Resilienz und Anpassungsfähigkeit lässt sich sagen: Resilienz drückt sich in einem durch Anpassungsfähigkeit realisierbaren Übergang von einer Erneuerungs- in eine Entfaltungsphase aus.

Zudem lässt sich aus dem adaptive cycle auch die Art der Anpassungsfähigkeit ableiten, die für Resilienz von Bedeutung ist. Diese Interpretation ist ebenfalls konsistent mit der komplexitätstheoretischen Analyse. Systeme, die sich in der Erhaltungsphase befinden, verfügen über ein sehr hohes Maß an spezialisierter Anpassungsfähigkeit. Sie sind sehr gut in der Lage, mit relativ wahrscheinlichen Risiken, mit bekannten Ereignissen umzugehen und sich diesen dank spezifischer Maßnahmen adäquat anzupassen. Dabei schwankt die Systemleistung nur unbeträchtlich. Das Eintreten eines gravierenden, extrem unwahrscheinlichen oder unerwarteten Ereignisses führt dann zu einem rapiden Rückgang der Systemleistung, dem mit etablierten Maßnahmen, mithilfe spezialisierter Anpassungsfähigkeit, nicht effektiv begegnet werden kann. Was macht die nun notwendige werdende Erneuerungsphase also aus? Was ermöglicht dem System einen Übergang in eine neue Entfaltungsphase? Lukesch spricht aus Sicht 
der ökonomischen Regionalplanung von einer „Neuorientierung“, von "grundlegende[n] Reformen“ und „radikale[n] Innovationen“, die notwendig seien. Dazu bedarf es einer relativ großen Menge an Ressourcen, die flexibel eingesetzt werden können, um das System an die in der Auflösungsphase eingetretenen Veränderungen anpassen zu können. In der komplexitätstheoretischen Analyse wurde das als ungenutzte Spielräume bzw. generische Anpassungsfähigkeit bezeichnet. Im adaptive cycle zeichnet sich die Erhaltungsphase gerade dadurch aus, dass keine ungenutzten Spielräume mehr vorhanden sind und Flexibilität durch Rigidität ersetzt wurde. Das System ist robust aber spröde, es ist „für situationsgerechtes Handeln zu unbeweglich geworden“ (Lukesch 2016: 301). Um den Zusammenhang zwischen Resilienz und Anpassungsfähigkeit präziser fassen zu können, muss Hollings ursprüngliches heuristisches Modell, so wie es in der vorliegenden Arbeit verstanden wird, daher an dieser Stelle erweitert werden. Demzufolge sind komplexe adaptive Systeme genau dann resilient, wenn es ihnen gelingt, in der Entfaltungs- und Erhaltungsphase ungenutzte Spielräume aufzubauen. Diese ungenutzten Spielräume können dann in einer durch ein widriges Ereignis (Auflösungsphase) notwendig werdenden Erneuerungsphase in Form generischer Anpassungsfähigkeit dazu genutzt werden, schnell in eine weitere Entfaltungsphase einzutreten. Generische Anpassungsfähigkeit ermöglicht letztlich eine „möglichst breit angelegte Vorsorge und Belastbarkeit gegen möglichst alle vorstellbaren Störeinwirkungen und Krisen über einen möglichst langen Zeitraum" (Lukesch 2016: 307). Für das Resilienz-Konzept der zivilen SiFo gilt damit: Für die Resilienz komplexer adaptiver Systeme ist entscheidend, dass sie in ibrem Lebenszyklus in der Entfaltungs- und Erhaltungsphase bewusst ungenutzte Spielräume aufbauen, um in einer an ein widriges Ereignis anschließenden Erneuerungsphase über generische Anpassungsfähigkeit zu verfügen.

Ähnlich wie in der gerade erfolgten Analyse, verwendet auch die sozial-ökologische Resilienzforschung den adaptive cycle als heuristisches Modell. Insofern trifft sie - und die Verwendung in der vorliegenden Arbeit - die Kritik an Hollings Idee nicht, wonach der adaptive cycle als analytisches Werkzeug aufgrund seiner zu großen Determiniertheit und einem zu starken Fokus auf Persistenz des Systems nicht gut geeignet sei (Folke 2006: 258, Leach 2008: 11). Carl Folke etwa nutzt den adaptive cycle als Inspiration, die ihn in seinem Denken über Resilienz voranbringe: „It has helped me to think about structures and processes in a dynamic fashion, to move away from a steady-state world where change is looked upon as an exception, to confront complexity and uncertainty" (Folke 2006: 258f). Nach Christmann und Ibert ist es insbesondere dieser Carl Folke, der die 
entscheidende Bedeutung von Anpassungsfähigkeit in den Resilienz-Diskurs einbringt und dazu Resilienz nicht länger als erreichbaren Zustand, sondern vielmehr als fortdauernden Prozess definiert (Christmann/Ibert 2016: 238). Folkes Ausgangspunkt entspricht dabei exakt dem, der für die vorliegende Arbeit anhand system- und komplexitätstheoretischer Überlegungen hergeleitet werden konnte. Demnach sind Unsicherheit und Überraschungen in komplexen adaptiven Systemen nicht zu vermeidende Phänomene, auf welche die Systeme sich einstellen müssen und mit denen sie zu leben lernen müssen (Folk 2006: 255). Resilienz als Fähigkeit zu verstehen, die Zeitspanne nach Eintreten eines widrigen Ereignisses bis zur Wiederherstellung des ursprünglichen Systemzustands zu minimieren, greift daher bei weitem zu kurz - und geht noch hinter Hollings Resilienz-Definition zurück. Die Komplexität der betrachteten Systeme genauso wie die Unerwartbarkeit von Ereignissen machen eine simple Wiederherstellung sowohl rein theoretisch als auch empirisch-praktisch unmöglich. Deshalb fokussieren Folke und die sozial-ökologische Forschung in ihren Untersuchungen komplexer adaptiver Systeme auf deren Fähigkeiten zur Erneuerung, Regeneration und Re-Organisation im Anschluss an unerwartet eintretende Störereignisse - oder mit anderen Worten: auf ihre Anpassungs- oder sogar Transformationsfähigkeit (Brand/Jax 2007: 5, Folke 2006: 253ff). Letzten Endes geht es darum, komplexe adaptive Systeme so zu gestalten, dass sie im Angesicht unerwartet auftretender Störereignisse kein sprödes Versagen an den Tag legen. Die Zukunft zeichnet sich durch unsichere Information, sich verändernde Bedingungen und das Auftreten neuartiger Ereignisse aus, die vorherige Annahmen, Pläne und Modelle relativ schnell nutzlos werden lassen können. Daher müssen die Systeme über ein „potential for adaptive action“ verfügen (Woods 2015: 6). In der sozialökologischen Forschung wird Resilienz als „capacity of a system to absorb disturbance and re-organize while undergoing change so as to still retain essentially the same function, structure, identity and feedbacks" definiert (Folke 2006: 259). Die Fähigkeit zur Re-Organisation als Reaktion auf extern oder intern getriggerte, abrupte und gravierende Veränderungen kann auch als Anpassungsfähigkeit bezeichnet werden. Im Wesentlichen wird Resilienz in dieser Debatte also mit Anpassungsfähigkeit, wenn nicht gleichgesetzt, so doch extrem eng verwandt gesehen. Anpassungsfähigkeit ist hier der entscheidende Bestandteil von Resilienz. Denn die Prozesse zur Anpassung verstanden als Fähigkeit, Veränderungen zu akzeptieren und damit umgehen zu können, resultieren aus dem Vermögen komplexer adaptiver Systeme zur Selbst- und Re-Organisation. „In this sense, resilience provides adaptive capacity“ (Folke 2006: 259). 
Folkes Überlegungen gehen deutlich über die ursprüngliche ResilienzDefinition von Holling hinaus. Dieser hatte Resilienz ja als Maß für die Persistenz, die Weiterexistenz des Systems verstanden, ausgedrückt in ihrer Fähigkeit, Veränderungen und Störungen zu absorbieren und immer noch relativ unverändert weiter zu existieren - zumindest was ihre internen Prozesse und Funktionen anbelangt. In dieser ursprünglichen Definition war der Erhalt der Systemleistung kein Ausweis von Resilienz. Es ging primär um das langfristige Überleben von Systemen, unabhängig von der Systemleistung (Kuhlicke 2010). Folke erweitert dieses Verständnis dahingehend, dass resiliente Systeme in der Lage sind, sich Veränderungen durch Re-Organisation anzupassen, ohne dabei ihre Systemleistung verringern zu müssen - zumindest nicht langfristig (Folke et al. 2002: 438). Veränderung als Voraussetzung für den Erhalt des Systems erscheint nach Folke auf den ersten Blick kontraintuitiv. „Yet the very dynamics between periods of abrupt and gradual change and the capacity to adapt and transform for persistence are at the core of the resilience of social-ecological systems" (Folke et al. 2010). Liegt der Fokus stärker darauf, Veränderungen verhindern bzw. vermeiden zu wollen, muss von einem „robusten“ statt einem resilienten System gesprochen werden. Dann erscheint Anpassungsfähigkeit gerade nicht als sinnvoll. In komplexen adaptiven Systemen mit multiplen möglichen Gleichgewichtszuständen wird dagegen ein hohes $\mathrm{Maß}$ an Variabilität benötigt, um die Persistenz und den Leistungserhalt der Systeme sicherstellen zu können. Für das Management komplexer adaptiver Systeme ergibt sich damit eine sinnvolle Herangehensweise, die auch im weiteren Verlauf der Arbeit bei den Überlegungen für Resilience Engineering eine Rolle spielen könnte. Da Veränderungen in komplexen adaptiven Systemen nicht verhindert werden können, muss die Maßgabe lauten, die Systeme durch (pro-)aktive Anpassung an Veränderungen resilient $\mathrm{zu}$ gestalten, statt nur zu reagieren. In diesem Gedanken von Folke spiegelt sich sowohl das Zitat von Brian Walker vom Beginn dieses Unterkapitels als auch die weiter oben herausgearbeitete Notwendigkeit zur Maximierung generischer Anpassungsfähigkeit komplexer adaptiver Systeme, wenn es um deren Resilienz geht (Folke 2006: 254f, Folke et al. 2010).

Anpassungsfähigkeit kann theoretisch noch weiter unterteilt werden. Birkmann schlägt zum Beispiel vor, einerseits von „Bewältigungskapazitäten" zu sprechen, die sich auf Fähigkeiten beziehen, die unmittelbar während des Eintretens eines widrigen Ereignisses wichtig sind, etwa Katastrophenhilfe, das Sicherstellen einer Versorgung mit dem Nötigsten (Strom, Wasser, Nahrung, etc.), die Bergung und Versorgung Verletzter, usw. Und andererseits mit „Anpassungskapazitäten“ eher langfristig benötigte Fähig- 
keiten zu meinen, die es dem betroffenen System ermöglichen, sich den neuen Anforderungen, die aus der veränderten System-Umwelt stammen, erfolgreich anzupassen und sein eigenes Überleben entsprechend langfristig sicherzustellen. Diese Anpassungskapazitäten können dann sowohl vor als auch nach widrigen Ereignissen wirksam werden (Birkmann 2008: 9). In der vorliegenden Arbeit wird diese explizite Unterscheidung so nicht getroffen. Unter dem Begriff der generischen Anpassungsfähigkeit werden Bewältigungs- wie auch Anpassungskapazitäten subsummiert. Diese müssen in Form ungenutzter Spielräume vor Eintreten eines widrigen Ereignisses aufgebaut werden, um dann während, unmittelbar danach und langfristig wirksam werden zu können. Damit gilt für das Resilienz-Konzept der zivilen SiFo: Generische Anpassungsfähigkeit ermöglicht komplexen adaptiven Systemen sowobl eine unmittelbare Bewältigung der Folgen extrem unwahrscheinlicher oder unerwarteter, die Belastungsgrenzen des Systems übersteigender Ereignisse als auch eine langfristig erfolgreiche Weiterentwicklung in einer veränderten System-Umwelt.

In der sozial-ökologischen Resilienzforschung wird die Diskussion um Resilienz und Anpassungsfähigkeit noch um einen weiteren Begriff ergänzt, den der Transformation komplexer adaptiver Systeme im Angesicht widriger Ereignisse. Daher muss auch dieser Begriff und sein Zusammenhang mit Resilienz für das Resilienz-Konzept der zivilen SiFo kurz näher beleuchtet werden. Es geht um die Fragen, was unter Transformation zu verstehen ist, inwiefern sich Transformation und Anpassung sauber voneinander unterscheiden lassen und ob Transformation oder besser gesagt Transformationsfähigkeit ein Teil von Resilienz sein kann oder muss. Als Ausgangspunkt eignet sich erneut Hollings Unterscheidung zwischen ecological resilience und engineering resilience. Laut Wink zeichnen sich diese beiden Konzepte gerade dadurch aus, dass sie beide primär darauf ausgerichtet sind, Veränderungen zu vermeiden. Er stellt sie deshalb den Begriffen Anpassungsfähigkeit und Transformationsfähigkeit gegenüber. Unter Anpassungsfähigkeit versteht er eine „Fähigkeit, Resilienz zu verändern“. Mit Transformationsfähigkeit meint er dagegen die „Fähigkeit, ein neues System zu schaffen“ (Wink 2011: 113). Generell bewegt sich diese Gegenüberstellung von Resilienz auf der einen und Anpassungs- sowie Transformationsfähigkeit auf der anderen Seite abseits der etablierten Diskussion in der Resilienzforschung, in der es stärker darum geht, inwiefern Resilienz gerade in Anpassungs- oder sogar Transformationsfähigkeit besteht. Nichtsdestotrotz bietet Winks Ansatz eine geeignete Definition des Begriffs Transformationsfähigkeit. Der Fokus liegt bei der Transformation auf der Schaffung von etwas Neuem, das sich fundamental vom bisheri- 
gen (System) unterscheidet (IRGC 2018: 16). Ein Resilienz-Begriff, der Transformation beinhaltet, ginge damit weit über Hollings ursprüngliche Ideen hinaus. Für Holling ging es ja gerade um Systemerhalt mithilfe von Veränderung bzw. spezifischer mithilfe generischer Anpassungsfähigkeit. Ihre Anpassungsfähigkeit erlaubt komplexen ökologischen Systemen den Übergang zwischen verschiedenen Gleichgewichtszuständen, wenn externe Reize ein Verbleiben im jeweils aktuellen Gleichgewichtszustand unmöglich machen. Die Systeme verändern dabei zwar ihre erbrachte Systemleistung, behalten dafür aber den Kern ihrer Identität, der unter anderem in den Beziehungsmustern und Regeln der Interaktion zwischen den Systemelementen besteht, bei. Sie wechseln zwischen verschiedenen, aber festgelegten Gleichgewichtszuständen. In diesem Bild lässt sich Transformationsfähigkeit dann als Fähigkeit zur Schaffung völlig neuartiger Gleichgewichtszustände verstehen, als eine fundamentale Veränderung der „stability landscape“ und das Beschreiten eines im bisherigen System so nicht möglichen, neuen Entwicklungspfads (Folke et al. 2010). Transformationsfähigkeit geht also über Anpassungsfähigkeit weit hinaus. Es geht nicht „nur“ um das Vermögen eines Systems, sich selbst angesichts von Veränderungen zielgerichtet weiterzuentwickeln. Es geht vielmehr darum, aus dem bestehenden System heraus etwas völlig Neuartiges zu kreieren (Folke 2006: 262). In der sozial-ökologischen Forschung definieren Walker et al. diesen Sachverhalt sehr prägnant: „Transformability: The capacity to create a fundamentally new system when ecological, economic, or social (including political) conditions make the existing system untenable" (Walker et al. 2004). „Untenable” lässt sich mit unhaltbar oder hinfällig übersetzen. Sinngemäß geht es um Situationen, in denen das bestehende System aufgrund externer oder interner Bedingungen (conditions) seine Systemleistung nicht mehr aufrechterhalten kann und in denen eine reine Anpassung an die Bedingungen im existierenden System nicht mehr ausreicht. Für das Resilienz-Konzept der zivilen SiFo lässt sich in Anlehnung an die Definition von Anpassungsfähigkeit Transformationsfähigkeit also wie folgt verstehen: Transformationsfähigkeit bezeichnet das Vermögen eines Systems, seine eigene Identität, Funktionalität und die bestimmenden Beziehungsmuster seiner Elemente angesichts von Veränderungen zielgerichtet so weiterzuentwickeln, dass ein neues System entsteht.

Damit scheint die Unterscheidung zwischen Anpassungs- und Transformationsfähigkeit eindeutig und klar. Anpassung erfolgt innerhalb eines bestehenden Systems, welches sich selbst weiterentwickelt, dabei aber seine Identität erhält. Transformation bedeutet den Übergang zu einem völlig neuen System, dessen Identität gerade nicht mehr dieselbe ist wie 
zuvor. Aus der system- und komplexitätstheoretischen Analyse lässt sich aber auch eine Gemeinsamkeit zwischen Anpassungs- und Transformationsfähigkeit herausarbeiten. Beide sind demzufolge Fähigkeiten, über die nur komplexe adaptive Systeme verfügen. Einfache Systeme verfügen nicht über Anpassungsfähigkeit, weil ihnen die dafür notwendigen Elemente - adaptive Agenten und Mechanismen, neue Signalverarbeitungsmuster durch Neuanordnung nützlicher Bausteine zu kreieren sowie in Interaktion mit anderen Agenten zu treten und so Feedback und Rückkopplungen zu produzieren - fehlen. Sie können ihren Entwicklungspfad nicht verlassen. Wenn sie von einem extremen widrigen Ereignis, das ihre Belastungsgrenzen übersteigt, getroffen werden, können sie - ohne Hilfe von außen - nicht weiter funktionieren und brechen zusammen. Nach der gleichen Logik lässt sich auch feststellen, dass einfache Systeme nicht über Transformationsfähigkeit verfügen können. Auch diese erfordert die Existenz adaptiver Agenten und die Möglichkeit, neue Signalverarbeitungsmuster zu kreieren. Nur dann ist eine Transformation in ein neues System, das sich gerade durch vollkommen andersartige Signalverarbeitungsmuster, andere Agenten und Bausteine auszeichnet, denkbar. Komplexität ist demnach eine notwendige Bedingung für das Vorhandensein von Anpassungsfähigkeit wie auch Transformationsfähigkeit. Die Komplexität von Systemen führt allerdings auch dazu, dass die Unterscheidung zwischen der Anpassung an Veränderungen, die noch innerhalb eines Systems erfolgt und der Anpassung an Veränderungen durch Transformation in ein neues System gerade doch nicht derart eindeutig und klar ist, wie es auf den ersten Blick erschien. Transformation bedeutet einen Wandel der Identität des Systems. In komplexen Systemen ist es allerdings sowohl theoretisch als auch empirisch/praktisch sehr schwer, eine eigentliche Identität des Systems auszumachen und fix zu definieren. Es ließe sich komplexitätstheoretisch formulieren: Die Identität eines komplexen adaptiven Systems ist eine emergente Eigenschaft dieses Systems. Aufgrund der fortlaufenden Weiterentwicklung komplexer adaptiver Systeme ist sie ebenfalls einem ständigen Wandel unterworfen. Wenn sich die Identität komplexer adaptiver Systeme ohnehin ständig verändert, fällt es schwer, die durch widrige Ereignisse hervorgerufene Weiterentwicklung des Systems trennscharf als entweder systeminterne Anpassung oder neues System konstituierende Transformation zu identifizieren. Lukesch formuliert treffend: „Die genaue Unterscheidung zwischen dem, was gerade noch Adaptation ist, und dem, was schon als Transformation zu bezeichnen ist, fällt $[\ldots]$ nicht leicht“" (Lukesch 2016: 302). Mit Bezug zur Systemtheorie ließe sich fragen: $\mathrm{Ab}$ wann differenziert ein neuer Entwicklungspfad, den ein komplexes 
adaptives System nach Eintreten eines gravierenden Reizes realisiert, das System so stark vom vor Eintreten des Reizes existierenden System, das von einer Transformation in ein neues System gesprochen werden muss? Ein Urteil darüber lässt sich nur retrospektiv treffen und es unterliegt notwendigerweise großen Interpretationsspielräumen. Anpassungs- und Transformationsfähigkeit lassen sich folglich nicht als diskrete Eigenschaften verstehen. Vielmehr bewegen sie sich auf einem Kontinuum, welches sich von einer leichten, kaum wahrnehmbaren Weiterentwicklung/Änderung des Systems angesichts externer Reize bis hin zu einem radikalen und tiefgreifenden Wandel aufspannt. Das heißt: In komplexen adaptiven Systemen sind Anpassungs- und Transformationsfähigkeit kontinuierlich miteinander verknüpft und bezeichnen unterschiedlich ausgeprägte Arten zielgerichteter Weiterentwicklung angesichts von Veränderungen.

Ist Transformationsfähigkeit dann - wie generische Anpassungsfähigkeit - ebenfalls ein notwendiger Bestandteil von Resilienz? Diesbezüglich gab es in der sozial-ökologischen Resilienzforschung in den letzten Jahren eine Entwicklung hin zur Bejahung dieser Frage. Walker et al. postulierten noch 2004, dass Resilienz und Anpassungsfähigkeit primär innerhalb von Systemen wichtig sind und Transformationsfähigkeit darüber hinausgeht (Walker et al. 2004). Folke et al. sprechen hingegen 2010 davon, sowohl Anpassungsfähigkeit als auch „the more radical concept of transformability" als Schlüsselkomponenten von Resilienz zu verstehen. In bewusster Abgrenzung zu und Weiterentwicklung von Holling ist es nach Folke und in seinem aktuellen Verständnis auch Walker sowie anderen Vertretern sozial-ökologischer Resilienzforschung auch und gerade Transformationsfähigkeit, die Resilienz ausmacht (Folke et al. 2010). Die Unterscheidung zwischen Anpassung und Transformation erfolgt dann zum Beispiel auch auf einer zeitlichen Ebene, auf der Anpassung als kurzfristige Reaktion und Transformation als langfristige Strategie verstanden wird (Lorenz 2010). Oder anhand einer Veränderung der „functionality of the system“, welche nach Rogers et al. dann auftritt, wenn ein widriges Ereignis zu gravierend ist, um systemerhaltend damit umgehen zu können. Resilienz drücke sich in einem solchen Fall in einer Metamorphose des Systems aus - was nichts anderes als ein weiterer Ausdruck für Transformation ist (Rogers et al. 2012: 81). Zusammenfassend steht für Folke et al. fest: „Deliberate transformation requires resilience thinking" (Folke et al. 2010). Das ist konsistent mit der weiter oben gegebenen Definition von Transformationsfähigkeit, wonach es stets um eine zielgerichtete Weiterentwicklung geht. Ein komplexes adaptives System reagiert auf ein widriges Ereignis durch Anpassung bzw. Transformation, um langfristig weiterbestehen zu 
können - in einer möglichst leistungserhaltenden Form. Folke weist übrigens auch bezüglich Transformationsfähigkeit auf den deskriptiven/analytischen Charakter des Resilienz-Verständnisses in diesem Kontext hin. Für ihn kann Resilienz auch normativ als negativ empfundene Strukturen aufrechterhalten helfen. Es könne sich als extrem schwierig herausstellen, ein resilientes System von einem aktuellen, normativ negativen Entwicklungspfad abzubringen und hin zu einem neuen, normativ als positiver empfundenen Entwicklungspfad zu „transformieren“ (Folke 2006: 259).

In der sozial-ökologischen Forschung hat sich Transformationsfähigkeit als Teil von Resilienz also in der Debatte etabliert. Gilt dies auch für das systemtheoretische Resilienz-Konzept der zivilen SiFo? Im Gegensatz zur Anpassungsfähigkeit, ergibt es bei Transformationsfähigkeit zunächst keinen Sinn, zwischen spezialisierter und generischer Ausprägung zu unterscheiden. Spezialisierte Anpassungsfähigkeit kommt in komplexen adaptiven Systemen immer dann zum Tragen, wenn es um den Umgang mit Risiken geht. Sie zeichnet sich gerade durch ihre Wirksamkeit innerhalb eingeübter Routinen im bestehenden System aus und findet sich daher rein logisch tendenziell eher am anderen Ende eines Kontinuums, auf dem Transformation enthalten ist. Transformationsfähigkeit bedeutet dagegen den Austausch wirkungslos gewordener Routinen - bzw. komplexitätstheoretisch Signalverarbeitungsmuster - durch völlig neue Herangehensweisen. Eine Verknüpfung von Spezialisierung und Transformationsfähigkeit ist insofern logisch inkonsistent. Für Resilienz spielt aber ohnehin die sogenannte generische Anpassungsfähigkeit die entscheidende Rolle. Nur mittels generischer Anpassungsfähigkeit wird Resilienz möglich. Transformationsfähigkeit lässt sich nach der bisherigen Diskussion nun als besonders ausgeprägte Form generischer Anpassungsfähigkeit verstehen. Ein zur Transformation fähiges komplexes adaptives System ist in der Lage, auf eine sehr viel größere Bandbreite an extrem unwahrscheinlichen oder unerwarteten, seine Belastungsgrenzen übersteigenden Ereignissen adäquat zu reagieren, als ein „nur“ anpassungsfähiges System. Denn systemtheoretisch bedeutet Transformationsfähigkeit ceteris paribus eine Ausweitung der überhaupt möglichen Entwicklungspfade nach Eintreten eines Reizes. Transformation ist die extremste Ausprägung der von Holling stammenden Maxime des „change to survive.“ Gleichzeitig gilt aber auch, dass eine Transformation des Systems nicht immer notwendig ist. Auf die meisten, auch extremen, widrigen Ereignisse lässt sich mittels generischer Anpassungsfähigkeit systemidentitätserhaltend adäquat reagieren. Dies wurde ausführlich sowohl system- als auch komplexitätstheoretisch hergeleitet. Für das Resilienz-Konzept der zivilen SiFo lässt sich also festhalten: Trans- 
formationsfähigkeit ist kein notwendiger Bestandteil von Resilienz. Allerdings erhöht das Vorhandensein von Transformationsfähigkeit - verstanden als besonders ausgeprägte Form generischer Anpassungsfähigkeit - ceteris paribus die Resilienz komplexer adaptiver Systeme.

Das führt noch einmal zurück zur Diskussion des Zusammenhangs zwischen Resilienz und generischer Anpassungsfähigkeit bzw. Anpassungsfähigkeit an sich. Die logisch notwendige Verknüpfung zwischen Systemtheorie, Komplexität und Anpassungsfähigkeit sowie die enge Beziehung dieser theoretischen Konzepte zu Resilienz legt den Gedanken nahe, Resilienz mit Anpassungsfähigkeit gleichzusetzen. Aufgrund der unterschiedlichen Arten von Anpassungsfähigkeit ergibt dies allerdings keinen Sinn. Zumal in der Komplexitätsforschung häufig auch die Ansicht vertreten wird, gerade Anpassungsfähigkeit mache Systeme erst komplex (Smith 2002). Bei einer Gleichsetzung der Begriffe wären damit alle komplexen Systeme immer auch resilient - gegeben die gesamte bisherige Diskussion eine unmittelbar theoretisch wie empirisch $\mathrm{zu}$ verwerfende Annahme. Denn es ist die Ausprägung der generischen Anpassungsfähigkeit, die kennzeichnend für das $\mathrm{Maß}$ an Resilienz ist, über das komplexe adaptive Systeme verfügen können. Generische Anpassungsfähigkeit ist aber ebenfalls nicht unmittelbar gleichzusetzen mit Resilienz. Über generische Anpassungsfähigkeit können komplexe adaptive Systeme prinzipiell auch Situationen verarbeiten, die keine Resilienz erfordern. Resilienz zeigt sich erst dann, wenn komplexe adaptive Systeme mittels generischer Anpassungsfähigkeit extrem unwahrscheinliche oder unerwartete, ihre Belastungsgrenzen übersteigenden Ereignisse so zu bewältigen in der Lage sind, dass sie ihre Systemleistung möglichst aufrechterhalten und langfristig sichern können. Das heißt: Resilienz ist nicht gleichbedeutend mit generischer Anpassungsfähigkeit.

Diese Erkenntnis ändert allerdings natürlich nichts an der zentralen Bedeutung von generischer Anpassungsfähigkeit für Resilienz. Was unter generischer Anpassungsfähigkeit system- und komplexitätstheoretisch $\mathrm{zu}$ verstehen ist, wurde bereits dargestellt. Bevor die Diskussion des $\mathrm{Zu}$ sammenhangs zwischen Resilienz und Anpassungsfähigkeit abgeschlossen werden kann, lohnt aber noch einmal ein näherer Blick auf Ideen aus der sozial-ökologischen Forschung bezüglich konkreterer Mechanismen und Organisationsformen, in denen sich generische Anpassungsfähigkeit in komplexen adaptiven Systemen ausprägen kann. Es ging in der Komplexitätstheorie immer wieder um ungenutzte Spielräume. Worin bestehen in ökologischen Systemen solche ungenutzten Spielräume, die ihnen zu generischer Anpassungsfähigkeit verhelfen? Folke nennt die Diversität, 
die er auch als Variabilität bezeichnet, als Prinzip, das in ökologischen Systemen dazu dient, mit Störungen und widrigen Ereignissen erfolgreich umzugehen. Mit dem Begriff der biologischen Diversität wird in Ökosystemen meist die vorhandene Artenvielfalt gemeint. Generell ist Artenvielfalt nach Folke eine essentielle Grundvoraussetzung für die Selbst-Organisation komplexer, adaptiver ökologischer Systeme. Erst die Artenvielfalt ermöglicht eine erfolgreiche Anpassung an Umweltveränderungen. Einzelne Arten tendieren dazu, sich an gegebene Umstände möglichst gut anzupassen (Maximierung der spezialisierten Anpassungsfähigkeit). Das erlaubt ihnen unter unveränderten Bedingungen eine Maximierung ihrer Population. Gleichzeitig führt es jedoch zu einer sehr hohen Verwundbarkeit gegenüber Veränderungen. Die sozial-ökologische Resilienzforschung argumentiert nun, dass deshalb möglichst viele, unterschiedliche Arten existieren sollten. Denn das maximiert die Wahrscheinlichkeit der erfolgreichen Anpassung des Gesamtsystems auch an gravierende Umweltveränderungen (Folke 2006: 257f, Folke et al. 2002: 438). Die Argumentation erinnert an eine Diskussion, die im Kontext der komplexitätstheoretischen Analyse weiter oben geführt wurde und in der es um die Eigenschaft der Agenten komplexer adaptiver Systeme ging, sich immer stärker zu spezialisieren. Stärkere Spezialisierung macht gleichzeitig stärkere Vernetzung notwendig, was zu immer größerer Komplexität des Systems führt. Einzelne Agenten sind durch stärkere Spezialisierung immer weniger dazu in der Lage, sich an gravierende Veränderungen anzupassen. Durch die Ausdifferenzierung der unterschiedlichen Agenten könnte das Gesamtsystem allerdings sogar besser dazu in der Lage sein, als zuvor. Welcher Effekt überwiegt - der Verlust generischer Anpassungsfähigkeit in den einzelnen Agenten oder der Zugewinn an generischer Anpassungsfähigkeit auf systemischer Ebene durch die Spezialisierung der Agenten - ließ sich a priori nicht feststellen. Diversität und Spezialisierung erscheinen nun auf den ersten Blick deckungsgleich. Wenn dem so wäre, könnte für ökologische Systeme nicht eindeutig festgestellt werden, ob größere Artenvielfalt unter sonst gleichen Bedingungen gleichbedeutend mit größerer generischer Anpassungsfähigkeit ist. Bei näherem Hinsehen lassen sich aber Unterschiede zwischen der Diversität eines komplexen adaptiven Systems und dem Grad an Spezialisierung seiner Agenten feststellen. Spezialisierung zeichnet sich notwendigerweise durch steigende Abhängigkeit von anderen Agenten aus. Je spezialisierter ein Agent ist, desto stärker ist er auf Leistungen anderer Agenten angewiesen, um seine Ziele zu erreichen. Innerhalb des Systems erfüllen die einzelnen Agenten sehr spezifische Funktionen. Fallen sie aufgrund eines widrigen Ereignisses aus, führt die Abhängigkeit der 
anderen Agenten von den genannten Funktionen dazu, dass das Gesamtsystem seine Leistungsfähigkeit verliert. Allerdings können in komplexen adaptiven Systemen diese Funktionen auch von sogenannten funktionalen Gruppen übernommen werden, innerhalb derer mehrere Agenten die gleiche Aufgabe erfüllen. Diversität bedeutet nach Folke dann eine möglichst unterschiedliche Reaktion funktional gleicher Agenten auf extrem unwahrscheinliche oder unerwartete Veränderungen. Je größer diese „variability in responses of species within functional groups to environmental change“ ist, desto ausgeprägter ist die generische Anpassungsfähigkeit des Systems (Folke 2006: 258). Das lässt sich auch wie folgt formulieren: Je größer die Diversität innerhalb funktional äquivalenter Gruppen komplexer adaptiver Systeme, desto größer ist ihre generische Anpassungsfähigkeit.

Derart gefasst, entspricht Diversität im Prinzip einem bestimmten Verständnis von Redundanz. Unter Redundanz wird, wie bereits zuvor erläutert, das mehrfache Vorhandensein von Ressourcen verstanden, die zur Erfüllung der gleichen Aufgabe - oder Funktion - dienen (siehe 2.6). Nach Effizienzgesichtspunkten sind diese Ressourcen unnötig und verzichtbar, da sie in einem ungestörten System prinzipiell keine eigenständige Aufgabe erfüllen und daher nicht zur Systemleistung beitragen. Sobald jedoch ein extrem unwahrscheinliches oder unerwartetes Ereignis dazu führt, dass Teile des Systems ausfallen, also Ressourcen überbeansprucht werden, erlaubt das Vorhandensein redundanter Ressourcen dem System eine leistungserhaltende Anpassung an das widrige Ereignis. Diese „springen“ sozusagen ein. In Ökosystemen können redundante Ressourcen beispielsweise in bestimmten Spezies bestehen, die für die „normale“ Funktionsfähigkeit des Systems scheinbar nicht benötigt werden, die aber für den Systemerhalt angesichts gravierender Veränderungen eine kritische Bedeutung haben. Das gilt besonders für Redundanz über verschiedene Skalen hinweg, also etwa für Spezies die nur lokal/regional auftreten im Vergleich zu Spezies, die überregional vorkommen. Oder Spezies, deren Lebenszyklen sich in kurzen Zeitspannen bemessen im Vergleich zu Spezies, die längere Zeitspannen überdauern. Existieren über diese Skalen hinweg jeweils Redundanzen, erhöht das die generische Anpassungsfähigkeit des Systems (Folke 2006: 258). Dieser Ansatz lässt sich auch auf komplexe adaptive Systeme an sich verallgemeinern. Wenn ein widriges Ereignis zum Aus- oder Wegfall bestimmter Agenten des Systems führt, kann dieses durch das Vorhandensein funktional äquivalenter Agenten kompensiert werden. Die Kompensation ist natürlich nur dann möglich, wenn die redundant vorhandenen Agenten nicht ebenfalls durch das widrige Ereignis betroffen sind (ENISA 2011: 48, Sterbenz et al. 2010: 
1250). Deshalb ist auch hier Redundanz über verschiedene Skalen hinweg sinnvoll. Ein Beispiel verdeutlicht diesen Sachverhalt. Wenn mehrere, geografisch verteilte Rechenzentren dieselbe Aufgabe innerhalb eines Unternehmens übernehmen, sind sie auf das Auftreten lokaler Störungen wie etwa einem Sturm oder einer Überflutung vorbereitet. Werden die Rechenzentren allerdings mithilfe der gleichen Software betrieben, sind sie gegenüber Cyber-Angriffen verwundbar. Redundanz kann also auch dazu dienen, spezialisierte Anpassungsfähigkeit (etwa gegenüber lokalen Extremwetterereignissen) zu erhöhen. Im Zusammenhang mit Resilienz geht es aber um die Frage danach, wie komplexe adaptive Systeme mit beliebigen, a priori unbestimmten widrigen Ereignissen umgehen können. Dabei können die gerade erwähnten skalenübergreifenden Redundanzen nützlich sein. Im Beispiel wäre neben der geografischen Verteilung auch noch unterschiedliche, aber dieselbe Funktion erfüllende, Steuerungssoftware notwendig. Das Beispiel vereinfacht zwar die Interaktion komplexer adaptiver Systeme mit ihrer Umwelt stark, hilft aber dabei, eine wichtige Erkenntnis für das Resilienz-Konzept der zivilen SiFo herauszuarbeiten: Je mehr skalenübergreifende Redundanzen vorhanden sind, desto größer ist die generische Anpassungsfähigkeit komplexer adaptiver Systeme.

Zusammenfassend lässt sich festhalten, dass Resilienz ohne Anpassungsfähigkeit nicht denkbar ist. Für das Resilienz-Konzept der zivilen SiFo ergeben sich daher aus der Diskussion des Zusammenhangs zwischen Resilienz und Anpassungsfähigkeit folgende Annahmen:

- Anpassungsfähigkeit meint das Vermögen, sich selbst angesichts von Veränderungen zielgerichtet weiterzuentwickeln, um die eigene Weiterexistenz sicherzustellen.

- Das Vorhandensein von Anpassungsfähigkeit ist eine zwingende Grundvoraussetzung für Resilienz. Aber Resilienz ist nicht gleichbedeutend mit Anpassungsfähigkeit. Resilienz ist auch nicht gleichbedeutend mit generischer Anpassungsfähigkeit.

- Komplexe adaptive Systeme durchlaufen verschiedene Phasen. In der Entfaltungsphase wachsen sie, der Grad an Vernetzung im System nimmt zu und damit auch seine Komplexität. Irgendwann geht das System in die Erhaltungsphase über, in dem es sich durch hohe Stabilität, geringe Variabilität aber eine sehr hohe und konstante Systemleistung auszeichnet. Erfährt ein derartiges System einen starken externen Reiz, geht es sehr wahrscheinlich in die Auflösungsphase über, die gekennzeichnet ist durch eine rapide und drastisch sinkende Systemleistung. Gelingt es dem System durch Anpassung seiner Funktionalitäten weiterzubestehen, tritt es in eine Erneuerungsphase ein. In dieser passt 
sich das System den veränderten Umweltbedingungen durch seinerseits rapide und umfassende Veränderungen an und die Systemleistung beginnt sich zu erholen.

- Resilienz drückt sich in einem durch Anpassungsfähigkeit realisierbaren Übergang von einer Erneuerungs- in eine Entfaltungsphase aus.

- Für die Resilienz komplexer adaptiver Systeme ist entscheidend, dass sie in ihrem Lebenszyklus in der Entfaltungs- und Erhaltungsphase bewusst ungenutzte Spielräume aufbauen, um in einer an ein widriges Ereignis anschließenden Erneuerungsphase über generische Anpassungsfähigkeit zu verfügen.

- Generische Anpassungsfähigkeit ermöglicht komplexen adaptiven Systemen sowohl eine unmittelbare Bewältigung der Folgen extrem unwahrscheinlicher oder unerwarteter, die Belastungsgrenzen des Systems übersteigender Ereignisse als auch eine langfristig erfolgreiche Weiterentwicklung in einer veränderten System-Umwelt.

- Transformationsfähigkeit bezeichnet das Vermögen eines Systems, seine eigene Identität, Funktionalität und die bestimmenden Beziehungsmuster seiner Elemente angesichts von Veränderungen zielgerichtet so weiterzuentwickeln, dass ein neues System entsteht.

- In komplexen adaptiven Systemen sind Anpassungs- und Transformationsfähigkeit kontinuierlich miteinander verknüpft und bezeichnen unterschiedlich ausgeprägte Arten zielgerichteter Weiterentwicklung angesichts von Veränderungen.

- Transformationsfähigkeit ist kein notwendiger Bestandteil von Resilienz. Allerdings erhöht das Vorhandensein von Transformationsfähigkeit - verstanden als besonders ausgeprägte Form generischer Anpassungsfähigkeit - ceteris paribus die Resilienz komplexer adaptiver Systeme.

- Diversität und Redundanz sind Prinzipien zur Erhöhung generischer Anpassungsfähigkeit in komplexen adaptiven Systemen. Diversität besteht in einer möglichst unterschiedlichen Reaktion funktional gleicher Agenten auf extrem unwahrscheinliche oder unerwartete Veränderungen. Je größer die Diversität innerhalb funktional äquivalenter Gruppen komplexer adaptiver Systeme, desto größer ist ihre generische Anpassungsfähigkeit.

- Derart gefasst, entspricht Diversität im Prinzip einem bestimmten Verständnis von Redundanz, nämlich skalenübergreifender Redundanz. Skalenübergreifende Redundanz bedeutet das Vorhandensein funktional äquivalenter Systemelemente, die sich in ihrer Abhängigkeit von unterschiedlichen widrigen Ereignissen unterscheiden. Je mehr skalen- 
übergreifende Redundanzen vorhanden sind, desto größer ist die generische Anpassungsfähigkeit komplexer adaptiver Systeme.

\subsubsection{Resilienz und Flexibilität}

Zum Abschluss der systemtheoretischen Analyse des Resilienz-Konzepts soll im Folgenden noch einmal ein Blick auf den Begriff der Flexibilität und seinen Zusammenhang mit Resilienz geworfen werden. „[M]aintaining flexibility above all else“ lautet eine der zentralen Aussagen Hollings, die weiter oben bereits prominent zitiert wurde (Holling 1973: 18). Die Aussage erfolgt vor dem Hintergrund unterschiedlich ausgestalteter ökologischer Systeme und deren jeweils entsprechend unterschiedlichem Vermögen zum erfolgreichen Umgang mit externen Störungen. Effiziente Ökosysteme weisen hohe Stabilität durch Rigidität auf und können mit bekannten Störungen gut und erfolgreich umgehen. Flexible Ökosysteme weisen dagegen hohe Resilienz durch Anpassungsfähigkeit auf und können - durch den Übergang von einem alten in einen neuen Gleichgewichtszustand - mit unbekannten, gravierenden und überraschend eintretenden Störungen gut und erfolgreich umgehen (siehe 2.3). Äquivalent zu diesen Überlegungen Hollings taucht der Begriff der Flexibilität immer wieder in der Resilienzforschung auf. Das gilt sowohl für psychologische, als auch ökologische, sozial-ökologische, sozialwissenschaftliche und organisationswissenschaftliche Ansätze (Brunner/Giroux 2009: 6, Elran 2012: 294, Folke 2006: 261, Folke et al. 2002: 438, Fooken 2016: 25, McDonald 2006: 168, Prior/Roth 2013: 62, Wink 2011: 115, Woods 2019: 56, 2015: 8). Dabei wird er sehr häufig explizit oder zumindest implizit direkt mit Anpassungsfähigkeit verknüpft. Für die vorliegende Arbeit stellen sich also zwei Fragen bezüglich Flexibilität, die beantwortet werden müssen, um das systemtheoretische Resilienz-Konzept für die zivile SiFo diesbezüglich zu vervollständigen. Zum einen geht es darum, was mit Flexibilität innerhalb der Resilienzforschung gemeint ist. Zum anderen darum, ob und wenn ja wie sich Flexibilität von Anpassungsfähigkeit, so wie sie im vorangegangenen Unterkapitel definiert wurde, unterscheidet. Neben Flexibilität und Anpassungsfähigkeit ist im selben Kontext zudem immer wieder von Lernfähigkeit, Improvisationsfähigkeit und Kreativität die Rede, so dass auch diese drei Begriffe noch kurz systemtheoretisch im Resilienz-Diskurs verortet werden.

Der Begriff Flexibilität taucht in der Resilienzforschung in einer expliziten Nennung deutlich seltener auf, als Begriffe wie Komplexität, Anpas- 
sungsfähigkeit, Unsicherheit oder Verwundbarkeit. Nichtsdestotrotz findet er immer wieder Erwähnung und wird von einigen Autoren zumindest teilweise auch (mit) im Zentrum ihres konzeptionellen Verständnisses verortet. Woods formuliert beispielsweise passend: „Central to resilience is identifying what basic architectural principles are preserved over these changes and provide the needed flexibility to continue to adapt over long scales" (Woods 2015: 8). In diesem Satz schlägt er die Brücke zwischen verschiedenen Aspekten von Resilienz, die bereits ausführlich diskutiert wurden. Er bewegt sich generell im Theoriegebäude der komplexen adaptiven Systeme und fragt sich daher hier, wie derartige Systeme ausgestaltet sein müssen, um langfristig erfolgreich mit Störungen bzw. Veränderungen umgehen zu können. Resilient ist ein komplexes adaptives System für Woods genau dann, wenn es so gestaltet wurde, dass es die benötigte Flexibilität aufweist, um sich Veränderungen anpassen zu können. Im Bereich der Katastrophenforschung verknüpfen Prior und Roth Flexibilität auf ähnliche Weise mit Resilienz: „[M]odern disaster preparedness and planning is increasingly characterized by the need to adapt to uncertainty and unpredictability. As a consequence, flexible strategies aimed at increasing social resilience are gaining ground in the preparedness practices of many cities" (Prior/Roth 2013: 65). Für Prior und Roth sind es Unsicherheit und Unvorhersagbarkeit (unpredictability) von widrigen Ereignissen bzw. Störungen oder gravierenden Veränderungen, die Anpassungsfähigkeit notwendig machen. Und um sich erfolgreich anzupassen, ist Flexibilität notwendig. Ihr Untersuchungsgegenstand sind an dieser Stelle Städte bzw. soziale Gemeinschaften, da es um soziale Resilienz geht. Diese sind unzweifelhaft als komplexe adaptive Systeme zu charakterisieren. Soziale Resilienz lässt sich also nach Prior und Roth mithilfe flexibler Strategien erhöhen. Flexibilität kommt aber auch in stärker technisch geprägten Resilienz-Konzepten vor. Nach Fooken gehört sie zu „zentralen Komponenten“, die Resilienz ausmachen, neben den Punkten Widerstandskraft, Selbstregulations-Kompetenz und Elastizität. Für ihn werden diese Eigenschaften eines Systems bzw. eines Materials genau dann zum Bestandteil von Resilienz bzw. notwendig, um ein System/Material als resilient zu kennzeichnen, wenn dieses „erheblichen Widrigkeiten sowie aversiven Einwirkungen und Störungen" ausgesetzt ist (Fooken 2016: 25). Ein Material lässt sich grundsätzlich nur schwerlich als komplexes adaptives System charakterisieren. Materialien haben eindeutige Eigenschaften, darunter a priori festgelegte Belastungsgrenzen innerhalb derer sie statischen, quasistatischen und dynamischen Lasteinwirkungen standzuhalten in der Lage sind. Sobald diese Belastungsgrenzen überschritten werden, versagt das 
entsprechende Material. Allerdings unterscheiden sich Materialien bezüglich ihrer Fähigkeiten, sich elastisch und/oder plastisch zu verformen, bevor sie versagen. In verschiedenen Teilbereichen der Resilienzforschung werden diese Fähigkeiten als Flexibilität verstanden und Resilienz darin erkannt, dass Materialien und/oder Systeme bei Lasteinwirkungen „biegen, aber nicht brechen" (bend, but not break) (Brunner/Giroux 2009: 7). Das Bild des in dieser Hinsicht flexiblen Materials, mit dessen Hilfe Belastungen absorbiert und Schäden minimiert werden können, scheint auf den ersten Blick attraktiv zur Erklärung von Resilienz. Für das Resilienz-Konzept der vorliegenden Arbeit ist es jedoch nicht geeignet. Es wurde bereits ausführlich dargelegt, warum Resilienz nur in komplexen adaptiven Systemen auftreten kann. Ohne die system- und komplexitätstheoretischen Argumente dafür wiederholen zu wollen, lässt sich kurz sagen: Die Fähigkeit eines Materials sich elastisch und/oder plastisch zu verformen ist exakt definiert und gibt dem Material bestimmte Belastungsgrenzen vor. Sobald eine Lasteinwirkung diese Grenze übersteigt, versagt das Material. Elastische und plastische Verformung können zudem als passive Reaktion des Materials auf eine Belastung verstanden werden. Resilienz besteht dahingegen gerade darin, Störungen und widrige Ereignisse, die extrem unwahrscheinlich oder unsicher sind und die Belastungsgrenzen des Systems übersteigen, erfolgreich zu überwinden. Dazu bedarf es einer aktiven Anpassung an veränderte Bedingungen der System-Umwelt. Um Flexibilität system- und komplexitätstheoretisch in das Resilienz-Konzept der zivilen SiFo einordnen zu können, gilt daher: Flexibilität meint nicht die Fähigkeit von (einfachen) Systemen, sich bei Lasteinwirkungen durch spezifisch definierte Belastungen elastisch und/oder plastisch zu verformen.

Was meint Flexibilität stattdessen? Für die vorliegende Arbeit liefert die sozial-ökologische Forschung dazu wieder verschiedene, wertvolle Anknüpfungspunkte. Das Zusammenspiel zwischen sozialen und ökologischen Systemen - beide Systemtypen sind zweifelsfrei als komplexe, adaptive Systeme zu charakterisieren - wird unter anderem durch überraschend auftretende Veränderungsprozesse in den verschiedenen Systemtypen gekennzeichnet. Um etwa überraschende Veränderungen von Ökosystemen erfolgreich bewältigen zu können, benötigen soziale Systeme sowohl Lernfähigkeit als auch Flexibilität (Folke 2006: 261). Konkrete Maßnahmen, um die Resilienz sozialer Systeme im Angesicht von deren Abhängigkeit von ökologischen (und anderen sozialen) Systemen zu erhöhen, oder anders gesagt „resilience-building management“, muss flexibel und „open to learning“ sein, da nur so mit Unsicherheit und Komplexität umgegangen werden kann (Folke et al. 2002: 438). Management so zu 
verstehen, entspricht Hollings Ideen des adaptiven Managements. Folke et al. sprechen konkret von "active adaptive management" und betonen also den aktiven Charakter von resilienzerhöhenden Maßnahmen. Ein aktives, adaptives Management zeichnet sich demzufolge gerade dadurch aus, dass es Raum für Flexibilität und Innovation lässt (Folke et al. 2002: 439). Auf das Thema adaptives Management wurde im vorangegangenen Unterkapitel zu Anpassungsfähigkeit bereits eingegangen. Damit wird die enge Verknüpfung zwischen Flexibilität und Anpassungsfähigkeit erneut deutlich. Ein weiteres Beispiel dafür ist das Resilienz-Verständnis in Teilen der Wirtschaftswissenschaften, in denen das Management komplexer ökonomischer Systeme im Zentrum steht. Nach Wink kann exogenen Störungen mithilfe verschiedener Systemeigenschaften erfolgreich begegnet werden. Diese Eigenschaften, zum Beispiel Redundanz und Diversität, machen Resilienz aus. Wink nennt an dieser Stelle auch Flexibilität. Durch Flexibilität werde eine „Anpassung an andere Anforderungen“ möglich (Wink 2011: 115). Der Zusammenhang zwischen Redundanz, Diversität und generischer Anpassungsfähigkeit wurde bereits analysiert. Skalenübergreifende Redundanzen verstanden als große Diversität in funktional äquivalenten Gruppen innerhalb komplexer adaptiver Systeme erhöhen deren generische Anpassungsfähigkeit und damit ihre Resilienz. Aber auch Flexibilität führt laut Wink zu größerer Anpassungsfähigkeit. Wie auch die sonstige Resilienzforschung differenziert er nicht zwischen spezialisierter und generischer Anpassungsfähigkeit. Und bei näherem Hinsehen meint er im Kontext Resilienz/Anpassungsfähigkeit/Flexibilität durchaus beide Arten (Wink 2011: 115f). Im Bereich des Managements komplexer adaptiver Systeme lässt sich Flexibilität also als Eigenschaft verstehen, deren Vorhandensein die Anpassungsfähigkeit der Systeme erhöht. Das gilt sowohl für ihre spezialisierte als auch ihre generische Anpassungsfähigkeit und damit auch für die Resilienz der Systeme. Brunner und Giroux fassen das prägnant zusammen, indem sie in Bezug auf Resilienz schreiben: „[D]ue to uncertainty, every system or social unit should strive to be as flexible as possible" (Brunner/Giroux 2009: 6). Resultierend aus verschiedenen Bereichen der Resilienzforschung ließe sich für das Resilienz-Konzept der zivilen SiFo also festhalten: Je flexibler komplexe adaptive Systeme sind, desto größer ist ihre Resilienz.

Resilienz zeichnet sich nach Bach et al. durch den Gebrauch gewöhnlicher Fähigkeiten unter außergewöhnlichen Umständen ab (Bach et al. 2011: 8). Ein Rückgriff auf Erkenntnisse der Katastrophenforschung hilft, diesen Aspekt mit Bezug auf das Thema Flexibilität zu verstehen. Die Menschen, die nach Extremwettereignissen, Terroranschlägen, Unfällen oder 
sonstigen widrigen Ereignissen als erste vor Ort sind, sind in den seltensten Fällen professionelle Rettungskräfte. Sehr viel häufiger sind diese Ersthelfer unbeteiligte Zivilisten, die rein zufällig am Ort des Geschehens sind (Flynn/Burke 2011: 5, 2011b: 131). Die Ersthelfer sind nicht ausgebildet, sie haben keine Erfahrung darin, mit derartigen Extremsituationen umzugehen. Professionelle Rettungskräfte trainieren dagegen ständig die richtigen Verhaltensweisen für solche Situationen und verfügen über erwiesenermaßen funktionierende Kompetenzen, Werkzeuge und Vorgehensweisen. Durch ihr Training und das Vertrauen in etablierte Verhaltensweisen versuchen professionelle Rettungskräfte die Notwendigkeit, vor Ort improvisieren zu müssen, zu minimieren (Al-Khudairy et al. 2012: 593). Um möglichst erfolgreich zu helfen, müssen jedoch beide involvierten Parteien, sowohl zufällige Ersthelfer als auch professionelle Rettungskräfte, möglichst flexibel (re-)agieren können. Zufälligen Ersthelfern steht eine Fülle an Handlungsmöglichkeiten zur Verfügung, zu denen beispielsweise auch Inaktivität oder Flucht zählen. Im Rahmen der vorliegenden Arbeit drückt sich Flexibilität darin aus, neben diesen Handlungsmöglichkeiten auch über Optionen zu verfügen, die es zufälligen Ersthelfern erlauben, sich einen Überblick über die Lage zu verschaffen, einzuschätzen, wie sie selbst konkret helfen können, gleichzeitig möglichst schnell professionelle Hilfe zu organisieren und bei all dem je nach Situation den Schutz ihres eigenen Lebens und ihrer eigenen Gesundheit sicherzustellen. Systemtheoretisch gesprochen entspräche eine vollumfängliche Erfüllung dieser Aufgaben der Realisierung eines Prozesses eigentlich extrem unerwartbarer Anschlussmöglichkeiten im Anschluss an das Auftreten eines sehr unwahrscheinlichen Reizes aus der Umwelt - oder anders gesagt Resilienz. Flexibilität ist also notwendig für Resilienz. Denn Flexibilität bedeutet das Vorhandensein - nicht notwendigerweise die Realisierung - eigentlich extrem unerwartbarer Prozesse an Anschlussmöglichkeiten, die dem System das Einschwenken auf einen neuen, leistungserhaltenden Entwicklungspfad ermöglichen.

Auch professionelle Rettungskräfte benötigen Flexibilität. Fekete et al. sprechen in diesem Zusammenhang von „überalleinsatzfähigen Fähigkeiten" und meinen damit Fähigkeiten, die es Rettungskräften erlauben „jede Lage [zu] meistern“ (Fekete et al. 2016: 223f). Im Gegensatz etwa zu Fachärzten lernen zum Beispiel Notärzte stärker generische Fähigkeiten zur akuten Versorgung völlig unterschiedlich verletzter oder kranker Menschen. Sie verfügen über eine größere Bandbreite unterschiedlicher, grundlegender Kompetenzen, sie sind grundsätzlich sehr flexibel, dafür aber weniger spezialisiert als ihre fachlich auf einen Bereich fokussierten Kollegen. In der vorliegenden Arbeit liegt der Fokus weniger auf Rettungs- 
kräften als Individuen, sondern vielmehr auf ihrer Rolle als Agenten in einem größeren, komplexen adaptiven System. Denn in diesem System sind professionelle Rettungskräfte stark spezialisierte Agenten, die eine ganz bestimmte Funktion - die akute Rettung und Versorgung verletzter und/oder kranker Menschen - übernehmen. Das Vorhandensein von Rettungskräften trägt grundsätzlich zur Resilienz des Systems bei. In diesem Fall ist die spezifische Form der Spezialisierung von Agenten so ausgestaltet, dass sie zu einer im Gesamtsystem höheren generischen Anpassungsfähigkeit führt. Rettungskräfte gehen im Alltag - wobei dieser Alltag nur für sie gilt, nicht aber für die von Notfällen betroffenen Personen einer Tätigkeit nach, die ihnen in einem systembedrohenden Notfall etwa einer großflächigen Naturkatastrophe - genau die Fähigkeiten an die Hand gibt, die sie zur Bewältigung einer solchen Situation benötigen. Die Resilienz des Systems profitiert also von diesen spezialisierten Agenten. Es gilt aber nicht, dass das System umso resilienter ist, je mehr professionelle Rettungskräfte vorhanden sind. Um Schäden - etwa in Form verletzter oder getöteter Menschen - zu vermeiden oder minimieren, können auch gänzlich andere Fähigkeiten benötigt werden. Beispielhaft kann hier auf einen Ingenieur verwiesen werden, der im Vergleich zu professionellen Rettungskräften sehr viel eher über die Fähigkeit verfügt, etwa ein außer Kontrolle geratenes Kernkraftwerk sicher herunterzufahren. Je nachdem, wie ein widriges Ereignis sich tatsächlich abspielt, werden also innerhalb komplexer adaptiver Systeme unterschiedliche, spezialisierte Fähigkeiten benötigt. Und da in komplexen adaptiven Systemen a priori nicht sicher ist, wie sich Ereignisse tatsächlich auswirken, benötigen sie eine möglichst große Bandbreite unterschiedlicher, spezialisierter Fähigkeiten. Das allein reicht aber noch nicht aus, um Resilienz im System zu erreichen. Im Moment des Eintretens widriger Ereignisse müssen die Systeme auch in der Lage sein, die richtig spezialisierten Agenten zum Einsatz zu bringen. Diese dürfen also nicht gleichzeitig mit anderen Aufgaben im System beschäftigt sein. Denn sonst stehen sie dem System gerade nicht zur Verfügung und das System kann sich höchstwahrscheinlich nicht die Agenten zunutze machen, die es zur Bewältigung der Situation benötigen würde. In der Komplexitätstheorie wurde zudem festgestellt, dass es in komplexen adaptiven System auch weiterhin Generalisten gibt, also Agenten, die sich nicht spezialisieren. Auch solche Agenten, die mit einer großen Bandbreite unterschiedlicher Anforderungen umzugehen in der Lage sind, werden vom System benötigt.

Spezialisierte, aber frei verfügbare Agenten und Generalisten lassen sich beim Blick auf das Gesamtsystem als eine bestimmte Art von Ressourcen 
verstehen. Im Resilienz-Diskurs ist immer wieder die Rede davon, sogenannte „slack resources“ aufzubauen. Dieser englische Begriff hat keine unmittelbar passende deutsche Entsprechung. Für die vorliegende Arbeit wird näherungsweise von „losen“ Ressourcen gesprochen. Lose Ressourcen sind Ressourcen, die innerhalb des Systems keinen spezifischen Aufgaben zugeordnet sind, ohne die das System nicht funktionieren bzw. eine geringere Leistung erbringen würde (Hutter 2011). Streng ökonomisch gesprochen wären diese Ressourcen zu jedem beliebigen Zeitpunkt überflüssig. Sie wirken sich negativ auf die Effizienz des Systems aus. Sobald das System jedoch von einem unerwarteten und seine eigentlichen Belastungsgrenzen übersteigenden, widrigen Ereignis getroffen wird, können lose Ressourcen zum Einsatz gebracht werden, um erfolgreich mit dem Ereignis umgehen zu können. Flexibilität wurde systemtheoretisch als Vorhandensein eigentlich extrem unerwartbarer Prozesse an Anschlussmöglichkeiten, die dem System das Einschwenken auf einen neuen, leistungserhaltenden Entwicklungspfad ermöglichen, definiert. Diese Definition kann nun komplexitätstheoretisch mit dem Begriff der losen Ressourcen ergänzt werden, um den Zusammenhang zwischen Flexibilität, Anpassungsfähigkeit und Resilienz zu spezifizieren. Weiter oben wurde festgehalten, dass mehr Flexibilität zu mehr Resilienz führt, da Flexibilität die Anpassungsfähigkeit ganz generell erhöht, und zwar sowohl die spezialisierte als auch die generische Anpassungsfähigkeit von Systemen. Präziser gesagt lässt sich Flexibilität besser als einer von zwei Bestandteilen von generischer Anpassungsfähigkeit verstehen. Je mehr eigentlich extrem unerwartbare Prozesse an Anschlussmöglichkeiten vorhanden sind, desto eher verfügt das System über eine ausgeprägte generische Anpassungsfähigkeit. Das Vorhandensein dieser Prozesse an Anschlussmöglichkeiten an sich macht aber generische Anpassungsfähigkeit erst möglich. Tatsächlich wirksam wird sie erst durch die Realisierung der Prozesse. Und dazu benötigt das System darüber hinaus noch lose Ressourcen. Denn lose Ressourcen sind auf systemischer Ebene Agenten, die im Normalfall - solange sich das System auf seinem normalen, erwartbaren Entwicklungspfad befindet - nicht systemkritisch sind. Das System kann seine Leistung prinzipiell im ungestörten Zustand auch ohne lose Ressourcen erbringen. Durch den Eintritt einer unerwarteten und die Belastungsgrenzen übersteigenden Störung wird das System dagegen auf einen neuen Entwicklungspfad gezwungen, den es - um seine Leistung zu erhalten bzw. wiederherzustellen - möglichst schnell wieder verlassen muss. Die gebundenen Ressourcen des Systems sind dazu nicht geeignet. Gebundene Ressourcen sind spezialisierte Agenten, deren Spezialisierung gerade darin besteht, die Systemleistung im ursprünglichen 
Entwicklungspfad zu erbringen. Von diesem Entwicklungspfad hat sich das System bereits entfernt und die dort erfolgreichen Strategien sind in der veränderten Situation mutmaßlich nutzlos. Lose Ressourcen hingegen können in diesem Moment dazu genutzt werden, einen Prozess eigentlich extrem unerwartbarer Anschlussmöglichkeiten zu realisieren, da sie frei verfügbar und zudem nicht auf eine Bewahrung des ursprünglichen Entwicklungspfads spezialisiert sind. Für das Resilienz-Konzept der zivilen SiFo lässt sich also festhalten: Die generische Anpassungsfähigkeit komplexer adaptiver Systeme setzt sich zusammen aus ibrer Flexibilität, also dem Vorhandensein eigentlich extrem unerwartbarer Prozesse an Anschlussmöglichkeiten, und der Verfügbarkeit loser Ressourcen, also spezialisierter Agenten sowie Generalisten, die für das Funktionieren des Systems auf dem ursprünglichen Entwicklungspfad nicht notwendig sind.

Damit kann auch der Zusammenhang zwischen Resilienz und Flexibilität noch einmal näher beleuchtet werden. In den vorangegangenen Unterkapiteln wurde detailliert hergeleitet, dass die Resilienz komplexer adaptiver Systeme abhängig ist vom Ausmaß der in diesen Systemen vorhandenen generischen Anpassungsfähigkeit. Je größer die generische Anpassungsfähigkeit, desto mehr Resilienz weisen komplexe adaptive Systeme auf. Und das Ausmaß der generischen Anpassungsfähigkeit hängt von der Flexibilität des Systems sowie der Menge an zur Verfügung stehenden losen Ressourcen ab. Bleibt die Menge an losen Ressourcen gleich, führt höhere Flexibilität dazu, dass eine größere Auswahl eigentlich extrem unerwartbarer Prozesse an Anschlussmöglichkeiten zur Verfügung steht, die mit Hilfe der losen Ressourcen realisiert werden können. Das führt ceteris paribus zu einer größeren generischen Anpassungsfähigkeit. Damit gilt auch weiterhin, wie oben bereits vor der ausführlicheren system- und komplexitätstheoretischen Analyse postuliert: Je flexibler komplexe adaptive Systeme sind, desto größer ist ibre Resilienz.

Im Kontext des Flexibilitäts-Begriffs und der Diskussion zur Anpassungsfähigkeit spielen innerhalb der Resilienzforschung auch immer wieder ähnliche, offensichtlich verwandte Begrifflichkeiten eine Rolle. Dazu zählen Lernfähigkeit, Improvisationsfähigkeit und Kreativität. Ihre Verwandtschaft mit Flexibilität besteht unter anderem darin, dass sie primär als menschliche Eigenschaften verstanden werden. Die bisherigen Ausführungen in der vorliegenden Arbeit gehen von Anpassungsfähigkeit als Eigenschaft sowohl von Agenten als auch der aus ihnen bestehenden komplexen adaptiven Systeme aus. Flexibilität wurde als Systemeigenschaft charakterisiert. Beide Begriffe sind nicht notwendigerweise mit menschlichen Trägern verbunden. In der zivilen SiFo geht es um sozio-techni- 
sche Systeme, die zwar häufig, aber ebenfalls nicht notwendigerweise Menschen beinhalten und die nichtsdestotrotz komplexe adaptive Systeme sind, die über Flexibilität und generische Anpassungsfähigkeit verfügen können. Rein aus Sicht der System- und Komplexitätstheorie haben diese Annahmen auch für die folgenden Ausführungen Bestand. Allerdings ist es durchaus interessant, sich den drei gerade genannten Begriffen über den Umweg eines Verständnisses als menschliche Eigenschaften zu nähern, um sie anschließend system- und komplexitätstheoretisch zu fassen. Auf diese Weise lässt sich herausarbeiten, inwiefern sie sich von den bereits dargestellten und definierten Konzepten (generische) Anpassungsfähigkeit und Flexibilität unterscheiden. Rahimi und Madni weisen dazu beispielsweise explizit darauf hin, dass viele reale komplexe Systeme gerade deshalb nicht völlig automatisiert betrieben werden, weil Menschen - und Rahimi und Madni verwenden hier den Begriff der "menschlichen Agenten“ (human agents) - im Zweifel besser in der Lage seien, unvorhergesehene Situationen zu entdecken und zu meistern „than their machine counterparts" (Rahimi/Madni 2014: 811). Die Antwort auf die Frage, warum das so gesehen werden könnte, gibt Edwards, wenn er sagt: „[A]s humans we have the capacity to learn and adapt" (Edwards 2009: 17). Implizit nimmt er dabei die Fähigkeit zum Lernen und zur Anpassung als exklusiv menschlich an. In ähnlicher Weise argumentieren Ouedraogo et al., wenn sie von der menschlichen Fähigkeit sprechen, ihr Verhalten durch das Gewinnen von Erfahrung und das Lernen durch wiederholtes Ausprobieren zu verbessern. Menschen können sich selbst an die dynamischen Veränderungen anpassen, denen sie in ihrer Umwelt begegnen. Und diese Anpassung erfolgt eben über ein durch Erfahrung und Ausprobieren vermitteltes Lernen (Ouedraogo et al. 2013: 26). Lernen hilft dabei, aus dem Bekannten heraus durch neue Kombination bekannter Bausteine neue Ideen, Prozesse oder Organisationsformen zu entwickeln. Lernen erfolgt in diesem Zusammenhang, wenn es um Resilienz und Anpassungsfähigkeit geht, also häufig durch Ausprobieren oder learning-by-doing. In der sozialökologischen Forschung wird hier eine Verbindung zwischen Lernen bzw. Lernfähigkeit und adaptivem Management gezogen, dessen Adaptivität oder Anpassungsfähigkeit - gerade im durch Ausprobieren ermöglichten Lernen besteht (Berkes 2007: 287, Longstaff 2012: 269). Das Unterkapitel zum Thema Resilienz und Unsicherheit hat wiederum gezeigt, wie Resilienz insbesondere beim Auftreten unerwarteter, die Belastungsgrenzen von Systemen übersteigender Ereignisse benötigt wird. Solche Ereignisse wurden auch als „beispiellos“ bezeichnet. Tritt etwas Beispielloses ein, können Menschen sich nicht spezifisch darauf vorbereiten. Brunner und 
Giroux formulieren dazu treffend: „In the field of resilience, best practices can never be established or achieved. They are constantly changing, and must be revised through continuous learning processes" (Brunner/Giroux 2009: 17). Auch sie betonen die Bedeutung andauernder und ständiger Lernprozesse, um Resilienz zu ermöglichen. Folke et al. wiederum sehen in der Verbindung zwischen Flexibilität und Lernfähigkeit den Schlüssel zu Management im Sinne einer Erhöhung von Resilienz. Flexibilität und Lernfähigkeit ermöglichen es ihnen zufolge mit Überraschungen, Unerwartbarkeit und Komplexität umzugehen (Folke et al. 2002: 438).

Damit verknüpfen Folke et al. die wesentlichen systemtheoretischen Begrifflichkeiten der vorliegenden Arbeit mit dem Aspekt des Lernens. Es liegt insofern nahe, diesen über ein Verständnis als rein menschliche Eigenschaft hinaus eher als generelle Eigenschaft komplexer adaptiver Systeme zu definieren. Das tun beispielsweise Carpenter et al., die Anpassungsfähigkeit als den Teil von Resilienz verstehen, der die Lernfähigkeit als Systemverhalten im Angesicht widriger Ereignisse widerspiegelt (Carpenter et al. 2001: 766). Auch Gunderson spricht nicht von Menschen, sondern von „Institutionen“, deren Fokus auf dem Aspekt des Lernens liegen sollte, da Lernfähigkeit eine zentrale Komponente sozialer Resilienz sei (Gunderson 2000: 436). Gegeben diese unterschiedlichen Definitionen und Bestandteile von Lernfähigkeit lässt sich fragen, was unter Lernfähigkeit system- und komplexitätstheoretisch zu verstehen ist und inwiefern und wie sie Teil des Resilienz-Konzepts der zivilen SiFo ist. Lernen heißt, durch Erfahrung und Ausprobieren herauszufinden, was funktioniert und was nicht. Im Kontext der Systemtheorie kann das als Fähigkeit übersetzt werden, aus der Vielzahl an zur Verfügung stehenden Prozessen an Anschlussmöglichkeiten diejenigen zu identifizieren, die für den Erhalt der Systemleistung am geeignetsten sind. Komplexitätstheoretisch gesprochen wäre Lernfähigkeit dann die Fähigkeit von Agenten, ungeeignete von geeigneten Signalverarbeitungsmustern zu unterscheiden. Lernen bedeutet eine Abschätzung darüber zu treffen, wie sich eine neue Kombination bekannter Bausteine zu Signalverarbeitungsmustern zusammensetzen und welchen Einfluss auf die Wahrscheinlichkeit zur eigenen Zielerreichung der Einsatz dieser neuen Signalverarbeitungsmuster hätte. Die Lernfähigkeit des Gesamtsystems setzt sich wiederum - allerdings in nichtlinearer Weise - aus der Lernfähigkeit der Agenten zusammen. Insgesamt lässt sich Lernfähigkeit als eine Art kognitiver Komponente von Anpassungsfähigkeit verstehen. Mithilfe des Lernens erlangen komplexe adaptive Systeme Wissen über die ihnen zur Verfügung stehenden Prozesse an Anschlussmöglichkeiten und mit welcher Systemleistung diese jeweils mutmaßlich 
verknüpft sein werden. Die Komplexität der Systeme verhindert zwar eine deterministische, eindeutige Zuordnung, nichtsdestotrotz ist Lernfähigkeit als Teil von Anpassungsfähigkeit notwendig für Resilienz. Wobei Lernfähigkeit dieser Definition zufolge generell Teil von Anpassungsfähigkeit ist, nicht nur von generischer, sondern auch von spezialisierter Anpassungsfähigkeit. Für das Resilienz-Konzept der zivilen SiFo gilt: Lernfähigkeit besteht darin, Wissen über die wahrscheinlichen Auswirkungen der Realisierung der verschiedenen zur Verfügung stehenden Prozesse an Anschlussmöglichkeiten erlangen zu können und ist damit ein Teil der Anpassungsfähigkeit komplexer adaptiver Systeme. Deshalb ist Lernfähigkeit eine notwendige Grundvoraussetzung für Resilienz.

Der zweite Begriff, der an dieser Stelle kurz beleuchtet werden soll, ist der der Improvisationsfähigkeit. Ähnlich wie Lernfähigkeit ist das ein Begriff, der immer wieder in der Resilienzforschung fällt. So zählt beispielsweise Hutter neben Lernen und Improvisieren auch Anpassungsfähigkeit, "capability“ und „competence“ als „fuzzy terms“ - also eher unklare Begrifflichkeiten - auf, die in Verbindung mit Resilienz bzw. präziser bei ihm „sozialer Resilienz“ genutzt würden (Hutter 2011). Für Brunner und Giroux geht es bei Resilienz ganz generell um die Fähigkeit zur Improvisation „in the face of unfamiliar challenges“ (Brunner/Giroux 2009: 7). Auch Vogt, der als christlicher Sozialethiker aus einer völlig anderen Forschungsrichtung stammt, zählt Improvisationsfähigkeit neben der „Offenheit für Überraschungen“ zu den „Leittugenden der Resilienz" (Vogt 2015: 15). Mit Improvisieren ist meistens ein Abweichen von einem vorherigen Plan, von der standardmäßig zu erwartenden Handlungsweise gemeint. Wenn Menschen nicht über geeignete Erfahrungen oder Routinen verfügen, es keine auf eine spezifische Situation passenden Pläne gibt, aber die Notwendigkeit besteht, sich an eine sich schnell verändernde Umwelt anzupassen - in solchen Fällen müssen sie „improvisieren.“ Aus den bisherigen Erkenntnissen der Arbeit ist zudem klar: zu detaillierte Planungen und Vorbereitungen können sogar kontraproduktiv sein, wenn sie zu einer den Blick verengenden Spezialisierung führen. Solcherart spezialisierte Agenten sind nur in der Lage, mit sehr spezifischen Begebenheiten umzugehen, nicht aber mit Situationen, die davon abweichen, die überraschend eintreten (Kuhlicke 2010, Longstaff 2012: 259). Improvisationsfähigkeit wird teilweise auch als eine dem Menschen eigene „unique property" verstanden, als Antwort auf Unsicherheit generell in der Lage dazu zu sein, Neues zu kreieren. Die Kreation von etwas Neuem sei demnach konstitutiv zur erfolgreichen Bewältigung unerwartet eintretender, die Belastungsgrenzen des Systems übersteigender Ereignisse. Und mit 
Improvisationsfähigkeit ist genau das gemeint (Gunderson 2000: 434). Wenn Improvisationsfähigkeit im Wesentlichen als dem Menschen eigen beschrieben und verstanden wird, gilt es in realen Situationen allerdings auch zu beachten, inwiefern ein tatsächliches Improvisieren überhaupt als akzeptable Alternative zum standardmäßigen Vorgehen gesehen wird. Diese Frage stellt sich in einer rein deskriptiv-analytischen Diskussion nicht. Sie ist jedoch für Menschen, die als verantwortlich Handelnde mit den Auswirkungen gravierender, disruptiver Ereignisse konfrontiert sind von großer Bedeutung. Improvisation ist nicht mit einer Erfolgsgarantie verbunden, so dass sich bei nicht-erfolgreicher Improvisation die Frage nach der Verantwortung stellt. Denn Menschen handeln durch Improvisieren anders, als eigentlich - im Rahmen etwa geregelter Vorgehensweisen was das Betreiben kritischer Infrastrukturen angeht - vorgesehen (Longstaff 2012: 278). Inwiefern hier rechtliche Fragen eine Rolle spielen und wie ein daraus möglicherweise entstehendes Dilemma gelöst werden könnte, ist nicht Gegenstand dieser Arbeit, sollte jedoch der Vollständigkeit halber an dieser Stelle kurze Erwähnung finden. Longstaffs Ideen sind aber noch in anderer Hinsicht sehr interessant. Sie definiert die Fähigkeit zur Improvisation noch prägnanter als etwa Gunderson. Ihr zufolge handelt es sich zunächst generell um die Fähigkeit von Menschen, neue Wege zu finden, ihre Bedürfnisse zu befriedigen im Angesicht unerwartet eintretender Ereignisse, die sich nicht mithilfe etablierter Methoden bewältigen lassen. Das stimmt mit Gunderson überein. Er spricht in einer aus der Katastrophenforschung gefärbten Sicht davon, dass Menschen in der Lage dazu seien, bei Bedarf alternative Möglichkeiten zu finden, sich mit lebensnotwendigen Dingen wie Wasser, Essen und einer Behausung zu versorgen. Und dazu nutzen sie unter anderem die Fähigkeit zur Improvisation indem sie die ihnen zur Verfügung stehenden Ressourcen neu oder anders verwenden (Longstaff 2012: 268). Damit wird eine unmittelbare Verbindung zwischen Improvisationsfähigkeit und der Definition von generischer Anpassungsfähigkeit in der vorliegenden Arbeit deutlich. Zur Verfügung stehende Ressourcen neu oder anders zu verwenden in einem Fall, wenn die standardmäßige Verwendung aufgrund des Eintretens gravierender widriger Ereignisse nicht mehr möglich ist, heißt nichts anderes, als lose Ressourcen zur Realisierung eigentlich extrem unerwartbarer Prozesse an Anschlussmöglichkeiten einzusetzen, um die Systemleistung zu erhalten. Für das Resilienz-Konzept der zivilen SiFo lässt sich festhalten: Improvisationsfähigkeit ist gleichbedeutend mit generischer Anpassungsfähigkeit. Während Improvisationsfähigkeit auf Menschen als Träger hindeutet, verfügen 
komplexe adaptive Systeme an sich abstrakter über generische Anpassungsfähigkeit.

Neben der Lern- und der Improvisationsfähigkeit wird im Kontext der Resilienzforschung und im Zusammenhang mit Anpassungsfähigkeit und Flexibilität auch immer wieder von Kreativität als einem möglichen Bestandteil von bzw. einer Notwendigkeit für Resilienz gesprochen. Colten et al. sehen etwa Improvisationsfähigkeit und Kreativität als Fähigkeiten an, die zur Bewältigung von Gefahren und daraus resultierenden Katastrophen generell benötigt werden, da diese dazu tendieren, Pläne über den Haufen zu werfen und Effekte zu verursachen, mit denen im Vorhinein nicht gerechnet wurde. Als Beispiele nennen sie etwa den Fakt, dass Rettungskräfte selbst betroffen und benötigte Ressourcen nicht zugänglich sind, Kommunikationsverbindungen zusammenbrechen, Verantwortlichkeiten und Führungsaufgaben unklar sind und die zur Verfügung stehenden Informationen verwirrend oder sogar gänzlich falsch (Colten et al. 2008: 4). Strambach und Klement wiederum unterscheiden ökonomische Akteure von ökologischen Systemen anhand der Fähigkeit ersterer, „kreativ zu handeln, zu lernen und sich an veränderte Umstände anzupassen“ und erkennen genau darin ein Spezifikum ökonomischer Resilienz (Strambach/Klement 2016: 269). Ein solcher Ansatz passt inhaltlich gut zu den oben diskutierten Ideen, wonach sowohl Lernfähigkeit als auch Improvisationsfähigkeit exklusiv menschliche Fähigkeiten seien. Bezogen auf Lernfähigkeit wurde diese Annahme für das Resilienz-Konzept der zivilen SiFo verworfen. Improvisationsfähigkeit steht dagegen als synonymer Begriff für die generische Anpassungsfähigkeit von Menschen. Was Kreativität angeht, geht die vorliegende Arbeit nun davon aus, dass diese nicht exklusiv Menschen vorbehalten ist. Gerade ökologische Systeme zeichnen sich zuweilen durch eine durchaus als kreativ zu bezeichnende Verhaltensweise angesichts gravierender externer Reize aus. Insofern soll an dieser Stelle Kreativität als etwas verstanden werden, über das die Agenten in komplexen adaptiven Systemen verfügen können. Strambach und Klement geben selbst darauf einen Hinweis, wenn sie für konkrete experimentelle Untersuchungsdesigns davon sprechen „Agenten von komplexen, adaptiven Systemen als selbstständige, kreative, interagierende Akteure" modellieren zu wollen „welche ihr Verhalten auf Basis der Kognition veränderter Umfeldbedingungen anpassen“ (Strambach/Klement 2016: 270f).

Klassische Definitionen von Kreativität beinhalten immer den Aspekt der Schaffung von etwas Neuem. Originalität ist zentral wichtig für Kreativität. Originalität oder Neuheit an sich reichen jedoch nicht aus zur Definition von Kreativität. Darüber hinaus muss das Originelle oder Neue 
auch nützlich oder brauchbar sein (Runco/Jaeger 2012: 92). Prägnant und im Kontext der Resilienzforschung besonders interessant ist eine Definition von Kreativität nach Torrance. Demnach ist Kreativität „an ability to think in an original and flexible manner to solve problems and adapt to real life situations" (Metzl 2009: 113). Die Ähnlichkeit zu zentralen Begrifflichkeiten der Resilienzforschung ist frappierend. Der Psychologe Ellis Paul Torrance entwickelte auch den sogenannten „Torrance Test of Creative Thinking”, der ursprünglich vier Kategorien enthielt. Mithilfe dieser Kategorien sollte die Kreativität von Menschen gemessen werden können. Die erste Kategorie war die "fluency“, womit die Menge an sinnvollen Ideen gemeint ist, die der zu Untersuchende als Antwort auf bestimmte Situationen zu geben in der Lage ist. Die zweite Kategorie bezeichnete man als „originality“, was ein Maß für die Häufigkeit der Antworten war, also mit anderen Worten wie originell im Vergleich zu anderen der zu Untersuchende antwortet. Die dritte Kategorie „elaboration“ bezeichnete die Detailtiefe der Antworten. Und die letzte Kategorie "flexibility“ die Menge an unterscheidbaren Bereichen, in die sich die Antworten einordnen lassen. Nach Metzl deuten aktuelle Erkenntnisse aus der neurologischen Forschung darauf hin, dass vor allen Dingen Flexibilität für Kreativität entscheidend wichtig ist (Metzl 2009: 113). Beim Blick auf die vier Kategorien fallen jedenfalls direkt Ähnlichkeiten zum Resilienz-Konzept auf. Die Frage ist, wie sich Kreativität als Begriff in die bisherigen system- und komplexitätstheoretischen Überlegungen zum Resilienz-Konzept der zivilen SiFo einordnen lässt. Dazu wird Kreativität klassisch als Schaffung von etwas originellem Neuen und gleichzeitig Nützlichen verstanden. Systeme, die sich auf ihrem normalen, ungestörten Entwicklungspfad befinden, benötigen in der Regel keine derartige Eigenschaft oder Fähigkeit. Die Abfolge an Ereignissen ist erwartbar und daher jeweils ohne Weiteres aus vorangegangenen Ereignissen ableitbar. Sobald jedoch ein extrem unwahrscheinlicher externer (oder interner) Reiz auftritt, benötigt das System dringend die Fähigkeit zur Schaffung originell nützlicher Anschlussmöglichkeiten. Oder anders gesprochen: die Fähigkeit zur Realisierung eigentlich extrem unerwartbarer Prozesse an Anschlussmöglichkeiten und den Übergang auf einen dann wieder erwartbaren Entwicklungspfad. In einem derartig systemtheoretischen Verständnis unterscheidet sich Kreativität nicht von generischer Anpassungsfähigkeit - und ebenfalls nicht von der menschlichen Improvisationsfähigkeit. Insofern muss der Begriff im Rahmen des Resilienz-Konzepts für die zivile SiFo nicht eigenständig genutzt werden. Allerdings wird Kreativität, so wie sie hier verstanden wird, immer mitgedacht, wenn es um generische Anpassungsfähigkeit geht. Deshalb gilt: 
Kreativität ist die Schaffung von etwas originellem Nützlichen und im Kontext eines systemtheoretischen Resilienz-Konzepts gleichbedeutend mit der Realisierung eines eigentlich extrem unerwartbaren Prozesses an Anschlussmöglichkeiten oder anders gesagt mit generischer Anpassungsfähigkeit.

Zusammenfassend ergeben sich für ein eigenständiges Resilienz-Konzept der zivilen Sicherheitsforschung aus der Diskussion des Zusammenhangs zwischen Resilienz und Flexibilität folgende Annahmen:

- Flexibilität meint nicht die Fähigkeit von (einfachen) Systemen, sich bei Lasteinwirkungen durch spezifisch definierte Belastungen elastisch und/oder plastisch zu verformen.

- Flexibilität bedeutet das Vorhandensein - nicht notwendigerweise die Realisierung - eigentlich extrem unerwartbarer Prozesse an Anschlussmöglichkeiten, die dem System das Einschwenken auf einen neuen, leistungserhaltenden Entwicklungspfad ermöglichen.

- Die generische Anpassungsfähigkeit komplexer adaptiver Systeme setzt sich zusammen aus ihrer Flexibilität, also dem Vorhandensein eigentlich extrem unerwartbarer Prozesse an Anschlussmöglichkeiten, und der Verfügbarkeit loser Ressourcen, also spezialisierter Agenten sowie Generalisten, die für das Funktionieren des Systems auf dem ursprünglichen Entwicklungspfad nicht notwendig sind.

- Je flexibler komplexe adaptive Systeme sind, desto größer ist ihre Resilienz.

- Lernfähigkeit besteht darin, Wissen über die wahrscheinlichen Auswirkungen der Realisierung der verschiedenen zur Verfügung stehenden Prozesse an Anschlussmöglichkeiten erlangen zu können und ist damit ein Teil der Anpassungsfähigkeit komplexer adaptiver Systeme. Deshalb ist Lernfähigkeit eine notwendige Grundvoraussetzung für Resilienz.

- Improvisationsfähigkeit ist gleichbedeutend mit generischer Anpassungsfähigkeit. Während Improvisationsfähigkeit auf Menschen als Träger hindeutet, verfügen komplexe adaptive Systeme an sich abstrakter über generische Anpassungsfähigkeit.

- Kreativität ist die Schaffung von etwas originellem Nützlichen und im Kontext eines systemtheoretischen Resilienz-Konzepts gleichbedeutend mit der Realisierung eines eigentlich extrem unerwartbaren Prozesses an Anschlussmöglichkeiten oder anders gesagt mit generischer Anpassungsfähigkeit. 


\section{Eigenständiges Resilienz-Konzept der zivilen Sicherheitsforschung}

\subsection{Zusammenführung der konzeptionellen Zugänge}

Resilienz ist ein Modewort. Das hat die ausführliche Analyse des Konzepts mithilfe konzeptioneller Zugänge gezeigt. Gleichzeitig führt das aber nicht dazu, dass Resilienz nicht zu einem fundierten wissenschaftlichen Konzept ausgearbeitet werden kann. Auch das wurde im vorangegangenen Kapitel mehr als deutlich. Resilienz ist eben mehr als „alter Wein in neuen Schläuchen“, wie von manchem behauptet. Warum und inwiefern Resilienz tatsächlich mehr ist, sollte gerade im Hinblick auf aktuelle Fragestellungen der zivilen Sicherheitsforschung beantwortet werden können. Insofern ist die Aufgabe, ein eigenständiges Resilienz-Konzept der zivilen SiFo zu entwickeln, kein Selbstzweck. Vielmehr kann sie als konsequente Adressierung der Fragestellungen verstanden werden, mit der sich die zivile SiFo seit einigen Jahren in immer stärkerem Maße konfrontiert sieht. Nämlich danach, welche Antworten die Forschung auf Herausforderungen wie zunehmende Komplexität, stärkere Vernetzung und größere Verwundbarkeit unserer Gesellschaften geben kann, wenn bisherige Strategien wie etwa klassisches Risikomanagement, das Vorsorge-Prinzip oder die Maximierung physischer Robustheit scheinbar nicht länger ausreichen. Gegeben seine Bedeutung in den unterschiedlichsten Disziplinen, werden diese Fragen in zunehmender Häufigkeit mit dem Modewort „Resilienz“ beantwortet. Lukesch formuliert dazu, wenn auch nicht im Kontext der zivilen SiFo, treffend: „Auf der Suche nach Konzepten und Modellen, die Widerstandsfähigkeit und Wandelbarkeit plausibel in sich vereinigen, bietet sich der Begriff der Resilienz als Leitstern an" (Lukesch 2016: 296, eigene Hervorhebung). Es reicht aber nicht aus, Resilienz als Leitstern oder auch als politische Zielstellung zu formulieren. Vielmehr muss der Begriff Resilienz innerhalb der zivilen SiFo konzeptionell fundiert werden.

Dazu wurde in der vorliegenden Arbeit zunächst ein klassischer, disziplinärer Zugang zu Resilienz gewählt und mit dessen Hilfe insgesamt zehn Begrifflichkeiten identifiziert, die disziplinübergreifend für Resilienz von Bedeutung sind. Um dem Anspruch an Resilienz, ein interdisziplinäres Brückenkonzept sein zu können, gerecht zu werden, wurde dann im nächsten Schritt der disziplinäre Zugang vollständig aufgelöst und 
durch einen Zugang über die identifizierten Begrifflichkeiten, die selbst jeweils eigenständige Konzepte sind, ersetzt. Mittels dieser konzeptionellen Zugänge konnte Resilienz als Konzept für die zivile SiFo präzisiert und entscheidende Aspekte identifiziert werden. Dabei hat sich gezeigt, dass es sich bei Resilienz selbst um ein komplexes Konzept handelt. Komplex in dem Sinne, dass es aus vielen Bestandteilen besteht, die sich aufeinander beziehen und nur als Gesamtkonstrukt als Resilienz wirkmächtig werden können. Aus diesem Grund scheint es auch nicht sinnvoll, als Ergebnis der ausführlichen Analyse des Konzepts Resilienz über disziplinäre und vor allen Dingen konzeptionelle Zugänge eine weitere, knappe und scheinbar prägnante Definition von Resilienz anzubieten. Derartige Definitionen, als Beispiel sei hier erneut auf Thoma 2014 verwiesen (Scharte et al. 2014: 17), können aus Sicht der vorliegenden Arbeit nicht mehr als ein Ausgangspunkt für eine detailliertere Beschäftigung mit Resilienz sein. Sie verkürzen das Konzept auf unmittelbar einsichtige Merkmale und erfüllen so eine wichtige kommunikative Funktion auch und gerade über die Wissenschaft hinaus. Allerdings folgt der inhaltlichen Verkürzung nicht selten eine Verzerrung des Konzepts, weil entscheidende Bestandteile notwendigerweise unter den Tisch fallen. Zu enge Definitionen können in dieser Hinsicht kontraproduktiv für die wissenschaftliche Diskussion sein (Walker et al. 2004).

Die Ergebnisse der Analyse mithilfe der konzeptionellen Zugänge werden daher im Folgenden bewusst breit präsentiert unter Inkaufnahme einer eventuellen Unübersichtlichkeit. Erst durch diese breite Präsentation werden die eigentlichen Charakteristika und vor allem auch Spezifika des Konzepts sichtbar. Durch jeden der zehn unterschiedlichen konzeptionellen Zugänge wurden spezifische Aspekte von Resilienz erkennbar, die für das Resilienz-Konzept der zivilen SiFo relevant sind. Zunächst war das eine Untersuchung von Resilienz anhand vier stärker normativ geprägter Konzepte. Einige Ergebnisse dieser Untersuchung, etwa das Resilienz ein Modewort und kein alter Wein in neuen Schläuchen ist, wurden in diesem Kapitel bereits dargestellt. Darüber hinaus konnten sowohl zur Frage nach dem Zusammenhang zwischen Resilienz und Neoliberalismus als auch Freiheit sowie Vertrauen jeweils Annahmen und Bedingungen identifiziert werden, die notwendiger Bestandteil eines Resilienz-Konzepts der zivilen SiFo sein müssen. Einige weitere Annahmen konnten aus einer näheren Untersuchung des Zusammenhangs zwischen Resilienz und Terrorismus als eine Art Spezialfall der Frage nach Resilienz und Freiheit gewonnen werden. Deskriptiv-analytisch erfolgte dann insbesondere eine Einordnung von Resilienz in eine übergreifende theoretische Denkschule. Hier konn- 
ten durch die Nutzung systemtheoretischer Annahmen Luhmanns sowie der Theorie komplexer adaptiver Systeme entscheidende Grundbestandteile des Resilienz-Konzepts entwickelt werden. Im nächsten Schritt wurden sowohl Unsicherheit und Vulnerabilität als Entwicklungen bzw. Umweltbedingungen, die eine Notwendigkeit für Resilienz motivieren können, als auch Anpassungsfähigkeit und Flexibilität als für Resilienz möglicherweise zentrale Eigenschaften, unter Zuhilfenahme system- und komplexitätstheoretischer Überlegungen diskutiert. Gerade durch die Verknüpfung bekannter Annahmen aus unterschiedlichen Disziplinen mit der im Rahmen der vorliegenden Arbeit erstmals vorgenommenen system- und komplexitätstheoretischen Einordnung der verschiedenen, für Resilienz relevanten konzeptionellen Zugänge, konnten neue und wertvolle Erkenntnisse gewonnen werden. Diese bauen zudem konsequent aufeinander auf, so dass ein aus deskriptiv-analytischer Sicht so umfassendes wie konsistentes Resilienz-Konzept entwickelt und damit der erste Teil der wissenschaftlichen Fragestellung der vorliegenden Arbeit beantwortet werden konnte.

Um als Grundlage für die Entwicklung von Hypothesen für Resilience Engineering dienen zu können, sollen die Erkenntnisse des vorangegangenen Kapitels, die am Ende der jeweiligen Unterkapitel in Form prägnanter Merksätze zusammengefasst wurden, im Folgenden in einer systematischen Ordnung aufgeführt werden. Dabei wird unterschieden zwischen Begrifflichkeiten, die in der vorliegenden Arbeit im Hinblick auf ihre Bedeutung für das Resilienz-Konzept der zivilen SiFo definiert wurden, auf der einen Seite und den eigentlichen Bestandteilen des Konzepts auf der anderen Seite. Erstere werden im nächsten Unterkapitel vorgestellt. Die Definitionen erheben keinesfalls den Anspruch umfassend zu sein und jeweils alle Aspekte der genutzten Begrifflichkeiten abzubilden. Es geht vielmehr darum, die Begrifflichkeiten so zu fassen, dass sie zur Präzisierung des Resilienz-Konzepts beitragen können. Die eigentlichen Bestandteile des Konzepts werden dann im darauffolgenden Unterkapitel dargestellt. Die Darstellung erfolgt unter Zuhilfenahme von Leitfragen, etwa „Worin besteht Resilienz aus system- und komplexitätstheoretischer Sicht?"“ oder „Wann wird Resilienz benötigt?" Zudem werden die einzelnen Annahmen im Hinblick auf ihre Gesamtkonsistenz mit den anderen Annahmen verglichen und wo es notwendig erscheint, entsprechend aktualisiert bzw. neu zugeordnet. Den Abschluss des Kapitels bildet dann eine kurze Diskussion über Grenzen des Resilienz-Konzepts der zivilen SiFo. Diese bezieht sich zum einen auf generelle Schwierigkeiten, die das Resilienz-Konzept mit sich bringt und die auch durch eine derart detaillierte Weiter- und Neu-Entwicklung des Konzepts, wie sie die vorliegende Arbeit 
leistet, nicht gänzlich zu vermeiden sind, etwa die Frage nach der Möglichkeit, sowohl resilient als auch effizient zu sein. Zum anderen bezieht sie sich aber auch auf die Grenzen des spezifischen Resilienz-Konzepts der zivilen SiFo und vor allen Dingen darauf, wie dieses sich im Verhältnis zu anderen Vorgehensweisen darstellt, etwa Methoden des klassischen Risikomanagements.

\subsection{Definition relevanter Begrifflichkeiten für das Resilienz-Konzept}

Die intensive Arbeit mit unterschiedlichen Konzeptionen von Resilienz hat gezeigt, dass eine umfassende Entwicklung eines neuen, eigenständigen Resilienz-Konzepts der zivilen SiFo nur dann gelingen kann, wenn die relevanten, mit Resilienz in enger Verbindung stehenden Begrifflichkeiten definiert werden. Dabei kann der Anspruch der vorliegenden Arbeit nicht darin bestehen, für jeden der folgenden Begriffe ebenfalls eine konzeptionell-wissenschaftlich erschöpfende Diskussion über geeignete Definitionen zu führen. Vielmehr geht es darum die Definitionen im Kontext des Resilienz-Konzepts der zivilen SiFo so zu verorten, dass damit insgesamt das Konzept an sich verständlich wird. Die folgenden Definitionen entstammen teilweise der Literatur, zum Teil sind sie aber auch direktes Ergebnis der konzeptionellen Analyse des vorangegangenen Kapitels. Zudem wird der Begriff „Definition“ hier relativ weit verstanden und umfasst beispielsweise auch Entwicklungen und Annahmen, die sich aus der Analyse ergeben haben und die von Bedeutung sind, um das Resilienz-Konzept der zivilen $\mathrm{SiFo}$ zu verstehen.

Bevor die einzelnen Definitionen nun vorgestellt werden, sollen noch einige Hinweise darauf erfolgen, wie mit ihnen in Bezug auf das Gesamtkonzept umzugehen ist. Demnach sind diese Definitionen zwar vorgelagert, aber nichtsdestoweniger Teil des eigenständigen Resilienz-Konzepts der zivilen SiFo. Ohne diesen Kontext lässt sich Resilienz nicht verstehen. Ein augenfälliges Beispiel dafür sind die Definitionen der Begriffe „Anpassungsfähigkeit“ und „generische Anpassungsfähigkeit“. Erstere wurde aus der Literatur entnommen und dann mithilfe der system- und komplexitätstheoretischen Überlegungen der vorliegenden Arbeit weiterentwickelt. Letztere basiert insofern auf ersterer, ist aber selbst eine Art „Übersetzung“ sowie Neuentwicklung und damit zentrale Erkenntnis der vorliegenden Arbeit. Ohne den Aspekt der generischen Anpassungsfähigkeit ist Resilienz nicht denkbar. Gleichzeitig ist Resilienz nicht gleichbedeutend mit 
generischer Anpassungsfähigkeit, da noch eine ganze Reihe weiterer Elemente beachtet werden müssen, um von Resilienz sprechen zu können.

Zudem muss an dieser Stelle bereits darauf verwiesen werden, dass die hier dargestellten Definitionen so auch in das nächste Kapitel übernommen werden, in dem es final darum gehen wird, aus dem eigenständigen Resilienz-Konzept der zivilen SiFo heraus Hypothesen für Resilience Engineering zu entwickeln. Dieses Vorgehen ist einerseits hilfreich, andererseits aber durchaus als nicht unkritisch zu werten. Nicht unkritisch ist es deshalb, weil in den Ingenieurwissenschaften, die mit den Hypothesen für Resilience Engineering adressiert werden sollen, zum Teil ein anderes Verständnis der im Folgenden definierten Begrifflichkeiten vorherrscht. Durch die explizite Verknüpfung der Hypothesen mit den Definitionen überwiegt jedoch die Nützlichkeit des Vorgehens, da die Ingenieurwissenschaften, wenn sie sich dem Verständnis von Resilience Engineering der vorliegenden Arbeit nähern wollen, selbst auf diese Definitionen schauen und sie - wo nötig - anpassen und „übersetzen“ können.

Für das eigenständige Resilienz-Konzept der zivilen SiFo sind die folgenden Begrifflichkeiten von definitorischer Bedeutung: 
Tabelle 1: Relevante Begrifflichkeiten für das Resilienz-Konzept der zivilen SiFo

Begriffe der system- und komplexitätstheoretischen Analyse

Begriff

System

\section{Definition}

Menge an kausal miteinander in Beziehung stehenden Elementen. Systeme haben eine eindeutige Grenze. Alle Elemente außerhalb des Systems sind für das System (Um-)Welt.

Systeme befinden sich auf einem sogenannten „Entwicklungspfad“, der den Zustand des Systems zu jedem beliebigen Zeitpunkt beschreibt. Zu jedem Zeitpunkt gibt es eine Reihe von Prozessen an „Anschlussmöglichkeiten“, was als nächstes im System passieren könnte. Von diesen wird jeweils genau ein Prozess realisiert. Ein Entwicklungspfad besteht aus der Realisierung der jeweils wahrscheinlichsten Prozesse an Anschlussmöglichkeiten. Systeme können von systemexternen wie -internen Reizen (im Kontext der zivilen SiFo zumeist als „Ereignis“ bezeichnet) getroffen werden, die eine Änderung des Entwicklungspfads nötig machen, indem sie die Wahrscheinlichkeiten der Prozesse an Anschlussmöglichkeiten verändern. ${ }^{38}$

38 Hier erfolgt bewusst eine Verknüpfung unterschiedlicher systemtheoretischer Ansätze. Zum einen werden Luhmanns Ideen genutzt, wonach Systeme aus Ereignissen bestehen, auf die stets neue Ereignisse folgen. Zum anderen der eher klassische Ansatz verwendet, wonach sie aus Elementen bestehen. Ersterer wird nur insoweit genutzt, als er als „Inspiration“ für den prozessualen, dynamischen Charakter von Systemen zu verstehen ist. Damit wird es möglich, Systeme auf einem Entwicklungspfad zu verorten und ihnen eine definierte Menge an "Anschlussmöglichkeiten“ zu geben. Beides erlaubt eine systemtheoretische Einordnung von Resilienz, ohne die komplexitätstheoretische Erweiterung zu verunmöglichen. 
Begriffe der system- und komplexitätstheoretischen Analyse

\section{Begriff Definition}

Einfache Systeme Ein einfaches System besteht aus Elementen, die eine eindeutige kausale Beziehung zueinander haben. Einfache Systeme zeichnen sich durch linear-deterministisches Verhalten aus. Ihre Funktionsweise kann reduktionistisch, also mit Blick auf die einzelnen Elemente des Systems erklärt werden.

Einfache Systeme verfügen über eindeutige Belastungsgrenzen. Einfache Systeme verfügen nicht über Anpassungsfähigkeit da ihnen die dafür notwendigen Elemente - adaptive Agenten - und Mechanismen - neue Signalverarbeitungsmuster durch Neuanordnung nützlicher Bausteine zu kreieren sowie in Interaktion mit anderen Agenten zu treten und so Feedback und Rückkopplungen zu produzieren - fehlen.

Komplexe adapti- Komplexe adaptive Systeme bestehen aus heterogenen, ve Systeme miteinander und mit der Systemumwelt interagierenden und anpassungsfähigen Agenten. Die vernetzte Interaktion der Agenten führt zu sogenannten „Feedbackschleifen“. Damit ist eine ständige Anpassung des Verhaltens eines Agenten an das wahrgenommene Verhalten anderer, die wiederum auf die Veränderungen des Verhaltens des ersten Agenten reagieren, usw., gemeint. Darüber hinaus reagieren Agenten - und damit das System - auch auf Veränderungen in der Systemumwelt.

Bedingt durch die Anpassungsfähigkeit der Agenten und die Existenz von Feedbackschleifen weisen komplexe adaptive Systeme Eigenschaften auf, die sich nicht durch Betrachtung der einzelnen Agenten erschließen, sondern erst auf Systemebene wirkmächtig werden. Diese werden als „emergente“ Eigenschaften bezeichnet. 
Begriffe der system- und komplexitätstheoretischen Analyse Begriff Definition

Komplexität, wei- Agenten haben Ziele, die sie in beschränkt rationaler tere Annahmen Weise zu erreichen versuchen. Bedingt durch endliche Ressourcen lohnt sich eine Spezialisierung. Spezialisierung macht Vernetzung mit anderen Agenten notwendig. Je größer die Vernetzung, desto mehr Feedbackschleifen gibt es und desto größer wird die Komplexität des Systems. Damit werden komplexe adaptive System über die Zeit komplexer.

Die Komplexität von Systemen wird als Eigenkomplexität bezeichnet. Auch die Umwelt komplexer Systeme besteht aus komplexen adaptiven Systemen. Hier ergibt es Sinn, von Umweltkomplexität zu sprechen. Auch diese steigt über die Zeit.

Systeme in der zi- Die für die zivile SiFo relevanten sozio-technischen vilen $\mathrm{SiFo}$ Systeme sind komplexe adaptive Systeme, weil sie die dafür relevanten Elemente - interagierende, heterogene, anpassungsfähige Agenten - und Eigenschaften Emergenz, Feedbackschleifen, Selbstorganisation - aufweisen.

Der Theorie komplexer adaptiver Systeme folgend, nimmt der Grad an Vernetzung und die Komplexität der für die zivile SiFo relevanten sozio-technischen Systeme kontinuierlich weiter zu. Dies gilt auch für die Umwelt dieser Systeme. 
Begriffe der system- und komplexitätstheoretischen Analyse

\begin{tabular}{|c|c|}
\hline Begriff & Definition \\
\hline $\begin{array}{l}\text { Unsicherheit } \\
\text { Risiko } \\
\text { Ungewissheit } \\
\text { Echte Unsicher- } \\
\text { heit } \\
\text { Unerwartete Er- } \\
\text { eignisse }\end{array}$ & $\begin{array}{l}\text { In den für die zivile SiFo relevanten komplexen adap- } \\
\text { tiven sozio-technischen Systemen sind Ereignisse, ihr } \\
\text { Eintreten und ihre Auswirkungen a priori unsicher. } \\
\text { Es gibt drei Arten von Unsicherheit. Die erste Art kann } \\
\text { als Risiko bezeichnet werden. Die Wahrscheinlichkeit } \\
\text { für das Auftreten von Ereignissen, die in diese Katego- } \\
\text { rie fallen, ist ebenso bekannt wie ihre Auswirkungen. } \\
\text { Die zweite Art kann als Ungewissheit bezeichnet wer- } \\
\text { den. Bei ungewissen Ereignissen sind die möglichen } \\
\text { Auswirkungen bekannt aber nicht die Wahrscheinlich- } \\
\text { keit, mit der das Ereignis eintritt. } \\
\text { Die dritte Art ist echte Unsicherheit. Echt unsichere Er- } \\
\text { eignisse sind nicht nur ihrer Wahrscheinlichkeit nach, } \\
\text { sondern auch im Hinblick auf ihre Auswirkungen un- } \\
\text { bekannt. } \\
\text { Ungewisse und unsichere können zusammenfassend } \\
\text { als unerwartete Ereignisse bezeichnet werden. }\end{array}$ \\
\hline
\end{tabular}

Vulnerabilität Vulnerabilität bezeichnet die physische, soziale, ökonomische und umweltbezogene Ausgesetztheit und Anfälligkeit eines Systems gegenüber widrigen Ereignissen. Vulnerabilität ist damit ein Ausmaß für Risikofaktoren, denen ein System ausgesetzt ist. Bewältigungskapazitäten und Schutzfaktoren sind nicht Teil von Vulnerabilität.

Vulnerabilität ist ein systemtheoretisches Konzept. Je höher die Vulnerabilität eines Systems ist, desto stärker weicht der von einem unwahrscheinlichen externen Reiz verursachte, unerwartbare neue Entwicklungspfad initial vom ohne externen Reiz eigentlich erwartbaren Entwicklungspfad ab.

Vulnerabilität ist ein multidimensionales Konzept. Deshalb ist a priori unklar, ob die in komplexen adaptiven Systemen inhärent steigende Eigenkomplexität auch zu steigender Vulnerabilität führt. 
Begriffe der system- und komplexitätstheoretischen Analyse

\begin{tabular}{|c|c|}
\hline egriff & tion \\
\hline $\begin{array}{l}\text { Anpassungsfähig- } \\
\text { keit }\end{array}$ & $\begin{array}{l}\text { Anpassungsfähigkeit meint das Vermögen, sich selbst } \\
\text { angesichts von Veränderungen zielgerichtet weiterzu- } \\
\text { entwickeln, um die eigene Weiterexistenz sicherzustel- } \\
\text { len. }\end{array}$ \\
\hline $\begin{array}{l}\text { Spezialisierte An- } \\
\text { passungsfähigkeit }\end{array}$ & $\begin{array}{l}\text { Spezialisierte Anpassungsfähigkeit liegt vor, wenn ein } \\
\text { komplexes adaptives System sich zielgerichtet an inkre- } \\
\text { mentelle, geringfügige und erwartete Veränderungen } \\
\text { anzupassen in der Lage ist. }\end{array}$ \\
\hline $\begin{array}{l}\text { enerische An- } \\
\text { assungsfähigkeit }\end{array}$ & $\begin{array}{l}\text { Generische Anpassungsfähigkeit liegt vor, wenn ein } \\
\text { komplexes adaptives System sich zielgerichtet an gra- } \\
\text { vierende, disruptive und unerwartete Veränderungen } \\
\text { anzupassen in der Lage ist. } \\
\text { Die generische Anpassungsfähigkeit komplexer adapti- } \\
\text { ver Systeme setzt sich zusammen aus ihrer Flexibili- } \\
\text { tät, also dem Vorhandensein eigentlich extrem uner- } \\
\text { wartbarer Prozesse an Anschlussmöglichkeiten, und } \\
\text { der Verfügbarkeit loser Ressourcen, also spezialisierter } \\
\text { Agenten sowie Generalisten, die für das Funktionieren } \\
\text { des Systems auf dem ursprünglichen Entwicklungspfad } \\
\text { nicht notwendig sind. } \\
\text { Generische Anpassungsfähigkeit ermöglicht komple- } \\
\text { xen adaptiven Systemen sowohl eine unmittelbare Be- } \\
\text { wältigung der Folgen extrem unwahrscheinlicher oder } \\
\text { unerwarteter, die Belastungsgrenzen des Systems über- } \\
\text { steigender Ereignisse als auch eine langfristig erfolg- } \\
\text { reiche Weiterentwicklung in einer veränderten System- } \\
\text { Umwelt. }\end{array}$ \\
\hline $\begin{array}{l}\text { Transformations- } \\
\text { fähigkeit }\end{array}$ & $\begin{array}{l}\text { Transformationsfähigkeit bezeichnet das Vermögen ei- } \\
\text { nes Systems, seine eigene Identität, Funktionalität und } \\
\text { die bestimmenden Beziehungsmuster seiner Elemente } \\
\text { angesichts von Veränderungen zielgerichtet so weiter- } \\
\text { zuentwickeln, dass ein neues System entsteht. } \\
\text { In komplexen adaptiven Systemen sind Anpassungs- } \\
\text { und Transformationsfähigkeit kontinuierlich miteinan- } \\
\text { der verknüpft und bezeichnen unterschiedlich ausge- } \\
\text { prägte Arten zielgerichteter Weiterentwicklung ange- } \\
\text { sichts von Veränderungen. }\end{array}$ \\
\hline
\end{tabular}


Begriffe der system- und komplexitätstheoretischen Analyse

\begin{tabular}{ll} 
Begriff & Definition \\
\hline Flexibilität & Flexibilität bedeutet das Vorhandensein - nicht not- \\
& wendigerweise die Realisierung - eigentlich extrem un- \\
erwartbarer Prozesse an Anschlussmöglichkeiten, die & dem System das Einschwenken auf einen neuen, leis- \\
& tungserhaltenden Entwicklungspfad ermöglichen. \\
& Flexibilität meint nicht die Fähigkeit von (einfachen) \\
& Systemen, sich bei Lasteinwirkungen durch spezifisch \\
& definierte Belastungen elastisch und/oder plastisch zu \\
& verformen. \\
\hline Lernfähigkeit & Lernfähigkeit besteht darin, Wissen über die wahr- \\
& scheinlichen Auswirkungen der Realisierung der ver- \\
& schiedenen zur Verfügung stehenden Prozesse an An- \\
& schlussmöglichkeiten erlangen zu können und ist da- \\
& mit ein Teil der Anpassungsfähigkeit komplexer adapti- \\
& ver Systeme. \\
\hline Kreativität & Kreativität ist die Schaffung von etwas originellem \\
& Nützlichen und im Kontext eines systemtheoretischen \\
& Resilienz-Konzepts gleichbedeutend mit der Realisie- \\
& rung eines eigentlich extrem unerwartbaren Prozesses \\
an Anschlussmöglichkeiten oder anders gesagt mit ge- & nerischer Anpassungsfähigkeit. \\
\hline Improvisations- & Improvisationsfähigkeit ist gleichbedeutend mit generi- \\
fähigkeit & scher Anpassungsfähigkeit. Während Improvisationsfä- \\
higkeit auf Menschen als Träger hindeutet, verfügen \\
komplexe adaptive Systeme an sich abstrakter über ge- \\
nerische Anpassungsfähigkeit.
\end{tabular}


Begriffe der normativen Analyse

Begriff

Definition

Neoliberalismus Neoliberalismus lässt sich - vereinfacht ausgedrückt und unter bewusster Verkürzung der wissenschaftlichen Diskussion - mithilfe folgender Annahmen beschreiben: Aufgrund der Komplexität von Systemen sind diese grundsätzlich nicht steuerbar, weshalb auf regulierende Eingriffe vonseiten des Staates verzichtet werden sollte. Allerdings existieren zugrundeliegende Mechanismen, die dazu führen, dass sich Systeme selbst regulieren. Individuen innerhalb dieser Systeme sind damit selbst für sich verantwortlich, auch im Fall des Eintretens gravierender Störungen.

\begin{tabular}{|c|c|}
\hline Freiheit & $\begin{array}{l}\text { In der zivilen SiFo bezieht sich der Begriff der Freiheit } \\
\text { laut BMBF auf den „freiheitlichen Lebensstil» in den } \\
\text { Gesellschaften, deren Schutz Ziel der zivilen SiFo ist. } \\
\text { Daher wird der Begriff in der vorliegenden Arbeit be- } \\
\text { wusst breit verstanden und umfasst prinzipiell die Auf- } \\
\text { rechterhaltung der Grund- bzw. Menschenrechte und } \\
\text { der liberalen und rechtsstaatlichen Demokratie. }\end{array}$ \\
\hline Terrorismus & $\begin{array}{l}\text { Straftaten, namentlich auch gegen Zivilpersonen, die } \\
\text { mit der Absicht begangen werden, den Tod oder } \\
\text { schwere Körperverletzungen zu verursachen, oder Gei- } \\
\text { selnahmen, die mit dem Ziel begangen werden, die } \\
\text { ganze Bevölkerung, eine Gruppe von Personen oder } \\
\text { einzelne Personen in Angst und Schrecken zu ver- } \\
\text { setzen, eine Bevölkerung einzuschüchtern oder eine } \\
\text { Regierung oder eine internationale Organisation zu } \\
\text { einem Tun oder Unterlassen zu nötigen. }\end{array}$ \\
\hline Vertrauen & $\begin{array}{l}\text { Vertrauen ist ein Mechanismus zur Reduktion von } \\
\text { Komplexität, der darin besteht, die Auswahl von Hand- } \\
\text { lungsentscheidungen in der Gegenwart aufgrund gene- } \\
\text { ralisierter Erwartungen über die gegenwärtige Zukunft } \\
\text { zu treffen. Generalisierte Erwartungen konstituieren } \\
\text { sich mithilfe ähnlicher Erfahrungen aus der Vergan- } \\
\text { genheit. }\end{array}$ \\
\hline
\end{tabular}


Begriffe der normativen Analyse

Begriff Definition

Systemvertrauen Systemvertrauen besteht im Vertrauen darin, dass das

betreffende System seine Funktion, die in einer spezi-

fischen Form der Reduktion von Umweltkomplexität

besteht, zuverlässig und ohne größere Störungen erfüllt.

\subsection{Bestandteile des Resilienz-Konzepts der zivilen Sicherheitsforschung}

$\mathrm{Zu}$ Beginn der vorliegenden Arbeit wurde Resilienz als „ordinary magic“ bezeichnet, verbunden mit dem Hinweis darauf, dass es - dank Resilienz manchmal besser kommt, als man denkt (siehe 1.1). Resilienz ist deshalb aber nicht als vollständige Immunität betroffener Systeme gegenüber disruptiven Ereignissen zu verstehen. Es geht immer darum, gegenüber derartigen Ereignissen relativ besser gewappnet zu sein, als es ohne Resilienz der Fall wäre (Duit et al. 2010: 3). Diese sehr grundsätzlichen Annahmen, die übergreifend für prinzipiell alle Forscher gelten, die sich mit Resilienz beschäftigen, bildeten den Ausgangspunkt der ausführlichen normativen wie auch deskriptiv-analytischen Analyse des Resilienz-Konzepts mithilfe disziplinärer und dann vor allen Dingen konzeptioneller Zugänge. Deren Ergebnisse teilen sich zum einen in eine Definition relevanter Begrifflichkeiten, wie sie im vorangegangenen Unterkapitel dargestellt wurden. Und zum anderen in die eigentlichen Bestandteile des Resilienz-Konzepts der zivilen Sicherheitsforschung, die in diesem Unterkapitel präsentiert werden. Um die unterschiedlichen Bestandteile, die sich aus der Analyse über konzeptionelle Zugänge ergeben haben, nicht unverbunden nebeneinander stehen zu lassen, werden die entwickelten Annahmen und Ideen anhand sogenannter „Leitfragen“ geordnet. Auf diese Weise wird erkennbar, wie die Erkenntnisse aus den unterschiedlichen konzeptionellen Zugängen zusammenhängen.

Die insgesamt sieben Leitfragen drehen sich zum einen ganz generell um die Frage, um was für eine Art Konzept es sich bei Resilienz handelt. Dann folgen jeweils drei Leitfragen zur Clusterung der Erkenntnisse aus der system- und komplexitätstheoretischen Analyse sowie drei Leitfragen für die Erkenntnisse der normativen Analyse. Die sieben Leitfragen lauten:

- Was für eine Art Konzept ist Resilienz?

- Wann wird Resilienz benötigt? 
- Welche Bedingungen sind aus system- und komplexitätstheoretischer Sicht notwendig für Resilienz?

- Worin besteht Resilienz aus system- und komplexitätstheoretischer Sicht?

- Worauf gilt es bei Resilienz im Rahmen der zivilen SiFo aus normativer Sicht zu achten?

- Worin besteht Resilienz aus normativer Sicht?

- Worin besteht Resilienz aus normativer Sicht nicht?

Die anhand dieser Leitfragen sortierten Ergebnisse beziehen sich auch da, wo es nicht explizit erwähnt wird, auf den Gegenstandsbereich der zivilen SiFo. Es steht zwar zu vermuten, dass ein großer Teil des Konzepts auch auf andere Forschungsbereiche übertragbar wäre. Allerdings steht diese Übertragbarkeit nicht im Fokus des Interesses dieser Arbeit. Sie wurde nicht ausdrücklich geprüft und kann daher nicht ohne weiteres angenommen werden. Eine Adaption durch andere Disziplinen könnte sich aber als sinnvoll erweisen, auch und gerade dann, wenn als Ergebnis eine NichtÜbertragbarkeit großer Teile des Konzepts stehen sollte.

Das eigenständige Resilienz-Konzept der zivilen Sicherheitsforschung besteht demzufolge aus folgenden Annahmen und Erkenntnissen:

Was für eine Art Konzept ist Resilienz?

- Resilienz ist ein systemtheoretisches Konzept.

- Sozio-technische Systeme sind die Träger von Resilienz.

- Resilienz ist eine Nicht-Selbstverständlichkeit, sie ist nicht erwartbar.

- Resilienz ist nicht das Gegenteil von Vulnerabilität. Die beiden Konzepte sind unabhängig voneinander. Größere Resilienz führt nicht notwendigerweise zu im Zeitverlauf sinkender Vulnerabilität.

- Resilienz ist kein neoliberales Paradigma.

- Resilienz ist nicht notwendigerweise normativ wünschenswert.

Wann wird Resilienz benötigt?

- Das Vorhandensein oder Nicht-Vorhandensein von Resilienz lässt sich nur beobachten, wenn Systeme von einem disruptiven, in der Systemumwelt verorteten, gravierenden und häufig extrem unwahrscheinlichen Reiz (bzw. Ereignis) getroffen werden. Der Theorie komplexer adaptiver Systeme folgend, wird die Welt immer komplexer. Je komplexer die Welt, desto wahrscheinlicher wird das Auftreten eigentlich extrem unwahrscheinlicher, disruptiver Ereignisse. Die steigende Komplexität der Welt macht deshalb Resilienz als Eigenschaft komplexer adaptiver Systeme immer notwendiger. 
- Neben Ereignissen, die aus der Systemumwelt resultieren, müssen komplexe adaptive Systeme auch mit dem Auftreten unsicherer interner, durch die Entscheidungen von Agenten ausgelöster, von diesen unintendierter oder intendierter Störungen umgehen können. Die Wahrscheinlichkeit des Auftretens solcher Störungen sowie ihre potentiellen Auswirkungen steigen mit der Eigenkomplexität der Systeme. Denn zum einen können Agenten die Konsequenzen ihrer eigenen Handlungen mit zunehmender Komplexität schlechter überblicken, was die Wahrscheinlichkeit für Unfälle steigert. Zudem steigt mit der Diversität des Systems die Wahrscheinlichkeit, dass Agenten auftreten, deren Ziele konträr zu denen des Systems laufen. Zum anderen erhöht sich die Wahrscheinlichkeit für das Auftreten von Kaskadeneffekten, die beim Eintreten widriger Ereignisse zu großflächigen und häufig langfristigen Schäden am System führen. Um diese verhindern zu können, benötigt das System Resilienz. Auch die steigende Eigenkomplexität komplexer adaptiver Systeme macht Resilienz als Systemeigenschaft also immer notwendiger.

- Um Risiken zu bewältigen, benötigen Systeme keine Resilienz. Das Ereignis an sich, die Wahrscheinlichkeit seines Auftretens und seine Auswirkungen auf das System sind bekannt, so dass spezifische Maßnahmen zum Umgang mit dem Risiko ausreichen.

- Es gibt Risiken, die komplexe adaptive Systeme ganz bewusst eingehen, weil ihre Eintrittswahrscheinlichkeit extrem gering ist, aber gleichzeitig die Auswirkungen so groß, dass die Ausbildung spezialisierter Anpassungsfähigkeit sehr viele Ressourcen in Anspruch nehmen würde. Zur Bewältigung von Risiken, für die komplexe adaptive Systeme keine spezialisierte Anpassungsfähigkeit entwickeln und die ihre Belastungsgrenzen übersteigen, ist Resilienz notwendig.

- In komplexen adaptiven Systemen besteht die Möglichkeit, dass unerwartete Ereignisse mit gravierenden Auswirkungen, sogenannte schwarze Schwäne bzw. beispiellose Ereignisse auftreten. Zur Bewältigung solcher ungewissen und echt unsicheren Ereignisse, welche die Belastungsgrenzen komplexer adaptiver Systeme übersteigen, benötigen diese Resilienz.

- Um mit langfristigen Veränderungsprozessen erfolgreich umgehen zu können, benötigen komplexe adaptive Systeme keine Resilienz. Erst wenn die langfristigen Prozesse durch Erreichen eines Schwellenwertes ein unerwartetes und die Belastungsgrenzen des Systems überschreitendes Ereignis auslösen, wird Resilienz notwendig. 
- Je höher die Vulnerabilität eines komplexen adaptiven Systems, desto wahrscheinlicher und stärker übersteigen widrige Ereignisse die eigentlichen Belastungsgrenzen des Systems, bzw. desto stärker weicht das System initial von seinem eigentlich erwartbaren Entwicklungspfad ab, und desto größer muss ceteris paribus seine Resilienz sein, um erfolgreich mit dem Ereignis umgehen zu können.

Welche Bedingungen sind aus system- und komplexitätstheoretischer Sicht notwendig für Resilienz?

- Einfache Systeme reagieren in deterministischer Weise auf Belastungen. Übersteigen diese die Grenzen ihrer Belastbarkeit, bricht das System zusammen. Es kann nicht mehr auf einen neuen Entwicklungspfad umschwenken, auch nicht auf einen mit verminderter Systemleistung. Dazu sind nur komplexe Systeme aufgrund ihrer Anpassungsfähigkeit in der Lage. Resilienz drückt sich durch das Betreten neuer Entwicklungspfade aus, obwohl die eigentlichen Belastungsgrenzen des Systems überstiegen werden. Die Komplexität von Systemen ist also eine notwendige Bedingung für Resilienz.

- A priori ist unklar, welchen Effekt steigende Komplexität auf das Ausmaß der im System vorhandenen bzw. auftretenden Resilienz hat. Steigende Komplexität kann sowohl zu mehr als auch zu weniger Resilienz führen.

- Das Vorhandensein von Anpassungsfähigkeit ist eine zwingende Grundvoraussetzung für Resilienz. Aber Resilienz ist nicht gleichbedeutend mit Anpassungsfähigkeit. Resilienz ist auch nicht gleichbedeutend mit generischer Anpassungsfähigkeit.

- Transformationsfähigkeit ist kein notwendiger Bestandteil von Resilienz. Allerdings erhöht das Vorhandensein von Transformationsfähigkeit - verstanden als besonders ausgeprägte Form generischer Anpassungsfähigkeit - ceteris paribus die Resilienz komplexer adaptiver Systeme.

- Je flexibler komplexe adaptive Systeme sind, desto größer ist ihre Resilienz.

- Lernfähigkeit ist eine notwendige Grundvoraussetzung für Resilienz.

Worin besteht Resilienz aus system- und komplexitätstheoretischer Sicht?

- Resilienz liegt dann vor, wenn ein System auf einen sehr unwahrscheinlichen Reiz (bzw. Ereignis) aus der Umwelt oder seinem Inneren, der das System initial auf einen Entwicklungspfad mit stark verminderter Systemleistung bringt, mit der Realisierung eines Prozes- 
ses eigentlich extrem unerwartbarer Anschlussmöglichkeiten reagiert, um anschließend auf einen so realisierbar gewordenen, neuen Entwicklungspfad erwartbarer Anschlussmöglichkeiten einzuschwenken, der sich durch eine (mindestens) vergleichbare Systemleistung auszeichnet, wie sie vor Eintritt des Ereignisses vorlag.

- In komplexen adaptiven Systemen muss zwischen spezialisierter und generischer Anpassungsfähigkeit unterschieden werden. Die Resilienz eines komplexen adaptiven Systems nimmt bei steigender Komplexität genau dann zu, wenn die generische Anpassungsfähigkeit des Systems steigt.

- Resilienz zeigt sich in der Fähigkeit komplexer adaptiver Systeme, mittels generischer Anpassungsfähigkeit extrem unwahrscheinliche oder unerwartete, ihre eigentlichen Belastungsgrenzen übersteigende Ereignisse so zu bewältigen, dass sie ihre Systemleistung möglichst aufrechterhalten und langfristig sichern können.

- Diversität und Redundanz sind Prinzipien zur Erhöhung generischer Anpassungsfähigkeit in komplexen adaptiven Systemen. Diversität besteht in einer möglichst unterschiedlichen Reaktion funktional gleicher Agenten auf extrem unwahrscheinliche oder unerwartete Veränderungen. Je größer die Diversität innerhalb funktional äquivalenter Gruppen komplexer adaptiver Systeme, desto größer ist ihre generische Anpassungsfähigkeit.

- Derart gefasst, entspricht Diversität im Prinzip einem bestimmten Verständnis von Redundanz, nämlich skalenübergreifender Redundanz. Skalenübergreifende Redundanz bedeutet das Vorhandensein funktional äquivalenter Systemelemente, die sich in ihrer Abhängigkeit von unterschiedlichen widrigen Ereignissen unterscheiden. Je mehr skalenübergreifende Redundanzen vorhanden sind, desto größer ist die generische Anpassungsfähigkeit komplexer adaptiver Systeme.

Worauf gilt es bei Resilienz im Rahmen der zivilen SiFo aus normativer Sicht zu achten?

- Strategien zur Erhöhung der Resilienz der für die zivile SiFo relevanten sozio-technischen Systeme müssen als Angebote formuliert werden, die für die Gesellschaft einen klaren, über ohnehin erfolgende Maßnahmen des Bevölkerungsschutzes und der Katastrophenhilfe hinausgehenden, Mehrwert haben.

- Auch Systeme, deren zugrundeliegende Identität sich durch unfreie Strukturen auszeichnet, scheinen in der Lage zu sein, Resilienz gegenüber disruptiven Ereignissen auszubilden. 
- Die Erhöhung der Resilienz eines Systems ist genau dann normativ wünschenswert, wenn die zugrundeliegende Identität des Systems normativ wünschenswert ist. Ob Lösungen zur Erhöhung der Resilienz eines Systems erforscht und entwickelt werden sollten, hängt von vorgelagerten gesellschaftlichen und politischen Aushandlungsprozessen zur normativen Erwünschtheit des Systems ab.

- Selbst, wenn ein System grundsätzlich normativ wünschenswert ist, gilt es bei der Entwicklung von Strategien zur Erhöhung seiner Resilienz darauf zu achten, dass möglicherweise bestehende Ungerechtigkeiten nicht perpetuiert oder sogar verstärkt werden.

- Lösungen zur Erhöhung der Resilienz eines Systems müssen einer Proportionalität im Hinblick auf das angemessene Verhältnis zwischen Kosten und Nutzen entsprechen, über deren Ausgestaltung ebenfalls in vorgelagerten gesellschaftlichen und politischen Aushandlungsprozessen entschieden werden muss.

- Vertrautheit als Orientierung am Gewesenen und Bekannten ist kein Mechanismus zur Erhöhung der Resilienz der für die zivile SiFo relevanten, komplexen adaptiven sozio-technischen Systeme.

- Je größer die Resilienz eines komplexen adaptiven Systems ist, desto größer ist das ihm entgegengebrachte Systemvertrauen. Gleichzeitig gilt: Je stärker das Systemvertrauen ausgeprägt ist, desto größer ist die Resilienz des Systems.

- Strategien zur Erhöhung der Resilienz der für die zivile SiFo relevanten sozio-technischen Systeme müssen so ausgestaltet sein, dass sie sich positiv zumindest aber nicht negativ auf das Vertrauen in die zuverlässige Funktionalität der jeweiligen Systeme auch und gerade im Fall des Eintretens extrem unwahrscheinlicher und unerwarteter, die eigentlichen Belastungsgrenzen der Systeme übersteigender disruptiver Ereignisse, auswirken.

Worin besteht Resilienz aus normativer Sicht?

- Resilienz ist ein post-neoliberales Paradigma. Denn Resilienz geht aufgrund der Komplexität von Systemen von der grundsätzlichen Unvorhersehbarkeit der Auswirkungen zielgerichteter, proaktiver Interventionen im System aus. Daher muss der Fokus auf der Maximierung von Reflexivität und (generischer) Anpassungsfähigkeit liegen, die dem System eine kontinuierliche und dynamische Weiterentwicklung - auch durch staatliches Handeln - im Fall des Eintretens von Problemen ermöglichen. 
- Resilienz besteht darin, aufgrund der Unsicherheit künftiger Ereignisse eine Strategie der Vorbereitung im Sinne der Maximierung von Störungsverarbeitungskapazitäten anzuwenden, unter gleichzeitiger Beibehaltung gesellschaftlicher Freiheiten.

- Resilienz lässt sich normativ als starkdemokratische Sicherheit verstehen. Mit „starkdemokratisch“ sind dabei nach Barber Strukturen gemeint, die eine aktive, transparente und partizipative Rolle von Bürgern bei gesellschaftlichen und politischen Aushandlungsprozessen bzgl. der normativen Erwünschtheit relevanter Systeme ermöglichen.

- Politikwissenschaftlich gesprochen besteht Resilienz dann normativ in einer durch Verantwortungsannahme aktiver Bürger möglich werdenden Aufrechterhaltung gesellschaftlicher Freiheiten bei gleichzeitiger Erhöhung der Sicherheit.

Worin besteht Resilienz aus normativer Sicht nicht?

- Eine unreflektierte Übernahme des Resilienz-Begriffs birgt die Gefahr, diesen als neoliberales Paradigma einer Verantwortungsverlagerung vom Staat auf den einzelnen Bürger misszuverstehen. Die Verwendung des Resilienz-Konzepts in der zivilen Sicherheitsforschung darf nicht dazu führen, dass von widrigen Ereignissen betroffenen Personen und Gruppen die Verantwortung für erlittene Schäden zugeschrieben wird.

- Resilienz besteht nicht darin, aufgrund der Unsicherheit künftiger Ereignisse eine Strategie der Vorsicht im Sinne vorbeugender Freiheitseinschränkung anzuwenden.

- Resilienz besteht nicht darin, aufgrund der Unsicherheit künftiger Ereignisse eine Strategie der Präemption im Sinne der Aushöhlung von Freiheit durch aktivistische Maßnahmen anzuwenden.

- Resilienz besteht nicht darin, aufgrund der Unsicherheit künftiger Ereignisse eine Strategie der maximalen physischen Robustheit der betreffenden Systeme anzuwenden.

- Die Resilienz der Gesellschaft gegenüber der Bedrohung durch das Phänomen des Terrorismus lässt sich nicht mithilfe rein physischer Schutzmaßnahmen erhöhen.

Bei den Antworten auf diese Leitfragen fehlen einige Erkenntnisse, die bei der Analyse durch konzeptionelle Zugänge im vorangegangenen Kapitel gewonnen werden konnten. Das hat jeweils unterschiedliche, spezifische Gründe, auf die kurz verwiesen werden soll. Zunächst wurden einige Erkenntnisse bereits zu Beginn dieses Kapitels verarbeitet (siehe 5.1). Einige andere werden noch im Bezug zu den Grenzen des Konzepts diskutiert (siehe 5.4). Die systemtheoretischen Erkenntnisse zur Eigenkomplexität 
von Systemen und deren Verhältnis zu Resilienz (siehe die Aufzählungspunkte 3, 5 und 6 am Ende von 4.3.1) konnten im weiteren Verlauf der Analyse durch komplexitätstheoretische Annahmen präzisiert werden. Der letzte Aufzählungspunkt in diesem Unterkapitel, in dem es um die Aufgaben der zivilen SiFo geht, ist generell als Aufruf zu verstehen und kein Bestandteil des Resilienz-Konzepts an sich. Die relativ ausführlichen Beschreibungen der verschiedenen Phasen, die komplexe adaptive Systeme durchlaufen und die Verknüpfung mit Resilienz und generischer Anpassungsfähigkeit entstammt sehr stark der sozial-ökologischen Resilienzforschung (siehe Aufzählungspunkte 3, 4 und 5 am Ende von 4.3.5). Sie waren primär als Hilfskonstrukte für das Verständnis des Themas „generische Anpassungsfähigkeit“ von Bedeutung und spielten im weiteren Verlauf der Analyse keine Rolle. In der Gesamtschau kommt das Resilienz-Konzept der zivilen SiFo auch ohne diese Annahmen aus, ohne dass diese deshalb ihre Gültigkeit verlieren. Der letzte Aspekt betrifft die spezifisch auf das Thema Terrorismus gemünzte Forderung, resilienzerhöhende Maßnahmen und Gestaltungsprinzipien bereits in der Designphase zu beachten. Diese kann auch weiterhin Gültigkeit für sich beanspruchen, sie ist jedoch ebenfalls eher als Aufruf zu verstehen und kein notwendiger Bestandteil des Resilienz-Konzepts an sich (siehe den Aufzählungspunkt 1 am Ende von 4.2.3).

Mithilfe der gerade dargestellten, umfassenden Liste lässt sich das häufig unpräzise und oberflächlich verstandene Konzept der Resilienz für die Zwecke der zivilen Sicherheitsforschung präzisieren. Die Antworten auf die sieben Leitfragen dienen insofern gemeinsam mit den zum Verständnis notwendigen Definitionen relevanter Begrifflichkeiten im Folgenden als Ausgangspunkt der weiteren Diskussion. Sie stellen die Vorlage dar, anhand derer im nächsten Kapitel - unter Zuhilfenahme einiger aktueller Erkenntnisse aus der ingenieurwissenschaftlichen Resilienzforschung Hypothesen für Resilience Engineering entwickelt werden.

\subsection{Diskussion der Grenzen des Konzepts}

Resilienz hat Grenzen. Auch resiliente Systeme sind nicht in der Lage, mit allen beliebigen, denkbaren und undenkbaren widrigen Ereignissen fertig zu werden. Die augenfälligen Beispiele der Eruption eines Super-Vulkans, des Einschlags eines großen Meteoriten auf der Erde (auch als „Global Killer" bezeichnet) oder der Invasion einer außerirdischen, technisch weit überlegenen Spezies dienten im Rahmen der Analyse durch konzeptionel- 
le Zugänge zur Verdeutlichung dieser Tatsache (Baum 2015: 231, Sikula et al. 2015: 225). Selbstverständlich ließen sich auch für derart existentielle Bedrohungen Überlegungen anstellen, wie ihnen zu begegnen wäre, etwa durch Besiedelung eines neuen Planeten, durch Meteoritenabwehr mithilfe unterschiedlicher Technologien oder durch radikale wehrtechnische Innovationen, die auch mit außerirdischer Technologie mithalten könnte. ${ }^{39}$ Solche Überlegungen sind jedoch nach aktuellem Stand eher akademischer Art und zudem jeweils immer nur geeignet, einzelne dieser existentiellen Bedrohungen zu bekämpfen. Denn selbst die Besiedelung eines neuen Planeten hilft nicht, wenn durch Mutation hoch-ansteckender und hoch-tödlicher Krankheitserreger eine über-weltweite Pandemie ausbricht. Und Meteoritenabwehr versagt, wenn im Rahmen teilchenphysikalischer Experimente versehentlich ein schwarzes Loch kreiert wird. Insofern bleibt die Erkenntnis bestehen, dass Resilienz Grenzen hat. Wobei es hier um Grenzen von Resilienz als tatsächlich vorhandener Eigenschaft komplexer adaptiver Systeme handelt. Das ließe sich auch als Grenzen das Ausmaß von Resilienz betreffend bezeichnen. Es gibt sozusagen ein - theoretisch nicht bestimmbares aber praktisch wohl vorhandenes - Maximalmaß an Resilienz bzw. ein Maximalausmaß an disruptivem Ereignis, dem mithilfe von Resilienz begegnet werden kann. Wenn als Konsequenz des Ereignisses die Auslöschung der Menschheit steht, wird dies unmittelbar einsichtig. Sobald ein kleiner Teil der Menschheit überlebt und sich den neuen Bedingungen (etwa weitgehende aber nicht komplette Unbewohnbarkeit des Planeten) erfolgreich anpasst, ließe sich zumindest theoretisch von der Resilienz dieser Menschen sprechen. Das wäre allerdings maximal noch konsistent mit einigen ursprünglichen Ideen Hollings, bei dem die pure Weiterexistenz (persistence) Ausweis von Resilienz war (Holling 1973: 14ff). Dem Resilienz-Konzept der zivilen SiFo, wie es in der vorliegenden Arbeit entwickelt wurde, zufolge, ließe sich in einem solchen Fall dagegen nicht von vorhandener Resilienz sprechen. Denn das Konzept enthält eine ganze Reihe weiterer Annahmen, die über die reine Existenz weit hinausgehen.

Neben diesen Grenzen bezüglich des Ausmaßes von Resilienz, hat auch das Konzept an sich aus konzeptioneller Sicht einige Grenzen bzw. gibt es Kritikpunkte, die nicht gänzlich unerwähnt bleiben und im Folgenden daher kurz diskutiert werden sollen. Die erste dieser Grenzen, lässt sich

39 Derartige Technologien werden beispielsweise aktuell in EU-Projekten wie etwa „NEOShield-2: Science and Technology for Near-Earth Object Impact Prevention" erforscht (siehe http://www.neoshield.net/)[Stand: 28.6.2019]. 
als eine Art „positive Abgrenzung“ formulieren. Eine wichtige Frage im Rahmen von Arbeiten der SiFo zum Resilienz-Konzept ist die nach der Abgrenzung zu anderen, existierenden Konzepten, Strategien und Vorgehensweisen im Bereich der zivilen Sicherheit. Hier werden immer wieder Risikomanagement oder klassische Schutzmaßnahmen, die auf einer Maximierung der Widerstandsfähigkeit bzw. Robustheit möglicherweise betroffener Systeme beruhen, genannt (Linkov et al. 2014: 407ff, Scharte et al. 2014: 9ff). Durch das nun spezifizierte Resilienz-Konzept der zivilen SiFo lässt sich diese Frage beantworten: Resilienz ist eine Ergänzung bestehender Konzepte, Strategien und Vorgehensweisen zur Erhöhung von Sicherheit (Baum 2015: 229, IRGC 2018: 50, Kete et al. 2018: 276, Park et al. 2013: 360, Sansavini 2016, Sikula et al. 2015: 221). Ähnlich wie bei Antonovsky seine „salutogenetische Orientierung“ keinesfalls zu einer „völlige[n] Aufgabe der pathogenetischen Orientierung“ führen soll, geht es bei Resilienz nicht um die Ersetzung bekannter und etablierter Methoden zur Risikovorsorge bzw. zum Schutz relevanter Systeme, sondern um eine Erweiterung. Antonovskys Aussagen bieten hier eine passende Analogie und sollen deshalb in Ansätzen skizziert werden. Ihm zufolge ist es selbstverständlich unabdingbar, weiter an der Heilung spezifischer, schwerer Krankheiten - wie etwa Krebs - zu forschen und nach „Wunderwaffen“ zu suchen. Antonovsky plädiert „dafür, die beiden Orientierungen als komplementär zu betrachten und dafür, dass die intellektuellen und materiellen Ressourcen ausgeglichener verteilt werden, als dies gegenwärtig der Fall ist" (Antonovsky 1997: 30). Ganz ähnlich verhält es sich mit Resilienz. Dem Resilienz-Konzept der zivilen SiFo zufolge sind Risikomanagement wie auch klassische Schutzmaßnahmen zur Erhöhung der Robustheit von Systemen kein Teil von Resilienz. Resilienz ist spezifisch dazu gedacht, erfolgreich mit extrem unwahrscheinlichen oder unerwarteten, die eigentlichen Belastungsgrenzen des Systems übersteigenden disruptiven Ereignissen umzugehen. Für diese Ereignisse ist keine spezifische Vorbereitung möglich und deshalb benötigen Systeme generische Anpassungsfähigkeit, um sie zu überstehen. Für andere Ereignisse und vor allen Dingen spezifische, bekannte und höchstwahrscheinlich wiederkehrende Bedrohungen sind dagegen Risikomanagement und klassische Schutzmaßnahmen nach wie vor das Mittel der Wahl. Dazu benötigen Systeme keine Resilienz und darin besteht eine Grenze des Konzepts bezüglich der Notwendigkeit seiner Anwendung. Wenn in der zivilen SiFo also von Resilienz gesprochen und ein Resilienz-Konzept eingeführt wird, geht es primär darum, den Blick für die Komplexität der betrachteten Systeme und die Unsicherheit im Sinne von Ungewissheit und echter Unsicherheit von Ereignissen zu 
weiten und die Entstehung einer reduktionistischen Fata Morgana der Sicherheit zu vermeiden. In diesem Sinne ergänzt und komplettiert das Resilienz-Konzept der zivilen SiFo etablierte und weiterhin valide Konzepte zur Erhöhung von Sicherheit wie Risikomanagement und klassische Schutzmaßnahmen.

Die sozusagen hehre Zielsetzung des gerade geschilderten „sowohl ... als auch" leitet direkt über zur zweiten Grenze des Konzepts, die in der Literatur sehr ausführlich diskutiert wird und auch in der vorliegenden Arbeit bereits an mehreren Stellen in unterschiedlicher inhaltlicher Ausprägung zur Sprache kam. Es geht um das Verhältnis bzw. den potentiellen Konflikt zwischen Resilienz und Effizienz. Wer sowohl Risikomanagement und klassischen Schutz betreibt, als auch Ressourcen für generische Anpassungsfähigkeit zur Verfügung hält, kommt beinahe zwangsweise in einen Zielkonflikt. Kaufmann und Blum bezeichnen Resilienz in diesem Kontext verkürzt als „ideology of mobilization which, in the name of security, is utterly ignorant of goal conflicts as well as conflicts of interest" (Kaufmann Blum 2012: 253f). Ressourcen lassen sich stets nur einmal verwenden und sie stehen nur begrenzt zur Verfügung. Je stärker man auf Risikomanagement und klassische Schutzmaßnahmen setzt, desto weniger Ressourcen bleiben zwangsweise für Resilienz übrig. Zumal es, so ein Ergebnis der Analyse des Zusammenhangs zwischen Resilienz und Unsicherheit, für Entscheidungsträger im Zweifel lohnenswerter scheinen kann, in Risikoreduktion zu investieren. Denn die Quantifizierbarkeit von Risiken (Eintrittswahrscheinlichkeit multipliziert mit den Auswirkungen bestimmter Ereignisse) kann in komplexen adaptiven Systemen dazu führen, dass Ressourcen zur Erhöhung spezialisierter Anpassungsfähigkeit an Risiken genutzt werden. Aufgrund von deren gerade erwähnter Endlichkeit führt dies ceteris paribus zu einer Verringerung generischer Anpassungsfähigkeiten und damit geringerer Resilienz (Kaufmann/Blum 2012: 253f).

Der genannte Zielkonflikt besteht aber nicht nur in der Wahl zwischen Risikomanagement und Resilienz, sondern noch sehr viel grundsätzlicher in der Wahl zwischen Resilienz einerseits und maximaler Effizienz andererseits. Dieser Zielkonflikt spielt, unter verschiedenen Vorzeichen, in der nicht-psychologischen Resilienzforschung bereits seit Jahrzehnten eine wichtige Rolle. So setzt Holling sein Resilienz-Verständnis bewusst als Gegenpol zu einer effizienz-zentrierten Sichtweise, während Wildavsky Resilienz sehr viel stärker als "marktkonforme“ und damit effizienz-optimale Strategie begreift (Holling 1973: 1f, Kaufmann/Blum 2012: 238ff). Mit Blick auf die Literatur lässt sich sagen, dass die erste Sichtweise, wonach 
Resilienz und Effizienz mindestens schwierig zu vereinen sind, deutlich überwiegt. Stellvertretend kann beispielsweise Longstaff zitiert werden, die schreibt: "Sometimes a resilience strategy comes at a cost because it can make the system less efficient" (Longstaff 2012: 275). David Woods analysiert das im Zusammenhang mit seiner Bewertung der Explosion des Space-Shuttle Columbia. Ihm zufolge wären Investitionen in Resilienz insbesondere dann vonnöten, wenn sie gesellschaftlich und vor allem ökonomisch am wenigsten akzeptiert sind (Woods 2005: 297). Das liegt vor allen Dingen daran, dass die Ereignisse, zu deren Bewältigung Ressourcen investiert werden müssten, unsicher sind, wohingegen die möglichen Gewinne effizienzmaximierenden Handelns sicher sind. Vor allen Dingen in Zeiten knapper Budgets stellt sich das als große Herausforderung dar (Boin/McConnell 2007: 52, Hutter 2011). Woods spricht davon, dass es nötig wäre "to sacrifice today's real production goals to consider uncertain evidence of possible future risks" (Woods 2005: 291, eigene Hervorhebung). Häufig werden dann Entscheidungen für Effizienz - und damit ein Stück weit gegen Resilienz - auch implizit getroffen, weil die Logik der Effizienzmaximierung unhinterfragt als Handlungsmaxime genutzt wird und keine explizite Beachtung der Komplexität der Systeme und der Unsicherheit der Zukunft unternommen wird. Daher agieren Menschen und Organisationen auch zum Teil risikoreicher (in einem eher alltagssprachlichen Sinn), als sie bei expliziter Berücksichtigung der gerade genannten Bedingungen agieren wollen würden (Woods 2005: 303). Insgesamt lässt sich festhalten: „Up to now, it is an open question whether an efficient organization can also be a resilient organization" (Hutter 2011). Es besteht also Klärungsbedarf was den möglichen Zielkonflikt zwischen Resilienz und Effizienz angeht, auch im Hinblick darauf, wie eine ausgeglichene Beachtung beider Zielstellungen gelingen könnte (von Gleich/Giese 2019: 94). Ob beispielsweise eine Gesellschaft bewusst darauf verzichtet, teure Investitionen zur Resilienz-Erhöhung vorzunehmen, weil die möglichen disruptiven Ereignisse, bei deren Eintreten Resilienz notwendig wäre, höchstwahrscheinlich - zumindest in nächster Zeit - nicht eintreten werden, wie von Baum vorgeschlagen, muss Gegenstand gesellschaftlicher Aushandlungsprozesse sein (Baum 2015: 233, Wachsmuth 2014: 8). Das eigenständige ResilienzKonzept der zivilen SiFo kann hier lediglich aus wissenschaftlicher Sicht Argumente liefern, warum es durchaus Sinn ergeben könnte, in Resilienz zu investieren bzw. dass zumindest explizit mit Komplexität und Unsicherheit gerechnet werden sollte.

Aus wissenschaftlicher Sicht könnte das Resilienz-Konzept auch aus einem stärker konstruktivistischen Blickwinkel hinterfragt werden. Kuhli- 
cke spricht hier von einem „Mythos Resilienz“ (myth of resilience). Er konstatiert eine erst retrospektiv feststellbare Existenz - oder Nicht-Existenz - von Resilienz und bezeichnet das Konzept als einen Weg, im Nachhinein dem Auftreten komplett überraschender, unerwarteter Ereignisse einen Sinn zu verleihen. Dieser Sinn besteht demnach in einer expliziten Betonung der Fähigkeiten zum erfolgreichen Umgang mit solchen Ereignissen durch Coping. Auch die immer wieder im Resilienz-Diskurs zu findende Annahme, dass durch Lernen und Anpassen Systeme von solchen Ereignissen langfristig sogar zu profitieren in der Lage sein sollen, rechnet Kuhlicke zum Resilienz-Mythos (Kuhlicke 2010). Auch wenn diese Bezeichnung als tendenziell überspitzt erscheint, ist die Frage danach, ob Resilienz immer erst retrospektiv sichtbar wird, durchaus gerechtfertigt, gerade in der stark anwendungsorientierten Sicherheitsforschung. Wenn a priori nicht feststellbar ist, inwiefern eine Maßnahme oder die Umsetzung einer bestimmten Strategie tatsächlich resilienzerhöhend wirkt, lassen sich Investitionen noch schwieriger rechtfertigen, als dies aufgrund des oben geschilderten Zielkonflikts ohnehin der Fall ist. Hier erlaubt ein detaillierterer Blick auf die Elemente des Resilienz-Konzepts der zivilen SiFo Abhilfe, da etwa generische Anpassungsfähigkeit zum Beispiel in Form skalenübergreifender Redundanz durchaus gemessen werden kann. Auch der Einfluss bestimmter Maßnahmen auf gesellschaftliche Freiheiten lässt sich sozialwissenschaftlich untersuchen, insbesondere auch mit Blick auf die von Kuhlicke ebenfalls zurecht gestellte Frage: „Who is benefitting from the ,myth of resilience' and to what end" (Kuhlicke 2010)?

Einige weitere generelle Kritikpunkte bei der Definition eines so komplexen Konzepts wie Resilienz wurden auch bereits im Rahmen der Konzeptentwicklung explizit adressiert und ein Stück weit zu beantworten versucht (MacAskill/Guthrie 2014: 669). MacAskill und Guthrie nennen hier beispielsweise die unpräzise Zuschreibung von Resilienz als Ergebnis, Prozess, physikalische Eigenschaft oder politische Strategie, die von Individuen über Organisationen bis zu Infrastruktursystemen verschiedene Träger hat. Oder den möglichen zirkulären Zusammenhang zwischen Resilienz und Vulnerabilität. Auch die Kontextabhängigkeit von Resilienz gilt es zu beachten. Und nicht zuletzt stellt sich die Frage, wie die unterschiedlichen Interpretationen von Resilienz in verschiedenen Disziplinen zu bewerten sind (MacAskill/Guthrie 2014: 670). Mit all diesen Aspekten hat sich die vorliegende Arbeit auseinandergesetzt und sie im Resilienz-Konzept der zivilen SiFo zu verorten gesucht. Nichtsdestotrotz - oder vielleicht auch gerade deshalb - lassen sich Zielkonflikte zwischen den system- und komplexitätstheoretischen und den normativen Annahmen, also innerhalb des 
Konzepts nicht ausschließen. Wenn beispielsweise generische Anpassungsfähigkeit nur zulasten gesellschaftlicher Freiheiten erhöht werden könnte, gilt es, dies klar zu benennen. Aufgrund der dem Konzept inhärenten Komplexität, lassen sich solche Zielkonflikte nicht a priori ausschließen, sondern müssen ebenfalls in gesellschaftlichen Aushandlungsprozessen entschieden werden. Das resultiert aus der Kombination aus einerseits system- und komplexitätstheoretischen Annahmen und andererseits stärker normativ geprägten Annahmen. Diese Kombination aufzugeben und ein rein deskriptiv-analytisches Resilienz-Konzept zu entwerfen, wäre zwar ohne weiteres möglich. Es würde jedoch den Spezifika der zivilen Sicherheitsforschung nicht entsprechen, die aufgrund ihrer Anwendungsnähe immer auch normative Diskussionen führen muss, um ihrer Zielstellung zu entsprechen (siehe zum Beispiel 4.2.2). Die Verwendung des in der vorliegenden Arbeit entwickelten, eigenständigen Resilienz-Konzepts der zivilen SiFo erlaubt es, diese Zielkonflikte explizit und im Rahmen des Konzepts zu benennen und zu bearbeiten. Das spielt auch vor dem Hintergrund der im abschließenden Schritt der Arbeit erfolgenden Entwicklung von Hypothesen für Resilience Engineering eine wesentliche Rolle. 


\section{Resilience Engineering - Hypothesen für die zivile Sicherheitsforschung}

\subsection{Diskussion von Resilience Engineering anhand von Leitfragen}

Wie können die Ingenieurwissenschaften dazu beitragen, dass es manchmal besser kommt, als man denkt? Wie schaffen sie das, was als „ordinary magic" bezeichnet wurde? Zumal wenn, wie die vorangegangenen Kapitel deutlich gemacht haben, diese Magie wohl gar nicht so alltäglich, jedenfalls nicht banal oder ganz normal ist. Wenn sie im Gegenteil, voraussetzungsreich, selbst komplex und ganz und gar unsicher ist. Wenn sie von einer Reihe an Bedingungen abhängt, vielfältige, miteinander verknüpfte oder vernetzte Bestandteile enthält und in sich unter Umständen nicht einmal widerspruchsfrei daherkommt. Wenn a priori überhaupt nicht klar ist, ob sie überhaupt wünschenswert ist oder welchen Einfluss sie auf die Ausgestaltung einer Gesellschaft hat. Mit anderen Worten: Was heißt es für die Ingenieurwissenschaften, wenn sie sich damit beschäftigen, wie sie die Resilienz eines Systems zielgerichtet verändern - zumeist erhöhen können? Oder noch spezifischer für die vorliegende Arbeit gefragt: Was heißt das für ingenieurwissenschaftliche zivile Sicherheitsforschung im Sinne eines Resilience Engineering? Die folgenden Ausführungen beziehen sich immer - äquivalent zur bisherigen Arbeit - auch da, wo es nicht explizit gemacht wird, auf die zivile SiFo.

Die Ingenieurwissenschaften beschäftigen sich schon länger mit Resilienz, das wurde in der Beschreibung des Stands der Forschung deutlich (siehe 2.6). Gleichzeitig wurden aber auch die Forschungslücken deutlich, die mit der bisherigen Ausrichtung ingenieurwissenschaftlicher Resilienzforschung einhergingen. Neben der generellen, über die Ingenieurwissenschaften hinausweisenden Lücke im Hinblick auf die Nicht-Existenz eines eigenständigen Resilienz-Konzepts der zivilen SiFo, waren dies das reduktionistische, stabilitätszentrierte Verständnis von Resilienz als engineering resilience, der Fokus auf Ansätze zur Quantifizierung, die mangelnde theoretische Fundierung der identifizierten Ideen und Mechanismen zur Umsetzung von Resilienz und die ungeeignete Verwendung des Begriffs Resilience Engineering in einem rein sozialwissenschaftlichen Sinne (siehe 3). Die erste und die vierte Forschungslücke hängen unmittelbar zusammen. Beide wurden in den letzten Kapiteln durch die detaillierte, theoretisch 
anspruchsvolle und neuartige Entwicklung eines system- und komplexitätstheoretischen Konzepts von Resilienz mit gleichzeitiger normativer Einbettung ausführlich adressiert. Dieses Konzept bildet nun die Grundlage für die nächsten Schritte hin zur Entwicklung von Hypothesen für Resilience Engineering. Altherr et al. bezeichnen ein solches Vorgehen als nächsten logischen Schritt. Zunächst bedarf es eines Verständnisses dafür, was Resilienz konzeptionell ausmacht, um dann eine Vorstellung davon zu entwickeln, wie sie ingenieurwissenschaftlich umgesetzt werden soll (Altherr et al. 2018: 189).

Die vorliegende Arbeit sucht diesen logischen Schritt nun dazu zu nutzen, die verbleibenden drei Forschungslücken direkt zu adressieren. Am deutlichsten wird das unmittelbar für die fünfte Forschungslücke. Der Begriff des Resilience Engineering wird explizit und in scharfer Abgrenzung von seinen organisationswissenschaftlichen Ursprüngen in einem ingenieurwissenschaftlichen, auf Technologie fokussierten Sinn gebraucht. Er wird somit spezifiziert oder - wie der Autor der vorliegenden Arbeit es in einigen Veröffentlichungen ausdrückt - bewusst eingeschränkt (Hiermaier et al. 2019: 158, Scharte/Thoma 2016: 134, Thoma et al. 2016: 11). Erstmals von Scharte und Thoma so formuliert, gilt nach wie vor, dass „[d]iese bewusste Einschränkung [...] eine - wenn auch notwendig unscharfe Trennung zwischen verschiedenen Optionen“ der Erhöhung von Resilienz erlaubt (Scharte/Thoma 2016: 134). Die Ingenieurwissenschaften und der Autor dieser Arbeit erheben zu keinem Zeitpunkt den Anspruch, Resilienz einzig und allein technologisch bzw. mittels ingenieurwissenschaftlicher Ideen und Ansätze schaffen zu können. Es geht immer darum, in Kombination und Kooperation mit anderen Disziplinen und Forschungsbereichen zu überlegen, wie die Gesellschaft und ihre relevanten Systeme, vor allem ihre kritischen Infrastrukturen, resilient(er) gegenüber plötzlichen, gravierenden und unerwartet eintretenden Störereignissen gestaltet werden können. Die bewusste Einschränkung bzw. Spezifizierung des Begriffs Resilience Engineering erlaubt es aber den Ingenieurwissenschaften, ihren Beitrag zur Erhöhung von Resilienz systematisch fassen und mit einer griffigen Überschrift versehen zu können. Engineering resilience kann diese Überschrift aufgrund der stabilitätszentrierten Bedeutung des Begriffs nicht lauten. Resilience Engineering bietet sich dagegen an, da der Begriff in den Ingenieurwissenschaften gerade noch nicht stark besetzt ist und nur in einigen wenigen Artikeln überhaupt explizit vorkommt. Und wenn er vorkommt, dann in einem systemischen Sinne, der dem Resilienz-Konzept der zivilen SiFo teilweise sehr nahekommt. Er ist dann anschlussfähig an die Erkenntnisse, die im Folgenden in Form von Hypothesen für Resilien- 
ce Engineering dargestellt werden (siehe z.B. Attoh-Okine 2016, ENISA 2011, Ouyang/Wang 2015, Rahimi/Madni 2014, Sansavini 2016). Das Fehlen eines ingenieurwissenschaftlichen Verständnisses von Resilience Engineering als Forschungslücke zu bezeichnen, ist selbstverständlich nicht als Geringschätzung der unter diesem Begriff versammelten organisationswissenschaftlichen Resilienzforschung zu verstehen. Im Gegenteil, deren Ideen sind zu einem guten Teil in das Resilienz-Konzept der zivilen SiFo geflossen. Mit den nun folgenden Ausführungen zu Resilience Engineering kann die dergestalt formulierte Forschungslücke aber als zumindest aus sozialwissenschaftlicher Sicht adressiert gelten. Für die Zukunft sollen darauf basierend natürlich auch konkrete, ingenieurwissenschaftliche Umsetzungen und Anwendungen erfolgen.

Die beiden verbleibenden Forschungslücken - unter den Stichworten engineering resilience und Dominanz von Quantifizierungsansätzen zusammenfassbar - werden ebenfalls durch die im nächsten Schritt entwickelten Hypothesen aus sozialwissenschaftlicher Sicht ein gutes Stück weit geschlossen. Darin liegt scheinbar ein Widerspruch in sich, wenn eine ingenieurwissenschaftliche Umsetzung sozialwissenschaftlich erfolgen soll. Der Widerspruch lässt sich aber dank der interdisziplinären Herangehensweise der Arbeit auflösen, deren Anspruch aufgrund ihrer sozialwissenschaftlichen Grundausrichtung nicht darin liegen kann, den Ingenieurwissenschaften mathematische Modelle oder technologische Methoden an die Hand zu geben. Vielmehr geht es darum, Wege, Bedingungen und Bestandteile aufzuzeigen, welche die Ingenieurwissenschaften berücksichtigen sollten, wenn sie versuchen, das Resilienz-Konzept der zivilen SiFo praktisch umzusetzen.

Dieses Resilienz-Konzept wurde im vorangegangenen Kapitel zum einen mithilfe der Definition relevanter Begrifflichkeiten konkretisiert. Die Definitionen sollen auch für die Hypothesen für Resilience Engineering weiterhin ihre Gültigkeit behalten. Die damit unter Umständen verbundenen Schwierigkeiten im Hinblick auf inkonsistente Begriffsverständnisse zwischen Sozial- und Ingenieurwissenschaften lassen sich zwar so nicht a priori aus der Welt schaffen. Sie werden aber zumindest explizit adressierbar, wenn die Ingenieurwissenschaften bei einer Verwendung der Hypothesen für Resilience Engineering zunächst prüfen können - und müssen - inwiefern die zugrundeliegenden Begrifflichkeiten in ihrer Disziplin äquivalent anwendbar sind. Wo dies nicht möglich ist, wäre dann im nächsten Schritt die Frage, ob die Definitionen in die Ingenieurwissenschaften „übersetzt“ werden können, ohne ihren wesentlichen Inhalt zu verlieren. Der wesentliche Inhalt ist ja für die einzelnen Bestandteile des Resilienz-Konzepts 
der zivilen SiFo unabdingbar. Eine Veränderung wirkt sich entsprechend unmittelbar auf das Konzept aus. Insofern scheint es sinnvoll, an dieser Stelle bei Bedarf einen direkten Dialog zwischen Ingenieurwissenschaftlern und Sozialwissenschaftlern zu forcieren. Denn ein gemeinsames oder aber zumindest adaptierbares Verständnis der relevantesten Begrifflichkeiten ist eine Grundbedingung für eine sinnvolle Anwendung des Resilienz-Konzepts mithilfe der Hypothesen für Resilience Engineering. Diese Überlegungen führen bereits zu einer ersten und sicherlich auch besonders relevanten Hypothese für Resilience Engineering. Diese lautet wie folgt:

Hypothese H1: Resilience Engineering erfordert ex ante eine Verständigung über die Bedeutung relevanter Begrifflichkeiten im Resilienz-Konzept der zivilen SiFo.

Nur wenn klar ist, wer mit welchem Begriff worüber redet, ist eine interdisziplinäre Verständigung möglich. Und ohne interdisziplinäre Verständigung lässt sich Resilienz nicht umsetzen, das hat die ausführliche Beschäftigung mit dem Konzept in der vorliegenden Arbeit mehr als deutlich gemacht.

Die Ergebnisse der bisherigen Analyse nutzend und auf die Ingenieurwissenschaften bezogen daraus Schlüsse ziehen. Mithilfe dieses gerade demonstrierten Vorgehens werden auch in den folgenden Unterkapiteln Hypothesen für Resilience Engineering abgeleitet. Neben der Definition relevanter Begrifflichkeiten, wurde das Resilienz-Konzept der zivilen SiFo anhand der Beantwortung von sieben sogenannten Leitfragen konkretisiert. Die Leitfragen erlaubten eine systematische Zuordnung der aus den konzeptionellen Zugängen gewonnenen Erkenntnisse zu besonders relevanten Aspekten des Konzepts. Etwa den Fragen danach, um was für eine Art Konzept es sich überhaupt handelt, warum es notwendig ist oder was den normativen Gehalt des Konzepts ausmacht. Erst in der systematischen Zuordnung und analytischen Kombination der einzelnen Merksätze ergab sich ein zusammenhängendes Konzept. Aus Merksätzen wurden so Konzept-Bestandteile, inhaltlich geordnet mithilfe der erwähnten Leitfragen. Wenn auch nicht gänzlich unkritisch zu sehen (siehe dazu 5.4), so bietet das Konzept doch aufgrund seines umfassenden Anspruchs und der breiten, theoretischen Basis vielfältige Anwendungsmöglichkeiten. Die Generierung von Hypothesen für Resilience Engineering ist eine derartige Anwendungsmöglichkeit. Diese soll ebenfalls in einer systematischen Weise und insofern nicht quasi zufallsbasiert erfolgen. Dazu wurde über unterschiedliche Wege nachgedacht, das Resilienz-Konzept der zivilen SiFo mit Hypothesen für Resilience Engineering zu verknüpfen. Ein Beispiel dafür 
wäre die Nutzung einer Liste an Prinzipien zur Umsetzung von Resilienz, wie sie etwa von Lovins und Lovins, aber auch von einer Reihe anderer Autoren vorgeschlagen werden (Dinh et al. 2012: 235f, Goessling-Reisemann/Thier 2019: 127, Lovins/Lovins 2001: 213, Madni/Jackson 2009: 189, Ouedraogo et al. 2013: 25). Aber weder sind diese Listen deckungsgleich noch theoretisch begründet, so dass in dieser Richtung eine Verknüpfung mit dem Resilienz-Konzept relativ schwer umzusetzen wäre. Ein weiteres Beispiel dafür wäre die Orientierung an den unterschiedlichen Bereichen der zivilen Sicherheitsforschung, etwa basierend auf dem aktuellen Sicherheitsforschungsprogramm. Auch hier wäre die Verknüpfung mit dem Resilienz-Konzept nur schwerlich herzustellen. Vor allen Dingen aber wäre bei einer Neuauflage des Sicherheitsforschungsprogramms in einigen Jahren davon auszugehen, dass - selbst bei einer einigermaßen großen Deckungsgleichheit - die Hypothesen zum Teil ihre Bedeutung verlieren. Und der Anspruch der vorliegenden Arbeit besteht durchaus darin, mittelund langfristig gültige Hypothesen zu entwickeln.

Um diese Schwierigkeiten zu umgehen, sollen die Hypothesen für Resilience Engineering im Folgenden äquivalent zum Resilienz-Konzept der zivilen SiFo anhand von Leitfragen diskutiert werden. Sinnigerweise handelt es sich dabei um die gleichen Leitfragen, die auch im vorangegangenen Kapitel genutzt wurden. Innerhalb der einzelnen Unterkapitel werden die Leitfragen dann noch zu den Bestandteilen des Resilienz-Konzepts der zivilen SiFo ausdifferenziert und zu diesen jeweils Hypothesen für Resilience Engineering gebildet. Dabei gilt zum einen, dass einzelne Bestandteile durchaus auch mehrere Hypothesen begründen können. Das gilt vor allem in Bezug auf die Frage danach, worin Resilienz aus systemund komplexitätstheoretischer Sicht besteht. Zum anderen werden nicht zu allen Bestandteilen Hypothesen aufgestellt bzw. mehrere Bestandteile zusammengefasst und dazu jeweils übergreifend ableitbare Hypothesen gebildet. Die jeweilige Vorgehensweise wird an den entsprechenden Stellen begründet. An allen Stellen werden, sofern in der Literatur dazu passende Erkenntnisse vorliegen, diese mit den theoretischen Annahmen des Resilienz-Konzepts der zivilen SiFo verknüpft, um das bereits vorhandene Wissen der relevanten Experten mit aufnehmen zu können (Jackson/Ferris 2013: 2). Zu den folgenden vier Leitfragen gibt es einzelne Unterkapitel:

- Was für eine Art Konzept ist Resilienz?

- Wann wird Resilienz benötigt? 
- Welche Bedingungen sind aus system- und komplexitätstheoretischer Sicht notwendig für Resilienz?

- Worin besteht Resilienz aus system- und komplexitätstheoretischer Sicht?

Die drei Leitfragen zu den normativ orientierten Bestandteilen des Resilienz-Konzepts der zivilen SiFo nach den Aspekten, auf die es bei Resilienz aus normativer Sicht zu achten gilt, sowie den Dingen, aus denen Resilienz aus normativer Sicht besteht und gerade nicht besteht, werden bewusst zu einem Unterkapitel zusammengefasst. Das soll nicht als eine Herabstufung hinsichtlich der Bedeutung normativer Fragen für Resilience Engineering missverstanden werden. Vielmehr bietet es die Möglichkeit, eine Reihe konsistenter Hypothesen aufzustellen, ohne der Gefahr einer Wiederholung und damit Verwässerung anheimzufallen. Nachdem die Hypothesen aufgestellt wurden, werden sie in einem weiteren Unterkapitel noch einmal zusammengefasst und übersichtlich dargestellt, da sie neben der Definition der relevanten Begrifflichkeiten in 5.2 und den Bestandteilen des Resilienz-Konzepts der zivilen SiFo in 5.3 den dritten zentralen Erkenntniskomplex der vorliegenden Arbeit bilden. Ähnlich wie im vorangegangenen Kapitel sind gleichzeitig auch Grenzen und mögliche Kritikpunkte in Bezug auf die Hypothesen sichtbar, die im abschließenden Unterkapitel noch kurz diskutiert werden.

\subsection{Art des Konzepts}

Resilienz ist ein systemtheoretisches Konzept.

Was für eine Art Konzept ist Resilienz? Der Beantwortung dieser Frage wurde in der vorliegenden Arbeit große Aufmerksamkeit geschenkt. Eine grundsätzliche Herausforderung bestand darin, unterschiedliche Erkenntnisse aus verschiedenen wissenschaftlichen Disziplinen nutzbar zu machen. Immerhin geht es hier um eine interdisziplinäre Analyse, die wenn auch primär darin verortet - nicht nur für die Sozialwissenschaften hilfreich sein soll. Deshalb wurde der Systembegriff eingeführt und damit auch die Systemtheorie als theoretische Basis für die deskriptiv-analytische Diskussion des Resilienz-Konzepts. Systemtheoretische Ansätze gibt es nicht nur in den Sozial- sondern auch in den Ingenieur- und Naturwissenschaften. Die Systemtheorie ist selbst grundsätzlich interdisziplinär oder besser über-disziplinär zu verstehen. Diese Möglichkeit hat die vorliegende Arbeit genutzt, um die Resilienz-Diskussionen aus verschiedenen Disziplinen in einem eigenständigen Konzept für die zivile SiFo zusammenzufüh- 
ren. Insbesondere durch die Verknüpfung der soziologischen Systemtheorie Luhmanns auf der einen Seite mit der sehr viel stärker ingenieur- bzw. naturwissenschaftlichen Theorie komplexer adaptiver Systeme auf der anderen Seite konnten so neuartige Erkenntnisse gewonnen und etablierte Ideen von Resilienz theoretisch noch stärker fundiert werden. So ließ sich dann insgesamt festhalten, dass Resilienz als systemtheoretisches Konzept verstanden werden kann. Welche Implikationen hat das für Resilience Engineering?

Zunächst sollten ingenieurwissenschaftliche Ansätze und Arbeiten in der zivilen SiFo von Anfang an berücksichtigen, um welche Art Konzept oder Definition von Resilienz es sich handelt. Dazu kann auf einen Artikel von Ayyub verwiesen werden, der zwischen „abstraktiven“ (abstractive) und "interpretativen" (interpretive) Definitionen unterscheidet (Ayyub 2015: 2). Das Verständnis dessen, was eine Definition ausmacht und welche Arten es gibt, ist sozusagen eine Wissenschaft für sich. Für den Zweck, den die vorliegende Arbeit an dieser Stelle verfolgt, bieten Ayyubs Bezeichnungen aber eine gute Grundlage. Eine abstraktive Definition ist ihm zufolge eine, die keine Zweideutigkeiten zulässt und die nur durch den Nachweis eines logischen Widerspruchs widerlegt werden kann. Mathematische Definitionen fallen für ihn in diesen Bereich. Und auch Metriken sind abstraktive Definitionen. Interpretative Definitionen sind hingegen primär deskriptiv, für eine generischere Nutzung geeignet, mit unterschiedlichen Graden an Nützlichkeit und Wert verbunden (Ayyub 2015: 2). Übertragen auf wissenschaftliche Konzepte lassen sich abstraktive Definitionen mit quantitativen Konzepten vergleichen und interpretative Definitionen eher mit qualitativen. Für Resilience Engineering, das auf dem eigenständigen Resilienz-Konzept der zivilen SiFo beruht, ist es von Bedeutung, die Art dieses Konzepts explizit in Rechnung zu stellen und zu adressieren. Resilienz hängt in vielfacher Hinsicht mit Komplexität zusammen und das schlägt sich selbstverständlich auch in den Hypothesen für Resilience Engineering nieder. Zunächst ist Resilienz, wie als Ergebnis der Analyse in 5.1 dargestellt, selbst ein komplexes Konstrukt. Aufgrund der unterschiedlichen, aufeinander bezogenen und wechselseitig voneinander abhängigen Bestandteile, kann es nur in einer Gesamtbetrachtung verstanden werden. Deshalb wurde auch bewusst auf die Aufstellung einer (weiteren), knappen Definition von Resilienz verzichtet. Auch die unter der Leitfrage woraus Resilienz besteht aufgeführten Bestandteile sind für sich genommen - ohne die zugrundeliegenden Begrifflichkeiten, ohne die Darstellung der Notwendigkeit von und der notwendigen Bedingungen für Resilienz oder ohne die normative Einordnung - nicht dazu geeignet, 
als Definition sozusagen alle weiteren Erkenntnisse zusammenzufassen (siehe 5.3). Bezogen auf die Frage nach der Art des Konzepts kann die Antwort also nur lauten, dass das Resilienz-Konzept der zivilen SiFo notwendig qualitativ und interpretativ sein muss. Resilience Engineering im Verständnis der vorliegenden Arbeit erfordert eine explizite Berücksichtigung dieser Tatsache. Die Verwendung einer verkürzten Arbeitsdefinition wie etwa der des mit dem Begriff engineering resilience verbundenen bounce back in einen ursprünglichen Gleichgewichtszustand, als Grundlage für eine ingenieurwissenschaftliche Umsetzung ist sicher attraktiv, da sie im system- und komplexitätstheoretischen Sinn „einfacher" ist, also lineare Kausalitäten und deterministisches Systemverhalten als Annahmen möglich macht. Sie widerspricht aber allen Erkenntnissen der vorangegangenen Analyse. Insofern sollte für Resilience Engineering folgende Hypothese gelten:

Hypothese H2: Resilienz ist ein qualitatives weil komplexes Konzept, dessen ingenieurwissenschaftliche Umsetzung dies explizit zu berücksichtigen hat.

Diese Überlegungen führen noch zu einer weiteren Hypothese. Wenn Resilienz sich nicht mittels einer einfachen Arbeitsdefinition fassen lässt. Wenn das Konzept komplex ist und demnach emergente, erst auf Konzeptebene wirksame Eigenschaften aufweist. Und wenn es in einer Verknüpfung deskriptiv-analytischer und normativer Bestandteile besteht. Dann lässt es sich nicht „einfach“ messen oder quantifizieren. Die Dominanz von Artikeln, die versuchen, Resilienz zu quantifizieren, wurde für die Ingenieurwissenschaften deutlich nachgewiesen (siehe 2.6). All diese Ansätze verfolgen eine völlig nachvollziehbare Zielstellung. Um eine Systemeigenschaft zu verändern, müssen Ingenieure zunächst wissen, welche Ausprägung diese Eigenschaft aktuell annimmt. Wie hoch, tief, klein, groß, gut, schlecht, rot, grün oder gelb sie ist. Das gilt so auch für Resilienz. Die ingenieurwissenschaftliche Begründung ist, dass die Resilienz eines Systems erst dann zielgerichtet verändert, meist erhöht, werden kann, wenn sie auch messbar ist. Und daher entwickeln die Ingenieurwissenschaften die unterschiedlichsten Ansätze, um Resilienz zu messen. Wobei diese Ansätze trotz ihrer Unterschiedlichkeit in vielen Fällen darauf hinauslaufen, ein einzelnes Maß für Resilienz zu definieren, zum Beispiel $R$. Dann gilt es für sie herauszufinden, wie hoch die Resilienz $R$ des Systems zu einem Zeitpunkt i ist: $R_{i}=X$. Wobei $X$ eine natürliche Zahl sein kann, häufiger aber in Prozenten ausgedrückt wird. Ein solches Maß kann durchaus hilfreich sein, um Systeme bewertend zu vergleichen und daraufhin über Maßnahmen zu entscheiden, welche das Maß in eine gewünschte Rich- 
tung zu verändern in der Lage sind. So wie Resilienz in der vorliegenden Arbeit verstanden wird, kann sie so aber nicht gemessen werden. Dazu vernachlässigen derart einfache Maße zu viele Dimensionen des komplexen Resilienz-Konzepts und verunmöglichen so, dass Resilienz seinen eigentlichen Mehrwert, der ja unter anderem gerade in der Akzeptanz von Komplexität, Unsicherheit und Mehrdeutigkeit besteht, ausspielen kann. Selbstverständlich müssen Systemzustände auch weiter gemessen werden. Resilience Engineering im Sinne der vorliegenden Arbeit verzichtet aber bewusst darauf, einfache Maße für Resilienz zu suchen. Die zugehörige Hypothese lautet:

Hypothese H3: Es ist nicht möglich, die Resilienz der für die zivile SiFo relevanten, komplexen adaptiven, sozio-technischen Systeme mittels einfacher Maße zu quantifizieren. Nichtsdestoweniger werden Maße für einfacher zu erhebende Größen benötigt, um den Systemzustand einschätzen und Resilienz erböhen zu können.

Mit Blick auf diese Hypothese sowie die Annahme, dass Resilienz ein systemtheoretisches Konzept ist, stellt sich für die Ingenieurwissenschaften zudem noch eine weitere, entscheidende Frage: Um welches System geht es? Gegeben seine vielfältige, interdisziplinäre Verwendung, ist der Systembegriff an sich bereits ein recht schwammiges Konzept (Hollnagel/Fujita 2013: 14). Trotzdem basieren alle Analysen der vorliegenden Arbeit auf ihm. Komplexe adaptive Systeme haben bestimmte Bestandteile, die bestimmte Verhaltensregeln befolgen. Es gibt aber immer eine Systemumwelt, etwas, was nicht zum System gehört. Die Systemumwelt besteht ihrerseits wieder aus weiteren Systemen, für die das gerade betrachtete System Umwelt ist. Im Hinblick auf Resilienz geht es immer darum zu fragen, wessen Resilienz untersucht bzw. mithilfe zielgerichteter Maßnahmen erhöht werden soll. Es muss also immer ein interessierendes System definiert werden (Francis/Bekera 2014: 92). Mit der Definition des Systems geht gleichzeitig die Notwendigkeit einher, Systemgrenzen abzustecken (Connelly et al. 2017: 47). Nur, wenn ein System über eindeutig festgelegte Grenzen verfügt, ist Resilience Engineering möglich. Natürlich sind Systemgrenzen in den für die zivile SiFo relevanten Systemen im Wesentlichen als artifizielle Konstrukte anzusehen (IRGC 2018: 21). Ohne Systemgrenzen ist aber keine Analyse möglich. Die Herausforderung für Resilience Engineering besteht nun darin, die „richtigen“ Systemgrenzen zu ziehen. Wird das System zu eng definiert, bleiben wichtige Einflussfaktoren außen vor und es können keine Erkenntnisse gewonnen werden, die dazu beitragen, seine Resilienz zu erhöhen. Werden die Systemgrenzen 
aber zu weit gesteckt, überfordert die daraus resultierende Komplexität der Einflussfaktoren jede ingenieurwissenschaftliche Methode. Die Kunst besteht also in der Wahl geeigneter Systemgrenzen einerseits. Hier setzen die vorhandenen Methoden und Modelle den Ingenieurwissenschaften Grenzen, die sie aber durch beständige Neu- und Weiterentwicklung derselben verschieben können. Andererseits gehört zu Resilience Engineering aber auch das Eingeständnis, dass die Wahl der Systemgrenzen einen signifikanten Einfluss auf jede Analyse hat. Mit dem Setzen von Systemgrenzen sind also Entscheidungen verbunden. Diese Entscheidungen sollten bewusst getroffen werden. Und sie sollten transparent getroffen werden. Gleichzeitig sollten Systemgrenzen als analytisches Instrument verstanden und nicht mit der Wirklichkeit verwechselt werden. Beispielsweise ist die Forderung nach der Verkleinerung von Systemen, um das Ausmaß widriger Ereignisse zu begrenzen, angesichts zunehmender Vernetzung und steigender Komplexität kaum zu erfüllen (Helbing 2013: 55). Als Hypothese formuliert, kann für Resilience Engineering gesagt werden:

Hypothese H4: Die bewusste und transparente Definition von Systemgrenzen als analytisches Instrument ist eine Voraussetzung für Resilience Engineering.

\section{Sozio-technische Systeme sind die Träger von Resilienz.}

Innerhalb der zivilen SiFo sind es vor allem sozio-technische Systeme, die von Interesse sind. Das wurde in der vorangegangenen Analyse deutlich. Diese sozio-technischen Systeme sind dann auch die Träger von Resilienz. Das hängt natürlich direkt mit der gerade geschilderten Notwendigkeit nach der Setzung geeigneter Systemgrenzen zusammen. Es ist ohne weiteres möglich, Systeme rein technisch - oder rein sozial - zu definieren. Ein augenfälliges Beispiel dafür sind Flugzeuge. Technisch betrachtet sind Flugzeuge äußerst komplizierte Systeme, mit einer Vielzahl an technischen Sicherheitsmechanismen, die das Flugzeug insgesamt zu einem der sichersten Verkehrsmittel haben werden lassen. Flugzeughersteller wie Boeing und Airbus arbeiten kontinuierlich daran, die Sicherheit mithilfe immer neuer Technologien noch weiter zu erhöhen und Abstürze, die zwar sehr selten vorkommen, dann aber fast immer den Tod aller Passagiere zur Konsequenz haben, zu verhindern. Ein rein technisches Verständnis des Systems kann allerdings auch zu unintendierten, negativen Konsequenzen führen, was darauf hinweist, dass die Systemgrenzen unter Umständen ungeeignet gewählt waren. Im Oktober 2018 und im März 2019 stürzten zwei Passagierflugzeuge vom Typ Boeing 737 Max ab. Dabei starben 346 Menschen. Die Ursachensuche liefert einen Hinweis darauf, dass die ver- 
antwortlichen Ingenieure bei Boeing beim Entwurf des Flugzeugs und seiner Sicherheitsmechanismen implizit ein rein technisches System vor Augen hatten und somit die menschliche Komponente, die Piloten, zu stark außer Acht ließen. Denn ersten Erkenntnissen zufolge, könnte eine Software namens "Maneuvering Characteristics Augmentation System“ (MCAS), die eigentlich dazu gedacht ist, einen Strömungsabriss zu verhindern und um dies zu erreichen, aktiv in die Steuerung des Flugzeugs eingreift, mitverantwortlich für die Abstürze sein. Die Piloten wurden nach Medienberichten unzureichend oder sogar gar nicht im Hinblick auf die Charakteristika dieser Software geschult, so dass sie in den konkreten Notfällen nicht in der Lage waren, die Flugzeuge in der Luft zu halten. ${ }^{40}$

Unabhängig davon, was weitere Untersuchungen zu diesen beiden Unglücken ergeben, eignen sie sich als Argumentationsgrundlage für eine Hypothese zu Resilience Engineering, die explizit den sozio-technischen Charakter der interessierenden Systeme betont. Trotz zunehmender Automatisierung aufgrund des technischen Fortschritts ist es sowohl empirisch zutreffend, als auch theoretisch sinnvoll, dass es einen „human in the loop" gibt (Jackson/Ferris 2013: 5). Menschen sind grundsätzlich lern- und anpassungsfähig, sie können improvisieren und kreativ sowie flexibel auf Situationen reagieren, die sie zuvor noch nicht erlebt haben (Ouedraogo et al. 2013: 26). All das sind Eigenschaften, die für die Resilienz eines Systems förderlich sind. Schon allein aus diesem Grund erscheint es sinnvoll, Menschen als Teil der interessierenden sozio-technischen Systeme in der SiFo zu begreifen. Zumal, wenn etwa die Anpassungsfähigkeit eines technischen Systems darin besteht, dass beschädigte Teile ersetzt werden (Altherr et al. 2018: 193). Aber auch unabhängig davon, ob es theoretisch zur Erhöhung der Resilienz von Systemen sinnvoll ist, wenn Menschen ein Teil davon sind, kann Resilience Engineering in der zivilen SiFo empirisch kaum davon absehen. Das Beispiel des Flugzeugs hat es gezeigt. Ohne Berücksichtigung der Piloten laufen Ingenieure Gefahr, Systeme im Hinblick auf eine vermeintlich technisch herstellbare Sicherheit hin zu optimieren, dabei aber gleichzeitig ihre Resilienz zu vermindern. Ein noch weit komplexeres Beispiel ist das Energiesystem. Dieses besteht natürlich zum einen aus rein technischen Bestandteilen, von Generatoren über Hochspannungsleitungen bis hin zu Steckdosen. Allerdings werden Kraftwerke von Menschen bedient, Strom von Menschen für unterschiedlichste Zwecke verbraucht und über die Ausgestaltung des Energiesystems

40 https:/www.spiegel.de/wissenschaft/mensch/boeing-737-max-von-ethiopian-airlines-erster-untersuchungsbericht-liegt-vor-a-1260637.html [Stand: 28.6.2019]. 
politisch durch Menschen entschieden. Das heißt nicht, dass Resilience Engineering immer dieses überkomplexe Gesamtsystem in den Blick nehmen sollte, um Mechanismen zur Erhöhung der Resilienz des Energiesystems zu entwickeln. Das ist durchaus auch auf kleineren Skalen möglich und sinnvoll. Es heißt aber doch, dass Resilience Engineering nicht sinnvollerweise rein technisch gedacht werden kann, bzw. zumindest immer mindestens näherungsweise in Form von Modellen auch menschliches Verhalten mitberücksichtigen sollte:

Hypothese H5: Dass die für die zivile SiFo relevanten Systeme sozio-technisch zu verstehen sind, macht es für Resilience Engineering notwendig, Menschen und menschliches Verhalten explizit mit zu berücksichtigen.

\section{Resilienz ist eine Nicht-Selbstverständlichkeit, sie ist nicht erwartbar.}

Diese Erkenntnis stammte primär aus der Psychologie, ließ sich aber auch auf die zivile SiFo übertragen. Wenn etwas außergewöhnlich Schlimmes passiert, mit dem zuvor niemand gerechnet hatte und das die eigentlichen Belastungsgrenzen des Systems übersteigt, ist rein logisch von einem $\mathrm{Zu}-$ sammenbruch des Systems auszugehen. Exakt so ergeht es auch einfachen Systemen, wenn diese über ihre Belastungsgrenzen hinaus beansprucht werden. Sie haben keine Möglichkeit, sich derart außergewöhnlichen Belastungen anzupassen. Komplexe adaptive Systeme verfügen dagegen über Anpassungsfähigkeit und sind damit grundsätzlich in der Lage, auch mit solchen Ereignissen fertig zu werden. Trotzdem ist das nicht selbstverständlich. Kammenhuber et al. beschreiben das treffend am Beispiel des Internets: „Ein ,resilientes“ Internet würde uns demnach erlauben, noch Meldungen bei www.heise.de zu lesen, auch wenn der entsprechende Webserver gerade einen Denial-of-Service-Angriff erleidet, bei Baggerarbeiten versehentlich ein wichtiges Backbone-Kabel durchtrennt wird, unser WLAN von dem des Nachbarn gestört wird oder wenn all das gleichzeitig geschieht“ (Kammenhuber et al. 2010: 132). Wenn all diese Störungen zeitgleich zutreffen, geht die intuitive Erwartung in Richtung einer Unerreichbarkeit der Webseite. Verfügt der Betroffene jedoch über ein Smartphone mit mobilem Internet über das Mobilfunknetz während die Betreiber der betreffenden Webseite gleichzeitig über eine redundante Serverarchitektur verfügen, die sowohl eine örtliche Trennung aufweist als auch funktional unterschiedliche Anfälligkeiten gegenüber einer Denial-of-Service-Attacke, steht einer weiteren Verfügbarkeit der Webseite nichts entgegen. Das kurze Beispiel zeigt aber: diese ist voraussetzungsreich und erfordert die Umsetzung unterschiedlicher technischer Maßnahmen. Welchen Prinzipien diese Maßnahmen folgen sollten, um die Resilienz des Systems zu 
erhöhen, wird in 6.5 aus den system- und komplexitätstheoretischen Annahmen des Konzepts abgeleitet. Daraus ergeben sich dann entsprechend weitere Hypothesen für Resilience Engineering.

Resilienz ist nicht das Gegenteil von Vulnerabilität. Die beiden Konzepte sind unabhängig voneinander. Größere Resilienz führt nicht notwendigerweise zu im Zeitverlauf sinkender Vulnerabilität.

Vulnerabilität ist so, wie es in der vorliegenden Arbeit verstanden wurde, selbst ein multidimensionales Konzept. Nichtsdestotrotz existieren im Vergleich zu Resilienz für Vulnerabilität mehr und vor allen Dingen etabliertere Wege zur Messung und Quantifizierung (Fekete et al. 2014: 14). Es scheint insofern für ein Resilience Engineering sinnvoll, sich an derartigen Maßen, wie sie etwa Cutters SoVI darstellt, zu orientieren und sie für die eigene Arbeit zu nutzen und/oder weiterzuentwickeln. Dies trifft umso mehr mit Blick auf Hypothese $\mathrm{H} 3$ zu. Vulnerabilität als physische, soziale, ökonomische und umweltbezogene Ausgesetztheit eines Systems gegenüber widrigen Ereignissen könnte eines der Maße sein, mit deren Hilfe Resilience Engineering arbeiten kann, ohne Resilienz selbst messen zu müssen. Dabei gilt es stets zu beachten, dass Resilienz und Vulnerabilität den system- und komplexitätstheoretischen Erkenntnissen zufolge tatsächlich unabhängig voneinander in Systemen existieren können. Hohe Resilienz und niedrige Vulnerabilität können genauso gemeinsam auftreten wie niedrige Resilienz und niedrige Vulnerabilität und alle weiteren Kombinationen von Resilienz und Vulnerabilität, die durch eine Verknüpfung zweier Kontinuen denkbar sind. Daraus ergeben sich Überlegungen für eine weitere Hypothese zu Resilience Engineering, die im nächsten Unterkapitel weitergeführt werden. Dort geht es um die Frage, wie Resilienz und Vulnerabilität, trotz der theoretischen Unabhängigkeit voneinander, doch in Form einer Notwendigkeit zusammenhängen.

Resilienz ist kein neoliberales Paradigma.

Wenn Resilienz als neoliberales Paradigma bezeichnet wird, so ist damit in den meisten Fällen eine normativ negative Bewertung verknüpft. Dem Konzept wird sozusagen der Vorwurf gemacht, den - als negativ empfundenen - Neoliberalismus unter dem Deckmäntelchen eines attraktiven Begriffs in gesellschaftliche Bereiche einzuführen, nämlich die zivile Sicherheitsforschung bzw. den Bevölkerungs- und Katastrophenschutz, in denen er aus Sicht derjenigen, welche die Vorwürfe erheben, nichts zu suchen habe. Die ausführliche Analyse der vorliegenden Arbeit hat jedoch ergeben: Resilienz ist gerade kein neoliberales Paradigma. Der Vorwurf 
geht bereits in dieser Hinsicht fehl. Welche detaillierteren normativen Konsequenzen das für Resilience Engineering hat, wird in 6.6 diskutiert. Grundsätzlich lässt sich aber festhalten, dass ingenieurwissenschaftliche Resilienzforschung den Vorwurf, sie leiste einer impliziten Verbreitung des Neoliberalismus Vorschub, mit Verweis auf das Resilienz-Konzept der vorliegenden Arbeit kontern kann. Darin findet sie eine fundierte Begründung, warum es keinen Sinn ergibt, Resilienz als neoliberales Paradigma zu verstehen. Insofern liefert die vorliegende Arbeit den Ingenieurwissenschaften an dieser Stelle eine Art theoretisches Rüstzeug, um eine Debatte mit sozialwissenschaftlichen Argumenten zu bestreiten. Im Fokus von Resilience Engineering steht dies allerdings nicht und eine Hypothese soll insofern an dieser Stelle nicht abgeleitet werden.

Resilienz ist nicht notwendigerweise normativ wünschenswert.

Diese Erkenntnis ist eine Grundlage, derer sich Resilience Engineering immer bewusst sein sollte. Gleichzeitig kann es nicht die Aufgabe der Ingenieurwissenschaften sein, über die normative Erwünschtheit der Resilienz des analysierten Systems zu urteilen. Weder liegt das in ihrem Erkenntnisinteresse noch sind ihre Methoden und Ansätze dazu geeignet, eine solche Bewertung vorzunehmen. Gleichwohl enthält das Resilienz-Konzept der zivilen SiFo eine ganze Reihe an normativen Bestandteilen, ohne deren Beachtung nicht von Resilienz gesprochen werden kann. Deshalb werden diese im Einzelnen in 6.6 näher diskutiert und wo geboten daraus auch Hypothesen für Resilience Engineering abgeleitet.

\subsection{Notwendigkeit für Resilience Engineering}

Das Vorhandensein oder Nicht-Vorhandensein von Resilienz lässt sich nur beobachten, wenn Systeme von einem disruptiven, in der Systemumwelt verorteten, gravierenden und häufig extrem unwahrscheinlichen Reiz (bzw. Ereignis) getroffen werden. Der Theorie komplexer adaptiver Systeme folgend, wird die Welt immer komplexer. Je komplexer die Welt, desto wahrscheinlicher wird das Auftreten eigentlich extrem unwahrscheinlicher, disruptiver Ereignisse. Die steigende Komplexität der Welt macht deshalb Resilienz als Eigenschaft komplexer adaptiver Systeme immer notwendiger.

\&

Neben Ereignissen, die aus der Systemumwelt resultieren, müssen komplexe adaptive Systeme auch mit dem Auftreten unsicherer interner, durch die Entscheidungen von Agenten ausgelöster, von diesen unintendierter oder intendier- 
ter Störungen umgehen können. Die Wahrscheinlichkeit des Auftretens solcher Störungen sowie ibre potentiellen Auswirkungen steigen mit der Eigenkomplexität der Systeme. Denn zum einen können Agenten die Konsequenzen ihrer eigenen Handlungen mit zunehmender Komplexität schlechter überblicken, was die Wabrscheinlichkeit für Unfälle steigert. Zudem steigt mit der Diversität des Systems die Wabrscheinlichkeit, dass Agenten auftreten, deren Ziele konträr zu denen des Systems laufen. Zum anderen erhöht sich die Wahrscheinlichkeit für das Auftreten von Kaskadeneffekten, die beim Eintreten widriger Ereignisse zu großflächigen und häufig langfristigen Schäden am System fübren. Um diese verhindern zu können, benötigt das System Resilienz. Auch die steigende Eigenkomplexität komplexer adaptiver Systeme macht Resilienz als Systemeigenschaft also immer notwendiger.

Die beiden gerade geschilderten Bestandteile des Resilienz-Konzepts, die eine zunehmende Notwendigkeit aufgrund der steigenden Komplexität der relevanten Systeme und ihrer Umwelt - die ja wieder aus Systemen besteht - kontastiert, können in Bezug auf Hypothesen für Resilience Engineering gemeinsam untersucht werden. Prinzipiell ist es egal, ob ein disruptives Ereignis seinen Ausgangspunkt außer- oder innerhalb des Systems hat. Unterschiedliche Entwicklungen führen für beide Fälle zu einer steigenden Wahrscheinlichkeit - auch wenn diese nicht quantitativ erfasst werden kann - für das Auftreten disruptiver Ereignisse. Und das ist der durch zunehmende Vernetzung der Systeme und wahrscheinlicher werdenden Kaskadeneffekten steigenden Komplexität geschuldet. Für Resilience Engineering ergibt sich daraus zunächst lediglich eine immer weiter steigende Notwendigkeit. Wenn Resilienz immer wichtiger wird, wird es auch immer wichtiger, geeignete ingenieurwissenschaftliche Ideen und Methoden zu entwickeln, mit deren Hilfe Resilienz gesteigert werden kann. Dieser Zusammenhang ist wichtig, er muss aber nicht in Form einer Hypothese verschriftlicht werden, da er auch bereits unmittelbar aus dem Resilienz-Konzept selbst ersichtlich wird.

Anders verhält es sich mit einer weiteren Annahme, die sich auf Basis des system- und komplexitätstheoretischen Verständnisses von Resilienz ergibt. Resilienz wurde und wird immer wieder als holistisches Konzept verstanden, dass in einem zyklischen Ansatz alle unterschiedlichen Phasen im Hinblick auf ein disruptives Ereignis umfasst. Das hat seinen Ursprung in klassischen Kreisläufen aus dem Bereich des KatastrophenManagements und wurde auch und gerade vom Autor der vorliegenden Arbeit immer wieder in eigenen Veröffentlichungen aufgeführt (Hiermaier/Scharte 2018: 302, Scharte et al. 2014: 17, Scharte/Thoma 2016: 128, 2015: 32, Thoma et al. 2016: 6). Demnach besteht Resilienz aus den fünf 
Phasen „prepare“, „prevent", „protect“, „respond“ und „recover“ (siehe Abbildung 4). In jeder dieser Phasen sollen Maßnahmen ergriffen werden, um die Resilienz von Systemen zu erhöhen. „Zunächst geht es um eine ernsthafte Vorbereitung auf Katastrophen, vor allem im Hinblick auf Frühwarnsysteme (prepare). Durch eine Reduzierung der zugrundeliegenden Risikofaktoren soll zudem - sofern möglich - das Eintreten des Ereignisses an sich verhindert werden (prevent). Tritt es trotzdem ein, kommt es darauf an, dass physische und virtuelle Schutzsysteme fehlerfrei funktionieren und die negativen Auswirkungen gering halten (protect). Zudem wird schnelle, gut organisierte und effektive Katastrophenhilfe benötigt. Hierbei muss das System - soweit möglich - seine essenzielle Funktionsfähigkeit aufrechterhalten können (respond). Nach dem Ende des unmittelbaren Schadensereignisses ist es wichtig, dass das System in der Lage ist, sich zu erholen und entsprechende Lehren aus dem Geschehen zu ziehen, um für künftige Bedrohungen besser gerüstet zu sein (recover)" (Scharte et al. 2014: 17f, eigene Hervorhebung). Ein dergestalt holistischer Charakter wurde von Scharte et al. in und anderen Artikeln des Autors sogar als der „zentrale Mehrwert, den die Verwendung des Begriffs der deutschen Gesellschaft langfristig bieten kann“ bezeichnet. Sein „Kern besteht demnach darin, dass resiliente Gesellschaften in der Lage sind, die menschlichen, ökonomischen und ökologischen Schäden, die durch widrige Ereignisse verursacht werden, so gering wie irgend möglich zu halten. Sie schaffen das, indem sie sich jedweder denk- und durchführbarer Lösungen bedienen“ (Scharte et al. 2014c: 123). 
Abbildung 4: Der Resilienz-Zyklus

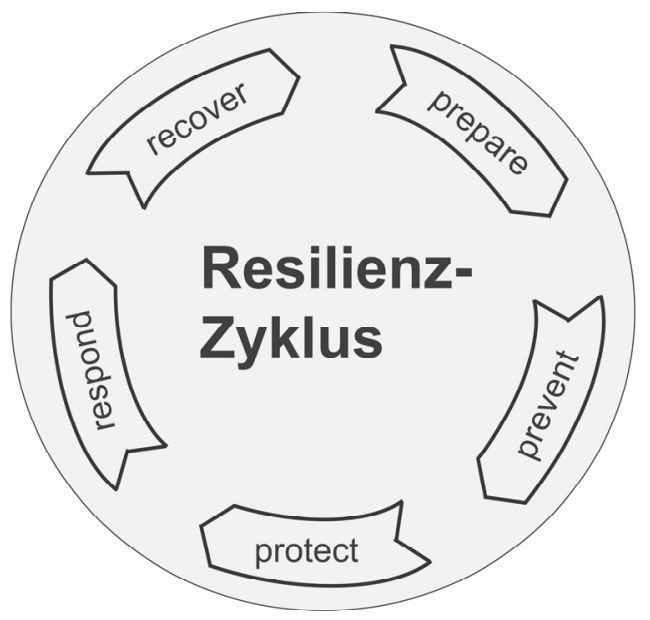

Quelle: eigene Darstellung nach Scharte et al. 2014.

Diese Aussage lässt sich nach der system- und komplexitätstheoretischen Analyse von Resilienz in der vorliegenden Arbeit nicht länger aufrechterhalten. Der zentrale Mehrwert von Resilienz besteht vielmehr in der theoretischen und praktischen Akzeptanz von Komplexität und Unsicherheit und den darauf basierenden Annahmen im Hinblick darauf, wann Resilienz notwendig ist, was für Resilienz notwendig ist und worin Resilienz besteht. Und als Situation, in der Resilienz notwendig wird, wurde gerade das Eintreten eines disruptiven Ereignisses bezeichnet. Nur wenn und erst nachdem ein derartiges Ereignis eintritt, kann sich Resilienz zeigen. Das führt ganz unmittelbar dazu, dass Prävention und präventive Maßnahmen kein Teil von Resilienz und damit auch nicht Teil von Resilience Engineering sein können. Der Hintergrund dessen ist nicht etwa ein Unwillen, widrige Ereignisse und Störungen zu verhindern. Wo immer dies möglich ist, sollte es unbedingt geschehen. Es geht lediglich darum, für das Konzept der Resilienz ein Mindestmaß an Trennschärfe zu entwickeln. Und diese besteht unter anderem darin, explizit zu akzeptieren: Prävention ist nicht immer und in allen Fällen möglich (Connelly et al. 2017: 46). Überraschungen, schwarze Schwäne, unexampled events treten auf und Systeme müssen damit umzugehen lernen. Genau dabei kann Resilienz helfen. Für Resilience Engineering ergibt sich folgende Hypothese: 
Hypothese H6: Prävention und präventive Maßnabmen sind kein Bestandteil von Resilience Engineering.

Diese Hypothese soll keinesfalls die Nutzlosigkeit präventiver Maßnahmen implizieren. Sie sind lediglich nicht als Teil von Resilience Engineering zu sehen, wie der Begriff im Rahmen der vorliegenden Arbeit verstanden wird. Mit der Hypothese $\mathrm{H} 6$ ist zudem ebensowenig verbunden, dass Resilience Engineering immer erst nach Eintreten eines disruptiven Ereignisses erfolgen kann. Das glatte Gegenteil ist der Fall. Zumal zunächst festzuhalten ist: komplexe adaptive Systeme sind dynamische Systeme. Sie entwickeln sich ständig in Anpassung an veränderte Umweltbedingungen weiter und erleben im Lauf ihrer Existenz teilweise multiple, ähnliche oder völlig unterschiedliche, gravierende oder weniger gravierende, überraschende oder erwartete, widrige Ereignisse und Störungen. Das gilt definitiv auch für die sozio-technischen Systeme, die in der zivilen SiFo eine Rolle spielen. Diese sind ja gerade komplexe adaptive Systeme und in vielen Fällen auf eine kontinuierliche Existenz ausgelegt, um gesellschaftlich relevante Funktionen auszufüllen. Dazu kommt noch die Komplexität, Unvorhersehbarkeit und Dynamik der Umwelt der betreffenden Systeme. All das zusammengenommen lässt sich häufig nur schwerlich zwischen einem Zeitpunkt vor einem disruptiven Ereignis und einem Zeitpunkt danach unterscheiden. Das davor eines Ereignisses, stellt häufig auch das danach eines zeitlich früher gelagerten Ereignisses dar. Schaffer und Schneider sprechen hier von einem Verwischen der Differenzierung (Schaffer/Schneider 2019: 23). Wenn es keine eindeutige Trennung zwischen davor und danach gibt, kann auch nicht zwischen proaktiven und reaktiven Maßnahmen zur Erhöhung von Resilienz unterschieden werden. Resilience Engineering kann also schon alleine aus diesem Grund auch und gerade vor dem Eintreten widriger Ereignisse erfolgen.

Dieser Gedanke ist auch konsistent mit dem Resilienz-Verständnis von ingenieurwissenschaftlichen Forschern wie Bruneau. Als Erdbebenforscher beschäftigt er sich mit einer Art disruptiver Ereignisse, die nach menschlichem Ermessen tatsächlich nicht verhindert werden kann. Trotzdem dürfen die Ingenieurwissenschaften Bruneau et al. zufolge nicht einfach abwarten, bis ein disruptives Ereignis eintritt und dann nach Wegen suchen, die Resilienz der betroffenen Systeme zu erhöhen. Die entsprechend ingenieurwissenschaftliche Resilienzforschung, das Resilience Engineering, muss vielmehr vor Eintreten eines widrigen Ereignisses erfolgen (Bruneau et al. 2003: 744). Die erforschten Maßnahmen unterscheiden sich dann lediglich bezüglich des Zeitraums in dem bzw. für den sie wirksam werden oder in dem sie angewendet werden. Auch wenn das von Bruneau et al. 
genannte Beispiel erhöhter Erdbebensicherheit mithilfe bauphysikalischer Maßnahmen (seismic retrofit) aufgrund seiner Verhaftung im Bereich spezialisierter Anpassungsfähigkeit nicht unter das Resilienz-Verständnis der vorliegenden Arbeit fällt, ist sein Gedanke passend (Bruneau et al. 2003: 744). Resilience Engineering besteht aus Maßnahmen, die unabhängig vom konkreten disruptiven Ereignis wirken sollen. Und der Zeitpunkt wie auch die Ausprägung des nächsten disruptiven Ereignisses sind inhärent unsicher. Somit befinden sich komplexe adaptive Systeme qua definitionem immer vor einem nächsten, disruptiven Ereignis. Es besteht demzufolge immer die Notwendigkeit für Resilience Engineering. Gleichzeitig besteht aber auch immer die Möglichkeit für Resilience Engineering. Von der Unmöglichkeit zur Prävention aller disruptiven Ereignisse abgesehen, kann Resilience Engineering zu allen Phasen des Resilienz-Zyklus beitragen, von prepare und protect zu respond und recover. Der Beitrag besteht dann allerdings weniger etwa in der Umsetzung klassischer Schutzmaßnahmen gegen spezifische Bedrohungen in der protect-Phase, sondern stärker in der Frage, welche generischen Schutzmaßnahmen gegen alle Arten von Bedrohungen helfen könnten. Maßnahmen zur Erhöhung der Resilienz in diesen unterschiedlichen Phasen können und müssen alle bereits vor dem nächsten widrigen Ereignis umgesetzt werden. Ihnen kann theoretisch eine Resilienz erhöhende Wirkung zugewiesen werden. Inwiefern sie tatsächlich auch empirisch zur Erhöhung der Resilienz beigetragen haben, lässt sich aber erst ex post feststellen. Für Resilience Engineering ergibt diese Diskussion folgende Hypothese:

Hypothese H7: Resilience Engineering erfolgt bereits vor dem Eintreten eines disruptiven Ereignisses und trägt zur Erhöhung von Resilienz in den Phasen prepare, protect, respond und recover bei.

Um Risiken zu bewältigen, benötigen Systeme keine Resilienz. Das Ereignis an sich, die Wahrscheinlichkeit seines Auftretens und seine Auswirkungen auf das System sind bekannt, so dass spezifische Maßnahmen zum Umgang mit dem Risiko ausreichen.

Risiko ist eine der zentralen Begrifflichkeiten der vorliegenden Arbeit. Wie die kurzen Erläuterungen des jeweiligen Stands der Forschung in den Sozial- wie Ingenieurwissenschaften gezeigt haben, gibt es dabei durchaus unterschiedliche Verständnisse mit einer unterschiedlichen Reichweite (siehe 2.4 und 2.6). Gerade Forscher wie Helbing oder Renn, die von systemischen und global vernetzten Risiken sprechen, oder Steen und Aven, die Unsicherheit statt Wahrscheinlichkeit als Schlüsselkonzept von Risiko bezeichnen, verstehen unter Risiko sehr viel mehr und etwas dezidiert 
anderes, als es in der klassischen, quantitativen Risikoanalyse der Fall ist (Helbing 2013: 51, Renn 2016: 30f, Steen/Aven 2011: 293). Nichtsdestoweniger dominieren die Ideen, die der quantitativen Risikoanalyse zugrunde liegen, in der ingenieurwissenschaftlichen SiFo immer noch an vielen Stellen. Genau aus diesem Grund, um den Unterschied zwischen einem klassischen, ingenieurwissenschaftlichen Verständnis von Risiko auf der einen Seite und einem systemischen Verständnis von Resilienz auf der anderen Seite herausarbeiten zu können, bedient sich die vorliegende Arbeit einer derart klassischen Definition von Risiko. Basierend auf Unterscheidungen aus den Wirtschaftswissenschaften wird Risiko als eine von drei Arten von Unsicherheit charakterisiert und sozusagen als vergleichsweise geringe Unsicherheit verstanden. Die Wahrscheinlichkeit für das Auftreten von Ereignissen, die in die Kategorie Risiko fallen, ist ebenso bekannt wie ihre Auswirkungen. Davon zu unterscheiden sind ungewisse und echt unsichere Ereignisse, die zusammenfassend als unerwartete Ereignisse bezeichnet wurden. In Übereinstimmung beispielsweise mit Linkov et al. wurde dann in der system- und komplexitätstheoretischen Analyse deutlich, dass Systeme zur Bewältigung von Risiken gerade keine Resilienz benötigen (Linkov et al. 2014: 407ff). Aufgrund von deren determinierter Erwartbarkeit und den ebenfalls determinierten Auswirkungen, lassen sich Risiken a priori mithilfe spezifischer Vorbereitungsmaßnahmen adressieren. Und spezifische Vorbereitungsmaßnahmen sind nicht Bestandteil von Resilienz.

Für Resilience Engineering ergibt sich daraus, dass Methoden zur Entwicklung spezifischer Schutzmaßnahmen nicht berücksichtigt werden. Äquivalent zum Thema Prävention ist damit in keiner Weise eine Geringschätzung spezifischer Schutzmaßnahmen verbunden. Im Gegenteil, wenn sich Risiken - in komplexen adaptiven Systemen immer nur näherungsweise - bestimmen lassen und gesellschaftlich als inakzeptabel eingeschätzt werden, gebietet sich ein klassisches Risikomanagement, bei dem das spezifische Risiko mittels spezifischer wirkender Maßnahmen zu minimieren versucht wird. Das gilt für viele Bedrohungen, deren Wahrscheinlichkeiten und mögliche Auswirkungen aufgrund historischer Erfahrungen gut bekannt sind, beispielsweise wiederkehrende Hochwasser an Flüssen. Ein geeigneter Hochwasserschutz in Form von Dämmen und Deichen ist hier ohne jeden Zweifel geboten. Es ist aber weder notwendig noch theoretisch vertretbar, derartiges Risikomanagement als Teil von Resilience Engineering zu verstehen. Selbiges gilt auch für andere Methoden, die ähnlich wie die quantitative Risikoanalyse auf einem linearen und deterministischen Systemverständnis beruhen. Sie als Teil von Resilience Engineering zu verstehen, kann dazu führen, relevante Faktoren und Un- 
sicherheiten zu vernachlässigen und so eine scheinbare Sicherheit auf Kosten der Resilienz zu produzieren (Rahimi/Madni 2014: 813, Steen/Aven 2011: 296). Ähnlich wie von Linkov et al. gefordert, ist es so auch möglich, dezidierter zwischen klassischem Risikomanagement und Resilience Engineering zu unterscheiden (Linkov et al. 2014: 407ff). Daraus ergeben sich gleich zwei Hypothesen für Resilience Engineering:

Hypothese H8: Die quantitative Risikoanalyse und ähnliche, linear und deterministisch arbeitende Methoden sind nicht dazu geeignet, $z u$ Resilience Engineering beizutragen.

Hypothese H9: Die Entwicklung bedrohungsspezifischer Schutzmaßnahmen ist kein Teil von Resilience Engineering.

Es gibt Risiken, die komplexe adaptive Systeme ganz bewusst eingehen, weil ibre Eintrittswahrscheinlichkeit extrem gering ist, aber gleichzeitig die Auswirkungen so groß, dass die Ausbildung spezialisierter Anpassungsfähigkeit sehr viele Ressourcen in Anspruch nehmen würde. Zur Bewältigung von Risiken, für die komplexe adaptive Systeme keine spezialisierte Anpassungsfähigkeit entwickeln und die ibre Belastungsgrenzen übersteigen, ist Resilienz notwendig.

Die Absolutheit der Hypothese H8 muss mit Blick auf diesen nächsten Bestandteil des Resilienz-Konzepts der zivilen SiFo ein Stück weit relativiert werden. Quantitative Risikoanalysen sind zwar nach wie vor kein Teil von Resilience Engineering. Wenn mit Hilfe quantitativer Risikoanalysen aber eine bestimmte Art von Risiken identifiziert wird, ergibt sich daraus wieder eine Notwendigkeit für Resilience Engineering. Diese Risiken sind diejenigen, die einerseits eine extrem geringe Eintrittswahrscheinlichkeit aufweisen, andererseits aber mit ebenfalls extremen, negativen Auswirkungen verknüpft sind. Aufgrund der extremen Schäden, die beim Eintreten des so charakterisierten Ereignisses zu erwarten wären, können Gesellschaften diese Art von Risiken nicht einfach ignorieren. Eine angemessene, spezifische Vorbereitung würde jedoch den Einsatz einer Vielzahl an Ressourcen verschlingen. Die extrem geringe Wahrscheinlichkeit lässt eine derartige Investition überwiegend unrentabel erscheinen. An dieser Stelle kommt die Möglichkeit von Resilienz ins Spiel und daher auch die Notwendigkeit für Resilience Engineering. Gerade, weil Resilienz einen unspezifischen, generischen Ansatz verfolgt, der szenario-unabhängig, vom konkreten Ereignis abstrahierend, funktioniert, ist eine Resilienz-orientierte Herangehensweise auch dazu geeignet, Risiken mit extrem geringer Eintrittswahrscheinlichkeit aber extrem großen Auswirkungen zu adressieren. Die generischen Maßnahmen und Mechanismen wirken eben unabhängig 
vom disruptiven Ereignis, auch wenn im Vorhinein bereits damit gerechnet wurde.

Das Argument könnte scheinbar konsequent zu Ende geführt werden, indem dann generell jedes konkrete Risikomanagement zugunsten von Resilience Engineering unterlassen werden könnte. Es wurde aber bereits dargelegt, dass das gerade nicht im Sinne der vorliegenden Arbeit ist, die Resilience Engineering als Ergänzung zu Risikomanagement versteht und nicht als Ersetzung. Genauso wie das Eintreten disruptiver Ereignisse a priori unsicher ist, können auch Maßnahmen und Mechanismen, die zur Erhöhung der Resilienz ergriffen wurden, nicht deterministisch als in jedem Falle in erwarteter Weise wirksam verstanden werden. Dahingegen wirken spezifische Maßnahmen auch in komplexen adaptiven Systemen durchaus, zumindest näherungsweise, häufig in der intendierten Weise. Risiken mittels spezifischer Maßnahmen zu adressieren, ergibt also auch weiterhin Sinn. Allerdings sollten komplexe adaptive Systeme im Bereich der zivilen SiFo nicht ausschließlich mithilfe von Risikomanagement sicherer gestaltet werden, da weder das Eintreten extrem unwahrscheinlicher, aber mit extremen negativen Auswirkungen verknüpfter Ereignisse ausgeschlossen werden kann, noch das Eintreten unerwarteter Ereignisse. Für Resilience Engineering heißt das:

Hypothese H10: Risiken mit extrem geringen Eintrittswabrscheinlichkeiten und extrem großen Auswirkungen können und sollten durch Resilience Engineering adressiert werden, da spezifische Vorbereitung aufgrund der Kostenintensität zumeist unterbleibt.

In komplexen adaptiven Systemen besteht die Möglichkeit, dass unerwartete Ereignisse mit gravierenden Auswirkungen, sogenannte schwarze Schwäne bzw. beispiellose Ereignisse auftreten. Zur Bewältigung solcher ungewissen und echt unsicheren Ereignisse, welche die Belastungsgrenzen komplexer adaptiver Systeme übersteigen, benötigen diese Resilienz.

In der vorliegenden Arbeit wurde der Zusammenhang zwischen Resilienz und Unsicherheit, der in der Resilienzforschung allgemein eine sehr große Rolle spielt, mithilfe der system- und komplexitätstheoretischen Analyse detailliert untersucht. Neben der Verknüpfung von Resilienz und extremen Risiken, wurde so auch die Verknüpfung zwischen Resilienz und unerwarteten, die eigentlichen Belastungsgrenzen der betroffenen Systeme übersteigenden Ereignissen offenbar. Damit ist es auch für Resilience Engineering wichtig zu diskutieren, was die Unsicherheit von Ereignissen und ihren Auswirkungen für ingenieurwissenschaftliche Resilienzforschung bedeutet. Generell zeigt sich in aktuellen Artikeln aus diesem Bereich 
eine Art Trend, Unsicherheit zumindest explizit anzuerkennen (siehe zum Beispiel Altherr et al. 2018). Teilweise wird dann auch an Methoden geforscht, welche mit (echter) Unsicherheit umzugehen in der Lage sind und Mechanismen entwickelt, die Unsicherheit in Rechnung stellen. Das kann sehr gut als Teil von Resilience Engineering verstanden werden und wird daher an späterer Stelle noch detaillierter diskutiert (siehe 6.5). Generell gilt für komplexe adaptive Systeme ein Hinweis von Madni und Jackson, den diese schon 2009 formulieren. Demnach sollen Systeme grundsätzlich mit adäquaten Sicherheitsmargen entwickelt werden, um mit Unsicherheit umgehen zu können (Madni/Jackson 2009: 182). Wobei mit Sicherheitsmargen nicht die eher statischen Toleranzen gemeint sein können, die es Systemen erlauben, Leistungsschwankungen bedingt durch kleinere Störereignisse innerhalb kurzer Zeit auszugleichen und in ihren Gleichgewichtszustand zurückzukehren. Sicherheitsmargen sind hier viel eher die ungenutzten Spielräume, von denen bereits in 4.3.2 und 4.3.5 die Rede war. Wachsmuth wählt mit einem ähnlichen inhaltlichen Verständnis den Begriff der „heuristischen Prinzipien“, die genutzt werden sollten, um Überraschungen zu begegnen (Wachsmuth 2014: 6). Aus diesen Überlegungen lässt sich für Resilience Engineering eine weitere Hypothese ableiten, die zentral für die später dargestellten, konkreten Prinzipien und Mechanismen ist. Sie stellt sozusagen deren Grundlage dar. Damit sind die Hypothesen für Resilience Engineering auch weiter konsistent zum Resilienz-Konzept der zivilen SiFo, für das ebenfalls Komplexität einerseits und Unsicherheit andererseits die entscheidenden Phänomene für die Notwendigkeit von Resilienz sind. Die resultierende Hypothese lautet:

Hypothese H11: Aufgrund der Unsicherheit von Ereignissen besteht ingenieurwissenschaftliche Resilienzforschung wesentlich aus der Entwicklung heuristischer generischer Prinzipien.

Um mit langfristigen Veränderungsprozessen erfolgreich umgehen zu können, benötigen komplexe adaptive Systeme keine Resilienz. Erst wenn die langfristigen Prozesse durch Erreichen eines Schwellenwertes ein unerwartetes und die Belastungsgrenzen des Systems überschreitendes Ereignis auslösen, wird Resilienz notwendig.

Dieser Bestandteil des Resilienz-Konzepts liefert in der Diskussion eine sehr interessante Hypothese für Resilience Engineering. Zunächst ist er nicht unstrittig, wird Resilienz doch von vielen Forschern als geeignet angesehen, sowohl langfristige Veränderungsprozesse als auch kurzfristige, abrupte Störungen zu adressieren. Gerade in der organisationswissenschaftlichen Resilienzforschung spielen solche Prozesse eine wichtige Rol- 
le (siehe 2.5). Aber auch in den Sozial- und Ingenieurwissenschaften ist die Ansicht durchaus verbreitet (siehe 2.4 und 2.6). Mit Blick auf die ökologische und sozial-ökologische Resilienzforschung und als Ergebnis der system- und komplexitätstheoretischen Analyse wurde nichtsdestotrotz deutlich, dass der erfolgreiche Umgang mit langfristigen Veränderungsprozessen gerade keine Resilienz im Sinne der vorliegenden Arbeit erfordert. Der lange Zeithorizont und die graduellen Veränderungsprozesse lassen sich sehr gut spezifisch und mittels sich langsam weiterentwickelnder, spezialisierter Anpassungsfähigkeit bewältigen. Die langfristigen Prozesse an sich sind für Resilience Engineering also nicht von Interesse. Ganz anders sieht es aber mit den sogenannten Umkipp-Punkten oder Schwellenwerten aus, an denen ein System plötzlich und unerwartet eine gravierende Änderung erfährt. Umkipp-Punkte und Schwellenwerte spielen in verschiedenen Bereichen der Resilienzforschung, vor allem in der Ökologie und der sozial-ökologischen Forschung, eine sehr wichtige Rolle (Walker et al. 2004). Auch in der vorliegenden Arbeit wurden sie bereits an verschiedenen Stellen erwähnt. Ein Weg, die Resilienz von Systemen zu erhöhen, besteht darin, ein Operieren des Systems nahe Schwellenwerten möglichst zu vermeiden. Das ist nur möglich, wenn die Schwellenwerte bekannt sind. Die Identifikation solcher Schwellenwerte ist in komplexen adaptiven Systemen aber alles andere als trivial. Bedingt durch die Eigenschaft der Emergenz bedarf es eines systemischen Blicks, um hier Fortschritte zu erzielen. Es gibt bereits einige Ansätze, die genau das versuchen. Rahimi und Madni sehen beispielsweise generische Frühwarnsignale am Werk. So gebe es eine kritische Verlangsamung der Rückkehr des Systems in seinen Gleichgewichtszustand nach leichten Störungen, wenn sich das System einem kritischen Schwellenwert nähere. Diese kritische Verlangsamung könne mit Hilfe von statistischen Phänomenen wie Autokorrelation und Varianz beobachtet werden. Rahimi und Madni sehen aber selbst eine Schwierigkeit darin, diese interessierenden statistischen Größen in komplexen Systemen zu erfassen (Rahimi/Madni 2014: 813). Auch Folke et al., aus der sozial-ökologischen Resilienzforschung kommend, sprechen davon, dass komplexe Systeme nahe Schwellenwerten sozusagen „stottern“ (stutter) oder eben eine größere Varianz aufwiesen. Mithilfe dieser größeren Varianz ließen sich Schwellenwerte evtl. im Vorhinein identifizieren (Folke 2006: 262). Nicht zuletzt ist es die Theorie komplexer adaptiver Systeme selbst, deren formale und mathematische Umsetzung Ideen enthält, mit deren Hilfe kritische Schwellenwerte in Systemen erkannt werden können. Insofern erfolgt an dieser Stelle eine Art Zirkelschluss. Die Theorie komplexer adaptiver Systeme wurde dazu genutzt, das Resilienz- 
Konzept der zivilen SiFo zu entwickeln. Gleichzeitig können die Theorie komplexer adaptiver Systeme und vor allem auch darauf basierende, formale und mathematische Modelle und Methoden selbst als Bestandteil bzw. mögliche Umsetzung von Resilience Engineering verstanden werden. Für Resilience Engineering folgt dann eine weitere Hypothese:

Hypothese H12: Die Theorie komplexer adaptiver Systeme und die daraus entwickelten mathematischen Modelle und Methoden können als Teil von Resilience Engineering genutzt werden, um Umkipp-Punkte oder Schwellenwerte $z$ identifizieren.

Je höher die Vulnerabilität eines komplexen adaptiven Systems, desto wabrscheinlicher und stärker übersteigen widrige Ereignisse die eigentlichen Belastungsgrenzen des Systems, bzw. desto stärker weicht das System initial von seinem eigentlich erwartbaren Entwicklungspfad ab, und desto größer muss ceteris paribus seine Resilienz sein, um erfolgreich mit dem Ereignis umgehen zu können.

Im vorangegangenen Unterkapitel wurde bereits auf die Möglichkeit verwiesen, Indikatoren für Vulnerabilität auch als Bestandteil von Resilience Engineering zu verstehen. Ein einfaches Maß für Resilienz kann durch Resilience Engineering nicht erreicht werden, das wurde ebenfalls bereits klar. Aber für Vulnerabilität gibt es einigermaßen etablierte Methoden zur Messung und Quantifizierung. Und gegeben den gerade geschilderten Teil des Resilienz-Konzepts, wonach höhere Vulnerabilität auch höhere Resilienz erfordert, lässt sich diese Idee noch untermauern. Steigende Vulnerabilität führt aufgrund der Unabhängigkeit der Konzepte ja gerade nicht notwendigerweise zu sinkender Resilienz. Resilienz kann auch bei steigender Vulnerabilität mithilfe von Resilience Engineering erhöht werden. Und je höher die Vulnerabilität eines Systems ist, desto stärker wird Resilienz benötigt. Wissen über die Vulnerabilität von Systemen und vor allen Dingen Wissen über die unterschiedliche Vulnerabilität unterschiedlicher Systeme ermöglicht es dann, sich auf die Systeme zu fokussieren, deren Bedarf an Resilienz aufgrund hoher Vulnerabilität ceteris paribus am größten ist. Für Resilience Engineering ergibt sich folgendes:

Hypothese H13: Die Nutzung geeigneter, etablierter Indikatoren zur Messung von Vulnerabilität ist Teil von Resilience Engineering und kann dazu genutzt werden, Systeme zu identifizieren, deren Resilienz besonders dringend erhöht werden sollte. 


\subsection{Notwendige Bedingungen für Resilience Engineering}

Einfache Systeme reagieren in deterministischer Weise auf Belastungen. Übersteigen diese die Grenzen ihrer Belastbarkeit, bricht das System zusammen. Es kann nicht mehr auf einen neuen Entwicklungspfad umschwenken, auch nicht auf einen mit verminderter Systemleistung. Dazu sind nur komplexe Systeme aufgrund ihrer Anpassungsfähigkeit in der Lage. Resilienz drückt sich durch das Betreten neuer Entwicklungspfade aus, obwohl die eigentlichen Belastungsgrenzen des Systems überstiegen werden. Die Komplexität von Systemen ist also eine notwendige Bedingung für Resilienz.

\&

A priori ist unklar, welchen Effekt steigende Komplexität auf das Ausmaß der im System vorhandenen bzw. auftretenden Resilienz hat. Steigende Komplexität kann sowobl zu mehr als auch zu weniger Resilienz führen.

Der Zusammenhang zwischen Resilienz und Komplexität ist vielgestaltig, das haben die vorangegangenen Analysen eindrücklich gezeigt. So macht Komplexität Resilienz notwendig, gleichzeitig ist Komplexität aber auch notwendig für Resilienz. Im Hinblick auf Resilience Engineering ergeben sich daraus einige interessante Überlegungen. Steigende Komplexität erhöht die Notwendigkeit für Resilienz. Aber steigende Komplexität führt nicht notwendigerweise zu steigender oder sinkender Resilienz. Demnach kann ein Wirkprinzip, das innerhalb der Ingenieurwissenschaften immer wieder genannt wird, um die Resilienz von Systemen zu erhöhen, als ungeeignet für Resilience Engineering definiert werden. Nämlich das Prinzip der Reduktion von Komplexität. Reduzierte Komplexität führt in diesem Verständnis zu einer loseren und einfacher zu durchschauenden Kopplung des Systems. Störungen können weniger einfach im System kaskadieren und ihre Wirkungen sind besser vorauszusehen. Das alles spricht dafür, die Komplexität von Systemen zu reduzieren, um ihre Resilienz zu erhöhen. Die system- und komplexitätstheoretische Analyse hat aber gezeigt, dass der Wirkmechanismus zwischen Resilienz und Komplexität nicht derart linear verläuft. Zunehmende Komplexität ermöglicht Systemen auch, neue und geeignete Handlungsmuster für den Umgang mit unerwarteten Störungen zu entwickeln. Das Resilienz-Konzept der zivilen SiFo liefert also hier keine eindeutigen Empfehlungen für Resilience Engineering, ob es sinnvoll wäre, die Komplexität der relevanten Systeme zu erhöhen oder aber zu reduzieren. Neben diesen grundsätzlichen, theoretischen Erwägungen, ist es an vielen Stellen für ingenieurwissenschaftliche Resilienzforschung auch schlicht nicht möglich, eine Komplexitätsreduktion als Lösung vorzuschlagen, da die zunehmende Komplexität und Vernetzung 
eine Vielzahl wünschenswerter Konsequenzen aufweist und eine empirische Realität ist. Konträr zur Ansicht einiger Forscher gilt für Resilience Engineering basierend auf den theoretischen Erkenntnissen der vorliegenden Arbeit:

Hypothese H14: Die Reduktion von Komplexität ist als Prinzip kein geeigneter Bestandteil von Resilience Engineering.

Das Vorhandensein von Anpassungsfähigkeit ist eine zwingende Grundvoraussetzung für Resilienz. Aber Resilienz ist nicht gleichbedeutend mit Anpassungsfäbigkeit. Resilienz ist auch nicht gleichbedeutend mit generischer Anpassungsfäbigkeit.

Ohne Anpassungsfähigkeit keine Resilienz. Diese zentrale Erkenntnis der vorliegenden Arbeit ist auch für Resilience Engineering von größter Bedeutung. Anpassungsfähigkeit meint dabei mehr als eine Störungstoleranz im Rahmen vorgegebener Grenzen. Anpassungsfähigkeit, vor allem generische Anpassungsfähigkeit, meint das Vermögen, sich selbst zielgerichtet weiterzuentwickeln, wenn das bisherige Vorgehen nicht mehr dazu geeignet ist, das System und seine von ihm erbrachte Leistung aufrechtzuerhalten. Für den Bereich der Ingenieurwissenschaften formulieren Altherr et al. sehr treffend, worauf es bei der hier gemeinten Anpassungsfähigkeit als essentiellem Bestandteil von Resilienz ankommt: „[T]he next logical step is to design resilient structures that can even cope with failures of components or other effects disregarded during design phase" (Altherr et al. 2018: 187, eigene Hervorhebung). In den Ingenieurwissenschaften werden Systeme grundsätzlich so ausgelegt, dass sie mit einer bestimmten Menge und einem bestimmten Ausmaß an Störungen umgehen können. Welche das sein sollen ist im Normalfall kein Teil der ingenieurwissenschaftlichen Umsetzung, sondern wird in vorgelagerten Aushandlungsprozessen entweder sozial, finanziell, politisch und/oder technisch geklärt. Für die Ingenieurwissenschaften sind diese Designspezifikationen eine conditio sine qua non. Ohne Vorgaben darüber, wie das System ausgestaltet werden soll, welchen Belastungen es standzuhalten in der Lage sein soll und mit welchen Maßnahmen es auf Störungen reagieren soll, ist ein ingenieurwissenschaftliches Systemdesign quasi unmöglich. So lassen sich jedenfalls alle Gespräche zusammenfassen, die der Autor der vorliegenden Arbeit in den vergangenen ca. fünf Jahren mit Ingenieurwissenschaftlern zu diesem Thema geführt hat.

Das gezeichnete Bild entspricht wieder dem eines klassischen Risikomanagements. Das Resilienz-Konzept der zivilen SiFo fordert aber mehr, es fordert Systeme, die nicht nur gegenüber spezifischen Bedrohungen 
bestehen können, sondern im Prinzip „no matter what“ - unabhängig davon, was konkret passiert (Altherr et al. 2018: 187). Und zwar unter der Nebenbedingung, dass die Ressourcen zur Umsetzung dieses Ziels erstens endlich sind und zweitens auch für konkurrierende Zwecke - etwa eine Maximierung der Systemleistungen dank größerer Effizienz - ausgegeben werden können (Fritzon et al. 2007: 38). Systeme mit der Form von Anpassungsfähigkeit auszustatten, die für Resilienz vonnöten ist, ist also eine voraussetzungsreiche und herausfordernde Aufgabe für Resilience Engineering. An dieser Stelle haben es andere Bereiche der Resilienzforschung „einfacher“, etwa die Organisationswissenschaften. Denn deren primäres Interesse gilt Menschen und menschlichem Verhalten in komplexen Organisationen. Menschen verfügen per definitionem über generische Anpassungsfähigkeit und es bleibt dann „nur" noch die Frage, wie Organisationen ausgestaltet sein sollten, um die generische Anpassungsfähigkeit von Menschen zu aktivieren und zu unterstützen. Nichtsdestotrotz kann ingenieurwissenschaftliche Resilienzforschung im Sinne eines Resilience Engineering nur dann als konsistent mit dem Resilienz-Konzept der zivilen SiFo verstanden werden, wenn sie Wege erforscht und entwickelt, um die generische Anpassungsfähigkeit komplexer adaptiver Systeme auf technologische Weise zu erhöhen. Dazu lassen sich in einer Kombination aus Erkenntnissen aus der Literatur und der system- und komplexitätstheoretischen Analyse einige Prinzipien sowie geeignete Methoden identifizieren, mit deren Hilfe das gelingen kann. Diese werden im nächsten Unterkapitel eingehender beleuchtet. Generell lässt sich für Resilience Engineering aber schon an dieser Stelle festhalten:

Hypothese H15: Resilience Engineering besteht in der Erforschung und Entwicklung von Prinzipien, welche die generische Anpassungsfähigkeit komplexer adaptiver Systeme auf technologische Weise zu erhöhen vermögen.

Transformationsfähigkeit ist kein notwendiger Bestandteil von Resilienz. Allerdings erböht das Vorhandensein von Transformationsfähigkeit - verstanden als besonders ausgeprägte Form generischer Anpassungsfähigkeit - ceteris paribus die Resilienz komplexer adaptiver Systeme.

Der Begriff der Transformationsfähigkeit ist vor allen Dingen in der sozial-ökologischen Resilienzforschung verbreitet. Forscher wie Brian Walker erkennen in der Transformation hin zu einem quasi neuen System das eigentliche Charakteristikum von Resilienz. Dem folgt die vorliegende Arbeit nicht in diesem Ausmaß. Hier wird Transformationsfähigkeit als Ende eines Kontinuums gesehen. So formalisiert ergibt sich aus der Tatsache, 
dass Transformationsfähigkeit nicht notwendig, aber gut für Resilienz ist, keine gesonderte Hypothese für Resilience Engineering.

Je flexibler komplexe adaptive Systeme sind, desto größer ist ihre Resilienz.

Flexibilität wurde im Rahmen des Resilienz-Konzepts der vorliegenden Arbeit definiert als das Vorhandensein eigentlich extrem unerwartbarer Prozesse an Anschlussmöglichkeiten, die dem System das Einschwenken auf einen neuen, leistungserhaltenden Entwicklungspfad ermöglichen. Damit ist Flexibilität ein Teil der generischen Anpassungsfähigkeit, die mithilfe von Resilience Engineering auf technologische Weise erhöht werden soll. Flexibilität ist demzufolge kein eigenes, von den im folgenden Unterkapitel beschriebenen unterscheidbares Prinzip zur Umsetzung und Erhöhung von generischer Anpassungsfähigkeit. Je höher die Flexibilität eines Systems, desto größer ist ihre Resilienz, das gilt weiterhin. Da Flexibilität ein Teil von generischer Anpassungsfähigkeit ist, leiten sich an dieser Stelle aber keine weiteren, neuen Hypothesen für Resilience Engineering ab.

\section{Lernfähigkeit ist eine notwendige Grundvoraussetzung für Resilienz.}

So wie Flexibilität wird auch Lernfähigkeit im Rahmen der vorliegenden Arbeit als Teil von Anpassungsfähigkeit verstanden. Im Gegensatz zur gerade erfolgten Diskussion lassen sich aber in Bezug auf Lernfähigkeit und mehr oder weniger verwandte Ideen einige Hypothesen für Resilience Engineering entwickeln. Lernfähigkeit besteht darin, Wissen über die wahrscheinlichen Auswirkungen der Realisierung der verschiedenen zur Verfügung stehenden Prozesse an Anschlussmöglichkeiten erlangen zu können. Aufgrund der inhärenten Unsicherheit in komplexen adaptiven Systemen kann es dabei nicht um determiniertes Wissen gehen, so dass die gerade genannte Wahrscheinlichkeit nicht in Form probabilistisch fixierter Aussagen erfasst werden kann. Es sollte aber näherungsweise möglich sein, bei klar gesteckten Systemgrenzen und unter Zuhilfenahme geeigneter Methoden ein Verständnis davon zu gewinnen, wie sich das Ergreifen bestimmter Maßnahmen auf das untersuchte System auswirkt. Dazu kann erneut auf die Theorie komplexer adaptiver Systeme zurückgegriffen werden. Die vorliegende Arbeit schlägt vor, diese Theorie unter anderem dazu zu nutzen, Schwellenwerte oder Umkipp-Punkte zu identifizieren. Damit kann verhindert werden, dass Systeme zu nahe an diesen Schwellen operieren, bei deren Überschreiten ein abrupter und gravierender Schaden auftritt. In der Komplexitätstheorie geht es aber auch um sogenannte Hebelpunkte, an denen kleine, zielgerichtete Handlungen zu gravierenden aber vorhersagbaren Veränderungen im Systemverhalten führen. Beim Über- 
schreiten von Schwellenwerten ist das folgende Systemverhalten hingegen zumeist unvorhersagbar. Hebelpunkte unterscheiden sich also von Schwellenwerten und Umkipp-Punkten durch die weitgehende Vorhersagbarkeit ihrer Auswirkungen. Ihre Lokalisierung ist dagegen ebenso unsicher und voraussetzungsreich, wie die von Schwellenwerten und Umkipp-Punkten. Um die Resilienz von Systemen auf ingenieurwissenschaftliche Weise zu erhöhen, könnte die Identifikation von Hebelpunkten aber durchaus sehr interessant sein. Denn wenn mithilfe kleiner, zielgerichteter Veränderungen am System, gravierende und gleichzeitig vorhersagbare Auswirkungen verbunden sind, könnte nach den Hebelpunkten gesucht werden, die sozusagen „positive Kaskadeneffekte“ auslösen (IRGC 2018: 56). Hebelpunkte, durch deren Realisierung das System profitieren könnte und die seine Resilienz erhöhen würden, weil sie generisch im gesamten System wirksam werden, obwohl sie lediglich an einer Stelle ausgelöst wurden. Das ist auch aus Sicht eines möglichst effizienten Einsatzes von Ressourcen ein lohnenswertes Ziel. Und es entspricht system- und komplexitätstheoretisch gesprochen der Erhöhung bzw. der Ausnutzung der Lernfähigkeit von Systemen. Das führt für Resilience Engineering zu einer weiteren Hypothese:

Hypothese H16: Die Theorie komplexer adaptiver Systeme und die daraus entwickelten mathematischen Modelle und Methoden können als Teil von Resilience Engineering genutzt werden, um Hebelpunkte zu identifizieren und durch deren Anwendung positive Kaskadeneffekte zu realisieren.

Etwas weniger zentral mit Lernfähigkeit verknüpft, aber doch an dieser Stelle der Diskussion zu Resilience Engineering verortbar, ist die Frage danach, ob und wie es möglich sein kann, Frühwarnsignale zu identifizieren, die zuverlässig das Auftreten eines disruptiven Ereignisses vorherzusagen in der Lage sind. Ohne jede Vorwarnung sind unvorhergesehene Situationen noch schwerer zu beherrschen, als wenn zumindest eine kurze Zeit des Einstellens und Vorbereitens bleibt (Dinh et al. 2012: 235f). Das ist konsistent mit dem Resilienz-Konzept der zivilen SiFo, da die interessierenden disruptiven Ereignisse nach wie vor grundsätzlich unerwartet eintreten, aber zumeist nicht innerhalb von Sekunden, so dass unter Umständen eine Vorwarnzeit bleibt. Frühwarnsignale sind häufig nur schwach ausgeprägt und daher schwer zu identifizieren (Amanatidou et al. 2012: 209). Frühwarnung kann unterschiedlich verstanden werden. Erdbeben lassen sich beispielsweise nicht langfristig vorhersagen. Es gibt zwar Regionen, in denen die Gefahr für das Auftreten von Erdbeben besonders hoch ist. Wann ein solches aber eintritt, ist Stand heute nicht prognostizierbar. Nichtsdestotrotz gibt es etwa in Japan, einem der Länder mit der größten 
Erfahrung mit Erdbeben, Frühwarnsysteme, die bei den ersten Anzeichen eines schwereren Erdbebens aktiv werden. Ein beeindruckendes Beispiel liefert das Tōhoku-Erdbeben von 2011, das in Deutschland überwiegend mit der Nuklearkatastrophe von Fukushima verbunden wird, das aber in Japan sehr viel stärker mit dem stärksten Erdbeben der Geschichte und dem davon ausgelösten Tsunami verknüpft ist, der über 20.000 Menschen das Leben kostete. Im Rahmen dieses disruptiven Ereignisses mit schlimmstdenkbaren, katastrophalen Auswirkungen, gab es aber auch so etwas wie „Erfolgsgeschichten.“ In Japan gibt es mit dem Shinkansen ein Hochgeschwindigkeitszugsystem, das im Hinblick auf seine Pünktlichkeit und Zuverlässigkeit sehr hohe Leistungsstandards erfüllt. Selbiges gilt für die Sicherheit. Bedingt durch Vorerfahrungen mit Erdbeben wurde für den Shinkansen ein automatisches Frühwarnsystem eingerichtet, das bei den ersten Vorbeben, die einem großen Beben in der Regel vorausgehen, automatisch auslöst und auswertet, ob ein größeres Beben zu erwarten ist. Falls ja, wird in den betroffenen Gebieten automatisch die Stromversorgung der Züge gekappt und diese vollführen eine Notbremsung. So ist es 2011 gelungen, alle Züge innerhalb einer guten Minute nach den ersten Warnzeichen zu stoppen und so Schäden oder gar Todesopfer zu vermeiden (Janić 2018: 1118).

Diese spezifische Art von Frühwarnsystem kann selbstverständlich nicht als Resilience Engineering verstanden werden. Sie stellt vielmehr ein beeindruckendes Beispiel gelungenen Risikomanagements dar. Das Beispiel zeigt aber auf, wie hilfreich und zum Teil tatsächlich lebensrettend Frühwarnsysteme sein können. Die Identifikation von Schwellenwerten und Umkipp-Punkten stellt selbst eine Art Frühwarnsystem dar. Resilience Engineering kann demnach aus dieser Richtung bereits signifikant beitragen. Darüber hinaus ist es bei vielen disruptiven Ereignissen, die innerhalb der zivilen SiFo besonders relevant sind, eher schwierig, generische Frühwarnsysteme zu implementieren. Systeme zur Warnung vor Hochwassern, Extremwetter, Waldbränden oder ähnlichem wirken spezifisch und sind etabliert. Für Frühwarnsysteme im Bereich schwerwiegender Industrieunfälle gilt technisch gesprochen ähnliches. Auch dort gibt es eine Fülle an zur Verfügung stehender Sensorik, um den Systemzustand zu überwachen und kritische Abweichungen frühzeitig zu erkennen. Hier mangelt es eher an einem Verständnis der eigentlichen Ursachen von Systemversagen, wie sie von der organisationswissenschaftlichen Resilienzforschung untersucht werden (siehe 2.5). Damit bleibt der Bereich bewusst herbeigeführter disruptiver Ereignisse, in der zivilen SiFo häufig mit Terrorismus identifiziert. Hier arbeiten Geheimdienste und Polizeibehörden weltweit 
daran, mithilfe von Überwachungstechnologien Anschlagspläne im Vorhinein zu identifizieren und Anschläge so zu verhindern. Gegeben die Grundannahmen des Resilienz-Konzepts der zivilen SiFo ist dies allerdings kein Teil von Resilience Engineering. Über die Effektivität und die Rechtfertigung solcher Überwachungsmaßnahmen kann an dieser Stelle nicht entschieden werden. Sie sind Gegenstand vielfältiger gesellschaftlicher und wissenschaftlicher Debatten. Für Resilience Engineering kann festgehalten werden:

Hypothese H17: Die Entwicklung spezifischer Frühwarnsysteme ist kein Teil von Resilience Engineering.

Innerhalb der organisationswissenschaftlichen Resilienzforschung gibt es den Ansatz der generischen Kompetenzen, die Menschen dabei helfen, unerwartete und kritische Situationen erfolgreich meistern zu können (siehe Bergström et al. 2009). Eine Möglichkeit, sich generische Kompetenzen anzueignen, ist das möglichst realitätsnahe Einüben unbekannter Situationen, idealerweise unangekündigt und im Rahmen der normalen Arbeit. Die Experimente, die Bergström et al. durchgeführt haben, bieten bereits einen Hinweis darauf, wie so etwas aussehen kann. Diejenigen, die an diesen Experimenten teilgenommen haben, konnten für ihre praktische Arbeit daraus Erkenntnisse gewinnen und sich selbst allein durch die Teilnahme am Experiment und die zwischengeschalteten Schulungen ein Stück weit generische Kompetenzen aneignen (Bergström et al. 2009: 89). Noch effektiver als im künstlichen Umfeld eines Experiments wirken solche Übungen aber im Kontext der alltäglichen Arbeit. Hier muss zunächst zwischen der Art der Arbeit unterschieden werden. Nicht jeder Arbeitnehmer ist in einem kritischen Bereich tätig. Genauer gesagt gibt es Millionen Arbeitnehmer, bei denen dies nicht der Fall ist. Auf der anderen Seite gibt es aber auch eine Vielzahl an Menschen, die in kritischen Bereichen beschäftigt sind, beispielsweise im Betrieb kritischer Infrastrukturen. Deren generische Kompetenzen zu erhöhen, wäre ein direkter Weg, um die Resilienz der betroffenen Systeme zu erhöhen. Das ist keine primär technische Aufgabe. Resilience Engineering kann aber dazu beitragen, wie ein genauerer Blick auf die gerade beschriebene Art von Übungen zeigt.

Als Vorbild können hier große amerikanische Internetkonzerne wie Google und Amazon dienen. Dort wurde vor einigen Jahren das Konzept der sogenannten „GameDays" eingeführt. Gegeben die auf einer Kombination von Soft- und Hardware basierende Infrastruktur dieser Konzerne ist es möglich, bewusst gravierende Störungen in einen Teil des Systems einzubauen - natürlich unter Sicherstellung der Aufrechterhaltung des 
normalen Alltagsbetriebs - um zu prüfen, wie die verantwortlichen Personen darauf reagieren (Robbins et al. 2012). Die Grundannahme hinter den GameDays ist dieselbe, die auch hinter Resilienz steckt. Fehler und Störungen lassen sich nicht komplett verhindern und deshalb ist es notwendig, auf alle denkbaren und undenkbaren Arten von disruptiven Ereignissen vorbereitet zu sein. Und das ist nicht möglich, indem rein theoretische Notfallpläne geschrieben werden: „The most important of those lessons is that an untested disaster recovery plan isn't really a plan at all" (Robbins et al. 2012). Deshalb bieten GameDays die Möglichkeit, Notfallpläne zu testen. Sie setzen Mitarbeiter einer unbekannten Stresssituation aus, mit der sie umgehen müssen. Denn die GameDays werden nur grundsätzlich angekündigt, nicht aber spezifisch, so dass die Mitarbeiter zunächst von einem echten Notfall ausgehen müssen. Diese Realitätsnähe ist in IT-Netzen einfacher zu simulieren, als beispielsweise für Rettungskräfte oder Katastrophenschützer. Aber auch ohne eine hundertprozentige Realitätsnähe setzen GameDays die handelnden Personen unter Druck und ermöglichen es ihnen, ihr eigenes Verhalten in solchen Fällen kennenzulernen und zu verbessern. Resilience Engineering lässt sich nun in zweierlei Hinsicht mit der Idee der GameDays verknüpfen. Zum einen fördern sie „special systems" und „backdoor boxes" zu Tage, welche von den Verantwortlichen in außergewöhnlichen Notfällen besonders intensiv genutzt werden (Robbins et al. 2012). Wenn diese bekannt sind, können sie mittels Resilience Engineering noch besser gestaltet werden. Zum anderen kann Resilience Engineering direkt dazu beitragen, GameDays zu ermöglichen, indem innovative Methoden zur Modellierung und Simulation des Verhaltens komplexer adaptiver Systeme in unerwarteten Ausnahmefällen weiterentwickelt werden. Daraus ergeben sich folgende Hypothesen:

Hypothese H18: Mithilfe realitätsnaher Notfallübungen können Systembestandteile identifiziert werden, die mittels Resilience Engineering optimiert werden sollten.

Hypothese H19: Durch die Verwendung realitätsnaher Modelle zur Simulation des Verhaltens komplexer adaptiver Systeme im Ausnahmefall in Notfallübungen kann Resilience Engineering dazu beitragen, die generischen Kompetenzen der handelnden Personen zu erhöhen und so die Resilienz des Gesamtsystems zu steigern. 


\subsection{Resilience Engineering aus system- und komplexitätstheoretischer Sicht}

Resilienz liegt dann vor, wenn ein System auf einen sebr unwahrscheinlichen Reiz (bzw. Ereignis) aus der Umwelt oder seinem Inneren, der das System initial auf einen Entwicklungspfad mit stark verminderter Systemleistung bringt, mit der Realisierung eines Prozesses eigentlich extrem unerwartbarer Anschlussmöglichkeiten reagiert, um anschließend auf einen so realisierbar gewordenen, neuen Entwicklungspfad erwartbarer Anschlussmöglichkeiten einzuschwenken, der sich durch eine (mindestens) vergleichbare Systemleistung auszeichnet, wie sie vor Eintritt des Ereignisses vorlag.

Dieser zentrale Bestandteil des Resilienz-Konzepts der zivilen SiFo war ein Ergebnis der system- und komplexitätstheoretischen Analyse, das es in dieser expliziten Form so vorher innerhalb der Resilienzforschung nicht gab. Was das vermittelt über generische Anpassungsfähigkeit für konkrete Prinzipien bedeutet, mit deren Hilfe sich Resilience Engineering betreiben lässt, bzw. die einen Teil von Resilience Engineering darstellen, wird im nächsten Schritt diskutiert. Zunächst soll noch eine Hypothese erörtert werden, die sich aus der Betonung der Systemleistung in obenstehender Aussage ableiten lässt. Die vorliegende Arbeit fokussiert sich auch und vor allem auf die Frage danach, wie kritische Infrastruktursysteme möglichst resilient gestaltet werden können, indem Prinzipien im Sinne von Resilience Engineering Verwendung finden (Hickford et al. 2018: 278). Als kritisch wurden die Infrastrukturen vor allem deshalb bezeichnet, weil sie eine für die Überlebensfähigkeit der Gesellschaft kritische Funktion erfüllen und die Gesellschaft, sobald eine solche Infrastruktur ausfällt, mit erheblichen Schäden zu rechnen hat (siehe 1.3).

Mit Blick auf das Gesamtsystem Gesellschaft erfüllen deren kritische Infrastrukturen kritische Funktionen - was sich aus dem Begriff bereits ergibt, aber trotzdem für die weitere Argumentation wichtig ist (Fox-Lent et al. 2015: 211). Denn sobald die Systemgrenzen etwas eingeschränkt werden und beispielsweise die Energieinfrastruktur als System verstanden wird, ändert sich die Bedeutung des Begriffs der kritischen Funktionen bzw. kritischen Funktionalitäten etwas. Die kritische Funktionalität des Energiesystems ist die Bereitstellung von Strom, Wärme und Kraftstoffen für die Bevölkerung. Mithilfe welcher konkreten Systemstruktur das erfolgt, ist a priori zweitrangig. Wichtig ist dagegen die Versorgungssicherheit unter allen Umständen - oder zumindest unter so vielen Umständen, wie es irgend möglich ist (Flynn/Burke 2011: 4, Wachsmuth 2014: 4). Kritische Funktionalitäten trotz gravierender disruptiver Ereignisse zu erhalten, ist ein Kerninhalt von Resilienz. Programmatisch formuliert ließe sich 
bei einem systemischen Resilienz-Verständnis wie dem der vorliegenden Arbeit sagen: „What matters is preserving and even en hancing critical functionality, not the pre-existing system" (LRF 2015: 10, eigene Hervorhebung). Nach dem Eintreten einer Störung ist es zudem besonders wichtig, die kritischen Funktionalitäten zu kennen, da diese zunächst wiederhergestellt werden müssen (IRGC 2018: 49). Was heißt das für Resilience Engineering? Einen wichtigen Schritt stellte zunächst die Definition von Systemgrenzen dar, um überhaupt bestimmen zu können, wessen Resilienz mithilfe von ingenieurwissenschaftlichen Maßnahmen erhöht werden soll. Nachdem die Systemgrenzen feststehen, wird direkt im nächsten Schritt die Identifikation der kritischen Funktionalitäten des Systems wichtig. Aufgrund des systemischen Resilienz-Verständnisses der vorliegenden Arbeit, besteht Resilienz in einer Anpassung des Systems an gravierende disruptive Ereignisse. Die Anpassung kann auch mit einer Veränderung der Systemstrukturen einhergehen. Sie zielt auf den Erhalt der Systemleistung, oder nach der Diskussion nun anders gesagt, auf den Erhalt der kritischen Funktionalitäten (Connelly et al. 2017: 47, Ganin et al. 2016: 1, LRF 2015: 11). Das setzt voraus, dass die Ingenieurwissenschaften eine Idee davon haben, worin diese kritischen Funktionalitäten bestehen. Für Resilience Engineering lässt sich dann folgende Hypothese aufstellen:

Hypothese H20: Nachdem die Systemgrenzen definiert wurden, bedeutet Resilience Engineering im nächsten Schritt, die kritischen Funktionalitäten der untersuchten Systeme zu identifizieren.

Nicht in jedem Fall lassen sich kritische Funktionalitäten voll aufrechterhalten. Einige disruptive Ereignisse nehmen Ausmaße an, die das System nicht ohne Schaden überstehen kann. Jedenfalls können mindestens rein theoretisch katastrophale Ereignisse eintreten. Aber auch in solchen Fällen bietet Resilienz bzw. Resilience Engineering Möglichkeiten, dem System und den von den kritischen Funktionalitäten des Systems abhängigen Menschen zu helfen. Entscheidend ist hier die Art der leistungsreduzierenden Reaktion des Systems. Um die Idee zu verdeutlichen, wird vereinfachend angenommen, dass sich die Leistungsreduktion über die Zeit mittels einer einfachen Funktion darstellen lässt, äquivalent zu Bruneaus Ideen (siehe 2.6). Dann lassen sich unterschiedliche Formen dieser Funktion voneinander abgrenzen.

In Abbildung 5 ist auf der linken Seite ein System dargestellt, dessen Fähigkeit zur Erfüllung kritischer Funktionalitäten nach Eintreten eines widrigen Ereignisses abrupt massiv abnimmt. Im Gegensatz dazu wird auf der rechten Seite ein System dargestellt, in dem die Leistung zwar 
ebenfalls bis zum gleichen Niveau abnimmt, dies aber sehr viel weniger steil, also sehr viel weniger schnell. Unter sonst gleichen Bedingungen und unter bewusster Inkaufnahme der Verkürzung der eigentlichen Komplexität an dieser Stelle - der Erholungspfad des Systems wird nicht berücksichtigt - zeigt das rechte System eine deutlich resilientere Reaktion auf das disruptive Ereignis, als das linke (siehe Abbildung 5). Dieses Phänomen wird in der Resilienzforschung als „elegantes Abschmelzen“ (graceful degradation) bezeichnet (Dueñas-Osorio/Vemuru 2009: 166, Goerger et al. 2014: 868, Rahimi/Madni 2014: 811). Bereits Lovins und Lovins haben, wenn auch unter dem Begriff der Stabilität, ein elegantes Abschmelzen als sehr relevant für Resilienz erkannt. Damit erkaufen sich das betroffene System und die handelnden Personen sozusagen Zeit, um mit der Umsetzung von Gegenmaßnahmen zu beginnen. Bzw. Zeit, damit bereits implementierte Prinzipien zur Gegensteuerung, zur Wiederherstellung der kritischen Funktionalitäten, greifen können, bevor es zu einem Totalzusammenbruch des Systems kommt (Lovins/Lovins 2001: 248). Die Sicherstellung eines möglichst eleganten Abschmelzens sollte demzufolge ein essentielles Ziel für Resilience Engineering sein. Daraus ergibt sich dann:

Hypothese H21: Ein Design, das Systemen ein elegantes Abschmelzen kritischer Funktionalitäten ermöglicht, trägt zur Resilienz von Systemen bei und sollte insofern Ziel ingenieurwissenschaftlicher Resilienzforschung sein.

Abbildung 5: Abrupt vs, graceful degradation
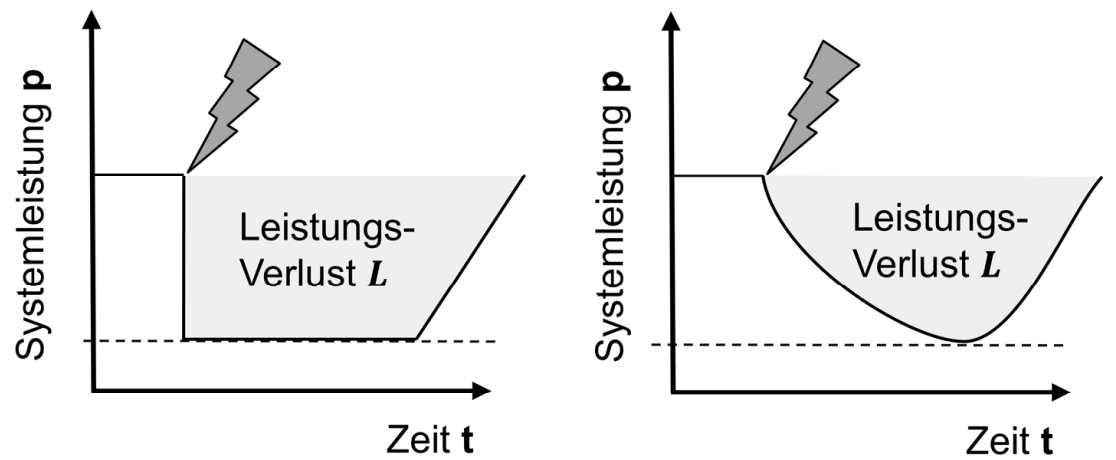

Quelle: eigene Darstellung.

In komplexen adaptiven Systemen muss zwischen spezialisierter und generischer Anpassungsfähigkeit unterschieden werden. Die Resilienz eines komplexen ad- 
aptiven Systems nimmt bei steigender Komplexität genau dann zu, wenn die generische Anpassungsfähigkeit des Systems steigt.

\&

Resilienz zeigt sich in der Fähigkeit komplexer adaptiver Systeme, mittels generischer Anpassungsfähigkeit extrem unwahrscheinliche oder unerwartete, ibre eigentlichen Belastungsgrenzen übersteigende Ereignisse so zu bewältigen, dass sie ibre Systemleistung möglichst aufrechterhalten und langfristig sichern können.

Generische Anpassungsfähigkeit ist neben Komplexität und Unsicherheit der zentrale Begriff des Resilienz-Konzepts der zivilen SiFo. Vor allem die Unterscheidung zwischen spezialisierter und generischer Anpassungsfähigkeit stellt eine theoretische Spezifizierung von Resilienz dar, wie es sie für die zivile SiFo bisher nicht gab. Insofern erscheint es auch notwendig und sinnvoll, einen Fokus der Hypothesen für Resilience Engineering darauf zu legen, wie generische Anpassungsfähigkeit ingenieurwissenschaftlich umgesetzt werden könnte. Es geht um die Beantwortung der Frage, wie komplexe adaptive Systeme so gestaltet werden können, dass ihre generische Anpassungsfähigkeit möglichst groß ist. Es geht um generische Designprinzipien, die sich aus der system- und komplexitätstheoretischen Analyse ableiten lassen und die gleichzeitig auch konsistent mit einigen Ideen aus der Literatur sind (Altherr et al. 2018: 193). Ein einfaches aber eindringliches Beispiel für die Umsetzung solcher Prinzipien liefern Park et al.: „It is not necessary to predict all potential causes of a ship sinking in order to provide life boats" (Park et al. 2013: 363). In einem ähnlichen Sinne werden beispielsweise auch militärische Systeme ausgelegt, die aufgrund ihrer häufig langen Lebensdauer mit dem Auftauchen neuer Technologien genauso zurechtkommen müssen, wie mit sich verändernden Anforderungen, neuen Einsatzumgebungen und dem Vorhandensein eines „adaptive foe“, eines selbst anpassungsfähigen Widersachers. Ein zu generischer Anpassungsfähigkeit ähnlicher Begriff, unter den diese Anforderungen zusammengefasst werden, ist der der „broad utility“ (Goerger et al. 2014: 867, 871). Systeme, die über diese broad utility verfügen, müssen letztlich den gleichen Designprinzipien folgen, wie Systeme, deren Resilienz maximiert werden soll. Diese Designprinzipien, sowie einige zugehörige Aspekte, werden im Folgenden näher beleuchtet. Die Reihenfolge der Beschreibung ist dabei nicht mit der Bedeutung der Prinzipien gleichzusetzen, da die besonders wichtigen Punkte Diversität und Redundanz erst weiter hinten kommen - bedingt durch die Tatsache, dass diese bereits Bestandteil des Resilienz-Konzepts der zivilen SiFo waren und daher in eigenen Aussagen spezifiziert wurden. 
Das erste Prinzip ist das der Modularität. Schon Lovins und Lovins nennen den modularen Aufbau von Systemen als eine Möglichkeit, seine Resilienz zu erhöhen. Seitdem hat sich dieses Systemprinzip etabliert und gilt als erfolgsversprechend dafür, resilienzfördernd zu sein, in dem es zum Management von Komplexität beiträgt (Helbing 2015, Lovins/Lovins 2001: 192, Lukesch 2016: 325). Modularität bezieht sich grundsätzlich ,auf die Möglichkeit der Herauslösung einzelner Bestandteile aus einer Gesamtmenge“" (Wink 2011: 115). Innerhalb eines komplexen adaptiven Systems bestehen Module aus Komponenten, die sehr eng miteinander vernetzt sind, aber eine weniger starke Vernetzung mit Systemkomponenten außerhalb des Moduls aufweisen. Auf diese Weise lassen sich Störungen und Fehler, die einen bestimmten Bereich des Systems treffen, besser isolieren. Sobald sie auftreten, werden vom System - oder seinem Operator - die Verbindungen zwischen dem betroffenen Modul und dem restlichen System gekappt. Das wird als „islanding“ bezeichnet. Wenn das Modul rechtzeitig vom restlichen System getrennt wird, kann sich die dort aufgetretene Störung nicht weiter im System verbreiten. So werden Kaskadeneffekte verhindert (Ash/Newth 2007: 677, 681, Dueñas-Osorio/Vemuru 2009: 165). Voraussetzung dafür ist allerdings, dass das System auch ohne einzelne Module - und im Vorhinein ist unsicher, welche der Systemmodule ausfallen - weiter in der Lage ist, seine kritischen Funktionalitäten aufrechtzuerhalten. Das führt zu einigen Überlegungen, die aus der System- und Komplexitätstheorie resultieren und die für das Prinzip der Modularität im Kontext der vorliegenden Arbeit wichtig sind. Komplexitätstheoretisch gesprochen bestehen Module in komplexen adaptiven Systemen aus einer Reihe von Agenten, die zur Erreichung ihrer Ziele eng miteinander kooperieren. Innerhalb des Moduls tendieren die Agenten dazu, sich stark zu spezialisieren und auf die zuverlässige Kooperation mit den anderen Agenten angewiesen zu sein. Nur so können die Agenten gemeinsam ihre Ziele erreichen - nur so kann das Modul seine Funktion innerhalb des Systems erbringen. Das führt zum einen dazu, dass Module ein Stück weit für sich selbst zu funktionieren imstande sind. Selbst ohne die vollumfängliche Einbettung in das Gesamtsystem sind sie in der Lage, eine gewisse Funktionalität zu erreichen. Zum anderen führt das aber auch dazu, dass das restliche System ein Stück weit in der Lage ist, ohne das entsprechende Modul zu funktionieren. Und da das Modul nicht spezifiziert wurde, gilt das für alle Module. Hintergrund dessen ist der geringere Grad an Feedback, der zwischen Modulen herrscht im Vergleich zum Ausmaß des Feedbacks innerhalb der Module. Damit wird auch deutlich, warum eine derart verstandene Modularität zu Systemen führt, die keinen „single point of 
failure" aufweisen. Ein single point of failure ist eine besonders kritische, wichtige Komponente eines Systems, deren Ausfall notwendigerweise zum Ausfall des gesamten Systems führt. Besteht ein System aber aus einer Vielzahl nur relativ lose gekoppelter Module, führt das Versagen einzelner Komponenten nur zum Ausfall einzelner Module, nicht aber des gesamten Systems. Die Agenten sind zur Erreichung ihrer Ziele nicht auf Agenten außerhalb des Moduls angewiesen. Und zwar ganz unabhängig davon, welches Modul des Systems aus welchem Grund ausfällt - Modularität ist daher ein generisch wirkender Mechanismus. Wenn einzelne Module ausfallen, kann das System die noch funktionsfähigen Module dazu nutzen, seine Leistung weiter zu erbringen. Die grundsätzliche Wirksamkeit des Prinzips wird also nicht nur in der Literatur behauptet - und ist unmittelbar einsichtig - sondern wurde auch system- und komplexitätstheoretisch erläutert. Demnach besteht Resilience Engineering unter anderem darin, komplexe adaptive Systeme möglichst modular zu gestalten. Die zugehörige Hypothese lautet dann:

Hypothese H22: Modularität, verstanden als Aufteilung eines Systems in funktional eigenständige Einheiten, bestehend aus einer Reihe stark miteinander vernetzter Agenten, die nur lose Kopplungen mit den anderen Einheiten des Systems aufweisen, ist ein geeignetes Designprinzip, um komplexe adaptive Systeme resilient $z$ u gestalten.

Das zweite Prinzip ist das der Dezentralität. Ähnlich wie das Modularitätsprinzip wird auch Dezentralität in der Literatur immer wieder als Möglichkeit genannt, die Resilienz eines Systems zu erhöhen. Dabei ist Dezentralität inhaltlich und logisch teilweise mit Modularität verknüpft. Systeme lassen sich generell entweder eher zentralisiert oder eher dezentralisiert organisieren. Klassische Beispiele sehr stark zentralisierter Systeme stellten die planwirtschaftlichen Ökonomien der ehemaligen kommunistischen Staaten dar. Sinnigerweise sind kapitalistisch organisierte Wirtschaftssysteme hingegen sehr viel stärker dezentralisiert ausgestaltet. Volkswirtschaften sind beliebig komplexe Systeme, die sich einfachen Kausalitäten völlig entziehen. Der Gedanke einer zielgerichteten, deterministischen Planbarkeit ist daher, wenn man der Komplexitätstheorie folgt, als geradezu absurd zu verwerfen (siehe 4.3.2). Das gilt auch innerhalb marktwirtschaftlich organisierter Wirtschaftssysteme, wie die Ideen und Ansätze von Chandler zur Frage, ob Resilienz ein neoliberales Paradigma sein kann, gezeigt haben. Obwohl in seiner wissenschaftlichen Ausrichtung rein sozialwissenschaftlich, arbeitet Chandler doch mit den gleichen komplexitätstheoretischen Annahmen, die auch dem Resilienz-Konzept der vorliegen- 
den Arbeit und somit den Hypothesen für Resilience Engineering zugrunde liegen (siehe 4.2.1). Der Fokus von Chandler lag auf der Frage nach der Steuerbarkeit komplexer adaptiver Systeme. Aufgrund ihrer Komplexität, aufgrund der in komplexen Systemen vorherrschenden Eigenschaft der Emergenz, ging er von einer grundsätzlichen Un-Steuerbarkeit derartiger Systeme aus. Die an dieser Stelle untersuchten Systemprinzipien der Zentralität bzw. Dezentralität haben einen anderen Ausgangspunkt. In ihnen geht es sehr viel stärker um die Frage, ob es zentrale Systembestandteile gibt, von denen das gesamte übrige System abhängt. Hier wiederum wäre ein klassisches Beispiel ein Energieversorgungssystem, das einzig auf wenigen, mit Kohle und anderen fossilen Energieträgern befeuerten Großkraftwerken beruht. Von diesen zentralen Elementen aus werden Strom, Wärme und Treibstoffe mithilfe von Leitungen, Pipelines und sonstigen Transportmitteln an die Empfänger - Privathaushalte, Industrie, Tankstellen, etc. - verteilt. Fällt am Ende dieser Kette ein Systemelement - etwa eine Tankstelle - aus, hat das auf das Gesamtsystem keinen messbaren Einfluss. Wenn aber eines der zentralen Elemente, wie etwa ein Großkraftwerk, ausfällt, kann das im gesamten System zu spürbaren Leistungseinbußen führen. Selbstverständlich könnten solche Einbußen durch andere Designprinzipien, etwa das der Redundanz, vermieden werden. Allerdings sind derart zentrale Systemelemente wie Großkraftwerke mit immensen Kosten verbunden, sie binden eine sehr große Menge an Ressourcen. Diese redundant vorzuhalten, schmälert die Effizienz des Systems sehr stark. Und je größer die Kosten für Resilienz, desto geringer ist die Wahrscheinlichkeit für ihre Umsetzung. Eine sehr umfassende und ausführliche Beschreibung eines derart zentralisiert organisierten Energieversorgungssystems findet sich bei Lovins und Lovins. Ihrem Urteil zufolge sind zentralisierte Systeme inhärent verwundbar, sie sind „brittle“, was sich mit spröde, brüchig und zerbrechlich, aber auch mit morsch übersetzen lässt (siehe Lovins/Lovins 2001).

Gleichzeitig haben zentralisierte Systeme auch große Vorteile. Einer Studie der European Network and Information Security Agency zufolge zeichnen sie sich durch eine sehr hohe Zuverlässigkeit und sehr hohe Verfügbarkeit aus - was für Energieversorgungssysteme fundamental wichtige Eigenschaften sind (ENISA 2011: 24). Eine geographische Zentralisierung, also die Unterbringung vieler wichtiger Systemkomponenten an einem Ort, ergibt zudem marktwirtschaftlich und aus Effizienzgründen Sinn (Flynn/Burke 2012: 12). Allerdings sehen auch die Autoren der gerade genannten Studie die Gefahr für ein katastrophales Versagen zentralisierter Systeme, wenn es eine Störung im Bereich der wenigen, zentralen Kompo- 
nenten gibt. Demgegenüber seien dezentralisierte Systeme, die sie als „horizontal skaliert" (scaled horizontally) bezeichnen, technisch zwar komplexer, dafür aber insgesamt resilienter, da das Versagen einzelner Komponenten nicht direkt zu katastrophalen Schäden führt (ENISA 2011: 24). Das sehen auch Flynn und Burke so, die von einem „security and resilience benefit" sprechen, wenn wichtige Systemelemente nicht geographisch zentriert werden. Mit Blick auf zwei Arten von widrigen Ereignissen, gezielte Attacken einerseits und lokal auftretende Extremwetterereignisse andererseits, wird der Vorteil eines nicht nur logisch, sondern auch geographisch dezentralisierten Netzes ebenfalls offensichtlich (Allenby/Fink 2005: 1035, Flynn/Burke 2012: 12). Ähnlich wie modulare Netze, vermeiden auch dezentralisiert organisierte Netze das Entstehen eines single point of failure. Dezentral bedeutet nicht notwendigerweise in Modulen organisiert. Es geht lediglich darum, dass die Bedeutung einzelner Komponenten für ein System nicht zu groß werden sollte (Jackson/Ferris 2013: 6). System- und komplexitätstheoretisch lässt sich das ebenfalls fassen. In zentralisierten Systemen gibt es einzelne, hochspezialisierte Agenten, deren Leistung für das Gesamtsystem kritisch ist. Ohne diese Agenten kann das System nicht funktionieren. Sobald sie ausfallen, bricht das gesamte System zusammen und kann seine kritischen Funktionalitäten nicht mehr erfüllen. Da sich in komplexen adaptiven Systemen aufgrund des Faktors Unsicherheit nicht sicher ausschließen lässt, dass gerade diese hochspezialisierten und hochkritischen Agenten versagen, ergibt es mehr Sinn, Systeme dezentralisiert zu organisieren. Das entspricht einer Verteilung der Aufgaben des vormals hochspezialisierten Agenten auf mehrere Agenten. Systeme dezentral zu organisieren, ist also ebenfalls ein Prinzip ingenieurwissenschaftlicher Resilienzforschung, das sich direkt aus dem Resilienz-Konzept der zivilen SiFo ableiten lässt und das als Möglichkeit, erfolgreich mit Komplexität umzugehen, auch in der Literatur immer populärer wird (Helbing 2013: $55)$.

Hypothese H23: Dezentralität, verstanden als Vermeidung des Auftretens hochkritischer Systemkomponenten bzw. hochspezialisierter Agenten, bei deren Ausfall das gesamte System unweigerlich zusammenbricht, ist ein geeignetes Designprinzip, um komplexe adaptive Systeme resilient zu gestalten.

Ein besonderer Typ dezentralisierter Systeme sind sogenannte „vermaschte Netzwerke" (meshed networks). Diese vereinen in sich viele der Eigenschaften und Prinzipien, die der Resilienz komplexer adaptiver Systeme zuträglich sind. So gehen vermaschte Netze nicht von einem zentralen Punkt aus, sondern verfügen über multiple, redundante Strukturen, die 
ausfallende Systembestandteile sofort umgehen bzw. ersetzen können. Die Systemkomponenten - da es um komplexe adaptive Systeme geht, sind dies definitionsgemäß Agenten - beobachten ihre Umwelt und können so auf Störungen und Fehler aufmerksam werden. Sobald in einem Teil des Netzes eine Störung auftaucht, rekonfiguriert das System sich selbst und ersetzt den wegfallenden Teil durch eine redundant vorhandene Komponente. Damit vermeidet es die Existenz eines single point of failure indem zu jedem Zeitpunkt mehrere alternative Möglichkeiten bestehen. Die Wahl zwischen mehreren Alternativen so wie sie in vermaschten Netzen möglich ist, entspricht dann dem system- und komplexitätstheoretischen Verständnis von generischer Anpassungsfähigkeit. Wenn die Störung behoben ist, kann das vermaschte Netz sich erneut rekonfigurieren und die wieder funktionierenden Teile integrieren, um seine Leistung zu optimieren (Dueñas-Osorio/Vemuru 2009: 166, ENISA 2011: 24). So konzipierte, vermaschte Netze beinhalten wie gesagt neben dem Prinzip der Dezentralität auch das Prinzip der Modularität sowie der Redundanz und außerdem Fähigkeiten wie die zur Selbst-Organisation. Allerdings sind nicht alle für die zivile SiFo relevanten Systeme in Form von Netzen strukturiert. Wo sie es aber sind, also vor allem im Bereich kritischer Infrastrukturen wie Energie- und Wasserversorgung sowie Telekommunikation, sollte Resilience Engineering dazu beitragen, eine vermaschte Netzstruktur aufzubauen. Oder anders gesagt:

Hypothese H24: Die Resilienz kritischer Netz-Infrastrukturen kann durch den Aufbau vermaschter Netze erböht werden.

Einen interessanten Sonderfall stellen mit Blick auf unterschiedliche Netztopologien sogenannte skalenfreie Netze bzw. Netzwerke dar. Im Vergleich zu zufallsbasierten Netzen, bei denen unterschiedliche Netzbestandteile im Schnitt alle gleich stark vernetzt sind, weisen skalenfreie Netzwerke eine Reihe an Elementen auf, die mit besonders vielen anderen Elementen vernetzt sind. Diese sind für die Funktionsfähigkeit des Netzes eminent wichtig. Um diese besonders wichtigen Elemente clustern sich sozusagen andere Systemelemente. Damit ist dieses Netz weder übergreifend zentralisiert organisiert, noch modular aufgebaut. Zentral sind die besonders vernetzten Elemente immer nur für einen lokal begrenzten Bereich des Systems. Und Modularität würde eine lokal sehr starke Vernetzung vieler Elemente miteinander, nicht nur mit einem - lokal - zentralen Element bedeuten. Der mathematischen Theorie zufolge sind derartige, skalenfreie Netzwerke nun ausfallsicher gegenüber zufälligen Fehlern und Störungen, wie sie beispielsweise von Unfällen oder Naturkatastrophen 
ausgehen können. Solche zufälligen Störungen treffen mit sehr hoher Wahrscheinlichkeit auf Elemente des Systems, die keine hohe Kritikalität aufweisen. Oder wenn sie ein kritisches Element treffen, dann nur ein einzelnes, was lediglich zu einem Teilausfall des Systems führt. Skalenfreie Netzwerke sind aber gleichzeitig sehr anfällig für zielgerichtete Attacken. Wer in der Lage ist, die wenigen, hochkritischen Elemente des Systems zu identifizieren und zu zerstören, kann mit relativ geringem Aufwand das gesamte System zum Zusammenbruch bringen. Das könnte besonders für Terroristen von Interesse sein, die grundsätzlich ebenfalls unter der Nebenbedingung begrenzter Ressourcen versuchen, mit ihren Aktionen einen möglichst großen Effekt zu erzielen (Ash/Newth 2007: 673, Cox et al. 2011: 314). Auch wenn das Phänomen des Infrastrukturterrorismus bisher nicht zu größeren Schäden in der Gesellschaft geführt hat, kann diese Möglichkeit für die Zukunft nicht vernachlässigt werden, wie unter anderem eine von Ortwin Renn herausgegebene Studie zur Resilienz des deutschen Energiesystems und des Prozesses der Energiewende gezeigt hat, an der auch der Autor der vorliegenden Arbeit beteiligt war (siehe Renn 2017).

Aufgrund des All-Gefahren-Ansatzes, der dem Resilienz-Konzept der zivilen SiFo zugrunde liegt, lässt sich der gerade geschilderten Argumentation folgend nicht eindeutig festlegen, ob die Anpassung bestehender Systeme in Richtung skalenfreier Netzwerke ihre Resilienz schmälern oder stärken würde. Insofern kann hier im Bezug darauf keine Hypothese für Resilience Engineering abgeleitet werden. Anders sieht es jedoch mit Systemen aus, die bereits in Form skalenfreier Netzwerke organisiert sind. Das gilt etwa durchaus für einige Netze der kritischen Infrastruktur, wie etwa Energienetze. Da es zumeist nicht möglich ist, die Topologie bestehender Netze - zumindest nicht kurzfristig - grundlegend zu ändern, müssen andere Wege gefunden werden, um ihre Resilienz zu erhöhen. In skalenfreien Netzwerken geht es dann etwa darum, die besonders relevanten Elemente erstens zu identifizieren und zweitens entsprechend redundant oder divers (siehe unten) auszulegen (Ash/Newth 2007: 681). Daraus folgt für Resilience Engineering:

Hypothese H25: Um skalenfreie Netzwerke, wie sie für kritische Infrastrukturen teilweise typisch sind, resilient zu gestalten, müssen deren besonders relevante Elemente identifiziert und mittels geeigneter Prinzipien wie Redundanz und Diversität optimiert werden.

Um herausfinden zu können, ob komplexe adaptive Systeme modular und dezentral aufgebaut sind, inwiefern sie Kriterien eines vermaschten 
Netzes entsprechen oder eher skalenfreien Netzwerken ähneln, ob sie über adäquate Sicherheitsmargen verfügen und wie nah oder fern sie sich an Schwellenwerten oder Umkipp-Punkten befinden, reicht der Augenschein nicht aus. Die Komplexität besteht ja auch gerade darin, dass keine einfachen Kausalitäten erkennbar sind. Deshalb kann ingenieurwissenschaftliche Resilienzforschung, kann Resilience Engineering, nicht sinnvoll konzeptualisiert werden, ohne auf Methoden zur Modellierung und Simulation komplexer Systeme einzugehen. Modelle sind grundsätzlich vereinfachende, meist formale Beschreibungen des gerade interessierenden Teils der gesamten Realität. Simulationen wiederum können als „virtuelle Experimente" bezeichnet werden. Sie sind also Nachbildungen dynamischer, mehrdimensionaler Prozesse in einem Modell und ermöglichen Erkenntnisse, die auf die Wirklichkeit übertragen werden können. Derartige virtuelle Experimente werden überall dort benötigt, wo „reale“ Experimente aus verschiedenen Gründen nicht möglich sind. Beispiele sind hier etwa die Astrophysik, bei der sich die zu beobachtenden Phänomene dem menschlichen Vorstellungsvermögen in Bezug auf Zeit und Raum entziehen, oder die Medizin, bei der die Ausbreitung einer Epidemie nicht einfach erprobt werden kann (Bungartz et al. 2009: 1ff). Die Notwendigkeit von Modellen und Simulationen zur Vorhersage des Verhaltens komplexer adaptiver Systeme im Angesicht widriger Ereignisse in der zivilen SiFo ist vor diesem Hintergrund evident. Sie dienen dazu, mit einem ökonomisch machbaren und ethisch vertretbaren Aufwand eine hinreichend wirklichkeitsgetreue Verhaltensbeschreibung zu erreichen, mit deren Hilfe mögliche Schwachstellen und Probleme des Systems identifiziert werden können (Bossel 2004: 15). Modelle und Simulationen werden im Bereich der ingenieurwissenschaftlichen Risikoforschung bereits seit Jahrzehnten intensiv genutzt (siehe Renn 2008, 2008b). Klassisches Risikomanagement nutzt Modelle und Simulationen, um Vulnerabilitäten spezifischer Systemkomponenten gegenüber definierten und erwarteten widrigen Ereignissen vorhersagen und das Verhalten des Systems beim Eintreten solcher Ereignisse berechnen zu können. Im breiteren gesellschaftlichen Kontext hat beispielsweise die Volkswagen-Stiftung bereits vor über fünfzehn Jahren die Notwendigkeit erkannt, neue Methoden zur Modellierung und Simulation komplexer Systeme zu entwickeln. Von 2003 bis 2011 wurden zahlreiche Projekte durch die Initiative „Neue konzeptionelle Ansätze zur Modellierung und Simulation komplexer Systeme“ gefördert. Hierbei lag der Fokus allerdings nicht primär auf den komplexen adaptiven sozio-tech- 
nischen Systemen, die für die zivile SiFo von Interesse sind.$^{41}$ Nichtsdestotrotz gibt es auf dem Gebiet der Modellierung und Simulation komplexer Systeme sowohl eine breite Basis mathematischen und informationstechnischen Grundlagenwissens als auch eine Vielzahl an Anwendungsbeispielen, etwa was die Erklärung von Vorgängen im menschlichen Gehirn oder die Algenbildung in den Ozeanen betrifft.

Der Anspruch der vorliegenden Arbeit kann weder darin bestehen, in formeller Weise die Vor- und Nachteile bestimmter Methoden zu beschreiben, noch eine eigene, neuartige Methodik zur Modellierung und Simulation des Verhaltens komplexer adaptiver Systeme in extremen Ausnahmefällen zu entwickeln. Dazu ist sie aufgrund ihrer sozialwissenschaftlichen Grundausrichtung nicht in der Lage. Es kann aber mit Blick auf die system- und komplexitätstheoretischen Annahmen des Resilienz-Konzepts und unter Zuhilfenahme von Überblicksarbeiten aus der Literatur zumindest versucht werden, auf einige Methoden hinzuweisen, die für Resilience Engineering im Sinne der vorliegenden Arbeit besonders geeignet scheinen. Die oben beschriebene grundsätzliche Eigenschaft der Vereinfachung komplexer Realitäten, die allen Modellen innewohnt, darf dabei zunächst nicht dazu führen, das Verständnis der interessierenden Systeme als komplex und adaptiv zu unterwandern. Die Möglichkeit des Auftretens unerwarteter Ereignisse, unintendierter Nebenfolgen und überhaupt von Unsicherheit, muss von den Methoden berücksichtigt werden können, damit sie für Resilience Engineering geeignet sind (Park et al. 2013. 362). Gleichzeitig muss als Grundannahme gelten, dass die untersuchten Systeme physikalischen Gesetzen folgen. Nur dann lassen sich Modelle bilden, die eine hinreichend große Wirklichkeitstreue enthalten (IRGC 2018: 61). Autoren wie Ahmed et al. oder Ouyang beschreiben unterschiedliche Möglichkeiten, komplexe adaptive Systeme zu modellieren - und so letztlich ihr Verhalten simulieren zu können. Dazu zählen laut Ahmed et al. zum Beispiel partielle Differentialgleichungen, zelluläre Automaten, Netzwerk-Methoden und agentenbasierte Modellierung (ABM) (Ahmed et al. 2005: 4). Vor allem letztere scheint vor dem Hintergrund der Annahmen der Theorie komplexer adaptiver Systeme besonders relevant und vielversprechend für Resilience Engineering. Auf agentenbasierte Simulationsmethoden wird gleich noch einmal gesondert eingegangen.

41 https://www.volkswagenstiftung.de/unsere-foerderung/unser-foerderangebot-imueberblick/neue-konzeptionelle-ans\%C3\%A4tze-zur-modellierung-und-simulation-komplexer-systeme [Stand: 28.6.2019]. 
Auch Ouyang beschreibt unterschiedliche Methoden und Ansätze zur Modellierung komplexer adaptiver Systeme. Sein Artikel bietet einen guten und auch für Sozialwissenschaftler in weiten Teilen sehr verständlichen Überblick (siehe Ouyang 2014). Ihm zufolge sind beispielsweise empirische Ansätze gekennzeichnet durch die Nutzung historischer Datensätze und von Expertenwissen, mit deren Hilfe auf künftiges Systemverhalten geschlossen werden kann. Stärker formalisiert sind etwa FuzzylogikMethoden, welche die unterschiedlichen Einschätzungen von Experten durch mathematisch formulierte Unschärfe in Modelle überführen können. Einen großen Einfluss und weite Verbreitung bei der Modellierung komplexer Systeme haben wiederum Netzwerk-basierte Methoden. Deren Logik wurde bereits in der komplexitätstheoretischen Analyse von Resilienz erläutert und im aktuellen Unterkapitel in Bezug auf skalenfreie Netzwerke genutzt (siehe 4.3.2). Mit ihrer Hilfe werden komplexe Systeme als Netzwerke verstanden, die aus Knoten und Kanten bestehen. Verschiedene Knoten interagieren direkt miteinander, wenn sie über Kanten verbunden sind. Das erlaubt es durchaus auch, Feedback und Rückkopplungen zwischen verschiedenen Knoten im Netzwerk zu modellieren. Netzwerkbasierte Methoden können vor allen Dingen dazu genutzt werden, den technisch klar abgrenzbaren Teil von Netzwerkinfrastrukturen wie etwa der Energieversorgung zu modellieren. Netzwerk-basierte Methoden können noch weiter aufgeteilt werden, unter anderem in sogenannte "flussbasierte" (flow-based), also Methoden, deren Fokus auf dem Fluss von Informationen bzw. Dienstleistungen oder Produkten liegt, der zwischen verschiedenen Knoten des Netzwerks besteht (Ouyang 2014: 46-53). Ouyang beschreibt auch noch einige weitere, weniger verbreitete Modellierungsmethoden. Gemeinsam ist ihnen allen aber ein großer bis sehr großer Datenhunger. Um realitätsnahe Ergebnisse zu liefern, benötigen die meisten dieser Methoden ein großes Maß an zur Verfügung stehenden Daten, auf deren Grundlage sie das Modell des untersuchten Systems aufbauen. Die flussbasierten Methoden benötigen beispielsweise detaillierte Informationen über die spezifischen Charakteristika der Systembestandteile, die sie zu modellieren suchen. Wenn es um kritische Infrastrukturen geht, sind dies sehr häufig Daten, welche von den Betreibern der kritischen Infrastruktur aufgrund von Datenschutz- und Sicherheitsbedenken kaum zur Verfügung gestellt werden können (Ouyang 2014: 53).

Das gilt auch für die gerade bereits genannte agentenbasierte Modellierung komplexer Systeme. Auch dort werden Daten benötigt, die von den verantwortlichen Personen als vertraulich eingeschätzt und nicht ohne Weiteres weitergegeben werden. Aber noch stärker als andere Methoden 
ist eine agentenbasierte Modellierung von der Quantität und der Qualität der zur Verfügung stehenden Daten abhängig. Denn über technische und strukturelle Daten hinaus werden auch Informationen zu politischen Entscheidungen oder auch menschlichem Verhalten in bestimmten Situationen benötigt, die nur sehr schwer zu erheben und systematisieren sind. Zudem ist die Qualität der Simulation auch stark abhängig von den Annahmen bzgl. der namensgebenden Agenten, die von demjenigen zu treffen sind, der die Modellierung vornimmt (Ouyang 2014: 48, 53). Nichtsdestotrotz bieten agentenbasierte Modellierungsansätze viele Vorteile, die sie zur Anwendung im Bereich komplexer adaptiver Systeme prädestinieren. Zunächst folgen agentenbasierte Methoden einem bottom-upAnsatz, der davon ausgeht, dass die Komplexität des zu modellierenden Systems sich als emergente Eigenschaft aus den relativ simplen Interaktionen autonomer Agenten untereinander ergibt. Agentenbasierte Methoden modellieren ein System als bestehend aus Agenten, die mit anderen und der Systemumwelt interagieren, indem sie bestimmten, nachvollziehbaren Regeln folgen. Aufgrund des durch die kontinuierliche Interaktion hervorgerufenen Feedbacks, wird das durch die Agenten gebildete System komplex. Die agentenbasierte Modellierung hat sich selbst ständig weiterentwickelt, so dass mittlerweile - auch dank vorhandener und dafür benötigter Rechenkapazitäten - Agenten auch als sich selbst weiterentwickelnde Elemente modelliert werden können. Dazu werden etwa mathematische Methoden wie stochastische Simulationen verwendet. Oder es wird darauf verzichtet, bestimmte Szenarien und Systemzustände zu simulieren und auch deren Auftreten als Emergenz verstanden, die stochastisch und nicht deterministisch abhängt von den getroffenen Grundannahmen für das modellierte System (Al-Khudhairy et al. 2012: 577, Duit et al. 2010: 2, Helbing 2013: 57, Ouyang 2014: 48).

Die Beschreibung der Methode der agentenbasierten Modellierung erinnert nicht nur zufällig an die Grundannahmen der Theorie komplexer adaptiver Systeme (siehe 4.3.2). Es besteht eine enge Verwandtschaft zwischen deren theoretischen Annahmen und der Entwicklung von Methoden zur agentenbasierten Modellierung komplexer Systeme. Allein aus diesem Grund ist es sinnvoll, diese Art der Modellierung als relevant für Resilience Engineering zu verstehen. Darüber hinaus sieht aber auch Ouyang die agentenbasierte Modellierung gemeinsam mit flussbasierten Netzwerkmethoden als diejenigen an, mit deren Hilfe am besten die Resilienz von Systemen erhöht werden kann.

Nach Ouyang sind sowohl die agentenbasierte Modellierung als auch flussbasierte Netzwerk-Methoden dazu geeignet, alle vier unterschiedli- 
chen Arten von Vernetzung (physikalisch, cyber, geographisch, logisch), wie sie auch bereits beim Zusammenhang zwischen Resilienz und Komplexität geschildert wurden, in Betracht zu ziehen. Und sie sind relativ gesehen die Methoden, die zu den meisten Resilienz-Strategien, wie sie Ouyang definiert, beitragen können. Zudem sind die Methoden bereits relativ ausgereift, was durch den Grad an Maturität (maturity) ausgedrückt, wird, der für beide Methoden als relativ hoch angesehen wird (Ouyang 2014: 53f). Agentenbasierte Modellierung stellt die Komplexität von Systemen also explizit in Rechnung und kann so ein relativ realitätsnahes Abbild der interessierenden Systeme schaffen. Wenn derart modellierte Systeme dann in Simulationen von stochastisch hervorgerufenen, beliebigen Störereignissen getroffen werden, zeigt sich, ob und wie sie ihnen standhalten, wo Schwachstellen liegen und welche Bestandteile des Systems besonders erfolgreich sind. Mithilfe agentenbasierter Modellierung könnte das System im nächsten Schritt auch bewusst verändert werden, so dass es beispielsweise eher resilienzfördernden Prinzipien wie Modularität und Dezentralität entspricht. Wenn das System dann erneut simulierten Störungen ausgesetzt ist, sollte es sich tendenziell erfolgreicher zeigen. Auf diese Weise kann agentenbasierte Modellierung dazu beitragen, das Verständnis für die Funktionsweise komplexer adaptiver Systeme zu erhöhen und den Einfluss resilienzfördernder Gestaltungsprinzipien nachzuweisen sowie zu fördern. Aus den Ergebnissen von Ouyang und den Überlegungen der vorliegenden Arbeit lassen sich daher zwei Hypothesen für Resilience Engineering ableiten:

Hypothese H26: Mithilfe agentenbasierter Modellierung kann die Funktionsweise komplexer adaptiver Systeme auch im stochastisch bestimmten extremen Ausnahmefall verstanden und so aufgezeigt werden, wie Resilience Engineering die Resilienz dieser Systeme beeinflusst.

Hypothese H27: Methoden zur agentenbasierten Modellierung weiterzuentwickeln, ist Bestandteil von Resilience Engineering.

Der potentielle Kritikpunkt eines logischen Zirkelschlusses wird hier durchaus gesehen. Wenn Systeme mithilfe einer Agenten-Logik verstanden werden und daraus ein Resilienz-Konzept abgeleitet wird, scheint es selbstverständlich, dass Resilience Engineering sich sehr gut mithilfe eines auf Agenten-Logik basierenden Modellierungsansatzes umsetzen lässt. Es muss allerdings unterschieden werden zwischen der rein theoretischen Analyse zur Entwicklung des Resilienz-Konzepts der zivilen SiFo einerseits und einer ingenieurwissenschaftlichen Anwendung der agentenbasierten Modellierung andererseits. Erstere bildet zusammen mit den Annahmen 
der soziologischen Systemtheorie die Basis des Resilienz-Konzepts der vorliegenden Arbeit und damit auch für Resilience Engineering. Diese Basis führt neben der abgeleiteten Nützlichkeit agentenbasierter Modellierung zu einer Vielzahl weiterer Hypothesen. Letztere ist lediglich ein mögliches Werkzeug, um Resilience Engineering konkret zu betreiben, gleichzeitig aber bei weitem nicht das einzige. Denn auch wenn agentenbasierte Modellierung ein wichtiger Teil von Resilience Engineering ist, erfordert die reale Komplexität der betrachteten Systeme gleichzeitig eine Weitung der Perspektive. Ouyang stellt zurecht die Frage in den Mittelpunkt, mithilfe welcher Methoden Entscheidungsträger in Systemen am besten in ihren Entscheidungen unterstützt werden können. Da alle von ihm untersuchten Methoden jeweils nur einen - wenn auch unterschiedlich großen - Teil der genannten Resilienz-Strategien abdecken, plädiert er für die Nutzung mehrerer Methoden in einem "uniform framework" (Ouyang 2014: 54). In eine ähnliche Richtung argumentiert das International Risk Governance Center (IRGC) mit der Beschreibung eines Ansatzes, der als „modulare Modellierung” (modular modelling) bezeichnet wird. Da keine einzelne Methode als geeignet angesehen wird, komplexe adaptive Systeme in Gänze zu modellieren, sollen jeweils passende Ansätze für Subsysteme genutzt und dann mithilfe spezialisierter Routinen zu einem systemischen Modellierungsansatz verbunden werden (IRGC 2018: 63). Modulare Modellierung ergibt in Bezug auf Resilience Engineering schon allein deshalb Sinn, weil Modularität als Systemprinzip zu Resilienz beiträgt. Derselben Logik folgend, akzeptiert modulare Modellierung die Unmöglichkeit einer umfassenden, zentralistischen und erschöpfenden Methode zur Modellierung komplexer Systeme und setzt dagegen vielmehr auf den strategisch zielgerichteten Einsatz unterschiedlicher Methoden mit deren jeweils spezifischen Stärken an den passenden Stellen des Systems, um durch Kombination der Ergebnisse zu einer übergeordneten, systemischen Sichtweise zu gelangen. Das ist gleichzeitig konsistent mit den system- und komplexitätstheoretischen Elementen des Resilienz-Konzepts der vorliegenden Arbeit, so dass für Resilience Engineering folgende Hypothese gebildet werden kann:

Hypothese H28: Modulare Modellierung, verstanden als systemische Kombination unterschiedlicher Methoden zur Modellierung komplexer adaptiver Systeme, ist ein Bestandteil von Resilience Engineering.

Aus der gerade getätigten Analyse lässt sich noch eine weitere Überlegung ableiten. Insbesondere Methoden wie die agentenbasierte Modellierung sind sehr datenintensiv. Reale bzw. präzise Daten liegen jedoch aus unter- 
schiedlichen Gründen sehr häufig nicht vor (Ouyang 2014: 54f). Das führt dazu, dass in den Ingenieurwissenschaften Modellierungsansätze unter $\mathrm{Zu}$ hilfenahme ungenügender oder ungeeigneter Daten entwickelt werden. Dies wiederum beeinträchtigt die Qualität der entwickelten Modelle negativ und verhindert einen Einsatz im realen Umfeld, da der mögliche Mehrwert für Entscheidungsträger nur gering ist. An den grundsätzlichen Hürden in Bezug auf die Verfügbarkeit von Daten wird sich voraussichtlich auch künftig nichts ändern. Um nichtsdestotrotz bessere Modelle entwickeln zu können, könnte deshalb die Einrichtung einer open source Datenbank sinnvoll sein, die beispielsweise mit realistischen Daten eines fiktiven komplexen adaptiven Systems gefüttert wird. Das fiktive System sollte möglichst detailliert abgebildet werden im Hinblick auf kritische Infrastrukturen, soziale und sonstige technische Aspekte und dank eines offenen Zugangs von unterschiedlichen Forschern genutzt werden können (Alderson 2019: 77). Da die vorliegende Arbeit sich im Rahmen der zivilen SiFo in Deutschland bewegt, wäre es etwa überlegenswert, eine derartige Datenbank für Deutschland aufzubauen. Dies wäre unter anderem als ein von der Bundesregierung zu unterstützendes, kontinuierliches Forschungsprojekt denkbar. In anderen Ländern wie etwa den USA existieren ähnliche Datensätze bzw. Beispielsysteme bereits (siehe Alderson 2019). Der gerade gemachte Vorschlag entspricht eher einem praktischen Hinweis, als einem theoretisch abgeleiteten Bestandteil von Resilience Engineering. Insofern soll er hier nicht als eigenständige Hypothese formuliert werden.

Bevor gleich noch die beiden Prinzipien der Diversität und der Redundanz für Resilience Engineering analysiert werden, muss an dieser Stelle, nach der Diskussion von Möglichkeiten zur Modellierung und Simulation komplexer adaptiver Systeme, noch auf eine spezifische (informations-)technische Entwicklung verwiesen werden. Im Zusammenhang mit der eminenten Bedeutung von generischer Anpassungsfähigkeit für die Resilienz von Systemen drängen sich das Thema der künstlichen Intelligenz (KI) und mit ihm verwandte Stichworte wie Big Data, maschinelles Lernen oder neuronale Netzwerke geradezu auf. Denn künstliche Intelligenz ist ja gerade mit der Annahme verbunden, dass technische Systeme künftig Problemstellungen zu meistern in der Lage sein werden, für die sie nicht explizit geplant und entwickelt wurden. Eben indem die Systeme eigenständig, unter Zuhilfenahme der ihnen innewohnenden künstlichen Intelligenz, das Problem erfassen und eine passende Lösungsstrategie entwickeln. Menschen und soziale System verfügen zweifelsfrei über diese Fähigkeit. Eher klassische, nach strikten Vorgaben zur Erfüllung spezifischer Aufgaben gestaltete technische Systeme hingegen grund- 
sätzlich nicht. Das ändert sich durch den Prozess der Digitalisierung und die Einführung der Informationstechnologie bereits seit längerer Zeit. So schreiben Comfort et al. schon 2001 von technischen Systemen, die dank Informationstechnologie dazu in der Lage seien, sich an unerwartete Ereignisse anzupassen. Das Voranschreiten der Informationstechnologie führe dazu, dass komplexe technische Systeme besser, resilienter, gestaltet werden könnten (Comfort et al. 2001: 144ff). Ähnlich sieht das Longstaff, der zufolge informationstechnische Systeme schon lange in der Lage seien, für unbekannte Probleme durch Suchalgorithmen die besten Lösungen zu finden (Longstaff 2012: 266). Mittlerweite bieten sich vor allem in Kommunikationsnetzen und informationstechnischen Systemen wie dem Internet - hier nur verstanden als das technische System - sehr viele Möglichkeiten, generische Anpassungsfähigkeit gegenüber Störungen einzubauen. Das zugehörige Stichwort lautet hier häufig „self x“. Damit ist im weitesten Sinne die Fähigkeit zur Selbst-Organisation des Systems gemeint, die noch weiter ausdifferenziert wird in "self-reconfiguration, self-optimising, self-diagnosing, self-healing, self-protecting, self-organization, self-forming, self-adaptivity or self-management" mit deren Hilfe Feedbackschleifen in das System eingebaut werden (ENISA 2011: 31). Insgesamt sind die Möglichkeiten, die sich der Gesellschaft durch Informationstechnologie bieten, in den letzten Jahrzehnten und vor allen Dingen in den letzten Jahren exponentiell gewachsen. Künstliche Intelligenz stellt diesbezüglich einen konsequenten nächsten Schritt dar. Wobei künstliche Intelligenz nicht als monolithischer Block verstanden werden kann. Technische Systeme sind nicht in einem binären Sinne intelligent oder nicht intelligent. Es wird beispielsweise zwischen „schwacher" und „starker“ künstlicher Intelligenz unterschieden. Erstere ist in Form moderner Sprachassistenten bereits im Alltag vieler Menschen angekommen. Letztere hingegen, die als künstliche Intelligenz auf menschlichem Niveau verstanden wird, ist nach wie vor ein hochaktuelles Forschungsthema, in dem allerdings durchaus spektakuläre Fortschritte erzielt werden (Baezner et al. 2018: 2). Daraus ergeben sich vielfältige Chancen und Möglichkeiten, aber auch Herausforderungen und Gefahren. Diese auch nur im Hinblick auf die zivile Sicherheitsforschung in Deutschland zu erörtern, würde den Rahmen hier weit sprengen und steht nicht im Fokus der vorliegenden Arbeit.

Methoden, die mit künstlicher Intelligenz in Verbindung stehen, werden aber bereits heute auch in der ingenieurwissenschaftlichen Resilienzforschung verwendet. Das sind zum Beispiel das sogenannte maschinelle Lernen, mit dessen Hilfe unüberschaubare Mengen an Daten (big data) automatisiert erkannt und analysiert werden können (Helbing 2013: 57f). 
Autoren wie Ji et al. bezeichnen derartige Methoden als Schlüsselthemen zur Adressierung des „Resilienz-Problems“ (Ji et al. 2017: 1358). Auch Attoh-Okine diskutiert Ansätze und Ideen zu Big Data in seinem Werk zu Resilience Engineering (Attoh-Okine 2016: 83-93). Mithilfe von maschinellem Lernen und anderen Methoden künstlicher Intelligenz können technische Systeme demnach generische Anpassungsfähigkeit aufbauen und sich verändernden Umweltbedingungen - und damit zumindest theoretisch auch gravierenden widrigen Ereignissen - erfolgreich anpassen (IRGC 2018: 31). Diese Art von Selbst-Organisation oder Selbst-Heilung, von autonomem Verhalten, wird vielfach als notwendig für Resilienz verstanden (Helbing 2013: 54, IRGC 2018: 30, Meadows 1999: 15, Sterbenz et al. 2010: 1257). Überspitzt formuliert ließe sich daraufhin an dieser Stelle fragen, ob Resilience Engineering, verstanden als den sinnvollen Einsatz und die Weiterentwicklung von Technologie zur Erhöhung von Resilienz, überhaupt ohne künstliche Intelligenz möglich ist. In einem strikt und ausschließlich technischen Verständnis von Resilience Engineering hätte diese Frage einige Berechtigung und könnte nicht ohne Weiteres abgetan werden. Die interessierenden Systeme in der zivilen SiFo sind jedoch sozio-technische Systeme. Hier wird erneut deutlich, wie wichtig die sinnvolle Definition von Systemgrenzen ist. Zudem geht es bei Resilience Engineering nicht um autonome oder automatisierte Systeme, bei denen die Notwendigkeit für menschliche Intervention entfernt werden soll (Sterbenz et al. 2010: 1257). Vielmehr geht es um komplexe adaptive sozio-technische Systeme, bei denen die sozialen und technischen Aspekte dergestalt miteinander gekoppelt sind, dass sie nicht losgelöst voneinander verstanden werden können. Im Rahmen derartiger Systeme kann Resilience Engineering basierend auf dem eigenständigen Resilienz-Konzept der zivilen SiFo - nur verstanden werden als Entwicklung und Umsetzung von technologischen Lösungen zur Erhöhung der generischen Anpassungsfähigkeit der Systeme an sich - und nicht nur ihrer technischen Komponenten. Das schließt den Einsatz von Methoden und Ansätzen aus dem Bereich der künstlichen Intelligenz nicht aus, ganz im Gegenteil. Künstliche Intelligenz erlaubt ohne jeden Zweifel an vielen Stellen, die generische Anpassungsfähigkeit auch sozio-technischer Systeme zu erhöhen - nicht nur rein technischer. Wie sie dazu beitragen kann, lässt sich an dieser Stelle nicht abschließend und umfassend analysieren. Die Frage danach könnte selbst als Teil von Resilience Engineering gefasst werden. Gleichzeitig gibt es aber auch über künstliche Intelligenz hinaus eine Vielzahl an Ideen und Möglichkeiten für die Ingenieurwissenschaften, Resilience Engineering zu betreiben. Die- 
se Überlegungen führen dann insgesamt zu den folgenden beiden Hypothesen:

Hypothese H29: Die Beantwortung der Frage danach, wie KI-basierte Lösungen zur Erhöhung der Resilienz der für die zivile SiFo relevanten soziotechnischen Systeme beitragen können, ist Teil ingenieurwissenschaftlicher Resilienzforschung.

Hypothese H30: Resilience Engineering lässt sich in komplexen adaptiven, sozio-technischen Systemen auch obne den Einsatz künstlicher Intelligenz umsetzen.

Diversität und Redundanz sind Prinzipien zur Erhöhung generischer Anpassungsfähigkeit in komplexen adaptiven Systemen. Diversität besteht in einer möglichst unterschiedlichen Reaktion funktional gleicher Agenten auf extrem unwahrscheinliche oder unerwartete Veränderungen. Je größer die Diversität innerhalb funktional äquivalenter Gruppen komplexer adaptiver Systeme, desto größer ist ibre generische Anpassungsfähigkeit.

Während die beiden Prinzipien der Modularität und der Dezentralität erst in diesem Unterkapitel explizit aus der system- und komplexitätstheoretischen Analyse von Resilienz als Bestandteil von Resilience Engineering abgeleitet wurden, erfolgte das für die Prinzipien Diversität und Redundanz bereits im Rahmen der Entwicklung des Resilienz-Konzepts der zivilen SiFo. Das zeigt deren herausgehobene Bedeutung für Resilienz. Die Diskussion soll daher an dieser Stelle wieder aufgegriffen und auf je eine Hypothese für Resilience Engineering übertragen werden. Dabei ändert sich nichts an dem grundsätzlichen Verständnis, dass sowohl Diversität als auch Redundanz in komplexen adaptiven Systemen geeignete Prinzipien sind, um deren generische Anpassungsfähigkeit und damit ihre Resilienz zu erhöhen. In der system- und komplexitätstheoretischen Analyse entsprachen sich allerdings Diversität und sogenannte „skalenübegreifende“ Redundanz sehr stark und es konnte nicht trennscharf zwischen den beiden Prinzipien unterschieden werden. Um Hypothesen für Resilience Engineering zu bilden, wird daher noch einmal stärker auf die Unterschiede zwischen Diversität und Redundanz verwiesen.

Die Formulierung beider Prinzipien als Bestandteil des Resilienz-Konzepts der zivilen SiFo resultierte aus der Frage, wie die ungenutzten Spielräume, die in der Komplexitätstheorie immer wieder eine Rolle spielen, verstanden werden können. Was Diversität anging, wurden die Anleihen dafür sehr stark aus der sozial-ökologischen Resilienzforschung genommen. Dort wird (Bio-)Diversität, oder auch Artenvielfalt, immer wieder 
als eine der entscheidenden Bestandteile von Resilienz verstanden. Wenn möglichst viele unterschiedliche Arten existieren, erhöht das ceteris paribus auch die Wahrscheinlichkeit, dass Arten vorhanden sind, die mit den jeweils auftretenden disruptiven Ereignissen erfolgreich umzugehen in der Lage sind, bzw. davon gar nicht betroffen sind. Bereits Holling erkannte Diversität als entscheidend für Resilienz, wie seine Beispiele hinsichtlich der Bedeutung unterschiedlicher Baumarten für Wälder und deren Anfälligkeit für bestimmte Schädlinge oder aber Waldbrände zeigen (siehe 2.3). Eine besonders wichtige Rolle spielt Diversität auch in der wirtschaftswissenschaftlichen Resilienzforschung. Beim Blick auf regionale Wirtschaftssysteme entspricht Diversität dem Vorhandensein mehrerer unterschiedlicher, wichtiger Branchen innerhalb der betrachteten Regionen, um eine Anfälligkeit des Gesamtsystems gegenüber Störungen in einzelnen Branchen zu verringern (Wink 2011: 115). Schwere wirtschaftliche Krisen resultieren häufig daraus, dass einzelne Regionen gerade keine Branchendiversität aufweisen und dann zu großen Teilen vom Erfolg eines einzelnen Sektors abhängig sind. Diversität wird in den Wirtschaftswissenschaften aber auch noch anders verstanden, nämlich als Investitionsstrategie. Durch eine breite Streuung von Investitionen wird eine Abhängigkeit von einzelnen Wertentwicklungen vermieden. Holling selbst nutzt diese Analogie interessanterweise dazu, sein Prinzip der "funktionalen Diversität“ (functional diversity) für biologische Systeme zu erläutern (Holling 1996: 40). Vor diesem Hintergrund wurde Diversität dann für das Resilienz-Konzept der vorliegenden Arbeit unter Zuhilfenahme der Theorie komplexer adaptiver Systeme untersucht. Demnach ähnelt Diversität auf den ersten Blick der Eigenschaft der Agenten komplexer adaptiver Systeme, sich immer stärker zu spezialisieren. Denn das führt zu Agenten, die sich stark unterscheiden, die sehr divers sind. Die Spezialisierung macht jedoch stärkere Vernetzung notwendig, was zu einem immer komplexeren System führt und zu Agenten, die immer weniger dazu in der Lage sind, sich an gravierende Veränderungen anzupassen. Inwiefern stärkere Spezialisierung auf Systemebene zu mehr oder weniger generischer Anpassungsfähigkeit führt, ließ sich rein theoretisch nicht sagen. Diversität meint aber mehr, als nur möglichst unterschiedliche Agenten. Diversität meint die Existenz möglichst unterschiedlicher Agenten innerhalb funktionaler Gruppen. In funktionalen Gruppen erfüllen die Agenten alle dieselbe Aufgabe. Je unterschiedlicher aber die Art und Weise ist, in der sie das tun, desto größer ist die Diversität im System.

Was heißt das für die zivile SiFo und insbesondere für ingenieurwissenschaftliche Resilienzforschung? Das Prinzip der Diversität, des Vorhanden- 
seins unterschiedlicher Agenten in funktional äquivalenten Gruppen, lässt sich sehr eindrücklich am bereits vielfach als Beispiel genutzten Energiesystem veranschaulichen. Dessen Funktion besteht darin, die Bevölkerung zuverlässig und möglichst ohne Ausfälle mit Energie, also Strom, Wärme und Treibstoff, zu versorgen. Innerhalb des Systems gibt es Elemente, die für die Stromversorgung zuständig sind, genauso wie Elemente, die für die Versorgung mit Wärme und Treibstoff sorgen. In sehr stark abstrahierender Weise stellen diese jeweils funktional äquivalente Gruppen dar. Für den Endabnehmer ist es rein funktional gänzlich irrelevant, ob der Strom, den er für beinahe sämtliche Bereiche seines Lebens ständig benötigt, durch Windkraft erzeugt wurde, aus der Verfeuerung von Kohle stammt oder mittels Photovoltaik gewonnen wurde. Selbiges gilt für Treibstoff. Solange er chemisch identisch ist, unterscheidet sich Biokraftstoff funktional nicht von aus Rohöl raffiniertem Benzin. Mithilfe dieses Beispiels wird unmittelbar einsichtig, warum derart verstandene Diversität die generische Anpassungsfähigkeit und somit die Resilienz des Energiesystems erhöht. Je diverser die funktional äquivalenten Gruppen ausgestaltet sind, je mehr unterschiedliche Möglichkeiten es innerhalb eines Systems gibt, bestimmte Funktionen - vor allem die oben diskutierten kritischen Funktionalitäten - zu erfüllen, desto unwahrscheinlicher wird es, dass ein zufällig und unerwartet auftretendes widriges Ereignis alle unterschiedlichen Systembestandteile gleichzeitig betrifft. Für den Bereich der Stromversorgung kann von einer gewissen Diversität bzgl. der Produktion von Strom ausgegangen werden. Ganz anders sieht es hingegen bei der Verteilung aus. Hier besteht eine sehr große Abhängigkeit von der Funktionsfähigkeit des Hoch- und Höchstspannungsnetzes, dessen Ausfall kaum kompensiert werden kann. Allerdings bieten sich dann wiederum andere Prinzipien wie Dezentralität und Modularität an, um das System trotzdem resilient zu gestalten.

Diversität findet sich als Prinzip auch bereits in der ingenieurwissenschaftlichen Resilienzforschung. Dort wird Diversität neben einigen anderen Prinzipien als geeignet angesehen, um die Resilienz von Systemen zu erhöhen. Diversität ist dazu in der Lage „systemic adaptability and innovation" innerhalb von Systemen zu stärken und somit deren Resilienz (Helbing 2013: 55). Laut Autoren wie Helbing funktioniert das Prinzip mittels unterschiedlicher Mechanismen. Je größer die Diversität eines Systems, desto wahrscheinlicher existieren beim Eintritt disruptiver Ereignisse Bereiche, die nicht durch das Ereignis betroffen sind, deren Fehlermechanismen eben andere sind. Und gleichzeitig erhöht Diversität auch die Chance, dass geeignete Lösungsmöglichkeiten bereits im System vorhan- 
den sind, die zur Bewältigung der Folgen disruptiver Ereignisse genutzt werden können (Helbing 2015). Das erinnert nicht nur zufällig an die Erkenntnisse der system- und komplexitätstheoretischen Analyse von Resilienz. Das Prinzip der Diversität taucht in ähnlicher Weise auch bereits bei Lovins und Lovins auf, denen zufolge größere Diversität die Wahrscheinlichkeit reduziert, dass einzelne Fehlermechanismen das ganze System lahmzulegen imstande sind. Das wird durch eine Heterogenität der Systemkomponenten möglich. Sie bezeichnen Diversität auch als funktionale Flexibilität (Lovins/Lovins 2001: 192ff). Vor dem Hintergrund des systemund komplexitätstheoretischen Verständnisses von Flexibilität ist das sehr interessant. Dort wird Flexibilität als das Vorhandensein eigentlich extrem unerwartbarer Prozesse an Anschlussmöglichkeiten, die dem System das Einschwenken auf einen neuen, leistungserhaltenden Entwicklungspfad ermöglichen, definiert. Das lässt sich durchaus mit dem Diversitätsprinzip in Übereinstimmung bringen. Je heterogener das System, desto diverser ist es. Je heterogener das System, desto mehr Prozesse an Anschlussmöglichkeiten existieren aber auch. Insgesamt finden sich zwischen dem aus der Ökologie stammenden Prinzip der Diversität, seiner Anwendung in der ingenieurwissenschaftlichen Resilienzforschung und den aus der system- und komplexitätstheoretischen Analyse von Resilienz gewonnenen Erkenntnissen sehr große Übereinstimmungen. Insofern sind die Ergebnisse der vorliegenden Arbeit konsistent mit existierenden Ansätzen. Und es lässt sich sehr deutlich festhalten, dass größere Diversität eine erstrebenswerte Systemeigenschaft darstellt, wenn das betreffende System resilienter gestaltet werden soll. All das führt zu einem weiteren Prinzip, das Bestandteil von Resilience Engineering sein sollte. Um die Resilienz komplexer adaptiver Systeme auf ingenieurwissenschaftliche Weise zu erhöhen, sollten Wege erforscht werden, die Diversität dieser Systeme zu erhöhen. Und zwar die Diversität innerhalb funktional äquivalenter Gruppen. Einfach nur möglichst unterschiedliche Agenten innerhalb eines Systems erhöhen noch nicht notwendigerweise dessen Diversität, so wie sie hier verstanden wird. Ingenieurwissenschaftliche Resilienzforschung besteht also auch in der Identifikation der funktional äquivalenten Gruppen und der Entwicklung von möglichst heterogenen Systemkomponenten, welche dieselbe Funktion erfüllen. In einer Hypothese für Resilience Engineering zusammengefasst, lässt sich sagen:

Hypothese H31: Diversität, verstanden als Vorhandensein funktional äquivalenter Gruppen, zusammengesetzt aus möglichst verschiedenartigen Agenten, ist ein geeignetes Designprinzip, um komplexe adaptive Systeme resilient $z$ u gestalten. 
Skalenübergreifende Redundanz bedeutet das Vorhandensein funktional äquivalenter Systemelemente, die sich in ibrer Abhängigkeit von unterschiedlichen widrigen Ereignissen unterscheiden. Je mehr skalenübergreifende Redundanzen vorhanden sind, desto größer ist die generische Anpassungsfähigkeit komplexer adaptiver Systeme.

Diese Definition von Redundanz als Bestandteil des Resilienz-Konzepts der zivilen SiFo liest sich im Prinzip eins zu eins wie das Verständnis von Diversität, das gerade diskutiert wurde. Um für Resilience Engineering eine Abgrenzung zwischen Diversität und Redundanz zu ermöglichen, bedarf es also einer näheren Untersuchung. Zunächst verwundert aber die Platzierung der Diskussion von Redundanz im Kontext von Resilience Engineering. Denn Redundanz wird als eines der wenigen Prinzipien in jeder dem Autor bekannten Aufzählung von Prinzipien zur Erhöhung von Resilienz genannt. Und Redundanz kommt häufig an erster oder zumindest vorderer Stelle zur Sprache (Jackson/Ferris 2013: 5, Madni/Jackson 2009: 189). In der vorliegenden Arbeit wird es dagegen als letztes Prinzip untersucht. Das sagt jedoch nichts über die dem Prinzip zugemessene Bedeutung aus, sondern resultiert einzig aus den Ergebnissen der systemund komplexitätstheoretischen Analyse von Resilienz. Daraus konnte Redundanz, bzw. genauer gesagt skalenübergreifende Redundanz, neben der Diversität als zweites fundamental wichtiges Systemprinzip bereits für das Resilienz-Konzept an sich identifiziert werden. Gleichzeitig kann Redundanz trotz seiner zentralen Bedeutung für die Umsetzung von Resilienz nach der ausführlichen theoretischen Betrachtung in der vorliegenden Arbeit keinesfalls mit Resilienz gleichgesetzt werden. Resilienz ist definitiv sehr viel mehr als Redundanz (Brunner/Giroux 2009: 8). Das wird auch in der ingenieurwissenschaftlichen Resilienzforschung durchgängig so gesehen. Dort wird unter Redundanz ganz grundsätzlich das Vorhandensein von Alternativen, von Ausweichmöglichkeiten verstanden, ein Backup-System, das parallel zum primären System läuft und einen „safe fallback level“ garantiert. Oder noch anders gefasst, latent vorhandene Möglichkeiten, auf die bei Bedarf zurückgegriffen werden kann (Brunner/Giroux 2009: 8, Cimellaro et al. 2010: 3641, Helbing 2013: 55, Wink 2011: 115). Vor allem der Begriff des Backups weist dabei auf das spezifische Charakteristikum von Redundanz hin. Redundante Systembestandteile sind für den normalen Betrieb des Systems nicht erforderlich. Das System kann seine volle Leistung im Normalzustand auch ohne das Vorhandensein von Redundanz erbringen. Insofern entsteht unmittelbar ein mindestens scheinbarer Zielkonflikt zwischen der für Resilienz notwendigen Redundanz einerseits und der aus ökonomischen Gesichtspunkten häufig geforderten - und im 
Wettbewerb benötigten - Effizienz. Redundanz ist per definitionem ineffizient, eine Vergeudung von Ressourcen, die anderweitig besser und sinnvoller zur Erhöhung der Leistungsfähigkeit des Systems eingesetzt werden könnten. Daher ist es häufig sehr schwer, verantwortliche Stellen von der Notwendigkeit von Redundanz im System zu überzeugen (Brunner/ Giroux 2009: 8, Lovins/Lovins 2001: 44). Mögliche Zielkonflikte zwischen Resilienz und Effizienz wurden in der vorliegenden Arbeit an mehreren Stellen bereits adressiert und mit Blick auf die Grenzen des Resilienz-Konzepts diskutiert. Dass es trotz vermeintlicher Ineffizienz langfristig gesehen sehr sinnvoll sein kann, in Resilienz zu investieren, hat die gesamte Diskussion deutlich gezeigt (siehe 5.4).

Bei der Frage nach einer Abgrenzung zwischen Diversität und Redundanz kann der Rückgriff auf den gerade genannten Begriff des Backups aber sehr hilfreich sein. Diversität bedarf nicht notwendigerweise mehrfach vorhandener Systembestandteile, die nicht durchgängig genutzt werden. Das Beispiel des Energienetzes hat das gezeigt. Dieses kann durchaus sehr große Diversität aufweisen im Hinblick auf die zur Versorgung der Bevölkerung mit Strom genutzten Quellen. Das führt aber nicht notwendigerweise zu Redundanz. Das System weist auch dann hohe Diversität auf, wenn die unterschiedlichen Quellen zur Stromversorgung in effizienter Weise gerade ausreichen, um die Bevölkerung zu versorgen und keine zusätzlichen, redundanten und für die normale Funktionsfähigkeit nicht benötigten Reservekapazitäten enthalten. Diversität lässt sich auch als „avoid fate sharing“, „so that the same fate is unlikely to be shared by parts of the system undergoing correlated failures" zusammenfassen (Sterbenz et al. 2010: 1247, 1256). Redundanz ist dagegen ein prinzipiell nicht benötigter Zusatz an Ressourcen, der spezifisch darauf ausgelegt ist, beim Wegfall der eigentlich dafür vorgesehenen Systemkomponenten sozusagen einzuspringen und die kritische Funktionalität so gut es geht aufrechtzuerhalten. Neben dem klassischen Beispiel der Datensicherung, bei dem die exakt gleichen Daten an mehreren, physikalisch und häufig auch geographisch unterschiedlichen Stellen gespeichert werden und nur bei Verlust des primären Speicherorts auf die Sicherungskopien zurückgegriffen wird, stellt auch die Notstromversorgung kritischer Systeme einen sehr bekannten und geübten Anwendungsfall für Redundanz dar. Besonders eindrücklich lässt sich das am Beispiel von Kernkraftwerken veranschaulichen. Diese müssen auch dann, wenn sie selbst nicht laufen, zur Kühlung der Brennelemente ständig mit Strom versorgt werden und sind daher an das öffentliche Stromnetz angeschlossen. Sollte das Netz versagen, verfügen Kernkraftwerke über netzunabhängige Stromerzeugungs- 
aggregate, zumeist Dieselgeneratoren, mit deren Hilfe sie einen sicheren Zustand aufrechterhalten können. Sogar die Dieselgeneratoren sind selbst redundant ausgelegt, nicht alle vorhandenen werden gleichzeitig benötigt, um die Kühlung der Brennelemente sicherzustellen. Sehr sicherheitskritische Systeme wie Kernkraftwerke verfügen also über ein großes Maß an Redundanz. Allerdings bedarf die gerade beschriebene Notkühlung einer konstanten Versorgung mit Dieselkraftstoff, die im Falle eines langanhaltenden und großflächigen Stromausfalls ebenfalls sehr schwer zu garantieren werden könnte. Deshalb wäre es sinnvoll, auch noch andere, funktional äquivalente Möglichkeiten im Sinne von Redundanz vorhalten zu können. In einem fiktiven Beispiel könnten das Photovoltaik- und Windkraftanlagen kombiniert mit einem stationären Energiespeicher in Form großer Batterien sein, die ergänzend zu Dieselgeneratoren die Notstromversorgung garantieren könnten.

Das weist darauf hin, dass es unterschiedliche Arten von Redundanz gibt. In der system- und komplexitätstheoretischen Analyse etwa wurde skalenübergreifende Redundanz als relevant für Resilienz identifiziert. Dieser Ansatz wurde auf komplexe adaptive Systeme generell verallgemeinert. Führt ein widriges Ereignis zum Ausfall bestimmter Agenten, kann das System trotzdem weiterfunktionieren, wenn funktional äquivalente Agenten vorhanden sind, die nicht selbst durch das widrige Ereignis betroffen sind. Deshalb ist Redundanz über verschiedene Skalen hinweg sinnvoll, eben gerade verstanden als unterschiedliche Abhängigkeit bzw. Betroffenheit von widrigen Ereignissen. Im geschilderten Beispiel des Kernkraftwerks hängen Photovoltaik-Anlage und Dieselgeneratoren von sehr unterschiedlichen Bedingungen ab und sind jeweils unterschiedlich anfällig für bestimmte widrige Ereignisse. Ergebnis der Analyse war dann ein positiver Zusammenhang zwischen skalenübergreifender Redundanz und generischer Anpassungsfähigkeit. Je mehr skalenübergreifende Redundanzen in einem System vorhanden sind, desto größer ist seine generische Anpassungsfähigkeit - und damit seine Resilienz.

In der ingenieurwissenschaftlichen Resilienzforschung wird nicht von skalenübergreifender Redundanz gesprochen, sondern von physischer Redundanz einerseits und funktionaler Redundanz andererseits. Physische Redundanz ist dabei das Vorhalten mehrfach vorhandener, aber identischer Systemkomponenten, die beim Ausfall der primären Komponente diese substituieren können. Das trifft auf Dieselgeneratoren zur Notstromversorgung klassischerweise zu, oder auch auf Datensicherung durch regelmäßige Backups. Dieses Prinzip der physischen Redundanz wird im Bereich der Ingenieurwissenschaften durchgängig als sinnvoll und wir- 
kungsvoll verstanden und beschrieben und dieser Einschätzung schließt sich auch die vorliegende Arbeit grundsätzlich an (Jackson/Ferris 2013: 5, Madni/Jackson 2009: 189). Physische Redundanz funktioniert erst einmal unabhängig davon, welches konkrete widrige Ereignis das System trifft, sie ist ein generischer Mechanismus. Fällt eine kritische Systemkomponente aus, wird sie umgehend durch eine vorgehaltene, gleichartige ersetzt und das System kann seine kritischen Funktionalitäten weiter erbringen. Physische Redundanz hat jedoch auch Grenzen in ihrer sinnvollen Anwendbarkeit, wie ebenfalls bereits geschildert wurde und wie auch Linkov und Kott für den Bereich der Cybersicherheit veranschaulichen. Wenn identische redundante Systemkomponenten genutzt werden, sind diese eben auch gegenüber denselben widrigen Ereignissen anfällig (Linkov/Kott 2019: 13). Daher kann Resilience Engineering nicht ohne die zweite Art von Redundanz gedacht werden, die so genannte funktionale Redundanz. Funktionale Redundanz entspricht im Prinzip der Idee der skalenübergreifenden Redundanz. Einfach formuliert sollte es immer mindestens zwei Wege geben, kritische Aufgaben zu erfüllen (Jackson/Ferris 2013: 5). Der Fokus liegt auf der mehrfach vorhandenen Fähigkeit zur Erfüllung der kritischen Funktionalitäten - aber auf unterschiedliche Weisen. Das ist eng mit dem Diversitätsverständnis der vorliegenden Arbeit verknüpft, entspricht ihm aber aus den oben genannten Gründen nicht. Wenn kritische Systemkomponenten funktional redundant ausgelegt sind, sind sie von unterschiedlichen Fehlermechanismen abhängig (Altherr et al. 2018: 193, Madni/Jackson 2009: 189). Das erhöht die generische Anpassungsfähigkeit der entsprechenden Systeme. Insgesamt gesehen sollte ingenieurwissenschaftliche Resilienzforschung, sollte Resilience Engineering, unter anderem darin bestehen, die physische und funktionale Redundanz der Systemelemente der für die zivile SiFo relevanten komplexen adaptiven sozio-technischen Systeme zu erhöhen, die für die Aufrechterhaltung der kritischen Funktionalitäten dieser Systeme bei Eintreten gravierender und unerwarteter disruptiver Ereignisse verantwortlich sind. Die Hypothese für Resilience Engineering lautet dann:

Hypothese H32: Physische und funktionale Redundanz, verstanden als das zusätzliche Vorhandensein für die Funktionsfähigkeit im Normalfall nicht benötigter, entweder physisch oder funktional gleicher Systemkomponenten, sind geeignete Designprinzipien, um komplexe adaptive Systeme resilient zu gestalten. 


\subsection{Normative Aspekte für Resilience Engineering}

Die Erhöhung der Resilienz eines Systems ist genau dann normativ wünschenswert, wenn die zugrundeliegende Identität des Systems normativ wünschenswert ist. Ob Lösungen zur Erhöhung der Resilienz eines Systems erforscht und entwickelt werden sollten, hängt von vorgelagerten gesellschaftlichen und politischen Aushandlungsprozessen zur normativen Erwünschtheit des Systems ab.

¿o

Selbst, wenn ein System grundsätzlich normativ wünschenswert ist, gilt es bei der Entwicklung von Strategien zur Erhöhung seiner Resilienz darauf zu achten, dass möglicherweise bestehende Ungerechtigkeiten nicht perpetuiert oder sogar verstärkt werden.

do

Lösungen zur Erhöhung der Resilienz eines Systems müssen einer Proportionalität im Hinblick auf das angemessene Verhältnis zwischen Kosten und Nutzen entsprechen, über deren Ausgestaltung ebenfalls in vorgelagerten gesellschaftlichen und politischen Aushandlungsprozessen entschieden werden muss.

\&

Resilienz lässt sich normativ als starkdemokratische Sicherheit verstehen. Mit „starkdemokratisch" sind dabei nach Barber Strukturen gemeint, die eine aktive, transparente und partizipative Rolle von Bürgern bei gesellschaftlichen und politischen Aushandlungsprozessen bzgl. der normativen Erwünschtheit relevanter Systeme ermöglichen.

¿r

Politikwissenschaftlich gesprochen besteht Resilienz dann normativ in einer durch Verantwortungsannabme aktiver Bürger möglich werdenden Aufrechterhaltung gesellschaftlicher Freiheiten bei gleichzeitiger Erhöhung der Sicherheit.

do

Resilienz besteht nicht darin, aufgrund der Unsicherheit künftiger Ereignisse eine Strategie der Vorsicht im Sinne vorbeugender Freibeitseinschränkung anzuwenden.

\&

Resilienz besteht nicht darin, aufgrund der Unsicherheit künftiger Ereignisse eine Strategie der Präemption im Sinne der Aushöhlung von Freiheit durch aktivistische Maßnahmen anzuwenden.

Das eigenständige Resilienz-Konzept der zivilen SiFo, das in der vorliegenden Arbeit entwickelt wurde, enthält neben den aus der system- und komplexitätstheoretischen Analyse stammenden Bestandteilen auch eine ganze Reihe stärker normativ geprägter Annahmen. Diese sind unabdingbar für das stark interdisziplinär geprägte Anwendungsfeld der zivilen Si- 
Fo. Gegeben ihre im Wesentlichen rein sozialwissenschaftliche Herleitung ist es aber nicht möglich, ähnlich konkrete Hypothesen für Resilience Engineering daraus abzuleiten, wie etwa beispielsweise mit Blick auf die konkreten Prinzipien Redundanz, Diversität, Modularität und Dezentralität. Vielmehr kann die Übersetzungsleistung zwischen den normativen Aspekten des Resilienz-Konzepts der zivilen SiFo und Resilience Engineering nur in einer Art Bewusstseinsbildung bestehen. Ingenieurwissenschaftliche Resilienzforschung sollte nur dann dazu beitragen, die Resilienz bestimmter, komplexer adaptiver Systeme zu erhöhen, wenn diese nicht im Widerspruch zu den Werten stehen, die in vorgelagerten gesellschaftlichen und politischen Aushandlungsprozessen vereinbart wurden. Das ist eine Quintessenz aus all den hier skizzierten normativen Bestandteilen von Resilienz. Mithilfe von Resilienz kann und soll primär die Sicherheit der Gesellschaft erhöht werden. Gesellschaften sollen dank Resilienz besser mit unerwarteten, überraschend auftretenden und gravierenden disruptiven Ereignissen umzugehen in der Lage sein. Sicherheit zu erhöhen bzw. Sicherheit aufrechtzuerhalten, dazu beizutragen, Sicherheit als gesellschaftlichen Wert zu verstehen und mittels adäquater Ideen und Lösungen zu verstetigen, wurde als grundlegendes Ziel und grundlegender Daseinszweck der zivilen SiFo definiert (siehe 1.2). Sicherheit ist aber, wie die normative Analyse des Resilienz-Konzepts gezeigt hat, ganz sicher nicht der einzige Wert, den Gesellschaften als fundamental wichtig erachten. Als mindestens gleichrangig wurde im Kontext der Diskussion der Wert der Freiheit definiert. Und durch die Interpretation von Resilienz als einer Art starkdemokratischer Sicherheit konnte eine Verknüpfung zwischen Sicherheit und Freiheit aufgezeigt werden. Wenn Resilienz im Sinne der vorliegenden Arbeit verstanden wird, führt eine Erhöhung von Resilienz rein gesellschaftlich gesprochen nicht zu einer Einschränkung von Freiheit. Der entscheidende Prozess ist hier die Verantwortungsannahme durch aktive Bürger, die selbst darüber entscheiden, welche Balance zwischen Freiheit und Sicherheit sie für richtig erachten. Und die selbst darüber entscheiden, welche Systeme und Systembestandteile sie als normativ negativ oder aber normativ wünschenswert werten. Dazu bedarf es geeigneter demokratischer Prozesse. Dazu müssen die genannten gesellschaftlichen und politischen Aushandlungsprozesse so ausgestaltet sein, dass sich Bürger aktiv und sinnvoll daran beteiligen können. Zumal Resilienz mit Kosten verbunden ist. Resilienz zu erhöhen, erfordert den Einsatz knapper Ressourcen, die dann nicht mehr für andere, gesellschaftlich gewünschte Zwecke verwendet werden können. 
Was heißt das für Resilience Engineering? Zunächst sollte festgehalten werden, dass ingenieurwissenschaftliche Resilienzforschung kein Selbstzweck sein kann und sich außerdem auch nicht selbst genug sein kann. Ingenieurwissenschaftliche Resilienzforschung sollte nur dann Systeme resilient gestalten, wenn es gesellschaftlich gewünscht ist. Und die Ingenieurwissenschaften an sich verfügen nicht über die notwendigen Methoden und Instrumente, um über die gesellschaftliche, die normative Erwünschtheit des Systems und seiner konkreten Ausgestaltung zu entscheiden. Sie sind auf eine enge Zusammenarbeit mit den Sozialwissenschaften angewiesen. Resilienz ist insofern tatsächlich genau jene Art von boundary object oder Brückenkonzept, auf das bereits in der Einleitung der vorliegenden Arbeit verwiesen wurde (siehe 1.1). Und das unterscheidet Resilience Engineering auch von klassischen ingenieurwissenschaftlichen Ansätzen, die häufig ohne einen Rückbezug auf gesellschaftliche Fragestellungen von einem technologischen Problem ausgehen, welches mithilfe ebenfalls technologischer Lösungsoptionen behoben werden soll und kann. Eher in diesem Sinne formulieren zum Beispiel Perrig et al., wenn sie sagen „security must pervade every aspect of system design“ (Perrig et al. 2004: 54). Aus rein technischer Sicht und mit der Zielstellung einer Maximierung der Sicherheit ein sinnvoller Hinweis. Als Teil von Resilience Engineering sollte eine solche Maxime aber nicht gesehen werden, da sie zu einseitig die Sicherheit von Systemen betont, ohne die Konsequenzen einer derartigen Fokussierung auf andere, für das System bzw. die Gesellschaft relevante Werte zu berücksichtigen.

Resilience Engineering weist insgesamt also durchaus einen dominanten Fokus auf Technologie auf. Ansonsten wäre es weder notwendig noch sinnvoll, von Resilience Engineering zu sprechen. Im Unterschied zu einer rein technologischen Sichtweise umfasst Resilience Engineering aber die Möglichkeiten, die sich aufgrund der Funktion des Resilienz-Konzepts als boundary object bieten und nutzt bewusst auch sozialwissenschaftliche Erkenntnisse. Und zwar nicht nur im Sinne vorgelagerter Prozesse, sondern sehr stark auch im Sinne einer Bewertung der Auswirkungen der Entwicklung und Umsetzung ingenieurwissenschaftlicher Ideen und Maßnahmen zur Erhöhung der Resilienz komplexer adaptiver Systeme. Das entspricht einer konstanten Rückbindung ingenieurwissenschaftlicher Erkenntnisse an die durch gesellschaftliche und politische Aushandlungsprozesse gesetzten Rahmenbedingungen, denen Lösungen entsprechen sollten, wenn sie als resilienzerhöhend gelten wollen. Gerade in der zivilen SiFo entspricht ein solches, interdisziplinäres Vorgehen mittlerweile durchaus der favorisierten und häufig praktizierten Herangehensweise und 
stellt insofern kein Spezifikum von Resilience Engineering dar. Auch in anderen ingenieurwissenschaftlichen Bereichen werden gesellschaftliche Konsequenzen der Entwicklung und Anwendung neuer Technologien immer stärker von Beginn an mitgedacht. Das kann weniger stark für grundlagenorientierte Wissenschaft gelten, die zunächst die Frage beantworten möchte, was überhaupt wissenschaftlich möglich ist. Für anwendungsnahe Gebiete wie die zivile SiFo und ganz sicher für Resilience Engineering ist eine solche Kombination rein ingenieurwissenschaftlicher Forschung mit Orientierungswissen aus den Sozialwissenschaften aber unerlässlich. Für Resilience Engineering ergibt sich daraus die folgende Hypothese:

Hypothese H33: Resilience Engineering zeichnet sich durch eine Kombination aus Technologiefokus einerseits und der Berücksichtigung und konsequenten Einbeziehung sozialwissenschaftlich vermittelter normativer Aspekte andererseits aus.

Auch Systeme, deren zugrundeliegende Identität sich durch unfreie Strukturen auszeichnet, scheinen in der Lage zu sein, Resilienz gegenüber disruptiven Ereignissen auszubilden.

Diese grundsätzliche Erkenntnis wird von den meisten sozialwissenschaftlichen Ansätzen der Resilienzforschung geteilt. Empirisch lässt sich die Resilienz von Systemen, die auf unfreien Strukturen basieren, leicht an diversen im Lauf der Geschichte aufgetretenen despotisch herrschenden Diktatoren demonstrieren, etwa Fidel Castro, Saddam Hussein oder ganz aktuell auch Baschar al-Assad. Dann ist mit Resilienz die Fähigkeit des Systems an sich gemeint, sich politischen und wirtschaftlichen Veränderungen so anzupassen, dass die eigene Herrschaft gesichert bleibt. Hier geht es aber um die Resilienz derart unfreier Systeme gegenüber disruptiven Ereignissen. Wenn Menschen durch den Glauben an einen fundamentalistischen Führer vereint sind, können sie durchaus ein starkes Kohärenzgefühl ausbilden und so über generalisierte Widerstandsressourcen verfügen, die ihnen ermöglichen, disruptive Ereignisse gut zu überstehen. Das ist der Fall, weil die Bevölkerung die Realität mithilfe der vorgegebenen Ideologie als sinnhaft, handhabbar und bedeutsam begreift, deshalb zur Ordnung des Alltags weniger Ressourcen benötigt und diese im Fall des Auftretens eines widrigen Ereignisses nutzen kann (Antonovsky 1997: 104). Inwiefern dies zutrifft, oder ob diktatorische Herrschaftsformen aufgrund mangelnder Flexibilität nicht doch eher zu weniger Resilienz führen, kann nur sozialund nicht ingenieurwissenschaftlich untersucht werden. Nichtsdestotrotz ergeben sich auch für Resilience Engineering aus dieser Analyse interessante Überlegungen. Nämlich die nach der Anwendbarkeit von Ergebnissen 
ingenieurwissenschaftlicher Resilienzforschung auch in Gesellschaften, deren Strukturen sich durch Unfreiheit auszeichnen oder jedenfalls nicht dem entsprechen, was in einer starkdemokratischen Sichtweise als normativ wünschenswert erachtet wird. Indem Ergebnisse ingenieurwissenschaftlicher Resilienzforschung auch in unfreien Gesellschaften Anwendung finden, könnten sie mindestens indirekt dazu beitragen - wenn diese Gesellschaften disruptive Ereignisse besser überstehen, als ohne Resilienz - die Unfreiheit zu perpetuieren. Allerdings führen sie gleichzeitig auch zu einer Reduktion von Schäden, die sich zumeist in Todesopfern und verletzten Personen ausdrücken. Insofern lässt sich eine Nutzung von Ergebnissen ingenieurwissenschaftlicher Resilienzforschung in unfreien Gesellschaften erstens nicht vermeiden und führt zweitens auch dort - wenigstens in Bezug auf das eingetretene disruptive Ereignis - zu einer Verringerung menschlichen Leids. Für Resilience Engineering heißt das:

Hypothese H34: Dass die Ergebnisse von Resilience Engineering sich auch in Gesellschaften anwenden lassen, deren innere Strukturen durch Unfreibeit gekennzeichnet sind, lässt sich ingenieurwissenschaftlich nicht ausschließen, führt aber auch in solchen Gesellschaften grundsätzlich zunächst zu einer wünschenswerten Verringerung menschlichen Leids.

Eine unreflektierte Übernabme des Resilienz-Begriffs birgt die Gefahr, diesen als neoliberales Paradigma einer Verantwortungsverlagerung vom Staat auf den einzelnen Bürger misszuverstehen. Die Verwendung des Resilienz-Konzepts in der zivilen Sicherheitsforschung darf nicht dazu führen, dass von widrigen Ereignissen betroffenen Personen und Gruppen die Verantwortung für erlittene Schäden zugeschrieben wird.

Das Motiv einer unzulässigen Verlagerung der Verantwortung für Vorsorge, Vorbereitung, Abwehr und Erholung bzgl. gravierender, widriger Ereignisse vom Staat auf den einzelnen Bürger ist vor allem in der sozialwissenschaftlichen Forschung in Deutschland präsent. In einer polemischen Zuspitzung wird Resilienz dort teilweise in den größeren Kontext einer „Weltanschauung des Individualismus“ eingeordnet und als normativ negativ zu verstehendes, neoliberales Konzept gefasst. Die Analyse der vorliegenden Arbeit hat gezeigt, dass ein solches Resilienz-Verständnis international nicht anschlussfähig ist. Zwar basiert es auf einer durchaus sehr verbreiteten Sichtweise auf das Thema Resilienz, die unter dem Stichwort community resilience firmiert. Allerdings betonen die meisten Forscher, die in diesem Bereich tätig sind, die Chancen, die sich daraus ergeben, Menschen zu befähigen, mit gravierenden widrigen Ereignissen besser umgehen zu können. Die Aufgabe der Resilienzforschung kann und 
sollte demzufolge darin bestehen, Ideen und Ansätze zu entwickeln, mit deren Hilfe diese community resilience erhöht werden kann. Legt man dieser Einsicht das Resilienz-Konzept der zivilen SiFo zugrunde, geht es unter anderem um die Frage, wie die generische Anpassungsfähigkeit von Menschen sich verändern lässt. Und zwar zum einen ganz generell, da prinzipiell jeder von widrigen Ereignissen betroffen sein könnte. Darüber hinaus aber auch spezifisch, wenn es etwa um Personen geht, die verantwortlich für Management und Betrieb kritischer Infrastrukturen sind. $\mathrm{Zu}$ dieser Aufgabe können zweifelsohne die Sozialwissenschaften, aber auch die Psychologie und die Organisationswissenschaften erheblich beitragen. Es stellt sich die Frage, ob das auch für die Ingenieurwissenschaften gilt. Aus Sicht der vorliegenden Arbeit muss diese Frage bejaht werden. Zwar können ingenieurwissenschaftliche Ansätze nicht direkt die generische Anpassungsfähigkeit von Menschen erhöhen. Es ist aber möglich, technologische und sozio-technische Systeme und Lösungen zu entwickeln, welche es betroffenen Personen und verantwortlich Handelnden ermöglichen und leichter machen, ihre generische Anpassungsfähigkeit im Fall des Eintretens eines gravierenden widrigen Ereignisses besser nutzen zu können (Lechner et al. 2016: 84). Ein Beispiel dafür könnte im Bereich der kritischen Infrastrukturen etwa die Bereitstellung zuverlässiger Echtzeitinformationen sein, die mithilfe geeigneter Sensortechnologien einerseits und nutzerfreundlicher Visualisierungsmethoden gestützt durch Modelle zur Simulation komplexer adaptiver Systeme andererseits erfolgen könnte. Auf diese Weise verfügen die Verantwortlichen auch im Ausnahmefall über das benötigte Wissen zum aktuellen Zustand ihres Systems. Stärker auf der Ebene von Einzelpersonen bzw. Gruppen von Personen gedacht, könnten redundant ausgelegte Notstromaggregate helfen, sich auf das Eintreten widriger Ereignisse vorzubereiten. Einfacher wäre es an dieser Stelle aber vermutlich, bei der Installation von Photovoltaik-Anlagen auf Dächern die technische Möglichkeit zu schaffen, den erzeugten Strom direkt zu nutzen und ihn nicht notwendigerweise ins Stromnetz einspeisen zu müssen. Insgesamt bieten sich eine Vielzahl an Ansätzen und Möglichkeiten, über deren konkrete Ausgestaltung und Umsetzung sinnvollerweise in interdisziplinären Teams entschieden werden sollte. Für Resilience Engineering lässt sich festhalten:

Hypothese H35: Resilience Engineering besteht auch darin, Verantwortlichen und Betroffenen mithilfe technologischer und sozio-technischer Lösungen dabei zu helfen, ibre generische Anpassungsfähigkeit zu erhöhen. 
Strategien zur Erhöhung der Resilienz der für die zivile SiFo relevanten soziotechnischen Systeme müssen als Angebote formuliert werden, die für die Gesellschaft einen klaren, über obnehin erfolgende Maßnahmen des Bevölkerungsschutzes und der Katastrophenhilfe hinausgehenden, Mehrwert haben.

In konsequenter Weiterführung der gerade geschilderten Diskussionen gilt an dieser Stelle die Annahme, dass ingenieurwissenschaftliche Resilienzforschung dazu da ist, mögliche, technologische Lösungsvorschläge zur Erhöhung der Resilienz der relevanten Systeme zu entwickeln und deren Anwendung vorzuschlagen. Außerdem geht es darum, betroffene Personen oder verantwortlich Handelnde durch innovative technologische und sozio-technische Lösungen darin zu unterstützen, ihre generische Anpassungsfähigkeit zu erhöhen bzw. möglichst gut nutzen zu können. Gleichzeitig wurde aus der bisherigen Diskussion aber auch deutlich, worum es bei Resilience Engineering nicht geht. Nämlich darum, Entscheidungen über den tatsächlichen Einsatz, über die konkrete Umsetzung der erforschten und entwickelten Lösungen zu treffen. Die Verantwortung dafür, derartige Entscheidungen zu treffen, lässt sich nicht sinnvollerweise als Teil von Resilience Engineering fassen. Wie im Unterkapitel zum Stand der Forschung im Bereich ingenieurwissenschaftlicher Resilienzforschung formuliert wurde, entwickeln Ingenieure Lösungen. Basierend auf Missständen, die ihnen von gesellschaftlicher Seite aufgezeigt werden bzw. die sie selbst mithilfe technologischer Methoden identifizieren - auch das im Zweifel im Auftrag anderer gesellschaftlicher Akteure - entwickeln sie Mechanismen und Methoden, um den Missständen entgegenzuwirken (siehe 2.6). Darin liegt die Verantwortung der Ingenieurwissenschaften. Darin erschöpft sich aber auch die Verantwortung der Ingenieurwissenschaften. $\mathrm{Ob}$ und inwiefern die von ihnen entwickelten Lösungen im realen Umfeld eingesetzt werden, kann nicht ingenieurwissenschaftlich entschieden werden. In leichter Abwandlung des gerade aufgezeigten Aspekts des Resilienz-Konzepts der zivilen SiFo, wonach Strategien zur Erhöhung der Resilienz als Angebote formuliert werden müssen, lässt sich selbiges eben auch für ingenieurwissenschaftliche Lösungen zur Erhöhung von Resilienz sagen. Diese unterliegen gesellschaftlichen und politischen Aushandlungsprozessen, die wiederum unter anderem auf vorgelagerten Wertentscheidungen beruhen. Nichtsdestotrotz spielt Resilience Engineering auch in diesem Zusammenhang eine wichtige Rolle. Strategien oder Maßnahmen zur Erhöhung der Resilienz müssen einen klar erkennbaren Mehrwert haben, um implementiert zu werden. Das ist eine logische und sicherlich weithin akzeptable Schlussfolgerung der normativen Analyse der vorliegenden Arbeit. Inwiefern das aber auf konkrete, ingenieurwissenschaftli- 
che Lösungsvorschläge zutrifft, ist nicht einfach zu erkennen. Im Bereich der komplexen adaptiven Systeme, indem sich ingenieurwissenschaftliche Resilienzforschung notwendigerweise bewegt, sind ja gerade keine einfachen und offensichtlichen Kausalitäten am Werk. Zu verstehen, warum eine bestimmte Maßnahme zu einem bestimmten Resultat führen sollte ist - wenn überhaupt möglich - alles andere als trivial. Um informierte Entscheidungen treffen zu können, benötigen Entscheidungsträger, von einzelnen Bürgern über Rettungskräfte und Betreiber kritischer Infrastrukturen bis hin zur ganzen Gesellschaft, aber möglichst zuverlässige Informationen darüber, wie sich die Umsetzung bestimmter Lösungsvorschläge auf die jeweiligen Systeme auswirkt. Hier kommt Resilience Engineering ins Spiel. Auch wenn sich die Auswirkungen bestimmter Maßnahmen in komplexen adaptiven Systemen nicht deterministisch vorhersagen lassen, verfolgen ja die Ingenieurwissenschaften mit deren Entwicklung bestimmte Ziele. Sie versuchen, bestimmte Missstände mithilfe ihnen technologisch geeignet erscheinender Ansätze zu beseitigen bzw. dem System zu ermöglichen, erfolgreicher damit umgehen zu können. Aufgrund ihrer fachlichen Expertise haben sie dabei einen notwendigen und sehr großen Wissensvorsprung vor Entscheidungsträgern. Insofern besteht Resilience Engineering auch ein Stück weit aus einer Informationsfunktion. Bei der Entwicklung technologischer Lösungen zur Erhöhung der Resilienz der Gesellschaft bzw. relevanter komplexer adaptiver Systeme innerhalb der Gesellschaft, kommt es auch darauf an, in systematischer Weise allgemein verständliches und zugängliches Wissen zur Funktionsweise der Lösungen mitzudenken und vorzuhalten. Mit dessen Hilfe können die zuständigen Entscheidungsträger informierte und damit bessere Entscheidungen über deren Anwendung treffen. Als Hypothese resultiert daraus:

Hypothese H36: Resilience Engineering umfasst eine Informationsfunktion, die darin besteht, allgemein verständliches Wissen über die Funktionsweise technologischer Lösungen zur Erhöhung von Resilienz zu entwickeln, mit dessen Hilfe gesellschaftliche Akteure informierte Entscheidungen über deren Anwendung treffen können.

Vertrautheit als Orientierung am Gewesenen und Bekannten ist kein Mechanismus zur Erhöhung der Resilienz der für die zivile SiFo relevanten, komplexen adaptiven sozio-technischen Systeme.

\&

Je größer die Resilienz eines komplexen adaptiven Systems ist, desto größer ist das ihm entgegengebrachte Systemvertrauen. Gleichzeitig gilt: Je stärker das Systemvertrauen ausgeprägt ist, desto größer ist die Resilienz des Systems. 
\&

Strategien zur Erhöhung der Resilienz der für die zivile SiFo relevanten soziotechnischen Systeme müssen so ausgestaltet sein, dass sie sich positiv zumindest aber nicht negativ auf das Vertrauen in die zuverlässige Funktionalität der jeweiligen Systeme auch und gerade im Fall des Eintretens extrem unwahrscheinlicher und unerwarteter, die eigentlichen Belastungsgrenzen der Systeme übersteigender disruptiver Ereignisse, auswirken.

Diese drei Annahmen zum Thema Vertrauen weisen eine enge Verknüpfung mit der Diskussion zu Hypothese H36 auf. Die darin geschilderte Informationsfunktion zu erfüllen, kann signifikant zum Vertrauen der Bevölkerung in ingenieurwissenschaftliche Resilienzforschung beitragen. Dazu muss die Informationsfunktion allerdings in einer bestimmten Weise ausgeführt werden und bestimmte Eigenschaften aufweisen. Vertrauen wurde in der vorliegenden Arbeit definiert als Mechanismus zur Reduktion von Komplexität, der darin besteht, die Auswahl von Handlungsentscheidungen in der Gegenwart aufgrund generalisierter Erwartungen über die gegenwärtige Zukunft zu treffen. Generalisierte Erwartungen konstituieren sich mithilfe ähnlicher Erfahrungen aus der Vergangenheit. Systemvertrauen bestand dann im Vertrauen darin, dass das betreffende System seine Funktion, die in einer spezifischen Form der Reduktion von Umweltkomplexität besteht, zuverlässig und ohne größere Störungen erfüllt. Wendet man diese Definitionen auf die Informationsfunktion von Resilience Engineering an, lässt sich sagen, dass durch Vertrauen in die von den Ingenieurwissenschaften vorgeschlagenen Lösungen zur Erhöhung der Resilienz, letztere bereits erhöht werden könnte. Das setzt ein Nicht-Enttäuschen dieses Vertrauens voraus. Wenn sich ingenieurwissenschaftliche Lösungen wiederholt als ungeeignet erweisen, verlieren Entscheidungsträger sowie die Bevölkerung ihr Vertrauen in deren Wirksamkeit. Gleichzeitig ist es für die Ingenieurwissenschaften nicht möglich, die erwünschte Wirksamkeit ihrer Lösungen deterministisch zu garantieren. Komplexität und Unsicherheit machen ihr hier einen Strich durch die Rechnung. Deshalb bedarf es transparenter Kommunikation in mehrfacher Hinsicht. Wie in der Diskussion zur Informationsfunktion dargestellt, sollten die Ingenieurwissenschaften ihre Annahmen über die Funktionsweise der von ihnen entwickelten Lösungen möglichst allgemeinverständlich zu kommunizieren in der Lage sein. Dazu zählt beispielsweise auch, Grundannahmen, auf deren Basis überhaupt erst Lösungen entstehen, transparent zu machen. Das kann als grundlegender Anspruch an jede Kommunikation gelten, die sich im Rahmen des Verständnisses von Resilience Engineering, wie es in der vorliegenden Arbeit gesehen wird, bewegt. 
Aufgrund von Komplexität und Unsicherheit reicht diese Art von Transparenz aber nicht aus, um das Vertrauen in Resilience Engineering und daraus resultierend Systemvertrauen aufrechtzuerhalten bzw. erst zu gewinnen. Darüber hinaus gehört auch eine Aufrichtigkeit und Transparenz in Bezug auf Grenzen des Wissens, vielleicht sogar auf Grenzen des Wissbaren. Vertrauen erfordert eben auch eine transparente Kommunikation über die Grenzen dessen, was Ingenieurwissenschaften sicher wissen und voraussagen können. Dazu bedarf es zunächst auch einer Akzeptanz innerhalb der Ingenieurwissenschaften, dass eine vollständige Kontrolle in komplexen adaptiven Systemen nicht zu erreichen ist und dass Unsicherheit immer Teil der Realität sein wird. Determinismus ist nicht zu erreichen, jedenfalls dann nicht, wenn die Ingenieurwissenschaften den Anspruch erheben, auch im Rahmen der zivilen SiFo zu umsetzbaren Lösungsideen beitragen zu wollen. Ein „Kontrolloptimismus“ oder „Kontrollwahn“ geht notwendig fehl. Wird das als Grundannahme akzeptiert, kann auch entsprechend kommuniziert werden. Es ließe sich aber fragen, inwiefern eine aufrichtige und transparente Kommunikation über Grenzen des Wissens, über die reale Komplexität der Welt, nicht selbst eher zu Misstrauen führt. Wenn nichts mit Sicherheit gesagt werden kann, warum sollte man überhaupt noch etwas Gesagtem vertrauen? Hier muss zwischen Information über spezifische technische Lösungen und ihre Wirksamkeit einerseits und Kommunikation im Sinne von Resilience Engineering andererseits unterschieden werden. Das Vertrauen in die Wirksamkeit spezifischer Lösungen könnte durchaus sinken, wenn entsprechend offen informiert wird. Das Vertrauen in ingenieurwissenschaftliche Resilienzforschung an sich wird aber eher gestärkt, wenn insgesamt transparent kommuniziert wird. Denn erstens funktionieren spezifische technische Lösungen häufig so, wie sie sollen. Komplexe adaptive Systeme sind zwar nicht deterministisch vorhersehbar, weisen aber durchaus Muster auf und es lassen sich Wege finden, ihre Resilienz zielgerichtet zu erhöhen - insbesondere mittels der im vorangegangenen Unterkapitel skizzierten Prinzipien und Ansätze. Das zu beobachten, stärkt das Systemvertrauen. Und zweitens wird bei transparenter Kommunikation auch das Nicht-Funktionieren spezifischer Lösungen nicht $\mathrm{zu}$ einem Grund für Misstrauen, da es nicht automatisch einem Versagen der Ingenieurwissenschaften zugerechnet wird, sondern viel eher erkannt werden kann, dass sozusagen Komplexität dahintersteckt. Grenzen des Wissens zu kommunizieren, entspricht für Resilience Engineering außerdem einer bewussten Einordnung in einen größeren Rahmen interdisziplinärer Resilienzforschung. Resilience Engineering ist eben nur ein Teil 
des ganzen Bildes. Zusammengefasst ergibt sich für den Zusammenhang zwischen Resilience Engineering und Vertrauen folgende Hypothese:

Hypothese H37: Durch Transparenz in der Kommunikation im Hinblick auf durch Komplexität und Unsicherheit bedingte Grenzen des Wissens und des Wissbarens kann Resilience Engineering Vertrauen schaffen und aufrechterhalten.

Resilienz ist ein post-neoliberales Paradigma. Denn Resilienz geht aufgrund der Komplexität von Systemen von der grundsätzlichen Unvorhersehbarkeit der Auswirkungen zielgerichteter, proaktiver Interventionen im System aus. Daher muss der Fokus auf der Maximierung von Reflexivität und (generischer) Anpassungsfähigkeit liegen, die dem System eine kontinuierliche und dynamische Weiterentwicklung - auch durch staatliches Handeln - im Fall des Eintretens von Problemen ermöglichen.

¿。

Resilienz besteht darin, aufgrund der Unsicherheit künftiger Ereignisse eine Strategie der Vorbereitung im Sinne der Maximierung von Störungsverarbeitungskapazitäten anzuwenden, unter gleichzeitiger Beibehaltung gesellschaftlicher Freibeiten.

do

Resilienz besteht nicht darin, aufgrund der Unsicherheit künftiger Ereignisse eine Strategie der maximalen physischen Robustheit der betreffenden Systeme anzuwenden.

do

Die Resilienz der Gesellschaft gegenüber der Bedrohung durch das Phänomen des Terrorismus lässt sich nicht mithilfe rein physischer Schutzmaßnahmen erhöhen.

All diese Annahmen verweisen mehr oder weniger explizit auf die Bedeutung von generischer Anpassungsfähigkeit, wenn es um die Erhöhung von Resilienz geht. Die diesbezüglichen Prinzipien und Hypothesen wurden im vorangegangenen Unterkapitel identifiziert.

\subsection{Zusammenfassung der Hypothesen für Resilience Engineering}

Die Ingenieurwissenschaften können signifikant dazu beitragen, dass es manchmal besser kommt, als man denkt. Das kann als eine zentrale Erkenntnis der vorliegenden Arbeit gelten. Resilience Engineering ist möglich und sinnvoll. Es ist allerdings auch voraussetzungsreich und in sich komplex. Das hat die Analyse und die darauffolgende Bildung von Hypothesen in den vorangegangenen Unterkapiteln gezeigt. Unter Zuhil- 
fenahme der system- und komplexitätstheoretischen sowie der normativen Bestandteile des Resilienz-Konzepts der zivilen SiFo wurden insgesamt 37 Hypothesen entwickelt, die Resilience Engineering als Ansatz für ingenieurwissenschaftliche Resilienzforschung deutlich konkretisieren. Die Hypothesen werden im Folgenden in Tabelle 2 zusammengefasst. Damit konnten drei der Forschungslücken adressiert werden, die für die vorliegende Arbeit als relevant identifiziert wurden.

Zunächst basieren die Hypothesen für Resilience Engineering sämtlich auf einem systemischen Resilienz-Verständnis, das Komplexität und Unsicherheit explizit berücksichtigt und als entscheidende Faktoren in vielfacher Hinsicht definiert. Das führt zu Hypothesen wie beispielsweise H3, H6, H8 und H9 (siehe Tabelle 2). Diese widersprechen sehr bewusst vielen, eher klassischen, stabilitätszentrierten Ansätzen aus dem Bereich der ingenieurwissenschaftlichen Resilienzforschung. Deren Nützlichkeit soll keinesfalls in Abrede gestellt werden. Die vorliegende Arbeit formuliert aber durchaus deutlich, dass derartige klassische Ansätze nicht dazu geeignet sind, ein systemisches Resilienz-Verständnis ingenieurwissenschaftlich umzusetzen. Deshalb können sie kein Bestandteil von Resilience Engineering sein, das auf dem Resilienz-Konzept der zivilen SiFo beruht. Der systemische Fokus führt aber auch zu Hypothesen wie H2, H4, H11, H14, H15 und H20, die Resilience Engineering noch stärker system- und komplexitätstheoretisch verorten. Und es führt zu Hypothesen wie H33, H36 und $\mathrm{H} 37$, die aufzeigen, wie interdisziplinär Resilience Engineering zu denken ist und wie notwendig die Einbeziehung auch normativer Aspekte in ingenieurwissenschaftliche Resilienzforschung ist (siehe Tabelle 2).

Die Hypothesen für Resilience Engineering sind auch dazu geeignet, der Dominanz von Ansätzen zur Quantifizierung von Resilienz im Bereich der Ingenieurwissenschaften entgegenzuwirken. Zumal es nach $\mathrm{H} 3$ überhaupt nicht möglich ist, Resilienz mittels einfacher Maße zu quantifizieren. Diese zugegeben überspitzt formulierte Hypothese dient sozusagen als Ausgangspunkt der weiteren Diskussion. Wenn ingenieurwissenschaftliche Resilienzforschung nicht in der Entwicklung von Maßen zur Messung von Resilienz besteht, worin besteht sie dann? Darauf geben Hypothesen wie beispielsweise H22, H23, H30 und H31 Antwort, die konkrete Prinzipien beinhalten, mit deren Hilfe in komplexen adaptiven Systemen generische Anpassungsfähigkeit und so Resilienz erhöht werden kann. Aber auch Hypothesen wie H12, H16, H19, H26, H27 und H28 tragen zu einer Konkretisierung von Resilience Engineering im Hinblick darauf bei, wie sich Resilienz ingenieurwissenschaftlich umsetzen lässt. Der Modellierung und 
Simulation komplexer adaptiver Systeme kommt dabei eine besondere Bedeutung zu (siehe Tabelle 2).

Die fünfte Forschungslücke wird mithilfe der durchgängigen - mit Ausnahme des Unterkapitels 2.5 - Nutzung des Begriffs Resilience Engineering für ingenieurwissenschaftliche, technologiefokussierte Resilienzforschung adressiert. Das spiegelt sich letztlich in jeder einzelnen der 37 Hypothesen wider. Ziel dieser konsequenten Zuschreibung ingenieurwissenschaftlicher Inhalte für den Begriff Resilience Engineering ist sicher nicht, die organisationswissenschaftliche Forschung in diesem Bereich zu kritisieren oder ersetzen. Zumal der Resilienz-Begriff dieser Forschungsrichtung klar ein systemischer ist, aus dem Anleihen und Ansätze für die hier entwickelten Hypothesen für Resilience Engineering übertragen werden konnten. Das Ziel der vorangegangenen Ausführungen besteht aber durchaus darin, kritisch zu hinterfragen, ob Resilience Engineering für die organisationswissenschaftliche Resilienzforschung der geeignete Begriff sein kann, oder nicht generell stärker im hier verwendeten Sinne verstanden werden sollte.

\section{Tabelle 2: Hypothesen für Resilience Engineering}

\section{Nr. Beschreibung}

H1 Resilience Engineering erfordert ex ante eine Verständigung über die Bedeutung relevanter Begrifflichkeiten im Resilienz-Konzept der zivilen SiFo.

H2 Resilienz ist ein qualitatives weil komplexes Konzept, dessen ingenieurwissenschaftliche Umsetzung dies explizit zu berücksichtigen hat.

H3 Es ist nicht möglich, die Resilienz der für die zivile SiFo relevanten, komplexen adaptiven, sozio-technischen Systeme mittels einfacher Maße zu quantifizieren. Nichtsdestoweniger werden Maße für einfacher zu erhebende Größen benötigt, um den Systemzustand einschätzen und Resilienz erhöhen zu können.

H4 Die bewusste und transparente Definition von Systemgrenzen als analytisches Instrument ist eine Voraussetzung für Resilience Engineering.

H5 Dass die für die zivile SiFo relevanten Systeme sozio-technisch zu verstehen sind, macht es für Resilience Engineering notwendig, Menschen und menschliches Verhalten explizit mit zu berücksichtigen. 
Nr. Beschreibung

H6 Prävention und präventive Maßnahmen sind kein Bestandteil von Resilience Engineering.

H7 Resilience Engineering erfolgt bereits vor dem Eintreten eines disruptiven Ereignisses und trägt zur Erhöhung von Resilienz in den Phasen prepare, protect, respond und recover bei.

H8 Die quantitative Risikoanalyse und ähnliche, linear und deterministisch arbeitende Methoden sind nicht dazu geeignet, zu Resilience Engineering beizutragen.

H9 Die Entwicklung bedrohungsspezifischer Schutzmaßnahmen ist kein Teil von Resilience Engineering.

H10 Risiken mit extrem geringen Eintrittswahrscheinlichkeiten und extrem großen Auswirkungen können und sollten durch Resilience Engineering adressiert werden, da spezifische Vorbereitung aufgrund der Kostenintensität zumeist unterbleibt.

H11 Aufgrund der Unsicherheit von Ereignissen besteht ingenieurwissenschaftliche Resilienzforschung wesentlich aus der Entwicklung heuristischer generischer Prinzipien.

H12 Die Theorie komplexer adaptiver Systeme und die daraus entwickelten mathematischen Modelle und Methoden können als Teil von Resilience Engineering genutzt werden, um Umkipp-Punkte oder Schwellenwerte zu identifizieren.

H13 Die Nutzung geeigneter, etablierter Indikatoren zur Messung von Vulnerabilität ist Teil von Resilience Engineering und kann dazu genutzt werden, Systeme zu identifizieren, deren Resilienz besonders dringend erhöht werden sollte.

H14 Die Reduktion von Komplexität ist als Prinzip kein geeigneter Bestandteil von Resilience Engineering.

H15 Resilience Engineering besteht in der Erforschung und Entwicklung von Prinzipien, welche die generische Anpassungsfähigkeit komplexer adaptiver Systeme auf technologische Weise zu erhöhen vermögen.

H16 Die Theorie komplexer adaptiver Systeme und die daraus entwickelten mathematischen Modelle und Methoden können als Teil von Resilience Engineering genutzt werden, um Hebelpunkte zu identifizieren und durch deren Anwendung positive Kaskadeneffekte $\mathrm{zu}$ realisieren. 
Nr. Beschreibung

H17 Die Entwicklung spezifischer Frühwarnsysteme ist kein Teil von Resilience Engineering.

H18 Mithilfe realitätsnaher Notfallübungen können Systembestandteile identifiziert werden, die mittels Resilience Engineering optimiert werden sollten.

H19 Durch die Verwendung realitätsnaher Modelle zur Simulation des Verhaltens komplexer adaptiver Systeme im Ausnahmefall in Notfallübungen kann Resilience Engineering dazu beitragen, die generischen Kompetenzen der handelnden Personen zu erhöhen und so die Resilienz des Gesamtsystems zu steigern.

H20 Nachdem die Systemgrenzen definiert wurden, bedeutet Resilience Engineering im nächsten Schritt, die kritischen Funktionalitäten der untersuchten Systeme zu identifizieren.

H21 Ein Design, das Systemen ein elegantes Abschmelzen kritischer Funktionalitäten ermöglicht, trägt zur Resilienz von Systemen bei und sollte insofern Ziel ingenieurwissenschaftlicher Resilienzforschung sein.

H22 Modularität, verstanden als Aufteilung eines Systems in funktional eigenständige Einheiten, bestehend aus einer Reihe stark miteinander vernetzter Agenten, die nur lose Kopplungen mit den anderen Einheiten des Systems aufweisen, ist ein geeignetes Designprinzip, um komplexe adaptive Systeme resilient zu gestalten.

H23 Dezentralität, verstanden als Vermeidung des Auftretens hochkritischer Systemkomponenten bzw. hochspezialisierter Agenten, bei deren Ausfall das gesamte System unweigerlich zusammenbricht, ist ein geeignetes Designprinzip, um komplexe adaptive Systeme resilient zu gestalten.

H24 Die Resilienz kritischer Netz-Infrastrukturen kann durch den Aufbau vermaschter Netze erhöht werden.

H25 Um skalenfreie Netzwerke, wie sie für kritische Infrastrukturen teilweise typisch sind, resilient zu gestalten, müssen deren besonders relevante Elemente identifiziert und mittels geeigneter Prinzipien wie Redundanz und Diversität optimiert werden. 
Nr. Beschreibung

H26 Mithilfe agentenbasierter Modellierung kann die Funktionsweise komplexer adaptiver Systeme auch im stochastisch bestimmten extremen Ausnahmefall verstanden und so aufgezeigt werden, wie Resilience Engineering die Resilienz dieser Systeme beeinflusst.

H27 Methoden zur agentenbasierten Modellierung weiterzuentwickeln, ist Bestandteil von Resilience Engineering.

H28 Modulare Modellierung, verstanden als systemische Kombination unterschiedlicher Methoden zur Modellierung komplexer adaptiver Systeme, ist ein Bestandteil von Resilience Engineering.

H29 Die Beantwortung der Frage danach, wie KI-basierte Lösungen zur Erhöhung der Resilienz der für die zivile SiFo relevanten sozio-technischen Systeme beitragen können, ist Teil ingenieurwissenschaftlicher Resilienzforschung.

H30 Resilience Engineering lässt sich in komplexen adaptiven, soziotechnischen Systemen auch ohne den Einsatz künstlicher Intelligenz umsetzen.

H31 Diversität, verstanden als Vorhandensein funktional äquivalenter Gruppen, zusammengesetzt aus möglichst verschiedenartigen Agenten, ist ein geeignetes Designprinzip, um komplexe adaptive Systeme resilient zu gestalten.

H32 Physische und funktionale Redundanz, verstanden als das zusätzliche Vorhandensein für die Funktionsfähigkeit im Normalfall nicht benötigter, entweder physisch oder funktional gleicher Systemkomponenten, sind geeignete Designprinzipien, um komplexe adaptive Systeme resilient zu gestalten.

H33 Resilience Engineering zeichnet sich durch eine Kombination aus Technologiefokus einerseits und der Berücksichtigung und konsequenten Einbeziehung sozialwissenschaftlich vermittelter normativer Aspekte andererseits aus.

H34 Dass die Ergebnisse von Resilience Engineering sich auch in Gesellschaften anwenden lassen, deren innere Strukturen durch Unfreiheit gekennzeichnet sind, lässt sich ingenieurwissenschaftlich nicht ausschließen, führt aber auch in solchen Gesellschaften grundsätzlich zunächst zu einer wünschenswerten Verringerung menschlichen Leids. 
Nr. Beschreibung

H35 Resilience Engineering besteht auch darin, Verantwortlichen und Betroffenen mithilfe technologischer und sozio-technischer Lösungen dabei zu helfen, ihre generische Anpassungsfähigkeit zu erhöhen.

H36 Resilience Engineering umfasst eine Informationsfunktion, die darin besteht, allgemein verständliches Wissen über die Funktionsweise technologischer Lösungen zur Erhöhung von Resilienz zu entwickeln, mit dessen Hilfe gesellschaftliche Akteure informierte Entscheidungen über deren Anwendung treffen können.

H37 Durch Transparenz in der Kommunikation im Hinblick auf durch Komplexität und Unsicherheit bedingte Grenzen des Wissens und des Wissbarens kann Resilience Engineering Vertrauen schaffen und aufrechterhalten.

Die 37 Hypothesen, die in Tabelle 2 aufgeführt werden, lassen sich alle in systematischer Weise auf die normative sowie die system- und komplexitätstheoretische Analyse und Entwicklung des Resilienz-Konzepts der zivilen SiFo beziehen. Abbildung 6 macht diese Verknüpfungen noch einmal grafisch anschaulich. Damit zeigt sich, dass die vorliegende Arbeit insgesamt konsistent vom Stand der Forschung ausgehend Lücken identifiziert hat, darauf aufbauend mithilfe konzeptioneller Zugänge ein eigenständiges Resilienz-Konzept entwickelt und dieses wiederum dazu nutzt, Hypothesen für Resilience Engineering aufzustellen, so dass die fünf gefundenen Forschungslücken adressiert werden können. 
Abbildung 6: Hypothesen für Resilience Engineering und konzeptionelle Zugänge zu Resilienz

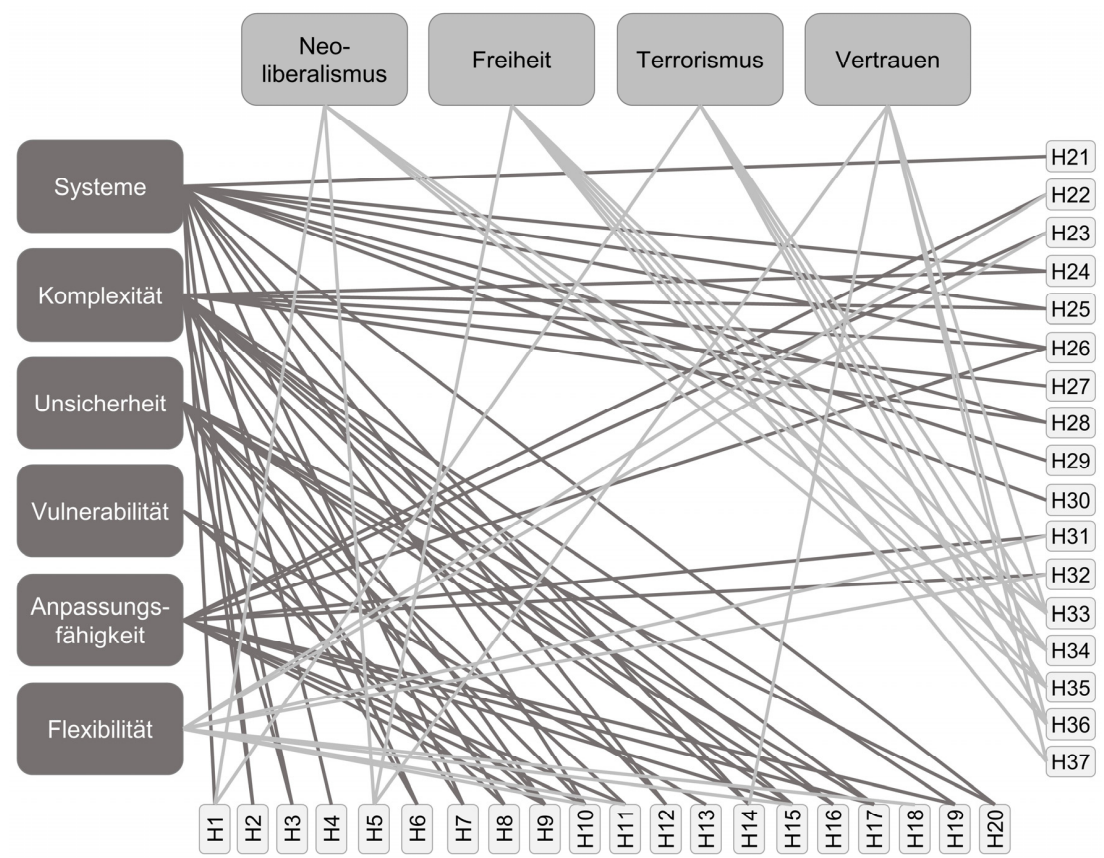

Quelle: eigene Darstellung.

\subsection{Grenzen für Resilience Engineering aus sozialwissenschaftlicher Sicht}

Resilience Engineering hat Grenzen. Ebenso wie Resilienz an sich nicht endlos ausweitbar ist, können auch ingenieurwissenschaftliche Ideen und Ansätze nicht immer und in jedem Fall dazu beitragen, die Resilienz der Gesellschaft zu erhöhen. Zunächst muss festgehalten werden, dass die 37 Hypothesen keine eins zu eins umsetzbare Anleitung für die Ingenieurwissenschaften darstellen, mit deren Hilfe sie konkrete Systeme resilient(er) gestalten können. Diesen Anspruch kann und möchte die vorliegende Arbeit nicht erheben. Vielmehr sind die 37 Hypothesen als Hinweise zu verstehen, die sich konsistent aus dem eigenständigen Resilienz-Konzept der zivilen SiFo ableiten lassen. Die enge Verknüpfung zwischen den Hypothesen und dem Resilienz-Konzept führt aber auch zu einer grundsätzlichen Übernahme von dessen Grenzen und Kritikpunkten. Res- 
ilience Engineering verstanden als ingenieurwissenschaftliche Umsetzung von Resilienz in einem systemischen Sinne, lässt sich beispielsweise nicht gegenüber allen beliebigen Arten von widrigen Ereignissen anwenden. Die genannten Beispiele der Eruption eines Super-Vulkans, des Einschlags eines großen Meteoriten auf der Erde oder der Invasion einer außerirdischen, technisch weit überlegenen Spezies haben das deutlich gemacht. Ein weiterer Punkt war eine theoretische Abgrenzung zwischen Risiko und Resilienz. Auch das wurde auf Resilience Engineering übertragen und vor allem in Form der Hypothesen H8, H9 und H10 aufgearbeitet. Der mögliche Konflikt zwischen Resilienz und Effizienz wiederum wurde vor dem Hintergrund begrenzter Ressourcen diskutiert und spielt für Resilience Engineering ebenfalls eine maßgebliche Rolle. Denn zur Umsetzung ingenieurwissenschaftlicher Maßnahmen zur Erhöhung von Resilienz werden Ressourcen benötigt. Gerade mit Blick auf das Prinzip der Redundanz wird ein Zielkonflikt zwischen Effizienz und Resilienz deutlich. Aber auch Prinzipien wie Modularität, Dezentralität und Diversität sind nicht notwendigerweise dazu geeignet, knappe Ressourcen nutzenmaximierend im Sinne kurzfristiger Effizienzsteigerung - einzusetzen. Der Zielkonflikt bleibt also bestehen und kann weiterhin nicht aufgelöst werden. Allerdings lässt er sich mittels der Hypothesen H36 und H37 zumindest explizit adressieren. Die Informationsfunktion von Resilience Engineering kann auch dazu beitragen, in geeigneter Weise die Kosten von Resilienz zu kommunizieren und der Gesellschaft so die Möglichkeit zu bieten, auf Grundlage sachlicher Informationen über die Umsetzung konkreter Maßnahmen zu entscheiden. Damit lässt sich gleichzeitig eine aus konstruktivistischer Sicht an Resilienz herangetragene Kritik zumindest ein Stück weit entkräften. Demnach lässt sich Resilienz immer nur retrospektiv beobachten und sei nicht mehr als ein Mythos, um zufällig aufgetretenen Ereignissen im Nachhinein Sinn zu verleihen. Die Hypothesen für Resilience Engineering sind aber keinesfalls auf das Eintreten eines widrigen Ereignisses angewiesen. Sie sollen im Gegenteil ja gerade dazu dienen, Gesellschaften im Vorhinein, prospektiv, resilienter zu gestalten. Mit Blick auf die Bestandteile des Konzepts stellt sich dann allerdings immer noch die Frage, was das konkret heißt. Denn es könnten durchaus auch Zielkonflikte innerhalb des Konzepts entstehen. Das gilt auch für Resilience Engineering, weshalb die Informationsfunktion und die Hypothesen $\mathrm{H} 1, \mathrm{H} 2$ und $\mathrm{H} 5$ besonders wichtig sind. Mit ihrer Hilfe lässt sich Resilience Engineering im größeren, interdisziplinären und transdisziplinären Kontext verorten.

Neben den gerade geschilderten, aus dem Resilienz-Konzept der zivilen SiFo abgeleiteten Kritikpunkten, spielen für Resilience Engineering 
noch eine Reihe weiterer Aspekte eine Rolle, wenn es um Grenzen der Gültigkeit der Hypothesen oder noch offene Fragestellungen geht. Zunächst ist Resilience Engineering immer kontextabhängig und in seiner konkreten Ausgestaltung nur für spezifische Systeme anwendbar. Das steht nur scheinbar im Widerspruch zu den generischen Prinzipien und der Betonung der Bedeutung generischer Anpassungsfähigkeit für Resilienz. Denn um die Resilienz realer, komplexer adaptiver Systeme zielgerichtet erhöhen zu können, müssen diese detailliert untersucht und möglichst verstanden werden - etwa mittels Methoden zur Modellierung und Simulation. Nur dann kann durch Resilience Engineering die generische Anpassungsfähigkeit des spezifischen Systems optimiert werden. Oder anders gesagt, es gibt keine „silver bullet solution“, kein über alle Systeme hinweg gültiges Patentrezept, wie die Hypothesen umgesetzt werden können (Kröger 2011: 75). Zumal nach wie vor das Problem der Unsicherheit vorhanden ist. Kneer und Nassehi formulieren das in ihrer Analyse der Systemtheorie Luhmanns mit Bezug zum Risikobegriff so: „Dass aber die Zukunft unbekannt bleibt, korrumpiert letztlich die Möglichkeit von Sicherheit" (Kneer/Nassehi 2000: 171). In komplexen adaptiven Systemen lassen sich Ursache und Wirkung nicht deterministisch und linear bestimmen. Es bleiben immer Unsicherheiten. Auch wenn Resilience Engineering das mithilfe der Hypothesen H36 und H37 im gesellschaftlichen Kontext durch Kommunikation zumindest adressieren kann, gilt das nicht unbedingt für die technologische Dimension. Wie bestimmte technologische Ansätze und Maßnahmen tatsächlich im konkreten System wirken, lässt sich nicht mit Sicherheit vorhersagen. Zwar sind die in diesem Kapitel identifizierten Prinzipien und Methoden für Resilience Engineering grundsätzlich wirkungsvoll und dazu geeignet, die Resilienz von Systemen zu erhöhen. Eine kontraintuitive und nicht-intendierte Wirkung lässt sich aber nicht deterministisch ausschließen. Rein aus der Theorie betrachtet, lassen sich nicht mal Wahrscheinlichkeiten für eine Wirksamkeit oder Unwirksamkeit präzise quantifizieren. Das ändert sich auch durch die Verwendung von Modellen nicht, die durch die Reduktion von Komplexität dazu beitragen sollen, Wissen für konkrete Entscheidungen bereitzustellen. Auch wenn das Modell daraufhin präzise Angaben zu machen imstande ist, lässt sich die Unsicherheit bei der Rückübersetzung in die Realität nicht vermeiden (Lukesch 2016: 299).

Nichtsdestotrotz müssen Modelle die Realität ein Stück weit vereinfachen, da sie sonst selbst zu komplex werden, um sinnvoll genutzt werden zu können (Ji et al. 2017: 1358). Das weist bereits in Richtung einer grundlegenden Kritik, die an Resilience Engineering geübt werden könn- 
te. Denn die zunehmende Komplexität der Gesellschaft resultiert unter anderem auch aus dem technischen Fortschritt. Wenn aber technischer Fortschritt zu größerer Komplexität führt, was Resilienz immer notwendiger macht, und Resilienz wiederum durch die Entwicklung technologischer Lösungen erhöht werden soll, scheint eher die Technologie selbst das „Problem“ zu sein. Oder wie Luhmann es ausdrückt: „Die Probleme der Technik zeigen sich an den Versuchen, die Probleme der Technik mit technischen Mitteln zu lösen“ (Luhmann 2003: 100). Gerade wenn Technologie aufgrund ihrer Komplexität sogar dazu führt, die Improvisations- und Anpassungsfähigkeit von Menschen in Ausnahmesituationen zu verringern, da sie ihre kognitiven Kompetenzen weit überschreitet (Schulmann/Roe 2007: 46ff). Zudem haben Technologien häufig gravierende und zunächst übersehene, unintendierte Nebenfolgen. Hier sei nur auf den durch die Verbrennung fossiler Brennstoffe wesentlich mitverursachten Klimawandel verwiesen. Ein reiner "technological fix“ ist also in komplexen adaptiven Systemen immer mit Unsicherheiten verbunden. Gerade deshalb gibt es in der Resilienzforschung und vor allem natürlich deren sozialwissenschaftlicher Spielart die Meinung, dass Resilienz primär als soziales Phänomen zu verstehen sei und Menschen die wichtigste Rolle spielen sollten (Bach et al. 2011: 28). Das widerspricht dem Verständnis von Resilience Engineering, wie es in der vorliegenden Arbeit entwickelt wurde, aber auch nicht, wie unter anderem die Hypothese $\mathrm{H} 2$ und $\mathrm{H} 5$ zeigen. Resilience Engineering stellt immer nur einen Baustein einer umfassenderen Resilienz-Strategie dar und ist selbst immer schon interdisziplinär und nicht rein technologisch zu verstehen. Das bannt die Gefahr unintendierter Nebenfolgen technologischer Entwicklungen nicht. Mit dieser muss Resilience Engineering immer rechnen und grundsätzlich umgehen. Es ordnet Resilience Engineering aber in einen größeren Kontext ein und ermöglicht daher, technologisch bedingte negative Entwicklungen frühzeitig zu erkennen.

Ein Beispiel für solche negativen Entwicklungen könnte etwa in einem Zuviel an Redundanz und Diversität bestehen. Longstaff zufolge könnte das zu mehr Komplexität führen und damit genau zu der Unbeherrschbarkeit für Anwender und Betreiber des Systems, von der gerade die Rede war (Longstaff 2012: 276). Das führt zu einem weiteren möglichen Kritikpunkt an den hier vorgeschlagenen Hypothesen für Resilience Engineering. Selbst wenn das Problem der endlichen Verfügbarkeit von Ressourcen nicht bestünde, ist fraglich, ob Sterbenz et al. mit ihrer Aussage "maximum resilience can be obtained with unlimited cost" recht haben (Sterbenz et al. 2010: 1255). Darüber sagen die 37 Hypothesen nicht direkt 
etwas aus, denn das ließ sich aus der normativen wie auch der system- und komplexitätstheoretischen Analyse von Resilienz nicht direkt ableiten. Es ist unklar, ob und wenn ja ab welchem Punkt mehr Redundanz vielleicht nicht mehr zu mehr generischer Anpassungsfähigkeit führt, sondern zu einem im Gegenteil eher unbeweglichen und starren System, das mit unerwarteten Ereignissen nicht umzugehen weiß. Ebensowenig wurde in den Hypothesen klargestellt, ob Redundanz grundsätzlich wichtiger ist als Diversität, oder ob vielleicht Dezentralität das wichtigste Prinzip ist. Es konnte nicht geklärt werden, ob eher Ressourcen in die Entwicklung neuer, agentenbasierter Simulationsmethoden investiert werden sollten oder in die Erforschung einer Konkretisierung der Informationsfunktion von Resilience Engineering. Mit anderen Worten bleibt theoretisch unscharf, welche Teile von Resilience Engineering wieviel zu Resilienz beitragen. Dieser Kritikpunkt gilt nicht nur für die vorliegende Arbeit. Er stellt vielmehr eine generelle Forschungslücke im Bereich ingenieurwissenschaftlicher Resilienzforschung dar (Goessling-Reisemann/Thier 2019: 132f). Deshalb wird darauf im abschließenden Kapitel im Hinblick auf den interdisziplinär-forschungsleitenden Ausblick noch einmal eingegangen.

Davor soll allerdings noch ein letzter, prinzipieller Kritikpunkt an den Hypothesen für Resilience Engineering beleuchtet werden. Die vorliegende Arbeit nutzt Resilienz bewusst als Brückenkonzept, als boundary object, um aus einer sozialwissenschaftlichen Perspektive heraus, ingenieurwissenschaftliche Resilienzforschung zu analysieren und konzeptionell weiterzuentwickeln. Das kann im ersten Schritt bereits an der unterschiedlichen Bedeutung von Begriffen scheitern, die es in den verschiedenen Disziplinen gibt (Alderson 2019: 69). Dem wurde unter anderem durch die Hypothese H1 proaktiv zu begegnen versucht, ohne damit diese Problemstellung gänzlich beseitigen zu können. Aus Sicht der Ingenieurwissenschaften lässt sich aber noch viel grundlegender fragen, ob ein Ansatz, wie die vorliegende Arbeit ihn wählt, überhaupt sinnvoll und statthaft ist. Resilienz ist selbst ein komplexes, ein qualitatives Konzept und auch die Hypothesen für Resilience Engineering können nicht einfach genommen und für konkrete, reale Systeme angewendet werden. Resilience Engineering besteht nicht aus Gleichungen, Formeln oder sonstigen mathematischen Modellen. Genau das stellt für die Ingenieurwissenschaften aber eine Herausforderung dar. Im interdisziplinären Austausch mit den Sozialwissenschaften sind deren Erkenntnisse für die Ingenieurwissenschaften zumeist zu unkonkret, zu vage, um tatsächlich umsetzbar zu sein (Altherr et al. 2018: 189, Woods 2019: 63). Woods spricht von einer "Sackgasse“ (impasse), in die interdisziplinäre Diskussionen immer wieder geraten, 
wenn einerseits vonseiten der Sozialwissenschaften auf die Bedeutung der Beachtung von Faktoren wie Komplexität, Unsicherheit und normativen Aspekten gedrängt und andererseits vonseiten der Ingenieurwissenschaften nach anwendbaren Lösungsmöglichkeiten zur Gestaltung realer Systeme gefragt wird (Woods 2019: 63). Dieser Diskussion kann sich die vorliegende Arbeit nicht entziehen. Und den daraus resultierenden Vorwurf einer nur begrenzten Nützlichkeit ihrer Ergebnisse für ingenieurwissenschaftliche Resilienzforschung kann sie letztlich nicht gänzlich entkräften. So sehr sie einem interdisziplinären Ansatz folgt, um den Austausch zwischen Ingenieur- und Sozialwissenschaften zu ermöglichen, so sehr ist sie doch gleichzeitig methodisch in den Sozialwissenschaften verhaftet. Allerdings gilt auch an dieser Stelle - wie in der Diskussion zu Hypothese H37 die Transparenz über in dem Fall in disziplinären Unterschieden begründete Grenzen des Konzepts als erster Schritt zu deren Überwindung. Die vorliegende Arbeit ist sich der Herausforderung an jeder Stelle bewusst und unternimmt nichtsdestoweniger den Versuch, für den Bereich der zivilen Sicherheitsforschung zum Dialog zwischen sozialwissenschaftlicher und ingenieurwissenschaftlicher Resilienzforschung beizutragen. Durch die Entwicklung eines eigenständigen, normativ wie system- und komplexitätstheoretisch fundierten Resilienz-Konzepts der zivilen SiFo und der darauf aufbauenden Formulierung von 37 Hypothesen für Resilience Engineering, konnte sie eine signifikant erweiterte Grundlage für diesen Dialog schaffen. 


\section{Fazit und Ausblick}

\subsection{Zusammenfassung der Ergebnisse}

Einen Beitrag dafür zu leisten, dass es besser kommt, als man denkt. Dass es besser kommt, als zu erwarten wäre. So lässt sich Forschung zum Thema Resilienz zusammenfassen. So lässt sich aber auch zivile Sicherheitsforschung verstehen. Und genau darin bestand auch die Motivation für die vorliegende Arbeit. Um das umsetzen zu können, wurde in den vorangegangenen Kapiteln eine interdisziplinäre, sowohl normative als auch system- und komplexitätstheoretische Analyse von Resilienz und Resilience Engineering unternommen, wie es sie in dieser Kombination aus wissenschaftlicher Breite und Tiefe bisher nicht gab. Damit steuert die vorliegende Arbeit zum interdisziplinären Resilienz-Diskurs insgesamt ein eigenständiges Resilienz-Konzept für die zivile SiFo und daraus abgeleitete Hypothesen für Resilience Engineering bei.

Das war nur durch ein systematisches Vorgehen in mehreren, aufeinanderfolgenden Schritten möglich. Zunächst wurden einige wichtige, übergreifende Grundannahmen untersucht und die Genese von Resilienz als Begriff und wissenschaftliches Konzept nachgezeichnet. Anschließend wurde der Stand der Forschung zu Resilienz in der Psychologie, der Ökologie und der sozial-ökologischen Forschung, verschiedenen Bereichen der Sozialwissenschaften, den Organisationswissenschaften sowie den Ingenieurwissenschaften analysiert. Dieser disziplinäre Zugang wurde aus zwei Gründen genutzt. Zum einen konnten insbesondere aus der Darstellung des Stands der Forschung in den Ingenieurwissenschaften und $\mathrm{zu}$ einem kleineren Teil den Sozialwissenschaften und den Organisationswissenschaften fünf Forschungslücken ausgemacht werden, zu deren SchlieBung die vorliegende Arbeit beitragen wollte. Die erste Forschungslücke bestand im Fehlen eines eigenständigen, theoretisch fundierten ResilienzKonzepts für die zivile SiFo. Als zweite Forschungslücke wurde das häufig reduktionistische, mechanische und Komplexität sowie Unsicherheit ausklammernde Resilienz-Verständnis der Ingenieurwissenschaften ausgemacht. Zumal dort, und das war die dritte Forschungslücke, eine Dominanz von Ansätzen zur Quantifizierung zu beobachten ist, die zulasten einer Konzentration auf Ideen zur konkreten Umsetzung von Resilienz geht. Wo solche Ideen existieren, sind sie zumeist theoretisch nicht hin- 
reichend fundiert. Auch das stellt eine Forschungslücke dar. Und schließlich schien die bisherige Verwendung des Begriffs Resilience Engineering in der Forschung zu stark mit organisationswissenschaftlichen Inhalten verknüpft. Aus den Forschungslücken heraus konnte wiederum die wissenschaftliche Fragestellung präzisiert werden: Was bedeutet Resilienz als Konzept in der zivilen Sicherheitsforschung und welche Hypothesen ergeben sich daraus für ein ingenieurwissenschaftliches Resilience Engineering?

Zum anderen wurde der disziplinäre Zugang genutzt, um Begrifflichkeiten und dahinterstehende Konzepte zu identifizieren, die für Resilienzforschung in mehreren Disziplinen von Bedeutung sind und die insofern dem interdisziplinären Charakter sowohl von Resilienz als Brückenkonzept als auch der zivilen SiFo entsprechen. Insgesamt wurden zehn derartige Konzepte als Ausgangspunkt für die weitere Analyse gewählt. Im ersten zentralen Teil der Arbeit wurden diese zehn Konzepte dann als sogenannte konzeptionelle Zugänge genutzt, mit deren Hilfe das eigenständige Resilienz-Konzept der zivilen SiFo entwickelt wurde. Dabei ergab sich eine logische Trennung zwischen stärker normativ konnotierten konzeptionellen Zugängen einerseits und stärker deskriptiv-analytischen Zugängen andererseits. Um letztere theoretisch fundiert untersuchen zu können, wurden die soziologische Systemtheorie nach Niklas Luhmann sowie die Theorie komplexer adaptiver Systeme, die einen stärker naturwissenschaftlichen Hintergrund hat, gewählt. Die konzeptionellen Zugänge der normativen Analyse waren Neoliberalismus, Freiheit, Terrorismus und Vertrauen. Die konzeptionellen Zugänge der system- und komplexitätstheoretischen Analyse waren System, Komplexität, Unsicherheit, Vulnerabilität, Anpassungsfähigkeit und Flexibilität. $\mathrm{Zu}$ jedem dieser Konzepte erfolgte eine detaillierte Untersuchung, eine Einordnung in den Kontext der zivilen SiFo und eine Ableitung daraus sich ergebender Bestandteile eines eigenständigen Resilienz-Konzepts. Um die Entstehung unverbunden nebeneinanderstehender Bestandteile zu verhindern, bauten die einzelnen Analysen jeweils explizit oder mindestens implizit aufeinander auf. Das Ergebnis der normativen sowie system- und komplexitätstheoretischen Analysen war das Resilienz-Konzept der zivilen SiFo, das aus zwei wesentlichen Teilen besteht. Einerseits einer Reihe von Definitionen für relevante Begrifflichkeiten, ohne die Resilienz nicht verstanden werden kann. Die Begrifflichkeiten waren die folgenden:

- System, Einfache Systeme, Systeme in der zivilen SiFo

- Komplexität, Komplexe adaptive Systeme 


\section{Fazit und Ausblick}

- Unsicherheit, Risiko, Ungewissheit, Echte Unsicherheit, Unerwartete Ereignisse

- Vulnerabilität

- Anpassungsfähigkeit, Spezialisierte \& Generische Anpassungsfähigkeit

- Transformationsfähigkeit, Flexibilität, Lernfähigkeit, Kreativität, Improvisationsfähigkeit

- Neoliberalismus

- Freiheit

- Terrorismus

- Vertrauen, Systemvertrauen

Andererseits wurden aus der normativen sowie system- und komplexitätstheoretischen Analyse heraus die eigentlichen Bestandteile des ResilienzKonzepts herausgearbeitet. Dabei stellte es sich als durchaus herausfordernd dar, diese Bestandteile in eine systematische Ordnung zu bringen. Der gewählte Lösungsweg war die Darstellung des Resilienz-Konzepts anhand von insgesamt sieben Leitfragen. Die Leitfragen lauteten:

- Was für eine Art Konzept ist Resilienz?

- Wann wird Resilienz benötigt?

- Welche Bedingungen sind aus system- und komplexitätstheoretischer Sicht notwendig für Resilienz?

- Worin besteht Resilienz aus system- und komplexitätstheoretischer Sicht?

- Worauf gilt es bei Resilienz im Rahmen der zivilen SiFo aus normativer Sicht zu achten?

- Worin besteht Resilienz aus normativer Sicht?

- Worin besteht Resilienz aus normativer Sicht nicht?

Auf Grundlage der Antworten auf die Leitfragen bestand der zweite zentrale Teil der Arbeit daraufhin in der Entwicklung von Hypothesen für Resilience Engineering im Sinne ingenieurwissenschaftlicher Resilienzforschung in der zivilen SiFo. Insgesamt konnten 37 Hypothesen gebildet werden, mit deren Hilfe Resilience Engineering deutlich konkretisiert wurde. Alle Hypothesen beruhen auf einer kombinierten Analyse der mithilfe von Leitfragen geordneten Bestandteile des Resilienz-Konzepts der zivilen SiFo sowie weiteren, aktuellen Erkenntnissen ingenieurwissenschaftlicher Resilienzforschung. Die normativen Leitfragen wurden zu einem Themenkomplex zusammengefasst, so dass letztlich fünf Themenkomplexe die Hypothesen ordneten. Die fünf Themenkomplexe werden nun in etwas erweiterter Weise auch dazu genutzt, die zentralen Erkenntnisse der vorliegenden Arbeit in systematisch gekoppelter Weise darzustellen. Zu jedem Themenkomplex gibt es auf der einen Seite relevante Bestandteile des 
Resilienz-Konzepts der zivilen SiFo und auf der anderen Seite zugehörige Hypothesen für Resilience Engineering. Die Themenkomplexe lauten:

- Art des Konzepts

- Notwendigkeit für Resilienz und Resilience Engineering

- Notwendige Bedingungen für Resilienz und Resilience Engineering

- Resilienz und Resilience Engineering aus system- und komplexitätstheoretischer Sicht

- Normative Aspekte von Resilienz und Resilience Engineering

Tabelle 3: Art des Konzepts

\begin{tabular}{|c|c|}
\hline Resilienz & Resilience Engineering \\
\hline $\begin{array}{l}\text { Resilienz ist } \\
\text { ein system- } \\
\text { theoretisches } \\
\text { Konzept. }\end{array}$ & $\begin{array}{l}\text { Hypothese H2: Resilienz ist ein qualitatives weil komple- } \\
\text { xes Konzept, dessen ingenieurwissenschaftliche Umset- } \\
\text { zung dies explizit zu berücksichtigen hat. } \\
\text { Hypothese H3: Es ist nicht möglich, die Resilienz der für } \\
\text { die zivile SiFo relevanten, komplexen adaptiven, sozio- } \\
\text { technischen Systeme mittels einfacher Maße zu quantifi- } \\
\text { zieren. Nichtsdestoweniger werden Maße für einfacher } \\
\text { zu erhebende Größen benötigt, um den Systemzustand } \\
\text { einschätzen und Resilienz erhöhen zu können. } \\
\text { Hypothese H4: Die bewusste und transparente Definition } \\
\text { von Systemgrenzen als analytisches Instrument ist eine } \\
\text { Voraussetzung für Resilience Engineering. }\end{array}$ \\
\hline $\begin{array}{l}\text { Sozio-techni- } \\
\text { sche Systeme } \\
\text { sind die Träger } \\
\text { von Resilienz. }\end{array}$ & $\begin{array}{l}\text { Hypothese H5: Dass die für die zivile SiFo relevanten } \\
\text { Systeme sozio-technisch zu verstehen sind, macht es } \\
\text { für Resilience Engineering notwendig, Menschen und } \\
\text { menschliches Verhalten explizit mit zu berücksichtigen. }\end{array}$ \\
\hline \multicolumn{2}{|c|}{ Resilienz ist eine Nicht-Selbstverständlichkeit, sie ist nicht erwartbar. } \\
\hline \multicolumn{2}{|c|}{$\begin{array}{l}\text { Resilienz ist nicht das Gegenteil von Vulnerabilität. Die beiden Kon- } \\
\text { zepte sind unabhängig voneinander. Größere Resilienz führt nicht } \\
\text { notwendigerweise zu im Zeitverlauf sinkender Vulnerabilität. }\end{array}$} \\
\hline \multicolumn{2}{|c|}{ Resilienz ist kein neoliberales Paradigma. } \\
\hline
\end{tabular}




\section{Fazit und Ausblick}

Tabelle 4: Notwendigkeit für Resilienz und Resilience Engineering

Resilienz $\quad$ Resilience Engineering

Das Vorhandensein oder Nicht-Vorhandensein von Resilienz lässt sich nur beobachten, wenn Systeme von einem disruptiven, in der Systemumwelt verorteten, gravierenden und häufig extrem unwahrscheinlichen Reiz (bzw. Ereignis) getroffen werden. Der Theorie komplexer adaptiver Systeme folgend, wird die Welt immer komplexer. Je komplexer die Welt, desto wahrscheinlicher wird das Auftreten eigentlich extrem unwahrscheinlicher, disruptiver Ereignisse. Die steigende Komplexität der Welt macht deshalb Resilienz als Eigenschaft komplexer adaptiver Systeme immer notwendiger.

Neben Ereignissen, die aus der Systemumwelt resultieren, müssen komplexe adaptive Systeme auch mit dem Auftreten unsicherer interner, durch die Entscheidungen von Agenten ausgelöster, von diesen unintendierter oder intendierter Störungen umgehen können. Die Wahrscheinlichkeit des Auftretens solcher Störungen sowie ihre potentiellen Auswirkungen steigen mit der Eigenkomplexität der Systeme. Denn zum einen können Agenten die Konsequenzen ihrer eigenen Handlungen mit zunehmender Komplexität schlechter überblicken, was die Wahrscheinlichkeit für Unfälle steigert. Zudem steigt mit der Diversität des Systems die Wahrscheinlichkeit, dass Agenten auftreten, deren Ziele konträr zu denen des Systems laufen. Zum anderen erhöht sich die Wahrscheinlichkeit für das Auftreten von Kaskadeneffekten, die beim Eintreten widriger Ereignisse zu großflächigen und häufig langfristigen Schäden am System führen. Um diese verhindern zu können, benötigt das System Resilienz. Auch die steigende Eigenkomplexität komplexer adaptiver Systeme macht Resilienz als Systemeigenschaft also immer notwendiger.
Hypothese H6: Prävention und präventive Maßnahmen sind kein Bestandteil von Resilience Engineering.

Hypothese H7: Resilience Engineering erfolgt bereits vor dem Eintreten eines disruptiven Ereignisses und trägt zur Erhöhung von Resilienz in den Phasen prepare, protect, respond und recover bei. 


\section{Resilienz}

Um Risiken zu bewältigen, benötigen Systeme keine Resilienz. Das Ereignis an sich, die Wahrscheinlichkeit seines Auftretens und seine Auswirkungen auf das System sind bekannt, so dass spezifische Maßnahmen zum Umgang mit dem Risiko ausreichen.

Es gibt Risiken, die komplexe adaptive Systeme ganz bewusst eingehen, weil ihre Eintrittswahrscheinlichkeit extrem gering ist, aber gleichzeitig die Auswirkungen so groß, dass die Ausbildung spezialisierter Anpassungsfähigkeit sehr viele Ressourcen in Anspruch nehmen würde. Zur Bewältigung von Risiken, für die komplexe adaptive Systeme keine spezialisierte Anpassungsfähigkeit entwickeln und die ihre Belastungsgrenzen übersteigen, ist Resilienz notwendig.

In komplexen adaptiven Systemen besteht die Möglichkeit, dass unerwartete Ereignisse mit gravierenden Auswirkungen, sogenannte schwarze Schwäne bzw. beispiellose Ereignisse auftreten. Zur Bewältigung solcher ungewissen und echt unsicheren Ereignisse, welche die Belastungsgrenzen komplexer adaptiver Systeme übersteigen, benötigen diese Resilienz.

Um mit langfristigen Veränderungsprozessen erfolgreich umgehen zu können, benötigen komplexe adaptive Systeme keine Resilienz. Erst wenn die langfristigen Prozesse durch Erreichen eines Schwellenwertes ein unerwartetes und die Belastungs-
Hypothese H10: Risiken mit extrem geringen Eintrittswahrscheinlichkeiten und extrem großen Auswirkungen können und sollten durch Resilience Engineering adressiert werden, da spezifische Vorbereitung aufgrund der Kostenintensität zumeist unterbleibt.

Hypothese H11: Aufgrund der Unsicherheit von Ereignissen besteht ingenieurwissenschaftliche Resilienzforschung wesentlich aus der Entwicklung heuristischer generischer Prinzipien.

\section{Hypothese H12: Die Theo-} rie komplexer adaptiver Systeme und die daraus entwickelten mathematischen Modelle und Methoden können als Teil von Resilience Engineering genutzt 


\begin{tabular}{|c|c|}
\hline \multicolumn{2}{|c|}{ Resilience Engineering } \\
\hline $\begin{array}{l}\text { grenzen des Systems überschreitendes Er- } \\
\text { eignis auslösen, wird Resilienz notwendig. }\end{array}$ & $\begin{array}{l}\text { werden, um Umkipp-Punk- } \\
\text { te oder Schwellenwerte zu } \\
\text { identifizieren. }\end{array}$ \\
\hline $\begin{array}{l}\text { Je höher die Vulnerabilität eines komple- } \\
\text { xen adaptiven Systems, desto wahrschein- } \\
\text { licher und stärker übersteigen widrige Er- } \\
\text { eignisse die eigentlichen Belastungsgren- } \\
\text { zen des Systems, bzw. desto stärker weicht } \\
\text { das System initial von seinem eigentlich } \\
\text { erwartbaren Entwicklungspfad ab, und } \\
\text { desto größer muss ceteris paribus seine Re- } \\
\text { silienz sein, um erfolgreich mit dem Ereig- } \\
\text { nis umgehen zu können. }\end{array}$ & $\begin{array}{l}\text { Hypothese H13: Die Nutzung } \\
\text { geeigneter, etablierter Indi- } \\
\text { katoren zur Messung von } \\
\text { Vulnerabilität ist Teil von } \\
\text { Resilience Engineering und } \\
\text { kann dazu genutzt werden, } \\
\text { Systeme zu identifizieren, } \\
\text { deren Resilienz besonders } \\
\text { dringend erhöht werden } \\
\text { sollte. }\end{array}$ \\
\hline
\end{tabular}

Tabelle 5: Notwendige Bedingungen für Resilienz und Resilience Engineering

Resilienz

Resilience Engineering

Einfache Systeme reagieren in deterministischer Weise auf Hypothese Belastungen. Übersteigen diese die Grenzen ihrer Belast- H14: Die Rebarkeit, bricht das System zusammen. Es kann nicht mehr duktion von auf einen neuen Entwicklungspfad umschwenken, auch nicht auf einen mit verminderter Systemleistung. Dazu Komplexität ist als Prinzip sind nur komplexe Systeme aufgrund ihrer Anpassungsfä- kein geeignehigkeit in der Lage. Resilienz drückt sich durch das Betreten neuer Entwicklungspfade aus, obwohl die eigentlichen Belastungsgrenzen des Systems überstiegen werden. Die Komplexität von Systemen ist also eine notwendige Bedingung für Resilienz.

A priori ist unklar, welchen Effekt steigende Komplexität auf das Ausmaß der im System vorhandenen bzw. auftretenden Resilienz hat. Steigende Komplexität kann sowohl zu mehr als auch zu weniger Resilienz führen. 


\section{Resilienz}

Das Vorhandensein von Anpassungsfähigkeit ist eine zwingende Grundvoraussetzung für Resilienz. Aber Resilienz ist nicht gleichbedeutend mit Anpassungsfähigkeit. Resilienz ist auch nicht gleichbedeutend mit generischer Anpassungsfähigkeit.

\section{Resilience Engineering}

Hypothese H15: Resilience Engi-

neering besteht in der Erforschung und Entwicklung von Prinzipien, welche die generische Anpassungsfähigkeit komplexer adaptiver Systeme auf technologische Weise zu erhöhen vermögen.

Transformationsfähigkeit ist kein notwendiger Bestandteil von Resilienz. Allerdings erhöht das Vorhandensein von Transformationsfähigkeit - verstanden als besonders ausgeprägte Form generischer Anpassungsfähigkeit - ceteris paribus die Resilienz komplexer adaptiver Systeme.

Je flexibler komplexe adaptive Systeme sind, desto größer ist ihre Resilienz.

Lernfähigkeit Hypothese H16: Die Theorie komplexer adaptiver Systeist eine notwen- me und die daraus entwickelten mathematischen Modige Grundvo- delle und Methoden können als Teil von Resilience raussetzung für Resilienz. Engineering genutzt werden, um Hebelpunkte zu identifizieren und durch deren Anwendung positive Kaskadeneffekte zu realisieren.

Hypothese H17: Die Entwicklung spezifischer Frühwarnsysteme ist kein Teil von Resilience Engineering. Hypothese H18: Mithilfe realitätsnaher Notfallübungen können Systembestandteile identifiziert werden, die mittels Resilience Engineering optimiert werden sollten.

Hypothese H19: Durch die Verwendung realitätsnaher Modelle zur Simulation des Verhaltens komplexer adaptiver Systeme im Ausnahmefall in Notfallübungen kann Resilience Engineering dazu beitragen, die generischen Kompetenzen der handelnden Personen zu erhöhen und so die Resilienz des Gesamtsystems zu steigern. 
Tabelle 6: Resilienz und Resilience Engineering aus system- und komplexitätstheoretischer Sicht

\section{Resilienz}

Resilienz liegt dann vor, wenn ein System auf einen sehr unwahrscheinlichen Reiz (bzw. Ereignis) aus der Umwelt oder seinem Inneren, der das System initial auf einen Entwicklungspfad mit stark verminderter Systemleistung bringt, mit der Realisierung eines Prozesses eigentlich extrem unerwartbarer Anschlussmöglichkeiten reagiert, um anschließend auf einen so realisierbar gewordenen, neuen Entwicklungspfad erwartbarer Anschlussmöglichkeiten einzuschwenken, der sich durch eine (mindestens) vergleichbare Systemleistung auszeichnet, wie sie vor Eintritt des Ereignisses vorlag.

In komplexen adaptiven Systemen muss zwischen spezialisierter und generischer Anpassungsfähigkeit unterschieden werden. Die Resilienz eines komplexen adaptiven Systems nimmt bei steigender Komplexität genau dann zu, wenn die generische Anpassungsfähigkeit des Systems steigt.

Resilienz zeigt sich in der Fähigkeit komplexer adaptiver Systeme, mittels generischer Anpassungsfähig-

\section{Resilience Engineering}

Hypothese H20: Nachdem die Systemgrenzen definiert wurden, bedeutet Resilience Engineering im nächsten Schritt, die kritischen Funktionalitäten der untersuchten Systeme zu identifizieren. Hypothese H21: Ein Design, das Systemen ein elegantes Abschmelzen kritischer Funktionalitäten ermöglicht, trägt zur Resilienz von Systemen bei und sollte insofern Ziel ingenieurwissenschaftlicher Resilienzforschung sein.
Hypothese H22: Modularität, verstanden als Auf- teilung eines Systems in funktional eigenstän- dige Einheiten, bestehend aus einer Reihe stark miteinander vernetzter Agenten, die nur lose Kopplungen mit den anderen Einheiten des Systems aufweisen, ist ein geeignetes Design- prinzip, um komplexe adaptive Systeme resili- ent zu gestalten.
Hypothese H23: Dezentralität, verstanden als Vermeidung des Auftretens hochkritischer Systemkomponenten bzw. hochspezialisierter Agenten, bei deren Ausfall das gesamte System unweigerlich zusammenbricht, ist ein geeigne- tes Designprinzip, um komplexe adaptive Sys- teme resilient zu gestalten. Hypothese H24: Die Resilienz kritischer Netz- Infrastrukturen kann durch den Aufbau ver- maschter Netze erhöht werden. 


\section{Resilienz}

keit extrem unwahrscheinliche oder unerwartete, ihre eigentlichen Belastungsgrenzen übersteigende Ereignisse so zu bewältigen, dass sie ihre Systemleistung möglichst aufrechterhalten und langfristig sichern können.

\section{Resilience Engineering}

Hypothese H25: Um skalenfreie Netzwerke, wie sie für kritische Infrastrukturen teilweise typisch sind, resilient zu gestalten, müssen deren besonders relevante Elemente identifiziert und mittels geeigneter Prinzipien wie Redundanz und Diversität optimiert werden. Hypothese H26: Mithilfe agentenbasierter Modellierung kann die Funktionsweise komplexer adaptiver Systeme auch im stochastisch bestimmten extremen Ausnahmefall verstanden und so aufgezeigt werden, wie Resilience Engineering die Resilienz dieser Systeme beeinflusst.

Hypothese H27: Methoden zur agentenbasierten Modellierung weiterzuentwickeln, ist Bestandteil von Resilience Engineering. Hypothese H28: Modulare Modellierung, verstanden als systemische Kombination unterschiedlicher Methoden zur Modellierung komplexer adaptiver Systeme, ist ein Bestandteil von Resilience Engineering. Hypothese H29: Die Beantwortung der Frage danach, wie KI-basierte Lösungen zur Erhöhung der Resilienz der für die zivile SiFo relevanten sozio-technischen Systeme beitragen können, ist Teil ingenieurwissenschaftlicher Resilienzforschung. Hypothese H30: Resilience Engineering lässt sich in komplexen adaptiven, sozio-technischen Systemen auch ohne den Einsatz künstlicher Intelligenz umsetzen.

Diversität und Redundanz sind Prinzipien zur Erhöhung generischer Anpassungsfähigkeit in komplexen adaptiven Systemen. Diversität besteht in einer möglichst unterschiedlichen Reaktion funktional gleicher Agenten auf extrem unwahrscheinliche oder unerwartete Veränderungen. Je größer die Diversität innerhalb funktional äquivalenter
Hypothese H31: Diversität, verstanden als Vorhandensein funktional äquivalenter Gruppen, zusammengesetzt aus möglichst verschiedenartigen Agenten, ist ein geeignetes Designprinzip, um komplexe ad- 


\section{Fazit und Ausblick}

Resilienz

Resilience Engineering

Gruppen komplexer adaptiver Systeme, desto aptive Systeme resilient zu größer ist ihre generische Anpassungsfähig- gestalten. keit.

Skalenübergreifende Redundanz bedeutet das Vorhandensein funktional äquivalenter Systemelemente, die sich in ihrer Abhängigkeit von unterschiedlichen widrigen Ereignissen unterscheiden. Je mehr skalenübergreifende Redundanzen vorhanden sind, desto gröBer ist die generische Anpassungsfähigkeit komplexer adaptiver Systeme.
Hypothese H32: Physische und funktionale Redundanz, verstanden als das zusätzliche Vorhandensein für die Funktionsfähigkeit im Normalfall nicht benötigter, entweder physisch oder funktional gleicher Systemkomponenten, sind geeignete Designprinzipien, um komplexe adaptive Systeme resilient zu gestalten.

\section{Tabelle 7: Normative Aspekte von Resilienz und Resilience Engineering}

\section{Resilienz $\quad$ Resilience Engineering}

Die Erhöhung der Resilienz eines Systems ist genau dann normativ wünschenswert, wenn die zugrundeliegende Identität des Systems normativ wünschenswert ist. Ob Lösungen zur Erhöhung der Resilienz eines Systems erforscht und entwickelt werden sollten, hängt von vorgelagerten gesellschaftlichen und politischen Aushandlungsprozessen zur normativen Erwünschtheit des Systems ab.

Selbst, wenn ein System grundsätzlich normativ wünschenswert ist, gilt es bei der Entwicklung von Strategien zur Erhöhung seiner Resilienz darauf zu achten, dass möglicherweise bestehende Ungerechtigkeiten nicht perpetuiert oder sogar verstärkt werden.

Lösungen zur Erhöhung der Resilienz eines Systems müssen einer Proportionalität im Hinblick auf das angemessene Verhältnis zwischen Kosten und Nutzen entsprechen, über deren Ausge-
Hypothese H33: Resilience Engineering zeichnet sich durch eine Kombination aus Technologiefokus einerseits und der Berücksichtigung und konsequenten Einbeziehung sozialwissenschaftlich vermittelter normativer Aspekte andererseits aus. 


\begin{abstract}
Resilienz Resilience Engineering

staltung ebenfalls in vorgelagerten gesellschaftlichen und politischen Aushandlungsprozessen entschieden werden muss.

Resilienz lässt sich normativ als starkdemokratische Sicherheit verstehen. Mit „starkdemokratisch" sind dabei nach Barber Strukturen gemeint, die eine aktive, transparente und partizipative Rolle von Bürgern bei gesellschaftlichen und politischen Aushandlungsprozessen bzgl. der normativen Erwünschtheit relevanter Systeme ermöglichen.

Politikwissenschaftlich gesprochen besteht Resilienz dann normativ in einer durch Verantwortungsannahme aktiver Bürger möglich werdenden Aufrechterhaltung gesellschaftlicher Freiheiten bei gleichzeitiger Erhöhung der Sicherheit. Resilienz besteht nicht darin, aufgrund der Unsicherheit künftiger Ereignisse eine Strategie der Vorsicht im Sinne vorbeugender Freiheitseinschränkung anzuwenden.

Resilienz besteht nicht darin, aufgrund der Unsicherheit künftiger Ereignisse eine Strategie der Präemption im Sinne der Aushöhlung von Freiheit durch aktivistische Maßnahmen anzuwenden.
\end{abstract}

Auch Systeme, deren zugrundeliegende Identität sich durch unfreie Strukturen auszeichnet, scheinen in der Lage zu sein, Resilienz gegenüber disruptiven Ereignissen auszubilden.
Hypothese H34: Dass die Ergebnisse von Resilience Engineering sich auch in Gesellschaften anwenden lassen, deren innere Strukturen durch Unfreiheit gekennzeichnet sind, lässt sich ingenieurwissenschaftlich nicht ausschließen, führt aber auch in solchen Gesellschaften grundsätzlich zunächst zu einer wünschenswerten Verringerung menschlichen Leids.
Eine unreflektierte Übernahme des ResilienzBegriffs birgt die Gefahr, diesen als neoliberales Paradigma einer Verantwortungsverlagerung vom Staat auf den einzelnen Bürger
Hypothese H35: Resilience Engineering besteht auch darin, Verantwortlichen und Betroffenen 
Resilienz

misszuverstehen. Die Verwendung des Resilienz-Konzepts in der zivilen Sicherheitsforschung darf nicht dazu führen, dass von widrigen Ereignissen betroffenen Personen und Gruppen die Verantwortung für erlittene Schäden zugeschrieben wird.

Strategien zur Erhöhung der Resilienz der für die zivile SiFo relevanten sozio-technischen Systeme müssen als Angebote formuliert werden, die für die Gesellschaft einen klaren, über ohnehin erfolgende Maßnahmen des Bevölkerungsschutzes und der Katastrophenhilfe hinausgehenden, Mehrwert haben.

\section{Resilience Engineering}

mithilfe technologischer und sozio-technischer Lösungen dabei zu helfen, ihre generische Anpassungsfähigkeit zu erhöhen.
Vertrautheit als Orientierung am Gewesenen und Bekannten ist kein Mechanismus zur Erhöhung der Resilienz der für die zivile SiFo relevanten, komplexen adaptiven sozio-technischen Systeme. Je größer die Resilienz eines komplexen adaptiven Systems ist, desto größer ist das ihm entgegengebrachte Systemvertrauen. Gleichzeitig gilt: Je stärker das Systemvertrauen ausgeprägt ist, desto gröBer ist die Resilienz des Systems.

Strategien zur Erhöhung der Resilienz der für die zivile SiFo relevanten sozio-technischen Systeme müssen so ausgestaltet sein, dass sie sich positiv zumindest aber nicht negativ auf das Vertrauen in die zuverlässige Funktionalität der jeweiligen Systeme auch und gerade im Fall des Eintretens extrem unwahrscheinlicher und unerwarteter, die eigentlichen Belastungsgrenzen der Systeme übersteigender disruptiver Ereignisse, auswirken.

Resilienz ist ein post-neoliberales Paradigma. Denn Resilienz geht aufgrund der Komplexität von Systemen von der grundsätzlichen Unvorhersehbarkeit der Auswirkungen zielgerichteter, proaktiver Interventionen im System aus. Daher muss der Fokus auf der Maximierung von 


\section{Resilienz}

Resilience Engineering

Reflexivität und (generischer) Anpassungsfähigkeit liegen, die dem System eine kontinuierliche und dynamische Weiterentwicklung auch durch staatliches Handeln - im Fall des Eintretens von Problemen ermöglichen.

Resilienz besteht darin, aufgrund der Unsicherheit künftiger Ereignisse eine Strategie der Vorbereitung im Sinne der Maximierung von Störungsverarbeitungskapazitäten anzuwenden, unter gleichzeitiger Beibehaltung gesellschaftlicher Freiheiten.

Resilienz besteht nicht darin, aufgrund der Unsicherheit künftiger Ereignisse eine Strategie der maximalen physischen Robustheit der betreffenden Systeme anzuwenden.

Die Resilienz der Gesellschaft gegenüber der Bedrohung durch das Phänomen des Terrorismus lässt sich nicht mithilfe rein physischer Schutzmaßnahmen erhöhen.

\subsection{Offene Fragestellungen und Kritik}

Die vorliegende Arbeit konnte einen Beitrag zur Adressierung einer Reihe von Forschungslücken im Bereich der zivilen SiFo und ingenieurwissenschaftlicher Resilienzforschung leisten. Dabei wurde auch die grundlegende wissenschaftliche Fragestellung beantwortet. Im Gesamtkontext dieser beiden Bereiche bleiben jedoch weiterhin eine Vielzahl an offenen Fragestellungen zu deren Beantwortung weitere Forschung notwendig ist. Nicht zuletzt hat die vorliegende Arbeit selbst eine Reihe von neuen Fragen aufgeworfen, die sich stellen, wenn das Resilienz-Konzept der zivilen SiFo und die Hypothesen für Resilience Engineering kritisch betrachtet werden. Auf mögliche Grenzen von Resilienz und Resilience Engineering wurde in den entsprechenden Unterkapiteln bereits detaillierter eingegangen, so dass diese Kritik nun hier nur noch einmal überblicksartig zusammengefasst werden soll.

Resilienz und Grenzen: Dieser erste Kritikpunkt bezieht sich darauf, dass auch sehr resiliente Systeme nicht mit jedem beliebigen Ereignis fertig werden können. Zwar ist theoretisch nicht bestimmbar, wo diese Grenzen liegen. Es lassen sich aber Beispiel finden - Invasion Außerirdischer, Einschlag eines großen Meteoriten, etc. - bei denen jedes noch so resiliente System notwendig versagen muss. Denn die pure Weiterexistenz der Spezi- 


\section{Fazit und Ausblick}

es Mensch ist definitionsgemäß in der vorliegenden Arbeit kein Ausweis von Resilienz.

Resilienz und Risiko: Resilienz als Konzept und Resilience Engineering als ingenieurwissenschaftliche Umsetzung sind nicht dazu geeignet und sollen auch nicht dazu dienen, etablierte Methoden wie etwa Risikomanagement oder klassische Schutzmaßnahmen zu ersetzen. Vielmehr sind sie eine Erweiterung der Möglichkeiten ziviler SiFo, um auch mit extrem unwahrscheinlichen oder unerwarteten, die eigentlichen Belastungsgrenzen des Systems übersteigenden disruptiven Ereignissen erfolgreich umgehen zu können. Indem sie Komplexität und Unsicherheit explizit berücksichtigen, unterscheiden sich Resilienz und Resilience Engineering deutlich von anderen Strategien im Bereich der zivilen SiFo.

Resilienz und Effizienz: Um die Resilienz komplexer adaptiver Systeme zu erhöhen, bedarf es des Einsatzes knapper Ressourcen. Oder einfacher gesagt: Resilienz kostet Geld. Und Ressourcen können immer nur einmal eingesetzt werden. Mit Blick auf Resilienz-Prinzipien wie Redundanz und Diversität wird ein Zielkonflikt zwischen maximaler Effizienz einerseits und Erhöhung von Resilienz andererseits deutlich. Es ist nach wie vor ungeklärt, wie eine optimale Balance zwischen den beiden Zielen erreicht werden könnte bzw. ob es eine solche überhaupt geben kann. Die Entscheidungen darüber, in Resilienz zu investieren oder nicht, sind letztlich nur in gesellschaftlichen und politischen Aushandlungsprozessen zu treffen.

Resilienz und Mythen: Überspitzt formuliert, stellt die vorliegende Arbeit ein exzellentes Beispiel dafür da, wie Wissenschaft dazu tendiert, großen Aufwand zu treiben, um dem Auftreten komplett überraschender, unerwarteter Ereignisse retrospektiv einen Sinn zu verleihen. Wenn Menschen oder Systeme es schaffen, sich solchen Ereignissen erfolgreich anzupassen, daraus zu lernen und langfristig sogar gestärkt daraus hervorzugehen, muss mehr dahinterstecken, als purer Zufall. Und dieses mehr wird als Resilienz bezeichnet. Obschon die vorliegende Arbeit ihr Resilienz-Konzept in systematischer Weise entwickelt, lässt sich diese konstruktivistische Kritik an Resilienz nicht gänzlich von der Hand weisen.

Resilienz und Zielkonflikte: Das Resilienz-Konzept der vorliegenden Arbeit ist selbst komplex. Es besteht aus einer Vielzahl normativer sowie system- und komplexitätstheoretischer Annahmen, die erst gemeinsam Resilienz ausmachen. In diesem Sinne ist es tatsächlich systemisch. Allerdings lassen sich deshalb innerhalb des Konzepts Zielkonflikte zwischen verschiedenen Bestandteilen nicht a priori und rein aus der Theorie heraus ausschließen. Das gilt auch und vor allem für Zielkonflikte zwischen den 
normativen Aspekten des Konzepts auf der einen Seite und den systemund komplexitätstheoretischen Annahmen auf der anderen Seite. Dieser Kritik muss sich die vorliegende Arbeit stellen.

Resilience Engineering und Unsicherheit: Die Nichtlinearität von Ursache und Wirkung ist ein charakteristisches Zeichen komplexer adaptiver Systeme. Aufgrund von Feedbackschleifen und der starken Vernetzung der Agenten untereinander, lässt sich nicht deterministisch festlegen, wie die Anwendung und Einführung bestimmter technologischer oder sonstiger Maßnahmen und Ideen auf das System wirkt. Nicht-intendierte Nebenfolgen oder sogar eine kontraintuitive Wirkung lassen sich nicht ausschließen. Dieser Herausforderung kann Resilience Engineering auch nicht durch die Verwendung von Methoden zur Modellierung und Simulation komplexer Systeme entgehen. Sie sollte vielmehr explizit mittels der Informationsfunktion adressiert werden.

Resilience Engineering und technischer Fortschritt: Die zunehmende Komplexität der Gesellschaft resultiert zu großen Teilen aus der Entwicklung moderner und innovativer Technologien, insbesondere im Zuge der Digitalisierung. Zunehmende Komplexität und wachsende Unsicherheit machen wiederum Resilienz immer notwendiger, wie die system- und komplexitätstheoretische Analyse gezeigt hat. Resilience Engineering sieht sich demzufolge durchaus der Kritik ausgesetzt, dass Probleme, die durch Technologie erst verursacht werden, durch mehr Technologie gelöst werden sollen. Dieser "technological fix" wird auch und gerade aus sozialwissenschaftlicher Sicht kritisiert, weshalb die vorliegende Arbeit Resilience Engineering auch in den größeren Kontext von Resilienz für die zivile SiFo einordnet.

Resilience Engineering und Entscheidungen: Wieviel Redundanz ist eigentlich genug Redundanz? Ist die Diversität eines Systems wichtiger als seine Modularität? Sollten begrenzte Ressourcen eher in die Entwicklung fortschrittlicher Methoden zur Modellierung und Simulation komplexer Systeme oder eine dezentrale Ausgestaltung konkreter Systeme investiert werden? All diese Fragen lassen sich mithilfe der 37 Hypothesen für Resilience Engineering nicht beantworten. Es bleibt theoretisch ungeklärt, wie stark die Umsetzung bestimmter Hypothesen zu Resilienz beiträgt und welche insofern besonders wichtig - oder aber zu vernachlässigen sind. Hier besteht weiterhin eine Forschungslücke und zwar nicht nur als Resultat der vorliegenden Arbeit, sondern ganz generell im Bereich der ingenieurwissenschaftlichen Resilienzforschung.

Resilience Engineering und Sozialwissenschaften: Vonseiten der Ingenieurwissenschaften ließe sich der Ansatz der vorliegenden Arbeit grundsätzlich 


\section{Fazit und Ausblick}

kritisieren. Denn die Hypothesen für Resilience Engineering bestehen nicht aus konkreten mathematischen oder ingenieurwissenschaftlichen Modellen und Methoden, die direkt anwendbar sind. Vielmehr sind die Hypothesen selbst qualitativ und ein Stück weit komplex. Für Ingenieurwissenschaften auf den ersten Blick zu unkonkret und vage. Den Vorwurf einer nur begrenzten Nützlichkeit ihrer Ergebnisse für ingenieurwissenschaftliche Resilienzforschung kann die vorliegende Arbeit letztlich nicht gänzlich entkräften. Nichtsdestoweniger unternimmt sie den Versuch, für den Bereich der zivilen Sicherheitsforschung zum Dialog zwischen sozialwissenschaftlicher und ingenieurwissenschaftlicher Resilienzforschung beizutragen.

\subsection{Interdisziplinär-forschungsleitender Ausblick}

Es bleibt viel zu tun. Das gilt für die zivile SiFo genauso wie für die Resilienzforschung. Und das gilt für die Arbeit an und mit Resilienz als theoretischem Konzept genauso wie für die Entwicklung anwendbarer und umsetzbarer Lösungen zur Erhöhung der Resilienz gesellschaftlich relevanter Systeme, wie sie etwa durch Resilience Engineering gelingen soll. Die Kritik, die an den Ergebnissen der vorliegenden Arbeit geübt werden kann und die offenen Fragestellungen, die sie nicht beantworten konnte oder sogar neu aufgeworfen hat, sind gleichzeitig die Ausgangsbasis für neue Forschungsansätze. Bevor also die Arbeit mit einem finalen Fazit ihr Ende findet, sollen noch einige mögliche Themen kurz andiskutiert werden, an denen in Zukunft geforscht werden könnte. Dem interdisziplinären Anspruch soll auch hier Genüge getan werden, indem sowohl stärker sozialwissenschaftlich geprägte Fragen aufgeworfen werden, als auch stärker ingenieurwissenschaftlich geprägte Fragen - und nicht zuletzt natürlich auf Themen hingewiesen wird, die sich nur durch eine Kooperation unterschiedlicher Disziplinen sinnvoll bearbeiten lassen. Dabei beanspruchen die folgenden Ausführungen keinesfalls irgendeine Art der Vollständigkeit, sondern dienen vielmehr als Schlaglichter, um die große Zahl möglicher Forschungsthemen zu illustrieren, die sich aus dem eigenständigen Resilienz-Konzept der zivilen SiFo und den 37 Hypothesen für Resilience Engineering ergeben. Es ließe sich sogar formulieren, dass eine tiefergehende, systematische Analyse des Konzepts und der Hypothesen im Hinblick darauf, welche offenen Fragestellungen sich darin noch verbergen, bereits selbst ein erstes Forschungsthema darstellt. Beide, Konzept wie Hypothesen, sind voraussetzungsreich und in ihrer Gesamtheit jeweils 
wesentlich von Grundannahmen abhängig, die sich durchaus auch anders formulieren ließen. Etwa aus einem anderen Theoriegebäude als der soziologischen Systemtheorie und der Theorie komplexer adaptiver Systeme heraus. Oder durch eine stärkere Betonung anderer Resilienzforschungstraditionen als der ökologischen und sozial-ökologischen, an denen sich die vorliegende Arbeit stark orientiert hat. Inwiefern daraus Ergebnisse folgen würden, die konsistent mit dem Resilienz-Konzept der zivilen SiFo und den 37 Hypothesen für Resilience Engineering wären, kann a priori nicht beantwortet werden und stellt insofern eine interessante Forschungsfrage dar.

Ein ganzer Komplex an unbeantworteten Fragen ergibt sich aus den beiden geschilderten Kritikpunkten hinsichtlich Zielkonflikten und Entscheidungen. Für das Resilienz-Konzept der zivilen SiFo ließe sich untersuchen, inwiefern seine einzelnen Bestandteile in sich und untereinander widerspruchsfrei sind. Größtmögliche Konsistenz war zwar der grundlegende Anspruch der vorliegenden Arbeit. Diese kann jedoch für ein derart komplexes Konzept nicht garantiert werden und bedarf zusätzlicher Untersuchungen. Das gilt in noch stärkerem Maße für die Frage nach der Bedeutung, nach dem Gewicht einzelner Hypothesen im Gesamtkontext Resilience Engineering. Auf diesen Aspekt wurde bereits in der Kritik verwiesen. Hier existiert eine Vielzahl an Fragestellungen, was das Verhältnis der einzelnen Hypothesen untereinander angeht. Und insbesondere was den Beitrag der einzelnen Prinzipien Redundanz, Diversität, Modularität und Dezentralität angeht. Wie von Goessling-Reisemann und Thier in einer aktuellen Publikation betont, sind diese Fragen generell noch unbeantwortet (Goessling-Reisemann/Thier 2019: 132f). Es ist zudem unklar, ob sie sich überhaupt generisch beantworten lassen oder aber - aufgrund der Komplexität der Systeme, in denen sie zur Anwendung kommen sollen - nur spezifisch erfassbar sind. Diese Frage ist durchaus auch empirisch zu verstehen. Hier werden systematische, vergleichende Analysen unterschiedlicher, realer, komplexer adaptiver Systeme benötigt. Diese, wie auch darüber hinaus gehende Analysen der weiteren Hypothesen, lassen sich nicht disziplinär umsetzen. Sie erfordern interdisziplinäre Zusammenarbeit, für die die vorliegende Arbeit die Basis für ein gemeinsames Grundverständnis der Untersuchungsgegenstände zu schaffen versucht hat.

Gerade für die normativen Aspekte von Resilienz stellen sich aber auch Fragen, die primär sozialwissenschaftlich beforscht werden können. In Anlehnung an Überlegungen von Riescher wurde Resilienz beispielsweise als starkdemokratische Sicherheit verstanden. Damit war eine aktive, transparente und partizipative Rolle von Bürgen bei gesellschaftlichen und poli- 


\section{Fazit und Ausblick}

tischen Aushandlungsprozessen zur normativen Erwünschtheit relevanter Systeme gemeint. Es wurde postuliert, dass so durch die Verantwortungsannahme aktiver Bürger eine Aufrechterhaltung gesellschaftlicher Freiheiten bei gleichzeitiger Erhöhung der Sicherheit möglich wird. Dieser innovative Ansatz, mithilfe von Resilienz Freiheit und Sicherheit zu kombinieren und einen scheinbaren Gegensatz zwischen den beiden aufzulösen, ist ein vielversprechender Ausgangspunkt für weitergehende Analysen. Denn in den Sozialwissenschaften und gerade in der Politikwissenschaft spielt die Frage nach dem Verhältnis zwischen Freiheit und Sicherheit immer wieder eine wichtige Rolle. Gegeben aktuelle soziale, politische und technologische Entwicklungen, vom Erstarken des Populismus über den Trend zur Automatisierung auch normativ besetzter Entscheidungen bis zur zunehmenden Bedeutung sozialer Medien, wird es wohl sogar wieder wichtiger, Freiheit in Bezug auf Sicherheit und vice versa zu diskutieren. Resilienz verstanden als starkdemokratische Sicherheit bietet hier einen geeigneten Ausgangspunkt. Aus sozusagen umgekehrter Richtung interessant wäre auch eine Untersuchung von Systemen, die sich durch unfreie Strukturen auszeichnen, trotzdem aber eine hohe Resilienz aufweisen. Es ließe sich die Frage stellen, ob generelle Mechanismen identifiziert werden können, die zur Resilienz derartiger Systeme beitragen und ob diese sich auch in freien, demokratischen Systemen sinnvoll einsetzen ließen.

Für die Ingenieurwissenschaften ergeben sich aus den 37 Hypothesen eine Reihe von Hinweisen, an welchen Herausforderungen verstärkt geforscht werden könnte, um zur Resilienz von komplexen adaptiven Systemen beizutragen. Ein Beispiel ist die Entwicklung neuer bzw. die Weiterentwicklung bestehender Methoden zur Modellierung und Simulation komplexer Systeme. Die Modelle können dazu genutzt werden, das Verhalten komplexer adaptiver Systeme im Normalfall zu simulieren. Sie sollen aber auch dazu genutzt werden, die Reaktion solcher Systeme beim Auftreten unerwarteter, gravierender widriger Ereignisse zu testen. Letztlich geht es darum, Unsicherheit und Komplexität in Modellen besser abzubilden, als das bisher der Fall ist. Vor allem agentenbasierte Modellierungsmethoden bieten vielversprechende Möglichkeiten dafür, auch weil sie unter anderem dazu geeignet sind, menschliches Verhalten mit abzubilden. So genügen sie dem Anspruch an Resilience Engineering, sozio-technische Systeme zu untersuchen. Gleichzeitig besteht auch noch Forschungsbedarf was den Ansatz der modularen Modellierung angeht. In der Kombination unterschiedlicher Modellierungsmethoden liegt großes Potenzial, das aber nur ausgeschöpft werden kann, wenn diese innerhalb eines übergreifenden Modells vereint werden können. Dafür ist 
die Entwicklung geeigneter Schnittstellen notwendig. Außerdem kann die Weiterentwicklung von Modellen und Simulationsmethoden auch genutzt werden, um nach Wegen zu suchen, systematisch Umkipp-Punkte, Schwellenwerte oder auch Hebelpunkte in komplexen adaptiven Systemen identifizieren zu können. Nicht zuletzt bestehen vielfältige Forschungsfragen auch nach wie vor in der Klärung des Verhältnisses zwischen künstlicher Intelligenz und Resilience Engineering. Es sollte geklärt werden, wie Methoden der künstlichen Intelligenz zu Resilience Engineering beitragen können. Genauso sollte aber untersucht werden, ob und wo künstliche Intelligenz auch zu einer Gefahr für Resilienz im Bereich der zivilen SiFo werden kann, gerade mit Blick auf die normativen Aspekte des Konzepts.

In einer von Komplexität und Unsicherheit charakterisierten Welt gehen die Fragen für Resilienzforschung nicht aus. Ganz zu Beginn wurde postuliert, dass es manchmal besser kommt als man denkt. Dass Krisen gemeistert, Probleme gelöst und Gefahren gebannt werden können. Und dass dazu drei Sorten von Fragen beantwortet werden müssen. Nämlich erstens danach, um welche Art von Krisen, Problemen und Gefahren es sich handelt? Zweitens, wie diese gemeistert, gelöst und gebannt werden können? Und drittens, in welchem Kontext das alles erfolgt, welche Nebenbedingungen beachtet werden müssen und welche sonstigen Auswirkungen entstehen? Die vorliegende Arbeit hat versucht, diese Fragen für den Bereich der zivilen Sicherheitsforschung ein gutes Stück weit zu beantworten, indem sie ein eigenständiges Resilienz-Konzept entwickelt und daraus 37 Hypothesen für Resilience Engineering abgeleitet hat. Insgesamt bildet sie so einen wichtigen Baustein auf dem Weg hin zu einer Gesellschaft mit größerer Resilienz. 


\section{Literatur}

Adger, W. (2000): Social and ecological resilience: Are they related? In: Progress in Human Geography, 24:3, 347-364.

Ahmed, E. / Elgazzar, A. / Hegazi, A. (2005): An Overview of Complex Adaptive Systems. In: arXiv:nlin/0506059 [nlin.AO].

Alcabes, P. (2009): Dread. How fear and fantasy have fueled epidemics from the black death to avian flu. Philadelphia, PA: PublicAffairs.

Alderson, D. (2019): Overcoming barriers to greater scientific understanding of critical infrastructure resilience. In: Ruth, M. / Goessling-Reisemann, S. (Hrsg.): Handbook on resilience of socio-technical systems. Cheltenham: Edward Elgar Publishing Limited, 66-88.

Alexander, D. (2013): Resilience and disaster risk reduction: an etymological journey. In: Natural Hazards and Earth System Sciences, 1, 1257-1284.

Al-Khudhairy, D. / Axhausen, K. / Bishop, S. / Herrmann, H. / Hu. B. / Kröger, W. / Lewis, T. / MacIntsoh, J. / Nowak, A. / Pickl, S. / Stauffacher, D. / Tan, E. (2012): Towards Integrative Risk Management and More Resilient Societies. In: The European Physical Journal Special Topics, 214:1, 571-595.

Allan, J. / Howell, P. (1994): The Nile, sharing a scarce Resource: A historical and technical Review of water management and of economic and legal issues. Cambridge: Cambridge University Press.

Allenby, B. / Fink, J. (2005): Toward inherently secure and resilient societies. In: Science 309, 1034-1036.

Altherr, L. / Brötz, N. / Dietrich, I. / Gally, T. / Geßner, F. / Kloberdanz, H. / Leise, P. / Pelz, P. / Schlemmer, P. / Schmitt, A. (2018): Resilience in Mechanical Engineering - A Concept for Controlling Uncertainty during Design, Production and Usage Phase of Load-Carrying Structures. In: Applied Mechanics and Materials, 885, 187-198.

Amanatidou, E. / Butter, M. / Carabias, V. / Könnölä, T. / Leis, M. / Saritas, O. / van Rij, V. (2012): On concepts and methods in horizon scanning: Lessons from initiating policy dialogues on emerging issues. In: Science and Public Policy, 39:2, 208-221.

Antonovsky, A. (1997): Salutogenese: Zur Entmystifizierung der Gesundheit. Herausgegeben von A. Franke. Tübingen: Deutsche Gesellschaft für Verhaltenstherapie Verlag.

Ash, A. / Newth, D. (2007): Optimizing complex networks for resilience against cascading failure. In: Physica A: Statistical Mechanics and its Applications, 380, 673-683.

Attoh-Okine, N. (2016): Resilience Engineering: Models and Analysis. New York, NY: Cambridge University Press. 
Aven, T. (2011): On some recent definitions and analysis frameworks for risk, vulnerability, and resilience. In: Risk Analysis, 31:4, 515-522.

Aven, T. (2010): On how to define, understand and describe risk. In: Reliability Engineering and System Safety, 95, 623-631.

Ayyub, B. (2015): Practical Resilience Metrics for Planning, Design, and Decision Making. In: ASCE-ASME Journal of Risk and Uncertainty in Engineering Systems, Part A: Civil Engineering, 1:3, 1-11.

Ayyub, B. (2014): Systems Resilience for Multihazard Environments: Definition, Metrics, and Valuation for Decision Making. In: Risk Analysis 34, 340-355.

Bach, R. / Kaufman, D. / Settle, K. (2011): Policy Challenges in Supporting Community Resilience. Working Paper \#1. The Multinational Resilience Policy Group.

Baezner, M. / Maduz, L. / Prior, T. (2018): Intelligente Schutzsysteme für die Stadt der Zukunft. CSS Analysen zur Sicherheitspolitik 235, Zürich: Center for Security Studies (CSS), ETH Zürich.

Bara, C. (2010): Factsheet. Social Vulnerability to Disasters. CRN Report, Zürich: Center for Security Studies (CSS), ETH Zürich.

Barber, B. (1984): Strong Democracy: Participatory Politics for A New Age. Berkeley: University of California Press.

Bartl, G. / Gerhold, L. / Schiller, J. (2014): Resilienz: Nationale Perspektiven. In: Thoma, K. (Hrsg.): Resilien-Tech. Resilience by Design: Strategie für die technologischen Zukunftsthemen (acatech STUDIE). München: Herbert Utz Verlag, 19-51.

Baum S. (2015): Risk and Resilience for unkown, unquantifiable, systemic and unlikely / catastrophic threats. In: Environment, Systems, and Decisions, 35:2, 229-236.

Beck, U. (2016): Risikogesellschaft. Auf dem Weg in eine andere Moderne, 23. Auflage. Frankfurt a.M.: Suhrkamp.

Bergdolt, K. (2011): Der Schwarze Tod: die Große Pest und das Ende des Mittelalters, 3. Aufl. in der Beck'schen Reihe, Neuaufl. München: Beck.

Bergström, J. / Dahlström, N. / van Winsen, R. / Lützhöft, M. / Dekker, S. / Nyce, J. (2009): Rule- and role retreat: An empirical study of procedures and resilience. In: Journal of Maritime Studies, 6:1, 75-90.

Berkes, F. (2007): Understanding uncertainty and reducing vulnerability: lessons from resilient thinking. In: Natural Hazards, 41, 283-295.

Birkmann, J. (2008): Globaler Umweltwandel, Naturgefahren, Vulnerabilität und Katastrophenresilienz. Notwendigkeit der Perspektivenerweiterung in der Raumplanung. In: Raumforschung und Raumordnung, 66:1, 5-22.

Blum, S. / Endreß, M. / Kaufmann, S. / Rampp, B. (2016): Soziologische Perspektiven. In: Wink, R. (Hrsg.): Multidisziplinäre Perspektiven der Resilienzforschung (Studien zur Resilienzforschung). Wiesbaden: Springer, 151-177. 
Bocchini, P. / Frangopol, D. (2011): Resilience-driven Disaster Management of Civil Infrastructure. In: Papadrakakis, M. / Fragiadakis, M. / Plevris, V. (Hrsg.): Computational methods in structural dynamics and earthquake engineering. Proceedings of the Third International Conference on Computational Methods in Structural Dynamics and Earthquake Engineering, May 26-28, 2011, Corfu, $1-11$.

Boin, A. / McConnell, A. (2007): Preparing for Critical Infrastructure Breakdowns: The Limits of Crisis Management and the Need for Resilience. In: Journal of Contingencies and Crisis Management, 15:1, 50-59.

Bossel, H. (2004): Systeme, Dynamik, Simulation. Modellbildung, Analyse und Simulation komplexer Systeme. Norderstedt: Books on Demand.

Brand, F. / Jax, K. (2007): Focusing the meaning(s) of resilience: resilience as a descriptive concept and a boundary object. In: Ecology and Society 12:1, 1-14.

Brugger, P. / Hasenclever, A. / Kasen, L. (2013): Vertrauen lohnt sich. Über Gegenstand und Potential eines vernachlässigten Konzepts in den internationalen Beziehungen. In: Zeitschrift für Internationale Beziehungen, 20:2, 65-104.

Brühwiler, B. / Romeike, F. (2010): Praxisleitfaden Risikomanagement. Berlin: Erich Schmidt Verlag.

Bruneau, M. / Reinhorn, A. (2006): Overview of the Resilience Concept. In: Proceedings of the 8th US National Conference on Earthquake Engineering.

Bruneau, M. / Chang, S. / Eguchi, R. / Lee, G. / O’Rourke, T. / Reinhorn, A. / Shinozuka, M. / Tierney, K. / Wallace, W. / von Winterfeldt, D. (2003): A framework to quantitatively assess and enhance the seismic resilience of communities. In: Earthquake Spectra, 19:4, 733-752.

Brunner, E. / Giroux, J. (2009): Examining Resilience: A concept to improve societal security and technical safety. CRN Report, Zürich: Center for Security Studies (CSS), ETH Zürich.

Bundesamt für Bevölkerungsschutz und Katastrophenhilfe (BBK) (2017): Katastrophenalarm. Ratgeber für Notfallvorsorge und richtiges Handeln in Notsituationen.

Bundesministerium des Innern (BMI) (2009): Nationale Strategie zum Schutz Kritischer Infrastrukturen (KRITIS-Strategie).

Bundesministerium für Bildung und Forschung (BMBF) (2018): Forschung für die zivile Sicherheit 2018-2023. Rahmenprogramm der Bundesregierung.

Bundesministerium für Bildung und Forschung (BMBF) (2016): Forschung für die zivile Sicherheit 2012-2017. Rahmenprogramm der Bundesregierung, aktualisierte Auflage.

Bungartz, H. / Zimmer, S. / Buchholz, H. / Pflüger, D. (2009): Modellbildung und Simulation. Eine anwendungsorientierte Einführung. Berlin: Springer.

Carpenter, S. / Walker, B. / Anderies, J. / Abel, N. (2001): From metaphor to measurement: resilience of what to what? In: Ecosystems, 4, 765-781.

Chandler, D. (2014): Beyond neoliberalism: Resilience, the new art of governing complexity. In: Resilience: International Policies, Practices and Discourses, 2:1, 47-63. 
Chang, S. (2009): Infrastructure Resilience to Disasters. In: The Bridge, 39:4, 36-41.

Christmann, G. / Ibert, O. (2016): Eine sozialräumliche Perspektive auf Vulnerabilität und Resilienz. Sozialkonstruktivismus, Akteur-Netzwerk-Theorie und relationale Raumtheorie im Dialog. In: Wink, R. (Hrsg.): Multidisziplinäre Perspektiven der Resilienzforschung (Studien zur Resilienzforschung). Wiesbaden: Springer, 233-261.

Cimellaro, G. / Reinhorn, A. / Bruneau, M. (2010): Framework for analytical quantification of disaster resilience. In: Engineering Structures, 32:11, 3639-3649.

Coaffee, J. (2010): Protecting vulnerable cities: the UK's resilience response to defending everyday urban infrastructure. In: International Affairs, 86:4, 939-954.

Coaffee J. (2008): Risk, resilience, and environmentally sustainable cities. In: Energy Policy 36:12, 4633-4638.

Coaffee, J. / Bosher, L. (2008): Integrating Counter-Terrorist Resilience into Sustainability. In: Proceeding of the Institute of Civil Engineers: Urban Design and Planning, 161, 75-84.

Coaffee, J. / Rogers, P. (2008): Rebordering the City for New Security Challenges: From Counter-terrorism to Community Resilience. In: Space and Polity, 12:1, 101-118.

Colten, C. / Kates, R. / Laska, S. (2008): Community Resilience: Lessons from New Orleans and Hurricane Katrina. CARRI Research Report 3. Community \& Regional Resilience Initiative.

Comfort, L. / Sungu, Y. / Johnson, D. / Dunn, M. (2001): Complex systems in crisis: anticipation and resilience in dynamic environments. In: Journal on Contingency and Crisis Management, 9:3, 144-158.

Connelly, E. / Allen, C. / Hatfield, K. / Palma-Oliveira, J. / Woods, D. / Linkov, I. (2017): Features of resilience. In: Environment Systems and Decisions, 37:1, 46-50.

Cook, R. / Nemeth, C. (2006): Taking Things in One's Stride: Cognitive Features of Two Resilient Performances. In: Hollnagel, E. / Woods, D. / Leveson N. (Hrsg.): Resilience Engineering. Concepts and Precepts. Hampshire: Ashgate Publishing Limited, 205-221.

Cook, R. / Woods, D. (2006): Distancing through Differencing: An Obstacle to Organizational Learning Following Accidents. In: Hollnagel, E. / Woods, D. I Leveson N. (Hrsg.): Resilience Engineering. Concepts and Precepts. Hampshire: Ashgate Publishing Limited, 329-338.

Cox, A. / Prager, F. / Rose, A. (2011): Transportation security and the role of resilience: a foundation for operational metrics. In: Transport Policy, 18, 307-317.

Center for Security Studies (CSS) (2009): Resilienz: Konzept zur Krisen- und Katastrophenbewältigung. CSS Analysen zur Sicherheitspolitik 60, Zürich: Center for Security Studies (CSS), ETH Zürich.

Cutter, S. (2013): The Social Vulnerability Index. Handout used at the workshop «International Perspectives on Resilience», May 15-16, 2013, Berlin.

Cutter, S. / Boruff, B. / Shirley W. (2003): Social Vulnerability to Environmental Hazards. In: Social Science Quarterly, 84:2, 242-261. 
D'Lima, M. / Medda, F. (2015): A new measure of resilience: an application to the London Underground. In: Transportation Research Part A, 81, 35-46.

Dekker, S. (2014): The problems of vision zero in work safety. In: Malaysia Labour Review, 1:8, 25-36.

Dekker, S. (2006): Resilience Engineering: Chronicling the Emergence of Confused Consensus. In: Hollnagel, E. / Woods, D. / Leveson N. (Hrsg.): Resilience Engineering. Concepts and Precepts. Hampshire: Ashgate Publishing Limited, 77-92.

Dekker, S. (2004): The hindsight bias is not a bias and not about history. In: Human Factors and Aerospace Safety, 4:2, 87-99.

Dekker, S. / Woods, D. (2010): The high reliability organization perspective. In: Salas, E. / Maurino, D. (Hrsg.): Human Factors in Aviation, 2nd edn., New York: Wiley, 123-146.

Deppisch, S. (2016): Urbane sozial-ökologische Resilienz. In: Wink, R. (Hrsg.): Multidisziplinäre Perspektiven der Resilienzforschung (Studien zur Resilienzforschung). Wiesbaden: Springer, 199-213.

Descartes, R. (1986): Meditationes de Prima Philosophia. Meditationen über die Erste Philosophie. Lateinisch / Deutsch. Stuttgart: Reclam.

Dijkstra, A. (2006): Safety Management in Airlines. In: Hollnagel, E. / Woods, D. / Leveson N. (Hrsg.): Resilience Engineering. Concepts and Precepts. Hampshire: Ashgate Publishing Limited, 183-203.

Dillon, D. (2001): Review of the Santa Fe Institute: Institutional and individual qualities of expert interdisciplinary work. GoodWork Projekt Report Series, Number 22. Internal working paper prepared for the Harvard Interdisciplinary Studies Project, October 15, 2001.

Dinh, L. / Pasman, H. / Gao, X. / Mannan, M. (2012): Resilience engineering of industrial processes: Principles and contributing factors. In: Journal of Loss Prevention in the Process Industries, 25:2, 233-241.

Dombrowsky, W. (2012): Resilience from a sociological viewpoint. In: Gander, H.H. / Perron, W. / Poscher, R. / Riescher, G. / Würtenberger, T. (Hrsg.): Resilienz in der offenen Gesellschaft. Symposium des Centre for Security and Society. Reihe: Sicherheit und Gesellschaft. Freiburger Studien des Centre for Security and Society, Bd. 1. Baden-Baden: Nomos, 281-289.

Dombrowsky, W. (2010): Resilience from a sociological viewpoint. Paper presented at the International Workshop „Resilience - A new Paradigm for Civil Security in Open Societies”, July 8, 2010, Freiburg.

Dombrowsky, W. / Siedschlag, A. (2013): Sozialwissenschaftliche Katastrophenforschung und Sicherheitsdiskurs. In: Siedschlag, A. (Hrsg.): Methoden der sicherheitspolitischen Analyse. Wiesbaden: Springer, 223-236.

Dueñas-Osorio, L. / Vemuru, S. (2009): Cascading failures in complex infrastructure systems. In: Structural Safety, 31:2, 157-167.

Duit, A. / Galaz, V. / Eckerberg, K. / Ebbesson, J. (2010): Governance, complexity and resilience. In: Global Environmental Change, 20:3, 363-368.

Edwards, C. (2009): Resilient Nation, London: Demos. 
Elran, M. (2012): Societal Resilience: A Key Response to Severe Terror. In: Gander, H.-H. / Perron, W. / Poscher, R. / Riescher, G. / Würtenberger, T. (Hrsg.): Resilienz in der offenen Gesellschaft. Symposium des Centre for Security and Society. Reihe: Sicherheit und Gesellschaft. Freiburger Studien des Centre for Security and Society, Bd. 1. Baden-Baden: Nomos, 291-299.

Elsberg, M. (2012): Blackout. Morgen ist es zu spät. München: Blanvalet Verlag.

European Network and Information Security Agency (ENISA) (2011): Enabling and managing end-to-end resilience.

Farid, A. (2015): Static Resilience of Large Flexible Engineering Systems: Axiomatic Design Model and Measures. In: IEEE Systems Journal, 11:4, 2006-2017.

Fekete, A. / Grinda, C. / Norf, C. (2016): Resilienz in der Risiko- und Katastrophenforschung: Perspektiven für disziplinübergreifende Arbeitsfelder. In: Wink, R. (Hrsg.): Multidisziplinäre Perspektiven der Resilienzforschung (Studien zur Resilienzforschung). Wiesbaden: Springer, 215-231.

Fekete, A. / Hufschmidt, G. / Kruse, S. (2014): Benefits and challenges of resilience and vulnerability for disaster risk management. In: International Journal of Disaster Risk Science, 5:1, 3-20.

Ferris, T. / Specking, E. / Jackson, S. / Parnell, G. (2018): The Fundamental Nature of Resilience of Engineered Systems. In: 28th annual INCOSE international symposium, July 7-12, 2018, Washington, D.C.

Finch, C. / Emrich, C. / Cutter, S. (2010): Disaster disparities and differential recovery in New Orleans. In: Population \& Environment, 31, 179-202.

Flaherty, J. (2016): Gift of God. In: Digital Development Debates, 18.

Flin, R. (2006): Erosion of Managerial Resilience: From Vasa to NASA. In: Hollnagel, E. / Woods, D. / Leveson N. (Hrsg.): Resilience Engineering. Concepts and Precepts. Hampshire: Ashgate Publishing Limited, 223-233.

Flynn, S. (2011): A National Security Perspective on Resilience. Resilience: Interdisciplinary Perspectives on Science and Humanitarianism. 2, i-ii.

Flynn, S. (2011b). Recalibrating Homeland Security. Mobilizing American Society to Prepare for Disaster. In: Foreign Affairs, 90:3, 130-140.

Flynn, S. (2008): America the Resilient. Defying Terrorism and Mitigating Natural Disasters. In: Foreign Affairs, 87:2, 2-8.

Flynn, S. / Burke, S. (2012): Powering America's Energy Resilience. A report by the center for national policy.

Flynn, S. / Burke, S. (2011): Brittle Infrastructure, Community Resilience, and National Security. TR News 275, July-August 2011, 4-11.

Folke, C. (2006): Resilience: The emergence of a perspective for social-ecological systems analyses. In: Global Environmental Change, 16, 253-267.

Folke, C. / Carpenter, S. / Elmqvist, T. / Gunderson, L. / Holling, C. / Walker, B. (2002): Resilience and Sustainable Development: Building Adaptive Capacity in a World of Transformations. In: Journal of the Human Environment, 31:5, 437-440. 
Fooken, I. (2016): Psychologische Perspektiven der Resilienzforschung. In: Wink, R. (Hrsg.): Multidisziplinäre Perspektiven der Resilienzforschung (Studien zur Resilienzforschung). Wiesbaden: Springer, 13-45.

Fox-Lent, C. / Bates, M. / Linkov, I. (2015): A matrix approach to community resilience assessment: an illustrative case at Rockaway Peninsula. In: Environment Systems and Decisions, 35:2, 209-218.

Francis, R. / Bekera, B. (2014): A metric and frameworks for resilience analysis of engineered and infrastructure systems. In: Reliability Engineering and System Safety, 121, 90-103.

Frankel, J. (2010): Unforeseen Future Global Developments. Windsor Conference, Harvard Faculty Club.

Fraunhofer (2018): Biologische Transformation und Bioökonomie.

Fritzon, Å / Ljungkvist, K. / Boin, A. / Rhinard, M. (2007); Protecting Europe's critical infrastructures: Problems and prospects. In: Contingencies and Crisis Management, 15:1, 30-41.

Fujita, Y. (2006): Resilient Systems. In: Hollnagel, E. / Woods, D. / Leveson N. (Hrsg.): Resilience Engineering. Concepts and Precepts. Hampshire: Ashgate Publishing Limited, 67.

Fujita, Y. (2006b): Rules and Procedures. In: Hollnagel, E. / Woods, D. / Leveson N. (Hrsg.): Resilience Engineering. Concepts and Precepts. Hampshire: Ashgate Publishing Limited, 327.

Galland, J.-P. (2012): A critique of the notion of critical infrastructure. In: Gander, H.-H. / Perron, W. / Poscher, R. / Riescher, G. / Würtenberger, T. (Hrsg.): Resilienz in der offenen Gesellschaft. Symposium des Centre for Security and Society. Reihe: Sicherheit und Gesellschaft. Freiburger Studien des Centre for Security and Society, Bd. 1. Baden-Baden: Nomos, 301-319.

Galloway, G. (2013): Dimensions of Resilience: A United States Perspective. Handout used at the workshop «International Perspectives on Resilience», May 15-16, 2013, Berlin.

Ganin, A. / Massaro, E. / Gutfraind, A. / Steen, N. / Keisler, J. / Kott, A. / Mangoubi, R. / Linkov, I. (2016): Operational resilience: concepts, design and analysis. In: Scientific Reports, 6, 1-12.

Gell-Mann, M. (1994): The Quark and the Jaguar: Adventures in the Simple and the Complex. New York City, NY: W.H. Freeman and Company.

Goehl, K. / Mayer, J. / Staub, K. (2000): Was tun, wenn die Pest kommt: Göttern lästern oder Juden brennen? In: Goehl, K. / Mayer, J. (Hrsg.): Editionen und Studien zur lateinischen und deutschen Fachprosa des Mittelalters. Festgabe für Gundolf Keil zum 65. Geburtstag. Würzburg: Königshausen \& Neumann $\mathrm{GmbH}, 127-166$.

Goerger S. / Madni, A. / Eslinger, O. (2014): Engineered Resilient Systems: A DoD Perspective. In: Procedia Computer Science, 28, 865-872. 
Goessling-Reisemann, S. / Thier, P. (2019): On the difference between risk management and resilient management for critical infrastructures. In: Ruth, M. I Goessling-Reisemann, S. (Hrsg.): Handbook on resilience of socio-technical systems. Cheltenham: Edward Elgar Publishing Limited, 117-135.

Golder, B. / Williams, G. (2004): What is 'Terrorism'? Problems of Legal Definition. In: University of NSW Law Journal, 27:2, 270-295.

Gunderson, L. (2000): Ecological Resilience - in theory and application. In: Annual Review of Ecology and Systems, 31, 425-439.

Hale, A. / Guldenmund, F. / Goossens, L. (2006): Auditing Resilience in Risk Control and Safety Management Systems. In: Hollnagel, E. / Woods, D. / Leveson N. (Hrsg.): Resilience Engineering. Concepts and Precepts. Hampshire: Ashgate Publishing Limited, 289-314.

Hale, A. / Heijer, T. (2006): Defining Resilience. In: Hollnagel, E. / Woods, D. / Leveson N. (Hrsg.): Resilience Engineering. Concepts and Precepts. Hampshire: Ashgate Publishing Limited, 35-40.

Hale, A. / Heijer, T. (2006b): Is Resilience Really Necessary? The Case of Railways. In: Hollnagel, E. / Woods, D. / Leveson N. (Hrsg.): Resilience Engineering. Concepts and Precepts. Hampshire: Ashgate Publishing Limited, 125-147.

Hannig, N. (2016): Maschinenschäden. Versicherungen und die Prävention von Industrieunfällen um 1900. In: Zeitschrift für Unternehmensgeschichte, 61:1, 84-106.

Häring, I. / Scharte, B. / Hiermaier, S. (2016): Towards a novel and applicable approach for Resilience Engineering. In: Proceedings of the 6th International Disaster and Risk Conference, 272-276.

Häring, I. / Scharte, B. / Stolz, A. / Leismann, T. / Hiermaier, S. (2016b): Resilience Engineering and quantification for sustainable systems development and assessment: Socio-technical systems and critical infrastructure. In: IRGC (2016): Resource Guide on Resilience. Lausanne: EPFL International Risk Governance Center.

Härter, K. / Hannappel, T. / Tyrichter, J. / Walter, T. (2014): Terrorismus für die Rechtsgeschichte? Neuerscheinungen zur Geschichte politischer Gewalt im 19. und 20. Jahrhundert. In: Rechtsgeschichte - Legal History Rg, 22, 374-385.

Helbing, D. (2015): Responding to complexity in socio-economic systems: How to build a smart and resilient society?

Helbing, D. (2013): Globally networked risks and how to respond. In: Nature, 497:7447, 51-59.

Hellige, H. (2019): The metaphorical processes in the history of the resilience notion and the rise of the ecosystem resilience theory. In: Ruth, M. / GoesslingReisemann, S. (Hrsg.): Handbook on resilience of socio-technical systems. Cheltenham: Edward Elgar Publishing Limited, 30-51.

Hickford, A. / Blainey, S. / Hortelano, A. / Pant, R. (2018): Resilience engineering: theory and practice in interdependent infrastructure systems. In: Environment Systems and Decisions, 38, 278-291. 
Hiermaier, S. / Scharte, B. (2018): Ausfallsichere Systeme. Resilienz als Sicherheitskonzept im Zeitalter der Digitalisierung. In: Neugebauer, R. (Hrsg.): Digitalisierung. Schlüsseltechnologien für Wirtschaft und Gesellschaft. Fraunhofer-Forschungsfokus. München: Springer-Vieweg, 295-310.

Hiermaier, S. / Scharte, B. / Fischer, K. (2019): Resilience Engineering: chances and challenges for a comprehensive concept. In: Ruth, M. / Goessling-Reisemann, S. (Hrsg.): Handbook on resilience of socio-technical systems. Cheltenham: Edward Elgar Publishing Limited, 155-166.

Hoffman, B. (2008): Terrorismus - Der unerklärte Krieg: neue Gefahren politischer Gewalt. Frankfurt: Fischer-Taschenbuch-Verlag.

Höfler, M. (2016): Bewältigungskapazität als Bildungsauftrag. Resilienz aus Perspektive der Erwachsenenbildung. In: Wink, R. (Hrsg.): Multidisziplinäre Perspektiven der Resilienzforschung (Studien zur Resilienzforschung). Wiesbaden: Springer, 101-121.

Holland, J. (2014): Complexity. A very short introduction. Oxford: University Press.

Holling, C. (1996): Engineering Resilience vs. Ecological Resilience. In: Schulze, P. (Hrsg.): Engineering Within Ecological Constraints. Washington, D.C.: National Academy Press, 31-44.

Holling, C. (1986): The resilience of terrestrial ecosystems: local surprise and global change. In: Clark, W. / Munn, R. (Hrsg.): Sustainable Development of the Biosphere. London: Cambridge University Press, 292-317.

Holling, C. (1973): Resilience and Stability of Ecological Systems. In: Annual Review of Ecology and Systematics, 4, 1-23.

Holling, C. / Gunderson, L. (2002): Panarchy: Understanding transformations in human and natural systems. Washington, D.C.: Island Press.

Hollnagel, E. (2011): Prologue: The Scope of Resilience Engineering. In: Hollnagel, E. / Pariès, J. / Woods, D. / Wreathall, J. (Hrsg.): Resilience Engineering in Practice. A Guidebook. Farnham, Surrey: Ashgate, xxix-xxxix.

Hollnagel, E. (2006): Resilience: The challenge of the unstable. In: Hollnagel, E. / Woods, D. / Leveson N. (Hrsg.): Resilience Engineering. Concepts and Precepts. Hampshire: Ashgate Publishing Limited, 9-17.

Hollnagel, E. / Fujita, Y. (2013): The Fukushima disaster - systemic failure as the lack of resilience. In: Nuclear Engineering and Technology, 45:1, 13-20.

Hollnagel, E. / Pariès, J. / Woods, D. / Wreathall, J. (Hrsg.) (2011): Resilience Engineering in Practice. A Guidebook. Farnham, Surrey: Ashgate.

Hollnagel, E. / Sundström, G. (2006): States of Resilience. In: Hollnagel, E. / Woods, D. / Leveson N. (Hrsg.): Resilience Engineering. Concepts and Precepts. Hampshire: Ashgate Publishing Limited, 339-346.

Hollnagel, E. / Woods, D. (2006): Epilogue: Resilience Engineering Precepts. In: Hollnagel, E. / Woods, D. / Leveson N. (Hrsg.): Resilience Engineering. Concepts and Precepts. Hampshire: Ashgate Publishing Limited, 347-358.

Hollnagel, E. / Woods, D. / Leveson N. (Hrsg.) (2006): Resilience Engineering. Concepts and Precepts. Hampshire: Ashgate Publishing Limited. 
Holzinger, M. (2011): Die Schleifung des Rechts. 10 Jahre Anti-Terror-Krieg. In: Blätter für deutsche und internationale Politik, 2, 64-72.

Horn, C. (2013): Philosophie der Antike: Von den Vorsokratikern bis Augustinus. München: Beck.

Hosseini, S. / Barker, K. / Ramirez-Marquez, J. (2016): A review of definitions and measures of system resilience. In: Reliability Engineering and System Safety, $145,47-61$.

Huber, S. / van Wijgerden I. / de Witt, A. / Dekker, S. (2009): Learning from Organizational Incidents: Resilience Engineering for High-Risk Process Environments. In: Process Safety Progress, 28:1, 90-95.

Hutter, G. (2011): Organizing social resilience in the context of natural hazards: a research note. In: Natural Hazards. Published online.

International Risk Governance Center (IRGC) (2018): Guidelines for the Governance of Systemic Risks. Lausanne: International Risk Governance Center (IRGC).

Jabareen, Y. (2013): Planning the resilient city: Concepts and strategies for coping with climate change and environmental risk. In: Cities, 31, 220-229.

Jackson, S. / Ferris, T. (2013): Resilience principles for engineered systems. In: Systems Engineering, 16:2, 152-164.

Jakob, C. / Schorb, F (2008): Soziale Säuberung. Wie New Orleans nach der Flut seine Unterschicht vertrieb. Münster: Unrast.

Janić, M. (2018): Modelling the resilience of rail passenger transport networks affected by large-scale disruptive events: the case of HSR (high speed rail). In: Transportation, 45:4, 1101-1137.

Janić, M. (2015): Modelling the resilience, friability and costs of an air transport network affected by a large-scale disruptive event. In: Transportation Research Part A: Policy and Practice, 71, 1-16.

Ji, C. / Wie, Y. / Poor, H. (2017): Resilience of energy infrastructure and services: modeling, data analytics, and metrics. In: Proceedings of the IEEE, 105:7, 1354-1366.

Joffe, H. / Rossetto, T. / Bradley, C. / O'Connor, C. (2018): Stigma in science: the case of earthquake prediction. In: Disasters, 42:1, 81-100.

Kammenhuber, N. / Fessi, A. / Carle, G. (2010): Resilience: Widerstandsfähigkeit des Internets gegen Störungen - Stand der Forschung und Entwicklung. In: Informatik Spektrum, 33:2, 131-142.

Kaplan, S. / Garrick, B. (1981). On the quantitative definition of risk. In: Risk Analysis, 1:1, 11-27.

Kaufmann, S. / Blum, S. (2012): Governing (In)Security: The Rise of Resilience. In: Gander, H.-H. / Perron, W. / Poscher, R. / Riescher, G. / Würtenberger, T. (Hrsg.): Resilienz in der offenen Gesellschaft. Symposium des Centre for Security and Society. Reihe: Sicherheit und Gesellschaft. Freiburger Studien des Centre for Security and Society, Bd. 1. Baden-Baden: Nomos, 235-257. 
Kean, T. / Hamilton, L. / Ben-Veniste, R. / Kerrey, B. / Fielding, F. / Lehman, J. / Gorelick, J. / Roemer, T. / Gorton, S. / Thompson, J. (2004): The 9 / 11 Commission Report.

Kéfi, S. / Dakos, V. / Scheffer, M. / van Nes, E. / Rietkerk, M. (2013): Early warning signals also precede noncatastrophic transitions. In: Oikos, 122, 641-648.

Kete, N. / Punzo, G. / Linkov, I. (2018): Enhancing resilience within and between critical infrastructure systems. In: Environment Systems and Decisions, 38, 275-277.

Kneer, G. / Nassehi, A. (2000): Niklas Luhmanns Theorie sozialer Systeme, 4., unveränderte Auflage. Paderborn: Wilhelm Fink.

Knight, F. (2009): Risk, Uncertainty, and Profit. Kissimmee, FL: Signalman Publishing.

Kolliarakis, G. (2013): Resilience as an innovation policy objective: Blind spots and untapped potential for security research. In: Lauster, M. (Hrsg.): 8th future security research conference - proceedings, Stuttgart: Fraunhofer VVS, 107-116.

Kröger, W. (2011): An Overview of Swiss Research on Vulnerability of Critical Infrastructure. In: Thoma, K (Hrsg.): European perspectives on security research. Heidelberg: Springer, 67-79.

Kröger, W. (2008): Critical infrastructures at risk: A need for a new conceptual approach and extended analytical tools. In. Reliability Engineering and System Safety, 93, 1781-1787.

Kuhlicke, C. (2010): Resilience: a capacity and a myth: findings from an in-depth case study in disaster management research. In: Natural Hazards. Published online.

Laux, H. / Gillenkirch, R. / Schenk-Mathes, H. (2014): Entscheidungstheorie, 9. Auflage. Berlin Heidelberg: Springer.

Leach, M. (Hrsg.) (2008): Re-framing resilience: A symposium report. STEPS Working Paper 13, vol. 1-7. Brighton: STEPS.

Lechner, S. / Jacometti, J. / McBean, G. / Mitchison N. (2016): Resilience in a complex world - Avoiding cross-sector collapse.

Leven, K.-H. (Hrsg.) (2005): Antike Medizin. Ein Lexikon. München: Beck.

Leveson, N. / Dulac, N. / Zipkin, D. / Cutcher-Gershenfeld, J. / Carroll, J. / Barrett, B. (2006): Engineering Resilience into Safety-Critical Systems. In: Hollnagel, E. / Woods, D. / Leveson N. (Hrsg.): Resilience Engineering. Concepts and Precepts. Hampshire: Ashgate Publishing Limited, 95-123.

Linkov, I. / Bridges, T. / Creutzig, F. / Decker, J. / Fox-Lent, C. / Kröger, W. / Lambert, J. / Levermann, A. / Montreuil, B. / Nathwani, J. / Nyer, R. / Renn, O. / Scharte, B. / Scheffler, A. / Schreurs, M. / Thiel-Clemen, T. (2014): Changing the resilience paradigm. In: Nature Climate Change, 4:6, 407-409.

Linkov, I. / Kott, A. (2019): Chapter 1: Fundamental Concepts of Cyber Resilience: Introduction and Overview. In: Kott, A. / Linkov, I. (Hrsg.): Cyber Resilience of Systems and Networks. Cham: Springer, 1-25.

Linkov, I. / Trump, B. / Keisler, J. (2018): Don't conflate risk and resilience. In: Nature, 555:7694, 30. 
Lintz, G. / Wirth, P. / Harfst, J. (2016): Regionaler Strukturwandel und Resilienz. In: Wink, R. (Hrsg.): Multidisziplinäre Perspektiven der Resilienzforschung (Studien zur Resilienzforschung). Wiesbaden: Springer, 333-355.

Lloyd's Register Foundation (LRF) (2015): Foresight review of resilience engineering. Lloyd's Register Foundation Report Series: No. 2015.2.

Longstaff, P. (2012): Complexity and Resilience: Concepts for Community Security. In: Gander, H.-H. / Perron, W. / Poscher, R. / Riescher, G. / Würtenberger, T. (Hrsg.): Resilienz in der offenen Gesellschaft. Symposium des Centre for Security and Society. Reihe: Sicherheit und Gesellschaft. Freiburger Studien des Centre for Security and Society, Bd. 1. Baden-Baden: Nomos, 259-279.

Lorenz, D. (2010): The diversity of resilience: contributions from a social science perspective. In: Natural Hazards. Published online.

Lovins, A. / Lovins, L. (2001): Brittle Power. Energy Strategy for National Security. Energy Policies for Resilience and National Security. New edition. Andover, MA: Brick House.

Luhmann, N. (2014): Vertrauen, 5. Auflage. Konstanz und München: UVK.

Luhmann, N. (2003): Soziologie des Risikos. Unveränderter Nachdruck der Ausgabe von 1991. Berlin: de Gruyter.

Lukesch, R. (2016): Resiliente Regionen. Zur Intelligenz regionaler Handlungssysteme. In: Wink, R. (Hrsg.): Multidisziplinäre Perspektiven der Resilienzforschung (Studien zur Resilienzforschung). Wiesbaden: Springer, 295-331.

Lundberg, J. / Johansson, B. (2015): Systemic resilience model. In: Reliability Engineering and System Safety, 141, 22-32.

Luthar, S. / Cicchetti, D. (2000): The construct of resilience: Implications for interventions and social policies. In: Development and Psychopathology, 12:4, 857-885.

Luthar, S. / Cicchetti, D. / Becker, B. (2000): The Construct of Resilience: A Critical Evaluation and Guidelines for Future Work. In: Child Development, 71: 3, 543-562.

MacAskill, K. / Guthrie, P. (2014): Multiple interpretations of resilience in disaster risk management. In: Procedia Economics and Finance 18, 667-674.

Madni, A. / Jackson, S. (2009): Towards a Conceptual Framework for Resilience Engineering. In: IEEE Systems Journal, 3:2, 181-191.

Marsavelski, A. (2013): The Crime of Terrorism and the Right of Revolution. In: Connecticut Journal of International Law, 28, 243-295.

McDonald, N. (2006): Organisational Resilience and Industrial Risk. In: Hollnagel, E. / Woods, D. / Leveson N. (Hrsg.): Resilience Engineering. Concepts and Precepts. Hampshire: Ashgate Publishing Limited, 155-179.

Meadows, D. (1999): Leverage points: Places to intervene in a system. Hartland: The Sustainability Institute.

Metzl, E. (2009): The Role of Creative Thinking in Resilience after Hurricane Katrina. In: Psychology of Aesthetics, Creativity, and the Arts, 3:2, 112-123. 
Nan, C. / Sansavini, G. (2017): A quantitative method for assessing resilience of interdependent infrastructures. In: Reliability Engineering and System Safety, $157,35-53$.

Narzisi, G. / Mincer, J. / Smith, S. / Mishra, B. (2007): Resilience in the Face of Disaster: Accounting for Varying Disaster Magnitudes, Resource Topologies, and (Sub)Population Distributions in the PLAN C Emergency Planning Tool.

Nemeth, C. (2008): Resilience engineering: The birth of a notion. In Hollnagel, E. / Nemeth, C. / Dekker, S. (Hrsg.): Resilience engineering perspectives. Volume 1: Remaining sensitive to the possibility of failure. Farnham, Surrey: Ashgate, 3-9.

Neugebauer, R. (Hrsg.) (2018): Digitalisierung. Schlüsseltechnologien für Wirtschaft und Gesellschaft. Fraunhofer-Forschungsfokus. München: Springer-Vieweg.

O'Rourke, T. (2007): Critical Infrastructure, Interdependencies and Resilience. In: The Bridge, 37:1, 22-29.

Osterloh, M. / Weibel, A. (2006): Investition Vertrauen. Prozesse der Vertrauensentwicklung in Organisationen. Wiesbaden: Gabler.

Ouedraogo, K. / Enjalbert, S. / Vanderhaegen, F. (2013): How to learn from the resilience of Human-Machine Systems? In: Engineering Applications of Artificial Intelligence, 26:1, 24-34.

Ouyang, M. (2014): Review on modeling and simulation of interdependent critical infrastructure systems. In: Reliability Engineering and System Safety, 121, 43-60.

Ouyang, M. / Wang, Z. (2015): Resilience assessment of interdependent infrastructure systems: with a focus on joint restoration modeling and analysis. In: Reliability Engineering and System Safety, 141, 74-82.

Pariès, J. (2006): Complexity, Emergence, Resilience... In: Hollnagel, E. / Woods, D. / Leveson N. (Hrsg.): Resilience Engineering. Concepts and Precepts. Hampshire: Ashgate Publishing Limited, 43-53.

Park, J. / Seager, T. / Rao, P. / Convertino, M. / Linkov, I. (2013): Integrating risk and resilience approaches to catastrophe management in engineering systems. In: Risk analysis: an official publication of the Society for Risk Analysis, 33:3, 356-367.

Perrig, A. / Stankovic, J. / Wagner, D. (2004): Security in Wireless Sensor Networks.

Perrow, C. (1999): Normal Accidents: Living with High-Risk Technologies, 2. Auflage. Princeton: Princeton University Press.

Perrow, C. (1999b): Organizing to Reduce the Vulnerabilities of Complexity. In: Journal of Contingencies and Crisis Management 7:3, 150-155.

Pillay, M. (2017): Resilience Engineering: An Integrative Review of Fundamental Concepts and Directions for Future Research in Safety Management. In: Open Journal of Safety Science and Technology, 7, 129-160.

Plodinec, M. (2009): Definitions of Resilience: An Analysis, Community \& Regional Resilience Institute. 
Plöger, J. / Lang, T. (2016): Die Resilienz industriell geprägter Städte: Anpassung an Strukturkrisen in Bremen und Leipzig. In: Wink, R. (Hrsg.): Multidisziplinäre Perspektiven der Resilienzforschung (Studien zur Resilienzforschung). Wiesbaden: Springer, 357-371.

Popper, K. (1973): Objektive Erkenntnis: ein evolutionärer Entwurf. Hamburg: Hoffmann und Campe.

Prior, T. (2014): Measuring Critical Infrastructure Resilience: Possible Indicators. Risk and Resilience Report 9, Zürich: Center for Security Studies, ETH Zürich.

Prior, T. / Roth, F. (2013): Disaster, Resilience and Security in Global Cities. In: Journal of Strategic Security. 6:2, 59-69.

Pursiainen, C. (2009): The Challenges for European Critical Infrastructure Protection. In: European Integration, 31:6, 721-739.

Rahimi, M. / Madni, A. (2014): Toward a Resilience Framework for Sustainable Engineered Systems. In: Procedia Computer Science, 28, 809-817.

Reed, D. / Kapur, K. / Christie, R. (2009): Methodology for assessing the resilience of networked infrastructure. In: IEEE Systems Journal, 3:2, 174-180.

Renn, O. (Hrsg.) (2017): Das Energiesystem resilient gestalten: Szenarien - Handlungsspielräume - Zielkonflikte (Schriftenreihe Energiesysteme der Zukunft), München.

Renn, O. (2016): Systemic Risks: The New Kid on the Block. In: Environment Science and Policy for Sustainable Development, 58:2, 26-36.

Renn, O. (2008): Concepts of Risk: An Interdisciplinary Review. Part 1: Disciplinary Risk Concepts. In: GAiA, 17:1, 50-66.

Renn, O. (2008b): Concepts of Risk: An Interdisciplinary Review. Part 2: Integrative Approaches. In: GAiA, 17:2, 196-204.

Renn, O. / Benighaus, C. / Klinke, A. (o.J.): Die Bedeutung anthropogener Eingriffe in natürliche Prozesse: die Wechselwirkungen zwischen Naturgefahren und Risiken.

Riescher, G. (2013): Resilienz: Demokratietheoretische Überlegungen zu einem neuen Sicherheitskonzept. In: Heckmann, D. / Schenke, R. / Sydow, G. (Hrsg.): Verfassungsstaatlichkeit im Wandel. Berlin: Duncker \& Humblot, 1067-1077.

Riescher, G. (2012): Policy-making für demokratische Sicherheit (statement). In: Gander, H.-H. / Perron, W. / Poscher, R. / Riescher, G. / Würtenberger, T. (Hrsg.): Resilienz in der offenen Gesellschaft. Symposium des Centre for Security and Society. Reihe: Sicherheit und Gesellschaft. Freiburger Studien des Centre for Security and Society, Bd. 1. Baden-Baden: Nomos, 95-99.

Riescher, G. (Hrsg.) (2010): Sicherheit und Freiheit statt Terror und Angst. Perspektiven einer demokratischen Sicherheit. Baden-Baden: Nomos.

Riescher, G. (2010b): Demokratische Freiheit und die Sicherheit des Leviathan. In: Riescher, G. (Hrsg.): Sicherheit und Freiheit statt Terror und Angst. Perspektiven einer demokratischen Sicherheit. Baden-Baden: Nomos, 11-24.

Righi, A. / Saurin, T. / Wachs, P. (2015): A systematic literature review of resilience engineering: research areas and a research agenda proposal. In: Reliability Engineering and System Safety, 141, 142-152. 
Rijpma, J. (1997): Complexity, Tight-Coupling and Reliability: Connecting Normal Accidents Theory and High Reliability Theory. In: Journal of Contingencies and Crisis Management, 5: 1, 15-23.

Robbins, J. / Krishnan, K. / Allspaw, J. / Limoncelli, T. (2012): Resilience Engineering. Learning to embrace failure. GameDay Exercise Case Study. In: Quality Assurance, 10:9.

Rogers, C. / Bouch, C. / Williams, S. / Barber, A. / Baker, C. / Bryson, J. / Chapman, D. / Chapman, L. / Coaffee, J. / Jefferson, I. / Quinn, A. (2012): Resistance and resilience - paradigms for critical local infrastructure. In: Municipal Engineer, 165: ME2, 73-84.

Runco, M. / Jaeger, G. (2012): The Standard Definition of Creativity. In: Creativity Research Journal, 24:1, 92-96.

S.C. Res. 1566 (2004). (http://www.un.org/depts/german/sr/sr_04-05/sr1566.pdf).

Sansavini, G. (2016): Engineering resilience in critical infrastructure. In: IRGC resource guide on resilience. EPFL International Risk Governance Center (IRGC), Lausanne.

Schaffer, A. / Schneider, M. (2019): Towards a responsible resilience. In: Ruth, M. / Goessling-Reisemann, S. (Hrsg.): Handbook on resilience of socio-technical systems. Cheltenham: Edward Elgar Publishing Limited, 9-29.

Scharte, B. / Hiller, D. / Leismann, T. / Thoma, K. (2014): Einleitung. In: Thoma, Klaus (Hrsg.): Resilien-Tech. Resilience by Design: Strategie für die technologischen Zukunftsthemen (acatech STUDIE). München: Herbert Utz Verlag, 9-18.

Scharte, B. / Hiller, D. / Leismann, T. / Thoma, K. (2014b): Resilienz. Internationale Perspektiven. In: Thoma, Klaus (Hrsg.): Resilien-Tech. Resilience by Design: Strategie für die technologischen Zukunftsthemen (acatech STUDIE). München: Herbert Utz Verlag, 53-95.

Scharte, B. / Hiller, D. / Leismann, T. / Thoma, K. (2014c): Fazit. In: Thoma, Klaus (Hrsg.): Resilien-Tech. Resilience by Design: Strategie für die technologischen Zukunftsthemen (acatech STUDIE). München: Herbert Utz Verlag, 121-130.

Scharte, B. / Thoma, K. (2016): Resilienz - Ingenieurwissenschaftliche Perspektive. In: Wink, R. (Hrsg.): Multidisziplinäre Perspektiven der Resilienzforschung (Studien zur Resilienzforschung). Wiesbaden: Springer, 123-150.

Schulman, P. / Roe, E. (2007): Designing Infrastructures: Dilemmas of Design and the Reliability of Critical Infrastructures. In: Journal of Contingencies and Crisis Management, 15:1, 42-49.

Schulman, P. / Roe, E. / Eeten, M. / de Bruijne, M. (2004): High Reliability and the Management of Critical Infrastructures. In: Journal of Contingencies and Crisis Management, 12:1, 14-28.

Schweer, M. (Hrsg.) (1997): Interpersonales Vertrauen. Theorie und empirische Befunde. Opladen / Wiesbaden: Westdeutscher Verlag.

Shirali, G. / Mohammadfam, I. / Ebrahimipour, V. (2013): A new method for quantitative assessment of resilience engineering by PCA and NT approach: A case study in a process industry. In: Reliability Engineering and System Safety, $119,88-94$. 
Sikula, N. / Mancillas, J. / Linkov, I. / McDonagh, J. (2015): Risk management is not enough: a conceptual model for resilience and adaptation-based vulnerability assessments. In: Environment Systems and Decisions, 35:2, 219-228.

Smith, J. (2002): A Technical Report on Complex Systems. Department of Computing Science. Villanova University. Villanova, PA.

Smith, P. / Hutchison, D. / Sterbenz, J. / Schöller, M. / Fessi, A. / Karaliopoulos, M. / Lac, C. / Plattner, B. (2011): Network Resilience: A Systematic Approach. In: IEEE Communications Magazine, 88-97.

Steen, R. / Aven, T. (2011): A risk perspective suitable for resilience engineering. In: Safety Science, 49, 292-297.

Sterbenz, J. / Hutchison, D. / Cetinkaya, E. / Jabbar, A. / Rohrer, J. / Schöller, M. / Smith, P. (2010): Resilience and survivability in communication networks: Strategies, principles, and survey of disciplines. In: Computer Networks, 54, 1245-1265.

Strambach, S. / Klement, B. (2016): Resilienz aus wirtschaftsgeographischer Perspektive: Impulse eines «neuen» Konzepts. In: Wink, R. (Hrsg.): Multidisziplinäre Perspektiven der Resilienzforschung (Studien zur Resilienzforschung). Wiesbaden: Springer, 263-293.

Sundström, G. / Hollnagel, E. (2006): Learning how to create Resilience in Business Systems. In: Hollnagel, E. / Woods, D. / Leveson N. (Hrsg.): Resilience Engineering. Concepts and Precepts. Hampshire: Ashgate Publishing Limited, 235-252.

Taleb, N. (2007): The Black Swan: The Impact of the Highly Improbable. New York City, NY: Random House and Penguin.

Tamvakis, P. / Xenidis, Y. (2013): Comparative evaluation of resilience quantification methods for infrastructure systems. In: Procedia - Social and Behavioral Sciences, 74, 339-348.

Tamvakis, P. / Xenidis, Y. (2012): Resilience in transportation systems. In: Procedia - Social and Behavioral Sciences, 48, 3441-3450.

Teodorescu, H. (2015): Defining resilience using probabilistic event trees. In: Environment Systems and Decisions, 35:2, 279-290.

The National Academies (2012): Disaster Resilience. A National Imperative, Washington, D.C.

Thoma, K. (Hrsg.) (2014): Resilien-Tech. Resilience by Design: Strategie für die technologischen Zukunftsthemen (acatech STUDIE). München: Herbert Utz Verlag.

Thoma, K. / Drees, B. / Leismann, T. (2012): Zukunftstechnologien in der Sicherheitsforschung. In: Winzer, P. / Schnieder, E. / Bach, F.-W. (Hrsg.): Sicherheitsforschung. Chancen und Perspektiven (acatech DISKUTIERT). Berlin: Springer, 13-37. 
Thoma, K. / Leismann, T. / Hiller, D. (2012b): The Concept of Resilience in the Context of Technical Sciences. In: Gander, H.-H. / Perron, W. / Poscher, R. I Riescher, G. / Würtenberger, T. (Hrsg.): Resilienz in der offenen Gesellschaft. Symposium des Centre for Security and Society. Reihe: Sicherheit und Gesellschaft. Freiburger Studien des Centre for Security and Society, Bd. 1. BadenBaden: Nomos, 321-340.

Thoma, K. / Scharte, B. (2015): Building a resilient society. In: per Concordiam, 6:1, 30-35.

Thoma, K. / Scharte, B. / Hiller, D. / Leismann, T. (2016): Resilience Engineering as Part of Security Research: Definitions, Concepts and Science Approaches. In: European Journal for Security Research, 1:1, 3-19.

Tierney, K. / Bruneau, M. (2007): Conceptualizing and Measuring Resilience. A Key to Disaster Loss Reduction. In: TR News, 250: May-June, 14-18.

Tilly, C. (2004): Terror, Terrorism, Terrorists. In: Sociological Theory, 22: 1, 5-14.

Tobin, G. (1999): Sustainability and community resilience: the holy grail of hazards planning? In: Environmental Hazards, 1, 13-25.

Ulbig, E. / Hertel, R. / Böl, G.-F. (Hrsg.) (2009): Evaluierung der Kommunikation über die Unterschiede zwischen „risk“ und „hazard.“ Abschlussbericht. Bundesinstitut für Risikobewertung.

Ungericht, B. / Wiesner, M. (2011): Resilienz: Zur Widerstandskraft von Individuen und Organisationen. In: Zeitschrift Führung und Organisation, 03, 188-194.

Vogt, M. (2015): Zauberwort Resilienz. Einführung zur Tagung „Zauberwort Resilienz. Was stärkt in Zeiten des radikalen Wandels?", 27. Februar - 1. März, 2015, Tutzing, 1-20.

von Freyberg, T. (2011): Resilienz - mehr als ein problematisches Modewort? In: Zander, M. (Hrsg.): Handbuch Resilienzförderung. Wiesbaden: VS Verlag für Sozialwissenschaften, 219-239.

von Gleich, A. / Giese, B. (2019): Resilient systems as biomimetic guiding concept. In: Ruth, M. / Goessling-Reisemann, S. (Hrsg.): Handbook on resilience of socio-technical systems. Cheltenham: Edward Elgar Publishing Limited, 89-98.

Voss, M. / Dittmer, C. (2016): Resilienz aus katastrophensoziologischer Perspektive. In: Wink, R. (Hrsg.): Multidisziplinäre Perspektiven der Resilienzforschung (Studien zur Resilienzforschung). Wiesbaden: Springer, 179-197.

Wachsmuth, J. (2014): Vulnerabilität und Resilienz als Konzepte zum Umgang mit irreduziblen Unsicherheiten bei der Energiewende. Thesenpapier 1. RESYSTRA. Auf dem Weg zu resilienten Energiesystemen. Artec Forschungsforum Nachhaltigkeit, Bremen.

Wadenpohl, S. (2016): Resilienz - An der Schnittstelle von Public Health und Gerontologie. In: Wink, R. (Hrsg.): Multidisziplinäre Perspektiven der Resilienzforschung (Studien zur Resilienzforschung). Wiesbaden: Springer, 73-99.

Waidner, M. (2018): Safety und Security. Cybersicherheit als Basis erfolgreicher Digitalisierung. In: Neugebauer, R. (Hrsg.): Digitalisierung. Schlüsseltechnologien für Wirtschaft und Gesellschaft. Fraunhofer-Forschungsfokus. München: Springer-Vieweg, 275-293. 
Waldmann, P. (2003): Terrorismus und Bürgerkrieg. Der Staat in Bedrängnis. München: Gerling Akademie.

Walker, J. / Cooper, M. (2011): Genealogies of Resilience. From Systems Ecology to the Political Economy of Crisis Adaptation. In: Security Dialogue 42: 2, 143-160.

Walker, B. / Holling, C. / Carpenter, S. / Kinzig, A. (2004): Resilience, adaptability and transformability in social-ecological systems. In: Ecology and Society 9:2.

Werner, E. (1971): The children of Kauai: A longitudinal study from prenatal period to age ten. Honolulu: University of Hawaii Press.

Werner, E. / Smith, R. (2001): Journeys from childhood to midlife. Risk, resilience and recovery. New York: Cornell University.

Werner, E. / Smith, R. (1982): Vulnerable but invincible: A study of resilient children. New York: McGraw-Hill.

Westrum, R. (2006): A tyopology of resilience situations. In: Hollnagel, E. / Woods, D. / Leveson N. (Hrsg.): Resilience Engineering. Concepts and Precepts. Hampshire: Ashgate Publishing Limited, 55-65.

Wiater, P. (2013): Sicherheitspolitik zwischen Staat und Markt. Der Schutz kritischer Infrastrukturen. Reihe: Sicherheit und Gesellschaft. Freiburger Studien des Centre for Security and Society, Bd. 6. Baden-Baden: Nomos.

Wiens, M. (Hrsg.) (2013): Vertrauen in der ökonomischen Theorie. Eine mikrofundierte und verhaltensbezogene Analyse. Münster: LIT-Verlag.

Wildavsky, A. (1988): Searching for Safety. (Studies in social philosophy \& policy, no. 10). Piscataway: Transaction Publishers.

Windle, G. (2011): What is resilience? A review and concept analysis. In: Reviews in Clinical Gerontology. 21:2, 152-169.

Wink, R. (2016): Resilienzperspektive als wissenschaftliche Chance. Eine Einstimmung zu diesem Sammelband. In: Wink, R. (Hrsg.): Multidisziplinäre Perspektiven der Resilienzforschung (Studien zur Resilienzforschung). Wiesbaden: Springer, 1-11.

Wink, R. (2011): Evolution regionaler Resilienz: theoretischer Rahmen und Messkonzepte. In: Dreger, C. / Kosfeld, R. / Türck, M. (Hrsg.): Empirische Regionalforschung heute. Festschrift für Professor Hans-Friedrich Eckey. Wiesbaden: Gabler Verlag, 111-124.

Wissenschaftlicher Programmausschuss für zivile Sicherheitsforschung (WPA) (2017): Empfehlungen für die dritte Förderperiode der zivilen Sicherheitsforschung des BMBF.

Woods, D. (2019): Essentials of resilience, revisited. In: Ruth, M. / Goessling-Reisemann, S. (Hrsg.): Handbook on resilience of socio-technical systems. Cheltenham: Edward Elgar Publishing Limited, 52-65.

Woods, D. (2018): Resilience is a verb. In: IRGC resource guide on resilience (vol. 2): Domains of resilience for complex interconnected systems. EPFL International Risk Governance Center (IRGC), Lausanne.

Woods, D. (2015): Four concepts for resilience and the implications for the future of resilience engineering. In: Reliability Engineering and System Safety 141, 5-9. 
Woods, D. (2006): Essential characteristics of resilience. In: Hollnagel, E. / Woods, D. / Leveson N. (Hrsg.): Resilience Engineering. Concepts and Precepts. Hampshire: Ashgate Publishing Limited, 21-34.

Woods, D. (2006b): How to Design a Safety Organization: Test Case for Resilience Engineering. In: Hollnagel, E. / Woods, D. / Leveson N. (Hrsg.): Resilience Engineering. Concepts and Precepts. Hampshire: Ashgate Publishing Limited, 315-325.

Woods, D. (2005): Creating Foresight: Lessons for enhancing resilience from Columbia. In: Starbuck, W. / Farjoun, M. (Hrsg.): Organization at the limit. Lessons from the Columbia disaster. Oxford: Wiley-Blackwell, 289-308.

Woods, D. / Hollnagel, E. (2006): Prologue: Resilience Engineering Concepts. In: Hollnagel, E. / Woods, D. / Leveson N. (Hrsg.): Resilience Engineering. Concepts and Precepts. Hampshire: Ashgate Publishing Limited, 1-6.

Wreathall, J. (2006): Properties of Resilient Organizations: An Initial View. In: Hollnagel, E. / Woods, D. / Leveson N. (Hrsg.): Resilience Engineering. Concepts and Precepts. Hampshire: Ashgate Publishing Limited, 275-285.

Zander, M. / Roemer, M. (2016): Resilienz im Kontext von Sozialer Arbeit: Das Geheimnis der menschlichen Seele lüften? In: Wink, R. (Hrsg.): Multidisziplinäre Perspektiven der Resilienzforschung (Studien zur Resilienzforschung). Wiesbaden: Springer, 47-71.

Zinke, T. / Ummenhofer, T. (2014): Nachhaltigkeit und Resilienz - Zukünftige Kriterien bei der integrativen Bewertung von Brücken. In: Stahlbau, 83:2, 74-82.

Zio, E. (2007): From Complexity Science to Reliability Efficiency: A New Way of Looking at Complex Network Systems and Critical Infrastructures. In: International Journal of Critical Infrastructures, 3:3/4, 448-508. 
